\title{
Pneumoviruses and Metapneumoviruses
}

\author{
Edited by \\ Helene F. Rosenberg \& Joseph B. Domachowske
}

Printed Edition of the Special Issue Published in Viruses

MDPI www.mdpi.com/journal/viruses 
Helene F. Rosenberg and Joseph B. Domachowske (Eds.)

\section{Pneumoviruses and Metapneumoviruses}


This book is a reprint of the special issue that appeared in the online open access journal Viruses (ISSN 1999-4915) in 2013 (available at:

http://www.mdpi.com/journal/viruses/special_issues/pneumoviruses_metapneumoviruses).

Guest Editors

Helene F. Rosenberg

National Institute of Allergy and Infectious Diseases (NIAID)

Bethesda, MD, USA

Joseph B. Domachowske

SUNY Upstate Medical University

Syracuse, NY, USA

Editorial Office

MDPI AG

Klybeckstrasse 64

Basel, Switzerland

Publisher

Shu-Kun Lin

Production Editor

Matthias Burkhalter

\section{Edition 2013}

MDPI • Basel • Beijing

ISBN 978-3-03842-049-1

(C) 2013 by the authors; licensee MDPI, Basel, Switzerland. All articles in this volume are Open Access distributed under the Creative Commons Attribution 3.0 license (http://creativecommons.org/licenses/by/3.0/), which allows users to download, copy and build upon published articles even for commercial purposes, as long as the author and publisher are properly credited, which ensures maximum dissemination and a wider impact of our publications. However, the dissemination and distribution of copies of this book as a whole is restricted to MDPI, Basel, Switzerland. 


\section{Table of Contents}

Preface vii

\section{General Review}

Lenneke E. M. Haas, Steven F. T. Thijsen, Leontine van Elden and Karen A. Heemstra Human Metapneumovirus in Adults. 1

Reprinted from Viruses 2013, 5(1), 87-110; doi:10.3390/v501008

http://www.mdpi.com/1999-4915/5/1/87

\section{Experimental Models and Methods}

\section{Rachel J. Derscheid and Mark R. Ackermann}

Perinatal Lamb Model of Respiratory Syncytial Virus (RSV) Infection 26

Reprinted from Viruses 2012, 4(10), 2359-2378; doi:10.3390/v4102359

http://www.mdpi.com/1999-4915/4/10/2359

\section{Randy E. Sacco, Jodi L. McGill, Mitchell V. Palmer, John D. Lippolis, Timothy A.}

Reinhardt and Brian J. Nonnecke

Neonatal Calf Infection with Respiratory Syncytial Virus: Drawing Parallels to the Disease in

Human Infants 47

Reprinted from Viruses 2012, 4(12), 3731-3753; doi:10.3390/v4123731

http://www.mdpi.com/1999-4915/4/12/3731

\section{Kimberly D. Dyer, Katia E. Garcia-Crespo, Stephanie Glineur, Joseph B. Domachowske} and Helene F. Rosenberg

The Pneumonia Virus of Mice (PVM) Model of Acute Respiratory Infection 70

Reprinted from Viruses 2012, 4(12), 3494-3510; doi:10.3390/v4123494

http://www.mdpi.com/1999-4915/4/12/3494

\section{Sumana Fathima, Bonita E. Lee, Jennifer May-Hadford, Shamir Mukhi and Steven J.}

Drews

Use of an Innovative Web-Based Laboratory Surveillance Platform to Analyze Mixed

Infections Between Human Metapneumovirus (hMPV) and Other Respiratory Viruses

Circulating in Alberta (AB), Canada (2009-2012). 88

Reprinted from Viruses 2012, 4(11), 2754-2765; doi:10.3390/v4112754

http://www.mdpi.com/1999-4915/4/11/2754 


\section{Epidemiology and Virus Evolution}

Luiz Gustavo Araujo Gardinassi, Paulo Vitor Marques Simas, Deriane Elias Gomes, Caroline Measso do Bonfim, Felipe Cavassan Nogueira, Gustavo Rocha Garcia, Claudia Márcia Aparecida Carareto, Paula Rahal and Fátima Pereira de Souza

Diversity and Adaptation of Human Respiratory Syncytial Virus Genotypes Circulating in Two Distinct Communities: Public Hospital and Day Care Center 100

Reprinted from Viruses 2012, 4(11), 2432-2447; doi:10.3390/v4112432

http://www.mdpi.com/1999-4915/4/11/2432

\section{Rosa Elena Sarmiento-Silva, Yuko Nakamura-Lopez and Gilberto Vaughan}

Epidemiology, Molecular Epidemiology and Evolution of Bovine Respiratory Syncytial Virus

Reprinted from Viruses 2012, 4(12), 3452-3467; doi:10.3390/v4123452

http://www.mdpi.com/1999-4915/4/12/3452

Penelope Adamson, Sutthiwan Thammawat, Gamaliel Muchondo, Tania Sadlon and

David Gordon

Diversity in Glycosaminoglycan Binding Amongst hMPV G Protein Lineages 134

Reprinted from Viruses 2012, 4(12), 3785-3803; doi:10.3390/v4123785

http://www.mdpi.com/1999-4915/4/12/3785

\section{Viral Pathogenesis and Cellular Responses}

Peter Mastrangelo and Richard G. Hegele

RSV Fusion: Time for a New Model 154

Reprinted from Viruses 2013, 5(3), 873-885; doi:10.3390/v5030873

http://www.mdpi.com/1999-4915/5/3/873

\section{Evelyn Rivera-Toledo and Beatríz Gómez}

Respiratory Syncytial Virus Persistence in Macrophages Alters the Profile of Cellular Gene

Expression 167

Reprinted from Viruses 2012, 4(12), 3270-3280; doi:10.3390/v4123270

http://www.mdpi.com/1999-4915/4/12/3270

Jonas Bystrom, Nasra Al-Adhoubi, Mohammed Al-Bogami, Ali S. Jawad and Rizgar A.

Mageed

Th17 Lymphocytes in Respiratory Syncytial Virus Infection. 178

Reprinted from Viruses 2013, 5(3), 777-791; doi:10.3390/v5030777

http://www.mdpi.com/1999-4915/5/3/777 
Deepthi Kolli, Xiaoyong Bao and Antonella Casola

Human Metapneumovirus Antagonism of Innate Immune Responses 193

Reprinted from Viruses 2012, 4(12), 3551-3571; doi:10.3390/v4123551

http://www.mdpi.com/1999-4915/4/12/3551

\section{Reagan G. Cox and John V. Williams}

Breaking In: Human Metapneumovirus Fusion and Entry....

Reprinted from Viruses 2013, 5(1), 192-210; doi:10.3390/v5010192

http://www.mdpi.com/1999-4915/5/1/192

Ellen R. T. Watkiss, Pratima Shrivastava, Natasa Arsic, Susantha Gomis and Sylvia van Drunen Littel-van den Hurk

Innate and Adaptive Immune Response to Pneumonia Virus of Mice in a Resistant and a Susceptible Mouse Strain 235

Reprinted from Viruses 2013, 5(1), 295-320; doi:10.3390/v5010295

http://www.mdpi.com/1999-4915/5/1/295

Elske van den Berg, Job B.M. van Woensel and Reinout A. Bem

Apoptosis in Pneumovirus Infection 261

Reprinted from Viruses 2013, 5(1), 406-422; doi:10.3390/v5010406

http://www.mdpi.com/1999-4915/5/1/406

\section{Antonieta Guerrero-Plata}

Dendritic Cells in Human Pneumovirus and Metapneumovirus Infections 278

Reprinted from Viruses 2013, 5(6), 1553-1570; doi:10.3390/v5061553

http://www.mdpi.com/1999-4915/5/6/1553

\section{Vaccine Development}

Rajeev Rudraraju, Bart G. Jones, Robert Sealy, Sherri L. Surman and Julia L. Hurwitz Respiratory Syncytial Virus: Current Progress in Vaccine Development. 297

Reprinted from Viruses 2013, 5(2), 577-594; doi:10.3390/v5020577

http://www.mdpi.com/1999-4915/5/2/577 


\section{Preface}

This volume of Viruses includes seventeen independent contributions that focus on the biology of pneumovirus and metapneumovirus respiratory virus pathogens. As a group, these are all pathogens of the family Paramyxoviridae, which are enveloped viruses with non-segmented single stranded RNA genomes that cause acute infections of the upper and lower respiratory tract. Among the pneumovirus pathogens, first and foremost is the human respiratory syncytial virus (HRSV; genus, pneumovirus). HRSV is the leading cause of lower respiratory tract infection in infants and children, and is an emerging pathogen of the elderly (reviewed in [1]). Current management for HRSV disease includes prophylaxis with humanized monoclonal antibody, a regimen available to infants identified as highrisk only (i.e. those born prematurely, with bronchopulmonary dysplasia, immunodeficiency or congenital heart disease). At this time, there are no vaccines available to prevent HRSV infection. Two contributions to this volume describe interesting and relatively novel experimental models, including a perinatal lamb model (Dersheid and Ackermann) and neonatal calf model of HRSV infection (Nonnecke and colleagues), both drawing important parallels to infection in human infants. In another contribution, Pereira de Souza and colleagues provide an update on circulating HRSV (G-gene based) genotypes in São José do Rio Preto, Brazil. Also included are several studies focused on HRSV basic biology and pathogenesis including receptor-based fusion (Mastrangelo and Hegele), gene expression in macrophages (Rivera-Toledo and Gomez) and interactions with Th17 lymphocytes (Mageed and colleagues). Finally, Hurwitz and colleagues have contributed a crucial update on current progress towards development of a safe and effective vaccine for prevention of this HRSV infection.

Bovine RSV (BRSV) is a pathogen of considerable veterinary importance. Similar to the clinical impact of HRSV, BRSV infects cattle worldwide, with the disease being most prevalent in calves less than six months of age (reviewed in [2]). One interesting distinction between these pathogens, BRSV infection results in a pronounced predisposition to secondary bacterial infections with a variety of pathogens, including $M$. haemolytica or $M$. bovis, a condition known as bovine respiratory disease complex [3]. In their contribution, Vaughan and colleagues provide an updated review of the epidemiology and evolution of BRSV, and discuss the impact of these findings on development of novel vaccine formulations. Likewise, several contributions address the biology of Pneumonia Virus of Mice (PVM), a mouse pneumovirus pathogen first discovered serendipitously by Horsfall and Curnen in 1946. PVM has more recently been featured as an important model severe HRSV infection in inbred mouse strains [4]. Dyer and colleagues have presented an updated review PVM as a model of severe RSV in vivo; Bem and colleagues include findings on PVM infection in their contribution featuring pneumoviruses and apoptosis, and van Drunen Littel-van den Hurk and colleagues discuss strain-dependent differences in cytokine production and cell recruitment in response to acute PVM infection. 
Human metapneumovirus (hMPV; Genus Metapneumovirus) was discovered by van den Hoogen and colleagues [5] as a result of an evaluation of samples from otherwise undiagnosed patients with symptoms of acute viral respiratory tract infections. HMPV results in disease that is similar to HRSV, characterized by mild upper respiratory tract symptoms that can progress to more severe bronchiolitis and pneumonia. As with HRSV, there are no specific therapies available for treatment, nor is there a vaccine available for prevention of HMPV infection. This volume features a full review of HMPV disease in adults by Heemstra and colleagues, and a presentation of a novel surveillance method designed to analyze HMPV in the setting of mixed virus infection (Drews and colleagues). Other contributions include an epidemiologic analysis of the HMPV G protein lineages (Gordon and colleagues), the role of HMPV F protein in mediating cell fusion (Cox and William), and reviews of interactions of HMPV with Type I Interferons (Casola and colleagues) and lung dendritic cells (Guerrero-Plata).

We appreciate the time and effort put forth by the authors of these manuscripts, by those who reviewed the manuscripts prior to initial publication in the journal Viruses, and by the editorial staff at MDPI who made this volume possible.

Helene F. Rosenberg and Joseph B. Domachowske

Guest Editors

\section{References}

1. Rosenberg, H.F.; Domachowske, J.B. Inflammatory responses to respiratory syncytial virus (RSV) infection and the development of immunomodulatory pharmacotherapeutics. Curr. Med. Chem. 2012, 19, 1424-1431.

2. Sacco, R.E.; McGill, J.L,; Pillatzki, A.E.; Palmer, M.V.; Ackermann, M.R. Respiratory syncytial virus infection in cattle. Vet. Pathol. 2013, in press.

3. Larson, R.L.; Step, D.L. Evidence-based effectiveness of vaccination against Mannheimia haemolytica, Pasteurella multocida, and Histophilus somni in feedlot cattle for mitigating the incidence and effect of bovine respiratory disease complex. Vet. Clin. North Am. Food Anim. Pract. 2012, 28, 97 - 106.

4. Rosenberg, H.F.; Domachowske, J.B. Pneumonia virus of mice. Severe respiratory virus infection in a natural host. Immunol. Lett. 2008, 118, 6 - 12.

5. van den Hoogen, B.G.; de Jong, J.C.; Groen, J.; Kuiken, T.; de Groot, R.; Fouchier, R.A.M.; Osterhaus, A.D.M.E. A newly discovered human pneumovirus isolated from young children with respiratory disease. Nature Medicine 2001, 7, 719 - 724.

\footnotetext{
* Acknowledgments: Work in the Inflammation Immunobiology Section (Rosenberg) is funded by NIAID Division of Intramural Research.
} 
Reprinted from Viruses. Cite as: Haas, L.E.M.; Thijsen, S.F.T.; van Elden, L.; Heemstra, K.A. Human Metapneumovirus in Adults. Viruses 2013, 5, 87-110.

Review

\section{Human Metapneumovirus in Adults}

Lenneke E. M. Haas ${ }^{1, *}$, Steven F. T. Thijsen ${ }^{2}$, Leontine van Elden ${ }^{3}$ and Karen A. Heemstra ${ }^{2}$

1 Department of Intensive Care Medicine, Diakonessenhuis, Utrecht, $3582 \mathrm{KE}$, The Netherlands

2 Department of Microbiology, Diakonessenhuis, Utrecht, 3582 KE, The Netherlands;

E-Mails: sthijsen@diakhuis.nl (S.F.T.T.); K.A.Heemstra@umcutrecht.nl (K.A.H.)

3 Department of Pulmonary Diseases, Diakonessenhuis, Utrecht, 3582 KE, The Netherlands;

E-Mail: 1velden@diakhuis.nl

* Author to whom correspondence should be addressed; E-Mail: 1vlelyveld@diakhuis.nl; Tel.: +31-88-250-5000.

Received: 30 November 2012; in revised form: 17 December 2012 / Accepted: 19 December 2012 /

Published: 8 January 2013

Abstract: Human metapneumovirus (HMPV) is a relative newly described virus. It was first isolated in 2001 and currently appears to be one of the most significant and common human viral infections. Retrospective serologic studies demonstrated the presence of HMPV antibodies in humans more than 50 years earlier. Although the virus was primarily known as causative agent of respiratory tract infections in children, HMPV is an important cause of respiratory infections in adults as well. Almost all children are infected by HMPV below the age of five; the repeated infections throughout life indicate transient immunity. HMPV infections usually are mild and self-limiting, but in the frail elderly and the immunocompromised patients, the clinical course can be complicated. Since culturing the virus is relatively difficult, diagnosis is mostly based on a nucleic acid amplification test, such as reverse transcriptase polymerase chain reaction. To date, no vaccine is available and treatment is supportive. However, ongoing research shows encouraging results. The aim of this paper is to review the current literature concerning HMPV infections in adults, and discuss recent development in treatment and vaccination.

Keywords: human metapneumovirus; HMPV; respiratory tract infection; adults; intensive care; diagnosis; treatment; vaccination 


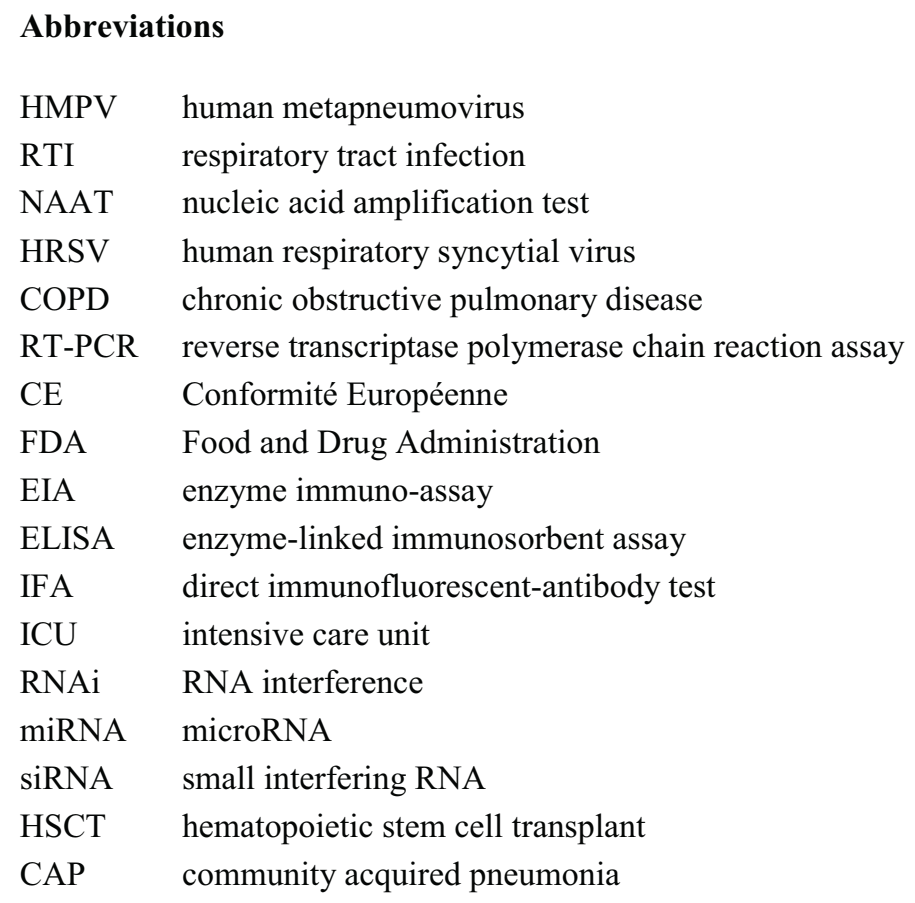

\section{Introduction}

The most common illness experienced by people of all ages worldwide is an acute respiratory tract infection (RTI). It is a leading cause of mortality and morbidity worldwide. Viruses are responsible for a large proportion of RTI's [1]. A significant portion of the infections with viral etiology can be attributed to the human metapneumovirus (HMPV), also in adults [2-9].

HMPV was first identified in the Netherlands in 2001, but serologic studies of antibodies against HMPV indicate that the virus is not new and circulated in humans for at least 50 years [4].

The aim of this paper is to review the current literature concerning HMPV infections in adults, and discuss recent development in treatment and vaccination.

\section{Virology}

HMPV is classified as the first human member of the Metapneumovirus genus in the Pneumovirinae subfamily within the Paramyxoviridae family. It is an enveloped negative-sense single-stranded RNA virus. The RNA genome includes 8 genes coding for 9 different proteins. HMPV is identical in gene order to the avian pneumovirus (AMPV), which also belongs to the Metapneumovirus genus [10].

Phylogenetic analysis has identified two genotypes of HMPV, namely A and B [4]. Both genotypes may co-circulate simultaneously, but during an epidemic, one genotype usually dominates $[11,12]$. Within each of these subgroups two clades are designated (designated A1, A2, B1 and B2 [12,13]. 
This classification is mainly based on the sequence variability of the attachment $(G)$ and fusion (F) surface glycoproteins [4]. The highly conserved $\mathrm{F}$ protein constitutes an antigenic determinant that mediates cross-lineage neutralization and protection [14]. In 2006, two further subgroups, A2a and $\mathrm{A} 2 \mathrm{~b}$, were described, but this further splitting was based on limited data and has not been confirmed by other groups [15]. In addition, no clinical significance of these subgroups has yet been shown.

\section{Pathogenesis and Susceptibility}

For extensive explanation about pathogenesis of HMPV and animal models, we refer to the review of Schildgen et al. [16]. The pathogenesis of HMPV infections in adults seems to be similar to that in children.

HMPV is associated with severe infection in patients with pulmonary disease and chronic obstructive pulmonary disease (COPD). Studies on HMPV in BALB/c in mice and cotton rats show airway obstruction and hyperresponsiveness after infection. Initially HMPV infection in the lung is characterized by interstitial inflammation with alveolitis starting on day 3 with a peak on day 5 and subsequently decreasing inflammation [17]. However, after 2-3 weeks this develops in a more prominent peribronchiolar and perivascular infiltrate. Hamelin et al. also show airway obstruction in BALB/c mice after a single HMPV challenge with a peak on day 5, but still present until day 70 [18]. In addition, significant hyperresponsiveness after methacholine challenge was also shown until day 70 , indicating long-term pulmonary inflammation after HMPV infection.

Darniot and colleagues demonstrated in a mice model that susceptibility to HMPV infection is age related with aged mice showing severer illness and mortality compared to young mice. Aged mice showed greater virus replication in the lung; however, viral clearance was not delayed. In addition, lower levels of virus specific antibodies, neutralizing antibodies and interferon gamma with a significant increase in IL4 and $\mathrm{CD}^{+}$lymphocytes were observed in aged mice after HMPV infection. This suggests an important role for the cellular immune response in controlling HMPV infection [19]. This hypothesis is partially confirmed by Ditt et al., who found that HMPV infection in aged mice results in a diminished TNF-alfa expression resulting in low levels of NF-Kb compared to young mice [20].

Lüsebrink et al. demonstrated that neutralizing antibodies seem to be present in all age groups in humans and that neutralizing capacities remain high, with a minor decrease for individuals over 69 years of age. Therefore, they hypothesized that the cellular response has a more important role in the clearing of HMPV infection than the neutralizing humoral immune response [21].

Sastre et al. used a recombinant fusion protein-based enzyme linked immunosorbent assay (F-ELISA) in the same set of sera. Their results support the hypothesis that it appears likely that neutralizing antibodies play a minor role in the control of HMPV infections in humans [22]. In addition, Falsey et al. found higher serum antibodies at baseline, a greater response in binding antibody and a trend towards greater neutralizing antibody responses in older adults compared to younger adults with HMPV illness of the same severity suggesting immune dysregulation in aged patients with an HMPV infection [23]. 
Overall, neutralizing antibodies seem to play a minor role in controlling HMPV infections. Cellular immune responses seem to be more important for the susceptibility of HMPV infections in aged patients.

\section{Epidemiology}

HMPV is distributed worldwide and has a seasonal distribution comparable to that of influenza viruses and RSV. It tends to strike in the late winter and early spring [11,24,25]. In young children, HMPV is the second most common cause of lower RTI after RSV, with children less than one year of age showing the highest rates of infection [26,27]. Seroprevalence at the age of 5 is almost $100 \%$ [4,25-36].

However, due to incompletely protective immune responses or infection with a new genotype reinfection occurs, especially in elderly and high risk patients [9,37]. Van den Hoogen et al. demonstrated that experimental HMPV infection induces transient protective immunity in cynomolgus macaques [38].

Walsh et al. found that the proportion of HMPV infections in adults varied between $3 \%-7.1 \%$ in four consecutive winters [9]. This is similar to the annual average infection rate for RSV (5.5\%) and greater than that of influenza A $(2.4 \%)$ in the same cohorts during the same time frame [39].

HMPV was identified in $2.2 \%$ of patients who visited a general practitioner because of community-acquired acute RTI who were negative for RSV and influenza virus [5].

HMPV infection is associated with hospitalization for acute RTI in adults in the study of Walsh et al. [9]. The incidence of HMPV infection in this hospitalized adults varied from year to year ranging from $4.3 \%-13.2 \%$. This is in accordance with the rates for RSV and influenza A. Average annual infection rates for RSV and influenza A were $9.6 \%$ and $10.5 \%$ in the same cohorts [39]. Two-third of these hospitalized patients had underlying disease. Twenty-tree percent of these patients had a co-infection with another respiratory virus.

Widmer et al. found that HMPV accounted for $4.5 \%$ of hospitalizations for acute RTI in adults older then 50 years during the winter season in 3 consecutive years [8]. Rates for RSV and influenza A were $6.1 \%$ and $6.5 \%$ respectively. Average annual rates of hospitalization for HMPV were 1.8/10,000 residents in adults from 50-65 years and 22.1/10,000 residents in adults $>65$ years. Patients with HMPV infections were older, had more cardiovascular disease and were more likely to be vaccinated with influenza vaccination compared to patients with influenza.

Boivin et al. found HMPV in 2.3\% of respiratory samples during the winter season 2000-2001 [29]. Of the $26 \mathrm{HMPV}$ infected hospitalized patient $35 \%$ was aged $<5$ years and $46 \%$ aged $>65$ years. One third of the hospitalized children aged $<5$ years, two-third of the patients aged 15-65 years and all patients aged $>65$ years had an underlying disease.

Data from our hospital suggest a comparable incidence in adult patients and pediatric patients. We analyzed all polymerase chain reaction (PCR) tests for respiratory viruses of the last 19 months in our hospital. A total of 283 adults were tested for HMPV because of symptoms of RTI and almost five percent of the patients (14 of 283 patients) tested positive for HMPV. 


\section{Transmission}

HMPV is thought to be transmitted by direct or close contact with contaminated secretions, which may involve saliva, droplets or large particle aerosols [40-42]. HMPV RNA is found in excretions five days to two weeks after initiation of symptoms [43]. However, the extent of contagiousness is unknown since detection of HMPV RNA in respiratory samples from patients recovering from infection does not per se indicate viable contagious viral particles.

Based on two single cases of nosocomial HMPV infections the incubation period of HMPV is estimated to be 4 to 6 days [44]. Another nosocomial HMPV infection study in a pediatric hemato-oncology ward found an estimated incubation period of 7-9 days. In a retrospective study HMPV transmission in households in Japan was studied. Of the 15 studied families all index-patients were children attending primary school, childcare or nursery homes. Contact cases developed symptoms with a median of five days (range 3-7 days) after the index case developed symptoms [40]. As this was a retrospective study including only symptomatic patients, no reliable exact number of transmissions in households could be determined. Two studies found HMPV carriage in $4.1 \%$ of asymptomatic adults, which suggests that asymptomatic adults might be a neglected source of HMPV transmission $[3,45]$. However, other studies found that the presence of HMPV RNA in excretions of asymptomatic persons is uncommon $[4,24,27,29,46]$.

\section{Clinical Manifestations}

In general, HMPV infection can not be distinguished from other respiratory viruses on clinical grounds only [5]. Adult patients with an HMPV infection might be asymptomatic or might have symptoms ranging from mild upper RTI symptoms to severe pneumonia [9]. Most patients present with cough, nasal congestion and dyspnoea. Purulent cough, wheezing, sore throat, fever, pneumonia, bronchi(oli)tis, conjunctivitis and otitis media are other reported symptoms [47]. Li et al. described a HMPV infection in an immunocompetent adult presenting as a mononucleosis-like illness [48]. Adults with HMPV infection were less likely to report fever in contrast to adults with RSV or influenza infection [8,49,50]. In addition, adults with an HMPV infection presented more often with wheezing compared to adults with RSV or influenza [9]. Falsey et al. showed that this is mainly in the elderly population ( $>65$ years) [49]. Elder patients also showed more dyspnoea compared to younger adults [49]. Young adults with HMPV infection had greater complaints of hoarseness [44]. In the frail elderly patients, the patients with pulmonary or cardiovascular disease and immunocompromised patients, infections can be severe [51-55].

Laboratory examination may show lymphopenia, neutropenia and elevated transaminases. Studies on imaging with chest X-ray and computed tomotography (CT) show initially signs of acute interstitial pneumonia (ground glass opacity and air-space consolidation) turning into signs of bronchiolitis/bronchitis (bronchial(ar) wall thickening or impaction) [56-58]. 
Compared to RSV and influenza, similar rates of intensive care unit (ICU) admission, mechanical ventilation, length of stay for hospitalization and length of stay in ICU were seen for HMPV infection in adults $[8,9]$.

\section{Diagnosis}

The diagnosis of HMPV infection can be made by several techniques, including culture, nucleic acid amplification tests (NAAT), antigen detection and serologic testing.

Virus culture is relatively difficult, because HMPV grows slowly in conventional cell culture and has mild cytopathic effects. The rapid culture technique is known as shell vial amplification [59].

Detection of viral RNA by NAAT such as reverse transcriptase-PCR (RT-PCR) assay is the most sensitive method for diagnosis of HMPV infection [53,60,61].

Methods for detection of HMPV antigens, such as enzyme immuno-assay (EIA) and enzyme-linked immunosorbent assay (ELISA) are not commonly used. No commercial immunochromatographic assays are available. A direct immunofluorescent-antibody (IFA) test - which is a rapid test in which labeled antibodies to detect specific viral antigens in direct patients materials are used - could be useful for the diagnosis of HMPV infections in outbreaks. The test results are known within two hours. However, the sensitivity of IFA is lower than that of RT-PCR and needs to be validated before use.

Detection of the immune response against the virus by serologic testing is only used for epidemiologic studies. One of the disadvantages of serology is the fact that the interval between virus spreading and detection of HMPV-specific IgM and IgG antibodies is relatively long. However, a combined approach of serology and RT-PCR has added diagnostic value in the diagnosis of HMPV infections in the case of investigating the magnitude of an outbreak for instance in long term care facilities $[43,61]$.

\section{Treatment and Prevention}

\subsection{Treatment}

To date, treatment of HMPV infection is mainly supportive. Several treatment regimes have been investigated. Most of these therapeutic options, like innovative approaches based on fusion inhibitors and on RNA interference, seemed effective in vitro and in animal studies.

Ribavirin is a nucleoside with broad spectrum inhibitory activity against a variety of RNA and DNA viruses, including HMPV. Ribavirin has demonstrated in vitro inhibition of tumor necrosis factor-alfa, interferon-gamma and interleukin (IL)-10, suggesting a down regulation in Th1 and Th2 cytokine production and an increase of IL-2 production by peripheral blood mononuclear cells $[43,62]$. Ribavirin may terminate T-cell immune-mediated damage caused by viral infections. It limits viral transcription and showed to have immunomodulatory effects [63]. The in vitro results are confirmed by an in vivo study in BALB/c mouse [64-69].

Immunoglobulins for therapeutical goals can be divided in specific and non specific. 
Palivizumab (Synagis ${ }^{\circledR}$ ) contains humanized monoclonal antibodies that can recognize a highly conserved neutralizing epitope on the fusion protein of RSV. It showed to have preventive effects in infants at high risk of severe hRSV infections; monthly palivizumab injections reduced RSV hospitalizations by $50 \%$ compared with placebo [70-73]. Motavizumab is another RSV specific monoclonal antibody preparation, which is developed after the success of palivizumab. It showed to be non-inferior to palivizumab for prevention of RSV hospitalization in high-risk children [74]. These data of effectiveness of humanized monoclonal Abs against RSV infection has prompted a similar approach for protection against HMPV.

MAb 338 is one of the antibodies that was developed to target the HMPV fusion protein. It appeared effective in animal models in which it neutralized the prototypic strains of the four subgroups of HMPV, significantly reduced the pulmonary viral titer, limited severe acute manifestations and limited bronchial hyper-reactivity. In mice, it appears to have both prophylactic and therapeutic benefits [75]. Hamelin et al. also showed that it could be useful after infection and not only as preventive measure [76].

Williams et al. tested a fully human monoclonal antibody fragment (Human Fab DS7) with biological activity against HMPV in vivo and in vitro and demonstrated a prophylactic and therapeutic potential against severe HMPV infection. When Fab DS7 was given intranasally to cotton rats, a $>1,500$-fold reduction in viral titer in the lungs and a modest 4 -fold reduction in the nasal tissues was found. A dose-response relationship between the dose of DS7 and virus titer was seen $[77,78]$.

Wyde et al. showed that standard immune globulin preparations (thus without selection for antibodies to a particular microorganism or its toxin), initially used as preventive measure against hRSV, also inhibit replication of HMPV in vitro [64].

The combination of oral and aerosolized ribavirin with polyclonal intravenous immune globulin (IVIG) seems an effective treatment for severe HMPV infections, but no randomized controlled trials in humans have been performed. Despite this lack of good trials in human, a lot of experience has been gained meanwhile in individual cases and small case series $[69,79,80]$.

Both ribavirin and IVIG are expensive and have disadvantages. Ribavirin is potential teratogen and administration by nebulization must be carried out via a small particle aerosol generator [81]. Therefore, in daily practice ribavirin nebulization is seldom used for HMPV infection. In addition, health care providers who are pregnant or attempting to become pregnant should avoid contact with patients receiving treatment with aerosolized ribavirin. Furthermore, IVIG requires large fluid volumes infusions, generates a high protein load and is associated with adverse side effects in children with congenital heart disease [82].

Fusion inhibitors target the first steps of the viral replication cycle. Deffrasnes and colleagues tested nine inhibitory peptides with sequences homology with the HRA en HRB domains of the HMPV fusion protein and demonstrated potent viral inhibitory activity in vitro of five of these peptides. One peptide, HRA2, displayed very potent activity against all four HMPV subgroups. BALB/c mice that received the HRA2 peptide and a lethal HMPV intranasal challenge simultaneously were completely protected from clinical symptoms and mortality [83]. The study of Miller and colleagues demonstrated 
that individual HR-1 peptides could lead to effective viral inhibition [84]. These peptides could be used in the prevention of severe infection in vulnerable patients after exposure, but the clinical role post-infection has to be investigated.

RNA interference (RNAi) is a recently discovered interesting approach for treatment of RNA virus infections. RNAi is a naturally occurring intracellular inhibitory process that regulates gene expression through the silencing of specific mRNAs. The small RNAs, the microRNA (miRNA) and small interfering RNA (siRNA), can down-regulate protein production by inhibiting targeted mRNA in a sequence-specific manner. RNAi therapeutics have been shown to be active in vitro and in vivo against respiratory syncytial virus, parainfluenza and influenza [85-87]. Deffrasnes et al. successfully identified two highly efficient siRNAs against HMPV in vitro, targeting essential components of the HMPV replication complex [88]. Very recently, Preston and colleagues designed and validate a siRNA molecule that is effective against the $\mathrm{G}$ gene of hMPV in vitro. Although, a significant reduction in $\mathrm{G}$ mRNA did not reduce viral growth in vitro or induce a significant type I interferon (IFN) response, hMPV G might still be a valid target for RNAi as G is required for viral replication in vivo [89].

Wyde et al. have also demonstrated that both the sulfated sialyl lipid (NMSO3) and heparin have antiviral activity against HMPV in vitro. NMSO3 acts most likely by inhibiting attachment and penetration of the virus and may inhibit cell-to-cell spread [90].

\subsection{Vaccination}

Several in vitro and animal studies have been performed investigating the development of an HMPV vaccine. However, no human studies have been performed yet and no vaccine is available up till now.

Results of studies performed in rodent and non-human primate models look promising, but very little research is performed in human volunteers. A variety of live attenuated, virus vectored, inactivated virus and subunit vaccines have been tested in animal models and showed to have immunogenicity and protective efficacy [91].

HMPV expresses the major surface glycoproteins $F$ and $G$. Two main genetic virus lineages exist worldwide which have a similar highly conserved F (fusion) protein. Immunization strategies have been targeted against these surface proteins. Immunization with monoclonal antibodies against the $\mathrm{F}$ protein shows a prophylactic effect [77,78,92,93]. Several animal studies investigating the immunization with a chimeric virus vector using a bovine parainfluenza virus 3 expressing the HMPV $\mathrm{F}$ protein, adjuvanted soluble $\mathrm{F}$ protein or $\mathrm{F}$ protein DNA show protective immunity after a HMPV challenge [92-96].

Immunization with the HMPV attachment $(\mathrm{G})$ glycoproteins did not show any production of antibodies or protection [97]. Ryder et al. also demonstrated that HMPV G is not a protective antigen. They evaluated the protective efficacy of immunization with a recombinant form of $\mathrm{G}$ ectodomain (GDeltaTM) in cotton rats. Although immunized animals developed high levels of serum antibodies to both recombinant and native $\mathrm{G}$ protein, they did not develop neutralizing antibodies and were not protected against virus challenge [98]. 
Studies investigating immunization with inactivated HMPV show an enhanced immune response with even lethal outcome following HMPV infection in animals [99-101]. Use of live-attenuated viruses generated by reverse genetics or recombinant proteins, tested in animals, showed encouraging results. Live vaccines are mimicking natural infection; however natural infection does only lead to transient protective immunity [38]. This makes an extra challenge for vaccine development.

The primary strategy is to develop a live-attenuated virus for intranasal immunization. Reverse genetics provides a means of developing highly characterized 'designer' attenuated vaccine candidates. To date, several promising vaccine candidates have been developed, each using a different mode of attenuation. The first candidate involves deletion of the $G$ glycoprotein, providing attenuation that is probably based on reduced efficiency of attachment. The second candidate involves deletion of the M2-2 protein, which participates in regulating RNA synthesis and whose deletion has the advantageous property of up-regulating transcription and increasing antigen synthesis. A third candidate involves replacing the P protein gene of HMPV with its counterpart from the related avian metapneumovirus, thereby introducing attenuation owing to its chimeric nature and host range restriction. Another live vaccine strategy involves using an attenuated parainfluenza virus as a vector to express HMPV protective antigens, providing a bivalent pediatric vaccine [102].

\subsection{Infection Control Measures}

As outbreaks with HMPV are frequently described, control measures to prevent HMPV transmission in hospitals and long-term care facilities seem justifiable [43,103-105]. When patients with HMPV infection are hospitalized, infection control measures similar to those taken in case of RSV infection should be taken including droplet isolation until clinical recovery. The Dutch working party on infection prevention advises to apply droplet isolation to all patients hospitalized with bronchiolitis until clinical recovery [106]. No specific advice is formulated for HMPV infections. The CDC advises contact and droplet precautions for infants and young children with respiratory infections; however no advice for adults is given [107]. In our hospitals (Diakonessenhuis Utrecht and the University Medical Centre Utrecht, Utrecht, The Netherlands), droplet isolation is applied to all patients with HMPV infection until clinical recovery. We do not routinely perform control RT-PCR on nasopharyngeal swabs after clinical recovery.

\section{Risk Groups}

HMPV infections may be more severe in older patients or patients with underlying medical conditions. It is a significant cause of acute respiratory diseases in adults over 65 years and adults with comorbid diseases, such as COPD, asthma, cancer, immunocompromised status, including HIV or post transplantation. 


\subsection{Adults with Pulmonary Disease or Congestive Heart Disease}

Respiratory viruses are a common trigger for exacerbations of COPD, and have been associated with respiratory failure in patients with cardiopulmonary disease such as COPD and congestive heart failure [108,109]. Walsh et al. performed a cohort-study during four winters to investigate the clinical outcome and incidence of HMPV infections [110]. Serum samples were taken before and after the observation period (November 15 to April 15) each year. In case of respiratory symptoms a nasopharyngeal swab for HMPV RNA analysis and serum were sampled. They showed that $71 \%$ of infections with HMPV were asymptomatic in the healthy young adults (19-40 years) in contrast to $39 \%$ in the high risk adults (patients with symptomatic lung disease, COPD, congestive heart failure). These patients were also more likely to use medical care service. Patients were ill for a mean of 10 days in the young adults versus 16 days in the high risk group. Johnstone et al. investigated the potential role of respiratory viruses in the natural history of community-acquired pneumonia (CAP). In $39 \%$ of the 193 patients who were admitted because of CAP, a pathogen was identified. Of these pathogens, 39\% were viruses and the easily transmissible viruses such as influenza, HMPV, and RSV were the most common (respectively 24, 24 and 17\%). There were few clinically meaningful differences in presentation and no differences in outcomes according to the presence or absence of viral infection. The patients with viral infection were, compared with bacterial infection, significant older, more likely to have cardiac disease and more frail [111].

This is in accordance with the results of Hamelin et al. who found HMPV in $4.1 \%$ of patients with CAP or exacerbation of chronic obstructive pulmonary disease [112]. Martinello et al. also showed that HMPV was frequently identified in patients hospitalized because of an exacerbation of COPD [113]. HMPV (both genotype A and B) was identified in nasopharyngeal specimens (by RT-PCR) in $12 \%$ of these patients (6/50). RSV, influenza A and parainfluenza type 3 were identified in respectively $8 \%, 4 \%$ and $2 \%$.

Along with these results, Williams and colleagues showed that HMPV was detected (by RT-PCR of nasal wash specimens) in almost 7\% (7/101) of the adults hospitalized for an acute asthma exacerbation, compared to $1.3 \%$ in follow-up patients $(p=0.03)$. While none of these patients tested positive for HMPV three months after discharge, a direct etiologic role of the virus seems very likely [114,115].

Recently, we reported a case series of adult patients, including two patients known with COPD, with severe HMPV infections with respiratory insufficiency and the need of ICU admission [116].

\subsection{Healthy Elderly Patients over 65 Years}

Since adults are not routinely screened for HMPV in the hospital and clinical course can be asymptomatic or mild, infections in the elderly are likely to be underreported. The reported yearly incidence in adults is between 4 and $11 \%$ and in adults aged over 50 years; hospitalization rates for HMPV were similar to those associated with influenza and RSV [8].

Walsh et al. showed that the risk for symptomatic severe HMPV infection was higher in the elderly. HMPV infection was asymptomatic in $44 \%$ of the healthy elderly in contrast to $71 \%$ of the healthy 
young adults. Thirty-eight $\%$ of the elderly with HMPV infection used medical care in contrast to $9 \%$ in the young adults [23]. Rates for hospitalization in elderly patients over 65 years were also significantly higher for HMPV infection (22.1/10,000 residents) compared to influenza virus (12.3/10,000 residents), but similar to those of RSV infection (25.4/10,000 residents) [8,23]. Antibody levels prior to infection were higher in elderly, suggesting possible immune dysregulation associated with decreased viral clearance in elderly [117].

\subsection{Outbreaks in Long Term Care Facilities}

Several studies have reported outbreaks in long-term care facilities for elderly. Boivin et al. studied a large outbreak in a long term care facility in Canada in which 96 (27\%) op the 364 residents had respiratory symptoms. Six out of 13 tested residents were HMPV positive by RT-PCR. Nine patients died, of which three residents tested HMPV positive [103]. In a 23-bed ward in a hospital for older people in Japan, all 8 residents with respiratory symptoms were HMPV positive by RT-PCR [118]. None of these residents died. Tu et al. found 10 of 13 tested residents of a 53-bed psychiatric ward of an armed forces general hospital in Taiwan HMPV positive by RT-PCR [41]. In a summer outbreak in a long term care facility in California $26(18 \%)$ of residents developed respiratory symptoms. Five of the 13 tested residents were HMPV positive [105]. In an outbreak that the authors of this review described, the attack rate was $13 \%$ in a long term care facility [43]. Three patients died, however these were only possible cases. Osbourn et al. found an attack rate of $16.4 \%$ in HMPV outbreak in a long-term care facility in Australia, in which two residents died [104]. Sixteen (36\%) of 44 residents in a long-term care facility in Oregon had respiratory symptoms of which 6 of 10 tested residents were HMPV positive by RT-PCR [119]. Another study in a community hospital in England reported an attack rate of $29.4 \%$. The different settings (residential care facilities for elderly versus hospital settings) and different case definitions might partly explain the difference in attack rate and mortality.

\subsection{Immunocompromised}

Several case reports and case series concerning HMPV infections in immunocompromised patients have been published reporting varying morbidity and mortality [67,120-124]. While immunocompromised patients, including patients with haematological malignancies and solid organ and hematopoietic stem cell transplant (HSCT) patients appear to acquire HMPV infection at the same frequency as immunocompetent individuals, they seem to be at risk for severe infections, probably due to poor viral clearance [125-127]. Clinical course is prolonged and respiratory failure may develop [121]. However, Debiaggi showed that HSCT recipients may frequently develop symptomless HMPV infection [128].

Sumino et al. examined a cohort of 688 patients who underwent a bronchoscopy. Of these patients, $72 \%$ were immunocompromised (mainly lung transplant patients) and 30\% were patients without acute illness who underwent routine bronchoscopy for surveillance after lung transplantation or follow-up of rejection. Six cases of HMPV infection were identified using RT-PCR; four of these were immunocompromised hosts. In the asymptomatic individuals, no cases were identified [129]. 
Kamboj et al. showed that HMPV is detected in $2.7 \%$ of cancer patients with respiratory disease. However, HMPV was associated with mild respiratory disease and RSV and influenza were more often found. In patients with hematologic malignancies HMPV was found more often [58].

Debur et al. showed that HMPV was present in $2.5 \%$ of hematologic stem cell transplant recipients with respiratory disease. Most patients presented with upper RTI, while $27 \%$ had a lower RTA. No patients died [130].

Englund et al. performed a retrospective survey to demonstrate the importance of HMPV in hematopoietic stem-cell transplant recipients [131]. In 3\% of these patients who underwent a BAL because of LRTI was HMPV detected (by RT-PCR). Clinical course in this group was severe and $80 \%$ died with acute respiratory failure.

Williams et al. showed that HMPV is found in the same frequency as RSV, influenza and parainfluenzavirus in hematologic malignancy patients with acute respiratory disease. All patients presented with an upper RTI, but $41 \%$ progressed to a lower RTI. One third (three patients) of these patients died, however in two of these patients potential bacterial pathogens were also found in their BAL fluid [125].

Cane et al. published a case report about a HSCT recipient who succumbed to progressive respiratory failure following an upper respiratory prodrome and where HMPV was detected as the sole pathogen in the nasopharyngeal aspirate [132].

In lung transplant patients, HMPV was found in $6 \%$ of adults with RTI. This was significantly lower then the most frequently found viral cause, namely parainfluenza virus (17\%) [133]. RSV and influenza were found in $12 \%$ and $14 \%$ respectively. The rate of required hospitalization and length of stay of hospitalization were not different between HMPV and other respiratory viruses. In this study, viral RTI was associated with acute graft rejection. However, this rate was significantly higher for RSV infection compared to HMPV infection.

Larcher et al. found HMPV in $25 \%$ of BAL fluids from lung transplant patients. Not al of them had respiratory symptoms at the time of the lavage. In this study, HMPV infection seems associated with acute graft rejection, but not with the development of bronchiolitis obliterans [134]. However, other studies suggest that viral RTI is associated with the risk of the development of bronchiolitis obliterans $[135,136]$.

\section{Complications}

Bacterial and fungal superinfections might complicate viral respiratory infections. To our knowledge no studies specific addressing this issue have been executed, although some studies report the presence of potential bacterial pathogens in the BAL fluid, sputum or blood cultures in those patients with sometimes lethal outcome $[9,50,114,125]$.

In a mouse model that HMPV infection predisposes to severe bacterial infections [137]. Higher levels of airway obstruction, pneumococcal replication and inflammatory cytokines and chemokines were observed in the lungs of superinfected mice, which were challenged with Streptococcus pneumoniae (S. pneumoniae) five days after HMPV infection. Inactivated HMPV did not result in 
these changes after a pneumococcal challenge, suggesting that HMPV replication rather than the host response to HMPV may be responsible for these effects. Mice infected with influenza A show long-term impairment of $S$. pneumoniae lung clearance, but the mechanism producing these effects might be different. In contrast to these findings, Ludewick et al. showed that BALB/c mice infected with HMPV had a normal bacterial lung clearance when they were challenged with $S$. pneumoniae 14 days after HMPV infection [138].

\section{Discussion}

The last years more knowledge is obtained about the significance of HMPV infection in adult patients.

Thanks to more sensitive diagnostic tools, like PCR, the proportion of known viral etiologies has increased and HMPV is recognized as a major cause of respiratory disease in patients of all ages. Reported yearly incidences in adults are up to $11 \%$, but the real incidence of HMPV infections is difficult to measure or estimate. First, because a great part of the HMPV infections is asymptomatic or mild and these patients do not present to the hospital. Secondly, the majority of the patients with respiratory symptoms presenting to our hospital are not tested for viral infections.

Epidemiological studies show that elderly over 65 years, patients with cardiac or pulmonary diseases and immunocompromised patients are at high risk for an HMPV infection presenting with severer disease than younger adults without co-morbidity [8,9,114,125]. As serious outbreaks of HMPV with mortality have been reported in long-term care facilities and among immunocompromised patients, infection control measures should be taken in case of a RTI with HMPV especially because these patient groups are at greater risk for severer disease and no proven treatment and/or vaccination strategies against HMPV are available up till now [42,103,134,139].

Till now a lot of experience on treatment of HMPV has been gained in individual cases and small case series [120,122,140-142]. The combination of ribavirin with IVIG seems to be very promising, although this combination is expensive and has disadvantages. Several other treatment regimes have been investigated and proven to be effective in vitro and in animal studies. Both immunoglobulins (like mAb 338 and Fab DS7) and synthetic fusion inhibitors showed to be efficient against HMPV. The recently discovered approach of RNA interference (RNAi) could be the technique of the future. However, up till now, no treatment proven to be effective in large clinical trials is available and treatment of HMPV infection is mainly supportive.

Since HMPV is an important cause of morbidity and mortality in frail patients, a vaccine is desirable and several in vitro and animal studies investigating the development of an HMPV vaccine have been performed. The development of a vaccine against HMPV is hampered by the fact that natural infection with HMPV does not elicits complete immunity and that studies in which is vaccinated with inactivated HMPV show an enlarged immune reaction with even lethal outcome. However, other studies showed promising results, although no vaccine is available up till now. 


\section{Conclusion}

HMPV is an important pathogen causing viral RTI. People at risk are the elderly, the immunocompromised patients and patients with cardiac or pulmonary diseases. While HMPV infections are mild and self-limiting in the majority of adults, clinical course can be complicated in these risk groups and associated morbidity and mortality are considerable.

\section{Key Issues}

- HMPV is an important pathogen causing viral RTI in adults.

- The elderly, immunocompromised patients and patients with cardiac or pulmonary diseases are at risk for severe infection.

- Distinguishing HMPV clinically from other respiratory viruses is difficult. Diagnosis relies mainly on RT-PCR.

- Although a lot of research has been performed last years, treatment of HMPV infection is mainly supportive and no vaccine is available up till now.

- In case of severe infections, treatment with ribavirin and IVIG might be considered.

\section{Conflicts of Interest}

The authors declare no conflict of interest.

\section{References}

1. Freymuth, F.; Vabret, A.; Gouarin, S.; Petitjean, J.; Charbonneau, P.; Lehoux, P.; Galateau-Salle, F.; Tremolieres, F.; Carette, M.F.; Mayaud, C.; et al. Epidemiology and diagnosis of respiratory syncitial virus in adults. Rev. Mal. Respir. 2004, 21, 35-42.

2. Osterhaus, A.; Fouchier, R. Human metapneumovirus in the community. Lancet 2003, 361, 890-891.

3. Falsey, A.R.; Erdman, D.; Anderson, L.J.; Walsh, E.E. Human metapneumovirus infections in young and elderly adults. J. Infect. Dis. 2003, 187, 785-790.

4. Van den Hoogen, B.G.; de Jong, J.C.; Groen, J.; Kuiken, T.; de Groot, R.; Fouchier, R.A.; Osterhaus, A.D. A newly discovered human pneumovirus isolated from young children with respiratory tract disease. Nat. Med. 2001, 7, 719-724.

5. Stockton, J.; Stephenson, I.; Fleming, D.; Zambon, M. Human metapneumovirus as a cause of community-acquired respiratory illness. Emerg. Infect. Dis. 2002, 8, 897-901.

6. Boivin, G.; Abed, Y.; Pelletier, G.; Ruel, L.; Moisan, D.; Cote, S.; Peret, T.C.; Erdman, D.D.; Anderson, L.J. Virological features and clinical manifestations associated with human metapneumovirus: A new paramyxovirus responsible for acute respiratory-tract infections in all age groups. J. Infect. Dis. 2002, 186, 1330-1334. 
7. Peret, T.C.; Boivin, G.; Li, Y.; Couillard, M.; Humphrey, C.; Osterhaus, A.D.; Erdman, D.D.; Anderson, L.J. Characterization of human metapneumoviruses isolated from patients in North America. J. Infect. Dis. 2002, 185, 1660-1663.

8. Widmer, K.; Zhu, Y.; Williams, J.V.; Griffin, M.R.; Edwards, K.M.; Talbot, H.K. Rates of hospitalizations for respiratory syncytial virus, human metapneumovirus, and influenza virus in older adults. J. Infect. Dis. 2012, 206, 56-62.

9. Walsh, E.E.; Peterson, D.R.; Falsey, A.R. Human metapneumovirus infections in adults: Another piece of the puzzle. Ann. Intern. Med. 2008, 168, 2489-2496.

10. Biacchesi, S.; Skiadopoulos, M.H.; Boivin, G.; Hanson, C.T.; Murphy, B.R.; Collins, P.L.; Buchholz, U.J. Genetic diversity between human metapneumovirus subgroups. Virology 2003, 315, 1-9.

11. Agapov, E.; Sumino, K.C.; Gaudreault-Keener, M.; Storch, G.A.; Holtzman, M.J. Genetic variability of human metapneumovirus infection: Evidence of a shift in viral genotype without a change in illness. J. Infect. Dis. 2006, 193, 396-403.

12. van den Hoogen, B.G.; Herfst, S.; Sprong, L.; Cane, P.A.; Forleo-Neto, E.; de Swart, R.L.; Osterhaus, A.D.; Fouchier, R.A. Antigenic and genetic variability of human metapneumoviruses. Emerg. Infect. Dis. 2004, 10, 658-666.

13. Mackay, I.M.; Bialasiewicz, S.; Jacob, K.C.; McQueen, E.; Arden, K.E.; Nissen, M.D.; Sloots, T.P. Genetic diversity of human metapneumovirus over 4 consecutive years in Australia. J. Infect. Dis. 2006, 193, 1630-1633.

14. Skiadopoulos, M.H.; Biacchesi, S.; Buchholz, U.J.; Riggs, J.M.; Surman, S.R.; Amaro-Carambot, E.; McAuliffe, J.M.; Elkins, W.R.; St Claire, M.; Collins, P.L.; et al. The two major human metapneumovirus genetic lineages are highly related antigenically, and the fusion (F) protein is a major contributor to this antigenic relatedness. J. Virol. 2004, 78, 6927-6937.

15. Huck, B.; Scharf, G.; Neumann-Haefelin, D.; Puppe, W.; Weigl, J.; Falcone, V. Novel human metapneumovirus sublineage. Emerg. Infect. Dis. 2006, 12, 147-150.

16. Schildgen, V.; van den Hoogen, B.; Fouchier, R.; Tripp, R.A.; Alvarez, R.; Manoha, C.; Williams, J.; Schildgen, O. Human metapneumovirus: Lessons learned over the first decade. Clin. Microbiol. Rev. 2011, 24, 734-754.

17. Hamelin, M.E.; Yim, K.; Kuhn, K.H.; Cragin, R.P.; Boukhvalova, M.; Blanco, J.C.; Prince, G.A.; Boivin, G. Pathogenesis of human metapneumovirus lung infection in BALB/c mice and cotton rats. J. Virol. 2005, 79, 8894-8903.

18. Hamelin, M.E.; Prince, G.A.; Gomez, A.M.; Kinkead, R.; Boivin, G. Human metapneumovirus infection induces long-term pulmonary inflammation associated with airway obstruction and hyperresponsiveness in mice. J. Infect. Dis. 2006, 193, 1634-1642.

19. Darniot, M.; Pitoiset, C.; Petrella, T.; Aho, S.; Pothier, P.; Manoha, C. Age-associated aggravation of clinical disease after primary metapneumovirus infection of BALB/c mice. J. Virol. 2009, 83, 3323-3332. 
20. Ditt, V.; Lusebrink, J.; Tillmann, R.L.; Schildgen, V.; Schildgen, O. Respiratory infections by HMPV and RSV are clinically indistinguishable but induce different host response in aged individuals. PLoS One 2011, 6, e16314.

21. Lusebrink, J.; Wiese, C.; Thiel, A.; Tillmann, R.L.; Ditt, V.; Muller, A.; Schildgen, O.; Schildgen, V. High seroprevalence of neutralizing capacity against human metapneumovirus in all age groups studied in Bonn, Germany. Clin. Vaccine Immunol. 2010, 17, 481-484.

22. Sastre, P.; Ruiz, T.; Schildgen, O.; Schildgen, V.; Vela, C.; Rueda, P. Seroprevalence of human respiratory syncytial virus and human metapneumovirus in healthy population analyzed by recombinant fusion protein-based enzyme linked immunosorbent assay. Virol. J. 2012, 9, 130-422X-9-130.

23. Falsey, A.R.; Hennessey, P.A.; Formica, M.A.; Criddle, M.M.; Biear, J.M.; Walsh, E.E. Humoral immunity to human metapneumovirus infection in adults. Vaccine 2010, 28, 1477-1480.

24. Van den Hoogen, B.G.; van Doornum, G.J.; Fockens, J.C.; Cornelissen, J.J.; Beyer, W.E.; de Groot, R.; Osterhaus, A.D.; Fouchier, R.A. Prevalence and clinical symptoms of human metapneumovirus infection in hospitalized patients. J. Infect. Dis. 2003, 188, 1571-1577.

25. Williams, J.V.; Wang, C.K.; Yang, C.F.; Tollefson, S.J.; House, F.S.; Heck, J.M.; Chu, M.; Brown, J.B.; Lintao, L.D.; Quinto, J.D.; et al. The role of human metapneumovirus in upper respiratory tract infections in children: A 20-year experience. J. Infect. Dis. 2006, 193, 387-395.

26. Esper, F.; Martinello, R.A.; Boucher, D.; Weibel, C.; Ferguson, D.; Landry, M.L.; Kahn, J.S. A 1 -year experience with human metapneumovirus in children aged $<5$ years. J. Infect. Dis. 2004, 189, 1388-1396.

27. Williams, J.V.; Harris, P.A.; Tollefson, S.J.; Halburnt-Rush, L.L.; Pingsterhaus, J.M.; Edwards, K.M.; Wright, P.F.; Crowe, J.E., Jr. Human metapneumovirus and lower respiratory tract disease in otherwise healthy infants and children. N. Engl. J. Med. 2004, 350, 443-450.

28. Williams, J.V.; Edwards, K.M.; Weinberg, G.A.; Griffin, M.R.; Hall, C.B.; Zhu, Y.; Szilagyi, P.G.; Wang, C.K.; Yang, C.F.; Silva, D.; et al. Population-based incidence of human metapneumovirus infection among hospitalized children. J. Infect. Dis. 2010, 201, 1890-1898.

29. Boivin, G.; De Serres, G.; Cote, S.; Gilca, R.; Abed, Y.; Rochette, L.; Bergeron, M.G.; Dery, P. Human metapneumovirus infections in hospitalized children. Emerg. Infect. Dis. 2003, 9, 634-640.

30. Mullins, J.A.; Erdman, D.D.; Weinberg, G.A.; Edwards, K.; Hall, C.B.; Walker, F.J.; Iwane, M.; Anderson, L.J. Human metapneumovirus infection among children hospitalized with acute respiratory illness. Emerg. Infect. Dis. 2004, 10, 700-705.

31. Van den Hoogen, B.G.; Osterhaus, D.M.; Fouchier, R.A. Clinical impact and diagnosis of human metapneumovirus infection. J. Pediatr. Infect. Dis. 2004, 23, S25-S32.

32. Crowe, J.E., Jr. Human metapneumovirus as a major cause of human respiratory tract disease. J. Pediatr. Infect. Dis. 2004, 23, S215-S221.

33. Debiaggi, M.; Canducci, F.; Ceresola, E.R.; Clementi, M. The role of infections and coinfections with newly identified and emerging respiratory viruses in children. Virol. J. 2012, 9, 247. 
34. Hustedt, J.W.; Vazquez, M. The changing face of pediatric respiratory tract infections: How human metapneumovirus and human bocavirus fit into the overall etiology of respiratory tract infections in young children. Yale J. Biol. Med. 2010, 83, 193-200.

35. Papenburg, J.; Boivin, G. The distinguishing features of human metapneumovirus and respiratory syncytial virus. Rev. Med. Virol. 2010, 20, 245-260.

36. Papenburg, J.; Hamelin, M.E.; Ouhoummane, N.; Carbonneau, J.; Ouakki, M.; Raymond, F.; Robitaille, L.; Corbeil, J.; Caouette, G.; Frenette, L.; et al. Comparison of risk factors for human metapneumovirus and respiratory syncytial virus disease severity in young children. J. Infect. Dis. 2012, 206, 178-189.

37. Pavlin, J.A.; Hickey, A.C.; Ulbrandt, N.; Chan, Y.P.; Endy, T.P.; Boukhvalova, M.S.; Chunsuttiwat, S.; Nisalak, A.; Libraty, D.H.; Green, S.; et al. Human metapneumovirus reinfection among children in thailand determined by elisa using purified soluble fusion protein. J. Infect. Dis. 2008, 198, 836-842.

38. Van den Hoogen, B.G.; Herfst, S.; de Graaf, M.; Sprong, L.; van Lavieren, R.; van Amerongen, G.; Yuksel, S.; Fouchier, R.A.; Osterhaus, A.D.; de Swart, R.L. Experimental infection of macaques with human metapneumovirus induces transient protective immunity. J. Gen. Virol. 2007, 88, 1251-1259.

39. Falsey, A.R. Respiratory syncytial virus infection in elderly and high-risk adults. Exp. Lung Res. 2005, 31, 77.

40. Matsuzaki, Y.; Itagaki, T.; Ikeda, T.; Aoki, Y.; Abiko, C.; Mizuta, K. Human metapneumovirus infection among family members. Epidemiol. Infect. 2012, 1-6.

41. Tu, C.C.; Chen, L.K.; Lee, Y.S.; Ko, C.F.; Chen, C.M.; Yang, H.H.; Lee, J.J. An outbreak of human metapneumovirus infection in hospitalized psychiatric adult patients in Taiwan. Scand. J. Infect. Dis. 2009, 41, 363-367.

42. Kim, S.; Sung, H.; Im, H.J.; Hong, S.J.; Kim, M.N. Molecular epidemiological investigation of a nosocomial outbreak of human metapneumovirus infection in a pediatric hemato-oncology patient population. J. Clin. Microbiol. 2009, 47, 1221-1224.

43. Te Wierik, M.J.; Nguyen, D.T.; Beersma, M.F.; Thijsen, S.F.; Heemstra, K.A. An outbreak of severe respiratory tract infection caused by human metapneumovirus in a residential care facility for elderly in Utrecht, the Netherlands, January to March 2010. Euro. Surveill. 2012, 17, 20132.

44. Peiris, J.S.; Tang, W.H.; Chan, K.H.; Khong, P.L.; Guan, Y.; Lau, Y.L.; Chiu, S.S. Children with respiratory disease associated with metapneumovirus in Hong Kong. Emerg. Infect. Dis. 2003, 9, 628-633.

45. Bruno, R.; Marsico, S.; Minini, C.; Apostoli, P.; Fiorentini, S.; Caruso, A. Human metapneumovirus infection in a cohort of young asymptomatic subjects. New Microbiol. 2009, 32, 297-301.

46. Falsey, A.R.; Criddle, M.C.; Walsh, E.E. Detection of respiratory syncytial virus and human metapneumovirus by reverse transcription polymerase chain reaction in adults with and without respiratory illness. J. Clin. Virol. 2006, 35, 46-50. 
47. Hall, W.B.; Kidd, J.M.; Campbell-Bright, S.; Miller, M.; Aris, R.M. Clinical manifestations and impact of human metapneumovirus in healthy adults: A retrospective analysis of 28 patients over 2 years. Am. J. Respir. Crit. Care Med. 2011, 183, 4927.

48. Li, I.W.; To, K.K.; Tang, B.S.; Chan, K.H.; Hui, C.K.; Cheng, V.C.; Yuen, K.Y. Human metapneumovirus infection in an immunocompetent adult presenting as mononucleosis-like illness. J. Infect. 2008, 56, 389-392.

49. Falsey, A.R.; Erdman, D.; Anderson, L.J.; Walsh, E.E. Human metapneumovirus infections in young and elderly adults. J. Infect. Dis. 2003, 187, 785-790.

50. Johnstone, J.; Majumdar, S.R.; Fox, J.D.; Marrie, T.J. Human metapneumovirus pneumonia in adults: Results of a prospective study. Clin. Infect. Dis. 2008, 46, 571-574.

51. Van den Hoogen, B.G. Respiratory tract infection due to human metapneumovirus among elderly patients. Clin. Infect. Dis. 2007, 44, 1159-1160.

52. Tu, C.C.; Chen, L.K.; Lee, Y.S.; Ko, C.F.; Chen, C.M.; Yang, H.H.; Lee, J.J. An outbreak of human metapneumovirus infection in hospitalized psychiatric adult patients in Taiwan. Scand. J. Infect. Dis. 2009, 41, 363-367.

53. O'Gorman, C.; McHenry, E.; Coyle, P.V. Human metapneumovirus in adults: A short case series. Euro. J. Clin. Micorbiol. Infect. Dis. 2006, 25, 190-192.

54. Boivin, G.; de Serres, G.; Hamelin, M.E.; Cote, S.; Argouin, M.; Tremblay, G.; Maranda-Aubut, R.; Sauvageau, C.; Ouakki, M.; Boulianne, N.; et al. An outbreak of severe respiratory tract infection due to human metapneumovirus in a long-term care facility. Clin. Infect. Dis. 2007, 44, 1152-1158.

55. Pelletier, G.; Dery, P.; Abed, Y.; Boivin, G. Respiratory tract reinfections by the new human metapneumovirus in an immunocompromised child. Emerg. Infect. Dis. 2002, 8, 976-978.

56. Syha, R.; Beck, R.; Hetzel, J.; Ketelsen, D.; Grosse, U.; Springer, F.; Horger, M. Humane metapneumovirus (HMPV) associated pulmonary infections in immunocompromised adults-initial ct findings, disease course and comparison to respiratory-syncytial-virus (RSV) induced pulmonary infections. Eur. J. Radiol. 2012, 81, 4173-4178.

57. Franquet, T.; Rodriguez, S.; Martino, R.; Salinas, T.; Gimenez, A.; Hidalgo, A. Human metapneumovirus infection in hematopoietic stem cell transplant recipients: High-resolution computed tomography findings. J. Comput. Assist. Tomo. 2005, 29, 223-227.

58. Kamboj, M.; Gerbin, M.; Huang, C.K.; Brennan, C.; Stiles, J.; Balashov, S.; Park, S.; Kiehn, T.E.; Perlin, D.S.; Pamer, E.G.; et al. Clinical characterization of human metapneumovirus infection among patients with cancer. J. Infect. 2008, 57, 464-471.

59. Hamelin, M.E.; Boivin, G. Development and validation of an enzyme-linked immunosorbent assay for human metapneumovirus serology based on a recombinant viral protein. Clin. Diagn. Lab. Immunol. 2005, 12, 249-253.

60. Cheng, M.F.; Chen, B.C.; Kao, C.L.; Kao, C.H.; Hsieh, K.S.; Liu, Y.C. Human metapneumovirus as a causative agent of lower respiratory tract infection in four patients: The first report of human metapneumovirus infection confirmed by rna sequences in Taiwan. Scand. J. Infect. Dis. 2006, 38, 392-396. 
61. Chiu, C.Y.; Alizadeh, A.A.; Rouskin, S.; Merker, J.D.; Yeh, E.; Yagi, S.; Schnurr, D.; Patterson, B.K.; Ganem, D.; DeRisi, J.L. Diagnosis of a critical respiratory illness caused by human metapneumovirus by use of a pan-virus microarray. J. Clin. Microbiol. 2007, 45, 2340-2343.

62. Sookoian, S.; Castano, G.; Flichman, D.; Cello, J. Effects of ribavirin on cytokine production of recall antigens and phytohemaglutinin-stimulated peripheral blood mononuclear cells. (Inhibitory effects of ribavirin on cytokine production). Ann. Hepatol. 2004, 3, 104-107.

63. Graci, J.D.; Cameron, C.E. Mechanisms of action of ribavirin against distinct viruses. Rev. Med. Virol. 2006, 16, 37-48.

64. Wyde, P.R.; Chetty, S.N.; Jewell, A.M.; Boivin, G.; Piedra, P.A. Comparison of the inhibition of human metapneumovirus and respiratory syncytial virus by ribavirin and immune serum globulin in vitro. Antivir. Res. 2003, 60, 51-59.

65. Hamelin, M.E.; Prince, G.A.; Boivin, G. Effect of ribavirin and glucocorticoid treatment in a mouse model of human metapneumovirus infection. Antimicrob. Agents Chemother. 2006, 50, 774-777.

66. Shachor-Meyouhas, Y.; Ben-Barak, A.; Kassis, I. Treatment with oral ribavirin and ivig of severe human metapneumovirus pneumonia (HMPV) in immune compromised child. Pediatr. Blood Cancer 2011, 57, 350-351.

67. Egli, A.; Bucher, C.; Dumoulin, A.; Stern, M.; Buser, A.; Bubendorf, L.; Gregor, M.; Servida, P.; Sommer, G.; Bremerich, J.; et al. Human metapneumovirus infection after allogeneic hematopoietic stem cell transplantation. Infection 2012, 40, 677-684.

68. Kroll, J.L.; Weinberg, A. Human metapneumovirus. Semin. Respir. Crit. Care Med. 2011, 32, 447-453.

69. Shahda, S.; Carlos, W.G.; Kiel, P.J.; Khan, B.A.; Hage, C.A. The human metapneumovirus: A case series and review of the literature. Transpl. Infect. Dis. 2011, 13, 324-328.

70. American Academy of Pediatrics Committee on Infectious Diseases and Committee on Fetus and Newborn. Revised indications for the use of palivizumab and respiratory syncytial virus immune globulin intravenous for the prevention of respiratory syncytial virus infections. Pediatrics 2003, $112,1442-1446$.

71. Ulbrandt, N.D.; Ji, H.; Patel, N.K.; Barnes, A.S.; Wilson, S.; Kiener, P.A.; Suzich, J.; McCarthy, M.P. Identification of antibody neutralization epitopes on the fusion protein of human metapneumovirus. J. Gen. Virol. 2008, 89, 3113-3118.

72. Feltes, T.F.; Cabalka, A.K.; Meissner, H.C.; Piazza, F.M.; Carlin, D.A.; Top, F.H., Jr.; Connor, E.M.; Sondheimer, H.M.; Cardiac Synagis Study Group. Palivizumab prophylaxis reduces hospitalization due to respiratory syncytial virus in young children with hemodynamically significant congenital heart disease. J. Pediatr. 2003, 143, 532-540.

73. The IMpact-RSV Study Group. Palivizumab, a humanized respiratory syncytial virus monoclonal antibody, reduces hospitalization from respiratory syncytial virus infection in high-risk infants. Pediatrics 1998, 102, 531-537. 
74. Carbonell-Estrany, X.; Simoes, E.A.; Dagan, R.; Hall, C.B.; Harris, B.; Hultquist, M.; Connor, E.M.; Losonsky, G.A.; Motavizumab Study Group. Motavizumab for prophylaxis of respiratory syncytial virus in high-risk children: A noninferiority trial. Pediatrics 2010, 125, e35-e51.

75. Ulbrandt, N.D.; Ji, H.; Patel, N.K.; Riggs, J.M.; Brewah, Y.A.; Ready, S.; Donacki, N.E.; Folliot, K.; Barnes, A.S.; Senthil, K.; et al. Isolation and characterization of monoclonal antibodies which neutralize human metapneumovirus in vitro and in vivo. J. Virol. 2006, 80, 7799-7806.

76. Hamelin, M.E.; Couture, C.; Sackett, M.; Kiener, P.; Suzich, J.; Ulbrandt, N.; Boivin, G. The prophylactic administration of a monoclonal antibody against human metapneumovirus attenuates viral disease and airways hyperresponsiveness in mice. Antivir. Ther. 2008, 13, 39-46.

77. Hamelin, M.E.; Gagnon, C.; Prince, G.A.; Kiener, P.; Suzich, J.; Ulbrandt, N.; Boivin, G. Prophylactic and therapeutic benefits of a monoclonal antibody against the fusion protein of human metapneumovirus in a mouse model. Antivir. Res. 2010, 88, 31-37.

78. Williams, J.V.; Chen, Z.; Cseke, G.; Wright, D.W.; Keefer, C.J.; Tollefson, S.J.; Hessell, A.; Podsiad, A.; Shepherd, B.E.; Sanna, P.P.; et al. A recombinant human monoclonal antibody to human metapneumovirus fusion protein that neutralizes virus in vitro and is effective therapeutically in vivo. J. Virol. 2007, 81, 8315-8324.

79. Wyde, P.R.; Moylett, E.H.; Chetty, S.N.; Jewell, A.; Bowlin, T.L.; Piedra, P.A. Comparison of the inhibition of human metapneumovirus and respiratory syncytial virus by NMSO3 in tissue culture assays. Antivir. Res. 2004, 63, 51-59.

80. Hamelin, M.E.; Prince, G.A.; Boivin, G. Effect of ribavirin and glucocorticoid treatment in a mouse model of human metapneumovirus infection. Antimicrob. Agents Chemother. 2006, 50, 774-777.

81. Kilham, L.; Ferm, V.H. Congenital anomalies induced in hamster embryos with ribavirin. Science 1977, 195, 413-414.

82. Wyde, P.R.; Chetty, S.N.; Jewell, A.M.; Boivin, G.; Piedra, P.A. Comparison of the inhibition of human metapneumovirus and respiratory syncytial virus by ribavirin and immune serum globulin in vitro. Antivir. Res. 2003, 60, 51-59.

83. Deffrasnes, C.; Hamelin, M.E.; Prince, G.A.; Boivin, G. Identification and evaluation of a highly effective fusion inhibitor for human metapneumovirus. Antimicrob. Agents Chemother. 2008, 52, 279-287.

84. Miller, S.A.; Tollefson, S.; Crowe, J.E., Jr.; Williams, J.V.; Wright, D.W. Examination of a fusogenic hexameric core from human metapneumovirus and identification of a potent synthetic peptide inhibitor from the heptad repeat 1 region. J. Virol. 2007, 81, 141-149.

85. Sah, D.W. Therapeutic potential of rna interference for neurological disorders. Life Sciences 2006, 79, 1773-1780.

86. Alvarez, R.; Elbashir, S.; Borland, T.; Toudjarska, I.; Hadwiger, P.; John, M.; Roehl, I.; Morskaya, S.S.; Martinello, R.; Kahn, J.; et al. RNA interference-mediated silencing of the respiratory syncytial virus nucleocapsid defines a potent antiviral strategy. Antimicrob. Agents Chemother. 2009, 53, 3952-3962. 
87. DeVincenzo, J.; Lambkin-Williams, R.; Wilkinson, T.; Cehelsky, J.; Nochur, S.; Walsh, E.; Meyers, R.; Gollob, J.; Vaishnaw, A. A randomized, double-blind, placebo-controlled study of an rnai-based therapy directed against respiratory syncytial virus. Proc. Natl. Acad. Sci. USA 2010, 107, 8800-8805.

88. Deffrasnes, C.; Cavanagh, M.H.; Goyette, N.; Cui, K.; Ge, Q.; Seth, S.; Templin, M.V.; Quay, S.C.; Johnson, P.H.; Boivin, G. Inhibition of human metapneumovirus replication by small interfering RNA. Antivir. Ther. 2008, 13, 821-832.

89. Preston, F.M.; Straub, C.P.; Ramirez, R.; Mahalingam, S.; Spann, K.M. SiRNA against the g gene of human metapneumovirus. Virol. J. 2012, 9, 105-422X-9-105.

90. Wyde, P.R.; Moylett, E.H.; Chetty, S.N.; Jewell, A.; Bowlin, T.L.; Piedra, P.A. Comparison of the inhibition of human metapneumovirus and respiratory syncytial virus by NMSO3 in tissue culture assays. Antivir. Res. 2004, 63, 51-59.

91. Herfst, S.; Fouchier, R.A. Vaccination approaches to combat human metapneumovirus lower respiratory tract infections. J. Clin. Virol. 2008, 41, 49-52.

92. Tang, R.S.; Mahmood, K.; Macphail, M.; Guzzetta, J.M.; Haller, A.A.; Liu, H.; Kaur, J.; Lawlor, H.A.; Stillman, E.A.; Schickli, J.H.; et al. A host-range restricted parainfluenza virus type 3 (PIV3) expressing the human metapneumovirus (hMPV) fusion protein elicits protective immunity in african green monkeys. Vaccine 2005, 23, 1657-1667.

93. Skiadopoulos, M.H.; Biacchesi, S.; Buchholz, U.J.; Riggs, J.M.; Surman, S.R.; Amaro-Carambot, E.; McAuliffe, J.M.; Elkins, W.R.; St Claire, M.; Collins, P.L.; et al. The two major human metapneumovirus genetic lineages are highly related antigenically, and the fusion (F) protein is a major contributor to this antigenic relatedness. J. Virol. 2004, 78, 6927-6937.

94. Herfst, S.; Fouchier, R.A. Vaccination approaches to combat human metapneumovirus lower respiratory tract infections. J. Clin. Virol. 2008, 41, 49-52.

95. Herfst, S.; de Graaf, M.; Schrauwen, E.J.; Ulbrandt, N.D.; Barnes, A.S.; Senthil, K.; Osterhaus, A.D.; Fouchier, R.A.; van den Hoogen, B.G. Immunization of syrian golden hamsters with $f$ subunit vaccine of human metapneumovirus induces protection against challenge with homologous or heterologous strains. J. Gen. Virol. 2007, 88, 2702-2709.

96. Cseke, G.; Wright, D.W.; Tollefson, S.J.; Johnson, J.E.; Crowe, J.E., Jr.; Williams, J.V. Human metapneumovirus fusion protein vaccines that are immunogenic and protective in cotton rats. J. Virol. 2007, 81, 698-707.

97. Mok, H.; Tollefson, S.J.; Podsiad, A.B.; Shepherd, B.E.; Polosukhin, V.V.; Johnston, R.E.; Williams, J.V.; Crowe, J.E., Jr. An alphavirus replicon-based human metapneumovirus vaccine is immunogenic and protective in mice and cotton rats. J. Virol. 2008, 82, 11410-11418.

98. Ryder, A.B.; Tollefson, S.J.; Podsiad, A.B.; Johnson, J.E.; Williams, J.V. Soluble recombinant human metapneumovirus $\mathrm{g}$ protein is immunogenic but not protective. Vaccine 2010, 28, 4145-4152. 
99. Yim, K.C.; Cragin, R.P.; Boukhvalova, M.S.; Blanco, J.C.; Hamlin, M.E.; Boivin, G.; Porter, D.D.; Prince, G.A. Human metapneumovirus: Enhanced pulmonary disease in cotton rats immunized with formalin-inactivated virus vaccine and challenged. Vaccine 2007, 25, 5034-5040.

100. Hamelin, M.E.; Couture, C.; Sackett, M.K.; Boivin, G. Enhanced lung disease and th2 response following human metapneumovirus infection in mice immunized with the inactivated virus. J. Gen. Virol. 2007, 88, 3391-3400.

101. De Swart, R.L.; van den Hoogen, B.G.; Kuiken, T.; Herfst, S.; van Amerongen, G.; Yuksel, S.; Sprong, L.; Osterhaus, A.D. Immunization of macaques with formalin-inactivated human metapneumovirus induces hypersensitivity to hMPV infection. Vaccine 2007, 25, 8518-8528.

102. Buchholz, U.J.; Nagashima, K.; Murphy, B.R.; Collins, P.L. Live vaccines for human metapneumovirus designed by reverse genetics. Expet. Rev. Vaccine. 2006, 5, 695-706.

103. Boivin, G.; De Serres, G.; Hamelin, M.E.; Cote, S.; Argouin, M.; Tremblay, G.; Maranda-Aubut, R.; Sauvageau, C.; Ouakki, M.; Boulianne, N.; et al. An outbreak of severe respiratory tract infection due to human metapneumovirus in a long-term care facility. Clin. Infect. Dis. 2007, 44, 1152-1158.

104. Osbourn, M.; McPhie, K.A.; Ratnamohan, V.M.; Dwyer, D.E.; Durrheim, D.N. Outbreak of human metapneumovirus infection in a residential aged care facility. Comm. Dis. Intell. 2009, $33,38-40$.

105. Louie, J.K.; Schnurr, D.P.; Pan, C.Y.; Kiang, D.; Carter, C.; Tougaw, S.; Ventura, J.; Norman, A.; Belmusto, V.; Rosenberg, J.; et al. A summer outbreak of human metapneumovirus infection in a long-term-care facility. J. Infect. Dis. 2007, 196, 705-708.

106. http://www.wip.nl.

107. Siegel, J.D.; Rhinehart, E.; Jackson, M.; Chiarello, L.; Health Care Infection Control Practices Advisory Committee. 2007 guideline for isolation precautions: Preventing transmission of infectious agents in health care settings. Am. J. Infect. Control 2007, 35, S65-S164.

108. Duncan, C.B.; Walsh, E.E.; Peterson, D.R.; Lee, F.E.; Falsey, A.R. Risk factors for respiratory failure associated with respiratory syncytial virus infection in adults. J. Infect. Dis. 2009, 200, 1242-1246.

109. Beckham, J.D.; Cadena, A.; Lin, J.; Piedra, P.A.; Glezen, W.P.; Greenberg, S.B.; Atmar, R.L. Respiratory viral infections in patients with chronic, obstructive pulmonary disease. J. Infect. 2005, 50, 322-330.

110. Walsh, E.E.; Falsey, A.R.; Hennessey, P.A. Respiratory syncytial and other virus infections in persons with chronic cardiopulmonary disease. Am. J. Respir. Crit. Care Med. 1999, 160, 791-795.

111. Johnstone, J.; Majumdar, S.R.; Fox, J.D.; Marrie, T.J. Viral Infection in Adults Hospitalized with Community-Acquired Pneumonia: Prevalence, Pathogens, and Presentation. Chest 2008, 134, 1141-1148.

112. Hamelin, M.E.; Boivin, G. Human metapneumovirus: A ubiquitous and long-standing respiratory pathogen. J. Pediatr. Infect. Dis. 2005, 24, S203-S207. 
113. Martinello, R.A.; Esper, F.; Weibel, C.; Ferguson, D.; Landry, M.L.; Kahn, J.S. Human metapneumovirus and exacerbations of chronic obstructive pulmonary disease. J. Infect. 2006, $53,248-254$.

114. Williams, J.V.; Crowe, J.E., Jr.; Enriquez, R.; Minton, P.; Peebles, R.S., Jr; Hamilton, R.G.; Higgins, S.; Griffin, M.; Hartert, T.V. Human metapneumovirus infection plays an etiologic role in acute asthma exacerbations requiring hospitalization in adults. J. Infect. Dis. 2005, 192, $1149-1153$

115. Williams, J.V.; Crowe, J.E.,Jr; Enriquez, R.; Minton, P.; Peebles, R.S.,Jr; Hamilton, R.G.; Higgins, S.; Griffin, M.; Hartert, T.V. Human metapneumovirus infection plays an etiologic role in acute asthma exacerbations requiring hospitalization in adults. J. Infect. Dis. 2005, 192, $1149-1153$

116. Haas, L.E.; de Rijk, N.X.; Thijsen, S.F. Human metapneumovirus infections on the ICU: A report of three cases. Ann. Intensive Care 2012, 2, 30.

117. Miller, R.A. The aging immune system: Primer and prospectus. Science 1996, 273, 70-74.

118. Honda, H.; Iwahashi, J.; Kashiwagi, T.; Imamura, Y.; Hamada, N.; Anraku, T.; Ueda, S.; Kanda, T.; Takahashi, T.; Morimoto, S. Outbreak of human metapneumovirus infection in elderly inpatients in Japan. J. Am. Geriatr. Soc. 2006, 54, 177-180.

119. Liao, R.S.; Appelgate, D.M.; Pelz, R.K. An outbreak of severe respiratory tract infection due to human metapneumovirus in a long-term care facility for the elderly in Oregon. J. Clin. Virol. 2012, 53, 171-173.

120. Raza, K.; Ismailjee, S.B.; Crespo, M.; Studer, S.M.; Sanghavi, S.; Paterson, D.L.; Kwak, E.J.; Rinaldo, C.R., Jr.; Pilewski, J.M.; McCurry, K.R.; et al. Successful outcome of human metapneumovirus (hMPV) pneumonia in a lung transplant recipient treated with intravenous ribavirin. J. Heart Lung Transplant. 2007, 26, 862-864.

121. Huck, B.; Egger, M.; Bertz, H.; Peyerl-Hoffman, G.; Kern, W.V.; Neumann-Haefelin, D.; Falcone, V. Human metapneumovirus infection in a hematopoietic stem cell transplant recipient with relapsed multiple myeloma and rapidly progressing lung cancer. J. Clin. Microbiol. 2006, 44, 2300-2303.

122. Kamble, R.T.; Bollard, C.; Demmler, G.; LaSala, P.R.; Carrum, G. Human metapneumovirus infection in a hematopoietic transplant recipient. Bone Marrow Transplant. 2007, 40, 699-700.

123. Muller, A.; Kupfer, B.; Vehreschild, J.; Cornely, O.; Kaiser, R.; Seifert, H.; Viazov, S.; Tillmann, R.L.; Franzen, C.; Simon, A.; et al. Fatal pneumonia associated with human metapneumovirus (HMPV) in a patient with myeloid leukemia and adenocarcinoma in the lung. Eur. J. Med. Res. 2007, 12, 183-184.

124. Kamboj, M.; Gerbin, M.; Huang, C.K.; Brennan, C.; Stiles, J.; Balashov, S.; Park, S.; Kiehn, T.E.; Perlin, D.S.; Pamer, E.G.; et al. Clinical Characterization of Human Metapneumovirus Infection among Patients with Cancer. J. Infect. 2008, 57, 464-471. 
125. Williams, J.V.; Martino, R.; Rabella, N.; Otegui, M.; Parody, R.; Heck, J.M.; Crowe, J.E., Jr. A prospective study comparing human metapneumovirus with other respiratory viruses in adults with hematologic malignancies and respiratory tract infections. J. Infect. Dis. 2005, 192, 1061-1065.

126. Martino, R.; Porras, R.P.; Rabella, N.; Williams, J.V.; Ramila, E.; Margall, N.; Labeaga, R.; Crowe, J.E., Jr.; Coll, P.; Sierra, J. Prospective study of the incidence, clinical features, and outcome of symptomatic upper and lower respiratory tract infections by respiratory viruses in adult recipients of hematopoietic stem cell transplants for hematologic malignancies. Biol. Blood Marrow Transplant. 2005, 11, 781-796.

127. Peck, A.J.; Englund, J.A.; Kuypers, J.; Guthrie, K.A.; Corey, L.; Morrow, R.; Hackman, R.C.; Cent, A.; Boeckh, M. Respiratory virus infection among hematopoietic cell transplant recipients: Evidence for asymptomatic parainfluenza virus infection. Blood 2007, 110, 1681-1688.

128. Debiaggi, M.; Canducci, F.; Sampaolo, M.; Marinozzi, M.C.; Parea, M.; Terulla, C.; Colombo, A.A.; Alessandrino, E.P.; Bragotti, L.Z.; Arghittu, M.; et al. Persistent symptomless human metapneumovirus infection in hematopoietic stem cell transplant recipients. J. Infect. Dis. 2006, 194, 474-478.

129. Sumino, K.C.; Agapov, E.; Pierce, R.A.; Trulock, E.P.; Pfeifer, J.D.; Ritter, J.H.; Gaudreault-Keener, M.; Storch, G.A.; Holtzman, M.J. Detection of severe human metapneumovirus infection by real-time polymerase chain reaction and histopathological assessment. J. Infect. Dis. 2005, 192, 1052-1060.

130. Debur, M.C.; Vidal, L.R.; Stroparo, E.; Nogueira, M.B.; Almeida, S.M.; Takahashi, G.A.; Rotta, I.; Pereira, L.A.; Silveira, C.S.; Delfraro, A.; et al. Impact of human metapneumovirus infection on in and outpatients for the years 2006-2008 in Southern Brazil. Memorias do Instituto Oswaldo Cruz 2010, 105, 1010-1018.

131. Englund, J.A.; Boeckh, M.; Kuypers, J.; Nichols, W.G.; Hackman, R.C.; Morrow, R.A.; Fredricks, D.N.; Corey, L. Brief communication: Fatal human metapneumovirus infection in stem-cell transplant recipients. Ann. Intern. Med. 2006, 144, 344-349.

132. Cane, P.A.; van den Hoogen, B.G.; Chakrabarti, S.; Fegan, C.D.; Osterhaus, A.D. Human metapneumovirus in a haematopoietic stem cell transplant recipient with fatal lower respiratory tract disease. Bone Marrow Transplant. 2003, 31, 309-310.

133. Weinberg, A.; Lyu, D.M.; Li, S.; Marquesen, J.; Zamora, M.R. Incidence and morbidity of human metapneumovirus and other community-acquired respiratory viruses in lung transplant recipients. Transpl. Infect. Dis. 2010, 12, 330-335.

134. Larcher, C.; Geltner, C.; Fischer, H.; Nachbaur, D.; Muller, L.C.; Huemer, H.P. Human metapneumovirus infection in lung transplant recipients: Clinical presentation and epidemiology. J. Heart Lung Transplant. 2005, 24, 1891-1901.

135. Kumar, D.; Erdman, D.; Keshavjee, S.; Peret, T.; Tellier, R.; Hadjiliadis, D.; Johnson, G.; Ayers, M.; Siegal, D.; Humar, A. Clinical impact of community-acquired respiratory viruses on bronchiolitis obliterans after lung transplant. Am. J. Transplant. 2005, 5, 2031-2036. 
136. Sharples, L.D.; McNeil, K.; Stewart, S.; Wallwork, J. Risk factors for bronchiolitis obliterans: A systematic review of recent publications. J. Heart Lung Transplant. 2002, 21, 271-281.

137. Kukavica-Ibrulj, I.; Hamelin, M.E.; Prince, G.A.; Gagnon, C.; Bergeron, Y.; Bergeron, M.G.; Boivin, G. Infection with human metapneumovirus predisposes mice to severe pneumococcal pneumonia. J. Virol. 2009, 83, 1341-1349.

138. Ludewick, H.P.; Aerts, L.; Hamelin, M.E.; Boivin, G. Long-term impairment of streptococcus pneumoniae lung clearance is observed after initial infection with influenza a virus but not human metapneumovirus in mice. J. Gen. Virol. 2011, 92, 1662-1665.

139. Louie, J.K.; Schnurr, D.P.; Pan, C.Y.; Kiang, D.; Carter, C.; Tougaw, S.; Ventura, J.; Norman, A.; Belmusto, V.; Rosenberg, J.; et al. A summer outbreak of human metapneumovirus infection in a long-term-care facility. J. Infect. Dis. 2007, 196, 705-708.

140. Bonney, D.; Razali, H.; Turner, A.; Will, A. Successful treatment of human metapneumovirus pneumonia using combination therapy with intravenous ribavirin and immune globulin. Br. J. Haematol. 2009, 145, 667-669.

141. Safdar, A. Immune modulatory activity of ribavirin for serious human metapneumovirus disease: Early i.v. therapy may improve outcomes in immunosuppressed SCT recipients. Bone Marrow Transplant. 2008, 41, 707-708.

142. Shachor-Meyouhas, Y.; Ben-Barak, A.; Kassis, I. Treatment with oral ribavirin and IVIG of severe human metapneumovirus pneumonia (HMPV) in immune compromised child. Pediatr. Blood Canc. 2011, 57, 350-351. 
Reprinted from Viruses. Cite as: Derscheid, R.J.; Ackermann, M.R. Perinatal Lamb Model of Respiratory Syncytial Virus (RSV) Infection. Viruses 2012, 4, 2359-2378.

Article

\title{
Perinatal Lamb Model of Respiratory Syncytial Virus (RSV) Infection
}

\section{Rachel J. Derscheid and Mark R. Ackermann *}

Department of Veterinary Pathology, 2738 College of Veterinary Medicine, Iowa State University, Ames, IA 50011, USA; E-Mail: rdersch@iastate.edu

* Author to which correspondence should be addressed; E-Mail: mackerma@iastate.edu; Tel.: +1-515-294-3647; Fax: +1-515-294-5423.

Received: 25 September 2012; in revised form: 17 October 2012 / Accepted: 18 October 2012 /

Published: 23 October 2012

\begin{abstract}
Respiratory syncytial virus (RSV) is the most frequent cause of bronchiolitis in infants and children worldwide. Many animal models are used to study RSV, but most studies investigate disease in adult animals which does not address the unique physiology and immunology that makes infants more susceptible. The perinatal (preterm and term) lamb is a useful model of infant RSV disease as lambs have similar pulmonary structure including airway branching, Clara and type II cells, submucosal glands and Duox/lactoperoxidase (LPO) oxidative system, and prenatal alveologenesis. Lambs can be born preterm ( $90 \%$ gestation) and survive for experimentation although both preterm and term lambs are susceptible to ovine, bovine and human strains of RSV and develop clinical symptoms including fever, tachypnea, and malaise as well as mild to moderate gross and histologic lesions including bronchiolitis with epithelial injury, neutrophil infiltration and syncytial cell formation. RSV disease in preterm lambs is more severe than in term lambs; disease is progressively less in adults and age-dependent susceptibility is a feature similar to humans. Innate and adaptive immune responses by perinatal lambs closely parallel those of infants. The model is used to test therapeutic regimens, risk factors such as maternal ethanol consumption, and formalin inactivated RSV vaccines.
\end{abstract}

Keywords: airways; bronchiolitis; infants; innate immunity; lambs; lung; ovine; perinatal; premature; preterm; respiratory syncytial virus (RSV) 


\section{Introduction}

Respiratory syncytial virus (RSV) is the most frequent cause of bronchiolitis in infants and children worldwide [1]. RSV was first discovered in 1955 in chimpanzees and two years later was isolated from an outbreak of respiratory disease in infants $[2,3]$. In the 57 years since that time, only one specific preventative measure, the humanized monoclonal antibody Palivizumab, and one therapy, the antiviral ribavirin, have been successfully developed for use in a clinical setting [4-7]. Vaccines are also not available as a result of vaccine trials of the 1960s, when enhanced disease occurred in infants, resulting in severe disease including two mortalities and hospitalization of $80 \%$ of those vaccinated [8]. Despite 50 years of research since these studies, the cellular and molecular mechanisms underlying severe disease are not fully understood [9].

Risk factors of severe RSV disease in infants include premature birth, congenital heart defects, neuromuscular deficits, Down's syndrome, immunodeficiency/compromise, and bronchopulmonary dysplasia [9-11]. Of these at-risk populations, the largest group is those born premature. A survey in the United States reported premature birth rate at $12.3 \%$, or approximately one half million [12] and worldwide, there are an estimated 13 million preterm births annually [13]. While epidemiologic evidence can identify individuals at increased risk for RSV, the mechanisms of severe disease are poorly understood. The vast majority of studies in animal models are performed in mature/adult animals, and many studies of human RSV infection are also performed in adults. Rodents, non-human primates, ferrets, cattle, and several other species have been utilized to study RSV; however, studies investigating disease in mature/adult animals fail to address the unique structure, cellularity, physiology, and immunology that contribute to the increased disease severity that can occur in perinatal infants infected with RSV.

The perinatal (preterm and term) lamb model has been used by several investigators to study RSV disease and paramyxoviral infections of infants as lambs have similar pulmonary development and are susceptible to ruminant and human strains of RSV and parainfluenza virus-3 [14-20]. Lambs develop mild clinical symptoms including fever, tachypnea or increased expiratory effort (wheeze), and malaise as well as mild to moderate gross and histologic lesions when experimentally infected with bovine (bRSV) or human (hRSV) strains of RSV [14-16,19,20]. Bovine strains infect cattle and sheep and have much homology with hRSV strains. Disease severity is increased in preterm lambs infected with bRSV which is similar to the increased disease severity that can occur in human infants born preterm infected with human strains of RSV [19,21]. Thus, the perinatal lamb model has a strong foundation and forms a valid model for RSV as evidenced by clinical alteration of respiration as well as gross and histologic lesions.

\section{Perinatal and Adult Lung in Regards to RSV Infection}

The structural differences between infant and adult lung at the alveolar level include fewer as well as thicker-walled alveoli in the infant [22]. The primary effect of this difference is on overall decreased efficiency of gas exchange; its implication in disease is more severe clinical symptoms with less 
inflammation. Airway submucosal glands are present in the human trachea at 13 weeks' gestation with rapid progressive appearance in the more distal areas; however, the gland is present but its structure is primitive, lacking the branching of more mature glands [23]. Submucosal glands are becoming increasingly recognized for their role in innate defense through expression of secretory products such as lactoperoxidase into the airways. Goblet cells form as early as 13 weeks of fetal age and contribute significantly to airway mucin production. With RSV infection of mice with the Line 19 strain, increased Goblet cell production occurs through a Toll-like receptor 7 (TLR7)-Interleukin-17 (IL-17) and IL-23 pathway [24]. The number and type of Clara cells increase in the lung during fetal and perinatal development $[25,26]$. As progenitor cells, Clara cells at different stages of maturity are identified by ultrastructural characteristics [27]. While Clara cell secretory protein (CSSP; also known as CC10) can be expressed as early as 10 weeks of gestation in humans [28], and almost certainly by 15 weeks gestation [25] the production of CCSP of Clara cells indicates a presence of cells but not necessarily maturity. Clara cells serve as a common progenitor for Clara and type II cells, serve a role in innate immunity through expression of immunomodulatory products such as $\mathrm{CC} 10$, and have a high level of cytochrome P450 enzymes that function in detoxification of xenobiotics [29,30]. Type II cells, which produce surfactant, surfactant proteins, other anti-RSV peptides and proteins, and serve as a progenitor for type I cells, are present by 20 weeks of gestation and continually form with increasing fetal age. Type II cells, Clara cells, pseudostratified ciliated cells and other airway epithelial subtypes are targets for RSV replication. Airway epithelium of the neonate, once infected by RSV, alters re-epithelialization and subsequent airway responses in the adult [31].

Cells of the innate immune system are decreased in number and often operate with decreased efficiency in infants. Dendritic cells (DCs) in neonates are reduced and the ratio of myeloid to plasmacytoid DCs is inversed as compared to adults [32]. Preterm DCs have a reduced ability to produce interferon (IFN) and decreased ability to stimulate a T helper-1 (Th1) response, although contrary to observations in mice this difference alone does not predispose infants to a Th2-skewed response [32]. Dendritic cells are integral to immune response to RSV infection and increased numbers are present in nasal washes of infants with severe RSV [33]. Neonates have a reduced proliferation pool as well as a reduced storage pool of neutrophils and those in circulation have an impaired response to chemotaxins and deficiencies at multiple stages of migration including rolling adhesion, transmigration, and lamellipodia formation. Once at the site of infection, neutrophils of infants do not function at the same level as those of adults [34]. Neutrophils of term infants have about half the amount of lactoferrin, a 30\% reduction in bactericidial/permeability increasing protein (BPI), and impaired oxidase activity [34-36]. Neutrophils are a key component in RSV pathology, with an influx into bronchioles and, to a lesser extent, alveoli [21]. Neutrophils undergoing NETosis (a form of neutrophil apoptotic-like death mediated by pathogens) can release neutrophilic extracellular traps (NETs) composed of DNA strands, histones, and antimicrobial peptides such as cathelicidins which can entrap bacteria and have antibacterial activity [37]. NETs have not been linked with RSV, but illustrate a mechanism of innate protection induced by neutrophils and may have a role against bacterial infections secondary to RSV. Neutrophilic inflammation in any individual is always in a 
precarious balance between killing of pathogen and damage to native tissue. Impaired recruitment and function may be in some degree preventative of collateral damage to lung tissue, but it also decreases the efficiency of pathogen killing, while the presence of neutrophils in small airways contributes to the clinical symptoms of labored breathing (increased expiratory effort, abdominal breathing) and wheeze.

Both the inflammatory mediators that are elicited upon RSV infection as well as the response to these can be different between neonates and adults. Co-cultures of RSV-infected DCs and T cells of either adults or umbilical cord blood elicited markedly different cytokine profiles with the primary differences attributed to differences in response to transforming growth factor beta (TGF- $\beta$ ) [33]. The response to chemokines in neonates varies from adult response due to the altered number and function of leukocytes as well as altered receptor expression. In vitro stimulation of term infant monocytes and antigen presenting cells shows decreased expression of tumor necrosis factor alpha (TNF- $\alpha$ ), IFN- $\alpha$, IFN- $\gamma$, interleukin 12 (IL-12), and IL-1 $\beta$, but increased expression of IL-6, IL-8, and IL-10 [38].

\section{Lambs as a Model of RSV Infection of Infants}

As recently reviewed by Bem, Domachowske, and Rosenberg, a number of animal models, including chimpanzee, cotton rat, mice and cattle have been used to recapitulate aspects of the different manifestations of human RSV disease [39-41]. While no animal model perfectly mimics all forms of human disease, the perinatal lamb has features that are beneficial to studies with RSV (Table 1). Similar to humans, sheep are outbred allowing manifestation of the diverse nature of response to RSV that occurs in infants. Also, the ovine lung bears a close resemblance in development, airway structure and cellularity to human lung. Sheep (and cattle) have a bronchus that branches from the distal tracheal mucosa into the right cranial lobe. This bronchus can be used for fiberoptic bronchoscope inoculations if desired; however, it requires dexterity due to a near ninety degree turn as the bronchus comes off the trachea in somewhat of a perpendicular fashion. Lung lobes of lambs include a right cranial lobe (with a cranial and caudal part), a left cranial lobe, left and right middle and caudal lobes, and an accessory lobe. The lung lobes are somewhat similar in size to those of a human infant allowing substantial tissue for tissue sampling. Airway branching patterns of lambs resembles infants, unlike rodents [42] and alveolar development (alveologenesis) in human fetus and lambs begins prenatally, in contrast to the post-natal alveolar development that occurs in mice/rodents [42-46]. In addition, the trachea and bronchi are lined by pseudostratified ciliated epithelium and have submucosal glands which contribute to mucus secretion and lactoperoxidase production similar to human infants but is in contrast to rodents which have few or limited submucosal gland structures [42-47].

Airways of human and sheep express all components of an oxidative system that consists of two $\mathrm{H}_{2} \mathrm{O}_{2}$-generating enzymes of airway epithelia, dual oxidase (Duox) 1 and 2, along with a pseudohalide anion (thiocyanate, $\mathrm{SCN}^{-}$), and the enzyme lactoperoxidase (LPO) [47-50]. Hydrogen peroxide is produced by Duox enzymes onto the apical extracellular space where it reacts with $\mathrm{SCN}^{-}$in a LPO-catalyzed reaction to form hypothiocyanate molecule $\mathrm{OSCN}^{-}\left(\mathrm{H}_{2} \mathrm{O}_{2}+\mathrm{SCN}^{-} \rightarrow \mathrm{OSCN}^{-}\right)$[49-57]. The Duox/LPO/SCN ${ }^{-}$system generates sufficient $\mathrm{OSCN}^{-}$to eliminate bacteria in vitro and in vivo [58-60]. In in vitro assays the addition of iodide $(\Gamma)$ to the Duox/LPO enzyme products 
generates hypoiodous acid $\left(\mathrm{H}_{2} \mathrm{O}_{2}+\mathrm{I}^{-} \rightarrow \mathrm{HOI}\right)$ instead of the physiological product $\mathrm{OSCN}^{-}$which has potent microbicidal activity against bacteria and viruses, including activity against respiratory syncytial virus (RSV), whereas $\mathrm{OSCN}^{-}$exhibits little antiviral activity [61]. Inhibition of lactoperoxidase in sheep reduces clearance of bacteria such as Mannheimia haemolytica, and we have shown that oral administration of iodide in the form of potassium iodide greatly increases iodide levels on the surface airway liquid of lambs [62]. Thus, lambs have a fully functional Duox/LPO oxidative system [62] which can be used to assess Duox/LPO anti-RSV activity in vivo.

Epithelial cells of perinatal (preterm and term) lamb lung also have close similarities to human infants. This is especially important since RSV replication within bronchioles results in bronchiolitis and accumulation of neutrophils and cell debris which can occlude or partially occlude the airway lumen and also affect bronchiolar dilation and contraction. Thus, the bronchiole is both an important site for RSV replication within non-ciliated cells and type II cells at the bronchiolar/alveolar junction and also a location where RSV-induced cell injury and inflammation can greatly impair airflow and gaseous exchange. Clara cells are a major cell type of non-ciliated cells that line bronchiolar epithelium. Bronchiolar epithelium in lambs is $18 \%-22 \%$ Clara cells which is similar to human lung, whereas mouse lung has a large $(50 \%+)$ population of Clara cells in bronchiolar airway $[25-27,45,63,64]$. The Clara cells of sheep have ultrastructural features more similar to human Clara cells than most other species $[27,64]$.

Damage to or dysfunction of Clara cells creates a proinflammatory environment due to the loss of their immunomodulatory secretions. Clara cells secrete large amounts of CC10 (also known as CCSP, CC16, secretoglobin, and uteroglobin) which is increased in bronchoalveolar lavage fluid (BALF) and serum during acute injury such as smoke inhalation or application of pneumotoxicants (naphthalene, 4-ipomeanol (4-IM), chloroethylene), but decreased in chronic or dysplastic airway dysfunction (asthma, chronic obstructive pulmonary disease, or bronchopulmonary dysplasia (BPD) [25,29,63,65]. CC10-deficient mice have increased inflammatory responses and persistence of RSV after infection while restoration of $\mathrm{CC} 10$ abrogates these effects [30] and Clara cells damaged by 4-ipomeanol enhanced RSV disease severity in calves [66]. CC10 may not only have a protective effect on Clara cells, but also stimulate development of Clara cells [63]. Clara (and type II) cells also produce substances with known anti-RSV activity including: surfactant proteins A (SP-A) and D (SP-D) which bind and opsonize RSV, beta-defensins, beta-galactoside-binding protein, and RSV receptors such as Toll-like receptor-4 (TLR-4), retinoic acid inducible gene-I (RIG-I) which triggers epithelial responses as well as inflammatory/immunomodulatory substances [29,30,67-70]. CC10 is reduced in lambs preterm which may contribute to the increased susceptibility of preterm ovine lung to RSV and the reduced levels of innate immune gene expression such as CC10 and SP-A [71]. To our knowledge, Clara cell distribution and $\mathrm{CC} 10$ expression has not been assessed during the ontogeny of human fetal lung. If similar to lambs, reduced Clara cell numbers may affect RSV susceptibility.

Type II cells are present in distal lung bronchioles and alveoli and proliferate upon bronchiolar and alveolar injury to replace other type II cells or alveolar type I cells [22]. Type II cells produce surfactant, surfactant proteins, defensins, cytokines, chemokines, and RIG-I and are infected by RSV 
in both lambs and humans [21,72]. Similar to preterm human infants, numbers of type II (CD208+) cells in preterm lamb lung are low and immature (increased glycogen retention) but are increased and have increased maturity at birth [73].

Preterm lambs have reduced neutrophil expression of myeloperoxidase and reduced alveolar macrophage expression of nitric oxide compared to term lambs [74]. Pulmonary dendritic cells isolated from term lamb lung differ from those isolated from adult lung in terms of antigen expression and maturation [75]. Ovine pulmonary dendritic cells from term lung support bRSV replication and have enhanced interleukin (IL)-4 and IL-10 gene transcripts [76,77]. Alveolar macrophages of term and adult sheep have differential expression of cytokines in response to bRSV and Toll-like receptor ligation [75,77]. With bRSV and hRSV A2 infection, lymphocytic infiltration of the lung includes CD4+ and CD8+ cells and tracheobronchial lymph nodes undergo marked lymphocytic hyperplasia characterized by increased paracortical and follicular lymphocytes [19,78].

In terms of innate immune and adaptive responses in developing lamb lung, ontogeny studies of fetal and perinatal lung tissue identified reduced mRNA levels of SP-A, SP-D, and sheep beta defensin-1 (SBD-1) in preterm tissues [79], while another study showed no significant difference in surfactant mRNA and very low levels of SBD-1 in prenatal and term tissue versus significantly higher levels in adult sheep [71]. Messenger RNA expression of Toll-like receptor-4 and -8 (TLR-4 and -8) in the lung, increases throughout gestation but for a sharp drop in TLR-4 mRNA levels in term lambs [71]. TLR-4 is associated with CD14 that recognizes the F protein of RSV [10,80]. Binding of the TLR-4/CD14 complex activates NF- $\kappa \beta$, eventually leading to secretion of IL-8, IL-10, IL-6, as well as increased expression of TLR-4 on epithelial cell [80]. Pulmonary TLR-7 mRNA is also significantly lower in term lambs than in preterm or adult animals [37]. TLR-7 recognizes single-stranded RNA (viral); TLR-7 mRNA is increased in infants with naturally-occurring RSV compared to infants with non-RSV bronchiolitis [71]. TLR-3 binds double stranded RNA, a replication intermediate of RSV. Term and adult lambs have similar levels of TLR-3 expression in lung, but preterm lambs have significantly higher levels [71]. Interferon gamma TNF- $\alpha$, IL-6, IL-8, and monocyte chemotactic protein-1 (MCP-1) increased throughout prenatal development, peaking at birth and decreasing into adulthood [71]. Differences in TLR, cytokine and chemokine expression in perinatal lung compared to adult may affect RSV binding, replication, and immune responses.

Lambs, other ruminants, swine and other species receive maternal immunoglobulins only through ingestion of colostrum. This is because transplacental passage of immunoglobulins does not occur as in rodents and human infants. Therefore, lambs deprived of colostrum are devoid of maternal immunoglobulin and thereby lack maternal antibodies to RSV. This allows great flexibility in altering the serum levels of maternal immunoglobulins in terms lambs which lends itself well to studies assessing the role of immunoglobulins in protecting against RSV infection. In clean facilities with proper management, colostrum-deprived lambs survive and lack secondary bacterial infections unlike calves which often die in several days if not raised in gnotobiotic conditions. Thus, lambs lacking maternal immunoglobulin can be used to test vaccines without interference by maternal immunoglobulin. Because RSV is more severe in infants born preterm, and lack of antibody affinity 
maturation has a significant role in enhanced RSV disease [81], the extent to which fetal and preterm infants may respond to a particular vaccine (without interference by transplacental passage of maternal immunoglobulin) can be tested by vaccinated lambs in utero, preterm, at birth and a few weeks after birth. In addition, the effect of maternal immunoglobulin on perinatal vaccination can be further assessed by feeding lambs: colostrum lacking RSV antibody, colostrum with low neutralizing RSV antibody, colostrum with high neutralizing RSV titers, and colostrum with antibodies to formalin-inactivated RSV (FI-RSV) vaccine. Lambs can be infected with RSV by several routes, each having advantages and disadvantages. Intratracheal inoculation is a relatively rapid injection of RSV in fluid media that bypasses the nasal cavity and larynx allowing the fluid to be distributed directly into the tracheal lumen with drainage into the major bronchi. At the tracheal carinae, the inoculated fluid does not divide evenly and one side of the lung receives more or less than the other. The fluid volume inoculated can range from $1 \mathrm{~mL}$ to $10 \mathrm{~mL}$ or more. This volume of fluid delivered rushes across the tracheal luminal surface and can overwhelm the comparatively small amount of air-surface liquid and its antimicrobial contents (e.g., lactoferrin, antimicrobial peptides, oxidative products). Bronchoscopic inoculation of RSV in fluid media has the benefit of very precise delivery with a fluid volume of virus into the bronchi that is more accurately localized than intratracheal inoculation but is still is often rapidly injected resulting in a relatively large, single volume of a fluid inoculum that can locally overwhelm the air-surface liquid. Aerosolized RSV inoculum delivered using atomizer devices into the nares results in deposition of droplets in the upper and lower respiratory tract; however, these mists are often rapidly delivered and the particles (ranging from 20 to $100 \mathrm{um}$ in diameter) are too large for consistent accumulation into the distal bronchioles and alveoli. Both aerosolized inoculum and nebulized mists reduce the amount of fluid volume deposited onto the nasal mucosa and thereby decrease the chance of the fluid volume of the inoculum to overwhelm the air-surface liquid volume. Nebulized mist can be delivered over a specified time and deposits RSV inoculum in the upper and lower respiratory tract allowing deposition to the bronchioles and alveoli. Thus, there is much flexibility in the type of inoculation route to deliver RSV to the lung which can be tailored to assess anti-RSV drugs, vaccines, or RSV pathogenesis. We prefer nebulization for studies that require even distribution of virus throughout the lung especially studies assessing mucosal innate immune responses.

There are some hurdles to overcome in using lambs for RSV studies. First, a vendor that can supply healthy lambs or a healthy flock is needed. Second, availability is limited to spring and fall lambing seasons; however, the fall lambing season in some areas, including the midwest area of the United States, can occur from September to December and the "spring" lambing season can last from January to June. Investigators with access to multiple vendors that differ in breeding regimens can have a very broad availability. Third, facilities for handling lambs and some expertise in ruminant anatomy, physiology and medicine is beneficial especially for studies of colostrum-deprived lambs which require a very clean environment, although most facilities for dogs or other midsized animals and a veterinarian on staff can easily allow for studies in lambs. Fourth, reagents to ovine proteins can be difficult to obtain; however, some antibodies to bovine proteins cross-react with ovine and the advent of proteome profiles can overcome these issues. Gene expression assays are not an issue with ovine 
samples since the ovine genome is becoming further clarified, primers and probes to bovine work well, and next-generation sequencing (NGS) allows extensive coverage. Fifth, premature lambs do not survive but preterm lambs (90\% gestation) have a good survival rate [19].

Lambs are naturally susceptible to ovine and bovine RSV strains and can be experimentally infected with a number of strains. Respiratory syncytial virus (RSV) is in the Paramyxoviridae family, subfamily Pneumovirinae. The Pneumovirus genus includes human, bovine, ovine, and caprine respiratory syncytial viruses as well as murine pneumovirus (pneumonia virus of mice). The four respiratory syncytial viruses are closely related while murine pneumovirus shares less homology. Initial studies by Lehmkuhl, Cutlip, Belknap, Lapin and others determined susceptibility of lambs to bRSV experimentally and characterized histological and ultrastructural lesions as well as immunoglobulin responses to infection [14-17]. More recent studies have assessed infection by human strains of RSV (hRSV) [20,82]. There are two primary strains of RSV, A and B, with many genetically divergent substrains of each. Human RSV A2 strain replicates in lambs and causes disease but as this is a laboratory-adapted strain, other strains may have enhanced virulence [20,82]. Infection with RSV in term as well as older (6 month old) lambs will cause clinical disease exhibited as fever, listlessness, and tachypnea, as accompanied by gross and histologic lesions. Histologically, lesions caused by bRSV and strains of hRSV are similar to those seen in infants and cattle and include bronchiolitis with epithelial cell necrosis, syncytial cell formation, hyperplasia of nearby epithelium (subacutely) and infiltrates of neutrophils with occasional macrophages [15,19,20,83,84] (Figure 1). Neutrophil infiltration in lambs is associated with increased levels of IL-8 expression, which is similar to human infants; rodents do not produce IL-8 but instead express the chemokine $\mathrm{KC}$ which has some overlap with IL-8 in function [78]. The adventitia of infected bronchioles is infiltrated by lymphocytes (CD4+ and $\mathrm{CD} 8+$ ) and plasma cells and alveolar lumens contain small amounts of cell debris with occasional alveolar macrophages [15,19,21,78,80]. Tracheobronchial lymph nodes become enlarged due to follicular and paracortical hyperplasia [78]. Viral antigen is present in bronchiolar epithelium which contains Clara cells, occasional type II cells lining alveoli, and occasional alveolar macrophages $[19,20,82,85]$. Infection of term lambs with another paramyxovirus, parainfluenza-3 (PI-3), caused similar lesions and induced increased mRNA expression of sheep beta defensins-1 (SBD-1), SP-A, and SP-D that was associated with viral clearance; protein levels of SP-A did not change throughout the course of disease [34].

Preterm lambs can be derived by Caesarean section at $90 \%$ gestation with $80 \%$ survival for experimentation $[19,74]$. These lambs are not premature; however, innate immune gene responses, cellular differentiation, and responses to RSV by preterm lambs have significant differences from term lambs $[71,79]$. In contrast, rodents do not survive preterm birth. Infection of preterm lambs with bRSV resulted in enhanced disease with decreased viral clearance, increased viral antigen, mRNA levels $\mathrm{RSV}$, and infiltration of bronchioles by neutrophils compared to term lambs $[19,74]$. bRSV-infected cells retrieved from lung tissue by laser capture microscopy (LCM) had increased SP-A and MCP-1 mRNA in both term and preterm lambs but expression was significantly lower in infected preterm 
lambs compared to term lambs [72]. These studies suggest that preterm lambs have reduced expression and responses of innate immune genes to RSV which may contribute to the increased disease severity.

Figure 1. Image of lung from a lamb that at 2 days of age was infected with human respiratory syncytial virus strain, Memphis 37 by nebulizer $\left(6.4 \times 10^{7}\right.$ plaque-forming units $(\mathrm{PFU}) / \mathrm{mL})$. Six days after infection, lung tissue was collected. The bronchiole has moderately thickened epithelium due to hypertrophy and proliferation of epithelial cells and within the lumen are neutrophils (blue arrow) and large syncytial cell (arrow). Around the airway in the tunica adventitia are lymphocytes (short arrow). $\mathrm{H}$ and E. Bar $=120 \mathrm{um}$. Manuscript in preparation.

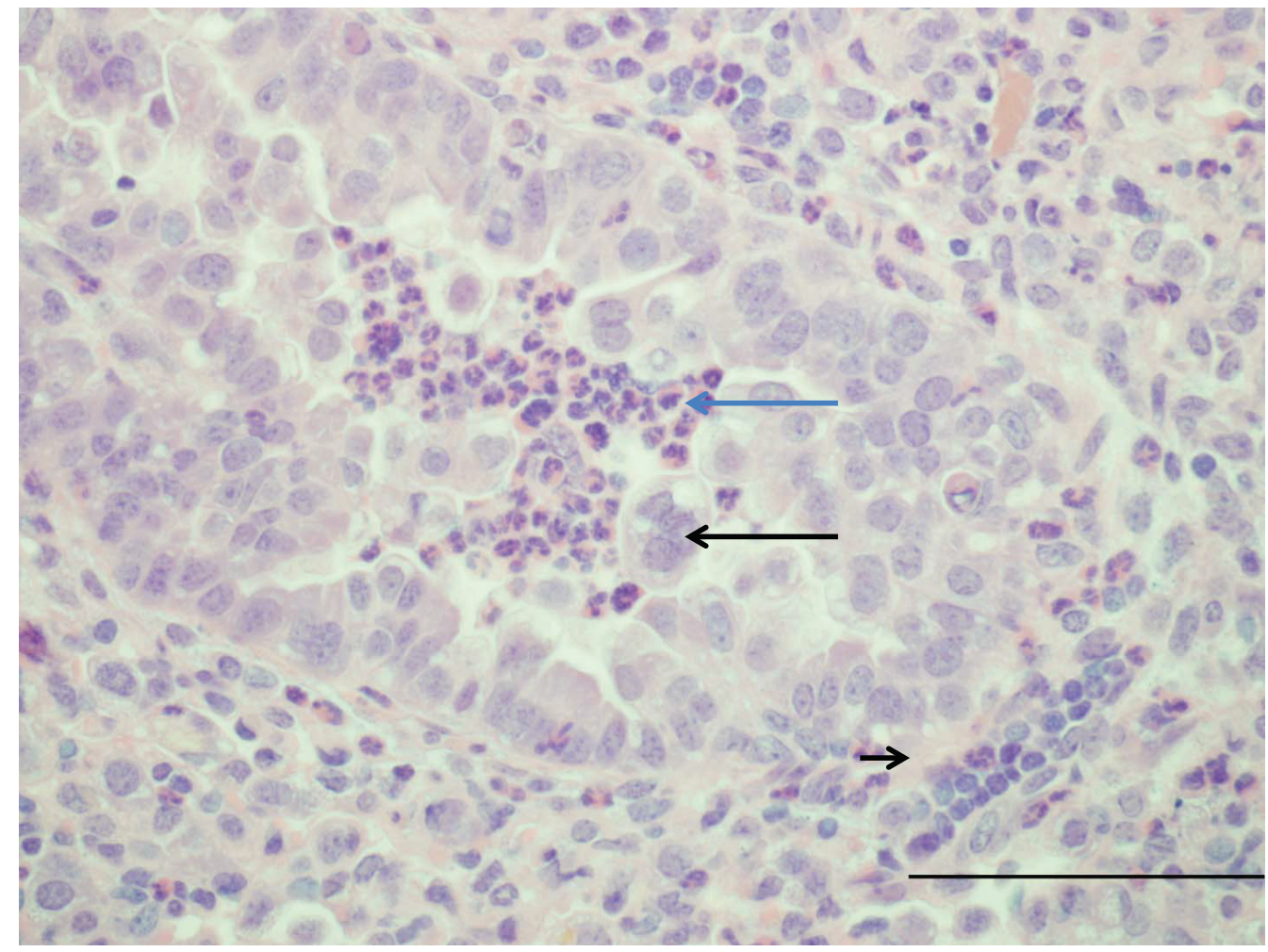

The Long strain of RSV-A and type B RSV will infect lambs and cause clinically detectable RSV disease [69]. Similar to bRSV, hRSV strain A2 infected term lambs, causing fever and inducing pulmonary lesions similar to those observed with bRSV in lambs [20,78]. In these lambs at day 3 post-inoculation (p.i.) there are increased mRNA levels of TNF- $\alpha$ and IL-10 as well as increased TNF- $\alpha$ protein in lung tissue. At 6 days p.i. IFN- $\gamma$, IL-8, MCP-1, and macrophage inflammatory protein-1 alpha (MIP-1 $\alpha$ ) mRNA levels are increased in infected levels compared to controls while IFN- $\beta$, TNF- $\alpha$, TGF- $\beta$, IL-10, and Regulated upon Activation, Normal T-cell Expressed, and Secreted 
(RANTES) mRNA are all decreased by infection with RSV. At 14 days p.i. the following mRNA levels are decreased in infected compared to control: IFN- $\beta$, IFN- $\gamma$, TNF- $\alpha$, TGF- $\beta$, IL-10, IL- 8 , MCP-1, and RANTES [78]. Memphis 37 (M-37) is a human RSV-A strain isolated from a pediatric case and used in studies in human adult subjects [86] and can replicate and cause disease in lambs. Recent studies have shown that M37 causes similar disease to that of both bRSV and hRSV A2 strains (personal observations). Lambs inoculated with M37 grown in Vero cells had reduced disease severity compared to lambs inoculated with M37 grown in HEp2 cells; this finding is consistent with studies showing that growth of RSV in Vero cells reduces G protein expression [87].

Figure 2. Image of lung from a lamb fed a milk replacer lacking iodide and infected at 2 days of age with human respiratory syncytial virus strain, Memphis 37 by nebulizer $\left(6.4 \times 10^{7} \mathrm{PFU} / \mathrm{mL}\right)$. Within the bronchiolar epithelium there is dense accumulation of RSV antigen detected by immunohistochemistry (primary antibody Meridan Biosciences). Hematoxlyn counterstain. Bar $=150$ um.

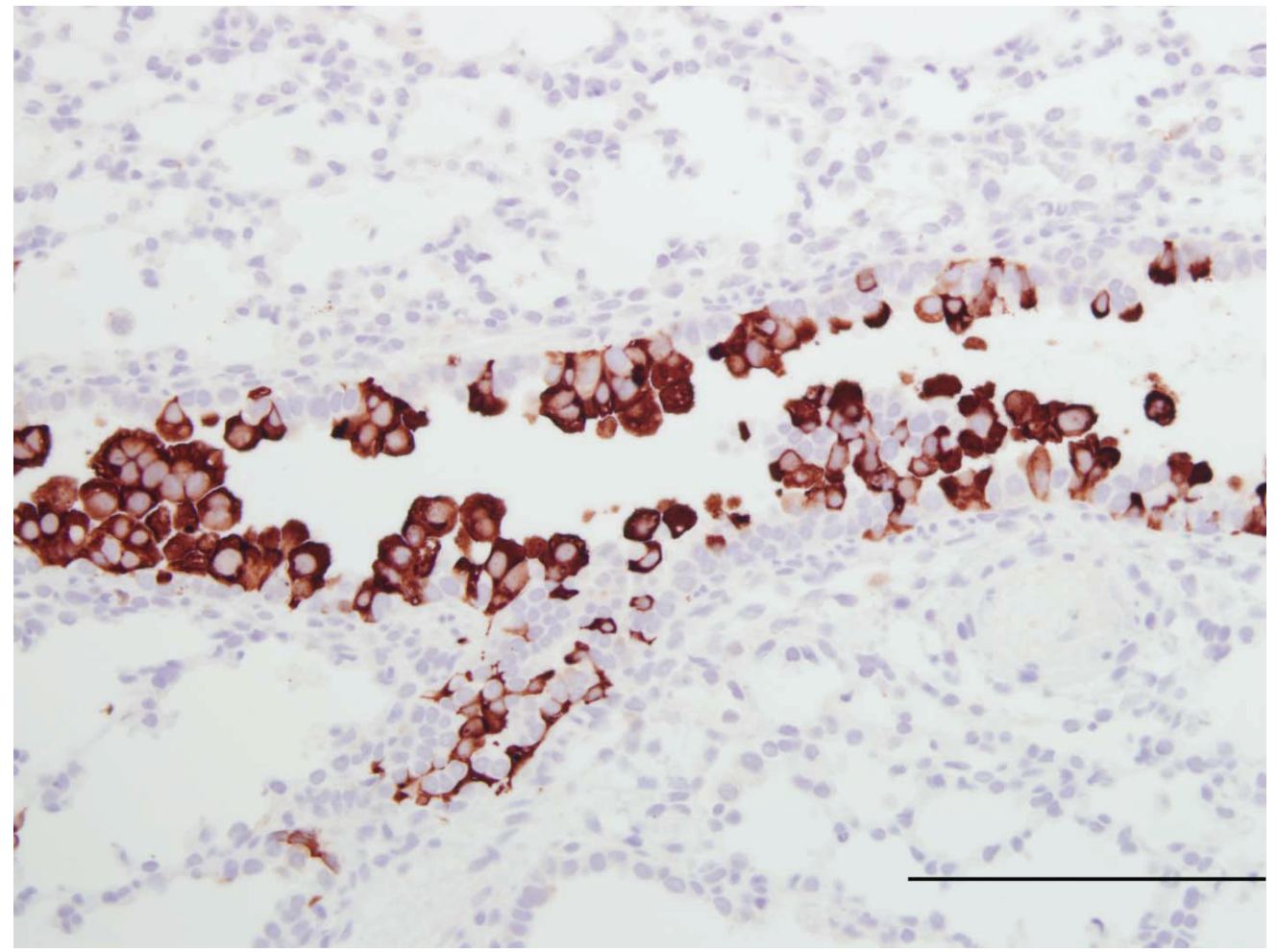

The lamb model has been used to test at therapeutic intervention. Vascular endothelial growth factor (VEGF) administered intratracheally before viral inoculation had a protective effect against bRSV characterized by decreased inflammation and decreased viral replication [85]. A similar protective effect of VEGF was shown using hRSV A2 and associated with increased SP-A and TLR-4 
gene expression and delays in expression of anti-inflammatory mediators TGF- $\beta$ and IL-10, decreasing their expression at 16 and $24 \mathrm{~h}$ then increasing expression at $32 \mathrm{~h}$ after VEGF administration as compared to control lambs $[82,88]$. The mechanism(s) by which VEGF reduces RSV severity is not known; however, VEGF increases expression of SP-A which opsonizes and aggregates RSV. VEGF also has other activities including those that may have anti-RSV effects such as: enhancement of monocyte infiltration, macrophage activation and induction of vascular leak. We are currently using the lamb model to assess efficacy of iodide enhancement of Duox/LPO-mediated innate defense (Figures 2 and 3).

Figure 3. Image of lung from a lamb fed a milk replacer lacking iodide but was supplemented with iodide by gastric gavage daily $(10 \mathrm{mg} / \mathrm{kg}$ body weight). At two days of age the lamb was infected with human respiratory syncytial virus strain, Memphis 37 by nebulizer $\left(6.4 \times 10^{7} \mathrm{PFU} / \mathrm{mL}\right)$. Within the bronchiolar epithelium there are a few cells containing viral antigen; however, significantly fewer cells are stained compared to the staining of the lung tissue from the lamb in Figure 3. Thus, with KI administration there is reduced viral antigen which is likely due to formation of iodide hypohalide (HOI) by dual oxidases and lactoperoxidase. RSV antigen was detected by immunohistochemistry (primary antibody Meridan Biosciences). Hematoxlyn counterstain. Bar $=150 \mathrm{um}$.

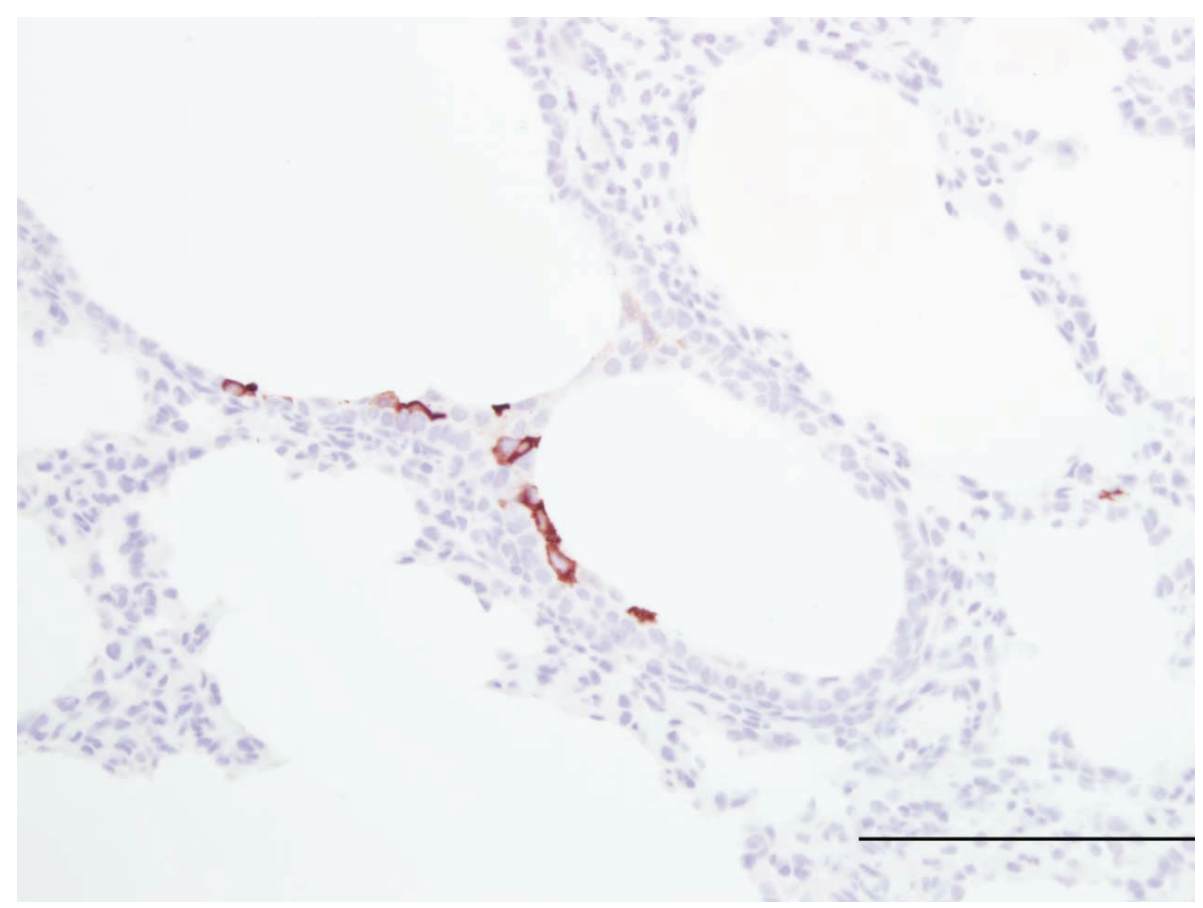

The perinatal lamb model of RSV infection has recently been used in studies assessing the effects of formalin-inactivated RSV vaccines (FI-RSV) [89]. Our laboratory has shown that term lambs 
receiving one FI-RSV vaccination have increased perivascular and peribronchiolar lymphocytic infiltrates with reduced viral titers and viral antigen, as well as low serum neutralizing antibodies all of which are consistent with features that occurred in infants and other models (Figure 4; manuscript in preparation). As indicated, since the lamb can be deprived of maternal immunoglobulin, the model could be used to assess novel, promising, vaccines [89,90] without maternal immunoglobulin interference and compare such promising vaccines to those with low-affinity antibodies such as FI-RSV [81].

Maternal ethanol consumption is a risk factor for preterm birth and infants born premature have increased risk for severe RSV infection. In development of a model to assess the effects of maternal ethanol consumption at the level of a moderate drinker on the developing fetal lung, it was determined that preterm lambs born from ewes receiving ethanol during the last three weeks of pregnancy had significant reductions in SP-A and also VEGF, VEGF receptors, VEGFR1 and VEGFR2 and VEGF transcription factors hypoxia inducible factor (HIF) HIF-1 $\alpha$ and HIF-2 $\alpha$ but not HIF-3 $\alpha$ [90,91]. Because VEGF is essential for lung vascular and epithelial cell growth, reductions in VEGF may contribute to the reduced SP-A expression. Since SP-A can opsonize and aggregate RSV and can also activate alveolar macrophages and infants with single nucleotide polymorphisms of SP-A have increased RSV disease severity, reductions in SP-A by exposure to ethanol in utero may exacerbate the susceptibility of preterm lung to severe RSV infection [51,93,94]; however, additional studies are needed to determine the extent to which ethanol exposure in utero may enhance RSV disease severity beyond that of preterm birth alone. Next Generation Sequencing (NGS) studies (unpublished) comparing preterm to term lambs with or without ethanol exposure in utero have identified transcripts with notable enhanced expression in preterm lung exposed to ethanol including genes related to (1) Inflammation, immunity and growth factors: lysozyme (19X), Secreted frizzled-related protein 2, SFRP2 which affects Wnt signaling (16X), Interleukin 12 A (9X), CXCL10 (6.8X), insulin-like growth factor-1, IGF-1 (6X), Fos (6X), IGF binding protein 5 (5.7X); early growth response protein 1, EGR1 (5X), CD28 (4X), CD4 (3.8X); (2) Metabolism/stress: leptin receptor, LEPR (5X), serotonin transporter, SERT (5X); (3) Cell proliferation inhibition: CDKN1C, p57, a Kip2 cell proliferation inhibitor (3.6X) and (4) Angiogenesis/vascularization: SERPINF1 (PEDF) was increased (4X) and this gene has anti-angiogenic properties which is consistent with findings demonstrating reductions in VEGF, VEGFR, and HIF $\alpha$ with ethanol exposure. Genes down-regulated by ethanol include: IL-8, TNF $\alpha$, ICAM-1, all of which are up-regulated by RSV infection. Thus, ethanol alters pulmonary transcripts that may underlie susceptibility to RSV. 
Table 1. Features of perinatal (preterm and term) lambs integral to studies of respiratory syncytial virus (RSV) infection.

\begin{tabular}{|c|c|c|}
\hline Feature & Advantage of Model & References \\
\hline Prenatal alveologenesis & Similar to infants & {$[43,44]$} \\
\hline Airway branching pattern & Similar to infants & [42] \\
\hline Submucosal glands in airways & Similar to infants & {$[43,45-47]$} \\
\hline Number/development of Clara cells & Similar to infants & {$[27,64]$} \\
\hline Number/development of type II cells & Similar to infants & {$[27,64,73]$} \\
\hline Lung size & Similar to infants & Generally known \\
\hline Can survive at $90 \%$ gestation & Similar to infants & [19] \\
\hline Susceptible to human RSV strains & Similar to infants & {$[16,20]$ Figure 1} \\
\hline Susceptible to bovine RSV strains & Permissible to RSV strains & {$[14-17,95]$} \\
\hline Susceptible to ovine PI-3 & Paramyxovirus susceptibility & {$[18]$} \\
\hline Bronchiolitis & & {$[15,18,21,82,85]$} \\
\hline Syncytial cell formation & & {$[19,20]$ Figure 1} \\
\hline Neutrophil infiltration & Similar to infants & {$[15,18-20,82,85]$} \\
\hline CD4, CD8 T cells & & {$[19,78]$} \\
\hline B cells and plasma cells & & {$[19,78]$} \\
\hline $\begin{array}{l}\text { Enhanced RSV disease severity in } \\
\text { preterm and newborn }\end{array}$ & Similar to infants & {$[19,20,74]$} \\
\hline Reduced immune responses preterm & Similar to infants & {$[71,75,76,79]$} \\
\hline Reduced neutrophils responses preterm & Similar to infants & [74] \\
\hline Dendritic cell responses to RSV & Similar to infants & [75-77] \\
\hline IL-8 gene expression & Similar to infants & {$[74,78]$} \\
\hline Functional Duox/LPO system & Similar to human/infants & {$[62]$} \\
\hline Innate immune responses & Similar to infants & {$[34,38,71,72,74,78,79]$} \\
\hline Adaptive immune responses & Similar to infants & {$[34,38,42,78]$} \\
\hline Outbred (genetic diversity) & Similar to infants & {$[34,38,42,78]$} \\
\hline $\begin{array}{l}\text { Newborn lamb can be deprived of maternal } \\
\text { immunoglobulin (Ig) }\end{array}$ & $\begin{array}{l}\text { Can vaccinate newborn } \\
\text { without interference by } \\
\text { maternal Ig }\end{array}$ & Generally known \\
\hline Jugular vein large and accessible & $\begin{array}{l}\text { Allows placement of } \\
\text { catheter to deliver drugs }\end{array}$ & Generally known \\
\hline Synchronized birth & $\begin{array}{l}\text { Allows groups of lambs } \\
\text { of similar age }\end{array}$ & Generally known \\
\hline VEGF reduces RSV severity & $\begin{array}{l}\text { Model can test anti-RSV } \\
\text { therapies and drugs }\end{array}$ & {$[82,85]$} \\
\hline $\begin{array}{l}\text { Fetal lambs exposed to ethanol in vivo have } \\
\text { reduced SP-A production, lung } \\
\text { development, HIF } 1 \alpha \text {, HIF } 2 \alpha \text {, VEGF, and } \\
\text { VEGFR }\end{array}$ & $\begin{array}{l}\text { Model can test drugs and } \\
\text { risk factors for lung } \\
\text { development and RSV } \\
\text { susceptibility }\end{array}$ & {$[89,90]$} \\
\hline $\begin{array}{l}\text { Enhanced lymphocytic responses following } \\
\text { FI-RSV vaccination }\end{array}$ & $\begin{array}{l}\text { Model can study FI-RSV } \\
\text { pathogenesis, mechanisms } \\
\text { and vaccines }\end{array}$ & $\begin{array}{l}\text { Manuscript in } \\
\text { preparation }\end{array}$ \\
\hline
\end{tabular}


Figure 4. Image of lung from a lamb from a lamb that at 3 days of age was vaccinated with a formalin-inactivated respiratory syncytial virus A2 strain followed three weeks later by nebulization of human respiratory syncytial virus strain, Memphis 37 by nebulizer $\left(6.4 \times 10^{7} \mathrm{PFU} / \mathrm{mL}\right)$. Six weeks after nebulization, this lung was collected. The image contains a bronchiole that is mildly dilated within the tunica adventitia are dense infiltrates of lymphocytes. $\mathrm{H}$ and $\mathrm{E} \mathrm{Bar}=185 \mathrm{um}$. Manuscript in preparation.

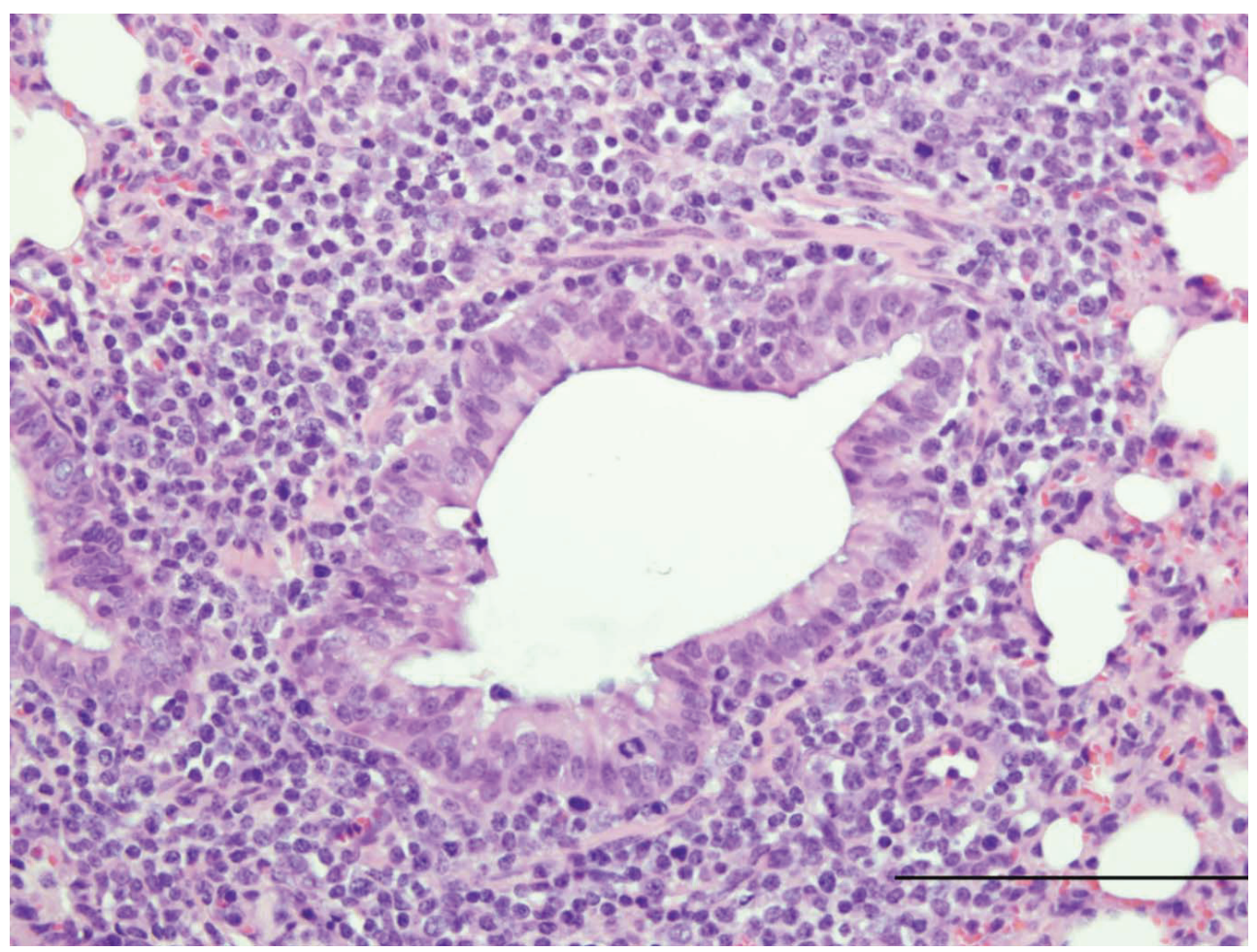

In summary, lambs provide a model of perinatal (preterm and term) RSV infection that is useful in exploring disease mechanisms due to their similar lung development, size, airway structure and epithelial (Clara and type II cell) composition, innate and adaptive immune response, their ability to survive if born preterm, and their ability to be deprived of maternal immunoglobulin and survive. Also, groups of lambs can be obtained for ease of experimental design. Lambs are used to study mechanisms of increased RSV disease severity in preterm lung, viral tropism to Clara and other bronchiolar epithelial cells, innate immune responses by epithelia and the Duox/LPO oxidative system, effects of anti-RSV immunoglobulins on RSV infection using colostrum-deprived lambs lacking maternal immunoglobulin, and persistent effects of RSV infection. Lambs are also valuable for use in pre-clinical trials of vaccines or therapeutics. 


\section{Acknowledgments}

This work was funded in part by NIH/NIAID 1R56AI091000-01A1, and NIH/NIAID R01AI062787, Gilead Sciences, Inc., and Meridian Bioscience, Inc, MedImmune, LLC. The authors thank Albert Van Geelen, Jack Gallup, and the many others who have contributed to the studies.

\section{Conflicts of Interest}

The authors declare no conflict of interest.

\section{References}

1. Nair, H.; Nokes, D.J.; Gessner, B.D.; Dherani, M.; Madhi, S.A.; Singleton, R.J.; O’Brien, K.L.; Roca, A.; Wright, P.F.; Bruce, N.; et al. Global burden of acute lower respiratory infections due to respiratory syncytial virus in young children: A systematic review and meta-analysis. Lancet 2010, 375, 1545-1555.

2. Chanock, R.; Roizman, B.; Myers, R. Recovery from infants with respiratory illness of a virus related to chimpanzee coryza agent (CCA). I. Isolation, properties and characterization. Am. J. Hyg. 1957, 66, 281-290.

3. Chanock, R.; Finberg, L. Recovery from infants with respiratory illness of a virus related to chimpanzee coryza agent (CCA). Ii. Epidemiologic aspects of infection in infants and young children. Am. J. Hyg. 1957, 66, 291-300.

4. Empey, K.M.; Peebles, R.S., Jr.; Kolls, J.K. Pharmacologic advances in the treatment and prevention of respiratory syncytial virus. Clin. Infect. Dis. 2010, 50, 1258-1267.

5. Meert, K.L.; Sarnaik, A.P.; Gelmini, M.J.; Lieh-Lai, M.W. Aerosolized ribavirin in mechanically ventilated children with respiratory syncytial virus lower respiratory tract disease: A prospective, double-blind, randomized trial. Crit. Care Med. 1994, 22, 566-572.

6. Wu, H.; Pfrarr, D.S.; Losonsky, G.A.; Kiener, P.A. Immunoprophylaxis of RSV infection: Advancing from RSV-IGIV to palivizumab and motavizumab. Curr. Top. Microbiol. Immunol. 2008, 317, 103-123.

7. Olszweska, W.; Openshaw, P. Emerging drugs for respiratory syncytial virus infection. Expert Opin. Ermg. Drugs 2009, 14, 207-217.

8. Graham, B.S. Pathogenesis of respiratory syncytial virus vaccine-augmented pathology. Am. J. Respir. Crit. Care Med. 1995, 152, S63-S66.

9. Collins, P.L.; Melero, J.A. Progress in understanding and controlling respiratory syncytial virus: Still crazy after all these years. Virus Res. 2011, 162, 80-99.

10. Hon, K.L.; Leung, T.F.; Cheng, W.Y.; Ko, N.M.; Tang, W.K.; Wong, W.W.; Yeung, W.H.; Chan, P.K. Respiratory syncytial virus morbidity, premorbid factors, seasonality, and implications for prophylaxis. J. Crit. Care 2012, ePub ahead of print.

11. Sommer, C.; Resch, B.; Simoes, E.A. Risk factors for severe respiratory syncytial virus lower respiratory tract infection. J. Open Microbiol. 2012, 5, 144-154. 
12. Martin, J.A.; Hamilton, B.E.; Sutton, P.D.; Ventura, S.J.; Mathews, T.J.; Osterman, M.J. Births: Final data for 2008. Natl. Vital Stat. Rep. 2010, 59, 3-71.

13. Lawn, J.E.; Gravett, M.G.; Nunes, T.M.; Rubens, C.E.; Stanton, C. Global Report on Preterm Birth and Stillbirth (1 of 7): Definitions, Description of the Burden and Opportunities to Improve Data; Report; BMC: London, UK, 2010.

14. Belknap, E.B.; Ciszewski, D.K.; Baker, J.C. Experimental respiratory syncytial virus infection in calves and lambs. J. Vet. Diagn. Investig. 1995, 7, 285-298.

15. Cutlip, R.C.; Lehmkuhl, H.D. Lesions in lambs experimentally infected with bovine respiratory syncytial virus. Am. J. Vet. Res. 1979, 40, 1479-1482.

16. Lapin, C.D.; Hiatt, P.W.; Langston, C.; Mason, E.; Piedra, P.T. A lamb model for human respiratory syncytial virus infection. Pediatr. Pulmonol. 1993, 15, 151-156.

17. Lehmkuhl, H.D.; Cutlip, R.C. Experimental respiratory syncytial virus infection in feeder-age lambs. Am. J. Vet. Res. 1979, 40, 1729-1730.

18. Grubor, B.; Gallup, J.M.; Meyerholz, D.K.; Crouch, E.; Evans, R.B.; Brogden, K.A.; Lehmkuhl, H.D.; Ackermann, M.R. Enhanced surfactant protein and defensin mRNA levels and reduced viral replication during paramyxoviral pneumonia in neonatal lambs. Clin. Vaccine Immunol. 2004, 11, 599-607.

19. Meyerholz, D.K.; Grubor, B.; Fach, S.J.; Sacco, R.E.; Lehmkuhl, H.D.; Gallup, J.M.; Ackermann, M.R. Reduced clearance of respiratory syncytial virus in a preterm lamb model. Microb. Infect. 2004, 6, 1312-1319.

20. Olivier, A.; Gallup, J.M.; de Macedo, M.M.; Varga, S.M.; Ackermann, M.R. Human respiratory syncytial virus A2 strain replicates and induces innate immune responses by respiratory epithelia of neonatal lambs. Int. J. Exp. Pathol. 2009, 90, 431-438.

21. Johnson, J.E.; Gonzales, R.A.; Olson, S.J.; Wright, P.F.; Graham, B.S. The histopathology of fatal untreated human respiratory syncytial virus infection. Mod. Pathol. 2007, 20, 108-119.

22. Burri, P.H. Fetal and postnatal development of the lung. Annu. Rev. Physiol. 1984, 46, 617-628.

23. Jeffery, P.K.; Gaillard, D.; Moret, S. Human airway secretory cells during development and in mature airway epithelium. Eur. Respir. J. 1992, 5, 93-104.

24. Lukacs, N.W.; Smitt, J.J.; Mukherjee, S.; Morris, S.B.; Nunez, G.; Lindell, D.M. Respiratory virus-induced TLR7 activation controls IL-17-associated increased mucus via IL-23 regulation. J. Immunol. 2010, 185, 2231-2239.

25. Barth, P.J.; Wolf, M.; Ramaswamy, A. Distribution and number of clara cells in the normal and disturbed development of the human fetal lung. Pediatr. Pathol. 1994, 14, 637-651.

26. Boers, J.E.; Ambergen, A.W.; Thunnissen, F.B. Number and proliferation of clara cells in normal human airway epithelium. Am. J. Respir. Crit. Care Med. 1999, 159, 1585-1591.

27. Plopper, C.G.; Mariassy, A.T.; Hill, L.H. Ultrastructure of the nonciliated bronchiolar epithelial (clara) cell of mammalian lung: I. A comparison of rabbit, guinea pig, rat, hamster, and mouse. Exp. Lung Res. 1980, 1, 139-154. 
28. Bernard, A.; Thielemans, N.; Lauwerys, R.; Langhendries, J.P.; van Lierde, M.; Freund, M.M. Clara cell protein in human amniotic fluid: A potential marker of fetal lung growth. Pediatr. Res. 1994, 36, 771-775.

29. Elizur, A.; Adair-Kirk, T.L.; Kelley, D.G.; Griffin, G.L.; de Mello, D.E.; Senior, R.M. Clara cells impact the pulmonary innate immune response to LPS. Am. J. Physiol. Lung Cell. Mol. Physiol. 2007, 293, L383-L392.

30. Wang, S.Z.; Rosenberger, C.L.; Bao, Y.X.; Stark, J.M.; Harrod, K.S. Clara cell secretory protein modulates lung inflammatory and immune responses to respiratory syncytial virus infection. J. Immunol. 2003, 171, 1051-1060.

31. You, D.; Becnel, D.; Wang, K.; Ripple, M.; Daly, M.; Cormier, S.A. Exposure of neonates to respiratory syncytial virus is critical in determining subsequent airway response in adults. Respir. Res. 2006, 7, 107-117.

32. Willems, F.; Vollstedt, S.; Suter, M. Phenotype and function of neonatal DC. Eur. J. Immunol. 2009, 39, 26-35.

33. Thornburg, N.J.; Shepherd, B.; Crowe, J.E., Jr. Transforming growth factor beta is a major regulator of human neonatal immune responses following respiratory syncytial virus infection. J. Virol. 2010, 84, 12895-12902.

34. Levy, O.; Martin, S.; Eichenwald, E.; Ganz, T.; Valore, E.; Carroll, S.F.; Lee, K.; Goldmann, D.; Thorne, G.M. Impaired innate immunity in the newborn: Newborn neutrophils are deficient in bactericidal/permeability-increasing protein. Pediatrics 1999, 104, 1327-1333.

35. Ambruso, D.R.; Bentwood, B.; Henson, P.M.; Johnston, R.B., Jr. Oxidative metabolism of cord blood neutrophils: Relationship to content and degranulation of cytoplasmic granules. Pediatr. Res. 1984, 18, 1148-1153.

36. Qing, G.; Rajaraman, K.; Bortolussi, R. Diminished priming of neonatal polymorphonuclear leukocytes by lipopolysaccharide is associated with reduced CD14 expression. Infect. Immun. 1995, 63, 248-252.

37. Remijsen, Q.; Kuijpers, T.W.; Wirawan, E.; Lippens, S.; Vandenabeele, P.; Vanden Berghe, T. Dying for a cause: Netosis, mechanisms behind an antimicrobial cell death modality. Cell Death Differ. 2011, 18, 581-588.

38. Levy, O. Innate immunity of the newborn: Basic mechanisms and clinical correlates. Nat. Rev. Immunol. 2007, 7, 379-390.

39. Bem, R.A.; Domachowske, J.B.; Rosenberg, H.F. Animal models of human respiratory syncytial virus disease. Am. J. Physiol. Lung Cell. Mol. Physiol. 2011, 301, L148-L156.

40. Thomas, L.H.; Cook, R.S.; Howard, C.J.; Gaddum, R.M.; Taylor, G. Influence of selective T-lymphocyte depletion on the lung pathology of gnotobiotic calves and the distribution of different T-lymphocyte subsets following challenge with bovine respiratory syncytial virus. Res. Vet. Sci. 1996, 61, 38-44.

41. Kalina, W.V.; Woolums, A.R.; Berghous, R.D.; Gerswin, L.J. Formalin-inactivated bovine RSV vaccine enhances a Th2 mediated immune response in infected cattle. Vaccine 2004, 22, 1465-1474. 
42. Scheerlinck, J.P.; Snibson, K.J.; Bowles, V.M.; Sutton, P. Biomedical applications of sheep models: From asthma to vaccines. Trends Biotechnol. 2008, 26, 259-266.

43. Alcorn, D.G.; Adamson, T.M.; Maloney, J.E.; Robinson, P.M. A morphologic and morphometric analysis of fetal lung development in the sheep. Anat. Rec. 1981, 201, 655-667.

44. Flecknoe, S.L.; Wallace, M.J.; Cock, M.L.; Harding, R.; Hooper, S.B. Changes in alveolar epithelial cell proportions during fetal and postnatal development in sheep. Am. J. Physiol. Lung. Cell Mol. Physiol. 2003, 285, L664-L679.

45. Plopper, C.G.; Mariassy, A.T.; Lollini, L.O. Structure as revealed by airway dissection. A comparison of mammalian lungs. Am. Rev. Respir. Dis. 1983, 128, S4-S7.

46. Smith, L.J.; McKay, K.O.; van Asperen, P.P.; Selvadurai, H.; Fitzgerald, D.A. Normal development of the lung and premature birth. Paediatr. Respir. Rev. 2010, 11, 135-142.

47. Borthwick, D.W.; West, J.D.; Keighren, M.A.; Flockhart, J.H.; Innes, B.A.; Dorin, J.R. Murine submucosal glands are clonally derived and show a cystic fibrosis gene-dependent distribution pattern. Am. J. Respir. Cell Mol. Biol. 1999, 20, 1181-1189.

48. Banfi, B. A novel host defense system of airways is defective in cystic fibrosis: Update. Am. J. Respir. Crit. Care Med. 2007, 175, 967.

49. Conner, G.E.; Salathe, M.; Forteza, R. Lactoperoxidase and hydrogen peroxide metabolism in the airway. Am. J. Respir. Crit. Care Med. 2002, 166, S57-S61.

50. Conner, G.E.; Wijkstrom-Frei, C.; Randell, S.H.; Fernandez, V.E.; Salathe, M. The lactoperoxidase system links anion transport to host defense in cystic fibrosis. FEBS Lett. 2007, 581, 271-278.

51. Fischer, H. Mechanism and function of DUOX in epithelia of the lung. Antioxid. Redox Signal. 2009, 11, 2453-2465.

52. Ampuero, S.; Luchsinger, V.; Tapia, L.; Palomino, M.A.; Larranaga, C.E. Sp-A1, SP-A2 and SP-D gene polymorphisms in severe acute respiratory syncytial infection in chilean infants. Infect. Genet. Evol. 2011, 11, 1368-1377.

53. Forteza, R.; Salathe, M.; Miot, F.; Forteza, R.; Conner, G.E. Regulated hydrogen peroxide production by Duox in human airway epithelial cells. Am. J. Respir. Cell Mol. Biol. 2005, 32, 462-469.

54. Fragoso, M.A.; Fernandez, V.; Forteza, R.; Randell, S.H.; Salathe, M.; Conner, G.E. Transcellular thiocyanate transport by human airway epithelia. J. Physiol. 2004, 561, 183-194.

55. Furtmüller, P.G.; Jantschko, W.; Regelsberger, G.; Jakopitsch, C.; Arnhold, J.; Obinger, C. Reaction of lactoperoxidase compound I with halides and thiocyanate. Biochemistry 2002, 41, 11895-11900.

56. Pedemonte, N.; Caci, E.; Sondo, E.; Caputo, A.; Rhoden, K.; Pfeffer, U.; Di Candia, M.; Bandettini, R.; Ravazzolo, R.; Zegarra-Moran, O.; et al. Thiocyanate transport in resting and IL-4-stimulated human bronchial epithelial cells: Role of pendrin and anion channels. J. Immunol. 2007, 178, 5144-5153. 
57. Wijkstrom-Frei, C.; El-Chemaly, S.; Ali-Rachedi, R.; Gerson, C.; Cobas, M.A.; Forteza, R.; Salathe, M.; Conner, G.E. Lactoperoxidase and human airway host defense. Am. J. Respir. Cell Mol. Biol. 2003, 29, 206-212.

58. Fischer, A.J.; Lennemann, N.J.; Krishnamurthy, S.; Pocza, P.; Durairaj, L.; Launspack, J.L.; Rhein, B.A.; Wohlford-Lenane, C.; Lorentzen, D.; Banfi, B.; et al. Enhancement of respiratory mucosal antiviral defenses by the oxidation of iodide. Am. J. Respir. Cell Mol. Biol. 2011, 45, 874-881.

59. Geiszt, M.; Witta, J.; Baffi, J.; Lekstrom, K.; Leto, T.L. Dual oxidases represent novel hydrogen peroxide sources supporting mucosal surface host defense. FASEB J. 2003, 17, 1502-1504.

60. Moskwa, P.; Lorentzen, D.; Excoffon, K.J.D.A.; Zabner, J.; McCray, P.B., Jr.; Nauseef, W.M.; Dupuy, C.; Banfi, B. A novel host defense system of airways is defective in cystic fibrosis. Am. J. Respir. Crit. Care Med. 2007, 175, 174-183.

61. Fischer, H.; Gonzales, L.K.; Kolla, V.; Schwarzer, C.; Miot, F.; Illek, B.; Ballard, P.L. Developmental regulation of Duox1 expression and function in human fetal lung epithelial cells. Am. J. Physiol. Lung Cell. Mol. Physiol. 2007, 292, L1506-L1514.

62. Gerson, C.; Sabater, J.; Scuri, M.; Torbati, A.; Coffey, R.; Abraham, J.W.; Lauredo, I.; Forteza, R.; Wanner, A.; Salathe, M.; et al. The lactoperoxidase system function in bacterial clearance of airways. Am. J. Respir. Cell Mol. Biol. 2000, 22, 665-671.

63. Barth, P.J.; Koch, S.; Muller, B.; Unterstab, F.; von Wichert, P.; Moll, R. Proliferation and number of clara cell 10-kda protein (CC10)-reactive epithelial cells and basal cells in normal, hyperplastic and metaplastic bronchial mucosa. Virchows. Arch. 2000, 437, 648-655.

64. Plopper, C.G.; Hill, L.H.; Mariassy, A.T. Ultrastructure of the nonciliated bronchiolar epithelial (clara) cell of mammalian lung. A study of man with comparison of 15 mammalian species. Exp. Lung Res. 1980, 1, 171-180.

65. Khoor, A.; Gray, M.E.; Singh, G.; Stahlman, M.T. Ontogeny of clara cell—specific protein and its mRNA: Their association with neuroepithelial bodies in human fetal lung and in bronchopulmonary dysplasia. J. Histochem. Cytochem. 1996, 44, 1429-1438.

66. Li, X.; Castleman, W.L. Effects of 4-ipomeanol on bovine parainfluenza type 3 virus-induced pneumonia in calves. Vet. Pathol. 1991, 28, 428-437.

67. Haynes, L.M.; Moore, D.D.; Kurt-Jones, E.A.; Finberg, R.W.; Anderson, L.J.; Tripp, R.A. Involvement of toll-like receptor 4 in innate immunity to respiratory syncytial virus. J. Virol. 2001, 75, 10730-10737.

68. Liu, P.; Jamaluddin, M.; Li, K.; Garofalo, R.P.; Casola, A.; Brasier, A.R. Retinoic acid-inducible gene I mediates early antiviral response and toll-like receptor 3 expression in respiratory syncytial virus-infected airway epithelial cells. $J$. Virol. 2007, 81, 1401-1411.

69. Scagnolari, C.; Midulla, F.; Pierangeli, A.; Moretti, C.; Bonci, E.; Berardi, R.; de Angelis, D.; Selvaggi, C.; Di Marco, P.; Girardi, E.; et al. Gene expression of nucleic acid-sensing pattern recognition receptors in children hospitalized for respiratory syncytial virus-associated acute bronchiolitis. Clin. Vaccine Immunol. 2009, 16, 816-823. 
70. Vareille, M.; Kieninger, E.; Edwards, M.R.; Regamey, N. The airway epithelium: Soldier in the fight against respiratory viruses. Clin. Microbiol. Rev. 2011, 24, 210-229.

71. Sow, F.B.; Gallup, J.M.; Derscheid, R.; Krishnan, S.; Ackermann, M.R. Ontogeny of the immune response in the ovine lung. Immunol. Investig. 2012, 41, 304-316.

72. Kawashima, K.; Meyerholz, D.K.; Gallup, J.M.; Grubor, B.; Lazic, T.; Lehmkuhl, H.D.; Ackermann, M.R. Differential expression of ovine innate immune genes by preterm and neonatal lung epithelia infected with respiratory syncytial virus. Viral Immunol. 2006, 19, 316-323.

73. Meyerholz, D.K.; DeGraaff, J.A.; Gallup, J.M.; Ackermann, M.R. Depletion of alveolar glycogen corresponds with immunohistochemical development of CD208 antigen expression in perinatal lamb lung. J. Histochem. Cytochem. 2006, 54, 1247-1253.

74. Sow, F.B.; Gallup, J.M.; Krishnan, S.; Patera, A.C.; Suzich, J.; Ackermann, M.R. Respiratory syncytial virus infection is associated with an altered innate immunity and a heightened pro-inflammatory response in the lungs of preterm lambs. Resp. Res. 2011, 12, 106.

75. Fach, S.J.; Olivier, A.; Gallup, J.M.; Waters, T.E.; Ackermann, M.R.; Lehmkuhl, H.D.; Sacco, R.E. Differential expression of cytokine transcripts in neonatal and adult ovine alveolar macrophages in response to respiratory syncytial virus or toll-like receptor ligation. Vet. Immunol. Immunopathol. 2010, 136, 55-64.

76. Fach, S.J.; Brockmeier, S.L.; Hobbs, L.A.; Lehmkuhl, H.D.; Sacco, S.E. Pulmonary dendritic cells isolated from neonatal and adult ovine lung tissue. Vet. Immunol. Immun. Pathol. 2007, 112, 171-182.

77. Fach, S.J.; Meyerholz, D.K.; Gallup, J.M.; Ackermann, M.R.; Lehmkuhl, H.D.; Sacco, R.E. Neonatal ovine pulmonary dendritic cells support bovine respiratory syncytial virus replication with enhanced interleukin (IL)-4 and IL-10 gene transcripts. Viral Immunol. 2007, 20, 119-130.

78. Sow, F.B.; Gallup, J.M.; Olivier, A.; Krishnan, S.; Patera, A.C.; Suzich, J.; Ackermann, M.R. Respiratory syncytial virus is associated with an inflammatory response in lungs and architectural remodeling of lung-draining lymph nodes of newborn lambs. Am. J. Physiol. Lung Cell. Mol. Physiol. 2011, 300, L12-L24.

79. Meyerholz, D.K.; Kawashima, K.; Gallup, J.; Grubor, B.; Ackermann, M.R. Expression of innate immune genes (SP-AD, SBD-1, TLR4) by respiratory epithelia at preterm gestation is less than full-term. Dev. Comp. Immunol. 2006, 30, 1060-1069.

80. Kurt-Jones, E.A.; Popova, L.; Kwinn, L.; Haynes, L.M.; Jones, L.P.; Tripp, R.A.; Walsh, E.E.; Freeman, M.W.; Golenbock, D.T.; Anderson, L.J.; et al. Pattern recognition receptors tlr4 and cd14 mediate response to respiratory syncytial virus. Nat. Immunol. 2000, 1, 398-401.

81. Delgado, M.F.; Coviello, S.; Monsalvo, A.C.; Melendi, G.A.; Hernandez, J.Z.; Batalle, J.P.; Diaz, L.; Trento, A.; Chang, H.Y.; Mitzner, W.; et al. Lack of antibody affinity maturation due to poor Toll-like receptor stimulation leads to enhanced respiratory syncytial virus disease. Nat. Med. 2009, 15, 34-41. 
82. Olivier, A.; Gallup, J.M.; van Geelen, A.; Ackermann, M.R. Exogenous administration of vascular endothelial growth factor prior to human respiratory syncytial virus A2 infection reduces pulmonary pathology in neonatal lambs and alters epithelial innate immune responses. Exp. Lung Res. 2011, 37, 131-143.

83. Neilson, K.A.; Yunis, E.J. Demonstration of respiratory syncytial virus in an autopsy series. Pediatr. Pathol. 1990, 10, 491-502.

84. Simoes, E.A.; Carbonell-Estrany, X.; Rieger, C.H.; Mitchell, I.; Fredrick, L.; Groothuis, J.R. The effect of respiratory syncytial virus on subsequent recurrent wheezing in atopic and nonatopic children. J. Allergy Clin. Immunol. 2010, 126, 256-262.

85. Meyerholz, D.K.; Gallup, J.M.; Lazic, T.; de Macedo, M.M.A.; Lehmkuhl, H.D.; Ackermann, M.R. Pretreatment with recombinant human vascular endothelial growth factor reduces virus replication and inflammation in a perinatal lamb model of RSV infection. Viral. Immunol. 2007, 20, 188-196.

86. DeVincenzo, J.P.; Wilkinson, T.; Vaishnaw, A.; Cehelsky, J.; Meyers, R.; Nochur, S.; Harrison, L.; Meeking, P.; Mann, A.; Moane, E.; et al. Viral load drives disease in humans experimentally infected with respiratory syncytial virus. Am. J. Respir. Crit. Care Med. 2010, 182, 1305-1314.

87. Kwilas, S.; Liesman, R.M.; Zhang, L.; Walsh, E.; Pickles, R.J.; Peeples, M.E. Respiratory syncytial virus grown in vero cells contains a truncated attachment protein that alters its infectivity and dependence on glycosaminoglycans. J. Virol. 2009, 83, 10710-10718.

88. Sow, F.B.; Gallup, J.M.; Meyerholz, D.K.; Ackermann, M.R. Gene profiling studies in the neonatal ovine lung show enhanced effects of VEGF on the immune response. Dev. Comp. Immunol. 2009, 33, 761-771.

89. Castillow, E.M.; Varga, S.M. Overcoming T cell-mediated immunopathology to achieve safe RSV vaccination. Future Virol. 2008, 3, 445-454.

90. Jones, B.G.; Sealy, R.E.; Rudraraju, R.; Traina-Dorge, V.L.; Finneryfrock, B.; Cook, A.; Takimoto, T.; Portner, A.; Hurwitz, J.L. Sendai virus-based RSV vaccine protects African green monkeys from RSV infection. Vaccine 2012, 30, 959-968.

91. Lazic, T.; Sow, F.B.; van Geelen, A.; Meyerholz, D.K.; Gallup, J.M.; Ackermann, M.R. Exposure to ethanol during the last trimester of pregnancy alters the maturation and immunity of the fetal lung. Alcohol 2011, 45, 673-680.

92. Lazic, T.; Wyatt, T.A.; Matic, M.; Meyerholz, D.K.; Grubor, B.; Gallup, J.M.; Kersting, K.W.; Imerman, P.M.; Almeida-De-Macedo, M.; Ackermann, M.R. Maternal alcohol ingestion reduces surfactant protein A expression by preterm fetal lung epithelia. Alcohol 2007, 41, 347-355.

93. LeVine, A.M.; Gwozdz, J.; Stark, J.; Bruno, M.; Whitsett, J.; Korfhagen, T. Surfactant protein-A enhances respiratory syncytial virus clearance in vivo. J. Clin. Investig. 1999, 103, 1015-1021.

94. LeVine, A.M.; Elliott, J.; Whitsett, J.A.; Srikiatkhachorn, A.; Crouch, E.; DeSilva, N.; Korfhagen, T. Surfactant protein-D enhances phagocytosis and pulmonary clearance of respiratory syncytial virus. Am. J. Respir. Cell Mol. Biol. 2001, 31, 193-199.

95. Meehan, J.T.; Cutlip, R.C.; Lehmkuhl, H.D.; Kluge, J.P.; Ackermann, M.R. Infected cell types in ovine lung following exposure to bovine respiratory syncytial virus. Vet. Pathol. 1994, 31, 229-236. 
Reprinted from Viruses. Cite as: Sacco, R.E.; McGill, J.L.; Palmer, M.V.; Lippolis, J.D.; Reinhardt, T.A.; Nonnecke, B.J. Neonatal Calf Infection with Respiratory Syncytial Virus: Drawing Parallels to the Disease in Human Infants. Viruses 2012, 4, 3731-3753.

Review

\title{
Neonatal Calf Infection with Respiratory Syncytial Virus: Drawing Parallels to the Disease in Human Infants
}

\author{
Randy E. Sacco ${ }^{1, *}$, Jodi L. McGill ${ }^{1}$, Mitchell V. Palmer ${ }^{2}$, John D. Lippolis ${ }^{1}$, \\ Timothy A. Reinhardt ${ }^{1}$ and Brian J. Nonnecke ${ }^{1}$
}

1 Ruminant Diseases and Immunology Unit, National Animal Disease Center,

Agricultural Research Service, United States Department of Agriculture, Ames, IA 50010, USA;

E-Mails: Jodi.McGill@ars.usda.gov (J.L.M.); John.Lippolis@ars.usda.gov (J.D.L.);

Tim.Reinhardt@ars.usda.gov (T.A.R.); Brian.Nonnecke@ars.usda.gov (B.J.N.)

2 Infectious Bacterial Diseases Research Unit, National Animal Disease Center,

Agricultural Research Service, United States Department of Agriculture, Ames, IA 50010, USA;

E-Mail: Mitchell.Palmer@ars.usda.gov

* Author to whom correspondence should be addressed; E-Mail: Randy.Sacco@ars.usda.gov;

Tel.: +1-515-337-7354; Fax: +1-515-337-7149.

Received: 1 November 2012; in revised form: 29 November 2012 / Accepted: 7 December 2012 /

Published: 13 December 2012

\begin{abstract}
Respiratory syncytial virus (RSV) is the most common viral cause of childhood acute lower respiratory tract infections. It is estimated that RSV infections result in more than 100,000 deaths annually worldwide. Bovine RSV is a cause of enzootic pneumonia in young dairy calves and summer pneumonia in nursing beef calves. Furthermore, bovine RSV plays a significant role in bovine respiratory disease complex, the most prevalent cause of morbidity and mortality among feedlot cattle. Infection of calves with bovine RSV shares features in common with RSV infection in children, such as an age-dependent susceptibility. In addition, comparable microscopic lesions consisting of bronchiolar neutrophilic infiltrates, epithelial cell necrosis, and syncytial cell formation are observed. Further, our studies have shown an upregulation of pro-inflammatory mediators in RSV-infected calves, including IL-12p40 and CXCL8 (IL-8). This finding is consistent with increased levels of IL- 8 observed in children with RSV bronchiolitis. Since rodents lack IL-8, neonatal calves can be useful for studies of IL-8 regulation in response to RSV infection. We have recently found that vitamin D in milk replacer diets can be manipulated
\end{abstract}


to produce calves differing in circulating 25-hydroxyvitamin $\mathrm{D}_{3}$. The results to date indicate that although the vitamin D intracrine pathway is activated during RSV infection, pro-inflammatory mediators frequently inhibited by the vitamin $\mathrm{D}$ intacrine pathway in vitro are, in fact, upregulated or unaffected in lungs of infected calves. This review will summarize available data that provide parallels between bovine RSV infection in neonatal calves and human RSV in infants.

Keywords: Bovine respiratory syncytial virus; human respiratory syncytial virus; innate immunity; adaptive immunity; vaccine

\section{Importance of Human and Bovine Respiratory Syncytial Viruses}

Human (hRSV) and bovine (bRSV) respiratory syncytial viruses are closely related viruses that are among the leading causes of acute serious lower respiratory infection (ALRI) in young children and calves, respectively. While estimated mortality rates in developing countries are low $(<0.02 \%)$, globally, it is estimated that new episodes of hRSV-associated disease in children younger than 5 years of age exceed 33 million annually, with more than 100,000 resultant deaths [1]. Severe hRSV infections during the first three years of life are frequently followed by recurrent episodes of childhood wheezing or asthma [2,3]. In addition, hRSV is increasingly seen as an important cause of morbidity and mortality in elderly adults $[4,5]$ and in immunocompromised patients [6].

BRSV is a cause of enzootic pneumonia in young dairy calves and summer pneumonia in nursing beef calves. In fact, worldwide estimates suggest the frequency of bRSV infections in some dairy and beef herds exceeds 50\% [7]. Furthermore, in combination with other viral and bacterial pathogens, bRSV plays a significant role in shipping fever or bovine respiratory disease complex (BRDC), the most prevalent cause of morbidity and mortality among feedlot cattle. Even in cases where animals do not succumb to the disease, there can be long-term losses in performance. This includes reductions in feed efficiency and rate of gain in the feedlot, as well as reproductive performance, milk production, and longevity in the breeding herd. As a result, economic costs to the cattle industry from BRDC have been estimated to approach $\$ 1$ billion annually due to death losses, reduced performance, and costs of vaccinations and treatment modalities.

\section{Historical Perspectives on Respiratory Syncytial Viruses}

In 1956, RSV was originally isolated as a causal agent of chimpanzee coryza [8]. However, at the time this virus was further linked with respiratory illness in a human working with the coryza agent that had close contact with the chimpanzees. The next year, there was a reported isolation of a virus from infants with respiratory illness that was indistinguishable from the chimpanzee coryza agent $[9,10]$. The first study to propose an involvement of RSV in respiratory disease in cattle came more than a decade later. Doggett et al. [11] found bovine sera that contained neutralizing antibody against hRSV, 
suggesting a similar virus might exist in cattle. In the early 1970s, two reports from respiratory disease outbreaks in Switzerland [12] and England [13] identified viruses isolated from cattle that were closely related to hRSV. At the same time, Inaba et al. [14] reported the isolation of what appeared to be a new virus from cases of acute BRDC that was initially referred to as Nomi virus, but which they subsequently identified as bRSV [15].

\section{Age and Seasonal Affects}

The most severe ALRI cases due to hRSV occur in infants and children less than 1 year of age, especially those born premature or with underlying cardiopulmonary conditions [4,5]. In the USA, by 24 months of age, nearly all children have been infected at least once with hRSV, and approximately half have experienced two infections [16]. BRSV-associated disease is most pronounced in calves less than 6 months of age, and infection can occur even in the presence of maternal antibodies. It has been estimated that more than $70 \%$ of calves have seroconverted by the age of 12 months [17]. As with humans, re-infections in calves are common. Seasonal periodicity is seen with hRSV and bRSV, with most common occurrences of infections in the fall and winter months [18].

\section{RSV Viral Proteins}

HRSV and bRSV belong to the family Paramixoviridae, subfamily Pneumoviridae, and genus Pneumovirus. Pneumoviruses are single-stranded, negative-sense RNA viruses with a genome of approximately $15.2 \mathrm{~kb}$. The RSV viral RNA is transcribed into 10 major subgenomic mRNAs encoding 11 proteins, due to the M2 gene encoding two proteins. Associated with the genomic RNA, are nucleocapsid $(\mathrm{N})$, phosphoprotein $(\mathrm{P})$, large polymerase $(\mathrm{L})$, and associated proteins, transcriptional anti-termination factor M2-1 and RNA regulatory protein M2-2. There are 3 transmembrane surface glycoproteins, attachment $(\mathrm{G})$, fusion (F), and small hydrophobic (SH). A non-glycosylated matrix or membrane protein, $\mathrm{M}$, is associated with the inner face of the envelope. Finally, there are 2 non-structural proteins that accumulate in infected cells NS1, NS2.

HRSV has been classified into two subgroups, A and B, based on antigenic and genetic differences $[19,20]$. BRSV isolates can be classified into subgroups based on reactivity of mAb to the $\mathrm{G}$ protein [21], although these may represent variants of a single major antigenic group [21,22]. The $\mathrm{F}$ protein is a type I viral fusion protein synthesized as a precursor that is proteolytically cleaved by furin into disulfide-linked fragments [23]. Among the hRSV subgroups, the cellular attachment G protein, a type II integral membrane protein, is more divergent than the F protein [24]. Although bRSV isolates possess antigenically heterogeneous $\mathrm{G}$ proteins, the nucleotide sequences are less variable than for hRSV [21,25]. The F and $\mathrm{G}$ glycoproteins contain the predominant neutralization and protective epitopes. For both hRSV and bRSV, the attachment protein is a major target of the host anti-RSV antibody response [26] and specific regions of the protein may be under immune selection [27]. However, immunological pressures may differ between bRSV and hRSV as suggested by differences in the variability of the central hydrophobic region of the $G$ protein of these viruses [28]. 


\section{Experimental Bovine RSV in Calves and Similarities to Lesions seen in Human Infants}

We have used bRSV strain 375 for inoculation of neonates in our studies [29-31]. The inoculum was prepared from virus stock re-isolated from the lung of an infected animal and passaged less than 4 times on bovine turbinate cells. Our bRSV aerosol challenge model [31] was adapted from that described by Woolums et al. [32]. Briefly, the challenge inoculum is delivered by nebulization into a mask covering the nostrils and mouth. The nebulization apparatus consists of a compressed air tank, a jet nebulizer, and a mask (Trudell Medical International, London, Ontario, Canada) modified to fit calves. Compressed air $\left(25 \mathrm{lb} / \mathrm{in}^{2}\right)$ is used to jet nebulize the challenge inoculum directly into a holding reservoir. Upon installation, the nebulized inoculum is inhaled through a one-way valve into the mask and directly into the nostrils. Each calf received a $5 \mathrm{~mL}$ challenge inoculum containing approximately $10^{4} \mathrm{TCID}_{50} / \mathrm{mL}$ of bRSV strain 375 during the nebulization period of $10-15 \mathrm{~min}$.

Figure 1. Lung from an RSV-infected calf at day 7 post-infection. Note multifocal to coalescing areas of plum-red consolidation in cranial and ventral aspects of right cranial and middle lobes. Consolidated areas surround and divide foci of pale pink hyperinflated lung (arrow). Scattered multifocal areas of consolidation are also present in the ventral third of the right caudal lobe (arrowheads). Original figure: [31].

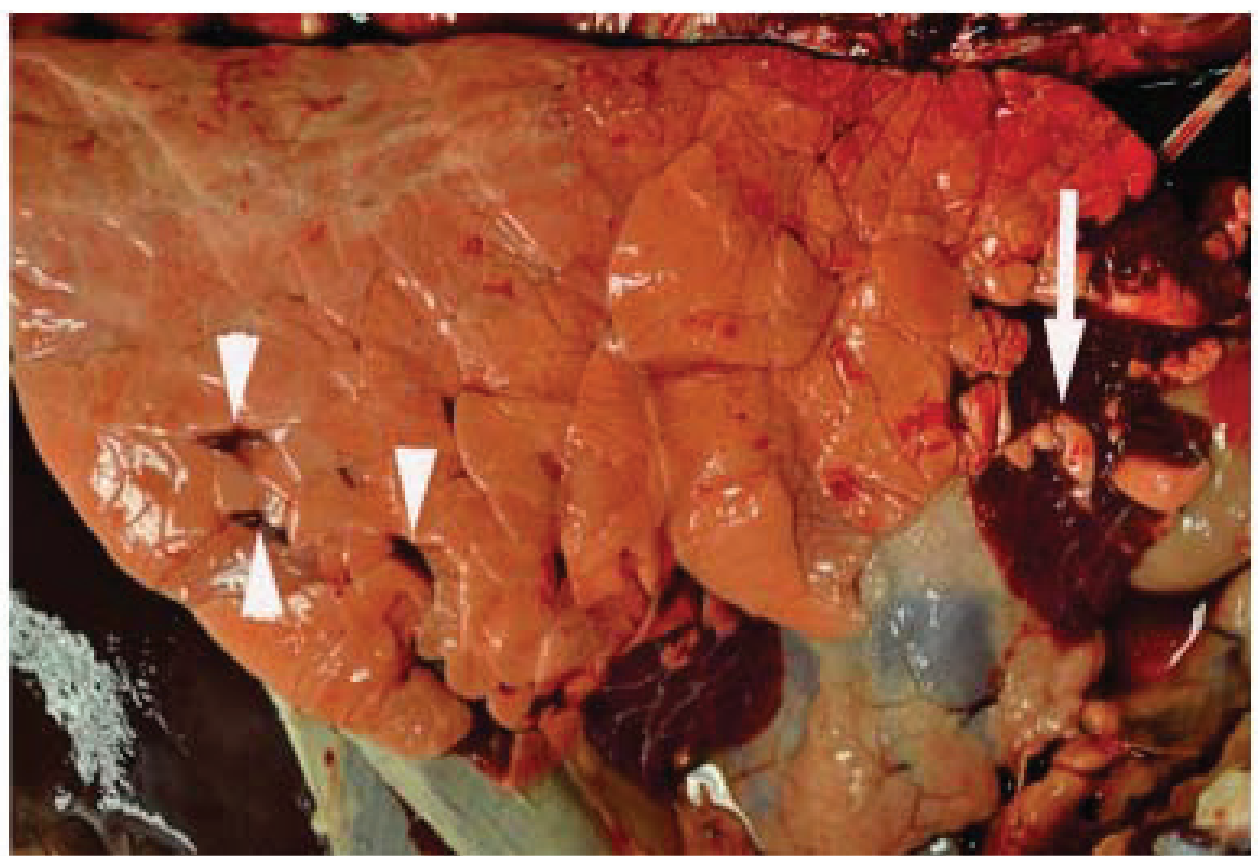


Figure 2. Histological lesions observed in lung of calves after experimental infection with bovine RSV. Calves were challenged via aerosol with bovine RSV. On day 7 post-infection, samples of lung were collected for histological evaluation. A representative image from a single calf is shown. (A) Moderate interstitial thickening and wide interlobular septae due to edema. Original magnification, 4X. (B) Alveolar septae are thickened due to cellular infiltrates found to be macrophages, lymphocytes and lesser numbers of neutrophils when viewed at higher magnification. Original magnification, 20X. (C) Bronchioles are filled with neutrophils, sloughed epithelial cells, and necrotic cell debris. Original magnification, 20X. (D) There is partial to complete loss of bronchiolar epithelial cells with attenuation of remaining cells. In some bronchioles, epithelial cells form multinucleated syncytial cells (arrows). Original magnification, 40X. Figure adapted from: [31].
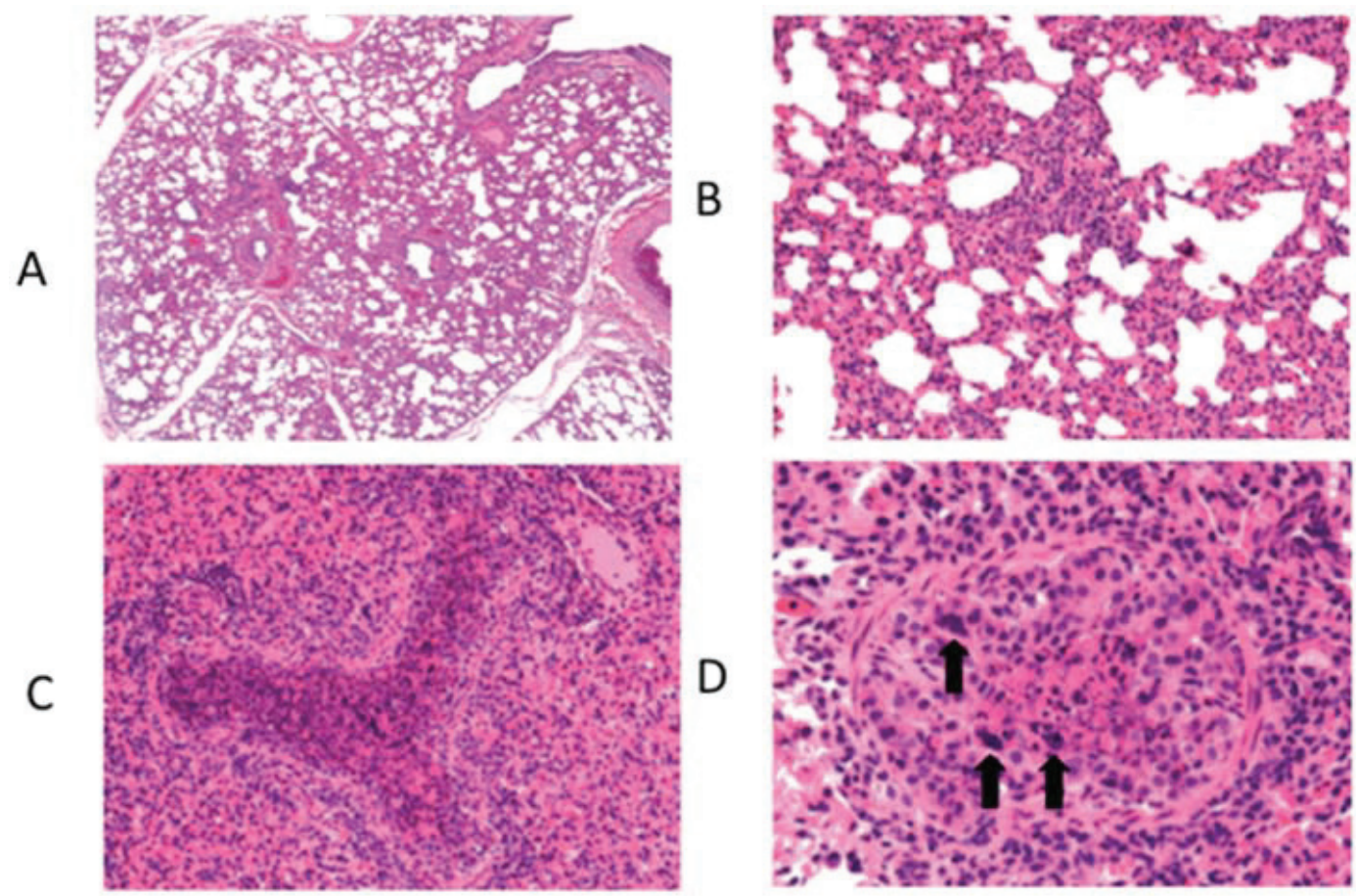

At necropsy, performed on day 7 post infection, gross lesions typically consist of bilateral, multifocal, firm, plum-red areas of consolidation that are of variable size and depressed compared to the adjacent normal appearing lung (Figure 1). Lesions are most frequently observed in cranioventral lung lobes. On cut surface, areas of consolidation are well delineated from adjacent normal lung. In some cases, areas of consolidation surround and divide regions of pink, hyperinflated lung. Significant microscopic lesions are apparent with representative photomicrographs shown (Figure 2). Microscopically, interlobular septa are expanded by clear space interpreted to be edema (Figure 2A). Alveolar septa are thickened due to infiltrates of macrophages, lymphocytes, and lesser numbers of neutrophils 
(Figure 2B). Intralesional bronchioles are filled with neutrophils, sloughed epithelial cells, and necrotic cellular debris (Figure 2C). Bronchiolar epithelial cell necrosis results in attenuation of remaining epithelial cells, or complete loss of airway epithelium. In some bronchioles, epithelial cells form multinucleated syncytial cells (Figure 2D). These lesions are similar to those described by others following experimental bRSV challenge.

In the rare instances where histopathological evaluation is available from fatal hRSV cases, it was noted that airway lumina contain cellular debris from leukocytes and sloughed epithelial cells, with fibrin and a minor amount of mucin [33-35]. Submucosal edema and peribronchiolar infiltrates, consisting predominately of mononuclear cells with minor numbers of neutrophils, further obstructed the airways. With interstitial pneumonia, there was evidence of significant alveolar involvement including edema and cellular infiltration, as well as epithelial cell attenuation or loss.

\section{Innate Immunity to RSV}

Critical to the induction of the innate immune response are pattern recognition receptors (PRRs) that recognize evolutionarily conserved pathogen-associated molecular patterns (PAMPs). Recognition of viral PAMPs involves at least three distinct classes of PRRs, toll-like receptors (TLRs), retinoic acid inducible gene-I (RIG-I)-like receptors (RLRs), and nucleotide-binding oligomerization domain (NOD)-like receptors (NLRs). Involvement of several TLRs has been described for hRSV infection, including TLR2, -3, -4, -7 and -8 [36,37]. Ligation of PRRs including TLR3, -7 and -8, and RLRs activates interferon regulatory factors (IRFs). These IRFs, in particular IRF3 and 7, interact with other transcription factors to control expression of type I IFNs and related molecules. Inactive IRFs reside in the cytoplasm in a latent form [38]. Viral infection triggers phosphorylation of serine and threonine residues and nuclear translocation of IRF3 and IRF7 where they associate with each other and complex with other co-activators (e.g., CREB-binding protein) to form a transcriptional complex that binds to promoter regions of type I IFN genes to activate transcription [39]. Virus-induced type I IFNs bind to the type I IFN receptor (IFNAR) activating Jak/STAT signaling leading to the upregulation of IFN-stimulated target genes (ISGs). Among ISGs are a number of antiviral mediators, including Mx, PKR, and 2'-5' oligoadenylate synthetase.

Paramixoviruses are known to utilize different mechanisms to affect IFN signaling. hRSV and bRSV have evolved strategies to inhibit the IFN-induced cellular response that are dependent upon nonstructural (NS) proteins. As is characteristic of pneumoviruses, these viruses have two genes that encode for NS proteins. It has been shown that NS1 and NS2 proteins cooperatively mediate resistance of bRSV and hRSV to IFN-stimulated responses in a species-specific manner [40,41]. In fact, the precise mechanism whereby type I IFN responses are altered, varies between these viruses. In the case of bRSV, NS proteins block phosphorylation and activation of IRF3 [42]. By comparison, hRSV NS1 and NS2 modulation of type I IFN responsiveness involves inhibition of Stat2 expression [43].

In addition to induction of IRFs, ligation of PRRs by viral PAMPs stimulates the release of inflammatory mediators. It has been shown that RSV induces cytokine/chemokine production in airway epithelial cells via signaling through TLR3 and RLRs, which have been linked to distinct 
pathways controlling NF- $\kappa \mathrm{B}$ activation [44]. In addition to respiratory epithelial cells, RSV infection induces inflammatory cytokines in antigen-presenting cells, the kinetics of which differs between cytokines. For example, we found peak induction of IL-1 $\beta$ and IL-12p40 mRNA in alveolar macrophages occurs on day 3 post-infection in the neonatal bRSV model, whereas IL-6 mRNA was higher at day 5 than day 3 of infection [29]. Recently, we have shown a significant upregulation of the innate chemokine IL-8 in lesioned lungs of bRSV-infected calves on day 7 post infection [31]. Importantly, our results fit well with data showing that IL-8 is elevated in the respiratory tract of children with hRSV bronchiolitis. Rodents lack a bona fide homologue of IL-8, although mice have what are considered to be functional homologues, CXCL1 (GRO/KC), CXCL2 (MIP-2) and CXCL5-6 (LIX), which belong to the same major cluster of chemokines. It is therefore evident that the neonatal calf model can prove useful as an in vivo platform for future exploration into pathways that specifically regulate innate immune responses during RSV infection.

Gamma delta $\mathrm{T}$ cells have been suggested to play a role in innate immunity to RSV based on their recognition of unprocessed and non-protein antigens independent of MHC restriction and their localization in epithelial tissues at the host-environmental interface [45]. In humans and mice, the frequency of $\gamma \delta \mathrm{T}$ cells in circulation or within secondary lymphoid tissues is low, generally representing less than $5 \%-10 \%$ of the circulating peripheral lymphocyte population [46]. However, in ruminants, $\gamma \delta \mathrm{T}$ cells are significantly more abundant, representing up to $70 \%$ of the circulating peripheral blood lymphocytes in very young animals $[47,48]$. Given their low frequency and the difficulty in obtaining sufficient cell numbers, relatively few studies exist which have examined the role of $\gamma \delta \mathrm{T}$ cells during hRSV infection. A study in patients hospitalized for hRSV bronchiolitis revealed a significant reduction in the numbers of $\gamma \delta \mathrm{T}$ cells circulating in the blood compared to control patients. Interestingly, this reduction was more pronounced in patients with severe disease [49]. Aoyagi et al. recently demonstrated that $\gamma \delta \mathrm{T}$ cells isolated from the blood of hRSV-infected infants produce less IFN $\gamma$ and more IL-4 in response to mitogen stimulation than equivalent cells isolated from rotavirus-infected infants [50], suggesting that $\gamma \delta$ T cells may be susceptible to the Th2-skewing often associated with hRSV infection (see section on Adaptive immunity to RSV). Data from mice suggests that $\gamma \delta \mathrm{T}$ cells contribute to immunopathology at the site of infection, as depletion of $\gamma \delta \mathrm{T}$ cells prior to challenge with hRSV results in reduced lung inflammation and disease severity [51]. However, $\gamma \delta \mathrm{T}$ cells may play a role in limiting viral replication in the lungs as their depletion also resulted in an increase in peak viral titers [51].

Despite their abundance in circulation in the blood of ruminants, studies of $\gamma \delta \mathrm{T}$ cells in the bovine during bRSV infection are also relatively scarce. A study by Taylor et al. reported that depletion of $\mathrm{WC}^{+} \gamma \delta \mathrm{T}$ cells from neonatal gnotobiotic calves infected with bRSV had little effect on clinical signs or viral clearance after 10 days of infection, but resulted in a significant increase in IgM and $\operatorname{IgA}$ in the bronchiolar alveolar lavage [52]. Similarly, $\gamma \delta \mathrm{T}$ cell depletion does not yield changes in macroscopic or microscopic lesions in the lungs of bRSV-infected calves compared to non-depleted control animals [53]. The results of these studies are difficult to interpret however, as the experimental numbers are very small and the infection failed to yield any clinical signs. Recent results from our 
laboratory suggest that bovine $\gamma \delta \mathrm{T}$ cell subsets respond to in vitro and in vivo BRSV infection with pro-inflammatory chemokine and cytokine production [54], suggesting $\gamma \delta \mathrm{T}$ cells may have a role in recruiting effector cells to the site of infection. Together, there appears a potential role for $\gamma \delta \mathrm{T}$ cells in altering lung pathology and viral clearance following both hRSV and bRSV infection, but much remains to be examined including the potential innate role of $\gamma \delta \mathrm{T}$ cells in regulating early virus recognition and lymphocyte recruitment.

\section{Adaptive Immunity to RSV}

The development of an adaptive immune response is required for the control and clearance of established RSV infections. Following infection, humans and cattle mount virus-specific antibody and $\mathrm{T}$ cell responses; however, these responses are weak and transient, as both species can be continuously re-infected throughout life. Clearance of RSV infection is primarily mediated by CD4 and CD8 $\alpha \beta \mathrm{T}$ cells. T cell responses are directed at epitopes within several RSV proteins including the N, M, NS2, M2-1, F and G [55]. The F and G proteins are the major HLA class II restricted targets in both humans and cattle $[55,56]$, with the F protein of hRSV being the most thoroughly studied and described to contain multiple antigenic regions [57]. To our knowledge, there are currently no specific BoLA class II epitopes defined for bRSV in the bovine.

While important in anti-viral immunity, the RSV-specific CD4 T cell response is also thought to contribute to disease pathogenesis and damaging immunopathology [58-60]. CD4 T cells, and the cytokines they produce, are key in shaping the nature of the adaptive virus-specific immune response. HRSV infection induces a mixed Th1 and Th2-type cytokine response [61]. Production of IL-12 by dendritic cells, and early IFN $\gamma$ is required for the priming of an effective Th1 type cytokine response; however, hRSV has been shown to interfere with dendritic cell cytokine production and their ability to initiate the development of a Th1 response $[56,62,63]$. The ensuing Th2 polarized response leads to increased disease severity and lung injury, and is thought to block the development of an effective CD8 $\mathrm{T}$ cell response during both primary and secondary challenge [56,64-66]. Much of our understanding about the adaptive immune response to hRSV is derived from small rodent models; however, evidence from humans, while limited, suggests a similar mechanism of disease pathogenesis. Infants infected with hRSV exhibit Th2 polarization with increased IL-4 production in the lungs and the establishment of eosinophilia [67,68]. Interestingly, eosinophilia was also apparent in the lungs of infants that exhibited vaccine enhanced disease after receiving a formalin-inactivated hRSV vaccine (see section on RSV Vaccines). However, cytokine balance and the resulting disease outcome likely depends upon genetic background, as some infants have also been shown to contain significant numbers IFN $\gamma$-producing cells in the lungs, and these increased levels did not correlate to disease severity or outcome [69].

Like humans, calves infected with bRSV develop a mixed cytokine response, but favor the development of a Th2-type immune response following infection. Studies of the cells and lymph fluid from bRSV infected calves reveal enhanced IL-4 and IL-13 production in the serum and tissues as early as day 4 post infection, and increased serum levels of virus-specific IgE, indicating the 
establishment of a Th2-type response [70-72]. Calves also develop IFN $\gamma$ producing cells and levels of the cytokine increase in the serum, but, as with humans, neither cell numbers nor IFN $\gamma$ levels correlate with positive disease outcome [73]. Evidence from humans has suggested that, due to the Th2 nature of the anti-viral immune response, hRSV infection may predispose children to the development of allergies and asthma later in life [74,75]. Interestingly, bRSV infection in calves has also been shown to predispose to allergic sensitization [76,77]. Gershwin et al. demonstrated that exposure to the model allergen ovalbumin during bRSV infection resulted in significantly increased levels of IL-4, IL-13 and ovalbumin-specific IgE compared to uninfected control calves [77].

Cytotoxic CD8 T cells play a critical role in the control and clearance of RSV infection. Infection of human infants results in a significant influx of activated CD8 T cells into the airways [78], and calves infected with bRSV exhibit increased CD8 T cell infiltration in the lungs, trachea and nasopharynx [79]. Depletion of CD8 T cells from mice [59] or bRSV infected calves [53] results in more severe disease and increased and sustained viral shedding compared to non-depleted control animals. In humans, the hRSV-specific HLA class I response is primarily targeted against the M2, $\mathrm{F}$ and $\mathrm{N}$ proteins [80-82]. Bovine CD8 T cells target the M2, F, N and G proteins of bRSV [83,84]. Interestingly, while G-specific CD8 $\mathrm{T}$ cells are readily detectable in cattle, they have not been demonstrated in humans [85]. It has been reported that the strong Th2 skewing that occurs during RSV infection acts to inhibit the development of an efficient CD8 $\mathrm{T}$ cell response and prevent the establishment of long-lived memory [56]. Anecdotally, this is evidenced by the recurring infections that occur commonly in both humans and calves [16,18]. In the mouse model, hRSV-specific CTLs appear impaired in both cytokine secretion and cytotoxicity $[56,86,87]$, while calves infected with bRSV exhibit a similar phenotype, displaying limited bRSV-specific cytotoxicity during primary infections and impaired memory responses following challenge or vaccination $[88,89]$.

Humoral immunity plays an important role in defending the host from RSV infection. While not fully effective, maternal antibodies may provide some level of protection from severe RSV infection in both humans [90-92] and calves [93]; however, their presence has also been described to suppress the development of antibody and $\mathrm{T}$ cell responses during acute infection [94,95]. Although humans initiate responses to several proteins of $\mathrm{hRSV}$, only antibodies that are reactive to the major surface glycoproteins $\mathrm{F}$ and $\mathrm{G}$ appear to be important for protection [96,97]. The F protein is highly conserved and the majority of the $\mathrm{F}$ specific response is cross-reactive, making it more important for protection across hRSV strains [98]. The G protein is more divergent, thus few antibodies cross-react between virus strains $[98,99]$. Further, the G-specific response is particularly inefficient and it has been proposed that this may be due to the secreted form of the G protein acting as a decoy for the host's humoral response [100,101].

Like humans, calves mount antibody responses to several bRSV antigens, but the primary targets for protective humoral responses are the F, G and NP proteins [84]. bRSV-specific IgM and IgA can be detected in the nasal secretions and serum of bRSV infected calves as early as 8 days post infection [95]. BRSV-specific IgG2, the antibody isotype associated with a Th1 response, is not detected in the serum until 1-3 months post infection. As evidence of the cytokine skewing that occurs 
during RSV infection of calves, virus-specific IgG1, the isotype associated with a Th2 phenotype, is detectable in the serum starting at 13 days post infection [95]. In a separate study, virus-specific $\operatorname{IgE}$, another antibody associated with Th2 skewing and airway hyperresponsiveness, was detectable in the serum concurrent with the development of clinical signs [71,72].

In humans, both neutralizing IgG and IgA are thought to have a role in protection from RSV. IgA is important in local immunity, particularly in the upper airways and nasopharynx, while serum $\operatorname{IgG}$ plays a significant role in protection of the lower airways. Adults who have been repeatedly infected with hRSV develop sustained high levels of $\operatorname{IgA}$ in nasal secretions which has been shown to prevent virus replication in the upper airways, regardless of serum Ig levels [102]. Further underlining the importance of both antibody types, passive immunization studies in rodents have shown that administration of neutralizing IgG provides complete protection from hRSV replication in the lungs, but not in nasal secretions [103-105]. However, the IgA response is often transient and neutralizing IgM and serum IgG are likely more important for long-term protection [94]. To date, the only licensed therapy available for hRSV infection in humans is the passive transfer of the virus-specific monoclonal antiserum, Palivizumab, which recognizes a conserved epitope in the F protein of hRSV. As proof of the importance of neutralizing antibodies in the immune response to $\mathrm{hRSV}$, the antiserum is effective as a prophylactic and known to reduce disease severity in hRSV-infected infants [106-108].

\section{RSV Vaccines}

In the 1960s, a formalin-inactivated hRSV vaccine was prepared and tested in infants and children. The Bernett strain of hRSV was initially propagated in human embryonic kidney cells and passaged in vervet monkey kidney cells. The infected cells were inactivated with formalin and concentrated by ultracentrifugation and alum precipitation. This preparation was known as lot 100. Lot 100 was administered as two or three intramuscular doses separated by 1 to 3 months to infants and children between 2 months and 7 years of age. Lot 100 not only failed to protect against hRSV disease, but also induced an exaggerated clinical response to hRSV infection in infants who were hRSV näive before vaccination [109-111]. Many vaccinates were hospitalized with LRI; in one study, the hospitalization rate of vaccinates approached $80 \%$ compared to $5 \%$ in controls [111]. Tragically, two infants who received lot 100 died following hRSV infection, one at 14 months of age and the second at 16 months of age [111]. HRSV was readily isolated from the lower respiratory tracts of these infants, whose lungs also contained eosinophilic infiltrates. The disastrous results of those clinical trials are still felt five decades later. To date there is no approved vaccine.

There are multiple vaccines currently marketed for bRSV (killed and modified-live) that are generally provided as part of multivalent products. As with humans, similar vaccine-enhanced disease has been reported from two cases of natural bRSV infections in calves [112,113]. In the former case, an outbreak of respiratory tract disease among 5- to 7-mo old calves on a beef-fattening farm in the Netherlands started two days after administration of a modified-live bRSV vaccine [112]. The disease was severe among vaccinates, but absent in non-vaccinated calves 8 mo of age or older. In the latter case, $30 \%$ of 8 -mo old Belgian Blue calves vaccinated with a beta-propiolactone inactivated bRSV 
vaccine died during a naturally occurring bRSV outbreak. Interestingly, no deaths were recorded among younger calves not vaccinated [113]. Experimentally, vaccine-enhanced disease has been reproduced in some studies of calves vaccinated with formalin-inactivated bRSV preparations [114,115], but not in others $[116,117]$. Where bRSV vaccine-enhanced disease has been observed, there is a bias toward a Th2-like response characterized by increases in serum IgE [114,118] and eosinophils in lavage fluid [114].

Given the significant disease burden associated with hRSV and bRSV infections, there is a profound interest in developing new and more efficacious vaccines for both species. RSV vaccine development in both cattle and humans has been the topic of several recent and excellent reviews $[55,101,119]$. As such, we will only briefly describe some of the approaches currently being pursued in the field.

RSV and its target populations pose several obstacles with respect to vaccine development, particularly the need to vaccinate populations with immature immune systems, to induce a response in the face of maternal antibodies, and to induce an appropriate, robust and long-lasting immune response. To address these challenges, a variety of new hRSV vaccines have recently been tested in animal models and, in some cases, clinical trials. The primary focus of these new platforms is to induce a more robust response using actual replicating agents, as opposed to the use of inactivated virus, and to induce Th1-type CD4 T cell response through the use of known CD4 T cell targeted proteins or Th1 activating adjuvants. Amongst these new candidates are synthetic and subunit vaccines that target whole or fragments of the F, N, G and M proteins, singly or in combination. Other examples include the use of live attenuated hRSV, such as strains lacking nonessential genes such as the NS1, NS2, SH, G or M2-2 genes and/or cold-passaged temperature-sensitive mutants [55,119]. One such temperature-sensitive mutant, which lacks the SH gene and contains a mutation in the L gene, proved promising in early phase I trials but lacked efficacy during phase II clinical trials in seronegative infants $[120,121]$. The stability of the mutant has recently been improved and shown success in trials using seronegative chimpanzees [121]. The use of nanoparticles, virosomes and virus-like particles, as well as vector-based approaches using DNA, virus or bacterial-based vectors that express hRSV proteins have all proven promising $[55,119]$. One of the vectors that has shown potential is a chimeric bovine/human parainfluenza virus type 3-based vaccine that has been engineered to express the hRSV F protein [122]. This vaccine has proven safe in phase I clinical trials and is currently being tested in a large international clinical trial in infants $[123,124]$.

Although there is widespread use of bRSV vaccines in calves, their efficacy is controversial and there is a definite need for improved technologies. Researchers are currently studying many of the same approaches as described for hRSV, including the use of subunit based vaccines and live attenuated bRSV [55]. One promising example reported by Valarcher et al. reported the success of two bRSV strains, one devoid of NS1 and the other lacking NS2 [125]. Calves vaccinated with either deletion mutant exhibited a robust virus-specific antibody and CD4 $\mathrm{T}$ cell response and were protected against virus challenge [125]. Interestingly, the NS2 mutant was more effective than its counterpart. Also being extensively pursued against bRSV is the use of new adjuvants coupled with inactivated bRSV 
or subunit vaccines. Amongst those showing promise is the use of $\mathrm{CpG}$ containing oligodeoxynucleotides (CpG-ODN) [126], and immunostimulating complexes (ISCOMS) [127,128] — both of which induce a robust Th1 skewing. ISCOMS are multimers composed of cholesterol, phospholipids, proteins and Quillaja saponins. Recent studies by Hagglund et al. have described the ability BRSV-ISCOMs to successfully induce bRSV-specific cellular and humoral responses and protect from virulent bRSV challenge in neonatal calves aged 3-8 weeks. Interestingly, this protection was robust despite the presence of significant levels of maternally derived antibodies $[127,128]$.

\section{Potential Role of Vitamin D as an Immunomodulator During RSV Infection}

Recent evidence has suggested a role for vitamin D in hematopoietic cell differentiation and immune function $[129,130]$. It is known that the nuclear vitamin D receptor (VDR) and the enzymes responsible for activation (CYP27B1; $1 \alpha$-hydroxylase) and degradation (CYP24A1; 24-hydroxylase) of vitamin D are expressed in subsets of immune cells. Moreover, several aspects of immune regulation are modified by the actions of vitamin D [129,131-134]. Based on these data and epidemiological evidence suggesting a connection between inadequate vitamin $\mathrm{D}$ levels and respiratory tract infections [135-137], there has been an examination of the ability of vitamin D to modulate the response to in vitro respiratory infections. In the case of hRSV, it was shown that treatment of cultured respiratory epithelial cells with $1,25(\mathrm{OH})_{2} \mathrm{D}_{3}$ decreases viral induction of pro-inflammatory gene expression [138]. In spite of the reduction of antiviral IFN- $\beta$, there was no concomitant increase in hRSV replication, suggesting that providing adequate vitamin $\mathrm{D}$ could reduce inflammation while maintaining antiviral activity.

We examined the influence of vitamin D status on the response to bRSV experimental challenge in calves [31]. Calves with high or low circulating $25(\mathrm{OH}) \mathrm{D}_{3}$ levels were challenged with RSV and subsequently, lung tissue samples examined at day 7 post infection. We showed, for the first time in vivo, that bRSV infection induced expression of the VDR and associated hydroxylase enzymes in the lung. Importantly, gene expression levels of pro-inflammatory cytokines were not suppressed in the presence of this induced vitamin D regulatory network, but rather specific pro-inflammatory cytokines were elevated in the high vitamin D group compared to the low vitamin D group of calves. Further examination of the potential effects of vitamin D status on bRSV disease resolution would require longer-term studies with immunologically sufficient and deficient vitamin D levels. Thus, preliminary bRSV challenge studies have been conducted with groups of calves with circulating levels considered sufficient $(40-60 \mathrm{ng} / \mathrm{mL})$ or deficient $(<20 \mathrm{ng} / \mathrm{mL})$ in $25(\mathrm{OH}) \mathrm{D}_{3}$. To date, we have not observed an alteration in the clinical course of bRSV as a result of providing differing levels of vitamin D supplementation.

\section{Summary}

In recent years, we have made significant progress in our knowledge of both bRSV and hRSV and the way each interacts with its respective host. To date, the disease process and lesions, as well as 
many aspects of the innate and adaptive immune response described during bRSV infection in the bovine parallel those described in human patients. See Table 1 for a summary of common features between bRSV and hRSV infection. From these data, we suggest that cattle serve as an excellent model for studying hRSV infection in humans. The field is currently pursuing several new approaches and vaccine platforms in both species that hold great promise for the future. Despite this, however, considerable challenges await with respect to our understanding of the RSV-specific immune response and the development of effective vaccine strategies.

Table 1. Features common to hRSV in infants and bRSV in calves.

\begin{tabular}{|c|c|c|c|}
\hline Feature & Human RSV & Bovine RSV & References \\
\hline Age-dependency & $\begin{array}{c}\text { More prevalent in } \\
\text { children }<2 \text { years old }\end{array}$ & $\begin{array}{l}\text { More prevalent in } \\
\text { calves }<6 \text { mo old }\end{array}$ & {$[16,17]$} \\
\hline Seasonal periodicity & More common in fall and winter & More common in fall and winter & [18] \\
\hline Histopathology & $\begin{array}{l}\text { Bronchiolitis; interstitial } \\
\text { pneumonia; } \\
\text { Prominent neutrophil and } \\
\text { macrophage infiltration }\end{array}$ & $\begin{array}{l}\text { Bronchiolitis; interstitial } \\
\text { pneumonia; } \\
\text { Prominent neutrophil and } \\
\text { macrophage infiltration }\end{array}$ & {$[31,33-35]$} \\
\hline CXC chemokines & CXCL8 (IL-8) upregulated & CXCL8 (IL-8) upregulated & {$[31,139]$} \\
\hline Adaptive immunity & Th2 cytokine bias & Th2 cytokine bias & {$[67,68,70-72]$} \\
\hline $\begin{array}{c}\text { Vaccine-enhanced } \\
\text { disease }\end{array}$ & $\begin{array}{c}\text { Observed with } \\
\text { formalin-inactivated vaccine }\end{array}$ & $\begin{array}{c}\text { Observed with } \\
\text { formalin-inactivated vaccine }\end{array}$ & [109-113] \\
\hline
\end{tabular}

\section{Acknowledgments}

The authors wish to thank Nancy Eischen, Emma Frimml-Morgan, Darrel Hoy, Tracy Porter, and Theresa Waters for their excellent technical assistance. Further, we thank our outstanding animal caretaker staff for the care of the calves. Finally, we thank Tyler C. Thacker for providing specific primer sequences for the work described herein.

\section{Conflicts of Interest}

The authors declare no conflict of interest.

\section{References}

1. Nair, H.; Nokes, D.J.; Gessner, B.D.; Dherani, M.; Madhi, S.A.; Singleton, R.J.; O’Brien, K.L.; Roca, A.; Wright, P.F.; Bruce, N.; et al. Global burden of acute lower respiratory infections due to respiratory syncytial virus in young children: A systematic review and meta-analysis. Lancet 2010, 375, 1545-1555.

2. Silvestri, M.; Sabatini, F.; Defilippi, A.C.; Rossi, G.A. The wheezy infant -- immunological and molecular considerations. Paediatr. Respir. Rev. 2004, 5 (Suppl. A), S81-S87. 
3. Stein, R.T.; Sherrill, D.; Morgan, W.J.; Holberg, C.J.; Halonen, M.; Taussig, L.M.; Wright, A.L.; Martinez, F.D. Respiratory syncytial virus in early life and risk of wheeze and allergy by age 13 years. Lancet 1999, 354, 541-545.

4. Falsey, A.R.; Hennessey, P.A.; Formica, M.A.; Cox, C.; Walsh, E.E. Respiratory syncytial virus infection in elderly and high-risk adults. N. Engl. J. Med. 2005, 352, 1749-1759.

5. Falsey, A.R.; Walsh, E.E. Respiratory syncytial virus infection in adults. Clin. Microbiol. Rev. 2000, 13, 371-384.

6. Raboni, S.M.; Nogueira, M.B.; Tsuchiya, L.R.; Takahashi, G.A.; Pereira, L.A.; Pasquini, R.; Siqueira, M.M. Respiratory tract viral infections in bone marrow transplant patients. Transplantation 2003, 76, 142-146.

7. Gershwin, L.J. Bovine respiratory syncytial virus infection: Immunopathogenic mechanisms. Anim. Health Res. Rev. 2007, 8, 207-213.

8. Blount, R.E., Jr.; Morris, J.A.; Savage, R.E. Recovery of cytopathogenic agent from chimpanzees with coryza. Proc. Soc. Exp. Biol. Med. 1956, 92, 544-549.

9. Chanock, R.; Finberg, L. Recovery from infants with respiratory illness of a virus related to chimpanzee coryza agent (CCA). II. Epidemiologic aspects of infection in infants and young children. Am. J. Hyg. 1957, 66, 291-300.

10. Chanock, R.; Roizman, B.; Myers, R. Recovery from infants with respiratory illness of a virus related to chimpanzee coryza agent (CCA). I. Isolation, properties and characterization. Am. J. Hyg. 1957, 66, 281-290.

11. Doggett, J.E.; Taylor-Robinson, D.; Gallop, R.G. A study of an inhibitor in bovine serum active against respiratory syncytial virus. Arch. Gesamte Virusforsch 1968, 23, 126-137.

12. Paccaud, M.F.; Jacquier, C. A respiratory syncytial virus of bovine origin. Arch. Gesamte Virusforsch. 1970, 30, 327-342.

13. Jacobs, J.W.; Edington, N. Isolation of respiratory syncytial virus from cattle in Britain. Vet. Rec. 1971, 88, 694.

14. Inaba, Y.; Tanaka, Y.; Sato, K.; Ito, H.; Omori, T. Nomi virus, a virus isolated from an apparently new epizootic respiratory disease of cattle. Jpn. J. Microbiol. 1970, 14, 246-248.

15. Inaba, Y.; Tanaka, Y.; Omori, T.; Matumoto, M. Isolation of bovine respiratory syncytial virus. Jpn. J. Exp. Med. 1970, 40, 473-474.

16. Glezen, W.P.; Taber, L.H.; Frank, A.L.; Kasel, J.A. Risk of primary infection and reinfection with respiratory syncytial virus. Am. J. Dis. Child. 1986, 140, 543-546.

17. Valarcher, J.F.; Schelcher, F.; Bourhy, H. Evolution of bovine respiratory syncytial virus. J. Virol. 2000, 74, 10714-10728.

18. Van der Poel, W.H.; Brand, A.; Kramps, J.A.; van Oirschot, J.T. Respiratory syncytial virus infections in human beings and in cattle. J. Infect. 1994, 29, 215-228.

19. Anderson, L.J.; Hierholzer, J.C.; Tsou, C.; Hendry, R.M.; Fernie, B.F.; Stone, Y.; McIntosh, K. Antigenic characterization of respiratory syncytial virus strains with monoclonal antibodies. J. Infect. Dis. 1985, 151, 626-633. 
20. Cristina, J.; Lopez, J.A.; Albo, C.; Garcia-Barreno, B.; Garcia, J.; Melero, J.A.; Portela, A. Analysis of genetic variability in human respiratory syncytial virus by the RNase A mismatch cleavage method: Subtype divergence and heterogeneity. Virology 1990, 174, 126-134.

21. Furze, J.M.; Roberts, S.R.; Wertz, G.W.; Taylor, G. Antigenically distinct G glycoproteins of BRSV strains share a high degree of genetic homogeneity. Virology 1997, 231, 48-58.

22. Deplanche, M.; Lemaire, M.; Mirandette, C.; Bonnet, M.; Schelcher, F.; Meyer, G. In vivo evidence for quasispecies distributions in the bovine respiratory syncytial virus genome. J. Gen. Virol. 2007, 88, 1260-1265.

23. McLellan, J.S.; Yang, Y.; Graham, B.S.; Kwong, P.D. Structure of respiratory syncytial virus fusion glycoprotein in the postfusion conformation reveals preservation of neutralizing epitopes. J. Virol. 2011, 85, 7788-7796.

24. Johnson, P.R.; Spriggs, M.K.; Olmsted, R.A.; Collins, P.L. The G glycoprotein of human respiratory syncytial viruses of subgroups A and B: Extensive sequence divergence between antigenically related proteins. Proc. Natl. Acad. Sci. USA 1987, 84, 5625-5629.

25. Valarcher, J.F.; Schelcher, F.; Bourhy, H. Evolution of bovine respiratory syncytial virus. J. Virol. 2000, 74, 10714-10728.

26. Garcia, O.; Martin, M.; Dopazo, J.; Arbiza, J.; Frabasile, S.; Russi, J.; Hortal, M.; Perez-Brena, P.; Martinez, I.; Garcia-Barreno, B.; et al. Evolutionary pattern of human respiratory syncytial virus (subgroup A): Cocirculating lineages and correlation of genetic and antigenic changes in the $\mathrm{G}$ glycoprotein. J. Virol. 1994, 68, 5448-5459.

27. Woelk, C.H.; Holmes, E.C. Variable immune-driven natural selection in the attachment (G) glycoprotein of respiratory syncytial virus (RSV). J. Mol. Evol. 2001, 52, 182-192.

28. Prozzi, D.; Walravens, K.; Langedijk, J.P.; Daus, F.; Kramps, J.A.; Letesson, J.J. Antigenic and molecular analyses of the variability of bovine respiratory syncytial virus $\mathrm{G}$ glycoprotein. J. Gen. Virol. 1997, 78 (Pt 2), 359-366.

29. Fach, S.J.; Meyerholz, D.K.; Gallup, J.M.; Ackermann, M.R.; Lehmkuhl, H.D.; Sacco, R.E. Neonatal ovine pulmonary dendritic cells support bovine respiratory syncytial virus replication with enhanced interleukin (IL)-4 And IL-10 gene transcripts. Viral. Immunol. 2007, 20, 119-130.

30. Meyerholz, D.K.; Grubor, B.; Fach, S.J.; Sacco, R.E.; Lehmkuhl, H.D.; Gallup, J.M.; Ackermann, M.R. Reduced clearance of respiratory syncytial virus infection in a preterm lamb model. Microbes Infect. 2004, 6, 1312-1319.

31. Sacco, R.E.; Nonnecke, B.J.; Palmer, M.V.; Waters, W.R.; Lippolis, J.D.; Reinhardt, T.A. Differential expression of cytokines in response to respiratory syncytial virus infection of calves with high or low circulating 25-hydroxyvitamin D3. PLoS One 2012, 7, e33074.

32. Woolums, A.R.; Anderson, M.L.; Gunther, R.A.; Schelegle, E.S.; LaRochelle, D.R.; Singer, R.S.; Boyle, G.A.; Friebertshauser, K.E.; Gershwin, L.J. Evaluation of severe disease induced by aerosol inoculation of calves with bovine respiratory syncytial virus. Am. J. Vet. Res. 1999, 60, 473-480.

33. Aherne, W.; Bird, T.; Court, S.D.; Gardner, P.S.; McQuillin, J. Pathological changes in virus infections of the lower respiratory tract in children. J. Clin. Pathol. 1970, 23, 7-18. 
34. Bem, R.A.; Domachowske, J.B.; Rosenberg, H.F. Animal models of human respiratory syncytial virus disease. Am. J. Physiol. Lung Cell. Mol. Physiol. 2011, 301, L148-L156.

35. Johnson, J.E.; Gonzales, R.A.; Olson, S.J.; Wright, P.F.; Graham, B.S. The histopathology of fatal untreated human respiratory syncytial virus infection. Mod. Pathol. 2007, 20, 108-119.

36. Bendelja, K.; Vojvoda, V.; Aberle, N.; Cepin-Bogovic, J.; Gagro, A.; Mlinaric-Galinovic, G.; Rabatic, S. Decreased Toll-like receptor 8 expression and lower TNF-alpha synthesis in infants with acute RSV infection. Respir. Res. 2010, 11, 143.

37. Zeng, R.; Cui, Y.; Hai, Y.; Liu, Y. Pattern recognition receptors for respiratory syncytial virus infection and design of vaccines. Virus Res. 2012, 167, 138-145.

38. Ning, S.; Pagano, J.S.; Barber, G.N. IRF7: Activation, regulation, modification and function. Genes Immun. 2011, 12, 399-414.

39. Yang, H.; Lin, C.H.; Ma, G.; Baffi, M.O.; Wathelet, M.G. Interferon regulatory factor-7 synergizes with other transcription factors through multiple interactions with p300/CBP coactivators. J. Biol. Chem. 2003, 278, 15495-15504.

40. Bossert, B.; Conzelmann, K.K. Respiratory syncytial virus (RSV) nonstructural (NS) proteins as host range determinants: A chimeric bovine RSV with NS genes from human RSV is attenuated in interferon-competent bovine cells. J. Virol. 2002, 76, 4287-4293.

41. Schlender, J.; Bossert, B.; Buchholz, U.; Conzelmann, K.K. Bovine respiratory syncytial virus nonstructural proteins NS1 and NS2 cooperatively antagonize alpha/beta interferon-induced antiviral response. J. Virol. 2000, 74, 8234-8242.

42. Bossert, B.; Marozin, S.; Conzelmann, K.K. Nonstructural proteins NS1 and NS2 of bovine respiratory syncytial virus block activation of interferon regulatory factor 3. J. Virol. 2003, 77, 8661-8668.

43. Lo, M.S.; Brazas, R.M.; Holtzman, M.J. Respiratory syncytial virus nonstructural proteins NS1 and NS2 mediate inhibition of Stat2 expression and alpha/beta interferon responsiveness. J. Virol. 2005, 79, 9315-9319.

44. Liu, P.; Jamaluddin, M.; Li, K.; Garofalo, R.P.; Casola, A.; Brasier, A.R. Retinoic acid-inducible gene I mediates early antiviral response and Toll-like receptor 3 expression in respiratory syncytial virus-infected airway epithelial cells. J. Virol. 2007, 81, 1401-1411.

45. Ferrick, D.A.; King, D.P.; Jackson, K.A.; Braun, R.K.; Tam, S.; Hyde, D.M.; Beaman, B.L. Intraepithelial gamma delta T lymphocytes: Sentinel cells at mucosal barriers. Springer Semin. Immunopathol. 2000, 22, 283-296.

46. Hayday, A.C. [gamma][delta] cells: A right time and a right place for a conserved third way of protection. Annu. Rev. Immunol. 2000, 18, 975-1026.

47. Hein, W.R.; Mackay, C.R. Prominence of gamma delta T cells in the ruminant immune system. Immunol. Today 1991, 12, 30-34.

48. Jutila, M.A.; Holderness, J.; Graff, J.C.; Hedges, J.F. Antigen-independent priming: A transitional response of bovine gammadelta T-cells to infection. Anim. Health Res. Rev. 2008, 9, 47-57. 
49. De Weerd, W.; Twilhaar, W.N.; Kimpen, J.L. T cell subset analysis in peripheral blood of children with RSV bronchiolitis. Scand. J. Infect. Dis. 1998, 30, 77-80.

50. Aoyagi, M.; Shimojo, N.; Sekine, K.; Nishimuta, T.; Kohno, Y. Respiratory syncytial virus infection suppresses IFN-gamma production of gammadelta T cells. Clin. Exp. Immunol. 2003, $131,312-317$.

51. Dodd, J.; Riffault, S.; Kodituwakku, J.S.; Hayday, A.C.; Openshaw, P.J. Pulmonary V gamma 4+ gamma delta $\mathrm{T}$ cells have proinflammatory and antiviral effects in viral lung disease. J. Immunol. 2009, 182, 1174-1181.

52. Taylor, G.; Thomas, L.H.; Wyld, S.G.; Furze, J.; Sopp, P.; Howard, C.J. Role of T-lymphocyte subsets in recovery from respiratory syncytial virus infection in calves. J. Virol. 1995, 69, 6658-6664.

53. Thomas, L.H.; Cook, R.S.; Howard, C.J.; Gaddum, R.M.; Taylor, G. Influence of selective T-lymphocyte depletion on the lung pathology of gnotobiotic calves and the distribution of different T-lymphocyte subsets following challenge with bovine respiratory syncytial virus. Res. Vet. Sci. 1996, 61, 38-44.

54. McGill, J.L.; Nonnecke, B.J.; Lippolis, J.D.; Reinhardt, T.A.; Sacco, R.E. Differential chemokine and cytokine production by neonatal bovine $\gamma \delta \mathrm{T}$ cell subsets in response to viral toll-like receptor agonists and in vivo RSV infection. Immunology Manuscript under review.

55. Meyer, G.; Deplanche, M.; Schelcher, F. Human and bovine respiratory syncytial virus vaccine research and development. Comp. Immunol. Microbiol. Infect. Dis. 2008, 31, 191-225.

56. Bueno, S.M.; Gonzalez, P.A.; Pacheco, R.; Leiva, E.D.; Cautivo, K.M.; Tobar, H.E.; Mora, J.E.; Prado, C.E.; Zuniga, J.P.; Jimenez, J.; et al. Host immunity during RSV pathogenesis. Int. Immunopharmacol. 2008, 8, 1320-1329.

57. Van Bleek, G.M.; Poelen, M.C.; van der Most, R.; Brugghe, H.F.; Timmermans, H.A.; Boog, C.J.; Hoogerhout, P.; Otten, H.G.; van Els, C.A. Identification of immunodominant epitopes derived from the respiratory syncytial virus fusion protein that are recognized by human CD4 T cells. J. Virol. 2003, 77, 980-988.

58. Cannon, M.J.; Openshaw, P.J.; Askonas, B.A. Cytotoxic T cells clear virus but augment lung pathology in mice infected with respiratory syncytial virus. J. Exp. Med. 1988, 168, 1163-1168.

59. Graham, B.S.; Bunton, L.A.; Wright, P.F.; Karzon, D.T. Role of T lymphocyte subsets in the pathogenesis of primary infection and rechallenge with respiratory syncytial virus in mice. J. Clin. Investig. 1991, 88, 1026-1033.

60. Graham, B.S.; Bunton, L.A.; Rowland, J.; Wright, P.F.; Karzon, D.T. Respiratory syncytial virus infection in anti-mu-treated mice. J. Virol. 1991, 65, 4936-4942.

61. Openshaw, P.J.; Tregoning, J.S. Immune responses and disease enhancement during respiratory syncytial virus infection. Clin. Microbiol. Rev. 2005, 18, 541-555.

62. Guerrero-Plata, A.; Casola, A.; Suarez, G.; Yu, X.; Spetch, L.; Peeples, M.E.; Garofalo, R.P. Differential response of dendritic cells to human metapneumovirus and respiratory syncytial virus. Am. J. Respir. Cell. Mol. Biol. 2006, 34, 320-329. 
63. Bartz, H.; Turkel, O.; Hoffjan, S.; Rothoeft, T.; Gonschorek, A.; Schauer, U. Respiratory syncytial virus decreases the capacity of myeloid dendritic cells to induce interferon-gamma in naive T cells. Immunology 2003, 109, 49-57.

64. Boelen, A.; Kwakkel, J.; Barends, M.; de Rond, L.; Dormans, J.; Kimman, T. Effect of lack of Interleukin-4, Interleukin-12, Interleukin-18, or the Interferon-gamma receptor on virus replication, cytokine response, and lung pathology during respiratory syncytial virus infection in mice. J. Med. Virol. 2002, 66, 552-560.

65. Hussell, T.; Baldwin, C.J.; O’Garra, A.; Openshaw, P.J. CD8+ T cells control Th2-driven pathology during pulmonary respiratory syncytial virus infection. Eur. J. Immunol. 1997, 27, 3341-3349.

66. Durbin, J.E.; Johnson, T.R.; Durbin, R.K.; Mertz, S.E.; Morotti, R.A.; Peebles, R.S.; Graham, B.S. The role of IFN in respiratory syncytial virus pathogenesis. J. Immunol. 2002, 168, 2944-2952.

67. Lindemans, C.A.; Kimpen, J.L.; Luijk, B.; Heidema, J.; Kanters, D.; van der Ent, C.K.; Koenderman, L. Systemic eosinophil response induced by respiratory syncytial virus. Clin. Exp. Immunol. 2006, 144, 409-417.

68. Kristjansson, S.; Bjarnarson, S.P.; Wennergren, G.; Palsdottir, A.H.; Arnadottir, T.; Haraldsson, A.; Jonsdottir, I. Respiratory syncytial virus and other respiratory viruses during the first 3 months of life promote a local TH2-like response. J. Allergy Clin. Immunol. 2005, 116, 805-811.

69. Van Benten, I.J.; van Drunen, C.M.; Koevoet, J.L.; Koopman, L.P.; Hop, W.C.; Osterhaus, A.D.; Neijens, H.J.; Fokkens, W.J. Reduced nasal IL-10 and enhanced TNFalpha responses during rhinovirus and RSV-induced upper respiratory tract infection in atopic and non-atopic infants. J. Med. Virol. 2005, 75, 348-357.

70. Miao, C.; Woolums, A.R.; Zarlenga, D.S.; Brown, C.C.; Brown, J.C., Jr.; Williams, S.M.; Scott, M.A. Effects of a single intranasal dose of modified-live bovine respiratory syncytial virus vaccine on cytokine messenger RNA expression following viral challenge in calves. Am. J. Vet. Res. 2004, 65, 725-733.

71. Stewart, R.S.; Gershwin, L.J. Role of $\operatorname{IgE}$ in the pathogenesis of bovine respiratory syncytial virus in sequential infections in vaccinated and nonvaccinated calves. Am. J. Vet. Res. 1989, 50, 349-355.

72. Stewart, R.S.; Gershwin, L.J. Detection of $\operatorname{IgE}$ antibodies to bovine respiratory syncytial virus. Vet. Immunol. Immunopathol. 1989, 20, 313-323.

73. Gershwin, L.J.; Gunther, R.A.; Anderson, M.L.; Woolums, A.R.; McArthur-Vaughan, K.; Randel, K.E.; Boyle, G.A.; Friebertshauser, K.E.; McInturff, P.S. Bovine respiratory syncytial virus-specific $\operatorname{IgE}$ is associated with interleukin-2 and -4, and interferon-gamma expression in pulmonary lymph of experimentally infected calves. Am. J. Vet. Res. 2000, 61, 291-298.

74. Sigurs, N.; Gustafsson, P.M.; Bjarnason, R.; Lundberg, F.; Schmidt, S.; Sigurbergsson, F.; Kjellman, B. Severe respiratory syncytial virus bronchiolitis in infancy and asthma and allergy at age 13. Am. J. Respir. Crit. Care Med. 2005, 171, 137-141. 
75. Sigurs, N.; Bjarnason, R.; Sigurbergsson, F.; Kjellman, B.; Bjorksten, B. Asthma and immunoglobulin $\mathrm{E}$ antibodies after respiratory syncytial virus bronchiolitis: A prospective cohort study with matched controls. Pediatrics 1995, 95, 500-505.

76. Gershwin, L.J.; Gunther, R.A.; Hornof, W.J.; Larson, R.F. Effect of infection with bovine respiratory syncytial virus on pulmonary clearance of an inhaled antigen in calves. Am. J. Vet. Res. 2008, 69, 416-422.

77. Gershwin, L.J.; Anderson, M.L.; Wang, C.; Berghaus, L.J.; Kenny, T.P.; Gunther, R.A. Assessment of IgE response and cytokine gene expression in pulmonary efferent lymph collected after ovalbumin inhalation during experimental infection of calves with bovine respiratory syncytial virus. Am. J. Vet. Res. 2011, 72, 134-145.

78. Heidema, J.; Lukens, M.V.; van Maren, W.W.; van Dijk, M.E.; Otten, H.G.; van Vught, A.J.; van der Werff, D.B.; van Gestel, S.J.; Semple, M.G.; Smyth, R.L.; et al. CD8+ T cell responses in bronchoalveolar lavage fluid and peripheral blood mononuclear cells of infants with severe primary respiratory syncytial virus infections. J. Immunol. 2007, 179, 8410-8417.

79. McInnes, E.; Sopp, P.; Howard, C.J.; Taylor, G. Phenotypic analysis of local cellular responses in calves infected with bovine respiratory syncytial virus. Immunology 1999, 96, 396-403.

80. Openshaw, P.J.; Anderson, K.; Wertz, G.W.; Askonas, B.A. The 22,000-kilodalton protein of respiratory syncytial virus is a major target for Kd-restricted cytotoxic $\mathrm{T}$ lymphocytes from mice primed by infection. J. Virol. 1990, 64, 1683-1689.

81. Cherrie, A.H.; Anderson, K.; Wertz, G.W.; Openshaw, P.J. Human cytotoxic T cells stimulated by antigen on dendritic cells recognize the $\mathrm{N}, \mathrm{SH}, \mathrm{F}, \mathrm{M}, 22 \mathrm{~K}$, and $1 \mathrm{~b}$ proteins of respiratory syncytial virus. J. Virol. 1992, 66, 2102-2110.

82. Bangham, C.R.; McMichael, A.J. Specific human cytotoxic T cells recognize B-cell lines persistently infected with respiratory syncytial virus. Proc. Natl. Acad. Sci. USA 1986, 83, 9183-9187.

83. Gaddum, R.M.; Cook, R.S.; Furze, J.M.; Ellis, S.A.; Taylor, G. Recognition of bovine respiratory syncytial virus proteins by bovine CD8+ T lymphocytes. Immunology 2003, 108, 220-229.

84. Taylor, G.; Thomas, L.H.; Furze, J.M.; Cook, R.S.; Wyld, S.G.; Lerch, R.; Hardy, R.; Wertz, G.W. Recombinant vaccinia viruses expressing the $\mathrm{F}, \mathrm{G}$ or $\mathrm{N}$, but not the $\mathrm{M} 2$, protein of bovine respiratory syncytial virus (BRSV) induce resistance to BRSV challenge in the calf and protect against the development of pneumonic lesions. J. Gen. Virol. 1997, 78 (Pt 12), 3195-3206.

85. Antonis, A.F.; Claassen, E.A.; Hensen, E.J.; de Groot, R.J.; de Groot-Mijnes, J.D.; Schrijver, R.S.; van der Most, R.G. Kinetics of antiviral CD8 T cell responses during primary and post-vaccination secondary bovine respiratory syncytial virus infection. Vaccine 2006, 24, 1551-1561.

86. Chang, J.; Braciale, T.J. Respiratory syncytial virus infection suppresses lung CD8+ T-cell effector activity and peripheral CD8+ T-cell memory in the respiratory tract. Nat. Med. 2002, 8, 54-60.

87. Chang, J.; Srikiatkhachorn, A.; Braciale, T.J. Visualization and characterization of respiratory syncytial virus F-specific CD8(+) T cells during experimental virus infection. J. Immunol. 2001, 167, 4254-4260. 
88. Gaddum, R.M.; Cook, R.S.; Thomas, L.H.; Taylor, G. Primary cytotoxic T-cell responses to bovine respiratory syncytial virus in calves. Immunology 1996, 88, 421-427.

89. Woolums, A.R.; Gunther, R.A.; McArthur-Vaughan, K.; Anderson, M.L.; Omlor, A.; Boyle, G.A.; Friebertshauser, K.E.; McInturff, P.S.; Gershwin, L.J. Cytotoxic T lymphocyte activity and cytokine expression in calves vaccinated with formalin-inactivated bovine respiratory syncytial virus prior to challenge. Comp. Immunol. Microbiol. Infect. Dis. 2004, 27, 57-74.

90. Piedra, P.A.; Jewell, A.M.; Cron, S.G.; Atmar, R.L.; Glezen, W.P. Correlates of immunity to respiratory syncytial virus (RSV) associated-hospitalization: Establishment of minimum protective threshold levels of serum neutralizing antibodies. Vaccine 2003, 21, 3479-3482.

91. Stensballe, L.G.; Ravn, H.; Kristensen, K.; Meakins, T.; Aaby, P.; Simoes, E.A. Seasonal variation of maternally derived respiratory syncytial virus antibodies and association with infant hospitalizations for respiratory syncytial virus. J. Pediatr. 2009, 154, 296-298.

92. Stensballe, L.G.; Ravn, H.; Kristensen, K.; Agerskov, K.; Meakins, T.; Aaby, P.; Simoes, E.A. Respiratory syncytial virus neutralizing antibodies in cord blood, respiratory syncytial virus hospitalization, and recurrent wheeze. J. Allergy. Clin. Immunol. 2009, 123, 398-403.

93. Kimman, T.G.; Zimmer, G.M.; Westenbrink, F.; Mars, J.; van Leeuwen, E. Epidemiological study of bovine respiratory syncytial virus infections in calves: Influence of maternal antibodies on the outcome of disease. Vet. Rec. 1988, 123, 104-109.

94. Crowe, J.E., Jr.; Williams, J.V. Immunology of viral respiratory tract infection in infancy. Paediatr. Respir. Rev. 2003, 4, 112-119.

95. Kimman, T.G.; Westenbrink, F.; Schreuder, B.E.; Straver, P.J. Local and systemic antibody response to bovine respiratory syncytial virus infection and reinfection in calves with and without maternal antibodies. J. Clin. Microbiol. 1987, 25, 1097-1106.

96. Groothuis, J.R. The role of RSV neutralizing antibodies in the treatment and prevention of respiratory syncytial virus infection in high-risk children. Antivir. Res. 1994, 23, 1-10.

97. Connors, M.; Collins, P.L.; Firestone, C.Y.; Murphy, B.R. Respiratory syncytial virus (RSV) F, G, M2 (22K), and N proteins each induce resistance to RSV challenge, but resistance induced by M2 and N proteins is relatively short-lived. J. Virol. 1991, 65, 1634-1637.

98. Oshansky, C.M.; Zhang, W.; Moore, E.; Tripp, R.A. The host response and molecular pathogenesis associated with respiratory syncytial virus infection. Future Microbiol. 2009, 4, 279-297.

99. Melero, J.A.; Garcia-Barreno, B.; Martinez, I.; Pringle, C.R.; Cane, P.A. Antigenic structure, evolution and immunobiology of human respiratory syncytial virus attachment $(\mathrm{G})$ protein. J. Gen. Virol. 1997, 78 (Pt 10), 2411-2418.

100. Bukreyev, A.; Yang, L.; Fricke, J.; Cheng, L.; Ward, J.M.; Murphy, B.R.; Collins, P.L. The secreted form of respiratory syncytial virus $\mathrm{G}$ glycoprotein helps the virus evade antibody-mediated restriction of replication by acting as an antigen decoy and through effects on Fc receptor-bearing leukocytes. J. Virol. 2008, 82, 12191-12204.

101. Graham, B.S. Biological challenges and technological opportunities for respiratory syncytial virus vaccine development. Immunol. Rev. 2011, 239, 149-166. 
102. Mills, J.T.; van Kirk, J.E.; Wright, P.F.; Chanock, R.M. Experimental respiratory syncytial virus infection of adults. Possible mechanisms of resistance to infection and illness. J. Immunol. 1971, 107, 123-130.

103. Prince, G.A.; Hemming, V.G.; Horswood, R.L.; Chanock, R.M. Immunoprophylaxis and immunotherapy of respiratory syncytial virus infection in the cotton rat. Virus Res. 1985, 3, 193-206.

104. Prince, G.A.; Horswood, R.L.; Chanock, R.M. Quantitative aspects of passive immunity to respiratory syncytial virus infection in infant cotton rats. $J$. Virol. 1985, 55, 517-520.

105. Singleton, R.; Etchart, N.; Hou, S.; Hyland, L. Inability to evoke a long-lasting protective immune response to respiratory syncytial virus infection in mice correlates with ineffective nasal antibody responses. J. Virol. 2003, 77, 11303-11311.

106. Singleton, R.; Dooley, L.; Bruden, D.; Raelson, S.; Butler, J.C. Impact of palivizumab prophylaxis on respiratory syncytial virus hospitalizations in high risk Alaska Native infants. Pediatr. Infect. Dis. J. 2003, 22, 540-545.

107. Malley, R.; DeVincenzo, J.; Ramilo, O.; Dennehy, P.H.; Meissner, H.C.; Gruber, W.C.; Sanchez, P.J.; Jafri, H.; Balsley, J.; Carlin, D.; et al. Reduction of respiratory syncytial virus (RSV) in tracheal aspirates in intubated infants by use of humanized monoclonal antibody to RSV F protein. J. Infect. Dis. 1998, 178, 1555-1561.

108. Simoes, E.A.; Groothuis, J.R.; Carbonell-Estrany, X.; Rieger, C.H.; Mitchell, I.; Fredrick, L.M.; Kimpen, J.L. Palivizumab prophylaxis, respiratory syncytial virus, and subsequent recurrent wheezing. J. Pediatr. 2007, 151, 34-42, 42 e31.

109. Chin, J.; Magoffin, R.L.; Shearer, L.A.; Schieble, J.H.; Lennette, E.H. Field evaluation of a respiratory syncytial virus vaccine and a trivalent parainfluenza virus vaccine in a pediatric population. Am. J. Epidemiol. 1969, 89, 449-463.

110. Kapikian, A.Z.; Mitchell, R.H.; Chanock, R.M.; Shvedoff, R.A.; Stewart, C.E. An epidemiologic study of altered clinical reactivity to respiratory syncytial (RS) virus infection in children previously vaccinated with an inactivated RS virus vaccine. Am. J. Epidemiol. 1969, 89, 405-421.

111. Kim, H.W.; Canchola, J.G.; Brandt, C.D.; Pyles, G.; Chanock, R.M.; Jensen, K.; Parrott, R.H. Respiratory syncytial virus disease in infants despite prior administration of antigenic inactivated vaccine. Am. J. Epidemiol. 1969, 89, 422-434.

112. Kimman, T.G.; Sol, J.; Westenbrink, F.; Straver, P.J. A severe outbreak of respiratory tract disease associated with bovine respiratory syncytial virus probably enhanced by vaccination with modified live vaccine. Vet. Q. 1989, 11, 250-253.

113. Schreiber, P.; Matheise, J.P.; Dessy, F.; Heimann, M.; Letesson, J.J.; Coppe, P.; Collard, A. High mortality rate associated with bovine respiratory syncytial virus (BRSV) infection in Belgian white blue calves previously vaccinated with an inactivated BRSV vaccine. J. Vet. Med. B Infect. Dis. Vet. Public Health 2000, 47, 535-550. 
114. Antonis, A.F.; Schrijver, R.S.; Daus, F.; Steverink, P.J.; Stockhofe, N.; Hensen, E.J.; Langedijk, J.P.; van der Most, R.G. Vaccine-induced immunopathology during bovine respiratory syncytial virus infection: Exploring the parameters of pathogenesis. J. Virol. 2003, 77, 12067-12073.

115. Gershwin, L.J.; Schelegle, E.S.; Gunther, R.A.; Anderson, M.L.; Woolums, A.R.; Larochelle, D.R.; Boyle, G.A.; Friebertshauser, K.E.; Singer, R.S. A bovine model of vaccine enhanced respiratory syncytial virus pathophysiology. Vaccine 1998, 16, 1225-1236.

116. West, K.; Petrie, L.; Haines, D.M.; Konoby, C.; Clark, E.G.; Martin, K.; Ellis, J.A. The effect of formalin-inactivated vaccine on respiratory disease associated with bovine respiratory syncytial virus infection in calves. Vaccine 1999, 17, 809-820.

117. Mohanty, S.B.; Rockemann, D.D.; Davidson, J.P.; Sharabrin, O.I.; Forst, S.M. Effect of vaccinal serum antibodies on bovine respiratory syncytial viral infection in calves. Am. J. Vet. Res. 1981, 42, 881-883.

118. Kalina, W.V.; Woolums, A.R.; Berghaus, R.D.; Gershwin, L.J. Formalin-inactivated bovine RSV vaccine enhances a Th2 mediated immune response in infected cattle. Vaccine 2004, 22, 1465-1474.

119. Hurwitz, J.L. Respiratory syncytial virus vaccine development. Expert Rev. Vaccines 2011, 10, 1415-1433.

120. Karron, R.A.; Wright, P.F.; Belshe, R.B.; Thumar, B.; Casey, R.; Newman, F.; Polack, F.P.; Randolph, V.B.; Deatly, A.; Hackell, J.; et al. Identification of a recombinant live attenuated respiratory syncytial virus vaccine candidate that is highly attenuated in infants. J. Infect. Dis. 2005, 191, 1093-1104.

121. Luongo, C.; Winter, C.C.; Collins, P.L.; Buchholz, U.J. Increased genetic and phenotypic stability of a promising live-attenuated respiratory syncytial virus vaccine candidate by reverse genetics. J. Virol. 2012, 86, 10792-10804.

122. Tang, R.S.; MacPhail, M.; Schickli, J.H.; Kaur, J.; Robinson, C.L.; Lawlor, H.A.; Guzzetta, J.M.; Spaete, R.R.; Haller, A.A. Parainfluenza virus type 3 expressing the native or soluble fusion (F) Protein of Respiratory Syncytial Virus (RSV) confers protection from RSV infection in African green monkeys. J. Virol. 2004, 78, 11198-11207.

123. Bernstein, D.I.; Malkin, E.; Abughali, N.; Falloon, J.; Yi, T.; Dubovsky, F. Phase 1 study of the safety and immunogenicity of a live, attenuated respiratory syncytial virus and parainfluenza virus type 3 vaccine in seronegative children. Pediatr. Infect. Dis. J. 2012, 31, 109-114.

124. Tang, R.S.; Spaete, R.R.; Thompson, M.W.; MacPhail, M.; Guzzetta, J.M.; Ryan, P.C.; Reisinger, K.; Chandler, P.; Hilty, M.; Walker, R.E.; et al. Development of a PIV-vectored RSV vaccine: Preclinical evaluation of safety, toxicity, and enhanced disease and initial clinical testing in healthy adults. Vaccine 2008, 26, 6373-6382.

125. Valarcher, J.F.; Furze, J.; Wyld, S.; Cook, R.; Conzelmann, K.K.; Taylor, G. Role of alpha/beta interferons in the attenuation and immunogenicity of recombinant bovine respiratory syncytial viruses lacking NS proteins. J. Virol. 2003, 77, 8426-8439. 
126. Mapletoft, J.W.; Oumouna, M.; Townsend, H.G.; Gomis, S.; Babiuk, L.A.; van Drunen Littel-van den Hurk, S. Formulation with $\mathrm{CpG}$ oligodeoxynucleotides increases cellular immunity and protection induced by vaccination of calves with formalin-inactivated bovine respiratory syncytial virus. Virology 2006, 353, 316-323.

127. Hagglund, S.; Hu, K.F.; Larsen, L.E.; Hakhverdyan, M.; Valarcher, J.F.; Taylor, G.; Morein, B.; Belak, S.; Alenius, S. Bovine respiratory syncytial virus ISCOMs--protection in the presence of maternal antibodies. Vaccine 2004, 23, 646-655.

128. Hagglund, S.; Hu, K.; Vargmar, K.; Pore, L.; Olofson, A.S.; Blodorn, K.; Anderson, J.; Ahooghalandari, P.; Pringle, J.; Taylor, G.; et al. Bovine respiratory syncytial virus ISCOMs-Immunity, protection and safety in young conventional calves. Vaccine 2011, 29, 8719-8730.

129. Hewison, M. Vitamin D and innate and adaptive immunity. Vitam. Horm. 2011, 86, 23-62.

130. Mora, J.R.; Iwata, M.; von Andrian, U.H. Vitamin effects on the immune system: Vitamins A and D take centre stage. Nat. Rev. Immunol. 2008, 8, 685-698.

131. Adams, J.S.; Liu, P.T.; Chun, R.; Modlin, R.L.; Hewison, M. Vitamin D in defense of the human immune response. Ann. N. Y. Acad. Sci. 2007, 1117, 94-105.

132. Bikle, D.D. Vitamin D regulation of immune function. Vitam. Horm. 2011, 86, 1-21.

133. Nelson, C.D.; Reinhardt, T.A.; Beitz, D.C.; Lippolis, J.D. In vivo activation of the intracrine vitamin D pathway in innate immune cells and mammary tissue during a bacterial infection. PLoS One 2010, 5, e15469.

134. Nelson, C.D.; Reinhardt, T.A.; Thacker, T.C.; Beitz, D.C.; Lippolis, J.D. Modulation of the bovine innate immune response by production of 1alpha,25-dihydroxyvitamin $\mathrm{D}(3)$ in bovine monocytes. J. Dairy Sci. 2010, 93, 1041-1049.

135. Grant, C.C.; Wall, C.R.; Gibbons, M.J.; Morton, S.M.; Santosham, M.; Black, R.E. Child nutrition and lower respiratory tract disease burden in New Zealand: A global context for a national perspective. J. Paediatr. Child. Health 2011, 47, 497-504.

136. Karatekin, G.; Kaya, A.; Salihoglu, O.; Balci, H.; Nuhoglu, A. Association of subclinical vitamin D deficiency in newborns with acute lower respiratory infection and their mothers. Eur. J. Clin. Nutr. 2009, 63, 473-477.

137. Wayse, V.; Yousafzai, A.; Mogale, K.; Filteau, S. Association of subclinical vitamin D deficiency with severe acute lower respiratory infection in Indian children under 5 y. Eur. J. Clin. Nutr. 2004, 58, 563-567.

138. Hansdottir, S.; Monick, M.M.; Lovan, N.; Powers, L.; Gerke, A.; Hunninghake, G.W. Vitamin D decreases respiratory syncytial virus induction of NF-kappaB-linked chemokines and cytokines in airway epithelium while maintaining the antiviral state. J. Immunol. 2010, 184, 965-974.

139. Abu-Harb, M.; Bell, F.; Finn, A.; Rao, W.H.; Nixon, L.; Shale, D.; Everard, M.L. IL-8 and neutrophil elastase levels in the respiratory tract of infants with RSV bronchiolitis. Eur. Respir. J. 1999, 14, 139-143. 
Reprinted from Viruses. Cite as: Dyer, K.D.; Garcia-Crespo, K.E.; Glineur, S.; Domachowske, J.B.; Rosenberg, H.F. The Pneumonia Virus of Mice (PVM) Model of Acute Respiratory Infection. Viruses 2012, 4, 3494-3510.

Review

\title{
The Pneumonia Virus of Mice (PVM) Model of Acute Respiratory Infection
}

\author{
Kimberly D. Dyer ${ }^{1, *}$, Katia E. Garcia-Crespo ${ }^{1}$, Stephanie Glineur ${ }^{1}$, Joseph B. Domachowske ${ }^{2}$ \\ and Helene F. Rosenberg ${ }^{1}$
}

1 Laboratory of Allergic Diseases, National Institute of Allergy and Infectious Diseases,

National Institutes of Health, Bethesda, MD 20892, USA;

E-Mails: garciacrespoke@niaid.nih.gov (K.E.G.-C.); stephanie.glineur@nih.gov (S.G.); hrosenberg@niaid.nih.gov (H.F.R.)

2 Department of Pediatrics, SUNY Upstate Medical University, Syracuse, NY 13210, USA;

E-Mail: domachoj@upstate.edu

* Author to whom correspondence should be addressed; E-Mail: kdyer@niaid.nih.gov;

Tel.: +1-302-402-2429; Fax: +1-301-480-8384.

Received: 7 November 2012; in revised form: 28 November 2012 / Accepted: 28 November 2012 /

Published: 5 December 2012

\begin{abstract}
Pneumonia Virus of Mice (PVM) is related to the human and bovine respiratory syncytial virus (RSV) pathogens, and has been used to study respiratory virus replication and the ensuing inflammatory response as a component of a natural host-pathogen relationship. As such, PVM infection in mice reproduces many of the clinical and pathologic features of the more severe forms of RSV infection in human infants. Here we review some of the most recent findings on the basic biology of PVM infection and its use as a model of disease, most notably for explorations of virus infection and allergic airways disease, for vaccine evaluation, and for the development of immunomodulatory strategies for acute respiratory virus infection.
\end{abstract}

Keywords: PVM; inflammation; leukocytes; eosinophils; respiratory syncytial virus; RSV; TLR; IFN; heterologous immunity; MIP-1 $\alpha$ 


\section{Introduction}

Pneumonia virus of mice (PVM), human respiratory syncytial virus (hRSV) and bovine respiratory syncytial virus (bRSV) are enveloped, negative sense, single-stranded RNA viruses of the family Paramyxoviridae, subfamily Pneumovirinae, genus Pneumovirus [1]. PVM was originally discovered in 1939 by researchers Horsfall and Hahn at The Rockefeller University as part of an attempt to identify pathogens from human clinical samples that would replicate in lung tissues of inbred mice. PVM was isolated from lung tissue of what had been presumed to be healthy control mice that had been subjected to serial mouse-to-mouse passage [2]. PVM virions are polymorphic and found in diverse shapes, from spheres of $80-120 \mu \mathrm{m}$ in diameter to filaments up to $3 \mu \mathrm{m}$ in length. The virus replicates over a period of $24-30 \mathrm{~h}$ in mouse lung tissue, with virus amplification proceeding at 16 -fold per cycle [2].

PVM is one of the many virus pathogens that are monitored in commercial and research rodent colonies [3,4]. In a study covering the years 2004-2007, Liang and colleagues [5] reported that $0.2 \%-1.0 \%$ of isolates from mouse colonies and $6.4 \%-25.8 \%$ of isolates from rat colonies tested were positive for PVM. Information on wild rodents is somewhat limited. However, an extensive three-year study performed by Kaplan and colleagues [6] documented over 40\% seropositivity for PVM in nearly 300 small wild rodents tested at 11 field sites in the United Kingdom. In contrast, Smith and colleagues [7] found no seropositivity for PVM among wild house mice in Southern Australia.

It is not yet clear how or if PVM replicates and induces pathology in non-rodent hosts. In a study carried out in 1986, Pringle and Eglin [8] found that more than $75 \%$ of adult sera had PVM-neutralizing activity that did not correlate with hRSV or parainfluenza virus (PIV)-3 neutralizing activity. More recently, Brock and colleagues [9] explored this question further, and determined that PVM did not replicate in situ when administered to the respiratory tracts of non-human primates, and that the PVM-neutralizing factor(s) in human sera did not interact specifically with virion components. In another recent development, Dubovi and colleagues [10,11] reported the isolation of canine pneumovirus $(\mathrm{CnPnV})$ from the respiratory tracts of shelter-confined dogs with apparent respiratory illness. CnPnV is very similar overall to PVM (Figure 1), replicates in the lungs of BALB/c mice and induces inflammatory pathology, morbidity and mortality similar to that elicited by PVM [12] but a much higher initial inoculum is required to elicit these effects. The specific virulence attributable to this virus in canine species remains to be explored.

There are two characterized strains of PVM, strain 15 (two variants) and strain J3666 in current use in the research community. The original studies by Horsfall and co-workers [2,13-15] were performed on an isolate named strain 15 , which was reported to be highly pathogenic in mice. Since that time, this strain had reportedly undergone tissue-culture passage, resulting in loss of its pathogenicity in vivo. Strain 15/Warwick is highly attenuated and elicits minimal inflammatory response [16] while strain 15/ATCC (American Tissue Culture Collection VR-25), in our hands, elicits inflammatory pathology in BALB/c mice but substantially less disease pathology in C57BL/6 mice [17]. Strain J3666 has reportedly been maintained via mouse passage [18] and thus retains full pathogenicity. The molecular organization of the PVM genome was elucidated by Easton and colleagues [19-22] and Krempl 
and colleagues [23,24]. The most significant differences between strains 15 and J3666 are in the G attachment protein. Anh and colleagues [25] documented the susceptibility of various strains of mice to strain J3666 as follows: $129 / \mathrm{Sv}>\mathrm{DBA}>\mathrm{C} 3 \mathrm{H} / \mathrm{HeJ}>\mathrm{BALB} / \mathrm{c}>\mathrm{C} 57 \mathrm{BL} / 6>\mathrm{SJL}$. Glineur and colleagues [26] have recently explored PVM infection in crosses between 129/Sv and SJL mice and have documented the polygenic nature of resistance and susceptibility to severe virus infection. A third strain, PVM strain Y, originally derived from a spontaneous infection in athymic mice [27] and featured in an early study of disease exacerbation in mice with severe combined immunodeficiency disease [28] has recently been sequenced (Figure 1; [29]).

Figure 1. (A) Although there is little direct amino acid sequence homology between PVM and hRSV, the two viruses share the same gene order. (B) Neighbor-joining tree featuring the amino acid sequences of the $\mathrm{G}$ glycoproteins of selected pneumoviruses; Genbank accession numbers include FJ614813.1; NC_001989.1; NC_006579; AY729016.1; JQ899033.1; HQ734815; AY743910.1. Panel A reprinted with permission from [1].

A.
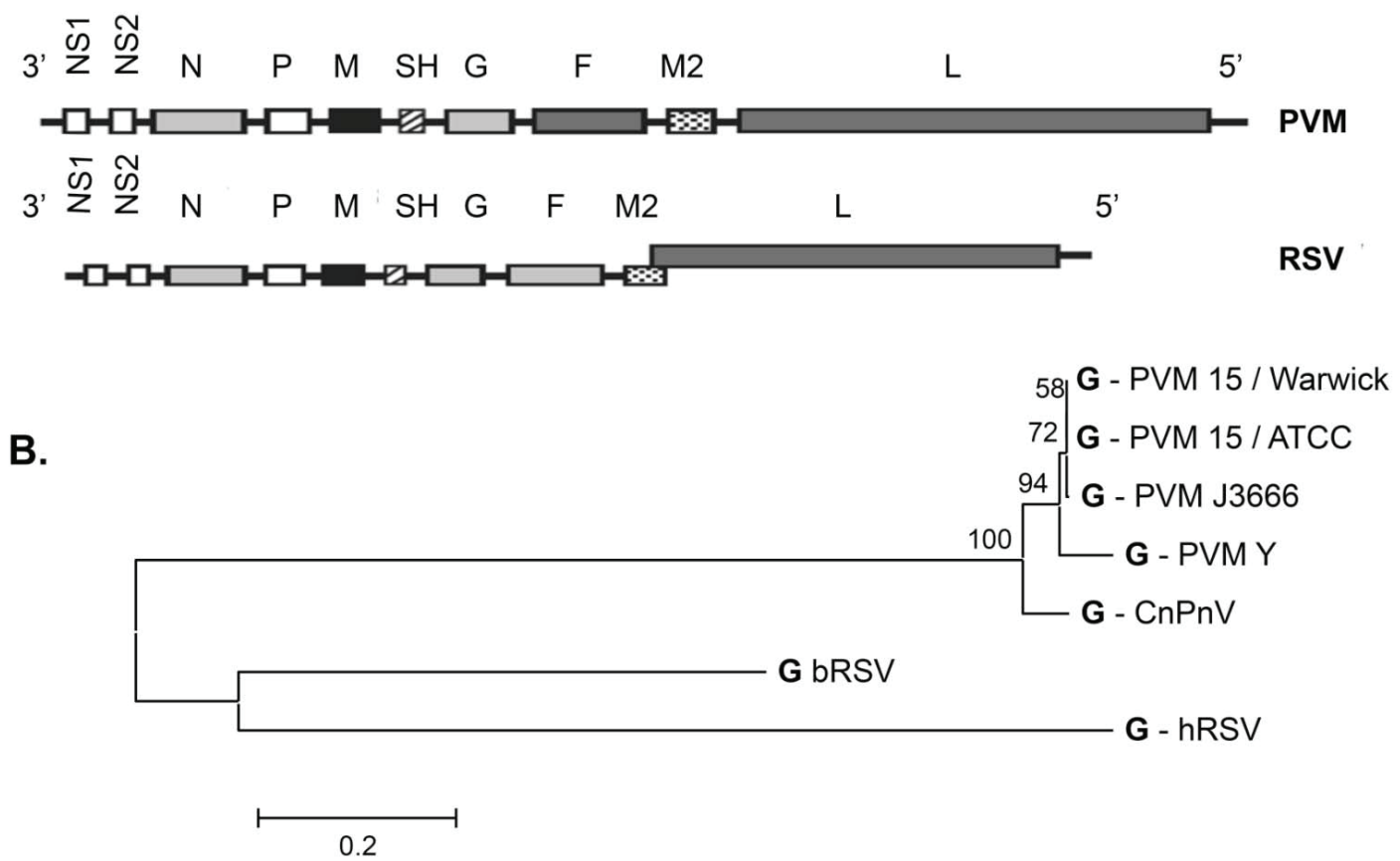

Horsfall and Ginsberg [14] recognized the potential of PVM for the exploration of acute respiratory virus infection in an evolutionarily relevant host. These authors were also the first to relate the development of lung lesions to ongoing virus replication and to evaluate altered morbidity and mortality in response to immunomodulatory therapy, specifically in response to administration of bacterial capsular polysaccharide [13-15]. We, and others, are using the PVM infection model to study 
the importance of virus-induced inflammatory responses in the development of severe respiratory virus disease and as a platform for the development of novel immunomodulatory strategies (see section on PVM and Disease, Heterologous Immunity).

\section{The PVM Model of Acute Respiratory Infection}

Our initial studies on PVM focused on the inflammatory responses to respiratory virus infection in a natural, evolutionarily relevant host [30,31]. We reproduced the findings of Horsfall and colleagues and reported robust virus replication in situ (to titers $>10^{8} \mathrm{pfu} / \mathrm{gm}$ lung tissue), progressing to marked morbidity (hunching, fur ruffling), weight loss, and mortality in response to a minimal virus inoculum of the highly pathogenic strain PVM J3666 [32,33]. We have localized immunoreactive PVM to the bronchiolar epithelium [34], in a distribution similar to what has been observed for RSV in human post-mortem specimens [35]. Profound inflammation of the lungs is evident and especially noteworthy is the recruitment of granulocytes and severe pulmonary edema. PVM replication in the mouse lung tissue is associated with local production of proinflammatory mediators including MIP-1 $\alpha$, MIP-2, and MCP-1 [34], consistent with those detected in lung and nasal washings in association with the more severe forms of RSV disease in human infants [1,36]. Although some features of the PVM model clearly conform to human pathophysiology, others do not. For example, neonatal mice exhibit little to no overt inflammation in response to PVM infection [37], nor can we establish a distinct pattern of infection in aged mice [38]. Similarly, it is crucial to recognize that PVM has no direct cross-reactivity with the human RSV pathogen, thus one's ability to perform studies of antigen-specific acquired immunity are limited.

\section{Host Immune Response to PVM Infection}

\subsection{Neutrophils and Eosinophils}

Microscopic examination of bronchoalveolar lavage fluid and lung tissue from morbid mice reveals profound inflammation, most notable for recruitment of granulocytes and progression to pulmonary edema (Figure 2). Similar to findings from the mouse model of influenza virus [39], MIP-1 $\alpha$ signaling through CC chemokine receptor (CCR)-1, its major receptor on neutrophils and eosinophils, is crucial for granulocyte recruitment in response to PVM infection [33]. We have built on this observation to explore immunomodulatory therapies for pneumovirus infection directed at limiting uncontrolled neutrophil influx $[40,41]$ as discussed below.

The role of eosinophils in respiratory virus infection is controversial and somewhat of a "double-edged sword" (reviewed in [42-44]). Eosinophils are among the granulocytes recruited at the earliest time points in response to PVM infection [32]. We and others have shown that eosinophils have antiviral properties against RSV [32,45,46]; recent findings from our laboratory demonstrate that activated eosinophils promote survival against lethal PVM infection [47]. PVM replicates in mouse eosinophils and promotes cytokine release [48]. 


\subsection{T Lymphocytes}

Although T cells have no apparent impact on the outcome of acute lethal PVM infection, both CD4 and $\mathrm{CD} 8^{+} \mathrm{T}$ cells are required for virus clearance in response to sublethal infection [49]. Claassen and colleagues [50] documented influx of activated $\mathrm{CD}^{+} \mathrm{T}$ cells into the lungs of infected mice and characterized PVM-specific responses against epitopes in the virus M (matrix; $\mathrm{M}_{43-51}$ ), F (fusion; $\mathrm{F}_{304-312}$ ) and $\mathrm{P}$ (phosphoprotein; $\mathrm{P}_{261-269}$ ) virion proteins. The relatively limited frequency of functional virus-specific $\mathrm{CD}^{+} \mathrm{T}$ cells suggested that PVM infection resulted in inactivation of effector $\mathrm{T}$ cells, similar to what has been reported in acute RSV infections [51]. Claassen and colleagues [52] have also identified a $\mathrm{CD}^{+} \mathrm{T}$ cell epitope in the $\mathrm{G}$ attachment protein, $\mathrm{G}_{381-385}$ and demonstrated protective immunity against lethal PVM challenge when mice were immunized simultaneously with both the $\mathrm{CD}^{+} \mathrm{G}_{381-385}$ and the $\mathrm{P}_{261-269} \mathrm{CD}^{+} \mathrm{T}$ cell epitope peptides.

While $\mathrm{CD}^{+}$and $\mathrm{CD}^{+} \mathrm{T}$ cells have been reported to promote virus clearance, IL-21, a type I cytokine produced primarily by activated $\mathrm{CD}^{+} \mathrm{T}$ cells, promotes pathology in response to PVM infection [53]. Mice devoid of the unique receptor for IL-21 (IL21R ${ }^{-/}$) have diminished levels of the proinflammatory chemokine $\mathrm{KC}$, and recruit fewer neutrophils, $\mathrm{CD} 4^{+}, \mathrm{CD}^{+}$and gamma-delta $\mathrm{T}$ cells to the lungs, and survive longer in response to PVM infection than their PVM-infected wild-type counterparts.

Figure 2. (A) Detection of PVM in bronchiolar epithelial cells, original magnification $63 \times$; (B,C) Histology of lung tissue from PVM-infected wild-type C57BL/6 mice, featuring multifocal acute alveolitis with intra-alveolar edema with scattered hemorrhage and moderate granulocytic infiltrates throughout; original magnifications $63 \times$ and $20 \times$, respectively; (D) Flow cytometric profiles of $\mathrm{Gr}^{+}$granulocytes in single cell suspensions from lung tissue of naïve and PVM-infected BALB/c mice. Reprinted with permission from (A) [34]; (B) and (C) [54].

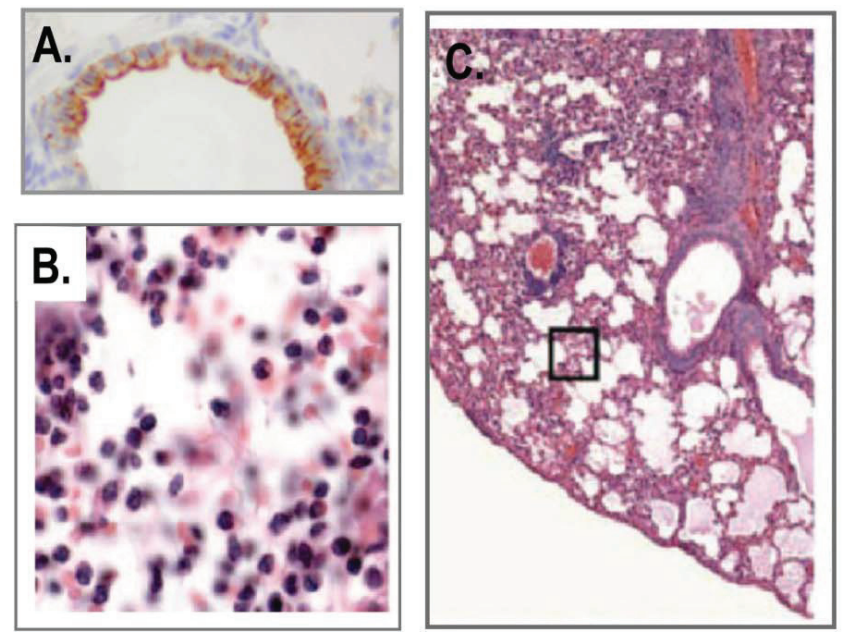

D.
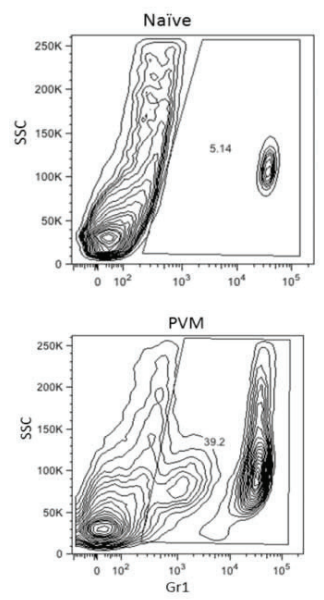


\subsection{Macrophages}

Macrophages are the main resident phagocytes in the lung and, working together with intact muco-ciliary to clear unwelcome debris including pathogens. Rigaux and colleagues [55] showed that depletion of alveolar macrophage prior to PVM infection resulted in a small increase in virus recovery and paradoxically prolonged survival. Macrophage depleted PVM-infected mice exhibited enhanced NK cell recruitment to the lungs accompanied by increased production of IFN $\gamma$ by recruited NK cells, $\mathrm{CD}^{+}$and $\mathrm{CD}^{+} \mathrm{T}$ cells. Interestingly, in similar studies featuring $\mathrm{RSV}$ challenge, Pribul and colleagues [56] found that macrophage depletion had no impact on virus-mediated $\mathrm{T}$ cell recruitment, weight loss or lung function, while Reed and colleagues [57] found that macrophage depletion prior to $\mathrm{RSV}$ challenge resulted in prominent airway occlusion in association with ongoing disease.

\subsection{Toll-Like Receptors}

Several studies have elucidated the nature and function of pattern recognition receptors (PRRs) that are involved in initiating the immune response to hRSV [58-60]. Despite findings that focused on hRSV signaling via TLR4, Faisca and colleagues [61] found that the sequelae of PVM infection - specifically, virus recovery, histopathology, body weight, and pulmonary function-were indistinguishable from one another when examined in wild-type and TLR4 gene-deleted mice. The results with the PVM model are consistent with recent findings from Marr and Turvey [62] who found that NF-kB activation mediated by infectious RSV particles in cell culture does not require the presence of a functional human TLR4/MD-2/CD14 complex.

Davidson and colleagues [63] utilized the PVM model to explore the role of TLR7 in promoting host defense against acute pneumovirus infection. Among their findings, PVM infection in TLR7 gene-deleted mice was associated with delayed induction of interferons and diminished recruitment of NK cells and neutrophils; adoptive transfer of TLR7-sufficient plasmacytoid dendritic cells restored innate antiviral responses and promoted virus clearance. Interestingly, TLR7-sufficient eosinophils also promote virus clearance in mouse models of RSV challenge [46].

\subsection{Type I Interferons}

Pneumoviruses have developed an efficient strategy to circumvent the host IFN response (reviewed in [64]). Among the most prominent of these findings, the non-structural NS1 and NS2 proteins of both human and bovine RSV inhibit the IFN alpha and beta (type I IFN) signaling pathways via several independent mechanisms [65-68], including degradation of the STAT2 signaling intermediate and blockade of activation and nuclear translocation of the transcription factor, interferon-regulatory factor 3 .

Garvey and colleagues [54] were the first to evaluate the interactions of PVM and type I interferons in their study of the sequelae of virus infection in mice devoid of the receptor for type I interferons (IFN $\alpha \beta \mathrm{R}^{-/-}$mice). PVM infection clearly elicited preferential expression of a wide spectrum of interferon-regulated and interferon-response genes, and virus replication in vivo was relatively 
suppressed in wild-type $v s$. IFN $\alpha \beta \mathrm{R}^{-/-}$mice. However, paradoxically prolonged survival was observed among the IFN $\alpha \beta \mathrm{R}^{-/}$mice, which may be attributed to the overriding impact of differential inflammatory pathology. Among the most striking differences, the IFN $\alpha \beta \mathrm{R}^{-/-}$mice developed tertiary mucosal associated lymphoid tissue (MALT, or B (bronchus) ALT) which has been associated with protection against virus pathogens in other settings (reviewed in [69]).

There are two studies that have directly addressed the role of PVM NS1 and NS2 using the recombinant virus (rPVM). In the first, Buchholz and colleagues [70] identified both NS1 and NS2 as virulence factors, as rPVM $\Delta \mathrm{NS} 1$ (i.e., with the NS1 gene-deletion), and more notably, $\mathrm{rPVM} \Delta \mathrm{NS} 2$, and $\mathrm{rPVM} \triangle \mathrm{NS} 1 \triangle \mathrm{NS} 2$ replicate less efficiently in BALB/c mice than the parent wild-type rPVM, and resulted in fewer clinical symptoms. Interestingly, the $\Delta \mathrm{NS} 1$ elicited production of both IFN $\alpha$ and

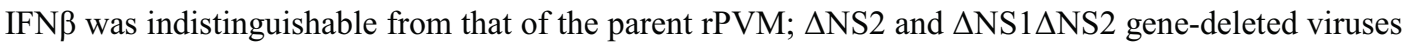
elicited higher levels of both IFN $\alpha$ and IFN $\beta$ than the parent rPVM at early time points during infection. In the second study, Heinze and colleagues [71] found that all three of the aforementioned deletion mutants replicated more effectively in IFN $\alpha \beta \mathrm{R}^{-/-}$mice than they did in wild type mice; replication of the mutant viruses was further enhanced in mice devoid of both IFN $\alpha \beta R$ and IL28R $\alpha$, the receptor for the type III interferon, IFN $\lambda$.

\section{Using the PVM Model to Explore Human Disease}

\subsection{Inflammation and Acute Infection}

Among the primary reasons to explore respiratory virus infection using the PVM model is to improve our understanding of the molecular basis of severe disease so as to design novel therapeutic strategies. As a natural rodent pathogen, PVM undergoes robust virus replication in lung tissue [31]. However, we have found that even highly effective antiviral therapy—strategies such as systemic ribavirin that result in immediate cessation of all further virus replication-do not provide tangible benefits when one is evaluating morbidity and mortality as endpoints (reviewed in [72]). Indeed, our experience with the PVM model mirrors the human clinical observations with ribavirin use for RSV infection. Ribavirin was once administered routinely to infants hospitalized with RSV disease; and although it was quite effective as an antiviral, clinical benefits were not observed [73,74]. As such, the PVM model has provided the impetus to explore several specific immunomodulatory strategies. Among the most promising directions are combined antivirals and immunomodulatory blockade of the proinflammatory cytokine, MIP-1 $\alpha$ [40,41]. Specifically, we have shown that antibody-mediated blockade of the actions of MIP- $1 \alpha$ resulted in improved survival, from $20 \%$ in response to ribavirin alone to $60 \%$ in response to ribavirin together with anti-MIP-1 $\alpha$ monoclonal antibody. Survival in response to acute PVM infection was also enhanced in response to increasing concentrations of metRANTES, which blocks MIP-1 $\alpha$ signaling via its primary receptor, CCR1.

A similar study documented the effectiveness of ribavirin in PVM infection in conjunction with montelukast a cysteinyl-leukotriene inhibitor [75]. Neither agent was effective at reducing morbidity or mortality when administered alone. However, significant improvements in long-term survival were 
observed when provided as combined therapy. Interestingly, montelukast had little impact on neutrophil recruitment, suggesting that the presence of neutrophils alone does not indicate inevitable progression to intractable disease.

The chemerin/ChemR23 (also known as CMKLR1) pathway is another potential therapeutic target [76]. pDCs preferentially express chemerin and prochemerin is processed by neutrophil proteases. Given that pDCs and neutrophils play an important role in the physiopathology of viral infections of the lung, the role of chemerin/ChemR23 in PVM was investigated. PVM-infected ChemR $23^{-/}$mice responded with augmented neutrophil recruitment, delayed virus clearance, and higher rates of morbidity and mortality than wild type counterparts, a response suggesting the therapeutic value of supplementation with the activated adipokine, chemerin, during acute virus infection.

Glucocorticoids are in general use as potent and non-specific anti-inflammatory agents but have only limited benefit for the treatment of severe hRSV-associated inflammation [77-80]. We have shown that hydrocortisone therapy has no effect on the production of MIP-1 $\alpha$ or on the influx of neutrophils during acute severe PVM infection. In fact, PVM-infected mice responded to hydrocortisone with enhanced viral replication and slightly accelerated mortality [81] suggesting that the added immunosupression of glucocortcoids in this context contributed to illness severity.

Bem and colleagues [82] explored the impact of mechanical ventilation on the acute inflammatory response in mice infected with PVM. In addition to increased levels of cytokines in the airways, mechanical ventilation activated caspase-dependent cell death pathways leading to acute lung injury in PVM-infected mice. In a subsequent study, van den Berg and colleagues [83] found that inflammatory injuries associated with mechanical ventilation were less severe in $\operatorname{lpr}$ (Fas-deficient) mice although all mice ultimately succumbed to infection.

\subsection{Asthma and Allergic Airway Disease}

The role of respiratory virus infection in promoting and exacerbating asthma and existing respiratory allergies is an area of significant medical concern [84,85]. Siegle and colleagues [86,87] have used PVM to determine how recovery from a respiratory virus infection early in life might alter subsequent responses to an unrelated allergen. Mice that recovered from a sublethal PVM infection displayed an exaggerated Th2 response to a chronic intranasal ovalbumin sensitization followed by a moderate challenge, with elevated levels of serum IgE and augmented expression of IL-4, IL-5 and IL-13; these responses were suppressed by a combination of neutralizing antibodies against both IL-4 and IL-25. Similarly, Barends and colleagues [88] found that PVM could exacerbate an ongoing allergic response. When PVM was administered to sensitized mice together with an intranasal antigen challenge, the virions elicited augmented eosinophil recruitment together with local elevation of Th2 cytokines. 


\subsection{Vaccines}

The first RSV vaccination trial, performed in the early 1960s with a formalin-inactivated preparation (FI-RSV, lot 100) resulted in an aberrant deleterious response following natural hRSV infection. Numerous vaccinated infants developed severe respiratory complications from subsequent natural RSV infection including two deaths, a phenomenon later referred to as "enhanced disease" [89]. Enhanced disease has been studied extensively, and has been modeled in BALB/c mice inoculated with formalin-inactivated RSV and RSV virion components (reviewed in [90,91]). PVM antigens, when prepared and administered in a manner analogous to the hRSV lot 100 vaccine also induces the enhanced disease response, likewise characterized by elevated levels of Th2 cytokines and eosinophil recruitment to airways and lung tissue [92]. Interestingly, the eosinophils, long perceived to be the cells promoting respiratory pathology in this setting, had no impact on virus recovery or weight loss in this experimental model.

Enhanced disease observed during the FI-RSV lot 100 study was among the issues that constrained further progress in the development of an RSV vaccine. Now, several decades later, a small number of human infant RSV vaccine trials are underway. Among the current vaccines under study, recent success with recombinant rodent Sendai virus (SeV) used to deliver RSV antigens [93,94] suggests that a similar approach may be feasible utilizing recombinant PVM [9,71]. Most recently, van Helden and colleagues [95] used the PVM model to explore the role of antigen-specific $\mathrm{CD}^{+} \mathrm{T}$ cells as a useful vaccine strategy. Among their findings, adoptive transfer of PVM-specific $\mathrm{CD} 8^{+} \mathrm{T}$ cells do provide at least partial protection against acute pneumovirus disease, and do not appear to contribute to immunopathology.

\subsection{Heterologous Immunity}

As part of an exploration of the immunomodulatory potential of probiotic Lactobacillus strains, we found that wild-type mice primed via intranasal inoculation with Lactobacillus plantarum or Lactobacillus reuteri were fully protected against lethal sequelae of a subsequent PVM infection ([96], Figure 3). These findings are a particularly robust example of heterologous immunity, a concept recently introduced into the literature that explains observations such as this, in which increased resistance (or susceptibility) to an unrelated (i.e., not cross-reactive) pathogen can be observed upon recovery from an inflammatory insult [97-99]. There are a number of examples in which PVM has been featured as a target pathogen in studies of heterologous immunity. One such study is that of Wiley and colleagues [100] who elicited protection against PVM (within a larger series of respiratory viruses) via instillation of protein cage nanoparticles, which are multi-subunit homopolymers of unique heat shock proteins from the thermophilic bacterium, Methanocaldococcus jannaschii). Likewise, Easton and colleagues [101] found that inoculation of mice with the defective interfering (DI) deletion mutant influenza 244/PR8 protects against subsequent infection with PVM. Interestingly, although each of these initial priming events_Lactobacillus, nanoparticles or defective interfering virus - all lead to a shared outcome, specifically, protection from the lethal sequelae of PVM 
infection, the cellular and biochemical mechanisms promoting these responses are unique and stimulus-specific [102].

Figure 3. (A) Mice primed via intranasal inoculation with L. plantarum or L. reuteri are fully $(100 \%)$ protected from the lethal sequelae of PVM infection. (B) Prolonged survival and significant long-term protection results even when virus challenge was delayed until 91 days (3 months) after initial Lactobacillus-mediated priming. Reprinted with permission from [96].

A.
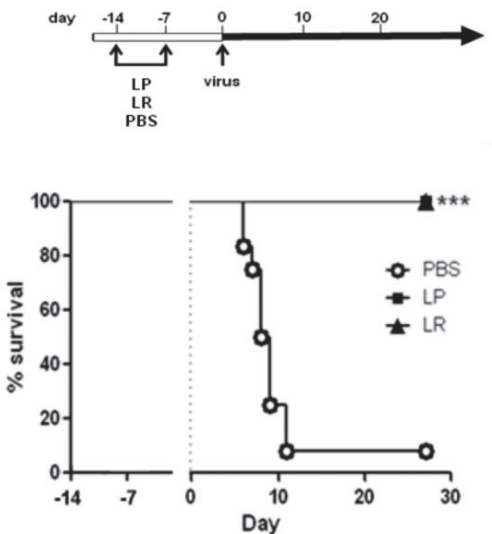

B.
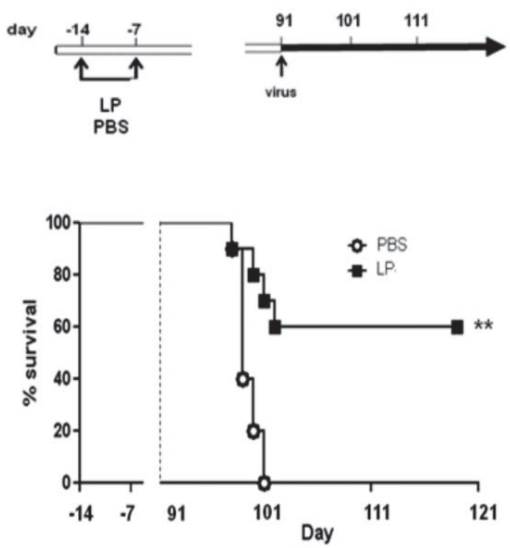

\section{Conclusions}

The PVM model holds great promise for the elucidation of inflammatory mechanisms associated with pneumovirus infection. Studies carried out to date have provided a rationale for the use of chemokine and/or chemokine receptor blockade alone and/or in conjunction with appropriate antiviral therapy as a means to reduce the inflammatory pathology in severe pneumovirus disease. Likewise, PVM is an excellent system in which to explore the molecular mechanisms of heterologous immunity to pneumovirus infection, information that may assist in the development of vaccines and other novel prevention strategies.

\section{Acknowledgments}

Funding for this work was provided by the NIAID Division of Intramural Research (AI000943 to HFR) and from the Children's Miracle Network of NY (to JBD).

\section{Conflicts of Interest}

The authors declare no conflict of interest. 


\section{References}

1. Easton, A.J.; Domachowske, J.B.; Rosenberg, H.F. Animal pneumoviruses: Molecular genetics and pathogenesis. Clin. Microbiol. Rev. 2004, 17, 390-412.

2. Horsfall, F.L.; Hahn, R.G. A latent virus in normal mice capable of producing pneumonia in its natural host. J. Exp. Med. 1940, 71, 391-408.

3. Miyata, H.; Kishikawa, M.; Kondo, H.; Kai, C.; Watanabe, Y.; Ohsawa, K.; Sato, H. New isolates of pneumonia virus of mice (PVM) from Japanese rat colonies and their characterization. Exp. Anim. 1995, 44, 95-104.

4. Zenner, L.; Regnault, J.P. Ten-year long monitoring of laboratory mouse and rat colonies in French facilities: A retrospective study. Lab. Anim. 2000, 34, 76-83.

5. Liang, C.T.; Shih, A.; Chang, Y.H.; Liu, C.W.; Lee, Y.T.; Hsieh, W.C.; Huang, Y.L.; Huang, W.T.; Kuang, C.H.; Lee, K.H.; et al. Microbial contaminations of laboratory mice and rats in Taiwan from 2004 to 2007. J. Am. Assoc. Lab. Anim. Sci. 2009, 48, 381-386.

6. Kaplan, C.; Healing, T.D.; Evans, N.; Healing, L.; Prior, A. Evidence of infection by viruses in small British field rodents. J. Hyg. 1980, 84, 285-294.

7. Smith, A.L.; Singleton, G.R.; Hansen, G.M.; Shellam, G. A serologic survey for viruses and Mycoplasma pulmonis among wild house mice (Mus domesticus) in southeastern Australia. J. Wildl. Dis. 1993, 29, 219-229.

8. Pringle, C.R.; Eglin, R.P. Murine pneumonia virus: Seroepidemiological evidence of widespread human infection. J. Gen. Virol. 1986, 67, 975-982.

9. Brock, L.G.; Karron, R.A.; Krempl, C.D.; Collins, P.L.; Buchholz, U.J. Evaluation of pneumonia virus of mice as a possible human pathogen. J. Virol. 2012, 86, 5829-5843.

10. Renshaw, R.; Laverack, M.; Zylich, N.; Glaser, A.; Dubovi, E. Genomic analysis of a pneumovirus isolated from dogs with acute respiratory disease. Vet. Microbiol. 2011, 150, 88-95.

11. Renshaw, R.W.; Zylich, N.C.; Laverack, M.A.; Glaser, A.L.; Dubovi, E.J. Pneumovirus in dogs with acute respiratory disease. Emerg. Infect. Dis. 2010, 16, 993-995.

12. Percopo, C.M.; Dubovi, E.J.; Renshaw, R.W.; Dyer, K.D.; Domachowske, J.B.; Rosenberg, H.F. Canine pneumovirus replicates in mouse lung tissue and elicits inflammatory pathology. Virology 2011, 416, 26-31.

13. Ginsberg, H.S.; Horsfall, F.L., Jr. Concurrent infection with influenza virus and mumps virus or pneumonia virus of mice as bearing on the inhibition of virus multiplication by bacterial polysaccharides. J. Exp. Med. 1949, 89, 37-52.

14. Ginsberg, H.S.; Horsfall, F.L., Jr. Therapy of infection with pneumonia virus of mice (PVM); effect of a polysaccharide on the multiplication cycles of the virus and on the course of the viral pneumonia. J. Exp. Med. 1951, 93, 161-171.

15. Horsfall, F.L., Jr.; Ginsberg, H.S. The dependence of the pathological lesion upon the multiplication of pneumonia virus of mice (PVM); kinetic relation between the degree of viral multiplication and the extent of pneumonia. J. Exp. Med. 1951, 93, 139-150. 
16. Thorpe, L.C.; Easton, A.J. Genome sequence of the non-pathogenic strain 15 of pneumonia virus of mice and comparison with the genome of the pathogenic strain J3666. J. Gen. Virol. 2005, 86, 159-169.

17. Ellis, J.A.; Martin, B.V.; Waldner, C.; Dyer, K.D.; Domachowske, J.B.; Rosenberg, H.F. Mucosal inoculation with an attenuated mouse pneumovirus strain protects against virulent challenge in wild type and interferon-gamma receptor deficient mice. Vaccine 2007, 25, 1085-1095.

18. Easton, A.J. University of Warwick, Coventry, UK. Personal communication, 1997.

19. Ahmadian, G.; Chambers, P.; Easton, A.J. Detection and characterization of proteins encoded by the second ORF of the M2 gene of pneumoviruses. J. Gen. Virol. 1999, 80, 2011-2016.

20. Barr, J.; Easton, A.J. Characterisation of the interaction between the nucleoprotein and phosphoprotein of pneumonia virus of mice. Virus Res. 1995, 39, 221-235.

21. Chambers, P.; Pringle, C.R.; Easton, A.J. Sequence analysis of the gene encoding the fusion glycoprotein of pneumonia virus of mice suggests possible conserved secondary structure elements in paramyxovirus fusion glycoproteins. J. Gen. Virol. 1992, 73, 1717-1724.

22. Easton, A.J.; Chambers, P. Nucleotide sequence of the genes encoding the matrix and small hydrophobic proteins of pneumonia virus of mice. Virus Res. 1997, 48, 27-33.

23. Krempl, C.D.; Collins, P.L. Reevaluation of the virulence of prototypic strain 15 of pneumonia virus of mice. J. Virol. 2004, 78, 13362-13365.

24. Krempl, C.D.; Wnekowicz, A.; Lamirande, E.W.; Nayebagha, G.; Collins, P.L.; Buchholz, U.J. Identification of a novel virulence factor in recombinant pneumonia virus of mice. J. Virol. 2007, 81, 9490-9501.

25. Anh, D.B.; Faisca, P.; Desmecht, D.J. Differential resistance/susceptibility patterns to pneumovirus infection among inbred mouse strains. Am. J. Physiol. Lung Cell Mol. Physiol. 2006, 291, L426-L435.

26. Glineur, S.; Tran Anh, D.B.; Sarlet, M.; Michaux, C.; Desmecht, D. Characterization of the resistance of SJL/J mice to pneumonia virus of mice, a model for infantile bronchiolitis due to a respiratory syncytial virus. PLoS One 2012, 7, e44581.

27. Weir, E.C.; Brownstein, D.G.; Smith, A.L.; Johnson, E.A. Respiratory disease and wasting in athymic mice infected with pneumonia virus of mice. Lab. Anim. Sci. 1988, 38, 133-137.

28. Roths, J.B.; Smith, A.L.; Sidman, C.L. Lethal exacerbation of Pneumocystis carinii pneumonia in severe combined immunodeficiency mice after infection by pneumonia virus of mice. J. Exp. Med. 1993, 177, 1193-1198.

29. Compton, C. Yale University, New Haven, CT, USA. Personal communication, 2012.

30. Bem, R.A.; Domachowske, J.B.; Rosenberg, H.F. Animal models of human respiratory syncytial virus disease. Am. J. Physiol. Lung Cell Mol. Physiol. 2011, 301, L148-L156.

31. Rosenberg, H.F.; Domachowske, J.B. Pneumonia virus of mice: Severe respiratory infection in a natural host. Immunol. Lett. 2008, 118, 6-12. 
32. Domachowske, J.B.; Bonville, C.A.; Dyer, K.D.; Easton, A.J.; Rosenberg, H.F. Pulmonary eosinophilia and production of MIP-1alpha are prominent responses to infection with pneumonia virus of mice. Cell Immunol. 2000, 200, 98-104.

33. Domachowske, J.B.; Bonville, C.A.; Gao, J.L.; Murphy, P.M.; Easton, A.J.; Rosenberg, H.F. The chemokine macrophage-inflammatory protein-1 alpha and its receptor CCR1 control pulmonary inflammation and antiviral host defense in paramyxovirus infection. J. Immunol. 2000, 165, 2677-2682.

34. Bonville, C.A.; Bennett, N.J.; Koehnlein, M.; Haines, D.M.; Ellis, J.A.; DelVecchio, A.M.; Rosenberg, H.F.; Domachowske, J.B. Respiratory dysfunction and proinflammatory chemokines in the pneumonia virus of mice (PVM) model of viral bronchiolitis. Virology 2006, 349, 87-95.

35. Welliver, T.P.; Garofalo, R.P.; Hosakote, Y.; Hintz, K.H.; Avendano, L.; Sanchez, K.; Velozo, L.; Jafri, H.; Chavez-Bueno, S.; Ogra, P.L.; et al. Severe human lower respiratory tract illness caused by respiratory syncytial virus and influenza virus is characterized by the absence of pulmonary cytotoxic lymphocyte responses. J. Infect. Dis. 2007, 195, 1126-1136.

36. Domachowske, J.B.; Bonville, C.A.; Rosenberg, H.F. Animal models for studying respiratory syncytial virus infection and its long term effects on lung function. Pediatr. Infect. Dis. J. 2004, 23, S228-S234.

37. Bonville, C.A.; Bennett, N.J.; Percopo, C.M.; Branigan, P.J.; Del Vecchio, A.M.; Rosenberg, H.F.; Domachowske, J.B. Diminished inflammatory responses to natural pneumovirus infection among older mice. Virology 2007, 368, 182-190.

38. Bonville, C.A.; Ptaschinski, C.; Percopo, C.M.; Rosenberg, H.F.; Domachowske, J.B. Inflammatory responses to acute pneumovirus infection in neonatal mice. Virol. J. 2010, 7, 320.

39. Cook, D.N.; Beck, M.A.; Coffman, T.M.; Kirby, S.L.; Sheridan, J.F.; Pragnell, I.B.; Smithies, O. Requirement of MIP-1 alpha for an inflammatory response to viral infection. Science 1995, 269, 1583-1585.

40. Bonville, C.A.; Easton, A.J.; Rosenberg, H.F.; Domachowske, J.B. Altered pathogenesis of severe pneumovirus infection in response to combined antiviral and specific immunomodulatory agents. J. Virol. 2003, 77, 1237-1244.

41. Bonville, C.A.; Lau, V.K.; DeLeon, J.M.; Gao, J.L.; Easton, A.J.; Rosenberg, H.F.; Domachowske, J.B. Functional antagonism of chemokine receptor CCR1 reduces mortality in acute pneumovirus infection in vivo. J. Virol. 2004, 78, 7984-7989.

42. Rosenberg, H.F.; Dyer, K.D.; Domachowske, J.B. Eosinophils and their interactions with respiratory virus pathogens. Immunol. Res. 2009, 43, 128-137.

43. Rosenberg, H.F.; Dyer, K.D.; Domachowske, J.B. Respiratory viruses and eosinophils: Exploring the connections. Antivir. Res. 2009, 83, 1-9.

44. Rosenberg, H.F.; Dyer, K.D.; Foster, P.S. Eosinophils: Changing perspectives in health and disease. Nat. Rev. Immunol. 2013, 13, 9-22. 
45. Adamko, D.J.; Yost, B.L.; Gleich, G.J.; Fryer, A.D.; Jacoby, D.B. Ovalbumin sensitization changes the inflammatory response to subsequent parainfluenza infection. Eosinophils mediate airway hyperresponsiveness, $\mathrm{m}(2)$ muscarinic receptor dysfunction, and antiviral effects. J. Exp. Med. 1999, 190, 1465-1478.

46. Phipps, S.; Lam, C.E.; Mahalingam, S.; Newhouse, M.; Ramirez, R.; Rosenberg, H.F.; Foster, P.S.; Matthaei, K.I. Eosinophils contribute to innate antiviral immunity and promote clearance of respiratory syncytial virus. Blood 2007, 110, 1578-1586.

47. Percopo, C.M.; Dyer, K.D.; Ochkur, S.I.; Lee, J.J.; Domachowske, J.B.; Rosenberg, H.F. Activated eosinophils protect against lethal respiratory virus infection. J. Immunol. 2013, to be submitted for publication.

48. Dyer, K.D.; Percopo, C.M.; Fischer, E.R.; Gabryszewski, S.J.; Rosenberg, H.F. Pneumoviruses infect eosinophils and elicit MyD88-dependent release of chemoattractant cytokines and interleukin-6. Blood 2009, 114, 2649-2656.

49. Frey, S.; Krempl, C.D.; Schmitt-Graff, A.; Ehl, S. Role of T cells in virus control and disease after infection with pneumonia virus of mice. J. Virol. 2008, 82, 11619-11627.

50. Claassen, E.A.; van der Kant, P.A.; Rychnavska, Z.S.; van Bleek, G.M.; Easton, A.J.; van der Most, R.G. Activation and inactivation of antiviral CD8 T cell responses during murine pneumovirus infection. J. Immunol. 2005, 175, 6597-6604.

51. Chang, J.; Braciale, T.J. Respiratory syncytial virus infection suppresses lung CD8+ T-cell effector activity and peripheral CD8+ T-cell memory in the respiratory tract. Nat. Med. 2002, 8, $54-60$.

52. Claassen, E.A.; van Bleek, G.M.; Rychnavska, Z.S.; de Groot, R.J.; Hensen, E.J.; Tijhaar, E.J.; van Eden, W.; van der Most, R.G. Identification of a CD4 T cell epitope in the pneumonia virus of mice glycoprotein and characterization of its role in protective immunity. Virology 2007, 368, $17-25$.

53. Spolski, R.; Wang, L.; Wan, C.K.; Bonville, C.A.; Domachowske, J.B.; Kim, H.P.; Yu, Z.; Leonard, W.J. IL-21 promotes the pathologic immune response to pneumovirus infection. J. Immunol. 2012, 188, 1924-1932.

54. Garvey, T.L.; Dyer, K.D.; Ellis, J.A.; Bonville, C.A.; Foster, B.; Prussin, C.; Easton, A.J.; Domachowske, J.B.; Rosenberg, H.F. Inflammatory responses to pneumovirus infection in IFN-alpha beta R gene-deleted mice. J. Immunol. 2005, 175, 4735-4744.

55. Rigaux, P.; Killoran, K.E.; Qiu, Z.; Rosenberg, H.F. Depletion of alveolar macrophages prolongs survival in response to acute pneumovirus infection. Virology 2012, 422, 338-345.

56. Pribul, P.K.; Harker, J.; Wang, B.; Wang, H.; Tregoning, J.S.; Schwarze, J.; Openshaw, P.J. Alveolar macrophages are a major determinant of early responses to viral lung infection but do not influence subsequent disease development. J. Virol. 2008, 82, 4441-4448.

57. Reed, J.L.; Brewah, Y.A.; Delaney, T.; Welliver, T.; Burwell, T.; Benjamin, E.; Kuta, E.; Kozhich, A.; McKinney, L.; Suzich, J.; et al. Macrophage impairment underlies airway occlusion in primary respiratory syncytial virus bronchiolitis. J. Infect. Dis. 2008, 198, 1783-1793. 
58. Klein Klouwenberg, P.; Tan, L.; Werkman, W.; van Bleek, G.M.; Coenjaerts, F. The role of Toll-like receptors in regulating the immune response against respiratory syncytial virus. Crit. Rev. Immunol. 2009, 29, 531-550.

59. Kurt-Jones, E.A.; Popova, L.; Kwinn, L.; Haynes, L.M.; Jones, L.P.; Tripp, R.A.; Walsh, E.E.; Freeman, M.W.; Golenbock, D.T.; Anderson, L.J.; et al. Pattern recognition receptors TLR4 and CD14 mediate response to respiratory syncytial virus. Nat. Immunol. 2000, 1, 398-401.

60. Ehl, S.; Bischoff, R.; Ostler, T.; Vallbracht, S.; Schulte-Monting, J.; Poltorak, A.; Freudenberg, M. The role of Toll-like receptor 4 versus interleukin-12 in immunity to respiratory syncytial virus. Eur. J. Immunol. 2004, 34, 1146-1153.

61. Faisca, P.; Tran Anh, D.B.; Thomas, A.; Desmecht, D. Suppression of pattern-recognition receptor TLR4 sensing does not alter lung responses to pneumovirus infection. Microbes Infect. 2006, 8, 621-627.

62. Marr, N.; Turvey, S.E. Role of human TLR4 in respiratory syncytial virus-induced NF-kappaB activation, viral entry and replication. Innate Immun. 2012, 18, 856-865.

63. Davidson, S.; Kaiko, G.; Loh, Z.; Lalwani, A.; Zhang, V.; Spann, K.; Foo, S.Y.; Hansbro, N.; Uematsu, S.; Akira, S.; et al. Plasmacytoid dendritic cells promote host defense against acute pneumovirus infection via the TLR7-MyD88-dependent signaling pathway. J. Immunol. 2011, 186, 5938-5948.

64. Fontana, J.M.; Bankamp, B.; Rota, P.A. Inhibition of interferon induction and signaling by paramyxoviruses. Immunol. Rev. 2008, 225, 46-67.

65. Bossert, B.; Conzelmann, K.K. Respiratory syncytial virus (RSV) nonstructural (NS) proteins as host range determinants: A chimeric bovine RSV with NS genes from human RSV is attenuated in interferon-competent bovine cells. J. Virol. 2002, 76, 4287-4293.

66. Ramaswamy, M.; Shi, L.; Monick, M.M.; Hunninghake, G.W.; Look, D.C. Specific inhibition of type I interferon signal transduction by respiratory syncytial virus. Am. J. Respir. Cell Mol. Biol. 2004, 30, 893-900.

67. Schlender, J.; Hornung, V.; Finke, S.; Gunthner-Biller, M.; Marozin, S.; Brzozka, K.; Moghim, S.; Endres, S.; Hartmann, G.; Conzelmann, K.K. Inhibition of toll-like receptor 7- and 9-mediated alpha/beta interferon production in human plasmacytoid dendritic cells by respiratory syncytial virus and measles virus. J. Virol. 2005, 79, 5507-5515.

68. Spann, K.M.; Tran, K.C.; Chi, B.; Rabin, R.L.; Collins, P.L. Suppression of the induction of alpha, beta, and lambda interferons by the NS1 and NS2 proteins of human respiratory syncytial virus in human epithelial cells and macrophages [corrected]. J. Virol. 2004, 78, 4363-4369.

69. Randall, T.D. Bronchus-associated lymphoid tissue (BALT) structure and function. Adv. Immunol. 2010, 107, 187-241.

70. Buchholz, U.J.; Ward, J.M.; Lamirande, E.W.; Heinze, B.; Krempl, C.D.; Collins, P.L. Deletion of nonstructural proteins NS1 and NS2 from pneumonia virus of mice attenuates viral replication and reduces pulmonary cytokine expression and disease. J. Virol. 2009, 83, 1969-1980. 
71. Heinze, B.; Frey, S.; Mordstein, M.; Schmitt-Graff, A.; Ehl, S.; Buchholz, U.J.; Collins, P.L.; Staeheli, P.; Krempl, C.D. Both nonstructural proteins NS1 and NS2 of pneumonia virus of mice are inhibitors of the interferon type I and type III responses in vivo. J. Virol. 2011, 85, 4071-4084.

72. Rosenberg, H.F.; Domachowske, J.B. Inflammatory responses to respiratory syncytial virus (RSV) infection and the development of immunomodulatory pharmacotherapeutics. Curr. Med. Chem. 2012, 19, 1424-1431.

73. Reassessment of the indications for ribavirin therapy in respiratory syncytial virus infections. American Academy of Pediatrics Committee on Infectious Diseases. Pediatrics 1996, 97, 137-140.

74. Van Woensel, J.B.; Kimpen, J.L.; Brand, P.L. Respiratory tract infections caused by respiratory syncytial virus in children. Diagnosis and treatment. Minerva Pediatr. 2001, 53, 99-106.

75. Bonville, C.A.; Rosenberg, H.F.; Domachowske, J.B. Ribavirin and cysteinyl leukotriene-1 receptor blockade as treatment for severe bronchiolitis. Antivir. Res. 2006, 69, 53-59.

76. Bondue, B.; Vosters, O.; de Nadai, P.; Glineur, S.; de Henau, O.; Luangsay, S.; van Gool, F.; Communi, D.; de Vuyst, P.; Desmecht, D.; et al. ChemR23 dampens lung inflammation and enhances anti-viral immunity in a mouse model of acute viral pneumonia. PLoS Pathog. 2011, 7, e1002358.

77. Buckingham, S.C.; Jafri, H.S.; Bush, A.J.; Carubelli, C.M.; Sheeran, P.; Hardy, R.D.; Ottolini, M.G.; Ramilo, O.; DeVincenzo, J.P. A randomized, double-blind, placebo-controlled trial of dexamethasone in severe respiratory syncytial virus (RSV) infection: Effects on RSV quantity and clinical outcome. J. Infect. Dis. 2002, 185, 1222-1228.

78. Patel, H.; Platt, R.; Lozano, J.M.; Wang, E.E. Glucocorticoids for acute viral bronchiolitis in infants and young children. Cochrane Database Syst. Rev. 2004, CD004878.

79. Van Woensel, J.B.; Lutter, R.; Biezeveld, M.H.; Dekker, T.; Nijhuis, M.; van Aalderen, W.M.; Kuijpers, T.W. Effect of dexamethasone on tracheal viral load and interleukin-8 tracheal concentration in children with respiratory syncytial virus infection. Pediatr. Infect. Dis. J. 2003, 22, 721-726.

80. Van Woensel, J.B.; van Aalderen, W.M.; de Weerd, W.; Jansen, N.J.; van Gestel, J.P.; Markhorst, D.G.; van Vught, A.J.; Bos, A.P.; Kimpen, J.L. Dexamethasone for treatment of patients mechanically ventilated for lower respiratory tract infection caused by respiratory syncytial virus. Thorax 2003, 58, 383-387.

81. Domachowske, J.B.; Bonville, C.A.; Ali-Ahmad, D.; Dyer, K.D.; Easton, A.J.; Rosenberg, H.F. Glucocorticoid administration accelerates mortality of pneumovirus-infected mice. J. Infect. Dis. 2001, 184, 1518-1523.

82. Bem, R.A.; van Woensel, J.B.; Bos, A.P.; Koski, A.; Farnand, A.W.; Domachowske, J.B.; Rosenberg, H.F.; Martin, T.R.; Matute-Bello, G. Mechanical ventilation enhances lung inflammation and caspase activity in a model of mouse pneumovirus infection. Am. J. Physiol. Lung Cell Mol. Physiol. 2009, 296, L46-56. 
83. Van den Berg, E.; van Woensel, J.B.; Bos, A.P.; Bem, R.A.; Altemeier, W.A.; Gill, S.E.; Martin, T.R.; Matute-Bello, G. Role of the Fas/FasL system in a model of RSV infection in mechanically ventilated mice. Am. J. Physiol. Lung Cell Mol. Physiol. 2011, 301, L451-L460.

84. Lukacs, N.W.; Smit, J.; Lindell, D.; Schaller, M. Respiratory syncytial virus-induced pulmonary disease and exacerbation of allergic asthma. Contrib. Microbiol. 2007, 14, 68-82.

85. Xepapadaki, P.; Papadopoulos, N.G. Viral infections and allergies. Immunobiology 2007, 212, 453-459.

86. Siegle, J.S.; Hansbro, N.; Dong, C.; Angkasekwinai, P.; Foster, P.S.; Kumar, R.K. Blocking induction of T helper type 2 responses prevents development of disease in a model of childhood asthma. Clin. Exp. Immunol. 2011, 165, 19-28.

87. Siegle, J.S.; Hansbro, N.; Herbert, C.; Rosenberg, H.F.; Domachowske, J.B.; Asquith, K.L.; Foster, P.S.; Kumar, R.K. Early-life viral infection and allergen exposure interact to induce an asthmatic phenotype in mice. Respir. Res. 2010, 11, 14.

88. Barends, M.; de Rond, L.G.; Dormans, J.; van Oosten, M.; Boelen, A.; Neijens, H.J.; Osterhaus, A.D.; Kimman, T.G. Respiratory syncytial virus, pneumonia virus of mice, and influenza A virus differently affect respiratory allergy in mice. Clin. Exp. Allergy 2004, 34, 488-496.

89. Kapikian, A.Z.; Mitchell, R.H.; Chanock, R.M.; Shvedoff, R.A.; Stewart, C.E. An epidemiologic study of altered clinical reactivity to respiratory syncytial (RS) virus infection in children previously vaccinated with an inactivated RS virus vaccine. Am. J. Epidemiol. 1969, 89, 405-421.

90. Castilow, E.M.; Olson, M.R.; Meyerholz, D.K.; Varga, S.M. Differential role of gamma interferon in inhibiting pulmonary eosinophilia and exacerbating systemic disease in fusion protein-immunized mice undergoing challenge infection with respiratory syncytial virus. J. Virol. 2008, 82, 2196-2207.

91. Castilow, E.M.; Olson, M.R.; Varga, S.M. Understanding respiratory syncytial virus (RSV) vaccine-enhanced disease. Immunol. Res. 2007, 39, 225-239.

92. Percopo, C.M.; Qiu, Z.J.; Phipps, S.; Foster, P.S.; Domachowske, J.B.; Rosenberg, H.F. Pulmonary eosinophils and their role in immunopathologic responses to formalin-inactivated pneumonia virus of mice. J. Immunol. 2009, 183, 604-612.

93. Hurwitz, J.L. Respiratory syncytial virus vaccine development. Expert Rev. Vaccines. 2011, 10, 1415-1433.

94. Jones, B.G.; Sealy, R.E.; Rudraraju, R.; Traina-Dorge, V.L.; Finneyfrock, B.; Cook, A.; Takimoto, T.; Portner, A.; Hurwitz, J.L. Sendai virus-based RSV vaccine protects African green monkeys from RSV infection. Vaccine 2012, 30, 959-968.

95. Van Helden, M.J.; van Kooten, P.J.; Bekker, C.P.; Grone, A.; Topham, D.J.; Easton, A.J.; Boog, C.J.; Busch, D.H.; Zaiss, D.M.; Sijts, A.J. Pre-existing virus-specific CD8(+) T-cells provide protection against pneumovirus-induced disease in mice. Vaccine 2012, 30, 6382-6388.

96. Gabryszewski, S.J.; Bachar, O.; Dyer, K.D.; Percopo, C.M.; Killoran, K.E.; Domachowske, J.B.; Rosenberg, H.F. Lactobacillus-mediated priming of the respiratory mucosa protects against lethal pneumovirus infection. J. Immunol. 2011, 186, 1151-1161. 
97. Didierlaurent, A.; Goulding, J.; Hussell, T. The impact of successive infections on the lung microenvironment. Immunology 2007, 122, 457-465.

98. Goulding, J.; Snelgrove, R.; Saldana, J.; Didierlaurent, A.; Cavanagh, M.; Gwyer, E.; Wales, J.; Wissinger, E.L.; Hussell, T. Respiratory infections: Do we ever recover? Proc. Am. Thorac. Soc. 2007, 4, 618-625.

99. Hussell, T.; Cavanagh, M.M. The innate immune rheostat: Influence on lung inflammatory disease and secondary bacterial pneumonia. Biochem. Soc. Trans. 2009, 37, 811-813.

100. Wiley, J.A.; Richert, L.E.; Swain, S.D.; Harmsen, A.; Barnard, D.L.; Randall, T.D.; Jutila, M.; Douglas, T.; Broomell, C.; Young, M. Inducible Bronchus-associated lymphoid tissue elicited by a protein cage nanoparticle enhances protection in mice against diverse respiratory viruses. PLoS One 2009, 4, e7142.

101. Easton, A.J.; Scott, P.D.; Edworthy, N.L.; Meng, B.; Marriott, A.C.; Dimmock, N.J. A novel broad-spectrum treatment for respiratory virus infections: Influenza-based defective interfering virus provides protection against pneumovirus infection in vivo. Vaccine 2011, 29, 2777-2784.

102. Garcia-Crespo, K.E.; Chan, C.C.; Gabryszewski, S.J.; Percopo, C.M.; Rigaux, P.; Dyer, K.D.; Domachowske, J.B.; Rosenberg, H.F. Lactobacillus priming of the respiratory tract: Heterologous immunity and protection against lethal pneumovirus infection. Antivir. Res. 2013, 97, 270-279. 
Reprinted from Viruses. Cite as: Fathima, S.; Lee, B.E.; May-Hadford, J.; Mukhi, S.; Drews, S.J. Use of an Innovative Web-Based Laboratory Surveillance Platform to Analyze Mixed Infections Between Human Metapneumovirus (hMPV) and Other Respiratory Viruses Circulating in Alberta (AB), Canada (2009-2012). Viruses 2012, 4, 2754-2765.

Article

\title{
Use of an Innovative Web-Based Laboratory Surveillance Platform to Analyze Mixed Infections Between Human Metapneumovirus (hMPV) and Other Respiratory Viruses Circulating in Alberta (AB), Canada (2009-2012)
}

\author{
Sumana Fathima ${ }^{1}$, Bonita E. Lee ${ }^{2}$, Jennifer May-Hadford ${ }^{3}$, Shamir Mukhi ${ }^{4}$ \\ and Steven J. Drews ${ }^{1,5, *}$
}

1 Provincial Laboratory for Public Health (ProvLab), 3030 Hospital Dr. NW, Calgary, AB T2N 4W4, Canada; E-Mail: sumana.fathima@albertahealthservices.ca

2 University of Alberta, Room 3-588B, ECHA, 11405 - 87 Avenue, Edmonton, AB T6G 1C9, Canada; E-Mail: bonita.lee@albertahealthservices.ca

3 Public Health Agency of Canada, 130 Colonnade Road A.L. 6501H Ottawa, ON K1A 0K9, Canada; E-Mail: jennifer.may-hadford@phac-aspc.gc.ca

4 Canadian Network for Public Health Intelligence, Public Health Agency of Canada, 1015 Arlington St, Winnipeg, MB R3E 3R2, Canada; E-Mail: shamir.nizar.mukhi@phac-aspc.gc.ca

5 University of Calgary, 2500 University Drive Northwest, Calgary, AB T2N 1N4, Canada;

E-Mail: steven.drews@albertahealthservices.ca

* Author to whom correspondence should be addressed;

E-Mail: steven.drews@albertahealthservices.ca; Tel.: +1-403-944-1068; Fax: +1-403-283-0142.

Received: 30 August 2012; in revised form: 12 October 2012 / Accepted: 26 October 2012/

Published: 5 November 2012

\begin{abstract}
We investigated the proportions of mono vs. mixed infections for human metapneumovirus (hMPV) as compared to adenovirus (ADV), four types of coronavirus (CRV), parainfluenza virus (PIV), RSV, and enterovirus/rhinovirus (ERV) in Alberta, Canada. Using the Data Integration for Alberta Laboratories (DIAL) platform, 26,226 respiratory specimens at ProvLab between 1 July 2009 and 30 June 2012 were selected and included in the study. Using the Respiratory Virus Panel these specimens tested positive for one or more respiratory virus and negative for influenza A and B. From our subset
\end{abstract}


hMPV was the fourth most common virus $(n=2,561)$ with $373(15 \%)$ identified as mixed infection using DIAL. Mixed infection with hMPV was most commonly found in infants less than 6 months old and ERV was most commonly found in mixed infection with hMPV $(230 / 373,56 \%)$ across all age groups. The proportion of mixed-infection $v s$. mono-infection was highest for ADV (46\%), followed by CRV 229E (32\%), CRV HKU1 (31\%), CRV NL63 (28\%), CRV OC43 (23\%), PIV (20\%), RSV (17\%), hMPV (15\%) and ERV (13\%). hMPV was significantly more likely to be identified in mono infection as compared with ADV, CRV, PIV, and RSV with the exception of ERV [p<0.05].

Keywords: hMPV; co-infection; testing; epidemiology; respiratory

\section{Introduction}

Respiratory tract infections are a global public health concern and in Canada is the eighth leading cause of death in 2009 [1]. Human metapneumovirus (hMPV) is an RNA virus belonging to Paramyxoviridae family. The virus was identified by researchers in the Netherlands in 2001 as an important cause of respiratory infections that affect all age groups [2]. A study from Saskatchewan, Canada by Liu et al. [3], using ELISA showed that seroprevalence for hMPV approaches 99\% by young adulthood. This virus can affect any age group but several studies have shown that hMPV is a leading cause in lower respiratory tract infections in children [4-6] but it also affects the elderly [7]. Clinical manifestations are similar to Respiratory Syncytial Virus (RSV) primarily leading to pneumonia and bronchiolitis [8]. In Canada, outbreaks associated with hMPV have been reported in Alberta, British Columbia and Quebec mainly in long term and senior care facilities [9-11]. Other common respiratory viruses in these settings include influenza A (FLUA), influenza B (FLUB), parainfluenza virus (PIV), enterovirus/rhinovirus (ERV), adenovirus (ADV), and coronavirus (CRV), which all can cause lower respiratory tract infections [12,13].

Provincial Laboratory for Public Health (ProvLab) in Alberta provides testing for all respiratory virus pathogens for the province of Alberta and surrounding Northern Territories (excluding Yukon) of Canada. The diagnostic testing algorithm for respiratory virus at ProvLab changed during the 2009 H1N1 pandemic. From April 2009, respiratory specimens arriving at ProvLab were screened by an in-house real-time reverse-transcriptase (RT)-PCR for influenza A [14]. Specimens that were positive for influenza A were also subtyped for seasonal H3, seasonal H1 and pandemic H1N1 (2009) genes by RT-PCR. A cost effective approach was adopted in June 2009, to only test specimens negative for both influenza A and B by either singleplex or multiplex real time PCR assays using the Respiratory Virus Panel (RVP) classic assay, a multiplexed assay which detects multiple respiratory viral pathogens including FLUA, FLUB, PIV, ERV, ADV, 4 types of CRV, RSV, and hMPV [15]. Exceptions to this testing policy include samples submitted from a provincial influenza-like-illness surveillance program 
(Tarrant Viral Watch) and some samples from patients with severe illness and admission to the intensive care units.

In Alberta, a unique platform was developed for laboratory-based surveillance called Data Integration of Alberta Laboratories (DIAL). DIAL is a secure web based platform, which is used to extract, interpret, collate and analyze respiratory virus testing data from ProvLab, Laboratory Information System (LIS) in real time [16]. DIAL has an automatic engine that extracts raw specimen-based laboratory data from ProvLab LIS, including patient demographics, information of physician and submitting agencies, and test data for specific targets. The second and most important component of DIAL is a built-in Automated Interpretation Engine (AIE) which provides clinically relevant interpretation and final target-specific classifications for each specimen. Finally, DIAL also has an analytical engine which allows users to select and create specific data sets for various targets by different factors, e.g., patient demographics, geographic distribution, time periods, testing methods and perform different types of analysis including graphical presentations, tables, maps, rate calculation and trending analysis. In the case of respiratory specimens from ProvLab, DIAL's AIE was designed to assign positive and negative classifications for each respiratory virus as well as a summary classification that classifies each specimen as: (1) only positive for one specific virus, (2) mixed infection with more than one respiratory virus or (3) negative for all respiratory viruses. Using these final classifications, positive and negative specimens for each virus can easily be selected in DIAL for further analysis.

In this study we used DIAL to select specimen-based data and investigated the proportions of mono vs. mixed infections for hMPV as compared to ADV, CRV, ERV, PIV and RSV for a period of three years, 1 July 2009 to 30 June 2012. In order to create a uniform dataset for this study, we excluded all samples that tested positive for influenza A or B by the in-house real-time PCR assays and included only samples that had undergone RVP testing.

\section{Results and Discussion}

Using DIAL, 36,824 specimens were identified as positive for one or more respiratory virus during the study period. A total of 10,598 were excluded from this study with 9,340 tested positive for influenza A, 1,065 for influenza B, 7 for both influenza A and B and 185 specimens not tested by RVP even though they were negative for influenza. For the 26,226 RVP positive specimens included in the study, 10,042 (38\%) were received between July 2009 and June 2010, 8,450 (32\%) between July 2010 and June 2011 and 7,734 (30\%) between July 2011 and June 2012. Mixed infection (having more than one virus identified) was found in 2,330 (9\%) specimens and 23,896 (91\%) had mono-infection (having only one virus identified). The majority of the specimens were collected from the upper respiratory tract as nasopharyngeal/nasal/throat swabs or nasopharyngeal aspirates $84 \%(n=22,013)$, $12 \%(n=3076)$ as respiratory samples with unspecified source, $4 \%(n=917)$ were from the lower respiratory tract e.g., endotracheal aspirates or bronchaveolar lavage, and remaining as unknown sample types and a few tissues and sterile body fluid. 
The age distribution of specimens submitted and tested positive for hMPV is summarized in Table 1. The highest number of specimens received was from patients less than 6 months old and the proportion of specimens tested positive for hMPV ranged from $4 \%-19 \%$ among the different age groups ( $\mathrm{p}<0.001$, Chi Square test). Overall, mixed infection was detected in $15 \%$ of specimens tested positive for hMPV. Using specimens from the youngest age group (less than six months old) as the reference age group, there was significant difference for the proportion of mixed hMPV infection among the various age groups ( $p<0.05$, Binary Logistic Regression) (Table 1). Mixed infection with hMPV was most commonly found in specimens from patients younger than six months and rarely in specimens submitted from older than 70 years old.

Among the 373 specimens with mixed infections with hMPV, the three most commonly found virus was ERV, RSV and PIV, which also were the three most common viruses detected in all the specimens (Table 2). In comparison with ADV, four types of CRV, PIV, and RSV, hMPV had a significantly lower proportion of mixed infection specimens $\left[\chi^{2}\right.$ with Bonferroni's correction, $\mathrm{p}=<0.05$ ] and there was no significant difference of mixed infection comparing hMPV and ERV $\left[\chi^{2}\right.$ with Bonferroni's correction, $\mathrm{p}=0.06]$.

The age distribution of virus found in mixed infection with hMPV is summarized in Table 3. ERV was the most commonly found virus in hMPV mixed infection across all age groups.

Table 1. Age distribution of specimens tested positive for metapneumovirus (hMPV) using Respiratory Virus Panel (RVP) and the number and \% of mixed infection with hMPV.

\begin{tabular}{|c|c|c|c|c|}
\hline Age groups & $\begin{array}{l}\text { Number of } \\
\text { specimens } \\
\text { tested } \\
(n=26,226)\end{array}$ & $\begin{array}{l}\text { Number of } \\
\text { specimens tested } \\
\text { positive for hMPV } \\
(\%)(n=2,561)\end{array}$ & $\begin{array}{l}\text { Number of specimens } \\
\text { with mixed hMPV } \\
\text { infection } \\
(n=373)\end{array}$ & $\begin{array}{l}\text { \% with } \\
\text { Mixed } \\
\text { infection }\end{array}$ \\
\hline Unknown & 83 & $13(16)$ & $5 *$ & $38 *$ \\
\hline Less than 6 months & 5636 & $389(7)$ & 90 & $23 \dagger$ \\
\hline 6 months to 1 year & 3398 & $350(10)$ & 75 & 21 \\
\hline 1 year & 4282 & $467(11)$ & 75 & $16 \dagger$ \\
\hline 2 years & 1808 & $203(11)$ & 34 & 17 \\
\hline 3 years & 1066 & $141(13)$ & 19 & $14 \dagger$ \\
\hline 4 years & 671 & $88(13)$ & 8 & $9 \dagger$ \\
\hline 5 to 9 years & 1386 & $153(11)$ & 19 & $12 \dagger$ \\
\hline 10 to 19 years & 1167 & $58(5)$ & 8 & 14 \\
\hline 20 to 29 years & 1026 & $43(4)$ & 4 & 9 \\
\hline 30 to 39 years & 1091 & $82(8)$ & 5 & $6 \dagger$ \\
\hline 40 to 49 years & 999 & $105(11)$ & 8 & $8 \dagger$ \\
\hline 50 to 59 years & 1224 & $116(10)$ & 8 & $7 \dagger$ \\
\hline 60 to 69 years & 996 & $110(11)$ & 13 & $12 \dagger$ \\
\hline 70 to 79 years & 595 & $102(17)$ & 0 & $0 \dagger$ \\
\hline 80 to 89 years & 562 & $104(19)$ & 2 & $2 \dagger$ \\
\hline 90 to 105 years & 236 & $37(16)$ & 0 & $0 \dagger$ \\
\hline
\end{tabular}




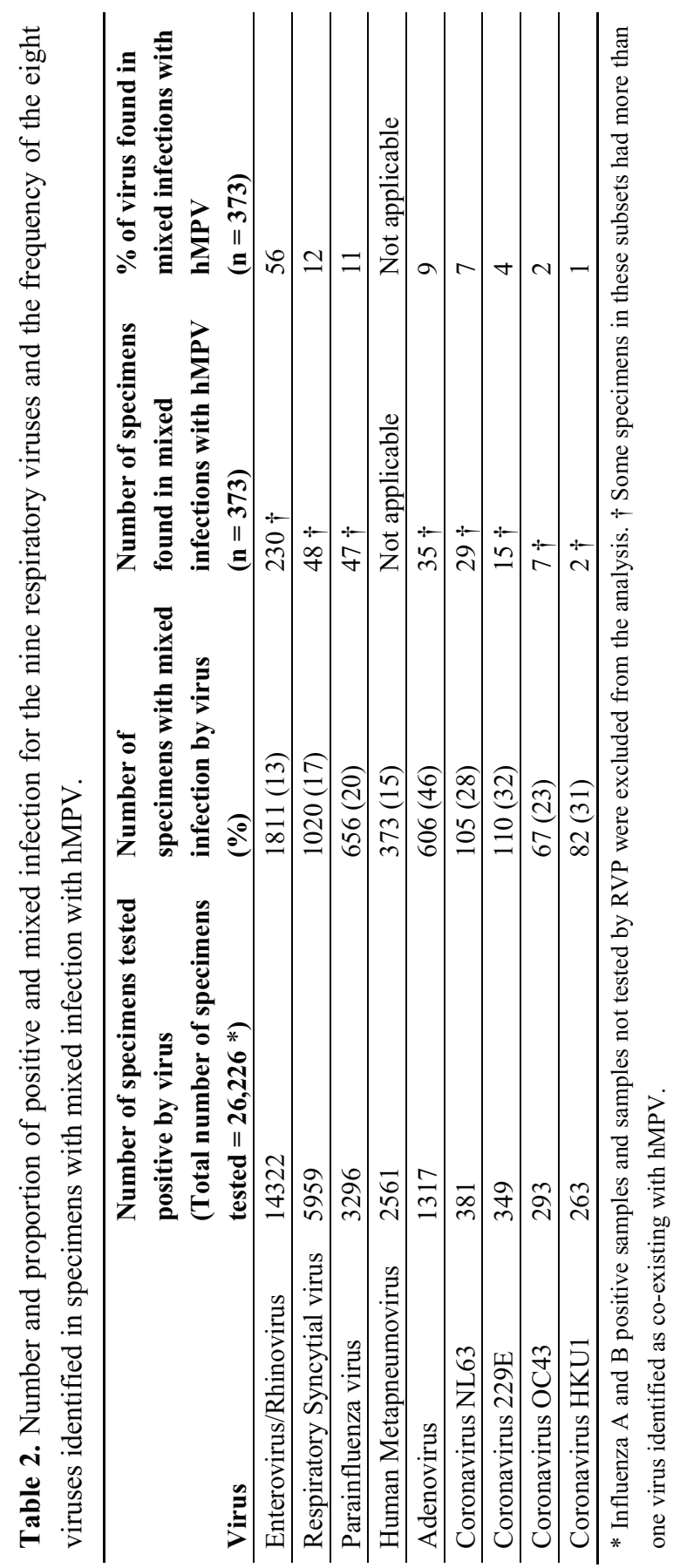




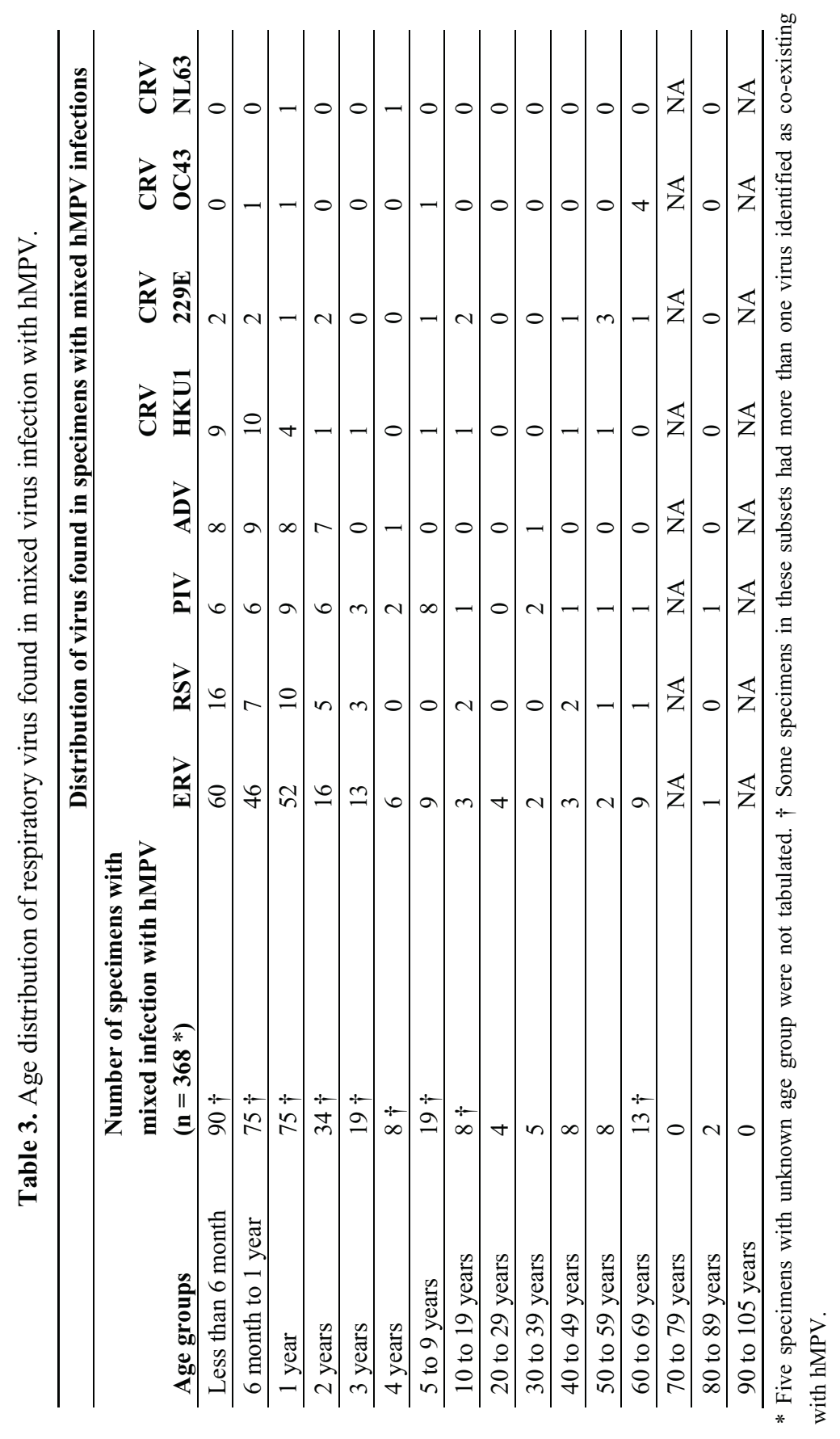


Peak hMPV activity was observed in February 2010, June 2011 and November 2011, whereas peak ERV activity was found in the month of September in three consecutive years (2009, 2010 and 2011) (Figure 1). Specimens with mixed infection with these two viruses followed the trend and circulatory pattern of hMPV.

Figure 1. Monthly distribution of hMPV and ERV along with mixed infection with these two viruses.

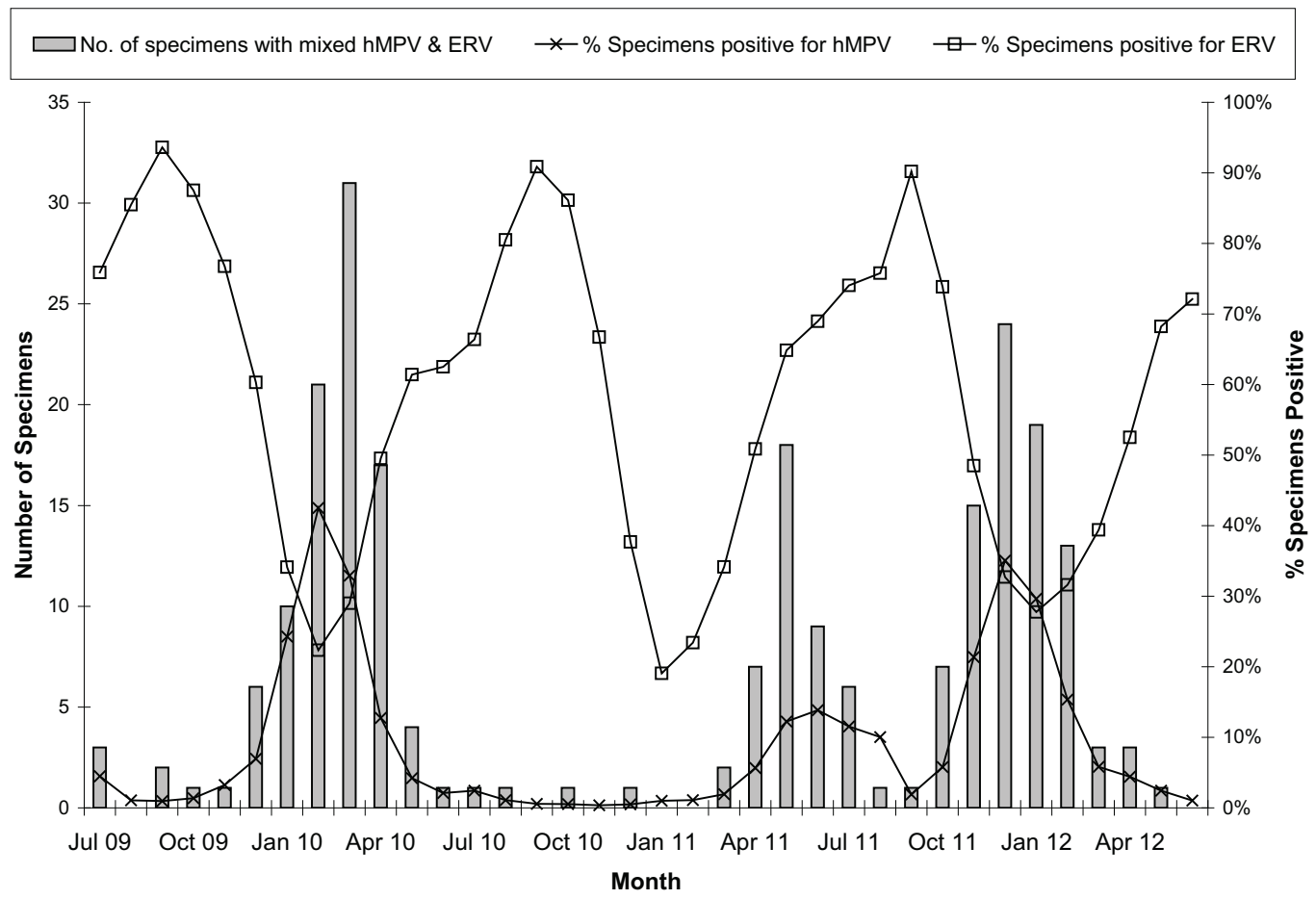

The objective of this study was to describe mono and mixed infections of hMPV in our jurisdiction for a three year period. All specimen data was acquired using ProvLab's DIAL application and we were able to identify that hMPV was more likely to occur in mono infection (85\%) than mixed (15\%). The results in this study were similar to a previous pilot study in Alberta which identified hMPV mixing with other pathogens in $15 \%$ of specimens (118/778) [17]. In other populations, namely hospitalized patients, mixed hMPV infections were in the minority.

Our study also determined that in hMPV mixed infections, the pathogen most likely to co-exist with hMPV is ERV (56\%). In contrast, other studies have identified RSV as the leading cause of mixed infection with hMPV [18-20]. There may be several reasons for the difference in findings including the type of diagnostic assays used and the detection of various respiratory targets, clinical setting, patient age, and timing of specimen collection. Many studies have focused on populations mostly consisting of hospitalized pediatric patients and these studies have shown that RSV was the leading 
pathogen identified in these young children who were between 16 months to 14 years old [21-23]. In contrast, our study examined all age groups, including community-based and hospital-based specimens. ERV and hMPV remained closely linked across all age groups. Mixed infection with hMPV was most commonly found in the age group less than 6 months old. Moreover, ERV was still the dominant virus found to be mixed with hMPV. Our data set included specimens collected during the second phase of 2009 H1N1 pandemic in Alberta and Northern Territories, which made up of more than one third of the specimens included in this study. We found $62 \%$ of the samples between July 2009 and June 2010 tested positive for ERV, significantly higher than that found in the other two time periods, July 2010 to June 2011 (49\%) and July 2011 to June 2012 (51\%) (p < 0.001, Binary Logistic Regression). Other studies have also shown that during the pandemic ERV was a very common circulating virus followed by CRV [22,24,25]. Moreover, this linkage between ERV and hMPV could also be due to the inclusion of a very large group of viruses identified as ERV since RVP could not distinguish between various strains of rhinovirus and enterovirus. There might also be factors related to host immune responses and viral infection kinetics [26-29]. We have only examined the seasonality of hMPV and ERV and the mixed infection between these two viruses because of the relatively lower number of hMPV mixed infections for the other six viruses. Annual variations in peak hMPV activity was observed in our study with one of the peak months occurring in the summer of 2011, which was different from other studies in various Canadian provinces showing peaks of hMPV in the winter (January to April) [30-32]. As expected, the mixed infection of hMPV and ERV followed the circulatory pattern of hMPV but differed from ERV.

Although our findings have shown some trends with hMPV and its ability to exist as mono versus mixed infection, there are some important limitations in this study. Firstly, influenza A and B positive samples were excluded mainly because ProvLab does not routinely test influenza positive specimens for hMPV since the pandemic 2009. Excluding influenza viruses from our database may have impacted viral co-infection rates identified in our study. On the other hand, a recent study in England showed a lower prevalence of influenza A (H1N1) in patients positive for hMPV, ADV, RSV, PIV and rhinovirus with statistical significance for this relationship with hMPV and rhinovirus [32]. Another limitation was that the analysis by age group was based on specimen-based data only with duplicate samples from some individuals. Moreover, only limited information is usually provided on the requisition submitted with the specimens so we were not able to study clinical manifestations of mono-infection versus mixed infections and explore different settings

Despite the limitations stated, this study has considerable public health implications. This study helps us better understand the frequency of hMPV mono versus mixed infection over a period of three years. Better understanding of what mechanisms or conditions support mono versus mixed infections and the difference in clinical presentations and prognosis is needed. The prevalence of this virus supports research efforts to develop vaccine strategies which may become available in the future [33]. This also brings us to question whether testing algorithms at ProvLab need to be changed to better understand the interaction between hMPV and influenza A and B. Since hMPV has been shown to be an important pathogen, it should be included in ongoing surveillance and public health strategies. 
Enhanced surveillance programs will help us better understand hMPV-associated diseases and maintain our awareness of trends in mono and mixed infections.

\section{Experimental Section}

ProvLab provides respiratory virus testing for the province of Alberta and surrounding Northern Territories (excluding Yukon) of Canada. Since June 2009, all respiratory specimens arriving at ProvLab was screened by an in-house (RT)-PCR for influenza A [14]. Specimens tested positive for influenza A were also subtyped for seasonal H3, seasonal H1 and pandemic H1N1 (2009) genes by RT-PCR. A multiplex assay to detect both influenza A and B was implemented in February 2010. Only specimens tested negative for both influenza A and B were tested using the RVP classic assay, a multiplexed assay which detects multiple respiratory viral pathogens including FLUA, FLUB, PIV, ERV, ADV, four types of CRV, RSV, and hMPV [14]. Exceptions to this testing policy included samples submitted from a provincial influenza-like-illness surveillance program (Tarrant Viral Watch) and some samples from patients with severe illness and admitted to the intensive care units. Specimens tested by RVP and tested positive for one or more respiratory virus excluding influenza A and B between July 2009 and June 2012 identified using the DIAL application was included in this study. DIAL provided classification of the specimens by each respiratory virus target as well as mono versus mixed infection. DIAL also allowed a user to select specimens based on the type of testing and over various geographic and time periods and age groups.

Statistical analysis of the proportion of mono versus mixed infections for the different virus was performed using Pearson Chi-squared $\left(\chi^{2}\right)$ test with Bonferroni's adjustment for multiple analyses. The proportion of hMPV as mixed infection by age group and ERV positive specimens by analysis of age group and time periods was analyzed using binary logistic regression. The SPSS software version 20.0 $\left(\right.$ IBM $^{\circledR}$ SPSS $^{\circledR}$ Statistics, IBM Corp., USA) was used for statistical analysis with the level of significance set at $\mathrm{p}<0.05$.

\section{Conclusions}

In this study we used DIAL to provide user-defined data sets of respiratory virus testing data and found that over a period of 3 years, July 2009 to June 2012, hMPV is significantly more likely to be identified in mono infection (86\%) as compared with ADV, four types of CRV, PIV, and RSV with the exception of ERV. The three viruses most likely to be found in mixed infection with hMPV in this study were ERV, PIV and RSV with a higher proportion of mixed infection found in young infants.

\section{Acknowledgments}

The authors would like to acknowledge the staff of the Provincial Laboratory of Public Health who performed all laboratory testing and the Canadian Network for Public Health Intelligence program for their development work on the DIAL platform. 


\section{Conflicts of Interest}

The authors declare no conflict of interest.

\section{References}

1. Statistics Canada. Leading causes of death in Canada 2009. Available online: http://www.statcan.gc.ca/pub/84-215-x/2012001/tb1/T001-eng.pdf (accessed 31 October 2012).

2. Van den Hoogen, B.G.; de Jong, J.C.; Groen, J.; Kuiken, T.; Fouchier, R.A.; Osterhaus, A.D. A newly discovered human pneumovirus isolated from young children with respiratory tract disease. Nat. Med. 2001, 7, 719-724.

3. Liu, L.; Bastien, N.; Sidaway, F.; Chan, E.; Li, Y. Seroprevalence of human metapneumovirus (hMPV) in the Canadian province of Saskatchewan analyzed by a recombinant nucleocapsid protein-based enzyme-linked immunosorbent assay. J. Med. Virol. 2007, 79, 308-313.

4. Manoha, C.; Espinosa, S.; Aho, S.L.; Huet, F.; Pothier, P. Epidemiological and clinical features of hMPV, RSV and RVs infections in young children. J. Clin. Virol. 2007, 38, 221-226.

5. Mullins, J.A.; Erdman, D.D.; Weinberg, G.A.; Edwards, K.; Hall, C.B.; Walker, F.J.; Iwane, M.; Anderson, L.J. Human metapneumovirus infection among children hospitalized with acute respiratory illness. Emerg. Infect. Dis. 2004, 10, 700-705.

6. Kaida, A.; Iritani, N.; Kubo, H.; Shiomi, M.; Kohdera, U.; Murakami, T. Seasonal distribution and phylogenetic analysis of human metapneumovirus among children in Osaka City, Japan. J. Clin. Virol. 2006, 35, 394-399.

7. Falsey, A.R.; Erdman, D.; Anderson, L.J.; Walsh, E.E. Human metapneumovirus infections in young and elderly adults. J. Infect. Dis. 2003, 187, 785-790.

8. Boivin, G.; Abed, Y.; Pelletier, G.; Ruel, L.; Moisan, D.; Cote, S.; Peret, T.C.; Erdman, D.D.; Anderson, L.J. Virological features and clinical manifestations associated with human metapneumovirus: A new paramyxovirus responsible for acute respiratory-tract infections in all age groups. J. Infect. Dis. 2002, 186, 1330-1334.

9. Boivin, G.; De, S.G.; Hamelin, M.E.; Cote, S.; Argouin, M.; Tremblay, G.; Maranda-Aubut, R.; Sauvageau, C.; Ouakki, M.; Boulianne, N.; Couture, C. An outbreak of severe respiratory tract infection due to human metapneumovirus in a long-term care facility. Clin. Infect. Dis. 2007, 44, 1152-1158.

10. Louie, J.K.; Schnurr, D.P.; Pan, C.Y.; Kiang, D.; Carter, C.; Tougaw, S.; Ventura, J.; Norman, A.; Belmusto, V.; Rosenberg, J.; Trochet, G. A summer outbreak of human metapneumovirus infection in a long-term-care facility. J. Infect. Dis. 2007, 196, 705-708.

11. Towgood, L.; Miller, M.; McKay, D.; Parker, R. Human metapneumovirus outbreak in a senior's care facility, British Columbia. Canada Communicable Disease Report (CCDR) Weekly 2008. 
12. Van Asten, L.; van den Wijngaard, C.; van Pelt, W.; van de Kassteele, J.; Meijer, A.; van der Hoek, W.; Kretzschmar, M.; Koopmans, M. Mortality attributable to 9 common infections: Significant effect of influenza a, respiratory syncytial virus, influenza B, norovirus, and parainfluenza in elderly persons. J. Infect. Dis. 2012, 206, 628-639.

13. Xie, Z.D.; Xiao, Y.; Liu, C.Y.; Hu, Y.H.; Yao, Y.; Yang, Y.; Qian, S.Y.; Geng, R.; Wang, J.W.; Shen, K.L. Three years surveillance of viral etiology of acute lower respiratory tract infection in children from 2007 to 2010. Zhonghua Er. Ke. Za Zhi. 2011, 49, 745-749.

14. Pabbaraju, K.; Tokaryk, K.L.; Wong, S.; Fox, J.D. Comparison of the Luminex xTAG respiratory viral panel with in-house nucleic acid amplification tests for diagnosis of respiratory virus infections. J. Clin. Microbiol. 2008, 46, 3056-3062.

15. Lee, B.E.; Mukhi, S.N.; May-Hadford, J.; Plitt, S.; Louie, M.; Drews, S.J. Determination of the relative economic impact of different molecular-based laboratory algorithms for respiratory viral pathogen detection, including Pandemic (H1N1), using a secure web based platform. Virol. J. 2011, 8, 277-278. Available online: http://www.virologyj.com/content/8/1/277 (accessed on 31 October 2012).

16. Mukhi, S.N.; May-Hadford, J.; Plitt, S.; Preiksaitis, J.; Lee, B.E. DIAL: A Platform for real-time Laboratory Surveillance. Online J. Public Health Inform. 2010, 2, doi:10.5210/ojphi.v2i3.3041.

17. Pabbaraju, K.; Wong, S.; McMillan, T.; Lee, B.E.; Fox, J.D. Diagnosis and epidemiological studies of human metapneumovirus using real-time PCR. J. Clin. Virol. 2007, 40, 186-192.

18. Boivin, G.; De, S.G.; Cote, S.; Gilca, R.; Abed, Y.; Rochette, L.; Bergeron, M.G.; Dery, P. Human metapneumovirus infections in hospitalized children. Emerg. Infect. Dis. 2003, 9, 634-640.

19. Kuypers, J.; Wright, N.; Corey, L.; Morrow, R. Detection and quantification of human metapneumovirus in pediatric specimens by real-time RT-PCR. J. Clin. Virol. 2005, 33, 299-305.

20. Wolf, D.G.; Greenberg, D.; Kalkstein, D.; Shemer-Avni, Y.; Givon-Lavi, N.; Saleh, N.; Goldberg, M.D.; Dagan, R. Comparison of human metapneumovirus, respiratory syncytial virus and influenza A virus lower respiratory tract infections in hospitalized young children. Pediatr. Infect. Dis. J. 2006, 25, 320-324.

21. Sung, C.C.; Chi, H.; Chiu, N.C.; Huang, D.T.; Weng, L.C.; Wang, N.Y.; Huang, F.Y. Viral etiology of acute lower respiratory tract infections in hospitalized young children in Northern Taiwan. J. Microbiol. Immunol. Infect. 2011, 44, 184-190.

22. Xiao, N.G.; Xie, Z.P.; Zhang, B.; Yuan, X.H.; Song, J.R.; Gao, H.C.; Zhang, R.F.; Hou, Y.D.; Duan, Z.J. Prevalence and clinical and molecular characterization of human metapneumovirus in children with acute respiratory infection in China. Pediatr. Infect. Dis. J. 2010, 29, 131-134.

23. Martin, E.T.; Kuypers, J.; Wald, A.; Englund, J.A. Multiple versus single virus respiratory infections: Viral load and clinical disease severity in hospitalized children. Influenza. Other Respir. Viruses 2012, 6, 71-77.

24. Esper, F.P.; Spahlinger, T.; Zhou, L. Rate and influence of respiratory virus co-infection on pandemic (H1N1) influenza disease. J. Infect. 2011, 63, 260-266. 
25. Thiberville, S.D.; Ninove, L.; Vu Hai, V; Botelho-Nevers, E.; Gazin, C.; Thirion, L.; Salez, N.; de Lamballerie, X.; Charrel, R.; Brouqui, P. The viral etiology of an influenza-like illness during the 2009 pandemic. J. Med. Virol. 2012, 84, 1071-1079.

26. Bitko, V.; Shulyayeva, O.; Mazumder, B.; Musiyenko, A.; Ramaswamy, M.; Look, D.C.; Barik, S. Nonstructural proteins of respiratory syncytial virus suppress premature apoptosis by an NF-kappaB-dependent, interferon-independent mechanism and facilitate virus growth. $J$. Virol. 2007, 81, 1786-1795.

27. Spann, K.M.; Tran, K.C.; Chi, B.; Rabin, R.L.; Collins, P.L. Suppression of the induction of alpha, beta, and lambda interferons by the NS1 and NS2 proteins of human respiratory syncytial virus in human epithelial cells and macrophages [corrected]. J. Virol. 2004, 78, 4363-4369.

28. Ditt, V.; Lusebrink, J.; Tillmann, R.L.; Schildgen, V.; Schildgen, O. Respiratory infections by HMPV and RSV are clinically indistinguishable but induce different host response in aged individuals. PLoS One 2011, 6, e16314.

29. Franz, A.; Adams, O.; Willems, R.; Bonzel, L.; Neuhausen, N.; Schweizer-Krantz, S.; Ruggeberg, J.U.; Willers, R.; Henrich, B.; Schroten, H.; et al. Correlation of viral load of respiratory pathogens and co-infections with disease severity in children hospitalized for lower respiratory tract infection. J. Clin. Virol. 2010, 48, 239-245.

30. Hamelin, M.E.; Abed, Y.; Boivin, G. Human metapneumovirus: A new player among respiratory viruses. Clin. Infect. Dis. 2004, 38, 983-990.

31. Robinson, J.L.; Lee, B.E.; Bastien, N.; Li, Y. Seasonality and clinical features of human metapneumovirus infection in children in Northern Alberta. J. Med. Virol. 2005, 76, 98-105.

32. Bastien, N.; Ward, D.; Van, C.P.; Brandt, K.; Lee, S.H.; McNabb, G.; Klisko, B.; Chan, E.; Li, Y. Human metapneumovirus infection in the Canadian population. J. Clin. Microbiol. 2003, 41, 4642-4646.

33. Kahn, J.S. Newly discovered respiratory viruses: Significance and implications. Curr. Opin. Pharmacol. 2007, 7, 478-483. 
Reprinted from Viruses. Cite as: Gardinassi, L.G.A.; Simas, P.V.M.; Gomes, D.E.; Bonfim, C.M.; Nogueira, F.C.; Garcia, G.R.; Carareto, C.M.A.; Rahal, P.; Souza, F.P. Diversity and Adaptation of Human Respiratory Syncytial Virus Genotypes Circulating in Two Distinct Communities: Public Hospital and Day Care Center. Viruses 2012, 4, 2432-2447.

Article

\title{
Diversity and Adaptation of Human Respiratory Syncytial Virus Genotypes Circulating in Two Distinct Communities: Public Hospital and Day Care Center
}

Luiz Gustavo Araujo Gardinassi ${ }^{3, \dagger}$, Paulo Vitor Marques Simas ${ }^{1, \dagger}$, Deriane Elias Gomes ${ }^{2, \dagger}$, Caroline Measso do Bonfim ${ }^{1}$, Felipe Cavassan Nogueira ${ }^{1}$, Gustavo Rocha Garcia ${ }^{3}$, Claudia Márcia Aparecida Carareto ${ }^{1}$, Paula Rahal ${ }^{1}$ and Fátima Pereira de Souza ${ }^{2, *}$

1 Universidade Estadual Paulista, Instituto de Biociências, Letras e Ciências Exatas de São José do Rio Preto, SP. Departamento de Biologia - Rua Cristóvão Colombo, 2265, Jardim Nazareth - Cep: 15054-000, Brazil; E-Mails: simaspvm@yahoo.com.br (P.V.M.S.); carolbonfim@yahoo.com.br (C.M.B.); cavassan@yahoo.com.br (F.C.N.); carareto@ibilce.unesp.br (C.M.A.C.); rahalp@yahoo.com.br (P.R.)

2 Universidade Estadual Paulista, Instituto de Biociências, Letras e Ciências Exatas de São José do Rio Preto, SP. Departamento de Física - Rua Cristóvão Colombo, 2265, Jardim Nazareth - Cep: 15054-000 Brazil; E-Mail: deribela@yahoo.com.br

3 Universidade de São Paulo, Faculdade de Medicina de Ribeirão Preto, SP. Departamento de Bioquímica e Imunologia - Av. dos Bandeirantes, 3900 Monte Alegre - Cep: 14049-900 Brazil; E-Mails: gugard@gmail.com (L.G.A.G.); gugarg10@usp.br (G.R.G.)

$\dagger$ These authors contributed equally to this work.

* Author to whom correspondence should be addressed; E-Mail: fatyssouza@yahoo.com.br; Tel.: +55-17-3221-2463; Fax: +55-17-3221-2247.

Received: 31 August 2012; in revised form: 16 October 2012 / Accepted: 17 October 2012 / Published: 24 October 2012

\begin{abstract}
HRSV is one of the most important pathogens causing acute respiratory tract diseases as bronchiolitis and pneumonia among infants. HRSV was isolated from two distinct communities, a public day care center and a public hospital in São José do Rio Preto - SP, Brazil. We obtained partial sequences from $G$ gene that were used on phylogenetic and selection pressure analysis. HRSV accounted for $29 \%$ of respiratory
\end{abstract}


infections in hospitalized children and $7.7 \%$ in day care center children. On phylogenetic analysis of $60 \mathrm{HRSV}$ strains, $48(80 \%)$ clustered within or adjacent to the GA1 genotype; GA5, NA1, NA2, BA-IV and SAB1 were also observed. SJRP GA1 strains presented variations among deduced amino acids composition and lost the potential O-glycosilation site at amino acid position 295, nevertheless this resulted in an insertion of two potential O-glycosilation sites at positions 296 and 297. Furthermore, a potential O-glycosilation site insertion, at position 293, was only observed for hospital strains. Using SLAC and MEME methods, only amino acid 274 was identified to be under positive selection. This is the first report on HRSV circulation and genotypes classification derived from a day care center community in Brazil.

Keywords: respiratory syncytial virus; attachment protein $(\mathrm{G})$; genetic variability; O-glycosilation; selection pressure

\section{Introduction}

Human Respiratory Syncytial Virus (HRSV) is a major viral agent causing serious respiratory tract diseases in the pediatric population worldwide [1]. Of the estimated 2 million children under the age of 5 years who require care for HRSV infections annually, 78\% are over the age of 1 year [2], although it has been recognized as a main cause of morbidity in children under 1 year of age [3]. HRSV infection results in several outcomes, ranging from common cold-like symptoms to more severe bronchiolitis and pneumonia in children, immunocompromised and elderly individuals [4,5].

HRSV is classified into the Pneumovirus genus of the Paramyxoviridae family and is composed of an envelope with a negative-sense single-stranded RNA genome, which encodes for 11 proteins. Based on reactions with monoclonal antibodies against the $\mathrm{G}$ and $\mathrm{F}$ glycoproteins, beyond molecular differences in several genes [6,7], two major groups, HRSVA and HRSVB, have been described [7-10]. Several HRSVA genotypes were identified in different geographical regions, which include GA1 to GA7 [9,10], SAA1 (South Africa, A1) [11], NA1 and NA2 [12], and most recently ON1 [13]. Thirteen HRSVB genotypes are currently known and designated as GB1 to GB4 [9], SAB1 to SAB3 (South Africa) [11], and BA1 to BA6 (Buenos Aires) [14]. Therefore, the antigenic variability of HRSV strains has been a relevant subject on discussions of the key features contributing to the ability of the virus to re-infect people and cause large-scale yearly outbreaks [15].

The sequence variability of the attachment $(\mathrm{G})$ protein gene, which shows the largest antigenic and genetic differences between the two HRSV groups [16], is commonly used for genotyping HRSVA and HRSVB viruses [13]. The G protein is a type II glycoprotein of 289 to 299 amino acids in length, consisting of the cytoplasmic tail (amino acids [AAs] 1-38), transmembrane domain (AA 38-66), and the ectodomain (AA 66-298) [17]. The C-terminal ectodomain of G protein is comprised of two variable regions, separated by a highly conserved region between amino acids 164 and 176 assumed to 
represent a receptor-binding site [17]. The two variable regions of the ectodomain contain high serine and threonine residues, which are potential acceptor sites for O-linked sugars affecting the antigenic structure of the G protein as well as impacting on virus infectivity $[18,19]$.

Few studies have evaluated the epidemiology and HRSV genotypes circulating in São Paulo State, Brazil [20-26] and furthermore this is the first report on HRSV genotypes isolated from children attending a day care center in Brazil. Accordingly, we aimed to identify HRSV strains features by analyzing the genetic variability in the second hypervariable region of the attachment $(\mathrm{G})$ gene of viruses isolated from clinical samples collected in a public day care center, and a public hospital in São José do Rio Preto-SP (SJRP), Brazil. Phylogenetic analyses were performed to establish the relation between SJRP's strains and previously described HRSV genotypes deposited in Genbank and further selection pressure analysis was performed to examine the replacement behavioral patterns of $\mathrm{G}$ protein ectodomain encoded amino acids.

\section{Results and Discussion}

\subsection{HRSV Epidemiology and Cohorts Characterization}

Brazil is a country of large territorial extension, but few studies have evaluated HRSV circulation patterns and genotypes, which are limited to hospital-based studies performed at Southeast and Northeast regions [20-22,24-26]. Studies including children that attended day care centers have been done mainly in Scandinavia, the United States and England [27], and evidenced that children who attended day care centers from the beginning of infancy present higher risks of respiratory infections comparing to children that did not attend day care centers [28]. Based on these works and due to the lack of informative data, we aimed to understand features, such as the diversity, genotypes and adaptation of HRSV strains circulating in two communities of São José do Rio Preto-SP, Brazil: children that attended a public day care center, and children that were hospitalized due to respiratory infections.

The day care center cohort was composed of 231 children, aged 1 to 78 months (average age of 30.85 months), $44.5 \%$ female and 55.5\% male, which presented an HRSV frequency of 7.7\%. From July 2003 to April 2004, this pathogen was mainly detected and isolated on winter and spring seasons, while in 2005 , outbreaks were observed in late autumn and winter seasons. These results contrasts with the previously reported HRSV frequency of 29\% [29], detected between May 2004 and September 2005 in clinical samples derived from the hospital, which was composed of 272 children including $57 \%$ male and $43 \%$ female, whose ages varied between 1 to 68 months. HRSV hospital outbreaks were observed in winter and spring 2004 and autumn 2005 [29].

Such variations on HRSV circulation patterns in both communities may have occurred due to environmental factors such as temperature or relative air humidity, co-circulation and competition with other respiratory pathogens as reported previously in São José do Rio Preto [29] or even due to virulence features as high infectivity and limited antigenic diversity of HRSV strains [30].

Episodes of respiratory infection, on HRSV positive children from the day care center, were characterized by the absence of severe symptoms and were generally limited to upper airways, whit no 
need of hospitalization during the respiratory infection. The most frequent symptom was runny nose (93.2\%), followed by cough (58.2\%), nasal obstruction (14.6\%), wheezing (3.7\%) and fever (2.4\%). In contrast, HRSV positive children from the hospital, developed severe diseases such as pneumonia (24.1\%), bronchiolitis (64\%), and acute wheezing (16.2\%) [29]. The most frequent symptoms were cough (93.1\%), fever (91\%) and coryza (62.1\%) and nasal obstruction (50\%) [29]. It is evident that strains isolated from the hospital were more pathogenic, accounting for severe symptoms, thus suggesting that multiple lineages co-circulated in São José do Rio Preto.

\subsection{Phylogenetic Analysis}

In order to understand the diversity and establish the relation between SJRP HRSV strains, we performed phylogenetic analyses of partial sequences obtained from the C-terminal ectodomain of the attachment protein $(\mathrm{G})$ gene $(\mathrm{n}=60)$. Results showed that SJRP's HRSV strains were grouped into three distinct clusters (Supplementary Figure 1) and evidenced the co-circulation of multiple HRSV antigenic groups and genotypes, as reported in several regions of Brazil and São Paulo State [20,21,25,29].

Therefore, we proceeded to an analysis of SJRP's HRSV sequences along with 32 HRSVA and 30 HRSVB reference strains derived from Genbank (Supplementary Table 1), including sequences derived from HRSV isolated in Brazil. The analysis confirmed the co-circulation of both HRSVA and HRSVB antigenic groups, however, most of the sequences $(n=55)$ were clustered to HRSVA group, while five sequences were associated to HRSVB group (Figure 1). Oliveira and collaborators (2008) also reported a predominance of HRSVA strains over HRSVB in Uberlândia-MG, which agrees with most of the studies performed to identify on antigenic group subtypes, while Cintra and collaborators (2001) have found higher frequencies of both antigenic groups circulating in Ribeirão Preto-SP. Although Ribeirão Preto, Uberlândia and São José do Rio Preto are nearly located (between 200-300 km of distance), different geographical and demographical characteristics must be accounted and also, HRSV antigenic group analysis were performed in different seasons and years, which may contribute for differences on the HRSV subgroups detection.

It has been widely recognized that both subtypes circulate concurrently [31]. Zlateva and collaborators (2004) found the presence of multiple identical sequences among Belgian isolates, which suggested that certain strains predominated in a given epidemic season. Peret and colleagues (2000) also found a dominance of HRSVA over HRSVB, and predominance of 1-2 genotypes in five communities [10]. The predominance of HRSV-A viruses has been attributed to the higher variability among the HRSV-A strains [32]. Usually, the dominant strains shift yearly, suggesting a mechanism for frequent re-infections by evasion of immunity induced by previous strains [33].

Genotype classification through phylogenies demonstrated that SJRP HRSVA isolates, derived from both communities, were more related to GA1 $(n=48)$, while GA5 $(n=2)$, NA1 $(n=3)$, NA2 $(n=2)$ were detected only in samples from hospitalized children (Figure 1a). These results contrast with previous reports, which showed major prevalence of GA2 genotype during 1999 in Salvador-Ba [21], and during 2004 in Campinas-SP [24]. Interestingly, three Brazilian HRSV reference sequences (RP221/5, BR266-05 and BR292-05), circulating in São Paulo State during 2005 epidemic season, that 
have been previously described as GA2 genotypes, were clustered together with NA2, a recently identified genotype in Japan, that is genetically close to GA2 [12]. The fact that the three Brazilian strains may be more related to NA2 genotype is supported by the absence of NA2 sequences on phylogenetic analysis performed at the time they were reported, thus contributing to the association with GA2 genotype.

Indeed, inter-continental circulation of HRSV had been reported [14]. Viruses of the BA-I genotype circulated extensively in Buenos Aires from June to August of 1999. In December of the same year, the first BA-I sequence with an exact copy of the duplicated 60 nucleotide segment on the third hypervariable domain of $\mathrm{G}$ gene, from a non-Argentinean sample, was found in Belgium. Thus, it is clear that HRSV crossed the Atlantic (in either direction) in a period of few months [14], supporting that NA2 strains may have circulated in Brazil and Japan.

There was a strong phylogenetic association (bootstrapping value of $75 \%$ ) between sequences obtained from GA1 isolates MMM05C and FAC03C from the day care center with the isolates 7004HB and 27905HB from the hospital. SJRP GA1 isolates were further divided into two major clusters, I and II, which suggested that individual lineages of GA1 genotype could be co-circulating in the period of analysis (Figure 1a), as also proposed by Eshaghi and collaborators (2012), that identified two NA1 lineages circulating in Ontario, Canada.

HRSVB strains were classified as SAB1 $(\mathrm{n}=1)$ (Figure $1 \mathrm{~b})$, also reported by Botosso and collaborators (2009) in São Paulo-SP and BA-IV $(n=4)$. Although, BA genotype had been isolated in Brazil [23], this is the first time HRSVB strains are associated to BA-IV, which was also identified in Buenos Aires, Argentina in 2004 epidemic season, Quebec, Canada through 2001-2003, Kenya in 2003, Belgium in 1999, 2001-2003 [14] and most recently in China through 2006-2009 epidemic seasons [16].

\subsection{Molecular Analysis of HRSV GA1 Genotype}

By comparing the nucleotide composition and the pattern of mutations among the $60 \mathrm{HRSV}$ isolates, remarkable genetic flexibility could be observed, as previously noted worldwide [31,34-36]. However, two major clusters comprising several groups of identical sequences were identified only among the GA1 genotype strains, thus further analysis were carried out on this group due the lack of sampling of other genotypes. The alignments of nucleotides of the second hypervariable region of HRSV G gene from representative isolates for each GA1 group, compared to the HRSVA reference strain A2 (originally isolated in Australia in 1961), showed possible deletion/insertion sites on nucleotide positions $853,854,864,865,866$ and 879 of the day care center strains, and on nucleotide position 877 of hospital strains (Supplementary Figure 2). Consequently, these variations accounted for changes on the deduced amino acid sequences of SJRP's GA1 isolates, compared to A2 strain amino acid sequence. High variability of day care center sequences was observed mainly from residue 285 to 299 , contrasting to hospital sequences which present high variability from residue 293 to the 299 (Figure 2). Such diversification is expected since a high level of genetic variation may be associated with the fact the G protein plays a key role in facilitating re-infections in HRSV-allowing 
evasion from cross-protective immune responses - and hence in the fluctuating patterns of viral circulation [25].

Since the two variable regions of the ectodomain contain high serine and threonine residues, which are potential acceptor sites for O-linked sugars affecting the antigenic structure of the G protein as well as impacting on virus infectivity [18,19], we performed an O-glycosylation site analysis on deduced aminoacid sequences from SJRP GA1 isolates. Third four sites were potentially O-glycosylated, among GA1 strains isolated from the day care center, while hospital GA1 strains retrieved third five potentially O-glycolsilated sites (G scores of 0.5-0.7) (Figure 2). Several amino acid positions that are likely to have O linked side chains (serine at 267, 270, 275, 283, 287 and threonine at 227, 231, 235, 253, and 282), reported by [37] were conserved in all SJRP GA1 isolates. SJRP GA1 strains lost the potential O-glycosilation site at amino acid position 295, nevertheless this resulted in an insertion of two potential O-glycosilation sites at positions 296 and 297. Furthermore, a potential O-glycosilation site insertion, at position 293, was only observed for hospital strains. It is possible that strains that have lost or changed $\mathrm{O}$ glycosylation can escape the immune system by losing recognition of a carbohydrate epitope [38]. We also performed N-glycosylation site analysis, which resulted in potential for $\mathrm{N}$-glycosilation at sites 237 (G score of 0.6340 ) and 251 (G score of 0.5601). These sites were conserved among all SJRP sequences.

Eshaghi and colleagues (2012) found 21 and 27 potentially O-glycosylation sites in ON/RSV89 and ON/RSV181 (GA5 genotypes isolates) respectively, whereas an average of 33 sites were potentially $\mathrm{O}$-glycosylated in NA1 isolates. By analyzing the same region, four putative N-glycosylation sites (Asn-X-Ser/Thr) were identified among Ontario circulating strains. Zlateva and collaborators (2004) predicted that serine residues at sites 117 and 290 are $\mathrm{O}$ glycosylated with a high potential and the positive selected threonine residue at site 225 was also predicted to be $\mathrm{O}$ - glycosylated (GA2 and GA5 genotypes).

Figure 1. (a) Phylogenetic tree of José do Rio Preto-SP (SJRP) HRSVA nucleotide sequences of 265-270 in length, from the second variable region of the G gene. Reference strains representing known genotypes are indicated by a solid circle. SW8/60 (subtype B) was used as outgroup, marked by an open square. (b) Phylogenetic tree of SJRP HRSVB nucleotide sequences from the second variable region of the $G$ gene. Reference strains representing known genotypes are indicated by a solid square. Long (subtype A) was used as outgroup, marked by an open circle. Multiple sequences alignment and phylogenetic tree was constructed using Clustal W and Neighbor-joining method running within MEGA 5.05 software. Tree topology was supported by bootstrap analysis with 1000 pseudo replicate datasets. Bootstrap values greater than 50 are shown at the branch nodes. HB refers to strains isolated from the hospital (indicated by a solid lozenge) and $\mathrm{C}$ refers to strains isolated in the day care center (indicated by an open triangle). 


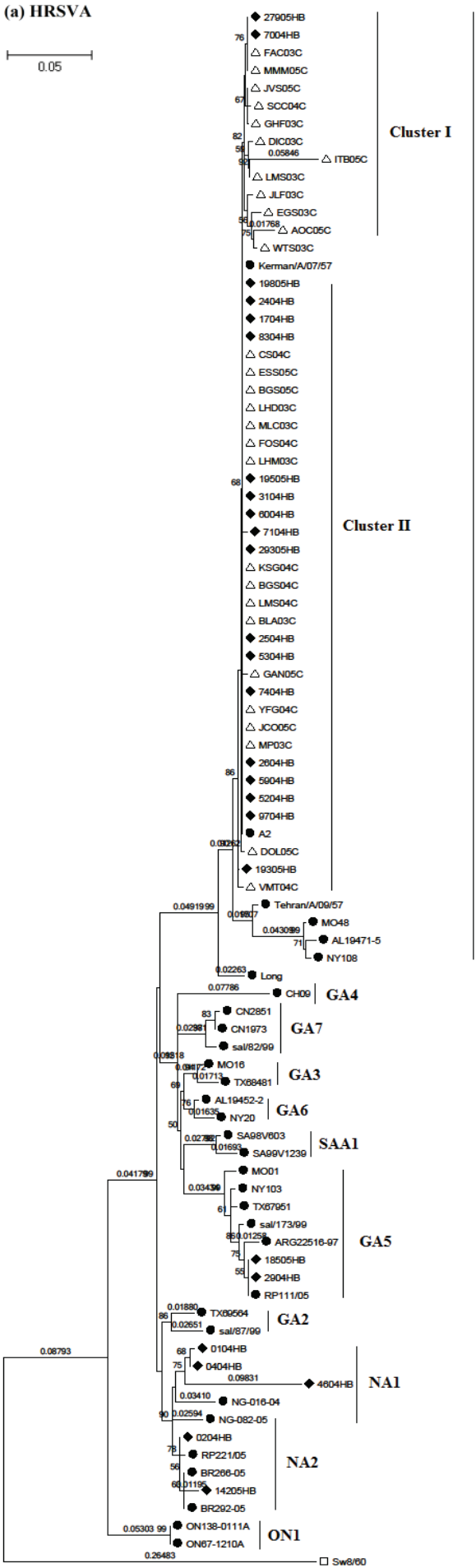


Figure 1. Cont.

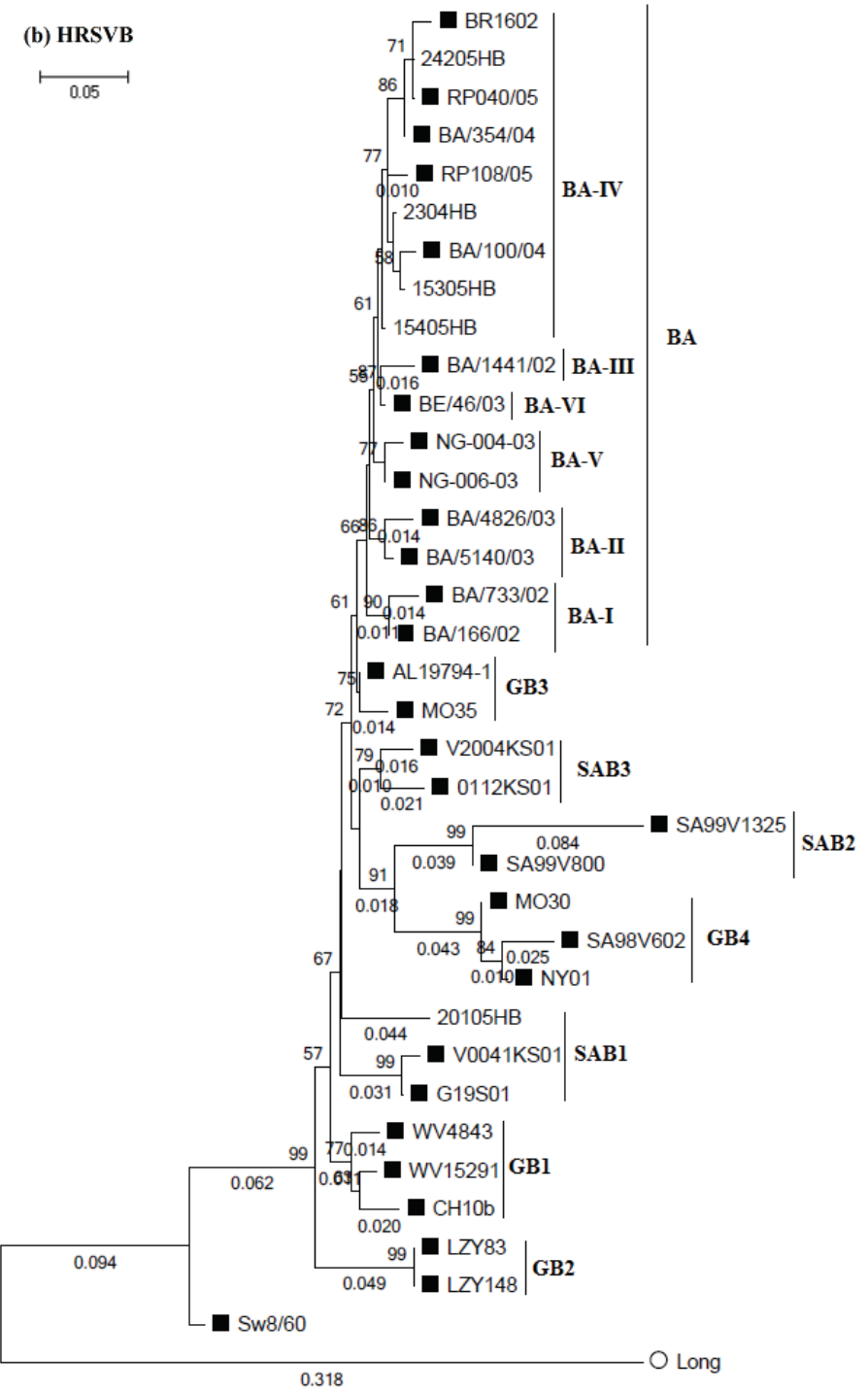


Figure 2. Alignment of deduced amino acid sequence of the G protein of HRSV GA1 genotype strains isolated in São José do Rio Preto-SP. Alignments are shown relative to the sequence of prototype strain A2. The amino acids shown correspond to positions 212 to 298 of the second hypervariable region of HRSVA strain A2 G protein. The alignment was done by the Clustal W algorithm running with BioEdit. Identical residues are represented as dots. Potential O-glycosylation sites conserved between HRSVA strain A2 and SJRP GA1 isolates are shaded in red. Potential O-glycosylation sites present only on SJRP GA1 isolates are shaded in yellow.

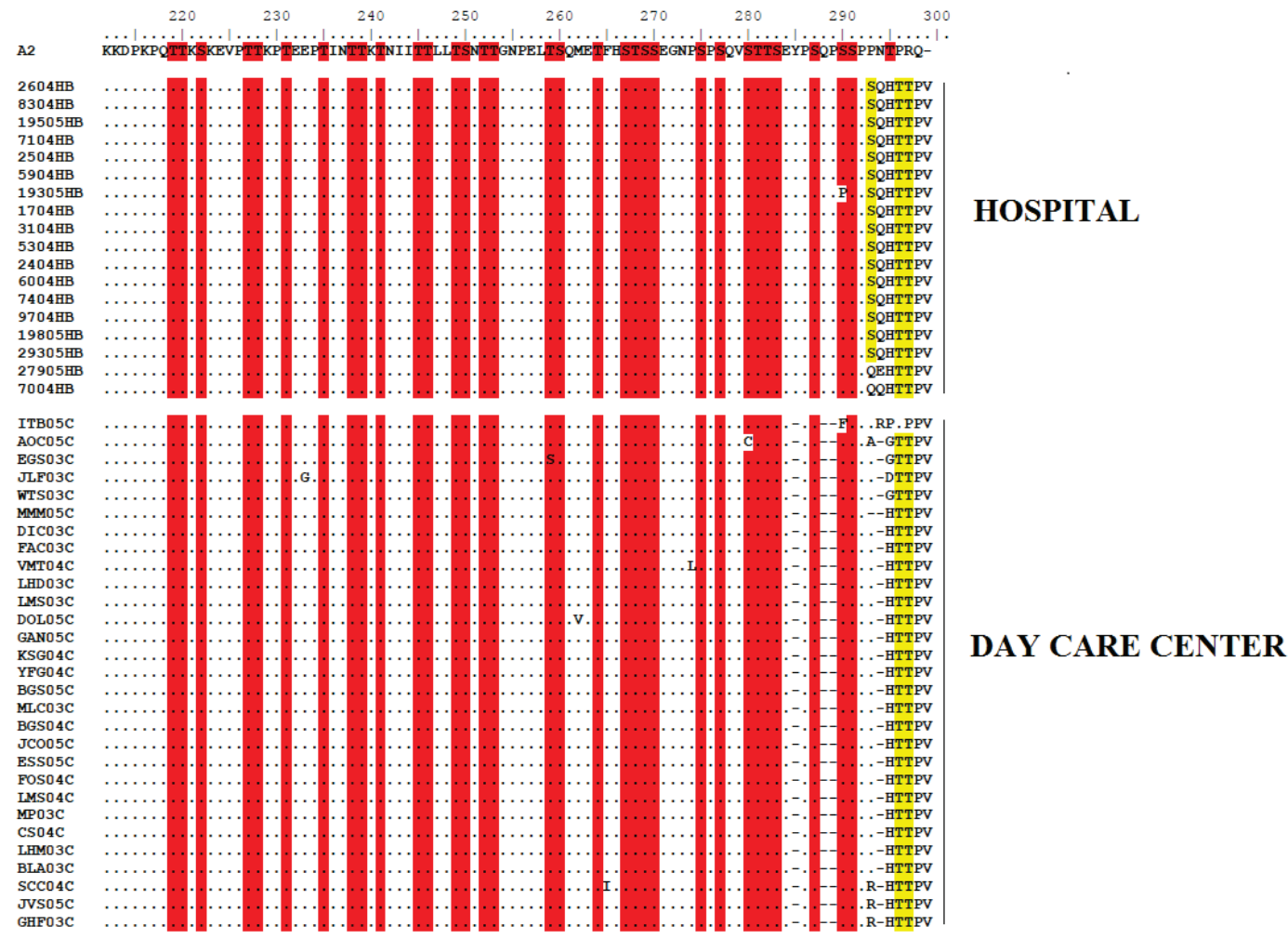

Virus infectivity has been shown to be sensitive to the limited removal of N-linked or O-linked oligosaccharides [19]. Thus, sequence changes may influence the location of carbohydrate side chains, which are important determinants of the $G$ glycoprotein antigenic structure [38]. Modifications observed on nucleotide sequences and consequently on the deduced amino acid residues composing the $\mathrm{G}$ protein ectodomain (Supplementary Figure 2 and Figure 2) of SJRP GA1 strains, also accounted for changes on potential O-glycosylation sites, thus suggesting that hospital and day care center strains could have different patterns of replication and virulence. 


\subsection{Selective Pressure}

The second hypervariable region of HRSV G gene has been reported to be an important domain of diversity and adaptation between HRSV strains [17,25,38,39]. Therefore, in order to test neutrality deviations on SJRP GA1 strains and obtain first evidence of selection pressure action on this region of the G gene, we performed the Tajima's Neutrality Test [40], which resulted in D value: $-2,32187$ with a p-value $<0.001$ and thus suggested the occurrence of sites under positive/purifying selection on GA1 genotype circulating in São José do Rio Preto (Table 1).

To test the hypothesis that the C-terminal hypervariable region of $\mathrm{G}$ proteins of SJRP's GA1 isolates could be under action of selection pressure forces, we estimated the ratio of non-synonymous $(\mathrm{dN})$ to synonymous $(\mathrm{dS})$ substitutions per site across the multiple aligned dataset of all SJRP GA1 sequences, including all 32 HRSVA sequences downloaded from GenBank (Supplementary Table 1). We used the Datamonkey webserver [41] to calculate global and site-specific non-synonymous $\left(d_{\mathrm{N}}\right)$ and synonymous $\left(d_{\mathrm{S}}\right)$ nucleotide substitution rate ratios $\left(\omega=d_{\mathrm{N}} / d_{\mathrm{S}}\right)$ using the SLAC and MEME methods, which are based on a neighbor-joining generated phylogenetic tree and the best-fit nucleotide substitution model.

We observed a high average ratio of non-synonymous to synonymous nucleotide substitutions in the $\mathrm{G}$ glycoprotein gene $(\omega=0.0 .645)$, however this value does not surpass the threshold of $\omega>1$, and it is therefore not indicative of positive selection [42]. Since the average $\omega$ is usually not sensitive enough to detect Darwinian selection at the molecular level [38], we used codon substitution models to detect sites under positive selection. Notably, amino acid residue 274 was the only site found to be subject of robust positive selection $(\mathrm{p}<0.05)$ by both methods, with posterior probability of 1.000 $(100 \%)$. In fact, previous works $[13,25,43]$ found several sites under adaptive evolution within the $G$ protein, including amino acid residue 274 , which is one of the sites that defines genotypes and lineages within genotypes, and correlate well with known epitopes described in escape-mutants selected with specific Mabs [44,45]. HRSV escape mutants that differ in their last 81 residues from the canonical Long prototype protein sequence, retain their compositions and hydropathy profiles, strongly suggesting that there may be indeed structural restrictions to changes in the G protein [25]. According to previous works $[13,38,43]$, the HRSV evolution is driven by positive selection operating at specific codon positions and the fact that we identified only one site under positive selection could be due to the method we used to identify positive selected sites, since these studies carried out the analysis on the PAML program.

Table 1. Nucleotide diversity ( $\pi$ ), Tajima D test (D), genetic distances (d) and statistical significance for Tajima D test (p) of identified human respiratory syncytial virus (HRSV) strains and GA1 genotype.

\begin{tabular}{cccccc}
\hline HRSV & N & $\Pi$ & D & d & p \\
\hline A & 55 & 0.03465 & -2.14299 & 0.048 & $\mathrm{p}<0.05$ \\
B & 5 & 0.03878 & -0.85185 & 0.040 & $\mathrm{p}>0.10$ \\
GA1 & 48 & 0.00924 & -2.32187 & 0.010 & $\mathrm{p}<0.01$ \\
\hline
\end{tabular}




\section{Experimental Procedures}

\subsection{Cohorts and Clinical Specimens}

A total of 1,089 respiratory samples were obtained from children aged between 0 and 6 years in the period of July 2003 to September 2005. Two cohorts were included, one composed of children that were hospitalized at São José do Rio Preto Public Hospital (HB) (272 samples), presenting lower respiratory tract diseases (such as bronchiolitis and pneumonia), and another composed of children presenting acute respiratory infections (ARI) (characterized by single or combined occurrence of the following physical signs and symptoms: cough, pharyngitis, rhinorrhea, nasal congestion, headache, low grade fever, facial pressure and sneezing), that attended the Maria Ines Arnal Day Care Center (C) (817 samples) in São José do Rio Preto-SP, Brazil. Nasopharyngeal washes were obtained after instillation of $0.5 \mathrm{~mL}$ of sterile PBS (Phosphate Buffered Saline - NaCl, Na2HPO4, NaH2PO4) into each nostril with immediate aspiration through a sterile neonatal canula inserted into the child's nasopharynx. The sample was transferred to a sterile vial and immediately transported to the laboratory, processed and frozen at $-80{ }^{\circ} \mathrm{C}$ in Trizol LS (Invitrogen, Carlsbad, CA) for later RNA extraction and RT-PCR testing.

\subsection{Viral Detection and Nucleotide Sequencing}

Total RNA was extracted from nasopharyngeal washes using guanidinium isothiocyanate phenol (Trizol LS, InvitrogenH, Carlsbad, CA, USA) according to the manufacturer's instructions. Reverse transcription was performed with High Capacity cDNA Archive Kit - Applied Biosystems (USA) according to the manufacturer's instructions.

Partial HRSV G gene amplification was performed by a semi-nested PCR procedure. First, cDNA was amplified using the primer FV: 5'- GTTATGACACTGGTATACCAACC-3' (based on sequences complementary to nucleotides 186 to 163 of the F protein gene [46] - and the forward primer GAB: 5'- YCAYTTTGAAGTGTTCAACTT-3' (G gene, 504-524 nt). A semi-nested PCR was then performed with primers F1AB 5'- CAACTCCATTGTTATTTGCC-3' (F gene, 3-22 nt) and GAB [9,10]. PCR assay was carried out in a reaction mixture containing $200 \mathrm{ng}$ of cDNA, $1 \mathrm{mM}$ $\mathrm{MgCl} 2,0,2 \mathrm{mM}$ dNTPs, 20 pmol of each primer, $2 \mathrm{U}$ of Taq DNA Polimerase (Biotools, ESP) in a final volume of $50 \mu \mathrm{L}$. Amplification was performed in a GeneAmp PCR System 9700 thermocycler (Applied Biosystems Inc.) with the following parameters: $95{ }^{\circ} \mathrm{C}$ for $5 \mathrm{~min}$, followed by 40 cycles of $1 \mathrm{~min}$ at $94{ }^{\circ} \mathrm{C}, 1 \mathrm{~min}$ at $55^{\circ} \mathrm{C}$ and $1 \mathrm{~min}$ at $72{ }^{\circ} \mathrm{C}$, and finally 7 min of extension at $72{ }^{\circ} \mathrm{C}$.

The semi nested PCR was carried under the same conditions, with 20 pmol of each primer on a final volume of $50 \mu \mathrm{L}$. Both cDNA synthesis and PCR followed strict procedures to prevent contamination, including redundant negative controls and segregated environments for pre- and post-amplification procedures. Amplified products of the G gene were purified with a commercial kit (Qiagen PCR Purification Kit (Qiagen - USA)), according to the manufacturer's instructions. 
Sanger sequencing, of a 490-bp fragment of the G gene, was carried out with the same primer pair used for semi-nested PCR amplification on the ABI PRISM 3100 and 377 DNA sequencers (Applied Biosystems Inc.) using the BigDye Terminator v3.1 cycle sequencing kit (Applied Biosystems).

\subsection{Phylogenetic Analysis}

Sequences were assembled with the Sequence Navigator program version 1.0 (Applied Biosystems Inc., EUA) resulting in contigs of 265 nucleotides on average, to isolates from the day care center, and contigs of 270 and 330 nucleotides on average to isolates from the hospital. These contigs were compared to HRSV G gene nucleotides from reference strains representing different HRSVA and HRSVB genotypes identified in other cities or states of Brazil, South America, South Africa as well as HRSV sequences from other countries available at GenBank (Supplementary Table 1). Multiple sequence alignments of the obtained fragments compared to globally sampled reference strains were performed using the Clustal W algorithm [47] and Bioedit sequence alignment-editing software. Phylogenetic associations were determined using Maximum Likelihood (ML) and Neighbor-Joining (NJ) methods running with MEGA 5.05 software [48]. Node support of each clade was evaluated using bootstrap analysis (1000 replicates) and the evolutionary distances were derived using the Kimura-2 parameter method [49].

\subsection{O-glycosylation and N-glycosilation Site Analysis}

Potential O-glycosylation and N-glycosylation sites were predicted using and NetOGlyc 3.1 [50] and NetNGlyc 1.0. The deduced AA sequences of the second hypervariable region of HRSV GA1 genotypes (encompassing AA 212 to the end of the G protein) were compared to those of HRSV-A2 strain.

\subsection{Selection Pressure Analysis}

Neutrality Tajima's D test [40] was performed by DnaSP version 5.10.01 software [51] to verify statistically significant deviations among sequences and provide first evidence of selection acting on SJRP HRSV isolates.

To determine the selection pressures acting on the ectodomain of the G gene of GA1 genotype isolates, we estimated the numbers of non-synonymous $(\mathrm{dN})$ to synonymous $(\mathrm{dS})$ nucleotide changes per site; when $\mathrm{dN}>\mathrm{dS}$, this was indicative of positive selection. Site-specific (that is, codon-specific) selection pressures were determined using the Single Likelihood Ancestral Counting (SLAC) available in the HyPhy package [52] and Mixed Effects Model of Evolution (MEME) [53] methods, accessed through the Datamonkey webserver [41]. These methods were run using best fit nucleotide model on a neighbor-joining phylogenetic tree. Using this procedure, only codon 274 contained statistically significant evidence for positive selection. 


\subsection{Ethics Statement}

A Written Consent signed by the parents or legal responsible guardians was obtained for each child. This study was approved by Research Ethics Committee from Unesp/IBILCE, Project Number 3777/2001 by opinion n ${ }^{\circ}$ 062/2001 on 11 June 2001 in São José do Rio Preto, Brazil.

\section{Acknowledgments}

The authors have received financial support from FAPESP, process $n^{\circ} .2010 / 50444-4$.

\section{Conflicts of Interest}

The authors declare no conflict of interest.

\section{References}

1. Glezen, P.; Denny, F.W. Epidemiology of acute lower respiratory disease in children. N. Engl. J. Med. 1973, 288, 498-505.

2. Hall, C.B.; Weinberg, G.A.; Iwane, M.K.; Blumkin, A.K.; Edwards, K.M.; Staat, M.A.; Auinger, P.; Griffin, M.R.; Poehling, K.A.; Erdman, D.; et al. The burden of respiratory syncytial virus infection in young children. N. Engl. J. Med. 2009, 360, 588-598.

3. Anderson, L.J.; Parker, R.A.; Strikas, R.L. Association between respiratory syncytial virus outbreaks and lower respiratory tract deaths of infants and young children. J. Infect. Dis. 1990, $161,640-646$.

4. Falsey, A.R.; Walsh, E.E. Respiratory syncytial virus infection in adults. Clin. Microbiol. Rev. 2000, 13, 371-384.

5. Widjojoatmodjo, M.N.; Boes, J.; van Bers, M.; van Remmerden, Y.; Roholl, P.J.; Luytjes, W. A highly attenuated recombinant human respiratory syncytial virus lacking the $G$ protein induces long-lasting protection in cotton rats. Virol. J. 2010, 7, 114.

6. Cane, P.A. Molecular epidemiology of respiratory syncytial virus. Rev. Med. Virol. 2001, 11, 103-116.

7. Mufson, M.A.; Orvell, C.; Rafnar, B.; Norrby, E. Two distinct subtypes of human respiratory syncytial virus. J. Gen. Virol. 1985, 66 (Pt 10), 2111-2124.

8. Anderson, L.J.; Hierholzer, J.C.; Tsou, C.; Hendry, R.M.; Fernie, B.F.; Stone, Y.; McIntosh, K. Antigenic characterization of respiratory syncytial virus strains with monoclonal antibodies. J. Infect. Dis. 1985, 151, 626-633.

9. Peret, T.C.; Hall, C.B.; Schnabel, K.C.; Golub, J.A.; Anderson, L.J. Circulation patterns of genetically distinct group A and B strains of human respiratory syncytial virus in a community. J. Gen. Virol. 1998, 79 (Pt 9), 2221-2229. 
10. Peret, T.C.; Hall, C.B.; Hammond, G.W.; Piedra, P.A.; Storch, G.A.; Sullender, W.M.; Tsou, C.; Anderson, L.J. Circulation patterns of group A and B human respiratory syncytial virus genotypes in 5 communities in North America. J. Infect. Dis. 2000, 181, 1891-1896.

11. Venter, M.; Madhi, S.A.; Tiemessen, C.T.; Schoub, B.D. Genetic diversity and molecular epidemiology of respiratory syncytial virus over four consecutive seasons in South Africa: Identification of new subgroup A and B genotypes. J. Gen. Virol. 2001, 82, 2117-2124.

12. Shobugawa, Y.; Saito, R.; Sano, Y.; Zaraket, H.; Suzuki, Y.; Kumaki, A.; Dapat, I.; Oguma, T.; Yamaguchi, M.; Suzuki, H. Emerging genotypes of human respiratory syncytial virus subgroup A among patients in Japan. J. Clin. Microbiol. 2009, 47, 2475-2482.

13. Eshaghi, A.; Duvvuri, V.R.; Lai, R.; Nadarajah, J.T.; Li, A.; Patel, S.N.; Low, D.E.; Gubbay, J.B. Genetic variability of human respiratory syncytial virus a strains circulating in ontario: A novel genotype with a 72 nucleotide $\mathrm{G}$ gene duplication. PLoS One 2012, 7, e32807.

14. Trento, A.; Viegas, M.; Galiano, M.; Videla, C.; Carballal, G.; Mistchenko, A.S.; Melero, J.A. Natural history of human respiratory syncytial virus inferred from phylogenetic analysis of the attachment (G) glycoprotein with a 60-nucleotide duplication. J. Virol. 2006, 80, 975-984.

15. Sullender, W.M. Respiratory syncytial virus genetic and antigenic diversity. Clin. Microbiol. Rev. 2000, 13, 1-15.

16. Zhang, R.F.; Jin, Y.; Xie, Z.P.; Liu, N.; Yan, K.L.; Gao, H.C.; Song, J.R.; Yuan, X.H.; Xiao, N.G.; Guo, M.W.; et al. Human respiratory syncytial virus in children with acute respiratory tract infections in China. J. Clin. Microbiol. 2010, 48, 4193-4199.

17. Johnson, P.R.; Spriggs, M.K.; Olmsted, R.A.; Collins, P.L. The G glycoprotein of human respiratory syncytial viruses of subgroups $\mathrm{A}$ and $\mathrm{B}$ : Extensive sequence divergence between antigenically related proteins. Proc. Natl. Acad. Sci. USA 1987, 84, 5625-5629.

18. Garcia-Beato, R.; Martinez, I.; Franci, C.; Real, F.X.; Garcia-Barreno, B.; Melero, J.A. Host cell effect upon glycosylation and antigenicity of human respiratory syncytial virus $\mathrm{G}$ glycoprotein. Virology 1996, 221, 301-309.

19. Lambert, D.M. Role of oligosaccharides in the structure and function of respiratory syncytial virus glycoproteins. Virology 1988, 164, 458-466.

20. Cintra, O.A.; Owa, M.A.; Machado, A.A.; Cervi, M.C.; Figueiredo, L.T.; Rocha, G.M.; Siqueira, M.M.; Arruda, E. Occurrence and severity of infections caused by subgroup A and B respiratory syncytial virus in children in southeast Brazil. J. Med. Virol. 2001, 65, 408-412.

21. Moura, F.E.; Blanc, A.; Frabasile, S.; Delfraro, A.; de Sierra, M.J.; Tome, L.; Ramos, E.A.; Siqueira, M.M.; Arbiza, J. Genetic diversity of respiratory syncytial virus isolated during an epidemic period from children of northeastern Brazil. J. Med. Virol. 2004, 74, 156-160.

22. Oliveira, T.F.; Freitas, G.R.; Ribeiro, L.Z.; Yokosawa, J.; Siqueira, M.M.; Portes, S.A.; Silveira, H.L.; Calegari, T.; Costa, L.F.; Mantese, O.C.; et al. Prevalence and clinical aspects of respiratory syncytial virus A and B groups in children seen at Hospital de Clinicas of Uberlandia, MG, Brazil. Mem. Inst. Oswaldo Cruz 2008, 103, 417-422. 
23. Da Silva, L.H.; Spilki, F.R.; Riccetto, A.G.; de Almeida, R.S.; Baracat, E.C.; Arns, C.W. Genetic variability in the $\mathrm{G}$ protein gene of human respiratory syncytial virus isolated from the Campinas metropolitan region, Brazil. J. Med. Virol. 2008, 80, 1653-1660.

24. Riccetto, A.G.; Silva, L.H.; Spilki, F.R.; Morcillo, A.M.; Arns, C.W.; Baracat, E.C. Genotypes and clinical data of respiratory syncytial virus and metapneumovirus in brazilian infants: A new perspective. Braz. J. Infect. Dis. 2009, 13, 35-39.

25. Botosso, V.F.; Zanotto, P.M.; Ueda, M.; Arruda, E.; Gilio, A.E.; Vieira, S.E.; Stewien, K.E.; Peret, T.C.; Jamal, L.F.; Pardini, M.I.; et al. Positive selection results in frequent reversible amino acid replacements in the $\mathrm{G}$ protein gene of human respiratory syncytial virus. PLoS Pathog. 2009, 5 , e1000254.

26. Lima, H.N.; Botosso, V.F.; Oliveira, D.B.; Campos, A.C.; Leal, A.L.; Silva, T.S.; Bosso, P.A.; Moraes, C.T.; Filho, C.G.; Vieira, S.E.; et al. Molecular epidemiology of the SH (small hydrophobic) gene of human respiratory syncytial virus (HRSV), over 2 consecutive years. Virus Res. 2012, $163,82-86$.

27. McCutcheon, H.; Fitzgerald, M. The public health problem of acute respiratory illness in childcare. J. Clin. Nurs. 2001, 10, 305-310.

28. Lu, N.; Samuels, M.E.; Shi, L.; Baker, S.L.; Glover, S.H.; Sanders, J.M. Child day care risks of common infectious diseases revisited. Child Care Health Dev. 2004, 30, 361-368.

29. Gardinassi, L.G.A.; Simas, P.V.M.; Salomão, J.B.; Durigon, E.L.; Trevisan, D.M.Z.; Cordeiro, J.A.; Lacerda, M.N.; Rahal, P.; Souza, F.P. Seasonality of viral respiratory infections in Southeast of Brazil: The influence of temperature and air humidity. Braz. J. Microbiol. 2012, 43, 98-108.

30. Collins, P.L.; Graham, B.S. Viral and host factors in human respiratory syncytial virus pathogenesis. J. Virol. 2008, 82, 2040-2055.

31. Hall, C.B.; Walsh, E.E.; Schnabel, K.C.; Long, C.E.; McConnochie, K.M.; Hildreth, S.W.; Anderson, L.J. Occurrence of groups A and B of respiratory syncytial virus over 15 years: Associated epidemiologic and clinical characteristics in hospitalized and ambulatory children. J. Infect. Dis. 1990, 162, 1283-1290.

32. Mufson, M.A.; Belshe, R.B.; Orvell, C.; Norrby, E. Respiratory syncytial virus epidemics: Variable dominance of subgroups A and B strains among children, 1981-1986. J. Infect. Dis. 1988, 157, 143-148.

33. Hall, C.B. Respiratory syncytial virus and parainfluenza virus. N. Engl. J. Med. 2001, 344, 1917-1928.

34. Matthijnssens, J.; Rahman, M.; van Ranst, M. Loop model: Mechanism to explain partial gene duplications in segmented dsRNA viruses. Biochem. Biophys. Res. Commun. 2006, 340, 140-144.

35. Ballard, A.; McCrae, M.A.; Desselberger, U. Nucleotide sequences of normal and rearranged RNA segments 10 of human rotaviruses. J. Gen. Virol. 1992, 73 (Pt 3), 633-638.

36. Kojima, K.; Taniguchi, K.; Kawagishi-Kobayashi, M.; Matsuno, S.; Urasawa, S. Rearrangement generated in double genes, NSP1 and NSP3, of viable progenies from a human rotavirus strain. Virus Res. 2000, 67, 163-171. 
37. Collins, P.L.; Chanock, R.M.; Murphy, B.R. Respiratory syncytial virus. In Fields Virology, 4th ed.; Howley, D.M.K.P.M., Ed.; Lippincott Williams \& Wilkins: Philadelphia, PA, USA, 2001; pp. 1443-1485.

38. Zlateva, K.T.; Lemey, P.; Vandamme, A.M.; van Ranst, M. Molecular evolution and circulation patterns of human respiratory syncytial virus subgroup a: Positively selected sites in the attachment g glycoprotein. J. Virol. 2004, 78, 4675-4683.

39. Zlateva, K.T.; Lemey, P.; Moes, E.; Vandamme, A.M.; van Ranst, M. Genetic variability and molecular evolution of the human respiratory syncytial virus subgroup B attachment G protein. J. Virol. 2005, 79, 9157-9167.

40. Tajima, F. Statistical method for testing the neutral mutation hypothesis by DNA polymorphism. Genetics 1989, 123, 585-595.

41. Pond, S.L.K.; Frost, S.D.W. Datamonkey: Rapid detection of selective pressure on individual sites of codon alignments. Bioinformatics 2005, 21, 2531-2533.

42. Yang, Z.; Nielsen, R.; Goldman, N.; Pedersen, A.M. Codon-substitution models for heterogeneous selection pressure at amino acid sites. Genetics 2000, 155, 431-449.

43. Gaunt, E.R.; Jansen, R.R.; Poovorawan, Y.; Templeton, K.E.; Toms, G.L.; Simmonds, P. Molecular epidemiology and evolution of human respiratory syncytial virus and human metapneumovirus. PLoS One 2011, 6, e17427.

44. Garcia, O.; Martin, M.; Dopazo, J.; Arbiza, J.; Frabasile, S.; Russi, J.; Hortal, M.; Perez-Brena, P.; Martinez, I.; Garcia-Barreno, B.; et al. Evolutionary pattern of human respiratory syncytial virus (subgroup A): Cocirculating lineages and correlation of genetic and antigenic changes in the $\mathrm{G}$ glycoprotein. J. Virol. 1994, 68, 5448-5459.

45. Rueda, P.; Delgado, T.; Portela, A.; Melero, J.A.; Garcia-Barreno, B. Premature stop codons in the $\mathrm{G}$ glycoprotein of human respiratory syncytial viruses resistant to neutralization by monoclonal antibodies. J. Virol. 1991, 65, 3374-3378.

46. Zheng, H.; Peret, T.C.; Randolph, V.B.; Crowley, J.C.; Anderson, L.J. Strain-specific reverse transcriptase PCR assay: Means to distinguish candidate vaccine from wild-type strains of respiratory syncytial virus. J. Clin. Microbiol. 1996, 34, 334-337.

47. Thompson, J.D.; Higgins, D.G.; Gibson, T.J. CLUSTAL W: Improving the sensitivity of progressive multiple sequence alignment through sequence weighting, position-specific gap penalties and weight matrix choice. Nucleic Acids Res. 1994, 22, 4673-4680.

48. Tamura, K.; Peterson, D.; Peterson, N.; Stecher, G.; Nei, M.; Kumar, S. MEGA5: Molecular evolutionary genetics analysis using maximum likelihood, evolutionary distance, and maximum parsimony methods. Mol. Biol. Evol. 2011, 28, 2731-2739.

49. Kimura, M. A simple method for estimating evolutionary rates of base substitutions through comparative studies of nucleotide sequences. J. Mol. Evol. 1980, 16, 111-120.

50. Julenius, K.; Molgaard, A.; Gupta, R.; Brunak, S. Prediction, conservation analysis, and structural characterization of mammalian mucin-type O-glycosylation sites. Glycobiology 2005, 15, 153-164. 
51. Librado, P.; Rozas, J. DnaSP v5: A software for comprehensive analysis of DNA polymorphism data. Bioinformatics 2009, 25, 1451-1452.

52. Pond, S.L.; Frost, S.D.; Muse, S.V. HyPhy: Hypothesis testing using phylogenies. Bioinformatics 2005, 21, 676-679.

53. Murrell, B.; Wertheim, J.O.; Moola, S.; Weighill, T.; Scheffler, K.; Kosakovsky Pond, S.L. Detecting individual sites subject to episodic diversifying selection. PLoS Genet. 2012, 8, e1002764. 
Reprinted from Viruses. Cite as: Sarmiento-Silva, R.E.; Nakamura-Lopez, Y.; Vaughan, G. Epidemiology, Molecular Epidemiology and Evolution of Bovine Respiratory Syncytial Virus. Viruses 2012, 4, 3452-3467.

Review

\title{
Epidemiology, Molecular Epidemiology and Evolution of Bovine Respiratory Syncytial Virus
}

\section{Rosa Elena Sarmiento-Silva ${ }^{1}$, Yuko Nakamura-Lopez ${ }^{2}$ and Gilberto Vaughan ${ }^{3, *}$}

1 Facultad de Medicina Veterinaria y Zootecnia, Universidad Nacional Autónoma de México, Circuito Exterior, Ciudad Universitaria, Delegación Coyoacán, México, D.F. 04510, Mexico;

E-Mail: rosass@unam.mx

2 Consejo Estatal para la Prevencion y Control del Sida-Centro Ambulatorio para la Prevencion y Atencion del Sida e ITS (COESIDA-CAPASITS) Oaxaca, Mexico; E-Mail: yuko@unam.mx

3 Instituto de Diagnóstico y Referencia Epidemiológicos, Carpio 470, Col. Santo Tomas, Mexico, D.F. 11340, Mexico

* Author to whom correspondence should be addressed; E-Mail: gilvaughan@yahoo.com; Tel.: +52-55-5342-7563; Fax: +52-55-5342-1230.

Received: 29 October 2012; in revised form: 22 November 2012 / Accepted: 23 November 2012 / Published: 30 November 2012

\begin{abstract}
The bovine respiratory syncytial virus (BRSV) is an enveloped, negative sense, single-stranded RNA virus belonging to the pneumovirus genus within the family Paramyxoviridae. BRSV has been recognized as a major cause of respiratory disease in young calves since the early 1970s. The analysis of BRSV infection was originally hampered by its characteristic lability and poor growth in vitro. However, the advent of numerous immunological and molecular methods has facilitated the study of BRSV enormously. The knowledge gained from these studies has also provided the opportunity to develop safe, stable, attenuated virus vaccine candidates. Nonetheless, many aspects of the epidemiology, molecular epidemiology and evolution of the virus are still not fully understood. The natural course of infection is rather complex and further complicates diagnosis, treatment and the implementation of preventive measures aimed to control the disease. Therefore, understanding the mechanisms by which BRSV is able to establish infection is needed to prevent viral and disease spread. This review discusses important information regarding the epidemiology and molecular epidemiology of BRSV worldwide, and it highlights the importance of viral evolution in virus transmission.
\end{abstract}


Keywords: BRSV; global distribution; genotypes; evolution

\section{Introduction}

The bovine respiratory syncytial virus (BRSV) has been recognized as a pathogen in cattle responsible of an acute respiratory disease syndrome in beef and dairy calves since the early 1970s [1,2]. The impact of BRSV infection on the cattle industry results in economic losses due to the morbidity, mortality, treatment and prevention costs that eventually lead to loss of production and reduced carcass value [3].

BRSV is an enveloped, non-segmented, negative-stranded RNA virus belonging to the Pneumovirus genus within the subfamily Pneumovirinae, family Paramyxoviridae [4]. The BRSV virion consists of a lipid envelope containing three surface glycoproteins (glycoprotein [G], the fusion protein $[\mathrm{F}]$ and the small hydrophobic protein $[\mathrm{SH}]$ ) (Figure 1). The envelope encloses a helical nucleocapsid composed by the nucleoprotein $(\mathrm{N})$, the phosphoprotein $(\mathrm{P})$, the viral RNA-dependent polymerase protein (L) the $\mathrm{M}$ protein and a transcriptional anti-termination factor known as M2-1. The genomic RNA ( $\sim 15,000$ nucleotides in length) also encodes an RNA regulatory protein M2-2 and two non-structural proteins, NS1 and NS2 [4].

Figure 1. Bovine respiratory syncytial virus (BRSV) genome scheme and commonly used region for molecular epidemiology studies. The areas encoding the BRSV proteins are represented in boxes. Targeting regions are following: region (1), N region (nt 1294 to nt 1984); region (2), SH complete genome (nt 4268 to 4513); region (3), G region (nt 4864 to 5353); region (3), F region (nt 6071 to nt 6812). Nucleotide positions were given in reference to strain NC_001989.

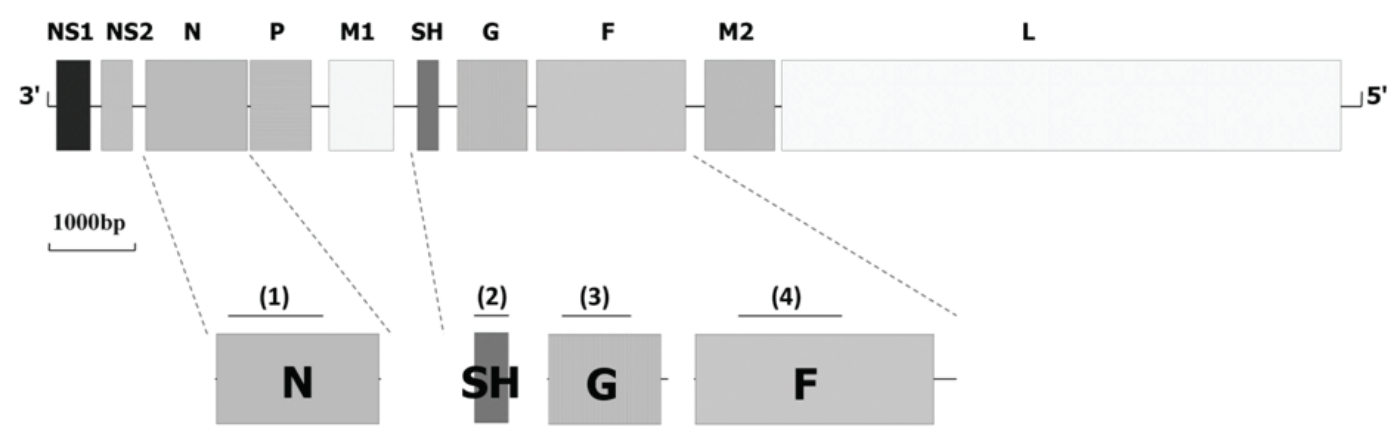

BRSV is closely related to human RSV (HRSV), and the epidemiology and pathogenesis of infection between these two viruses share some similarities and also many differences [5]. The similarities between the two viruses have facilitated the unveiling of some of the mechanisms by 
which BSRV can cause disease. However, the means used by the virus to warrant transmission among individuals within and between herds have remained elusive.

Understanding of the global epidemiology and molecular epidemiology of BRSV has significantly improved over recent years. In this review, we discuss various aspects of the epidemiology and molecular epidemiology of BRSV as well as their relationship with viral evolution.

\section{Epidemiology of BRSV}

BRSV infection is widely spread around the world, most likely as a direct result of the movement of cattle [6]. Regardless of the geographical location, infectivity rates are usually rather high, suggesting that viral transmission is a common event among herds. Cattle are the principal reservoir of infection; however, sheep can also become infected [7]. Intra-herd transmission usually occurs by aerosols, allowing the virus to enter susceptible cattle via the respiratory tract. However, local spread and airborne transmission between herds are not of great importance for inter-herd transmission despite the circulation of BRSV in a given geographical region [8]. On the other hand, direct transmission between herds is frequently a consequence of the introduction of new infected animals, while indirect transmission occurs by individuals visiting farms. Some of the main risk factors for BRSV transmission include large herd size and common farm practices such as not providing boots to visitors and dual-purpose farms $[9,10]$. Additionally, it has also been proposed that good management and better hygienic routines have a direct impact on overall health status [8].

BRSV outbreaks commonly occur during winter [11]. Thus, clinical disease is commonly diagnosed during autumn and winter in temperate regions [12]. Nevertheless, infection can also be observed during summer [12,13]. The sero-prevalence of BRSV infection varies greatly across different geographical regions [10,14-17]. The distribution of BRSV is most likely affected by the movement of cattle, as insect vectors are not believed to play a role in viral transmission [6]. The morbidity of the disease is quite high, and in some instances, it has been responsible for up to $60 \%$ of the clinical respiratory diseases among dairy herds $[13,18]$. In general, the frequency of BRSV is strongly associated with cattle population density in the region and with the age of the host $[13,19,20]$. Interestingly, BRSV infection is also associated with a high morbidity of up to $80 \%$ and with mortality that can reach up to $20 \%$ in some outbreaks.

BRSV outbreaks can become epidemics affecting animals in all age groups. However, the age distribution of BRSV infection seems to be a function of exposure. In other words, herds that have been previously exposed to the virus tend to experience infections that are limited to younger, more susceptible animals. In consequence, morbidity is commonly high during the occurrence of outbreaks [21]. Importantly, natural infection affects both beef and dairy cattle, although management practices can significantly impact the infectivity rates [22]. Climate also favors the dissemination of the virus during winter, after the sudden drop in temperature [11], although infection can occur throughout the year.

The mechanisms that are responsible for the survival of the virus within a given population are not fully understood. Controversial information has been reported about viral persistence. Nonetheless, 
chronicity has been proposed as a mechanism that might play role in disease spread [23]. BRSV can be isolated from asymptomatic animals and can persist for several months [6]. Thus, one possibility is the existence of persistently infected calves, which might start shedding the virus under specific conditions [24,25]. Therefore, latent infection among herds might occur, providing a possible explanation for the occurrence of outbreaks among relatively isolated calves. However, some reports have suggested that subclinical infection in cattle is not a plausible mechanism for the persistence of BRSV in dairy herds [26]. On the other hand, clinically ill animals are believed to be the most likely sources of infection, and therefore, the most likely explanation for recurrent infections is the reintroduction of the virus into the herd before the occurrence of a new outbreak. This controversial issue requires more detailed studies aiming to assess the role of persistence in virus spread to fully understand the mechanism exploited by the BRSV to warrant transmission.

The prevalence of the disease varies greatly in North America. For instance, in the United States, original studies conducted during the early 1970s showed a frequency of infection of $67 \%$ among adult cattle [27]. However, in a few instances, $100 \%$ of the animals within herds showed the presence of specific antibodies. Subsequently published reports showed that up to $81 \%$ of herds had specific antibodies against BRSV [28,29]. Additionally, the incidence of sero-conversion soon after the occurrence of an outbreak has been reported to be as high as 45\% [30]. Furthermore, the sero-positivity rate among asymptomatic cattle has been shown to reach up to $95 \%$ [15]. Importantly, the sero-positivity rate seems to be closely associated with the age of the host, showing a higher prevalence among older animals. Moreover, southern regions of the U.S. commonly exhibit a higher sero-prevalence than the northern parts of the country [31]. These discrepancies could be explained by differences in the vaccination practices in those regions, as well as by management practices or by sampling errors. Thus, the frequency of BRSV infection in a particular region is subject to a number of factors that can drastically change the prevalence of the disease. Interestingly, the mortality among herds experiencing respiratory disease in the U.S. can reach up to $13 \%$ [32]. While other viruses, such as the bovine viral diarrhea and parainfluenza viruses, could also account for the elevated mortality rates seen in the U.S., BRSV remains as a very important ethological agent and a probable cause of death due to respiratory disease in cattle.

In Canada, initial reports have shown that the overall frequency of BRSV infection might be nearly $36 \%[16,17]$. However, during outbreaks, the percentage of sero-positive individuals within a herd can range between $22 \%$ and $53 \%$. Subsequent studies confirmed the high frequency of the disease (40\%) among feedlot calves [33]. Similar to what has been observed in the U.S., BRSV is still an important cause of mortality among cattle in Canada [34]. In Mexico, the circulation of BRSV was recently reported in two different regions of the country $[35,36]$. Both studies reported rather high overall frequencies of BRSV infection (52\% and 90.8\%). The reasons for the significant differences in the distribution of BRSV infection between the two regions are not known; however, age played a major role in the distribution of infection. Additionally, BRSV has also been shown to circulate in other regions of the Americas [10,37,38]. 
In Europe, shortly after the virus's discovery, it was reported to have widely circulated in different parts of the continent [39]. In Sweden, different studies have shown high frequencies of antibodies in milk, ranging between $41 \%$ and $89 \%$ depending on the geographical region of the country [13]. In general, higher antibody frequencies in milk samples were observed in samples from the southern regions of Sweden, while lower frequencies were detected in samples from the northern part of the country. The authors attributed the discrepancies observed among differences herds to cattle population density, as highly populated regions were more common in the southern part of Sweden. More recently, the geographical distribution of BRSV in the country has been studied [40]. The aforementioned study showed that infection with BRSV occurs predominantly in the central-western and southern parts of Sweden. In particular, two regions of the country, Skaraborg and Skåne, displayed a high prevalence of BRSV infection. Similarly, Danish reports have also shown rather high frequencies of BRSV infection (54\%) [41]. Likewise, a high prevalence of BRSV infection has been observed in Belgium [42]. Circulation of the virus in Scotland has also been reported, although conclusive figures regarding BRSV prevalence are not available [43]. Furthermore, the presence of antibodies in bulk tank milk samples has been reported in herds from England [44]. Additionally, a high sero-prevalence of BRSV has also been reported in Northern Europe [21,45].

In Africa, Ethiopia and South Africa have also been shown to have high incidences of BRSV infection [14,46-48]. Other countries, in different regions, such as Turkey, have also been shown to a have high sero-prevalence, which can reach up to 43\% [49]. Unsurprisingly, high sero-prevalence has also been associated with large-capacity facilities, rather than with small farms. Interestingly, organic farms have been shown to exhibit lower antibody prevalence when compared to conventional farms [22]. These findings highlight the importance of management for the effective control of viral transmission and disease spread, which are closely associated with different farming methods

Thus, more information is required to understand the mechanisms that allow for viral survival in a given geographical region. Monitoring of outbreaks among herds is likely to provide valuable information that might help us to understand in greater detail the epidemiology of BRSV infection.

\section{Molecular Epidemiology and Evolution of BRSV}

Limited information is available regarding the molecular epidemiology of BRSV infection. However, the rapidly changing field of DNA sequencing might help to unveil the molecular mechanisms exploited by BRSV to assure transmission. Additionally, the field of phylogenetics has contributed to identify the existence of diverse BRSV genotypes and their genetic relationship while helping understand the molecular basis of BRSV genetic history [50,51]. Phylogeny approaches have also been particularly useful for studying the origins and subsequent evolution of BRSV [51]. Recent advances in phylogenetics have allowed for the analysis in greater detail of sequence information, which could also help to understand the patterns of BRSV infection [52]. Additionally, current phylogenetic methods facilitate the estimation of the time to the origin of a new viral strain, of the emergence of new species, identification of viral recombination, of population size, and of how the virus spread and evolve in particular settings [53]. Thus, the information obtained from phylogenetic 
studies could assist in the design and implementation of measures aimed to prevent BRSV transmission. Molecular epidemiological approaches have provided important insights into BRSV evolution, spread and transmission [50,54]. Phylogentic analysis have also helped to identify the presence of mutations mapping along the immunodominat region located on the G protein [55] and to elucidate the origin circulating strains in certain geographical regions [56]. Thus, the study of the molecular epidemiology of the virus will most likely improve our understanding of the dynamics of virus transmission and help us to implement informed public health policies.

The occurrence of antigenic variation among BRSV isolates was first suggested by the lack of reaction between a polyclonal serum made against one particular viral strain, which failed to recognize a different isolate [57]. Furthermore, viral heterogeneity was also inferred from the differences observed among the molecular sizes of some of the structural proteins, which implied that BRSV was composed of distinct subgroups [58,59]. The existence of diversity among BRSV isolates was further assessed by the reactivity of a set of strains against anti-HRSV monoclonal antibodies [58]. In that pioneering study, the authors showed the existence of different recognition patterns among different BRSV isolates, thus implying antigenic diversity. The aforementioned findings highlighted the limitations and efficacy of the BRSV vaccine, suggesting that vaccine failure could be at least partially attributed to a possible broader antigenic spectrum of the BRSV population [58]. Thus, these initial reports suggested the presence of two distinct BRSV subgroups. These observations were later confirmed by another study [60]. Estimation of BRSV antigenic variation further confirmed the presence of two major and one intermediate subgroups [61]. Currently, four antigenic subgroups (A, B, AB, untyped) have been identified in BRSV; however, they might only represent variants of a single major antigenic group [62].

The study of the molecular epidemiology of BRSV began with the identification of the nucleotide sequences of the glycoprotein $(\mathrm{G})$, fusion $(\mathrm{F})$, nucleocapsid $(\mathrm{N})$, matrix $(\mathrm{M})$, phosphoprotein $(\mathrm{P})$, small hydrophobic (SH) and M2 proteins during the early 1990s [55,63-67]. The initial assessment of the homology along the P protein between two isolates showed an identity of $97 \%$ at the nucleotide level [67]. Subsequent studies, which analyzed the nucleotide variation of a limited number of isolates at the $\mathrm{G}$ protein level, showed that the levels of identity between BRSV strains ranged between $84 \%$ and $95 \%$ [60,68]. This degree of heterogeneity was suggestive of only one single genetic group. The first direct comparison between the antigenic heterogeneity and molecular diversity among BRSV isolates showed that the antigenic divergence observed among BRSV strains was the result of inter-subgroup variation [60]. The characterization of the antigenic structure of the BRSV G protein provided further information about the mutations responsible for the distinctive BRSV groups reported at that time [69]. Subsequent studies further confirmed the existence of antigenic divergence and antigenic variability among wild BRSV isolates [70]. The partial nucleotide sequences of the $G$ protein from a set of isolates, along with the recognition pattern by monoclonal antibodies against the BRSV G, F, N, and P proteins, showed random antigenic differences among the isolates, although cross-reactivity to the viral protein epitopes was observed, particularly with the F protein. Moreover, structural differences between the $\mathrm{F}$ and $\mathrm{P}$ proteins were also observed. The $\mathrm{P}$ protein exhibited diverse 
patterns, due to differences in molecular size, when subjected to polyacrylamide gel electrophoresis analysis. However, the structural differences along the $\mathrm{P}$ protein were not correlated with the antigenic differences observed in the $\mathrm{F}$ and $\mathrm{N}$ proteins. Overall, the nucleotide sequence identity in the $\mathrm{G}$ protein ranged from $94.1 \%$ to $99.9 \%$. In comparison, the predicted amino acid sequence homology ranged from $89.9 \%$ to $99.6 \%$, supporting the theory that BRSV belonged to a monophyletic group [70].

More comprehensive studies, which analyzed the reaction patterns against anti-BRSV G protein and the genetic diversity occurring in a larger segment of the $G$ protein gene from several isolates obtained from different geographical regions, showed that the intragroup genetic variation among BRSV strains ranged between $88 \%$ and $100 \%$ [71]. The corresponding phylogenetic analysis revealed the presence of two main branches. Branch I was further subdivided into two groups, Ia and Ib, and then into five different lineages, each representing a geographic cluster. Thus, group Ia contained strains belonging to the antigenic subgroup A, whereas branch Ib consisted of strains of European origin belonging to the subgroup AB. Branch II, in contrast, grouped all viral strains classified as antigenic subgroup B. A third independent cluster included a set of Scandinavian strains that were not grouped with any of the aforementioned branches. A direct correlation was also observed between the positions along the phylogenetic tree and some of the strains and their antigenic patterns. Thus, this study can be considered to be the first research demonstrating that BRSV belongs to a single antigenic group with different genetic variants [71]. A subsequent study demonstrated that isolates of Danish origin formed three distinctive lineages within a separate cluster. These isolates were also closely related to the 220-69Bel strain, the prototype strain of the intermediate antigenic group [72]. Interestingly, viral isolates from the Czech Republic were closely related to the Danish strains isolated during the mid-1990s, a finding that was most likely due to animals being imported into the country, rather than due to virus evolution [73]. Moreover, a recently comparative analysis between Swedish and Danish isolates showed that the reduced sequence diversity among Swedish strains might be due to the relatively closed cattle population in Sweden as a consequence of the limited import of cows into the country [12]. In conjunction, these findings support the participation of import of cows in the increase of nucleotide sequence diversity of BRSV lineages in given regions.

A large-scale study addressing the global molecular epidemiology and evolution of BRSV included 54 European and North American isolates, in addition to previously reported sequences [50]. The study assessed the diversity of the N, F and G protein nucleotides and analyzed amino acid sequences, as well as their phylogenetic relationships. The average percentage of pairwise divergences was lowest for the $\mathrm{N}$ and $\mathrm{F}$ protein genes $(2 \%)$, in comparison to the $\mathrm{G}$ protein gene $(8 \%)$. In general, complete homology was observed among all of the BRSV proteins among animals within the same herd. A limited number of differences were detected within the $\mathrm{N}$ and $\mathrm{G}$ protein gene sequences among a few herds. This finding suggests that a single virus or a group of very closely related viruses would seem to infect predominantly a given herd at a given time [74]. Phylogenetic analysis, based on the G protein sequence, classified the isolates into six different groups (Figure 2A) [50]. The topology of the phylogeny was retained when the analysis of the $\mathrm{N}$ and $\mathrm{F}$ protein gene sequences was conducted; however, only five phylogenetic groups were observed for either protein (Figure 2B,C) [50]. As 
expected, the characteristic clustering of BRSV sequences, according to geographical origin and date of isolation, was observed, further supporting the theory of geographical and temporal clustering. Subgroup I consisted of old European strains isolated before 1976. Subgroup III included viruses exclusively from the United States. Subgroups II, IV, V, and VI were composed entirely of "younger" European isolates. Strains from northern Europe, Denmark, and Sweden were clustered in subgroup II, while those from the Netherlands, Belgium, and France were found in subgroups II, IV, V, and VI [75]. Interestingly, the study identified vaccine failure among animals harboring infections with BRSV groups V and VI, indicating that commercial vaccines performed poorly against infections by such viral groups. Moreover, continuous evolution of the BRSV N, G, and F proteins was also observed, which seemed to be correlated with the implementation of vaccination in different countries. Moreover, strong, positive selection was shown on the mucin-like region of the G protein and on particular sites of the $\mathrm{N}$ and $\mathrm{F}$ proteins. The analysis of French BRSV isolates included in this study also showed the presence of mutations located along the conserved central hydrophobic part of the ectodomain of the $\mathrm{G}$ protein, resulting in the loss of four Cys residues, two disulfide bridges and, consequently, a helix, which is critical for the three-dimensional structure of the G protein. These observations suggested the continuous modification of the highly conserved central region of the immunodominant $\mathrm{G}$ protein, thus highlighting the importance of considering BRSV evolution in the rational development of vaccines [50]. Overall, this work could be considered as the foundation of modern BRSV molecular epidemiology, it has also established the basis for subsequent studies looking at the molecular epidemiological patterns of BRSV infection in different geographical regions [37,56,76,77].

Importantly, BRSV intrahost population has been shown to exist as a complex mixture of viral variants, ambiguously referred as "quasispecies" [51]. Analyses of the BRSV G protein gene have demonstrated the spectrum of subpopulations co-existing in clinical isolates. Clonal analysis revealed the nucleotide heterogeneity along the G-coding region, exhibiting mutation frequencies ranging from $6.8 \times 10^{4}$ to $10.1 \times 10^{4}$ substitutions per nucleotide. These findings suggest that BRSV populations evolve as complex and dynamic mutant swarms, despite the virus's apparent genetic stability.

Molecular epidemiology approaches have shown the circulation of identical viruses among animals within the same herds, especially during the occurrence of outbreaks [74]. However, viral strains from recurrent outbreaks have varied significantly (up to 11\%), suggesting the circulation of different BRSV viral variants that can persistently infect calves within a herd. In consequence, and because of intrahost viral evolution, new, highly fit viruses became dominant and spread from a single, or a few, animals associated with each new outbreak. Alternatively, and based on the high level of diversity observed between outbreaks, this finding might suggest that BRSV outbreaks are the result of the introduction of new viral strains into the population [74]. Interestingly, the reduced exposure to new BRSV strains has been shown to limit the diversity of the circulating BRSV population [12]. Importantly, all aforesaid studies have focus on the nucleotide sequence of the gene coding for the $G$ protein. While the information obtained from the $G$ protein has been extremely valuable to understand the landscape and sequence space available to BRSV, the somehow limited sequence information provided by this region significantly handicaps our capacity to fully characterize viral strains. Future 
studies analyzing longer sections of the BRSV, and possibly the full-length of the viral genome, will likely provide a more accurate picture of the viral strains circulating worldwide.

Figure 2. Phylogenetic analysis of BRSV genomic regions. Maximum likelihood phylogenetic trees from the $\mathrm{G}(\mathbf{A}), \mathrm{F}(\mathbf{B})$ and $\mathrm{N}(\mathbf{C})$ proteins were generated using representative strains from each BRSV genotype [50].

A

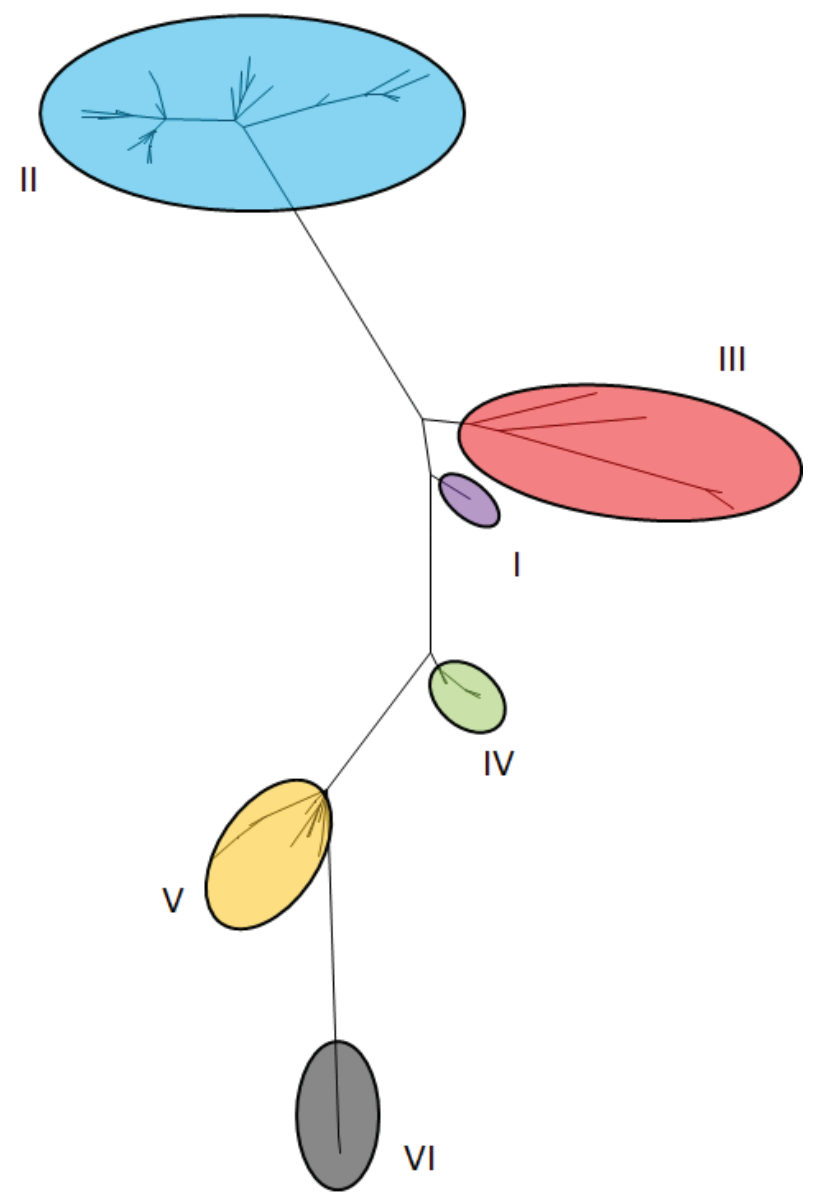


Figure 2. Cont.

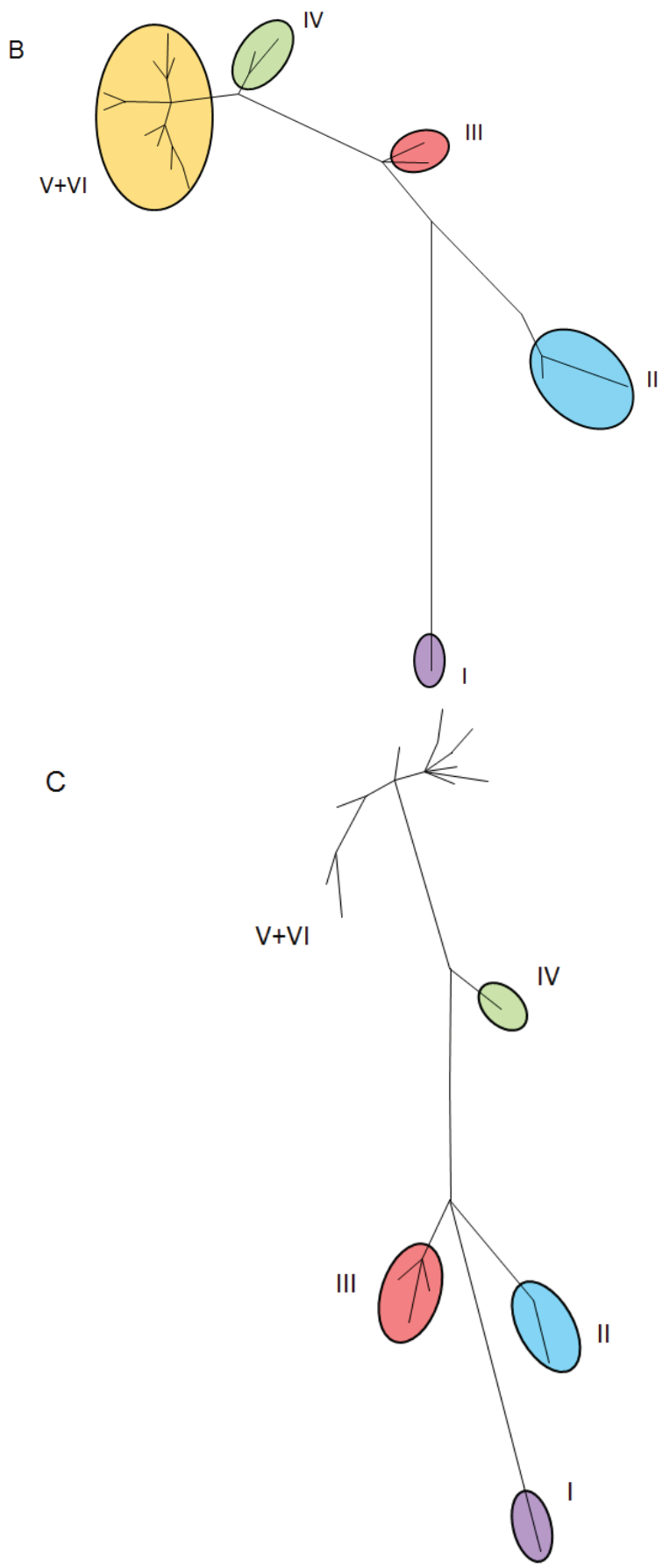


The global molecular epidemiology of BRSV has remained elusive. Only sparse reports addressing the distribution of BRSV groups are available in the literature. Consequently, the molecular characterization of BRSV isolates from representative regions of the world is still rather incomplete. A study conducted in Japan in the early 2000s reported the circulation of strains belonging to subgroup III. The isolates were further subdivided into two distinctive lineages [76]. Unfortunately, this study only accounted for a very limited number of isolates, which notably precluded us from fully understanding the molecular epidemiology of BRSV in the region. More recently, two Brazilian studies identified a handful of isolates belonging to BRSV group B [37,77]. Likewise, this information was limited, and the inference of the molecular epidemiology of BRSV in the country was not possible. Recently, a Swedish report was published describing the molecular epidemiology of BRSV from recent outbreaks (2007-2011) occurring among 30 different herds [12]. The report highlighted the circulation of BRSV strains belonging to subgroup II, for the most part, in the southern region of Sweden. A second study showed that isolates from England are related genetically to U.S. strains. [78]. Thus, both reports suggest that live cattle importation plays an important role in the global molecular epidemiology of BRSV.

In summary, the study of the molecular epidemiology of BRSV has evolved considerably over the past two decades. The information generated by the study of the molecular characterization, phylogenetics and evolution of BRSV strains have broadly advanced our understanding of the molecular mechanisms controlling virus transmission and disease spread. However, this field requires extensive research to unveil the means exploited by the virus to attain persistence in a given population. The establishment of molecular surveillance of BRSV in different geographical regions will likely improve the identification of outbreaks, resulting in the implementation of preventive measures aimed to control the disease. The advent of next-generation sequencing platforms on the eve of the "DNA-sequencing era" could also provide a unique opportunity for the discovery of the underlying processes responsible for viral replication and survival in the host.

\section{Concluding Remarks}

BRSV has been recognized as an important cause of respiratory disease in cattle for nearly four decades. The characteristic heterogeneity of the viral genome and its low fidelity in replication are some of the most important features that the virus exploits to assure its survival and persistence within the host. The instability of the viral particle usually leads to unsuccessful attempts to isolate viral strains in the laboratory from clinical specimens. Consequently, molecular approaches are rapidly becoming the gold standard for the correct identification and characterization of BRSV in clinical cases. As result, the field of the molecular epidemiology of BRSV has gained significant strength and has further enhanced our knowledge about BRSV distribution and transmission patterns worldwide. The arrival of new and more sophisticated molecular methods, including next-generation sequencing, will most likely help to unveil the genetic makeup of the circulating viral population in different geographical regions, as well as the mechanisms on which the virus relies for survival, persistence and transmission. 


\section{Acknowledgments}

This work was supported by the "Instituto de Ciencias y Tecnologías del Distrito Federal" (grant Icyt/248/2010).

\section{References}

1. Paccaud, M.F.; Jacquier, C. A respiratory syncytial virus of bovine origin. Arch. Gesamte Virusforsch. 1970, 30, 327-342.

2. Wellemans, G.; Leunen, J.; Luchsinger, E. Respiratory ailments of cattle: Isolation of a virus $(220 / 69)$ with serologic resemblance to the human respiratory syncytial virus. Ann. Med. Vet. 1970, 114, 89-93.

3. Smith, R.A. Effects of feedlot disease on economics, production and carcass value. Am. Assoc. Bov. Pract. Proc. 2000, 33, 125-128.

4. Lamb, R.A.; Parks, G.D. Paramyxoviridae: The Viruses and their Replication. In Fields Virology, 5th ed.; Knipe, D.M., Howley, P.M., Eds.; Lippincott Williams \& Williams: Philadelphia, PA, USA, 2007; Volume 2, pp. 1449-1496.

5. Van der Poel, W.H.; Brand, A.; Kramps, J.A.; van Oirschot, J.T. Respiratory syncytial virus infections in human beings and in cattle. J. Infect. 1994, 29, 215-228.

6. Ames, T.R. The epidemiology of BRSV infection. Vet. Med. 1993, 88, 881-885.

7. Masot, A.J.; Kelling, C.L.; Lopez, O.; Sur, J.H.; Redondo, E. In situ hybridization detection of bovine respiratory syncytial virus in the lung of experimentally infected lambs. Vet. Pathol. 2000, 37, 618-625.

8. Ohlson, A.; Emanuelson, U.; Traven, M.; Alenius, S. The relationship between antibody status to bovine corona virus and bovine respiratory syncytial virus and disease incidence, reproduction and herd characteristics in dairy herds. Acta Vet. Scand. 2010, 52, 37.

9. Ohlson, A.; Heuer, C.; Lockhart, C.; Traven, M.; Emanuelson, U.; Alenius, S. Risk factors for seropositivity to bovine coronavirus and bovine respiratory syncytial virus in dairy herds. Vet. Rec. 2010, 167, 201-66.

10. Saa, L.R.; Perea, A.; Jara, D.V.; Arenas, A.J.; Garcia-Bocanegra, I.; Borge, C.; Carbonero, A. Prevalence of and risk factors for bovine respiratory syncytial virus (BRSV) infection in non-vaccinated dairy and dual-purpose cattle herds in Ecuador. Trop. Anim. Health Prod. 2012, $44,1423-1427$.

11. Valarcher, J.F.; Taylor, G. Bovine respiratory syncytial virus infection. Vet. Res. 2007, 38, 153-180.

12. Bidokhti, M.R.; Traven, M.; Ohlson, A.; Zarnegar, B.; Baule, C.; Belak, S.; Alenius, S.; Liu, L. Phylogenetic analysis of bovine respiratory syncytial viruses from recent outbreaks in feedlot and dairy cattle herds. Arch. Virol. 2012, 157, 601-607.

13. Elvander, M. Severe respiratory disease in dairy cows caused by infection with bovine respiratory syncytial virus. Vet. Rec. 1996, 138, 101-105. 
14. Woldemeskel, M.; Kebede, E.; Yigezu, L.; Potgieter, L.N. Prevalence of bovine respiratory syncytial virus (BRSV) and bovine herpesvirus-4 (BHV-4) in cattle from Ethiopia. Dtsch. Tierarztliche Wochenschr. 2000, 107, 464-466.

15. Collins, J.K.; Teegarden, R.M.; MacVean, D.W.; Salman; Smith, G.H.; Frank, G.R. Prevalence and specificity of antibodies to bovine respiratory syncytial virus in sera from feedlot and range cattle. Am. J. Vet. Res. 1988, 49, 1316-1319.

16. Elazhary, M.A.; Silim, A.; Dea, S. Prevalence of antibodies to bovine respiratory syncytial virus, bovine viral diarrhea virus, bovine herpesvirus-1, and bovine parainfluenza-3 virus in sheep and goats in Quebec. Am. J. Vet. Res. 1984, 45, 1660-1662.

17. Elazhary, M.A.; Roy, R.S.; Champlin, R.; Higgins, R.; Marsolais, G. Bovine respiratory syncytial virus in Quebec: Antibody prevalence and disease outbreak. Can. J. Comp. Med. 1980, 44, 299-303.

18. Baker, J.C.; Ames, T.R.; Markham, R.J. Seroepizootiologic study of bovine respiratory syncytial virus in a dairy herd. Am. J. Vet. Res. 1986, 47, 240-245.

19. Kimman, T.G.; Zimmer, G.M.; Westenbrink, F.; Mars, J.; van Leeuwen, E. Epidemiological study of bovine respiratory syncytial virus infections in calves: Influence of maternal antibodies on the outcome of disease. Vet. Rec. 1988, 123, 104-109.

20. Hagglund, S.; Svensson, C.; Emanuelson, U.; Valarcher, J.F.; Alenius, S. Dynamics of virus infections involved in the bovine respiratory disease complex in Swedish dairy herds. Vet. J. 2006, 172, 320-328.

21. Raaperi, K.; Bougeard, S.; Aleksejev, A.; Orro, T.; Viltrop, A. Association of herd BRSV and BHV-1 seroprevalence with respiratory disease and reproductive performance in adult dairy cattle. Acta Vet. Scand. 2012, 54, 4.

22. Bidokhti, M.R.; Traven, M.; Fall, N.; Emanuelson, U.; Alenius, S. Reduced likelihood of bovine coronavirus and bovine respiratory syncytial virus infection on organic compared to conventional dairy farms. Vet. J. 2009, 182, 436-440.

23. Stott, E.J.; Thomas, L.H.; Collins, A.P.; Crouch, S.; Jebbett, J.; Smith, G.S.; Luther, P.D.; Caswell, R. A survey of virus infections of the respiratory tract of cattle and their association with disease. J. Hyg. (Lond.) 1980, 85, 257-270.

24. Brodersen, B.W. Bovine respiratory syncytial virus. Vet. Clin. North Am. Food Anim. Pract. 2010, 26, 323-333.

25. Baker, J.C.; Werdin, R.E.; Ames, T.R.; Markham, R.J.; Larson, V.L. Study on the etiologic role of bovine respiratory syncytial virus in pneumonia of dairy calves. J. Am. Vet. Med. Assoc. 1986, 189, 66-70.

26. De Jong, M.C.; van der Poel, W.H.; Kramps, J.A.; Brand, A.; van Oirschot, J.T. Quantitative investigation of population persistence and recurrent outbreaks of bovine respiratory syncytial virus on dairy farms. Am. J. Vet. Res. 1996, 57, 628-633.

27. Rossi, C.R.; Kiesel, G.K. Serological evidence for the association of bovine respiratory syncytial virus with respiratory tract disease in Alabama cattle. Infect. Immun. 1974, 10, 293-298. 
28. Smith, M.H.; Frey, M.L.; Dierks, R.E. Isolation, characterization, and pathogenicity studies of a bovine respiratory syncytial virus. Arch. Virol. 1975, 47, 237-247.

29. Baker, J.C.; Ames, T.R.; Markham, R.J. Serologic studies of bovine respiratory syncytial virus in Minnesota cattle. Am. J. Vet. Res. 1985, 46, 891-892.

30. Lehmkuhl, H.D.; Gough, P.M. Investigation of causative agents of bovine respiratory tract disease in a beef cow-calf herd with an early weaning program. Am. J. Vet. Res. 1977, 38, 1717-1720.

31. Grubbs, S.T.; Kania, S.A.; Potgieter, L.N. Prevalence of ovine and bovine respiratory syncytial virus infections in cattle determined with a synthetic peptide-based immunoassay. J. Vet. Diagn. Investig. 2001, 13, 128-132.

32. Fulton, R.W.; Purdy, C.W.; Confer, A.W.; Saliki, J.T.; Loan, R.W.; Briggs, R.E.; Burge, L.J. Bovine viral diarrhea viral infections in feeder calves with respiratory disease: Interactions with Pasteurella spp., parainfluenza-3 virus, and bovine respiratory syncytial virus. Can. J. Vet. Res. 2000, 64, 151-159.

33. Martin, S.W.; Bateman, K.G.; Shewen, P.E.; Rosendal, S.; Bohac, J.E. The frequency, distribution and effects of antibodies, to seven putative respiratory pathogens, on respiratory disease and weight gain in feedlot calves in Ontario. Can. J. Vet. Res. 1989, 53, 355-362.

34. Gagea, M.I.; Bateman, K.G.; van Dreumel, T.; McEwen, B.J.; Carman, S.; Archambault, M.; Shanahan, R.A.; Caswell, J.L. Diseases and pathogens associated with mortality in Ontario beef feedlots. J. Vet. Diagn. Investig. 2006, 18, 18-28.

35. Figueroa-Chavez, D.; Segura-Correa, J.C.; Garcia-Marquez, L.J.; Pescador-Rubio, A.; Valdivia-Flores, A.G. Detection of antibodies and risk factors for infection with bovine respiratory syncytial virus and parainfluenza virus 3 in dual-purpose farms in Colima, Mexico. Trop. Anim. Health Prod. 2012, 44, 1417-1421.

36. Solis-Calderon, J.J.; Segura-Correa, J.C.; Aguilar-Romero, F.; Segura-Correa, V.M. Detection of antibodies and risk factors for infection with bovine respiratory syncytial virus and parainfluenza virus-3 in beef cattle of Yucatan, Mexico. Prev. Vet. Med. 2007, 82, 102-110.

37. Almeida, R.S.; Domingues, H.G.; Spilki, F.R.; Larsen, L.E.; Hagglund, S.; Belak, S.; Arns, C.W. Circulation of bovine respiratory syncytial virus in Brazil. Vet. Rec. 2006, 158, 632-634.

38. Arns, C.W.; Campalans, J.; Costa, S.C.; Domingues, H.G.; D’Arce, R.C.; Almeida, R.S.; Coswig, L.T. Characterization of bovine respiratory syncytial virus isolated in Brazil. Braz. J. Med. Biol. Res. 2003, 36, 213-218.

39. Jacobs, J.W.; Edington, N. Isolation of respiratory syncytial virus from cattle in Britain. Vet. Rec. 1971, 88, 694.

40. Beaudeau, F.; Bjorkman, C.; Alenius, S.; Frossling, J. Spatial patterns of bovine corona virus and bovine respiratory syncytial virus in the Swedish beef cattle population. Acta Vet. Scand. 2010, 52,33 .

41. Uttenthal, A.; Larsen, L.E.; Philipsen, J.S.; Tjornehoj, K.; Viuff, B.; Nielsen, K.H.; Nielsen, T.K. Antibody dynamics in BRSV-infected Danish dairy herds as determined by isotype-specific immunoglobulins. Vet. Microbiol. 2000, 76, 329-341. 
42. Pardon, B.; de Bleecker, K.; Dewulf, J.; Callens, J.; Boyen, F.; Catry, B.; Deprez, P. Prevalence of respiratory pathogens in diseased, non-vaccinated, routinely medicated veal calves. Vet. Rec. 2011, 169, 278.

43. Hotchkiss, E.J.; Dagleish, M.P.; Willoughby, K.; McKendrick, I.J.; Finlayson, J.; Zadoks, R.N.; Newsome, E.; Brulisauer, F.; Gunn, G.J.; Hodgson, J.C. Prevalence of Pasteurella multocida and other respiratory pathogens in the nasal tract of Scottish calves. Vet. Rec. 2010, 167, 555-560.

44. Paton, D.J.; Christiansen, K.H.; Alenius, S.; Cranwell, M.P.; Pritchard, G.C.; Drew, T.W. Prevalence of antibodies to bovine virus diarrhoea virus and other viruses in bulk tank milk in England and Wales. Vet. Rec. 1998, 142, 385-391.

45. Raaperi, K.; Bougeard, S.; Aleksejev, A.; Orro, T.; Viltrop, A. Association of herd BHV-1 seroprevalence with respiratory disease in youngstock in Estonian dairy cattle. Res. Vet. Sci. 2012, 93, 641-648.

46. Van Vuuren, M. Serological studies of bovine respiratory syncytial virus in feedlot cattle in South Africa. J. S. Afr. Vet. Assoc. 1990, 61, 168-169.

47. Mahin, L.; Wellemans, G.; Shimi, A. Prevalence of antibodies to bovid herpesvirus 1 (IBR-IPV), bovine virus diarrhoea, bovine respiratory syncytial parainfluenza 3, adeno A and adeno B viruses in indigenous and imported Moroccan cattle. Ann. Rech. Vet. 1985, 16, 279-283.

48. Mahin, L.; Wellemans, G. Serological evidence for the intervention of bovine respiratory syncytial virus in a respiratory disease outbreak in Moroccan cattle. Zentralblatt Veterinarmed. B 1982, 29, 76-79.

49. Yesilbag, K.; Gungor, B. Seroprevalence of bovine respiratory viruses in North-Western Turkey. Trop. Anim. Health Prod. 2008, 40, 55-60.

50. Valarcher, J.F.; Schelcher, F.; Bourhy, H. Evolution of bovine respiratory syncytial virus. J. Virol. 2000, 74, 10714-10728.

51. Deplanche, M.; Lemaire, M.; Mirandette, C.; Bonnet, M.; Schelcher, F.; Meyer, G. In vivo evidence for quasispecies distributions in the bovine respiratory syncytial virus genome. J. Gen. Virol. 2007, 88, 1260-1265.

52. Lam, T.T.; Hon, C.C.; Tang, J.W. Use of phylogenetics in the molecular epidemiology and evolutionary studies of viral infections. Crit. Rev. Clin. Lab. Sci. 2010, 47, 5-49.

53. Khudyakov, Y. Coevolution and HBV drug resistance. Antivir. Ther. 2010, 15, 505-515.

54. Spilki, F.R.; Almeida, R.S.; Domingues, H.G.; D’Arce, R.C.F.; Ferreira, H.L.; Campalans, J.; Costa, S.C.B.; Arns, C.W. Phylogenetic relationships of Brazilian bovine respiratory syncytial virus isolates and molecular homology modeling of attachment glycoprotein. Virus Res. 2006, 116, 30-37.

55. Samal, S.K.; Zamora, M.; McPhillips, T.H.; Mohanty, S.B. Molecular cloning and sequence analysis of bovine respiratory syncytial virus mRNA encoding the major nucleocapsid protein. Virology 1991, 180, 453-456.

56. Kovarcik, K.; Valentova, V. Bovine respiratory syncytial virus strains currently circulating in the Czech Republic are most closely related to Danish strains from 1995. Acta Virol. 2004, 48, 57-62. 
57. Lerch, R.A.; Stott, E.J.; Wertz, G.W. Characterization of bovine respiratory syncytial virus proteins and mRNAs and generation of cDNA clones to the viral mRNAs. J. Virol. 1989, 63, 833-840.

58. Baker, J.C.; Wilson, E.G.; McKay, G.L.; Stanek, R.J.; Underwood, W.J.; Velicer, L.F.; Mufson, M.A. Identification of subgroups of bovine respiratory syncytial virus. J. Clin. Microbiol. 1992, 30, $1120-1126$.

59. Mallipeddi, S.K.; Samal, S.K. Structural difference in the fusion protein among strains of bovine respiratory syncytial virus. Vet. Microbiol. 1993, 36, 359-367.

60. Prozzi, D.; Walravens, K.; Langedijk, J.P.; Daus, F.; Kramps, J.A.; Letesson, J.J. Antigenic and molecular analyses of the variability of bovine respiratory syncytial virus G glycoprotein. J. Gen. Virol. 1997, 78, 359-366.

61. Furze, J.; Wertz, G.; Lerch, R.; Taylor, G. Antigenic heterogeneity of the attachment protein of bovine respiratory syncytial virus. J. Gen. Virol. 1994, 75, 363-370.

62. Meyer, G.; Deplanche, M.; Schelcher, F. Human and bovine respiratory syncytial virus vaccine research and development. Comp. Immunol. Microbiol. Infect. Dis. 2008, 31, 191-225.

63. Lerch, R.A.; Anderson, K.; Wertz, G.W. Nucleotide sequence analysis and expression from recombinant vectors demonstrate that the attachment protein $\mathrm{G}$ of bovine respiratory syncytial virus is distinct from that of human respiratory syncytial virus. J. Virol. 1990, 64, 5559-5569.

64. Walravens, K.; Kettmann, R.; Collard, A.; Coppe, P.; Burny, A. Sequence comparison between the fusion protein of human and bovine respiratory syncytial viruses. J. Gen. Virol. 1990, 71, 3009-3014.

65. Samal, S.K.; Zamora, M. Nucleotide sequence analysis of a matrix and small hydrophobic protein dicistronic mRNA of bovine respiratory syncytial virus demonstrates extensive sequence divergence of the small hydrophobic protein from that of human respiratory syncytial virus. J. Gen. Virol. 1991, 72, 1715-1720.

66. Zamora, M.; Samal, S.K. Sequence analysis of M2 mRNA of bovine respiratory syncytial virus obtained from an F-M2 dicistronic mRNA suggests structural homology with that of human respiratory syncytial virus. J. Gen. Virol. 1992, 73, 737-741.

67. Mallipeddi, S.K.; Samal, S.K. Sequence comparison between the phosphoprotein mRNAs of human and bovine respiratory syncytial viruses identifies a divergent domain in the predicted protein. J. Gen. Virol. 1992, 73, 2441-2444.

68. Mallipeddi, S.K.; Samal, S.K. Sequence variability of the glycoprotein gene of bovine respiratory syncytial virus. J. Gen. Virol. 1993, 74, 2001-2004.

69. Langedijk, J.P.; Meloen, R.H.; Taylor, G.; Furze, J.M.; van Oirschot, J.T. Antigenic structure of the central conserved region of protein $\mathrm{G}$ of bovine respiratory syncytial virus. J. Virol. 1997, 71, 4055-4061.

70. Stine, L.C.; Hoppe, D.K.; Kelling, C.L. Sequence conservation in the attachment glycoprotein and antigenic diversity among bovine respiratory syncytial virus isolates. Vet. Microbiol. 1997, 54, 201-221. 
71. Elvander, M.; Vilcek, S.; Baule, C.; Uttenthal, A.; Ballagi-Pordany, A.; Belak, S. Genetic and antigenic analysis of the $\mathrm{G}$ attachment protein of bovine respiratory syncytial virus strains. J. Gen. Virol. 1998, 79, 2939-2946.

72. Larsen, L.E.; Uttenthal, A.; Arctander, P.; Tjornehoj, K.; Viuff, B.; Rontved, C.; Ronsholt, L.; Alexandersen, S.; Blixenkrone-Moller, M. Serological and genetic characterisation of bovine respiratory syncytial virus (BRSV) indicates that Danish isolates belong to the intermediate subgroup: No evidence of a selective effect on the variability of $G$ protein nucleotide sequence by prior cell culture adaption and passages in cell culture or calves. Vet. Microbiol. 1998, 62, 265-279.

73. Kovarcik, K.; Valentova, V. Bovine respiratory syncytial virus strains currently circulating in the Czech Republic are most closely related to Danish strains from 1995. Acta Virol. 2004, 48, 57-62.

74. Larsen, L.E.; Tjornehoj, K.; Viuff, B. Extensive sequence divergence among bovine respiratory syncytial viruses isolated during recurrent outbreaks in closed herds. J. Clin. Microbiol. 2000, 38, 4222-4227.

75. Valentova, V.; Antonis, A.F.; Kovarcik, K. Restriction enzyme analysis of RT-PCR amplicons as a rapid method for detection of genetic diversity among bovine respiratory syncytial virus isolates. Vet. Microbiol. 2005, 108, 1-12.

76. Yaegashi, G.; Seimiya, Y.M.; Seki, Y.; Tsunemitsu, H. Genetic and antigenic analyses of bovine respiratory syncytial virus detected in Japan. J. Vet. Med. Sci. 2005, 67, 145-150.

77. Spilki, F.R.; Almeida, R.S.; Domingues, H.G.; D’Arce, R.C.; Ferreira, H.L.; Campalans, J.; Costa, S.C.; Arns, C.W. Phylogenetic relationships of Brazilian bovine respiratory syncytial virus isolates and molecular homology modeling of attachment glycoprotein. Virus Res. 2006, 116, 30-37.

78. Nettleton, P.F.; Gilray, J.A.; Caldow, G.; Gidlow, J.R.; Durkovic, B.; Vilcek, S. Recent isolates of bovine respiratory syncytial virus from Britain are more closely related to isolates from USA than to earlier British and current mainland European isolates. J. Vet. Med. B Infect. Dis. Vet. Public Health 2003, 50, 196-199. 
Reprinted from Viruses. Cite as: Adamson, P.; Thammawat, S.; Muchondo, G.; Sadlon, T.; Gordon, D. Diversity in Glycosaminoglycan Binding Amongst hMPV G Protein Lineages. Viruses 2012, 4, 3785 3803.

Article

\title{
Diversity in Glycosaminoglycan Binding Amongst hMPV G Protein Lineages
}

\author{
Penelope Adamson ${ }^{1,2, *}$, Sutthiwan Thammawat ${ }^{1}$, Gamaliel Muchondo ${ }^{1}$, Tania Sadlon ${ }^{1,2}$ and \\ David Gordon ${ }^{1,2}$
}

1 Department of Microbiology and Infectious Diseases, Flinders University, Flinders Medical Centre, Bedford Park, SA 5042, Australia; E-Mails: ant3714@hotmail.com (S.T.); gamaliel.muchondo@health.sa.gov.au (G.M.); tania.sadlon@health.sa.gov.au (T.S.); d.gordon@flinders.edu.au (D.G.)

2 Department of Microbiology and Infectious Diseases, SA Pathology, Flinders Medical Centre, Bedford Park, SA 5042, Australia

* Author to whom correspondence should be addressed;

E-Mail: penelope.adamson@health.sa.gov.au; Tel.: +61-8-8204-4284; Fax: +61-8-8204-4733.

Received: 31 October 2012; in revised form: 10 December 2012 / Accepted: 10 December 2012 /

Published: 14 December 2012

\begin{abstract}
We have previously shown that hMPV G protein (B2 lineage) interacts with cellular glycosaminoglycans (GAGs). In this study we examined subtypes A1, A2 and B1 for this interaction. GAG-dependent infectivity of available hMPV strains was demonstrated using GAG-deficient cells and heparin competition. We expressed the G protein ectodomains from all strains and analysed these by heparin affinity chromatography. In contrast to the B2 lineage, neither the A2 or B1 G proteins bound to heparin. Sequence analysis of these strains indicated that although there was some homology with the B2 heparin-binding domains, there were less positively charged residues, providing a likely explanation for the lack of binding. Although sequence analysis did not demonstrate well defined positively charged domains in G protein of the A1 strain, this protein was able to bind heparin, albeit with a lower affinity than G protein of the B2 strain. These results indicate diversity in GAG interactions between G proteins of different lineages and suggest that the GAG-dependency of all strains may be mediated by interaction with an alternative surface protein, most probably the conserved fusion (F)
\end{abstract}


protein. Analysis of both native and recombinant $\mathrm{F}$ protein confirmed that $\mathrm{F}$ protein binds heparin, supporting this conclusion.

Keywords: human metapneumovirus (hMPV); Glycosaminoglycan (GAG); G protein; F protein; infectivity

\section{Introduction}

Human metapneumovirus (hMPV) is responsible for causing serious respiratory illness, most commonly in infants and young children, but also in the elderly and immunocompromised patients [1-5]. hMPV is a member of the genus Metapneumovirus within the family Paramyxoviridae. Avian pnuemovirus is the only other member of this genus [5]. Respiratory syncytial virus (RSV), a member of the genus Pneumovirus, also within the family Paramyxoviridae, is the most closely related human pathogen to hMPV and as such they share many of the same symptoms [6-9]. These range from mild upper respiratory tract disease to severe lower tract diseases such as bronchiolitis and pneumonia [5] and together, hMPV and RSV, are responsible for at least $50 \%$ of all respiratory viral infections requiring hospitalisation in children $[10,11]$.

hMPV is an enveloped, single stranded, negative-sense RNA virus which contains 3 envelope glycoproteins, the fusion $(\mathrm{F})$, attachment $(\mathrm{G})$ and the small hydrophobic $(\mathrm{SH})$ protein. It is the organisation of the genome and the lack of non structural genes which separates hMPV from other paramyxoviruses, such as RSV [5,8]. Phylogenetic analysis of the nucleotide sequence of several hMPV genes has shown that hMPV is divided into two major groups, A and B, both of which can be further divided into two minor subgroups, 1 and 2 [12-16]. hMPV resembles other members of the Paramyxoviridae family genetically and morphologically, as determined by electron microscopy [17].

Whilst the fusion protein of hMPV is highly conserved, immunogenic and induces protective antibodies, the other surface glycoproteins, G and SH, unlike most other Paramyxoviridae, have been shown to be only weakly or negligibly immunogenic [18-22]. The G protein of hMPV is a type II membrane protein consisting of extracellular, transmembrane and intracellular domains [8] and is highly glycosylated [12,23]. It is highly variable, particularly the extracellular domain [13]. This diversity along with its extensive glycosylation probably aids in its evasion of the immune system [12]. The role of the G protein has not been fully elucidated, however previous studies indicate that it likely plays a role in viral attachment [24] and replication [25].

Glycosaminoglycans (GAGs) are linear polysaccharides which are comprised of repeating disaccharide units. The repeat units of GAGs consist of an amino sugar (N-acetylglucosamine or $\mathrm{N}$-acetylgalactosamine) and uronic acid (glucuronic or iduronic acid) or galactose which are variably sulphated, except hyaluronic acid which lacks sulphate groups [26]. They are covalently attached to core proteins to form proteoglycans which are found in tissue, the extracellular matrix (ECM) and on the inside and the surface of most cell types [26]. 
A number of viruses, including other paramyxoviruses, have been shown to interact with cell surface GAGs to facilitate cellular attachment and subsequent entry into cells [27-30]. Past studies of RSV and our previous study of hMPV have shown that infection is markedly or completely inhibited by the presence of soluble GAGs such as heparin, by removing GAGs from the surface of cells enzymatically or with the use of GAG deficient cells [24,31,32].

We have shown previously that attachment of hMPV to cells may be mediated by a G protein-GAG interaction. This initial work was carried out using G protein of the B2 lineage. Due to the high variability of $\mathrm{G}$ protein between strains we investigated if this is consistent for all hMPV strains. Using recombinantly expressed $\mathrm{G}$ protein we demonstrated that, other that the B2 strain, only the A1 strain binds to GAGs. By truncating the G proteins of the A1 and B2 subtypes, we have further characterised the functional domains within each protein involved in these interactions. Furthermore, using hMPV infected cell lysates and recombinant $\mathrm{F}$ protein, we show binding of the F protein to GAGs suggesting that this protein is also involved in virus attachment to the cell surface, and providing an explanation for the GAG-dependency of all hMPV strains.

\section{Results and Discussion}

\subsection{Susceptibility of hMPV Primary Isolates to Inhibition of Infection by Heparin}

To date our investigations have focused on G protein from the hMPV B2 strain. To determine if the infectivity of other strains is also GAG-dependent, we incubated primary isolates of hMPV A2, B1 and B2 strains with and without heparin before inoculation of LLC-MK2 cells. Infectivity was determined using quantitative real time PCR. Primary isolates were used to preclude the possibility that heparin binding was an adaptation of the virus during cell culture. hMPV A1 strain is not represented in these experiments as a primary isolate could not be obtained during the specimen collection period. Infection with all available hMPV strains was markedly or completely inhibited by heparin pre-incubation (Table 1).

Table 1. Susceptibility of hMPV primary isolates to inhibition of infection by heparin ${ }^{1}$.

\begin{tabular}{cccc}
\hline \multirow{2}{*}{ Isolate } & \multirow{2}{*}{ Subtype } & \multicolumn{2}{c}{ Ct value $^{2}$} \\
\cline { 3 - 4 } & & Heparin Pre-treated & Untreated virus \\
\hline V47041 & B2 & Negative & 24.1 \\
V52283 & A2 & 30.2 & 16.6 \\
V50569 & B1 & Negative & 13.1 \\
\hline
\end{tabular}

${ }^{1}$ LLC-MK2 cells were inoculated with primary isolates of hMPV, \pm 50 IU heparin pre-treatment. Infectivity was determined by a quantitative real-time RT-PCR for nucleoprotein gene. ${ }^{2}$ Results are represented as a cycle threshold $(\mathrm{Ct})$ value. Higher or negative $\mathrm{Ct}$ values after heparin pretreatment of the virus indicate reduced viral infectivity. 


\subsection{GAG-Deficient CHO Cells Are Resistant to hMPV Infection}

As soluble heparin was able to reduce or completely inhibit infection of LLC-MK2 cells, we examined the ability of the 3 primary isolates to infect $\mathrm{CHO}-\mathrm{K} 1$ and $\mathrm{CHO}-$ pgsA 745 cells. CHO-pgsA 745 cells lack xylosyltransferase activity and therefore are deficient in cellular GAGs [33]. The CHO-K1 and CHO-pgsA cells were incubated for 2 weeks with nasopharyngeal aspirates positive for hMPV by PCR. As the viral titres from $\mathrm{CHO}$ cultures were low, the wild type and mutant CHO-K1 cell supernatants were tested for infectious hMPV by subsequent incubation with LLC-MK2 cells followed by cell ELISA. Infectivity of all three strains, hMPV A2, B1 and B2, was dependent on GAGs as GAG deficient CHO-K1 cells had negligible evidence of infection (Figure 1).

Figure 1. Primary isolates of hMPV utilise GAGs to mediate infection. CHO-K1 and CHO-pgsA 745 cells were inoculated with hMPV PCR positive nasopharyngeal aspirates. LLC-MK2 cells were inoculated with the CHO cell supernatants and infectivity determined by cell ELISA using a hMPV matrix protein $\mathrm{mAb}$. Data represent mean values $\pm \mathrm{SD}$ of triplicate wells.

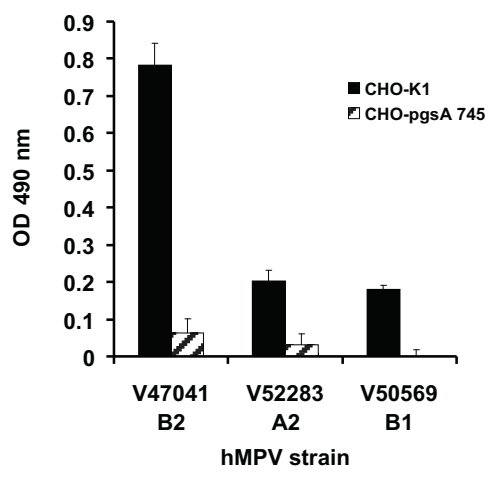

\subsection{Binding of hMPV G Glycoprotein to Immobilised Heparin}

Previous studies carried out in our laboratory demonstrated the binding of G protein of hMPV B2 strain to cellular GAGs [24]. To determine if this is consistent for all strains of hMPV, we expressed the extracellular domain of the G protein for the A1, A2 and B1 strains in the yeast Pichia pastoris $\mathrm{X} 33$. The recombinant $\mathrm{B} 1 \mathrm{G}$ protein migrated at an apparent molecular weight of $60 \mathrm{kDa}$ which is comparable to the recombinant B2 ectodomain, however the apparent molecular weights for the A1 and $\mathrm{A} 2$ protein migration were $100 \mathrm{kDa}$ and $75 \mathrm{kDa}$, respectively (Figure 2). The purified recombinant proteins were applied to heparin agarose columns and after extensive washing were eluted with a stepwise salt gradient. The recombinant G ectodomains of hMPV strains B2 and A1 bound to the heparin column while strains A2 and B1 did not (Figure 2). The recombinant hMPV B2 G protein appears to have a higher affinity for heparin than the recombinant hMPV A1 G protein as it requires a higher salt concentration for elution. 
Figure 2. Heparin agarose affinity chromatography of recombinant $\mathrm{G}$ ectodomain for A1, A2, B1 and B2 hMPV strains. The start (S), flow through (FT), final wash (W) and salt elution fractions were analyzed by $10 \%$ SDS-PAGE under reducing conditions and western blot analysis using anti-c-Myc monoclonal antibody. A1, A2, B1 and B2 indicate the strain type.

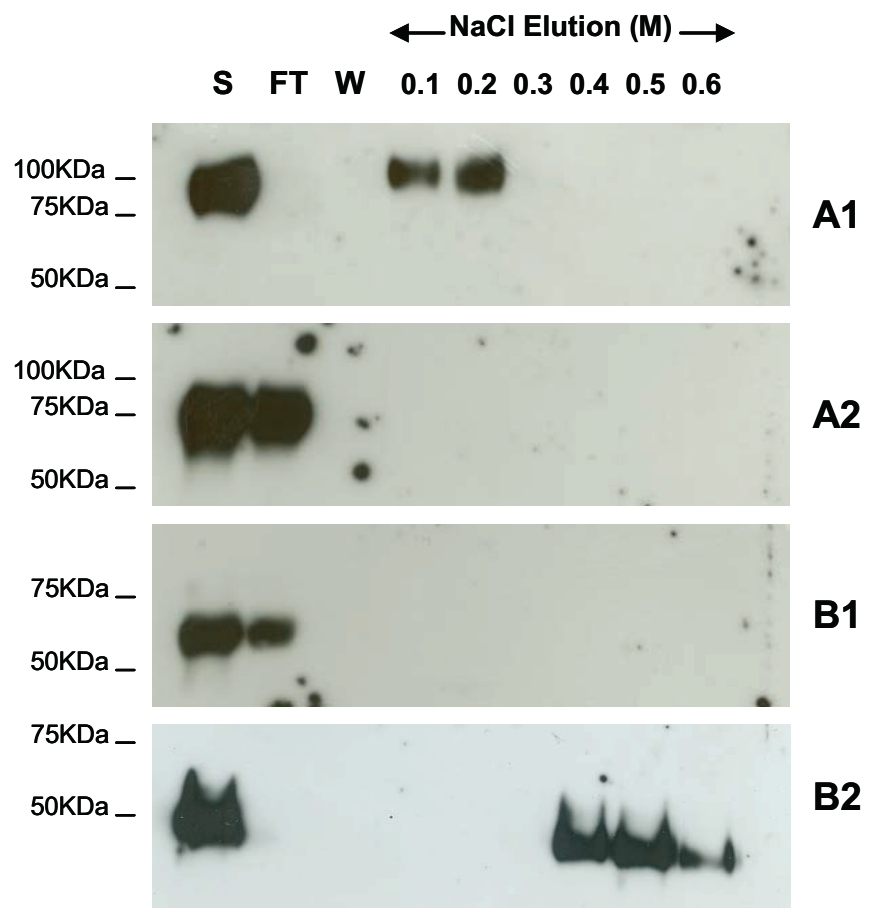

\subsection{Functional Domains in hMPV G A1 Protein Involved in GAG Interactions}

We have previously identified 2 adjacent regions of highly charged amino acids within the extracellular region of the B2 G protein which are important for heparin binding [24]. These domains are less well defined in other hMPV strains (Figure 3) and certainly appear to be lacking in the A1 and B1 strains. Despite this, the A1 strain G protein still interacts with heparin, perhaps due to the high number of positively charged residues adjacent to this region.

To identify the regions of A1 strain G protein involved in binding to heparin, we constructed 8 fragments of the hMPV G A1 ectodomain (Figure 4a), particularly targeting the region previously shown to be important for binding in hMPV G B2 strain (Figure 4b). These recombinant truncations migrated at sizes on SDS PAGE which varied depending on the length of the hMPV G (A1) fragment expressed. The purified hMPV G A1 fragments were applied to a heparin agarose column and following extensive washing were eluted with a stepwise salt gradient. hMPV-G A1 F3 and F4, but none of the other fragments, bound to heparin agarose (Figure 5, some data not shown). hMPV-G A1 
F4 (residues 93-142 of the extracellular domain) is the smallest fragment which binds heparin. A smaller fragment, hMPV-G A1 F6 (residues 108-142), was unable to bind heparin, which indicates that residues 93-108 are crucial for the interaction of hMPV-G A1 F4 with heparin. However, since the non-binding hMPV-G A1 F2 (residues 1-115) also incorporates residues 93-108, there must be additional residues required for the heparin-G protein interaction between residues 115 and 142 . These results indicate that the region of GAG binding in hMPV G A1 is similar to that identified in hMPV G B2 despite the fact that there are no well defined positively charged clusters in this protein.

Figure 3. Comparison of the predicted amino acid sequence for representatives of each strain of hMPV G protein (residues 98-136/137/142 of the extracellular domain). Strains are shown in blue and number of positively charged residues (shown in red in the sequence) is indicated in green at the end of each sequence. The yellow highlights in the B2 sequence indicate the previously identified heparin binding domains [24].

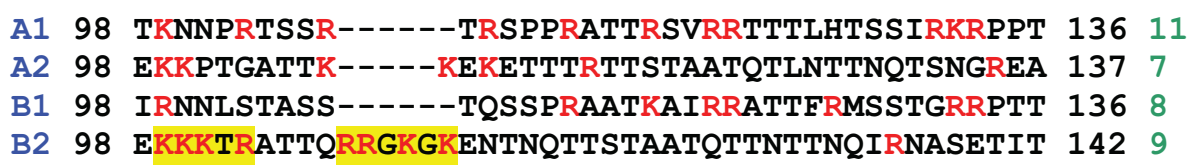

Figure 4. A schematic diagram of recombinant hMPV G protein from (a) the A1 strain and the fragments produced and (b) the B2 strain. hMPV-G A1 F1, F2, F3, F4, F5, F6, F7 and F8 indicate the 8 fragments of hMPV G A1 strain that were engineered. The sequence of the smallest fragment that binds to heparin (hMPV-G A1 F4) is shown with the positively charged residues in red. The red boxes represent the clusters of positively charged amino acids that are considered potential heparin binding sites.

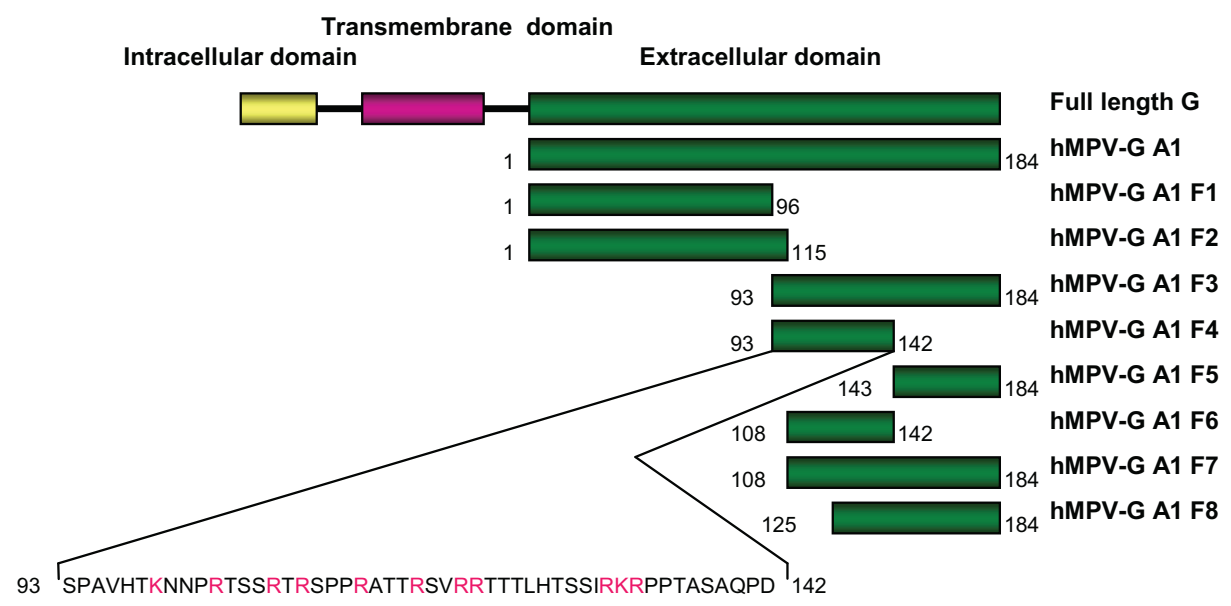

(a) 
Figure 4. Cont.

Intra-cellular domain Transmembrane domain Extracellular domain
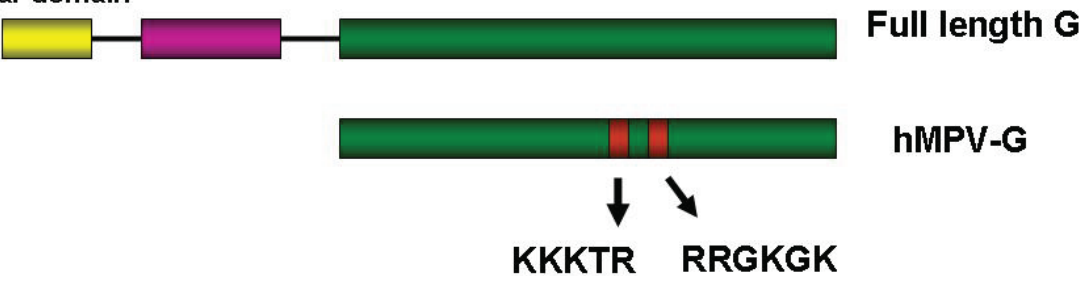

hMPV-G

(b)

Figure 5. Heparin agarose affinity chromatography of recombinant F2, F3, F4, and F6 fragments of the hMPV G A1 strain ectodomain. The start (S), flow through (FT), final wash (W) and salt elution fractions were analysed by 10\%-14\% SDS-PAGE under reducing conditions and western blot analysis using anti-c-Myc MAb.

$\longleftarrow \mathrm{NaCl}$ Elution $(\mathrm{M}) \longrightarrow$

$\begin{array}{lllllllll}S & \text { FT } & W & 0.1 & 0.2 & 0.3 & 0.4 & 0.5 & 0.6\end{array}$

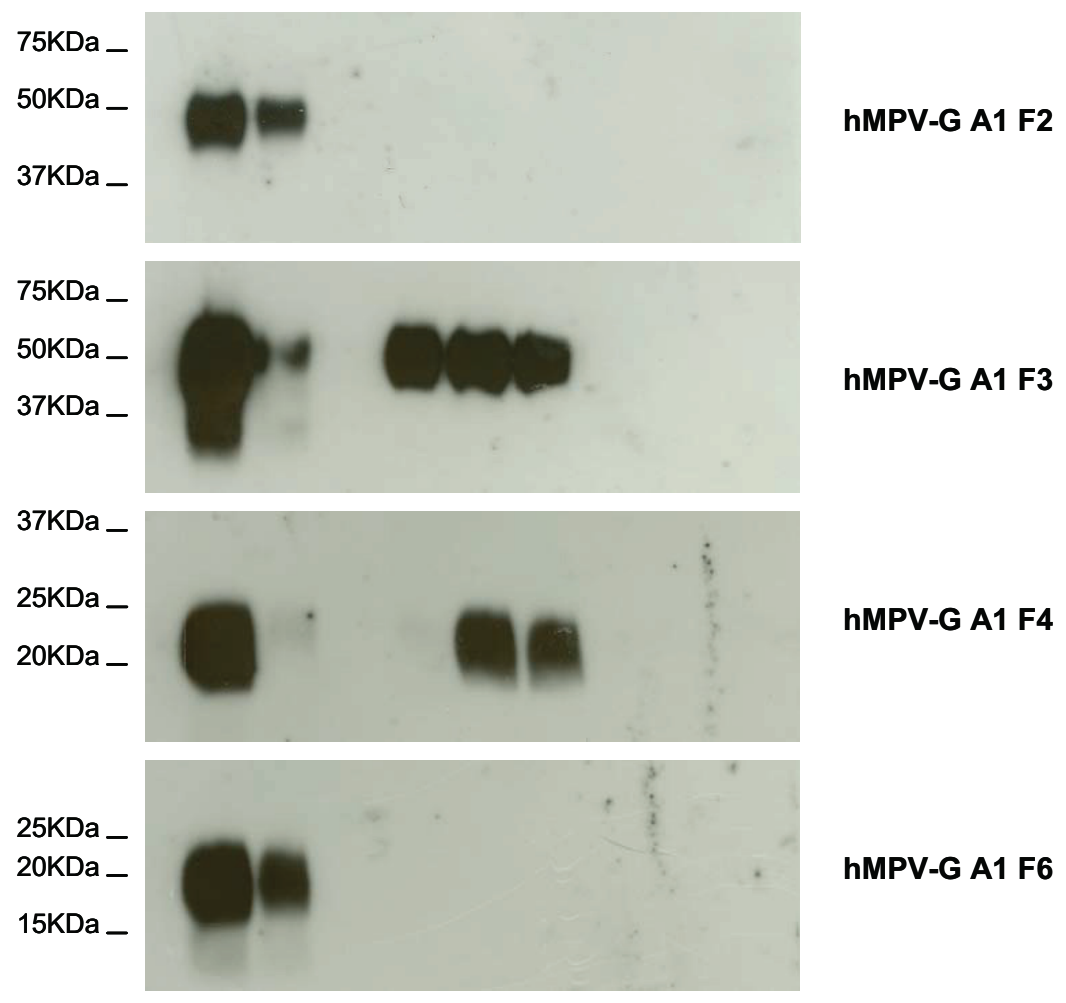




\subsection{Binding of hMPV F Protein to Immobilised Heparin}

The results of the infection experiments indicate that infection with the available strains of hMPV is dependent on the presence of GAGs; however not all of the hMPV G recombinant proteins bind to immobilised heparin (Figure 2). This implies that there are interactions occurring between GAGs and other surface exposed proteins on hMPV. An alternative to the attachment $(\mathrm{G})$ protein is the fusion $(\mathrm{F})$ protein which is highly conserved across all strains of hMPV. Fusion protein binding to GAGs was demonstrated by incubating hMPV infected cell lysates with heparin agarose and elution with salt (Figure 6). Both the precursor $\mathrm{F}$ protein, $\mathrm{F}_{0}$, and the biologically active cleaved $\mathrm{F}_{1}$ protein bound to heparin. No proteins were detected when uninfected cell lysate was subjected to heparin affinity precipitation (Figure 6).

Figure 6. Binding of native hMPV F protein to heparin. Start material (ST), flow through (FT), wash (W) and $2 \mathrm{M} \mathrm{NaCl}$ elution (E) fractions were analysed by $10 \%$ SDS-PAGE under reducing conditions and western blot analysis using anti-hMPV F antibody. Arrows indicate bands corresponding to the predicted sizes of full length precursor hMPV-F $\left(\mathrm{F}_{0}\right)$ and the cleavage fragment $h M P V F_{1}$. Molecular weight markers are shown in $\mathrm{kDa}$.

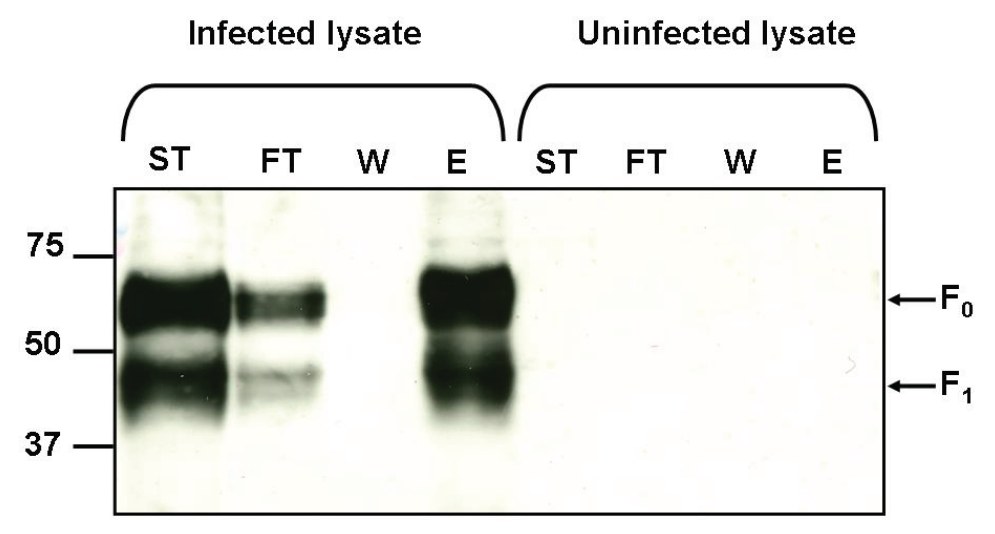

Additionally, the extracellular domain of $\mathrm{F}$ protein was cloned and expressed in a mammalian expression system. This resulted in a recombinant protein which migrated on SDS PAGE according to the predicted molecular weight of the uncleaved precursor form $\left(\mathrm{F}_{0}\right)$. Binding of soluble recombinant $\mathrm{F}$ protein to GAGs was investigated by heparin affinity chromatography. Recombinant hMPV-F protein bound to heparin with high affinity as demonstrated by protein elution with a high salt concentration (Figure 7). 
Figure 7. Heparin agarose affinity chromotography of recombinant soluble hMPV-F protein. Start material (ST), flow through (FT), wash (W) and elution (E) fractions, with $0.5 \mathrm{M} \mathrm{NaCl}(\mathrm{E} 1 \& \mathrm{E} 2), 1 \mathrm{M} \mathrm{NaCl}(\mathrm{E} 3 \& \mathrm{E} 4)$ and $1.5 \mathrm{M} \mathrm{NaCl}$ (E5 \& E6), were analysed by $10 \%$ SDS-PAGE under reducing conditions and western blot analysis using anti-hMPV F antibody. Molecular weight markers are shown in $\mathrm{kDa}$.

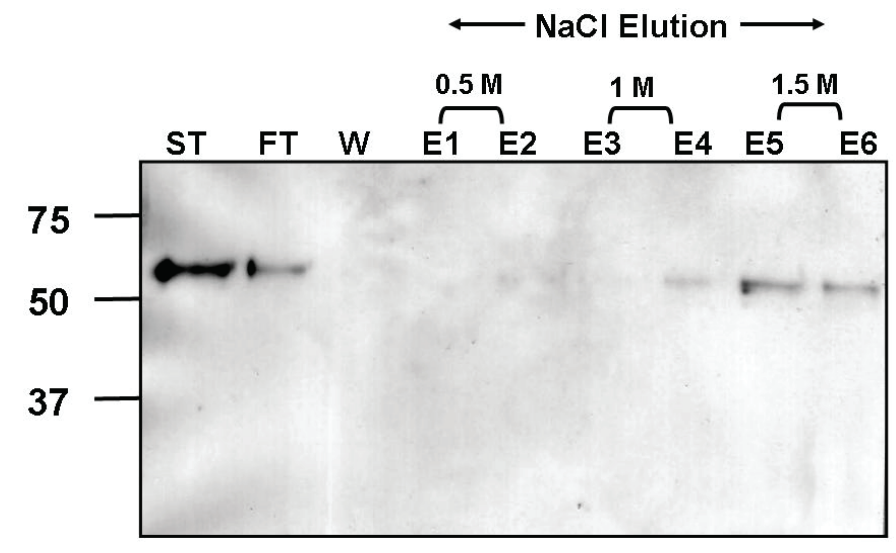

\section{Experimental Section}

\subsection{Cells and Viruses}

The rhesus monkey kidney cells (LLC-MK2) and the human epithelial tumour cell line (HEp-2) were grown in medium 199 (Invitrogen, Carlsbad, CA, USA) supplemented with $10 \%$ foetal bovine serum (FBS). Wild type Chinese hamster ovary cells (CHO-K1) and GAG-deficient CHO-pgsA745 cells [33] were grown in Hams F12 medium (Invitrogen) supplemented with 10\% FBS.

The A2 (V52283), B1 (V50569) and B2 (V47041) strains of hMPV were isolated from clinical samples by the Virology Laboratory, Flinders Medical Centre (FMC). These samples were positive only for hMPV and were not coinfected with influenza A, influenza B, RSV, adenovirus or parainfluenza 1, 2 or 3. Stocks of hMPV were prepared by inoculating LLC-MK2 cells with the hMPV strains and incubating for 14 to 21 days at $37{ }^{\circ} \mathrm{C}$ in $5 \% \mathrm{CO}_{2}$. Stocks were stored at $-70{ }^{\circ} \mathrm{C}$ until use. RNA extracted from the hMPV A1 strain was kindly provided to us by Dr. I. Mackay (Queensland Paediatric Infectious Diseases Laboratory, Sir Albert Sakzewski Virus Research Centre, Royal Children's Hospital, Queensland, Australia). hMPV strain subtyping was based on the Ishiguro classification [14].

\subsection{Heparin Inhibition of hMPV Infection}

Primary isolates of hMPV, A2, B1 and B2 strains (A1 was not available as it was not detected in South Australia during the collection period for these isolates), were pre-treated with or without heparin $\left(50 \mathrm{IU} / \mathrm{mL}\right.$ heparin) at $37{ }^{\circ} \mathrm{C}$ for $30 \mathrm{~min}$ before inoculation of LLC-MK2 cells. The cultures 
were incubated for 14 days with the maintenance medium changed every 3 days. Infectivity was determined after 14 days by a quantitative real-time RT-PCR for a $163 \mathrm{bp}$ region of the nucleoprotein gene of hMPV [34]. Viral RNA extracted from the cultures was reverse transcribed using $50 \mathrm{U}$ MMLV reverse transcriptase and $1 \mu \mathrm{M}$ NL-N-reverse following the manufacturer's protocol. Five $\mu \mathrm{L}$ cDNA was amplified using a Rotor Gene RG-3000 (Corbett Research, Sydney, Australia) in a $25 \mu \mathrm{L}$ reaction containing $1 \times$ PCR buffer, $5 \mathrm{mM} \mathrm{MgCl}_{2}, 200 \mu \mathrm{M}$ dNTPs, $0.4 \mu \mathrm{M}$ NL-N-forward, $0.2 \mu \mathrm{M}$ NL-N-reverse, $0.1 \mu \mathrm{M}$ NL-N-probe and $0.75 \mathrm{U}$ HotStar Taq polymerase (QIAGEN) with a 15 min Taq activation at $95{ }^{\circ} \mathrm{C}$ followed by 55 cycles of $10 \mathrm{~s}$ at $95{ }^{\circ} \mathrm{C}, 15 \mathrm{~s}$ at $55^{\circ} \mathrm{C}$ and $15 \mathrm{~s}$ at $72{ }^{\circ} \mathrm{C}$.

\subsection{GAG Dependence of hMPV Primary Isolates}

CHO-K1 and CHO-pgsA cells were incubated with $200 \mu \mathrm{L}$ of the primary hMPV isolates for strains A2, B1 and B2 in $1 \mathrm{~mL}$ CHO maintenance medium in $14 \mathrm{~mL}$ conical tubes (Cellstar, Greiner Bio-one, Frickenhausen, Germany) at $37{ }^{\circ} \mathrm{C}, 5 \% \mathrm{CO}_{2}$ for 14 days with the medium changed every 3 days. Two hundred microlitres of the culture supernatants, collected on day 14, were used to inoculate LLC-MK2 cells in 96 well tissue culture plates (Linbro, ICN Biomedicals, Aurora, OH, USA). After incubation for $2 \mathrm{~h}$ in $37{ }^{\circ} \mathrm{C}, 5 \% \mathrm{CO}_{2}$ the inoculum was replaced with $200 \mu \mathrm{L}$ maintenance medium and incubated for 7 days at $37{ }^{\circ} \mathrm{C}, 5 \% \mathrm{CO}_{2}$.

Infectivity was assessed using a cell based enzyme linked immunosorbent assay (ELISA) [24]. Briefly, confluent monolayers of Hep-2 cells in 96 well plates were inoculated with day 7 LLC-MK2 cell supernatants and incubated for $2 \mathrm{~h}$ at $37{ }^{\circ} \mathrm{C}, 5 \% \mathrm{CO}_{2}$. Unbound virus was removed by washing with medium 199 and the cells were cultured in medium 199 containing $1 \mu \mathrm{g} / \mathrm{mL}$ trypsin at $37{ }^{\circ} \mathrm{C}$, $5 \% \mathrm{CO}_{2}$. After $48 \mathrm{~h}$ the cells were fixed with $1 \%$ paraformaldehyde in phosphate buffered saline (PBS) for $30 \mathrm{~min}$ at room temperature. Cells were washed twice with PBS, permeabilised with $0.02 \%$ Triton X-100/PBS for $30 \mathrm{~min}$ at $4{ }^{\circ} \mathrm{C}$ and then washed twice with PBS. The cells were blocked with $5 \%$ skim milk/PBS for $1 \mathrm{~h}$ before incubation with $1 / 320(\mathrm{v} / \mathrm{v}) \mathrm{hMPV}$ matrix protein monoclonal antibody (Chemicon, Temecula, CA, USA) in $0.5 \%$ Tween 20/PBS followed by 1/10,000 (v/v) horseradish peroxidase (HRP) conjugated sheep anti-mouse immunoglobulin $\mathrm{G}$ (Chemicon) in $0.5 \%$ Tween 20/PBS. All incubations were $1 \mathrm{~h}$ at $37{ }^{\circ} \mathrm{C}$ and the wells were washed four times with PBS after each step. The wells were developed by incubating with $O$-phenylenediamine substrate (Sigma, St. Louis, MO, USA) for $30 \mathrm{~min}$. Reactions were stopped with $1 \mathrm{~N} \mathrm{H}_{2} \mathrm{SO}_{4}$ and the absorbance at $490 \mathrm{~nm}$ was determined. Wells were inoculated in triplicate and each experiment carried out at least twice.

\subsection{Construction and Expression of Recombinant hMPV G Proteins}

The ectodomains of A1, A2 and B1 were cloned and expressed for this study using similar methods as that described for the construction and expression of the extracellular domain of the $\mathrm{G}$ protein of the B2 strain of hMPV [24]. Briefly, RNA was extracted from hMPV (subtype A2 and B1) infected LLC-MK2 cells using the QIAamp Viral RNA purification kit (QIAGEN, Hilden, Germany) following the manufacturer's protocol. cDNA was synthesised using $500 \mu \mathrm{M}$ GB1R or GA2R, as appropriate, or 
$50 \mathrm{ng}$ random primer (Promega, Madison, WI, USA) for the A1 strain, using $200 \mathrm{U}$ Moloney murine leukaemia virus (MMLV) reverse transcriptase (Invitrogen) according to the manufacturer's conditions. The ectodomain of hMPV G protein was amplified using $0.8 \mu \mathrm{M}$ forward and reverse primers (Table 2) for individual strains of hMPV or the region of the G protein to be expressed. PCR was carried out in a reaction containing $1 \times$ PCR buffer, $1 \times$ Q-Solution, $1.5 \mathrm{mM} \mathrm{MgCl}_{2}, 400 \mu \mathrm{M}$ dNTPs and 0.75 U HotStar Taq Polymerase (QIAGEN) and $5 \mu \mathrm{L}$ cDNA. Forward and reverse primers incorporated restriction sites compatible with those in the multiple cloning site of the pPICZaA plasmid (Invitrogen) as underlined in Table 2. This allowed cloning of the PCR products downstream of the yeast $\alpha$ factor signal sequence, resulting in the secretion of the expressed protein into the growth medium. The PCR products were cloned in frame with the C-terminal tags, c-Myc and $6 \times \mathrm{HIS}$, for ease of detection and purification. Following transformation into Pichia pastoris X33 yeast, expression of the soluble recombinant protein was induced with methanol over 3-4 days. The hMPV G proteins were purified using immobilised metal affinity chromatography (IMAC) over HP fast flow chelating columns (GE Life Sciences, Uppsala, Sweden) loaded with nickel chloride. The sequence of each construct was verified prior to transformation into P. pastoris X33.

Table 2. Primers used to amplify the extracellular region of the hMPV strains A1, A2, B1 and B2, hMPV-G A1 strain fragments and the hMPV F extracellular region. Restriction sites incorporated for ease of cloning are underlined; EcoRI (GAATTC), MluI (ACGCGT) and XbaI (TCTAGA). Bases incorporated to remain in frame with the C-terminal tags are shown in italics.

\begin{tabular}{|c|c|c|}
\hline Primer Name & hMPV Strains & Primer Sequence \\
\hline GB2F & $\mathrm{B} 2$ & 5'-GGGGAATTCGATCATGCAACATTAAGAAACATG-3' \\
\hline GB2R & $\mathrm{B} 2$ & 5'-GGGTCTAGA $G C$ TCCTGCACCTCYCCGTGCAT-3' \\
\hline GB1F & B1 & 5'-AGAATTCGAATCAGAACATCACACCAG-3' \\
\hline GB1R & B1 & 5'-ATCTAGA $G C$ TGTGCTTGCAGATGCCTG-3' \\
\hline GA2F & $\mathrm{A} 2$ & 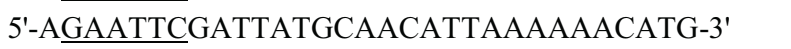 \\
\hline GA2R & $\mathrm{A} 2$ & 5'-ATCTAGA $G C A C T A C T T A G A G A A G A T G T G T C-3 '$ \\
\hline GA1F & A1 & 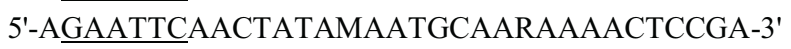 \\
\hline GA1R & A1 & 5'-TTCTAGA GCACTAGTTTGGTTGTATGTTGTTGA-3' \\
\hline GA1F2 & A1 & 5'-AGAATTCAGCCCAGCAGTCCACACAAAAAAC-3' \\
\hline GA1R1 & A1 & 5'-TTCTAGA $T C G A C T G C T G G G C T T G T C T T T G T T C-3 '$ \\
\hline GA1R2 & A1 & 5'-TTCTAGATCTGTTGTTGCCCGTGGTGGGGAAC-3' \\
\hline GA1F3 & A1 & 5'-AGAATTCGACAGCAGCGCAACAATCC-3' \\
\hline GA1F4 & A1 & 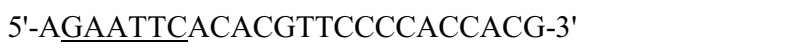 \\
\hline GA1F5 & A1 & 5'-AGAATTCCTCCACACAAGCAGCATAAG-3' \\
\hline GA1R4 & A1 & 5'-TTCTAGAGCGTCTGGTTGGGCTGATGC-3' \\
\hline FMPV-for & $\mathrm{B} 2$ & 5'-GGGACGCGTCTTAAAGAGAGCTAYYTAGAAG-3' \\
\hline FMPV-r2B & $\mathrm{B} 2$ & 5'-GGGTCTAGAGCRCCAGTGTTTCCTTTTTCTGC-3' \\
\hline
\end{tabular}




\subsection{Heparin Agarose Affinity Chromatography}

Heparin agarose chromatography was carried out in $50 \mathrm{mM}$ sodium phosphate buffer $\mathrm{pH} 7.4$ (PB) using $1 \mathrm{~mL}$ heparin agarose columns (Pierce Chemical Corporation, Rockford, IL, USA and Sigma). Recombinant proteins were dialysed into phosphate buffer overnight and then passed over the column 4-6 times. The columns were extensively washed with phosphate buffer and then eluted in a step-wise $\mathrm{NaCl}$ gradient. Start, flow-through, wash and elutions were all analysed using SDS-PAGE and western blotting.

\subsection{Detection of hMPV G Protein by Western Blot}

Proteins were separated by $10 \%-14 \%$ SDS PAGE (depending on the expected size of the protein) under reducing conditions and transferred onto Amersham ${ }^{\mathrm{TM}}$ Hybond $^{\mathrm{TM}}$-ECL membrane (GE Healthcare). Membranes were blocked with 5\% skim milk/PBS and incubated with $1 / 5$ (v/v) anti-c-Myc monoclonal antibody (from in-house hybridoma cell line 9E10) followed by 1/2,000 (v/v) HRP conjugated sheep anti-mouse immunoglobulin G. The proteins were visualised using enhanced chemiluminescence (ECL).

\subsection{Heparin Binding Studies Using hMPV Infected Cell Lysates}

Monolayers of LLC-MK2 cells were infected with hMPV (over 10 days) or virus/Chlamydia transport medium (VCTM), as a "mock" inoculum control. Cells were washed twice with PBS and lysed with $250 \mu \mathrm{L} 60 \mathrm{mM}$ n-octyl $\beta$-B-glucopyranoside (Sigma), $1 \times$ protease inhibitor cocktail tablet (Roche, Mannheim, Germany) in PBS. After $20 \mathrm{~min}$ on ice, cell debris was removed by centrifugation at $13,000 \times \mathrm{g}$ for $15 \mathrm{~min}$ at $4{ }^{\circ} \mathrm{C}$ and the supernatant collected. Infected and uninfected cell lysates were diluted 1:2 (v/v) in $\mathrm{PB}$ and incubated with heparin agarose, washed and eluted with salt. Fractions were analysed by SDS-PAGE and western blotting.

\subsection{Detection of hMPV F Protein by Western Blot}

Proteins were separated by $10 \%$ SDS PAGE under reducing conditions and transferred onto Amersham $^{\mathrm{TM}}$ Hybond $^{\mathrm{TM}}$-ECL membrane. Membranes were blocked with $5 \%$ skim milk/PBS and incubated with 1/2,000 (v/v) hamster polyclonal anti-hMPV F antibody (MedImmune, Gaithersburg, MD, USA) followed by $1 / 2,000(\mathrm{v} / \mathrm{v})$ HRP conjugated goat anti-Armenian hamster immunoglobulin (Rockland Immunochemicals, Gilbertsville, PA, USA). The proteins were visualised using ECL.

\subsection{Construction and Expression of hMPV F Protein}

The extracellular domain of the F gene of hMPV (B2 strain) was amplified as described previously using the primers shown in Table 2 and amplification conditions as described for amplification of hMPV G protein. The PCR product was cloned into the mammalian expression vector, BSR $\alpha$ EN [35] downstream of the factor $\mathrm{H}(\mathrm{fH})$ signal sequence. The hMPV F/BSR $\alpha \mathrm{EN}$ construct was verified by 
sequencing. CHO-K1 cells were transiently transfected with hMPV F/BSR $\alpha$ EN using Lipofectamine 2000 (Invitrogen) then grown in OPTI-MEM (Invitrogen) for $72 \mathrm{~h}$. Supernatant was harvested and analysed for protein expression using SDS PAGE and western blotting with anti-hMPV F antibody.

\section{Conclusions}

In this study we investigated whether sequence variability between different strains of hMPV G protein would affect the ability of their attachment proteins to interact with GAGs. Although infection with all strains tested (A2, B1 and B2) was inhibited by soluble heparin and required cell surface GAGs for infection, only recombinant G proteins from the A1 and B2 strains bound heparin agarose. Some viruses, including Sindbis and Ross River virus have been shown to adapt during cell culture to bind to heparin [36-38]. Sequence analysis of these serially cultured viruses, show the introduction of amino acid substitutions which are likely to create heparin binding domains to better adapt to cell culture and to expand the host range of the viruses. However, this may abrogate the ability of these viruses to retain their pathogenicity as has been shown in culture-adapted Sindbis virus [38]. We confirmed that the GAG dependency of each hMPV strain was not an artifact of serial cell culture passage by using non-passaged primary isolates for these experiments and also by sequencing the $G$ gene of a series of primary isolates (data not shown).

We observed differences in the apparent molecular weight of the G proteins expressed for each strain. The proteins expressed for the B1 and B2 strains migrated on SDS PAGE at apparent molecular weights of approximately $60 \mathrm{kDa}$ and $50 \mathrm{kDa}$, respectively, however $\mathrm{A} 1$ and $\mathrm{A} 2$ recombinant $\mathrm{G}$ protein migrated at approximately $100 \mathrm{kDa}$ and $75 \mathrm{kDa}$, respectively. This could be due to varying lengths of the hMPV G ectodomains expressed and/or differences in glycosylation between strains. It has been demonstrated that differential glycosylation of RSV G protein, dependent on the cell line of origin, results in changes in electrophoretic mobility on SDS PAGE [39]. Yeast expression systems, such as Saccharomyces cerevisiae, are known to hyperglycosylate secreted proteins, however this is less likely in glycoproteins expressed by Pichia pastoris which are thought to resemble those of higher eukaryotes [40].

The interactions between hMPV G protein and GAGs are most likely mediated by electrostatic interactions between positively charged residues in the $G$ ectodomain and negatively charged sulphates on the GAGs rather than at specific recognition sites. It appears from our results that hMPV B2 G protein has a higher affinity for heparin than hMPV A1 G protein. This could be due to the availability of positively charged residues on the protein surface after folding, the number of positively charged residues or the spacing of the residues. The conformation of Kaposi's sarcoma-associated herpesvirus complement control protein $(\mathrm{KCP})$ is important in the formation of a strong positive patch on the $\mathrm{KCP}$ which is then available to bind to heparin. Using docking simulations, Mark et al. [41] showed that the extended conformation of this protein presented a more favourable docking site than could be found in the bent conformation. Differences in conformation of the $G$ protein in each strain, due to high sequence variability, may explain the reduced affinity for heparin of hMPV A1 G protein compared to $\mathrm{G}$ protein of the B2 strain. To investigate regions of positively charged residues we produced a series 
of truncations of hMPV G (A1) strain which were tested for binding to heparin agarose. The results indicated a region between residues 93 and 142 may be responsible for G protein-GAG interactions. This domain contained a greater concentration of positively charged residues and is not dissimilar to the region identified for the B2 strain [24]. When the amino acid sequence of G protein from each hMPV strain is compared (Figure 3), GAG-binding strains had a higher number of positively charged residues in the tentative binding region (residues 98-136/137/142). The increased affinity of the $G$ protein of the B2 strain is probably not due to the total number of positively charged residues, as the A1 strain has more in this region, but more likely the clustering of the residues seen in the B2 strain. Consensus motifs, XBBXBX, XBBBXXBX and TXXBXXTBXXXTBB (where B is the probability of basic residues, $X$ is an uncharged hydrophobic residue and $T$ is an amino acid in a turn) have been proposed $[42,43]$ to mediate GAG recognition however these do not hold true for every heparin binding protein, including factor $H$. This suggests that the orientation and spacing of amino acids, as described by Margalit et al. [44], and the local absence of a negative charge are important. Although it is apparent from our previous study [24] that hMPV infection is inhibited by a wide range of GAGs, other viruses, such as RSV, Dengue virus and yellow fever virus, have a far more limited repertoire of cell surface GAGs [45,46]. It may be possible that different strains of hMPV are also this discriminatory and hence do not bind heparin, although our infection experiments suggest that this is unlikely. A complex interaction between the number and clustering of charged residues is ultimately likely to determine GAG-binding characteristics. Further mutagenesis of the A1 strain or engineering additional positively charged residues into this region within the non-binding strains may provide a greater insight into the residues involved.

We have shown that the dependency of hMPV infection on GAGs is not explained by a common interaction of G protein with GAGs. There is debate that the G protein of hMPV is not essential for virus infectivity. Replication of a mutant hMPV virus which was lacking the $\mathrm{G}$ protein was shown to be as efficient in cell culture as wild type virus, however replication in vivo was markedly reduced [25,47]. Similarly this has been investigated for RSV [48]; however the effect of deleting RSV G protein appears to be cell specific [49] and replication of mutant RSV in the respiratory tract of mice is highly restricted. We have shown in this study, and previously, that both hMPV infectivity and G protein binding can be mediated by GAGs, however it is unlikely that the mechanism of infection is solely reliant on the ability of $G$ protein to bind GAGs. There is evidence that some viruses recognise more than one receptor to gain entry to target host cells [50,51]. Wickham et al. [51] described the use of multiple receptors in adenovirus which firstly binds to the cell with a high affinity interaction through the fibre protein and then to integrins via RGD sequences present in the penton base. Another example is the cascade of interactions involved in the entry of HSV into cells which involves several virion glycoproteins, some of which interact with heparin sulphate [52-57]. Our results indicate a mechanism similar to that described for adenovirus. The inhibition of infection of the two strains not shown to bind heparin agarose via their attachment proteins, can be explained by the ability of the highly conserved $\mathrm{F}$ protein to bind to heparin. We showed that $\mathrm{F}$ protein, either in its native form, as part of a hMPV infected cell lysate, or expressed recombinantly, was able to bind to heparin with high affinity. 
This indicates that the GAG dependency of all hMPV strains for infectivity may be mediated by $\mathrm{F}$ protein-GAG interactions. These results have recently been confirmed by Chang et al. [58] who demonstrated that interactions between the hMPV F protein (of the A2 subtype) and the glycosaminoglycan heparan sulphate was essential for efficient binding of virus to cell surfaces and is most likely the initial step in virus binding and infection. Virus attachment and entry appears to require a combination of GAG binding with, or as a precursor to, other interactions, including integrin binding. Recently an integrin binding recognition Arg-Gly-Asp (RGD) sequence was identified in the F protein of hMPV which is conserved across all strains [19]. The F protein was shown to interact with the integrin $\alpha v \beta 1$ and this was suggested as a potential route for viral entry. Subsequently the same group has demonstrated that $F$ protein not only binds $\alpha \mathrm{v} \beta 1$ but will interact with a range of integrins known to bind RGD sequences [59]. Although not essential for virus attachment the interaction between the fusion protein and integrins is critical for efficient infection [58,59].

It is still unclear as to whether the binding of hMPV to GAGs is specific, however it is more likely due to non-specific electrostatic interactions and may represent the first step in a multivalent receptor process. In summary, we have shown that diversity exists in GAG binding amongst hMPV G protein lineages and that there is a high affinity interaction between GAGs and the hMPV fusion protein which could explain the dependency of hMPV on glycosaminoglycans.

\section{Acknowledgments}

The authors would like to thank Ian Mackay, Queensland Paediatric Infectious Diseases Laboratory, Sir Albert Sakzewski Virus Research Centre, Royal Children's Hospital, Queensland, Australia for kindly providing us with RNA extracted from the hMPV A1 strain. This work was supported by the Channel 7 Children's Research Foundation, the Flinders Medical Centre Foundation and SA Pathology. ST was supported by a Royal Thai Government Ph.D. scholarship.

\section{Conflicts of Interest}

The authors declare no conflict of interest.

\section{References}

1. Boivin, G.; Abed, Y.; Pelletier, G.; Ruel, L.; Moisan, D.; Cote, S.; Peret, T.C.; Erdman, D.D.; Anderson, L.J. Virological features and clinical manifestations associated with human metapneumovirus: A new paramyxovirus responsible for acute respiratory-tract infections in all age groups. J. Infect. Dis. 2002, 186, 1330-1334.

2. Boivin, G.; de Serres, G.; Cote, S.; Gilca, R.; Abed, Y.; Rochette, L.; Bergeron, M.G.; Dery, P. Human metapneumovirus infections in hospitalized children. Emerg. Infect. Dis. 2003, 9, 634-640.

3. Osterhaus, A.; Fouchier, R. Human metapneumovirus in the community. Lancet 2003, 361, 890-891. 
4. Peret, T.C.; Boivin, G.; Li, Y.; Couillard, M.; Humphrey, C.; Osterhaus, A.D.; Erdman, D.D.; Anderson, L.J. Characterization of human metapneumoviruses isolated from patients in North America. J. Infect. Dis. 2002, 185, 1660-1663.

5. Van den Hoogen, B.G.; de Jong, J.C.; Groen, J.; Kuiken, T.; de Groot, R.; Fouchier, R.A.; Osterhaus, A.D. A newly discovered human pneumovirus isolated from young children with respiratory tract disease. Nat. Med. 2001, 7, 719-724.

6. Kahn, J.S. Epidemiology of human metapneumovirus. Clin. Microbiol. Rev. 2006, 19, 546-557.

7. King, A.M.; Lefkowitz, E.; Adams, M.J.; Carstens, E.B. Virus taxonomy: Ninth report of the international committee on taxonomy of viruses; Ninth Report; Academic Press, Elsevier: Oxford, UK, 2012; pp. 672-682. Available online: http://ictvonline.org/virusTaxonomy.asp?version=2009 (accessed on 14 February 2012).

8. Van den Hoogen, B.G.; Bestebroer, T.M.; Osterhaus, A.D.; Fouchier, R.A. Analysis of the genomic sequence of a human metapneumovirus. Virology 2002, 295, 119-132.

9. Van den Hoogen, B.G.; van Doornum, G.J.; Fockens, J.C.; Cornelissen, J.J.; Beyer, W.E.; de Groot, R.; Osterhaus, A.D.; Fouchier, R.A. Prevalence and clinical symptoms of human metapneumovirus infection in hospitalized patients. J. Infect. Dis. 2003, 188, 1571-1577.

10. Cilla, G.; Onate, E.; Perez-Yarza, E.G.; Montes, M.; Vicente, D.; Perez-Trallero, E. Hospitalization rates for human metapneumovirus infection among 0- to 3-year-olds in Gipuzkoa (Basque Country), Spain. Epidemiol. Infect. 2009, 137, 66-72.

11. Ordas, J.; Boga, J.A.; Alvarez-Arguelles, M.; Villa, L.; Rodriguez-Dehli, C.; de Ona, M.; Rodriguez, J.; Melon, S. Role of metapneumovirus in viral respiratory infections in young children. J. Clin. Microbiol. 2006, 44, 2739-2742.

12. Bastien, N.; Liu, L.; Ward, D.; Taylor, T.; Li, Y. Genetic variability of the G glycoprotein gene of human metapneumovirus. J. Clin. Microbiol. 2004, 42, 3532-3537.

13. Biacchesi, S.; Skiadopoulos, M.H.; Boivin, G.; Hanson, C.T.; Murphy, B.R.; Collins, P.L.; Buchholz, U.J. Genetic diversity between human metapneumovirus subgroups. Virology 2003, 315, 1-9.

14. Ishiguro, N.; Ebihara, T.; Endo, R.; Ma, X.; Kikuta, H.; Ishiko, H.; Kobayashi, K. High genetic diversity of the attachment (G) protein of human metapneumovirus. J. Clin. Microbiol. 2004, 42, 3406-3414.

15. Ludewick, H.P.; Abed, Y.; van Niekerk, N.; Boivin, G.; Klugman, K.P.; Madhi, S.A. Human metapneumovirus genetic variability, South Africa. Emerg. Infect. Dis. 2005, 11, 1074-1078.

16. Van den Hoogen, B.G.; Herfst, S.; Sprong, L.; Cane, P.A.; Forleo-Neto, E.; de Swart, R.L.; Osterhaus, A.D.; Fouchier, R.A. Antigenic and genetic variability of human metapneumoviruses. Emerg. Infect. Dis. 2004, 10, 658-666.

17. Feuillet, F.; Lina, B.; Rosa-Calatrava, M.; Boivin, G. Ten years of human metapneumovirus research. J. Clin. Virol. 2012, 53, 97-105. 
18. Chanock, R.M.; Murphy, B.R.; Collins, P.L. Parainfluenza Viruses. In Fields Virology, 4th ed.; Knipe, D.M., Howley, P.M., Griffin, D.E., Martin, M.A., Lamb, R.A., Roizman, B., Straus, S.E., Eds.; Lippincott Williams and Wilkins: Philadelphia, PA, USA, 2001; Volume 1, pp. 1341-1379.

19. Cseke, G.; Maginnis, M.S.; Cox, R.G.; Tollefson, S.J.; Podsiad, A.B.; Wright, D.W.; Dermody, T.S.; Williams, J.V. Integrin $\alpha v \beta 1$ promotes infection by human metapneumovirus. Proc. Natl. Acad. Sci. USA 2009, 106, 1566-1571.

20. Skiadopoulos, M.H.; Biacchesi, S.; Buchholz, U.J.; Amaro-Carambot, E.; Surman, S.R.; Collins, P.L.; Murphy, B.R. Individual contributions of the human metapneumovirus F, G, and SH surface glycoproteins to the induction of neutralizing antibodies and protective immunity. Virology 2006, 345, 492-501.

21. Skiadopoulos, M.H.; Biacchesi, S.; Buchholz, U.J.; Riggs, J.M.; Surman, S.R.; Amaro-Carambot, E.; McAuliffe, J.M.; Elkins, W.R.; St Claire, M.; Collins, P.L.; et al. The two major human metapneumovirus genetic lineages are highly related antigenically, and the fusion (F) protein is a major contributor to this antigenic relatedness. J. Virol. 2004, 78, 6927-6937.

22. Tang, R.S.; Mahmood, K.; Macphail, M.; Guzzetta, J.M.; Haller, A.A.; Liu, H.; Kaur, J.; Lawlor, H.A.; Stillman, E.A.; Schickli, J.H.; et al. A host-range restricted parainfluenza virus type 3 (PIV3) expressing the human metapneumovirus (hMPV) fusion protein elicits protective immunity in African green monkeys. Vaccine 2005, 23, 1657-1667.

23. Liu, L.; Bastien, N.; Li, Y. Intracellular processing, glycosylation, and cell surface expression of human metapneumovirus attachment glycoprotein. J. Virol. 2007, 81, 13435-13443.

24. Thammawat, S.; Sadlon, T.A.; Hallsworth, P.G.; Gordon, D.L. Role of cellular glycosaminoglycans and charged regions of viral $\mathrm{G}$ protein in human metapneumovirus infection. J. Virol. 2008, 82, 11767-11774.

25. Biacchesi, S.; Pham, Q.N.; Skiadopoulos, M.H.; Murphy, B.R.; Collins, P.L.; Buchholz, U.J. Infection of nonhuman primates with recombinant human metapneumovirus lacking the $\mathrm{SH}, \mathrm{G}$, or M2-2 protein categorizes each as a nonessential accessory protein and identifies vaccine candidates. J. Virol. 2005, 79, 12608-12613.

26. Esko, J.D.; Kimata, K.; Lindahl, U. Proteoglycans and Sulfated Glycosaminoglycans. In Essentials of Glycobiology, 2nd ed.; Cold Spring Harbor Laboratory Press: Cold Spring Harbor, NY, USA, 2009; Chapter 16.

27. Baba, M.; Snoeck, R.; Pauwels, R.; de Clercq, E. Sulfated polysaccharides are potent and selective inhibitors of various enveloped viruses, including herpes simplex virus, cytomegalovirus, vesicular stomatitis virus, and human immunodeficiency virus. Antimicrob. Agents Chemother. 1988, 32, $1742-1745$.

28. Feldman, S.A.; Hendry, R.M.; Beeler, J.A. Identification of a linear heparin binding domain for human respiratory syncytial virus attachment glycoprotein G. J. Virol. 1999, 73, 6610-6617. 
29. Hosoya, M.; Balzarini, J.; Shigeta, S.; de Clercq, E. Differential inhibitory effects of sulfated polysaccharides and polymers on the replication of various myxoviruses and retroviruses, depending on the composition of the target amino acid sequences of the viral envelope glycoproteins. Antimicrob. Agents Chemother. 1991, 35, 2515-2520.

30. Zhu, Z.; Gershon, M.D.; Ambron, R.; Gabel, C.; Gershon, A.A. Infection of cells by varicella zoster virus: Inhibition of viral entry by mannose 6-phosphate and heparin. Proc. Natl. Acad. Sci. USA 1995, 92, 3546-3550.

31. Krusat, T.; Streckert, H.J. Heparin-dependent attachment of respiratory syncytial virus (RSV) to host cells. Arch. Virol. 1997, 142, 1247-1254.

32. Wyde, P.R.; Moylett, E.H.; Chetty, S.N.; Jewell, A.; Bowlin, T.L.; Piedra, P.A. Comparison of the inhibition of human metapneumovirus and respiratory syncytial virus by NMSO3 in tissue culture assays. Antivir. Res. 2004, 63, 51-59.

33. Esko, J.D.; Stewart, T.E.; Taylor, W.H. Animal cell mutants defective in glycosaminoglycan biosynthesis. Proc. Natl. Acad. Sci. USA 1985, 82, 3197-3201.

34. Maertzdorf, J.; Wang, C.K.; Brown, J.B.; Quinto, J.D.; Chu, M.; de Graaf, M.; van den Hoogen, B.G.; Spaete, R.; Osterhaus, A.D.; Fouchier, R.A. Real-time reverse transcriptase PCR assay for detection of human metapneumoviruses from all known genetic lineages. J. Clin. Microbiol. 2004, 42, 981-986.

35. Shenoy-Scaria, A.M.; Gauen, L.K.; Kwong, J.; Shaw, A.S.; Lublin, D.M. Palmitylation of an amino-terminal cysteine motif of protein tyrosine kinases $\mathrm{p} 56^{\text {lck }}$ and $\mathrm{p} 59^{\text {fyn }}$ mediates interaction with glycosyl-phosphatidylinositol-anchored proteins. Mol. Cell Biol. 1993, 13, 6385-6392.

36. Byrnes, A.P.; Griffin, D.E. Binding of sindbis virus to cell surface heparan sulfate. J. Virol. 1998, $72,7349-7356$.

37. Heil, M.L.; Albee, A.; Strauss, J.H.; Kuhn, R.J. An amino acid substitution in the coding region of the E2 glycoprotein adapts Ross River virus to utilize heparan sulfate as an attachment moiety. J. Virol. 2001, 75, 6303-6309.

38. Klimstra, W.B.; Ryman, K.D.; Johnston, R.E. Adaptation of Sindbis virus to BHK cells selects for use of heparan sulfate as an attachment receptor. J. Virol. 1998, 72, 7357-7366.

39. Garcia-Beato, R.; Martinez, I.; Franci, C.; Real, F.X.; Garcia-Barreno, B.; Melero, J.A. Host cell effect upon glycosylation and antigenicity of human respiratory syncytial virus G glycoprotein. Virology 1996, 221, 301-309.

40. Cregg, J.M.; Vedvick, T.S.; Raschke, W.C. Recent advances in the expression of foreign genes in Pichia pastoris. Biotechnology 1993, 11, 905-910.

41. Mark, L.; Lee, W.H.; Spiller, O.B.; Villoutreix, B.O.; Blom, A.M. The Kaposi's sarcoma-associated herpesvirus complement control protein $(\mathrm{KCP})$ binds to heparin and cell surfaces via positively charged amino acids in CCP1-2. Mol. Immunol. 2006, 43, 1665-1675.

42. Cardin, A.D.; Weintraub, H.J. Molecular modeling of protein-glycosaminoglycan interactions. Arteriosclerosis 1989, 9, 21-32. 
43. Hileman, R.E.; Fromm, J.R.; Weiler, J.M.; Linhardt, R.J. Glycosaminoglycan-protein interactions: Definition of consensus sites in glycosaminoglycan binding proteins. Bioessays 1998, 20, 156-167.

44. Margalit, H.; Fischer, N.; Ben-Sasson, S.A. Comparative analysis of structurally defined heparin binding sequences reveals a distinct spatial distribution of basic residues. J. Biol. Chem. 1993, 268, 19228-19231.

45. Germi, R.; Crance, J.M.; Garin, D.; Guimet, J.; Lortat-Jacob, H.; Ruigrok, R.W.; Zarski, J.P.; Drouet, E. Heparan sulfate-mediated binding of infectious dengue virus type 2 and yellow fever virus. Virology 2002, 292, 162-168.

46. Hallak, L.K.; Spillmann, D.; Collins, P.L.; Peeples, M.E. Glycosaminoglycan sulfation requirements for respiratory syncytial virus infection. $J$. Virol. 2000, 74, 10508-10513.

47. Biacchesi, S.; Skiadopoulos, M.H.; Yang, L.; Lamirande, E.W.; Tran, K.C.; Murphy, B.R.; Collins, P.L.; Buchholz, U.J. Recombinant human metapneumovirus lacking the small hydrophobic $\mathrm{SH}$ and/or attachment $\mathrm{G}$ glycoprotein: Deletion of $\mathrm{G}$ yields a promising vaccine candidate. J. Virol. 2004, 78, 12877-12887.

48. Karron, R.A.; Buonagurio, D.A.; Georgiu, A.F.; Whitehead, S.S.; Adamus, J.E.; Clements-Mann, M.L.; Harris, D.O.; Randolph, V.B.; Udem, S.A.; Murphy, B.R.; et al. Respiratory syncytial virus (RSV) $\mathrm{SH}$ and $\mathrm{G}$ proteins are not essential for viral replication in vitro: Clinical evaluation and molecular characterization of a cold-passaged, attenuated RSV subgroup B mutant. Proc. Natl. Acad. Sci. USA 1997, 94, 13961-13966.

49. Teng, M.N.; Whitehead, S.S.; Collins, P.L. Contribution of the respiratory syncytial virus G glycoprotein and its secreted and membrane-bound forms to virus replication in vitro and in vivo. Virology 2001, 289, 283-296.

50. Haywood, A.M. Virus receptors: Binding, adhesion strengthening, and changes in viral structure. J. Virol. 1994, 68, 1-5.

51. Wickham, T.J.; Mathias, P.; Cheresh, D.A.; Nemerow, G.R. Integrins $\alpha_{v} \beta_{3}$ and $\alpha_{v} \beta_{5}$ promote adenovirus internalization but not virus attachment. Cell 1993, 73, 309-319.

52. Fuller, A.O.; Lee, W.C. Herpes simplex virus type 1 entry through a cascade of virus-cell interactions requires different roles of $\mathrm{gD}$ and $\mathrm{gH}$ in penetration. J. Virol. 1992, 66, 5002-5012.

53. Herold, B.C.; Visalli, R.J.; Susmarski, N.; Brandt, C.R.; Spear, P.G. Glycoprotein C-independent binding of herpes simplex virus to cells requires cell surface heparan sulphate and glycoprotein B. J. Gen. Virol. 1994, 75, 1211-1222.

54. Herold, B.C.; WuDunn, D.; Soltys, N.; Spear, P.G. Glycoprotein C of herpes simplex virus type 1 plays a principal role in the adsorption of virus to cells and in infectivity. J. Virol. 1991, 65, $1090-1098$.

55. Johnson, R.M.; Spear, P.G. Herpes simplex virus glycoprotein D mediates interference with herpes simplex virus infection. J. Virol. 1989, 63, 819-827.

56. McClain, D.S.; Fuller, A.O. Cell-specific kinetics and efficiency of herpes simplex virus type 1 entry are determined by two distinct phases of attachment. Virology 1994, 198, 690-702. 
57. WuDunn, D.; Spear, P.G. Initial interaction of herpes simplex virus with cells is binding to heparan sulfate. J. Virol. 1989, 63, 52-58.

58. Chang, A.; Masante, C.; Buchholz, U.J.; Dutch, R.E. Human metapneumovirus (HMPV) binding and infection are mediated by interactions between the HMPV fusion protein and heparan sulphate. J. Virol. 2012, 86, 3230-3243.

59. Cox, R.G.; Livesay, S.B.; Johnson, M.; Ohi, M.D; Williams, J.V. The human metapneumovirus fusion protein mediates entry via an interaction with RGD-binding integrins. J. Virol. 2012, 86, $12148-12160$. 
Reprinted from Viruses. Cite as: Mastrangelo, P.; Hegele, R.G. RSV Fusion: Time for a New Model. Viruses 2013, 5, 873-885.

Review

\title{
RSV Fusion: Time for a New Model
}

\section{Peter Mastrangelo and Richard G. Hegele *}

Department of Laboratory Medicine and Pathobiology, University of Toronto, Toronto, ON M5S 1A8, Canada; E-Mail: peter.mastrangelo@utoronto.ca

* Author to whom correspondence should be addressed; E-Mail: richard.hegele@utoronto.ca; Tel.: +1-416-978-2557; Fax: +1-416-978-7361.

Received: 21 February 2013; in revised form: 11 March 2013 / Accepted: 15 March 2013 /

Published: 19 March 2013

\begin{abstract}
In this review we propose a partially hypothetical model of respiratory syncytial virus (RSV) binding and entry to the cell that includes the recently discovered RSV receptor nucleolin, in an attempt to stimulate further inquiry in this research area. RSV binding and entry is likely to be a two-step process, the first involving the attachment of the virus to the cell membrane, which may be enhanced by electrostatic interactions with cellular glycoproteins/heparin and the viral G protein, and the second involving fusion to the cell membrane mediated by the viral $\mathrm{F}$ protein and a specific cellular fusion receptor. With our recent discovery of nucleolin as a functional fusion receptor for RSV, comes the possibility of a number of new approaches to the development of novel strategies for RSV prophylaxis and therapy, as well as raising some new questions concerning the pathobiology of RSV infection and tropism.
\end{abstract}

Keywords: RSV; nucleolin; receptor

\section{Introduction}

Human respiratory syncytial virus (RSV) is found ubiquitously and a major cause of acute lower respiratory tract infections in children leading to hospitalization and occasionally death [1]. RSV also causes disease in adults, particularly in the elderly and in the immunocompromised [2]. Treatment and prophylaxis, given primarily to infants, are limited to Ribavirin and Palivizumab, respectively [2]. RSV is a negative-polarity, enveloped, single-stranded RNA virus from the Paramyxoviridae family. 
The viral genome encodes 11 proteins, three of which, small hydrophobic (SH), glycoprotein (G) and fusion (F) contribute to the viral coat. In spite of the fact that the virus was discovered in 1956, a safe, effective vaccine for RSV has remained elusive [3].

In this article we discuss aspects related to the discovery of nucleolin as a functional fusion receptor for RSV and propose a revised model for RSV fusion at the cell surface that incorporates nucleolin. Also, we discuss the implications this discovery has on the pathobiology of RSV infections and the development of novel prophylactic and therapeutic strategies.

\section{Viral Envelope Proteins and Fusion}

In order to understand attachment and fusion/entry into cells it is crucial to determine which viral envelope protein(s) are involved and what are their corresponding cellular ligands. The RSV G protein is a heterogeneous glycoprotein that defines the two subtypes of RSV (A and B). Besides its role in attachment of virus to the cell surface, the RSV G protein also helps the virus to elude the host immune system by mimicking cellular cytokines and through shedding [4]. Fusion of the virus to the cell membrane as well as the formation of syncytia, the characteristic cytopathic effect of RSV, is mediated by the viral F protein. The F protein unlike the $G$ protein is homologous to both subtypes of RSV. SH, the remaining viral envelope protein, is not required for RSV attachment or entry [5].

As will be discussed in further detail below, we propose that RSV binding and entry into cells is likely to be at least a two-step process: first, attachment of the virus to the cell membrane mediated by electrostatic interactions with cellular glycoproteins/heparin and the viral G protein, and secondly, fusion to the cell membrane mediated by the viral $\mathrm{F}$ protein and a specific cellular fusion receptor.

\section{Defining a Functional RSV Receptor}

Molecules proposed to be the "RSV receptor" include intercellular adhesion molecule (ICAM)-1 [6], heparin [7], annexin II [8], toll-like receptor (TLR) 4 [9] and fractalkine (CX3CL1) receptor, CX3CR1 [10]. Prior to the discovery of nucleolin as a functional receptor of RSV, no candidate receptor molecule met all of the following criteria of being a functional receptor, including: (i) decreased infection through antibody neutralization, competition with soluble candidate receptor molecule or decreased receptor expression through RNA interference; (ii) increased infection of non-permissive cells after ectopic expression of the candidate receptor molecule on the cell surface [11]. Despite a lack of definitive validation of these various candidate receptor molecules by all of the above criteria to date, one or more of them could function as co-receptors or co-factors for RSV and nucleolin.

Binding of viral $\mathrm{G}$ protein in an electrostatic fashion to the cell surface may be the first step in efficient viral attachment prior to fusion via nucleolin. Heparin, for example, binds the RSV G protein [7] on HEp-2 cells, however it turns out that human airway epithelium does not express heparin on the apical surface, the site of RSV attachment and cellular entry [12]. Thus the binding to heparin by the $\mathrm{G}$ protein may simply serve to demonstrate that the $\mathrm{G}$ protein has a general affinity for 
negatively charged carbohydrates on the cell surface. Further, mutant forms of RSV that lack the G protein $(\mathrm{RSV} \Delta \mathrm{G})$ are capable of causing productive infections, albeit at much lower efficiency than wild-type RSV [13].

The RSV F protein is necessary for infection, as mutant RSV lacking F protein $(\mathrm{RSV} \Delta \mathrm{F})$ cannot infect cells on its own but rather requires a helper virus to gain entry into cells [14]. In contrast to RSV $\mathrm{G}$, the $\mathrm{F}$ protein has a list of much more specific protein interactions. One example of such a protein interaction is RSV-F protein with intercellular adhesion molecule (ICAM)-1 expressed on the cell surface. Although it has not been definitively shown to be essential for viral fusion, ICAM-1 has been reported to bind the F protein and may as such still have a role in fusion [6]. Importantly, despite the apparently greater specificity of RSV F-protein interactions $v s$. RSV G, one must exercise caution over RSV F-protein interactions, as Toll-like receptor (TLR) 4 binds the purified RSV F protein, yet has no effect on viral infectivity in cell culture [9].

\section{Nucleolin: A Functional Fusion Receptor of RSV [15]}

\subsection{Identifying Nucleolin as a Ligand of Intact RSV}

To search for candidate RSV receptor molecules, we performed a number of preliminary experiments in which cell cultures from numerous mammalian species were treated with enzymes of cell surface components (protein, lipid, carbohydrate) and effects of enzyme pre-treatment on subsequent RSV infection were quantified. Results showed that pre-treatment of cells with trypsin, a protease, resulted in lower RSV infection than occurred in cells that did not undergo enzyme treatment, without affecting cell viability. These findings provided the rationale to use a Virus Overlay Protein Binding Assay (VOPBA) to identify candidate RSV receptor molecules [16]. VOPBA is essentially a modified Western blot where the virus substitutes for the primary antibody and is then detected with a virus-specific secondary. In protein extracts from various mammalian cell lines and numerous RSV isolates (including both laboratory-adapted and community strains of RSV A and RSV B), we reproducibly identified a VOPBA signal of approximately $100 \mathrm{kDa}$. As one would expect, a number of "hits" were obtained by mass spectrometry of this $100 \mathrm{kDa}$ band, but nucleolin was the most consistent, being found in every extract and viral isolate tested, and it also satisfied the requirement of being found at the cell surface [17,18]. As noted above, RSV F and G are the envelope proteins primarily involved in cell surface binding. To determine if nucleolin binds to RSV F or G, immunoprecipitations (IPs) were performed. Nucleolin co-precipitated only with the viral F protein in every instance tested. Addition of excess heparin to the IPs did not interfere with the nucleolin-protein $\mathrm{F}$ interactions in IPs although we also saw, as have others, a decrease in RSV infection in vitro [13].

The biological plausibility of RSV-nucleolin interaction in infection was confirmed in vitro and in vivo through a series of experiments that included: visualization of RSV-nucleolin co-localization on the cell surface by use of confocal fluorescence microscopy; decreased RSV infection of cells pre-treated with nucleolin-specific antibody and when cellular nucleolin expression was silenced by use of RNA interference, or when virus was incubated with soluble recombinant nucleolin prior to 
being added to cell cultures; increased RSV infection of a non-permissive cell type (Sf9) [36] that had been transfected with the human nucleolin gene and which showed ectopic expression of human nucleolin protein on the cell surface; decreased RSV infection of mouse lung, in animals that were pre-treated with short-interfering RNA of mouse nucleolin, delivered intranasally prior to RSV challenge.

\subsection{Nucleolin: Brief Overview}

First described in 1973, nucleolin is a multifunction protein that is found throughout the cell but it is primarily localized within the nucleolus, contributing up to $10 \%$ of the total protein in that compartment $[19,20]$. Although its predicted molecular weight is $77-78 \mathrm{kDa}$ (depending on the species), its relative molecular mobility in SDS-PAGE is 100-110 $\mathrm{kDa}$ [21], due to highly phosphorylated amino acids of the N-terminus [22]. Nucleolin has been shown to be more stable in proliferating cells due to inhibition of an auto-proteolytic activity more prominently found in quiescent cells [23]. Nucleolin is involved in diverse biological processes including cell proliferation, growth, cytokinesis, replication, embryogenesis and nucleogenesis and is considered necessary for cell survival and proliferation [24].

Nucleolin has a very high degree of evolutionary conservation [25] and can be divided into three structural/functional domains: (i) multiple acidic stretches in the N-terminus; (ii) multiple RNA recognition motifs (RRMs) in the central portion and (iii) a glycine/arginine rich domain in the C-terminal portion [21]. Although nucleolin is typically thought of first and foremost as an intranuclear protein [25], there is abundant evidence that it can also be found within the cytoplasm and on the cell surface and may play the role of a "molecular shuttle" between these compartments $[24,26]$. Nucleolin has a bipartite nuclear localization signal whose function is regulated by phosphorylation [27]. The actin cytoskeleton modulates the entry of substances via nucleolin into the cytoplasm [28]. The half-life of cell surface nucleolin is less than one hour and its expression is very sensitive to inhibition of transcription/translation, unlike nuclear nucleolin that has a half-life greater than eight hours [26].

In contrast to other cell surface proteins, nucleolin does not have a transmembrane domain or a glycosylphosphatidyl-inositol (GPI) anchor [26]; instead, nucleolin exists on the cell surface as part of a $500 \mathrm{kDa}$ protein complex that includes other membrane proteins [29]. Nucleolin functions as a receptor for a number of different molecules including DNA nanoparticles [30], apoB/E-containing lipoproteins, laminin-1, viruses (see below) [24] and bacteria [31,32].

Nucleolin also plays a role in viral replication and intracellular trafficking of viral components. For example nucleolin is required for HSV-1 DNA replication [33] and also for trafficking of the viral protein US11 out of the nucleus [34]. It also has been shown to bind the RNA-dependent RNA polymerase of HCV (NS5B) [35]. In HCMV nucleolin helps to maintain the architecture of viral replication compartments [36]. Similarly, nucleolin has been shown to bind the 3' untranslated regions and protease-polymerase NS6/7 of feline calicivirus again having a role in viral replication [37]. That these roles in viral replication and trafficking are connected to nucleolin's role as a viral receptor has yet to be determined. 


\section{A New Model for RSV Fusion/Entry}

In light of our findings of expression of cell surface nucleolin being sufficient for RSV infection, a revised model for RSV fusion can be generated. The present models for RSV fusion are in part inferred by analogy to data obtained for other enveloped viruses, particularly influenza virus [38,39]. The RSV F protein initially exists in a pre-fusion state that then undergoes conformational changes to a post-fusion form upon binding to receptor molecules expressed on the cell surface [31]. Current models reflect the fact that comparatively little is known about receptors or other factors involved in RSV fusion, and what triggers the conformational changes in the RSV F protein required for fusion. What seems evident from the results of our experiments is that nucleolin can allow this event to occur. We propose a model were nucleolin binds the pre-fusion conformation of the F protein possibly priming transition to the "extended" intermediate conformation [39]. Evidence that it is the pre-fusion form of RSV F that binds nucleolin is provided by results of our VOPBA experiments, in which live virus was shown to specifically bind nucleolin immobilized on a membrane in the absence of any cells [13]. It follows that this interaction would be with the pre-fusion conformation of the F protein, as "triggering" has not yet occurred. Furthermore, in so-called "competition" experiments in which RSV was incubated with soluble, recombinant nucleolin prior to being added to cell cultures, this state involved exogenous nucleolin saturating $\mathrm{F}$ protein binding sites in a pre-fusion state, since there were no cells present during this incubation step.

In our proposed model (Figure 1A), RSV binds cell surface nucleolin via the trimeric pre-fusion form of the $\mathrm{F}$ protein. Nucleolin is shown as part of a protein complex which includes either membrane proteins or glycosylphosphatidylinositol (GPI)-linked proteins that tether it to the cell surface. We postulate this binding event triggers the re-folding of the F protein into its extended conformation and once this has occurred, fusion can continue without further need of nucleolin. In this model as many as three molecules of nucleolin can bind one RSV F protein pre-fusion trimer. Refolding of the fusion protein from a pre-fusion to post-fusion conformation is irreversible making the temporal and spatial triggering of this event critical [38]. A fusion receptor like nucleolin helps to ensure that the optimal conditions for viral fusion are met.

Cell surface nucleolin has been found in association with lipid-rich rafts upon anchorage of HIV to permissive cells [40] and efficient RSV infection of human lung epithelial cells requires intact lipid-rich rafts [41]. Nucleolin-mediated trafficking of DNA nanoparticles is lipid raft dependent and nucleolin colocalizes with and co-immunoprecipitates with the raft protein, flotillin [42]. Thus nucleolin may serve to bring RSV in contact to raft domains that may themselves be preferred areas for virus fusion. Alternatively, RSV may be brought into the cell via caveolae or endosomes and triggering/fusion might occur in one of these intracellular compartments. Uptake of virus by bovine dendritic cells has been shown to involve caveolae and RSV colocalizes with caveolin in these cells [43]. However, unlike the case of influenza and Semliki forest virus (SFV) [44], RSV F protein does not appear to be triggered by $\mathrm{pH}$ change that occurs during the acidification of lysosomes $[45,46]$. Interestingly, it has been reported that the internalization of nucleolin on ligand binding is a calcium-dependent process [26] and it has been known for some years that RSV infection is also a 
calcium-dependent process [47]. On the other hand, there is a body of evidence that supports the idea of clatherin-mediated endocytosis being necessary for RSV infection [48]. Similarly, there are publications that show that nucleolin is internalized after ligand binding (i.e., endostatin) via a clatherin-mediated pathway [49]. It seems possible that RSV and nucleolin may use both types of endosomal pathways in different circumstances however this still remains to be resolved.

Figure 1. (A) Model of RSV F-protein binding nucleolin. On the left, the F-protein is shown in its trimeric pre-fusion conformation. The red circles are putative nucleolin binding sites. Nucleolin is shown in orange as part of a protein complex that includes proteins anchored to the membrane by either a transmembrane domain or a GPI anchor. Only one nucleolin molecule is shown binding the F-protein trimer for clarity but in this model as many as three could bind at once. On the right the F-protein is shown in the "extended" conformation with fusion peptides (yellow) inserted into the cell membrane. After this step virus-cell membrane fusion would proceed without nucleolin. (B) Diagram of virus binding to the cell surface. Indicated in light yellow are lipid-rich domains/rafts. The virus is shown in a dark magenta covered with F-protein binding to nucleolin that is preferentially located in lipid-rich rafts or caveolae. A caveola is shown covered with caveolin (dark red). This in turn can enter the cell to form a caveozome and join the endosomal or lysosomal pathway. Our proposed model leaves open the possibilities that viral fusion may occur at the cell surface or in a caveozome/endosome/lysosome. Another possibility (not shown) is that virus enters via clathirin-coated pits (see text).

A

cell

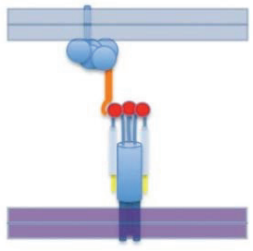

virus cell

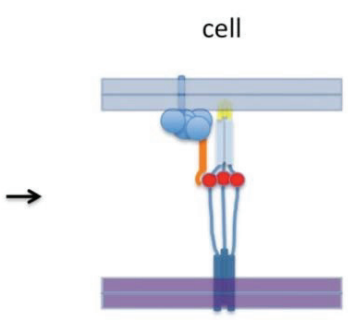

virus

B

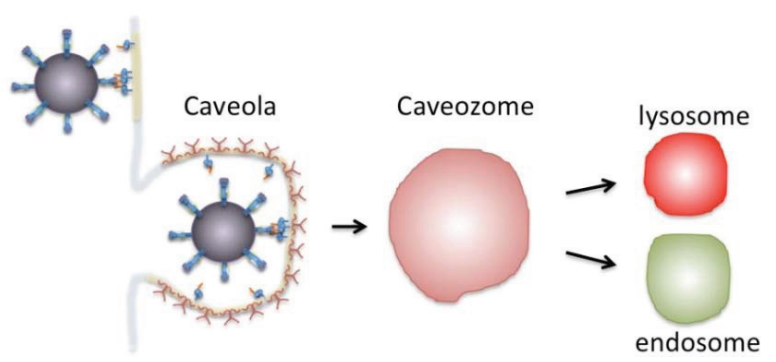


As depicted in Figure 1B, RSV binding to the cell surface is shown incorporating lipid-rich rafts or caveolae. Based on available evidence, including analogies to other pathogens, there exist a number of possibilities by which this can occur and how nucleolin may be involved. For example, nucleolin may either be located within lipid rafts or may become associated with these rafts after binding to the virus. Moreover, lipid raft formation may even be induced by the aggregation of nucleolin around the viral fusion protein. At this point either fusion occurs immediately or the virus is brought into the cell possibly via a caveosome and fusion is triggered later on. Overall, the model we show here, while incomplete, provides a framework with which these various possibilities can be tested experimentally.

\section{Future Directions}

\subsection{Targeting the Host in RSV Prophylaxis and Therapy?}

As has been demonstrated for Palivizumab [50], targeting RSV as a strategy for patient management has limitations, since resistant viral strains can arise through random genetic mutation and selective pressure. An alternative strategy is to target the host rather than the virus, the human genome being much more stable. This is not as radical an idea as it may sound if one considers that when immunizing a patient against infection the goal is "conversion" of the host to a virus-resistant state -in effect the host, not the virus, is being targeted. The challenge is to achieve an adequate anti-viral state in the host, and to avoid undesired off-target effects or other toxicity.

\subsection{Nucleolin as an Anti-RSV Target}

Nucleolin has a rapid half-life on the cell surface: exposure of cells to nucleolin-specific antibody results in rapid internalization of the nucleolin-antibody complex and replacement with "fresh" nucleolin [51]. The rapid turnover of the cell surface nucleolin may be advantageous in the context of designing RSV prophylaxis and/or therapy since it is unlikely there would be any long-lasting disruption of its normal functions. Targeting cell surface nucleolin with the guanine-rich oligonucleotide (GRO), AS1411 [52], is being evaluated in the therapy a variety of cancers in human clinical trials. Other nucleolin-binding compounds (e.g., midkine, pleiotropin, lactoferrin, pseudopeptide HB-19) have been described and in some cases, their safety profiles have already been established in humans. An important practical consideration is that, in contrast to use in chronic diseases such as cancer, nucleolin-binding compounds used for either prophylaxis or therapy of RSV infections (e.g., as a nasal spray formulation) would be administered for relatively short intervals and thus could avoid the development of some of the undesired drug-related effects associated with longer term use.

\subsection{RSV-Nucleolin Interaction Domain(s)}

Detailed inspection of the primary amino acid sequence of nucleolin does not provide any apparent clues about potential interaction domain(s) with RSV, although one might reasonably exclude the RRMs found in the central portion because these domains are known to be nucleic acid binding 
motifs [21]. While binding cell surface nucleolin with a specific compound that targets nucleolin and causing it to internalize may be sufficient to make it unavailable for binding to virus, determining the interaction domains of nucleolin and the RSV F protein will be essential for the rational design of small molecule inhibitors. Also, if cell surface nucleolin is part of a larger protein complex [29] that functions to tether it to the cell surface, one could potentially target the other proteins in this complex in order to destabilize it.

One potential site of RSV-F protein interaction with nucleolin may occur with the "head" around amino acids 429-437. This region of the protein binds mAb 19 and mAb 20, both monoclonal antibodies known to inhibit RSV fusion [53-55]. However, such associations based on antibody binding epitopes need to be interpreted cautiously as interference via an antibody may only require that it bind near enough to the interaction domain to be effective at neutralization.

Figure 2. Human airway epithelial cells from bronchial brushings stained with a rabbit polyclonal antibody against human nucleolin (H-250, Santa Cruz Biotechnology Inc., Santa Cruz, CA, USA). Note the positive nucleolin immunostaining (dark brown) at the apical surface of airway epithelial cells (arrows).

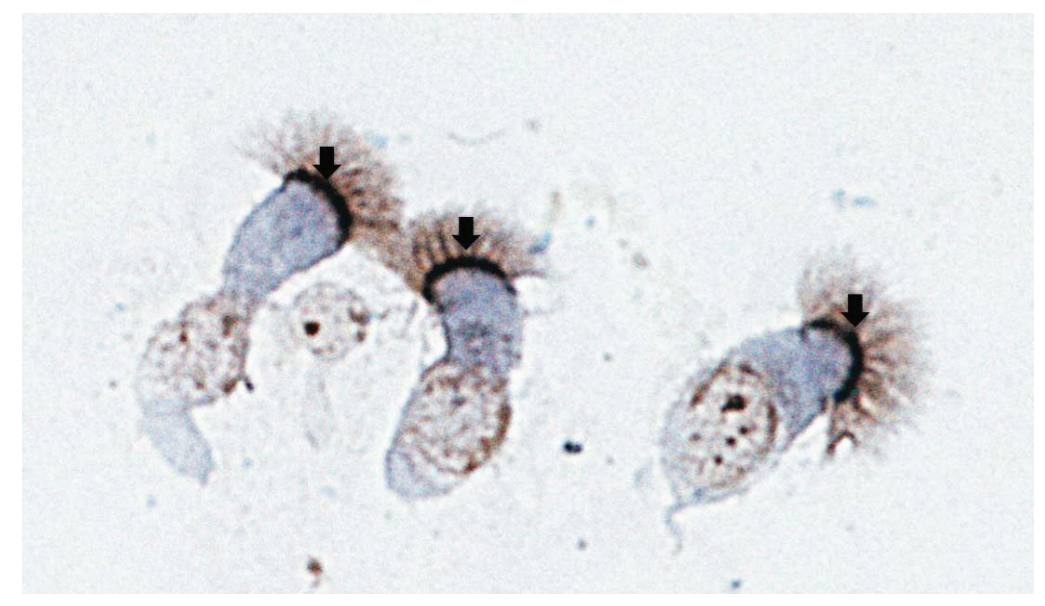

\subsection{Nucleolin and RSV Tropism}

Because nucleolin is an essential protein found in all cells, it does not by itself account for the apparent restricted tropism of RSV to the respiratory tract and while it is clearly expressed on the cell surface of airway epithelium, the major target for RSV infection, it has not yet been determined if this is the case for other cell types in vivo (Figure 2). However, this situation is not unique to RSV, as other viral receptors are widely expressed in various cells and tissues, while tropism is restricted [56]. For example, the cellular receptor of Hepatitis A virus, HAVcr-1, is expressed in various cells within the gastrointestinal tract, salivary glands, kidney, spleen and lymph nodes, yet clinically the virus shows marked tropism to the liver [57]. We speculate that the tropism of RSV for the respiratory tract could 
involve a combination of the local environment/context and possibly the involvement of more than one receptor/co-receptor, including other molecules postulated to be RSV receptors indicated above. In this context, the viral G protein may be an important determinant of RSV's tropism to the respiratory tract, in terms of the role of RSV G in initial attachment and enabling the virus to recognize nucleolin expressed on the cell surface. Given that the efficiency of RSV infection is markedly decreased in $\mathrm{RSV} \Delta \mathrm{G}$ compared to wild-type virus, a role for RSV G as a determinant of tropism is likely.

\section{Concluding Remarks}

Our discovery of nucleolin as a functional RSV fusion receptor in vitro and in vivo increases our understanding of the pathobiology of RSV infections, and presents a number of possibilities for novel interventions, including targeting RSV fusion from a "host" perspective. Although the mechanisms of RSV fusion require further study, we present a model of RSV fusion that has a number of testable assumptions and could lead to a deeper understanding of the events underlying RSV fusion and entry into cells.

\section{Conflicts of Interest}

The authors declare no conflict of interest.

\section{References}

1. Nair, H.; Nokes, D.J.; Gessner, B.D.; Dherani, M.; Madhi, S.A.; Singleton, R.J.; O'Brien, K.L.; Roca, A.; Wright, P.F.; Bruce, N.; et al. Global burden of acute lower respiratory infections due to respiratory syncytial virus in young children: A systematic review and meta-analysis. Lancet 2010, 375, 1545-1555.

2. Walsh, E.E. Respiratory syncytial virus infection in adults. Semin. Respir. Crit. Care Med. 2011, $32,423-432$.

3. Blount, R.E., Jr.; Morris, J.A.; Savage, R.E. Recovery of cytopathogenic agent from chimpanzees with coryza. Proc. Soc. Exp. Biol. Med. 1956, 92, 544-549.

4. Collins, P.L.; Melero, J.A. Progress in understanding and controlling respiratory syncytial virus: Still crazy after all these years. Virus Res. 2011, 162, 80-99.

5. Techaarpornkul, S.; Barretto, N.; Peeples, M.E. Functional analysis of recombinant respiratory syncytial virus deletion mutants lacking the small hydrophobic and/or attachment glycoprotein gene. J. Virol. 2001, 75, 6825-6834.

6. Behera, A.K.; Matsuse, H.; Kumar, M.; Kong, X.; Lockey, R.F.; Mohapatra, S.S. Blocking intercellular adhesion molecule-1 on human epithelial cells decreases respiratory syncytial virus infection. Biochem. Biophys. Res. Commun. 2001, 280, 188-195.

7. Krusat, T.; Streckert, H.J. Heparin-dependent attachment of respiratory syncytial virus (RSV) to host cells. Arch. Virol. 1997, 142, 1247-1254. 
8. Malhotra, R.; Ward, M.; Bright, H.; Priest, R.; Foster, M.R.; Hurle, M.; Blair, E.; Bird, M. Isolation and characterisation of potential respiratory syncytial virus receptor(s) on epithelial cells. Microbes Infect. 2003, 5, 123-133.

9. Marr, N.; Turvey, S.E. Role of human TLR4 in respiratory syncytial virus-induced NF-kappaB activation, viral entry and replication. Innate Immun. 2012, 18, 856-865.

10. Harcourt, J.; Alvarez, R.; Jones, L.P.; Henderson, C.; Anderson, L.J.; Tripp, R.A. Respiratory syncytial virus $\mathrm{G}$ protein and $\mathrm{G}$ protein $\mathrm{CX} 3 \mathrm{C}$ motif adversely affect $\mathrm{CX} 3 \mathrm{CR} 1+\mathrm{T}$ cell responses. J. Immunol. 2006, 176, 1600-1608.

11. Dimmock, N.J.; Easton, A.J.; Leppard, K. Introduction to Modern Virology, 6th ed.; Blackwell Pub.: Malden, MA, USA, 2007; p. 516.

12. Duan, D.; Yue, Y.; Yan, Z.; McCray, P.B., Jr.; Engelhardt, J.F. Polarity influences the efficiency of recombinant adenoassociated virus infection in differentiated airway epithelia. Hum. Gene Ther. 1998, 9, 2761-2776.

13. Techaarpornkul, S.; Collins, P.L.; Peeples, M.E. Respiratory syncytial virus with the fusion protein as its only viral glycoprotein is less dependent on cellular glycosaminoglycans for attachment than complete virus. Virology 2002, 294, 296-304.

14. Batonick, M.; Oomens, A.G.; Wertz, G.W. Human respiratory syncytial virus glycoproteins are not required for apical targeting and release from polarized epithelial cells. J. Virol. 2008, 82, 8664-8672.

15. Tayyari, F.; Marchant, D.; Moraes, T.J.; Duan, W.; Mastrangelo, P.; Hegele, R.G. Identification of nucleolin as a cellular receptor for human respiratory syncytial virus. Nat. Med. 2011, 17, 1132-1135.

16. Cao, W.; Henry, M.D.; Borrow, P.; Yamada, H.; Elder, J.H.; Ravkov, E.V.; Nichol, S.T.; Compans, R.W.; Campbell, K.P.; Oldstone, M.B. Identification of alpha-dystroglycan as a receptor for lymphocytic choriomeningitis virus and Lassa fever virus. Science 1998, 282, 2079-2081.

17. Reyes-Reyes, E.M.; Akiyama, S.K. Cell-surface nucleolin is a signal transducing P-selectin binding protein for human colon carcinoma cells. Exp. Cell Res. 2008, 314, 2212-2223.

18. Losfeld, M.E.; Khoury, D.E.; Mariot, P.; Carpentier, M.; Krust, B.; Briand, J.P.; Mazurier, J.; Hovanessian, A.G.; Legrand, D. The cell surface expressed nucleolin is a glycoprotein that triggers calcium entry into mammalian cells. Exp. Cell Res. 2009, 315, 357-369.

19. Orrick, L.R.; Olson, M.O.; Busch, H. Comparison of nucleolar proteins of normal rat liver and Novikoff hepatoma ascites cells by two-dimensional polyacrylamide gel electrophoresis. Proc. Natl. Acad. Sci. USA 1973, 70, 1316-1320.

20. Bugler, B.; Caizergues-Ferrer, M.; Bouche, G.; Bourbon, H.; Amalric, F. Detection and localization of a class of proteins immunologically related to a $100-\mathrm{kDa}$ nucleolar protein. Eur. J. Biochem. 1982, 128, 475-480.

21. Ginisty, H.; Sicard, H.; Roger, B.; Bouvet, P. Structure and functions of nucleolin. J. Cell Sci. 1999, 112, 761-772. 
22. Bicknell, K.; Brooks, G.; Kaiser, P.; Chen, H.; Dove, B.K.; Hiscox, J.A. Nucleolin is regulated both at the level of transcription and translation. Biochem. Biophys. Res. Commun. 2005, 332, $817-822$.

23. Chen, C.M.; Chiang, S.Y.; Yeh, N.H. Increased stability of nucleolin in proliferating cells by inhibition of its self-cleaving activity. J. Biol. Chem. 1991, 266, 7754-7758.

24. Srivastava, M.; Pollard, H.B. Molecular dissection of nucleolin's role in growth and cell proliferation: New insights. FASEB J. 1999, 13, 1911-1922.

25. Tajrishi, M.M.; Tuteja, R.; Tuteja, N. Nucleolin: The most abundant multifunctional phosphoprotein of nucleolus. Commun. Integr. Biol. 2011, 4, 267-275.

26. Hovanessian, A.G.; Soundaramourty, C.; El Khoury, D.; Nondier, I.; Svab, J.; Krust, B. Surface expressed nucleolin is constantly induced in tumor cells to mediate calcium-dependent ligand internalization. PLoS One 2010, 5, e15787.

27. Schwab, M.S.; Dreyer, C. Protein phosphorylation sites regulate the function of the bipartite NLS of nucleolin. EJCB 1997, 73, 287-297.

28. Hovanessian, A.G.; Puvion-Dutilleul, F.; Nisole, S.; Svab, J.; Perret, E.; Deng, J.S.; Krust, B. The cell-surface-expressed nucleolin is associated with the actin cytoskeleton. Exp. Cell Res. 2000, 261, 312-328.

29. Krust, B.; El Khoury, D.; Nondier, I.; Soundaramourty, C.; Hovanessian, A.G. Targeting surface nucleolin with multivalent HB-19 and related Nucant pseudopeptides results in distinct inhibitory mechanisms depending on the malignant tumor cell type. BMC Cancer 2011, 11, 333.

30. Chen, X.; Kube, D.M.; Cooper, M.J.; Davis, P.B. Cell surface nucleolin serves as receptor for DNA nanoparticles composed of pegylated polylysine and DNA. Mol. Ther. 2008, 16, 333-342.

31. Barel, M.; Hovanessian, A.G.; Meibom, K.; Briand, J.P.; Dupuis, M.; Charbit, A. A novel receptor-ligand pathway for entry of Francisella tularensis in monocyte-like THP-1 cells: Interaction between surface nucleolin and bacterial elongation factor Tu. BMC Microbiol. 2008, 8,145

32. Sinclair, J.F.; Dean-Nystrom, E.A.; O'Brien, A.D. The established intimin receptor Tir and the putative eucaryotic intimin receptors nucleolin and betal integrin localize at or near the site of enterohemorrhagic Escherichia coli $\mathrm{O} 157: \mathrm{H} 7$ adherence to enterocytes in vivo. Infect. Immun. 2006, 74, 1255-1265.

33. Calle, A.; Ugrinova, I.; Epstein, A.L.; Bouvet, P.; Diaz, J.J.; Greco, A. Nucleolin is required for an efficient herpes simplex virus type 1 infection. J. Virol. 2008, 82, 4762-4773.

34. Greco, A.; Arata, L.; Soler, E.; Gaume, X.; Coute, Y.; Hacot, S.; Calle, A.; Monier, K.; Epstein, A.L.; Sanchez, J.C.; et al. Nucleolin interacts with US11 protein of herpes simplex virus 1 and is involved in its trafficking. J. Virol. 2012, 86, 1449-1457.

35. Kusakawa, T.; Shimakami, T.; Kaneko, S.; Yoshioka, K.; Murakami, S. Functional interaction of hepatitis C Virus NS5B with Nucleolin GAR domain. J. Biochem. 2007, 141, 917-927.

36. Strang, B.L.; Boulant, S.; Kirchhausen, T.; Coen, D.M. Host cell nucleolin is required to maintain the architecture of human cytomegalovirus replication compartments. mBio 2012, 3, e00301-11. 
37. Cancio-Lonches, C.; Yocupicio-Monroy, M.; Sandoval-Jaime, C.; Galvan-Mendoza, I.; Urena, L.; Vashist, S.; Goodfellow, I.; Salas-Benito, J.; Gutierrez-Escolano, A.L. Nucleolin interacts with the feline calicivirus 3' untranslated region and the protease-polymerase NS6 and NS7 proteins, playing a role in virus replication. J. Virol. 2011, 85, 8056-8068.

38. Chang, A.; Dutch, R.E. Paramyxovirus fusion and entry: Multiple paths to a common end. Viruses 2012, 4, 613-636.

39. Harrison, S.C. Viral membrane fusion. Nat. Struct. Mol. Biol. 2008, 15, 690-698.

40. Nisole, S.; Krust, B.; Hovanessian, A.G. Anchorage of HIV on permissive cells leads to coaggregation of viral particles with surface nucleolin at membrane raft microdomains. Exp. Cell. Res. 2002, 276, 155-173.

41. Chang, T.H.; Segovia, J.; Sabbah, A.; Mgbemena, V.; Bose, S. Cholesterol-rich lipid rafts are required for release of infectious human respiratory syncytial virus particles. Virology 2012, 422, 205-213.

42. Chen, X.; Shank, S.; Davis, P.B.; Ziady, A.G. Nucleolin-mediated cellular trafficking of DNA nanoparticle is lipid raft and microtubule dependent and can be modulated by glucocorticoid. Mol. Ther. 2011, 19, 93-102.

43. Werling, D.; Hope, J.C.; Chaplin, P.; Collins, R.A.; Taylor, G.; Howard, C.J. Involvement of caveolae in the uptake of respiratory syncytial virus antigen by dendritic cells. J. Leukoc. Biol. 1999, 66, 50-58.

44. White, J.M.; Delos, S.E.; Brecher, M.; Schornberg, K. Structures and mechanisms of viral membrane fusion proteins: Multiple variations on a common theme. Crit. Rev. Biochem. Mol. Biol. 2008, 43, 189-219.

45. Kahn, J.S.; Schnell, M.J.; Buonocore, L.; Rose, J.K. Recombinant vesicular stomatitis virus expressing respiratory syncytial virus (RSV) glycoproteins: RSV fusion protein can mediate infection and cell fusion. Virology 1999, 254, 81-91.

46. Srinivasakumar, N.; Ogra, P.L.; Flanagan, T.D. Characteristics of fusion of respiratory syncytial virus with HEp-2 cells as measured by R18 fluorescence dequenching assay. J. Virol. 1991, 65, 4063-4069.

47. Shahrabadi, M.S.; Lee, P.W. Calcium requirement for syncytium formation in HEp-2 cells by respiratory syncytial virus. J. Clin. Microbiol. 1988, 26, 139-141.

48. Kolokoltsov, A.A.; Deniger, D.; Fleming, E.H.; Roberts, N.J., Jr.; Karpilow, J.M.; Davey, R.A. Small interfering RNA profiling reveals key role of clathrin-mediated endocytosis and early endosome formation for infection by respiratory syncytial virus. J. Virol. 2007, 81, 7786-7800.

49. Song, N.; Ding, Y.; Zhuo, W.; He, T.; Fu, Z.; Chen, Y.; Song, X.; Fu, Y.; Luo, Y. The nuclear translocation of endostatin is mediated by its receptor nucleolin in endothelial cells. Angiogenesis 2012, 15, 697-711.

50. Adams, O.; Bonzel, L.; Kovacevic, A.; Mayatepek, E.; Hoehn, T.; Vogel, M. Palivizumab-resistant human respiratory syncytial virus infection in infancy. Clin. Infect. Dis. 2010, 51, 185-188. 
51. Deng, J.S.; Ballou, B.; Hofmeister, J.K. Internalization of anti-nucleolin antibody into viable HEp-2 cells. Mol. Biol. Rep. 1996, 23, 191-195.

52. Bates, P.J.; Laber, D.A.; Miller, D.M.; Thomas, S.D.; Trent, J.O. Discovery and development of the G-rich oligonucleotide AS1411 as a novel treatment for cancer. Exp. Mol. Pathol. 2009, 86, 151-164.

53. Taylor, G.; Stott, E.J.; Furze, J.; Ford, J.; Sopp, P. Protective epitopes on the fusion protein of respiratory syncytial virus recognized by murine and bovine monoclonal antibodies. J. Gen. Virol. 1992, 73, 2217-2223.

54. Arbiza, J.; Taylor, G.; Lopez, J.A.; Furze, J.; Wyld, S.; Whyte, P.; Stott, E.J.; Wertz, G.; Sullender, W.; Trudel, M.; et al. Characterization of two antigenic sites recognized by neutralizing monoclonal antibodies directed against the fusion glycoprotein of human respiratory syncytial virus. J. Gen. Virol. 1992, 73, 2225-2234.

55. McLellan, J.S.; Yang, Y.; Graham, B.S.; Kwong, P.D. Structure of respiratory syncytial virus fusion glycoprotein in the postfusion conformation reveals preservation of neutralizing epitopes. J. Virol. 2011, 85, 7788-7796.

56. Schneider-Schaulies, J. Cellular receptors for viruses: Links to tropism and pathogenesis. J. Gen. Virol. 2000, 81, 1413-1429.

57. Asher, L.V.; Binn, L.N.; Mensing, T.L.; Marchwicki, R.H.; Vassell, R.A.; Young, G.D. Pathogenesis of hepatitis A in orally inoculated owl monkeys (Aotus trivirgatus). J. Med. Virol. 1995, 47, 260-268. 
Reprinted from Viruses. Cite as: Rivera-Toledo, E.; Gómez, B. Respiratory Syncytial Virus Persistence in Macrophages Alters the Profile of Cellular Gene Expression. Viruses 2012, 4, 3270-3280.

Review

\title{
Respiratory Syncytial Virus Persistence in Macrophages Alters the Profile of Cellular Gene Expression
}

\section{Evelyn Rivera-Toledo * and Beatríz Gómez}

Department of Microbiology and Parasitology, Faculty of Medicine, Universidad Nacional Autónoma de México, Circuito exterior s/n, Ciudad Universitaria, México D.F., C.P. 04510, Mexico; E-Mail: begomez@servidor.unam.mx

* Author to whom correspondence should be addressed; E-Mail: evelynmicro@gmail.com; Tel.: +52-55-5623-2469; Fax: +52-55-5623-2382.

Received: 23 October 2012; in revised form: 14 November 2012 / Accepted: 15 November 2012 / Published: 22 November 2012

\begin{abstract}
Viruses can persistently infect differentiated cells through regulation of expression of both their own genes and those of the host cell, thereby evading detection by the host's immune system and achieving residence in a non-lytic state. Models in vitro with cell lines are useful tools in understanding the mechanisms associated with the establishment of viral persistence. In particular, a model to study respiratory syncytial virus (RSV) persistence in a murine macrophage-like cell line has been established. Compared to non-infected macrophages, macrophages persistently infected with RSV show altered expression both of genes coding for cytokines and trans-membrane proteins associated with antigen uptake and of genes related to cell survival. The biological changes associated with altered gene expression in macrophages as a consequence of persistent RSV infection are summarized.
\end{abstract}

Keywords: Respiratory syncytial virus; viral persistence; macrophages; P388D1; altered gene expression

\section{The Virus: Characteristics, Pathogenesis, and Epidemiology}

Respiratory syncytial virus (RSV; family Paramyxoviridae, genus Pneumovirus) is a highly infectious agent-more so than other respiratory viruses - and worldwide is the principal cause of 
serious lower-respiratory tract illness in infants and young children [1]. Structurally, RSV is an enveloped and pleomorphic virus, with a single-stranded, negative-sense RNA genome encoding 11 proteins $[1,2]$. Epidemiological studies of RSV indicate that this pathogen is frequently isolated from children with bronchiolitis $[3,4]$ and is the most frequent cause of hospitalization of infants in industrialized countries [5]. Risk factors, such as premature birth, congenital heart disease, and immune deficiencies, predispose children $<6$ months of age to severe respiratory disease, thus increasing the frequency of RSV-related hospitalizations by as much as 56\% [6-8]. Most infants experience RSV infection during the first year of life and there exists an association between early severe RSV infection and recurrent wheezing or asthma in later childhood [9-11]. RSV is also an important cause of morbidity and mortality in the elderly and in immunocompromised patients $[12,13]$. In the elderly, RSV is the second leading cause of viral death, with an annual incidence up to 5\% [14]. The World Health Organization (WHO) reports 64 million cases and 160,000 deaths each year due to RSV - more than that caused by any other respiratory virus [15]. Seasonal RSV outbreaks occur each year throughout the world during the winter months: in the northern hemisphere, the annual epidemics normally start in November, peak in January and February and end in May; in the southern hemisphere, the epidemic season runs from May through September [16,17].

Prospective studies of cohorts of patients with chronic obstructive pulmonary disease (COPD) have revealed, through reverse-transcription polymerase chain reaction (RT-PCR), that RSV is the virus most frequently detected in nasopharyngeal aspirates during stable COPD and exacerbated episodes $[18,19]$. The effects of the sequelae of severe RSV disease may be explained, in part, by viral persistence, with the RSV infection causing an alteration of the airway structure and/or inducing an aberrant immune response $[9,10,19]$. Continuous stimulation of the immune system by persistent viral infections may cause chroniflanmation or alter the expression of immunoregulatory molecules [20-22]; such outcomes may explain the clinical manifestations that persist long after acute viral infection. Infected epithelial cells and macrophages secrete cytokines, chemokines, and other factors that attract lymphocytes and other cells to the site of infection, thus resulting in airway inflammation $[23,24]$.

\section{RSV Persistence}

Although RSV persistence in humans has not been demonstrated, some observations indicate that this may be the case: (1) the presence of RSV antigen in bone biopsies and in osteoclasts cultured from patients with Paget disease was detected by using immunohistological assays [25]; (2) RSV was isolated repeatedly from the nasopharynx of apparently healthy children [26]; (3) RSV nucleoprotein mRNA was detected in archival postmortem lung tissue from infants, who had died during the summer, without apparent clinical disease having been reported [27]; and (4) RSV genome has been detected in human naïve primary bone marrow stromal cells from adults (6/8) and children (3/3) [28].

Persistent RSV infection has been established in vivo in mouse and guinea pig models [29-31]. In studies using BALB/c mice, persistent RSV infection has been followed through kinetic studies, revealing that infectious virus can be isolated from bronchioalveolar fluid or lymph nodes only during 
the first 14 days post-infection, whereas in lung homogenates, viral genomic RNA and mRNA can still be detected after 100 days, even though signs of acute infection have disappeared [30]. In guinea pigs, after resolution of acute bronchiolitis and at 60 days post-infection, viral genomic RNA and RSV proteins, along with polymorphonuclear cell infiltrates, can be detected in lungs by RT-PCR and immunohistochemistry [29]. Although, in these models in vivo, the cell type that RSV is able to persistently infect has not been determined, studies in vitro indicate that RSV can establish persistent infection in epithelial cells, macrophages and dendritic cells [32-36].

The predominant cell type recovered from bronchioalveolar lavages from children with acute severe lower-respiratory tract symptoms is the alveolar macrophage; these macrophages express RSV antigens along with pro-inflammatory cytokines [37]. Also, experiments with calves acutely infected with bovine respiratory syncytial virus (BRSV), a virus closely related to RSV, indicate that upper and lower airway epithelial cells and alveolar macrophages are target cells for the virus, as they became productively infected [38]. In addition, experiments with isolated human alveolar macrophages have shown that this cell type can support prolonged RSV replication (up to 25 days post-infection) without an apparent effect on cell viability, suggesting that macrophages may be an important reservoir for RSV in vivo [39].

Succeeding in a persistent infection depends on the ability of the virus to regulate not only its own genes but also the host genes in order to avoid killing the host cell. This is achieved by an alternative viral strategy of replication and the ability to evade the immunologic surveillance system of the host. In this way, the continuous replication of a virus in a differentiated cell can alter the normal functions of said cell without destroying it; this in turn disturbs the homeostasis of the host, thus producing disease [40].

Given that macrophages are important target cells for RSV and that, once infected, they can support a persistent viral infection, this brief review is focused on alterations in the biological functions of a murine macrophage-like cell line persistently infected with RSV.

\section{Establishment and Characteristics of a Persistently RSV-Infected Macrophage-Like Culture}

A model to study the RSV persistence in macrophages was established by using the murine macrophage-like cell line, P388D1, which was derived from serial passages in mice of an original methylcholanthrene-induced lymphoid neoplasm in a DBA/2 mouse [41]. When this cell line was infected at a multiplicity of infection (m.o.i.) of 1.0 with the prototype RSV Long strain (wild-type $\mathrm{RSV}$ ), both a low frequency of syncytia and a high percentage of cell death during the first $48 \mathrm{~h}$ post infection were observed. Nevertheless, after $72 \mathrm{~h}$, the number of macrophages started to increase and the surviving cells were propagated. In the first few passages, $40 \%-60 \%$ of the cells presented viral antigen on their cell membrane; after cloning the cells by limited dilution and reinfecting the clones at an m.o.i. of 1.0, subsequent passages were stabilized, with a constant viral expression in $90 \%-95 \%$ of the cells being achieved [33]. Currently, after more than 85 passages, this line of macrophages persistently infected with RSV $(\mathrm{M} \varphi \mathrm{P})$ continues to express the viral genome: mRNA of the $\mathrm{N}$ viral 
gene is detected by RT-PCR and viral proteins are expressed on the cell membrane, as demonstrated by immunofluorescence [42].

One of the effects of persistent virus infection in immortalized cells is alteration of the viral genome, thus producing viral variants adapted for a prolonged period of replication without killing the host cell [40]. Similarly, the RSV in $\mathrm{M} \varphi \mathrm{P}$ shows genotypic changes, at least in the viral membrane fusion protein (F), compared to the wild-type RSV [43]. The genotypic change in persistent RSV was associated with a decreased fusogenic activity and was manifested by reduced size and frequency of syncytia, as well as with low extracellular viral titer in Vero cells, an RSV-permissive cell line [43]. When the deduced amino acid sequences of the F protein from the persistent and wild-type RSV were compared, changes in nine amino acids were observed, three of which are adjacent to the cleavage domain and the fusion peptide. The particular changes in the region of the cleavage domain suggest that the processing of the F0 precursor by cellular proteases may not be efficient, thus reducing its membrane fusion capacity. This hypothesis is supported by experiments in which the number of syncytia was augmented approximately five-fold when Vero cells infected with persistent RSV were cultured either in the presence of trypsin or in a low $\mathrm{pH}$ environment-conditions that have been shown to improve activation of viral fusogenic proteins [44-46]. However, it seems that the efficiency of F0 processing from persistent RSV is cell-line dependent, because when lung carcinoma cells H358 were used as target cells for the same persistent virus, neither the enzymatic nor acidic treatment improved the fusogenic activity; in fact, the fusogenic activity was similar to that obtained without treatment, indicating that the intracellular protease activation of the persistent RSV F protein is less efficient in Vero cells than in H358 cells [43].

\section{Persistent RSV Infection Alters Macrophage Gene Expression and Biological Activities}

Macrophages, important cells of the innate immune system, act as a first-line of defense against invading pathogens and help to initiate T-cell responses by processing and presenting antigens. The non-specific defense function of macrophages depends mainly on their ability to take up particulate material by phagocytosis [47]. Phagocytosis can be mediated either directly by receptors on the macrophages recognizing foreign structures of particles or indirectly by receptors that recognize self-ligands (e.g., when a foreign particle is opsonized by complement or by antibodies) [48,49].

Specific phagocytosis mediated by Fc $\gamma$ receptors (Fc $\gamma \mathrm{R}$ ) of IgG-opsonized sheep red blood cells is three- to six-fold enhanced in $\mathrm{M} \varphi \mathrm{P}$, compared to mock infected macrophages $(\mathrm{M} \varphi \mathrm{N})$; this relevant change is likely a consequence of an increased level of expression of FcyRII and Fc $\gamma$ RIII in the $\mathrm{M} \varphi \mathrm{P}$ [50]. Arrevillaga et al. [42] showed that non-opsonized phagocytosis is also altered in $\mathrm{M} \varphi \mathrm{P}$. In that work, $\mathrm{M} \varphi \mathrm{P}$ show a decreased efficiency in phagocytizing non-typeable Haemophilus influenzae (NTHi), a pathogen associated with exacerbations of COPD, with bacterial adhesion and ingestion being 1.7- and 11-fold less, respectively, than the values obtained with $\mathrm{M} \varphi \mathrm{N}$ [42]. This diminished uptake of bacteria by $\mathrm{M} \varphi \mathrm{P}$ is linked to a reduced expression ( $50 \%)$ of both the ICAM-1 mRNA and ICAM-1 protein on the cell membrane, the latter serving as a ligand to bind bacteria. Although ICAM-1 is not the only ligand for NTHi, the negative transcriptional regulation of this molecule, 
as a consequence of the persistent RSV infection, could contribute to inefficient bacterial clearance by macrophages.

Dendritic cells, macrophages, and B lymphocytes are "professional" antigen-presenting cells (APCs). Although dendritic cells and their subsets are the most potent stimulators of T lymphocytes, the relevance of particular APCs can be determined according to their abundance in a particular tissue [51]. Alveolar macrophages comprise $95 \%$ of the cells of the lung lavage with the remaining portion consisting mostly of leukocytes, thus indicating that macrophages may be important in establishing an early non-specific defense and by functioning as presenting cells to initiate the adaptive immune response in the lung [52]. A study by Guerrero-Plata et al. [53], which focused on determining whether $\mathrm{M} \varphi \mathrm{P}$ preserve their ability to present antigens, showed that persistent infection with RSV increases expression levels of alleles K and D of the MHC class-I molecules to levels similar to those obtained at 24-h post-acute infection. The augmented MHC-I expression in $\mathrm{M} \varphi \mathrm{P}$ correlates with an efficient processing and presentation of RSV antigens to RSV-specific CD8 T cells, as determined by cytotoxicity assays. Also, $\mathrm{M} \varphi \mathrm{P}$ maintain the ability to process and present other viral antigens, such as a peptide derived from the influenza virus nucleoprotein (NP147-155). In addition, the profiles of cytokine expression in supernatants of $\mathrm{M} \varphi \mathrm{P}$ and $\mathrm{M} \varphi \mathrm{N}$ cultures indicate that the cytokines IL-1 $\beta$ and IL-6 are statistically significantly augmented in the M $\varphi \mathrm{P}$, suggesting that persistent RSV infection keeps macrophages in a permanently activated state [50]. Acute RSV infection of lung epithelial cells and granulocytes induced prolonged survival of infected cells by increasing the expression of anti-apoptotic molecules of the Bcl-2 family [54,55]. M $\varphi \mathrm{P}$, under normal culture conditions, display similar viability as $M \varphi \mathrm{N}$ [56]. However, treatment of these macrophage cultures with staurosporine - an inhibitor of protein kinases, which induces cellular apoptosis in the original P388D1 cell line [57] — induces cell death of almost all $\mathrm{M} \varphi \mathrm{N}$ after $24 \mathrm{~h}$, whereas more than $75 \%$ of $\mathrm{M} \varphi \mathrm{P}$ are refractory [56]. $\mathrm{M} \varphi \mathrm{P}$ resistance to apoptosis is associated with reduced expression of the protein pro-caspase 9, although its mRNA levels are normal or even higher than in $\mathrm{M} \varphi \mathrm{N}$, suggesting that persistent infection regulates caspase 9 expression at a post-transcriptional level. Furthermore, chronic RSV infection of $\mathrm{M} \varphi \mathrm{P}$ up-regulates mRNA and the protein products of anti-apoptotic genes such as Bcl-2, Bcl-x, and XIAP, indicating that abrogation of the intrinsic pathway of apoptosis is a mechanism crucial for the establishment and maintenance of viral persistence [56]. Figure 1 summarizes changes in virus and $\mathrm{M} \varphi \mathrm{P}$ as a consequence of persistent infection. 
Figure 1. Changes in respiratory syncytial virus (RSV) and macrophages by persistent infection. RSV persistence in macrophages leads to genotypic changes, at least in the viral membrane fusion protein $\mathrm{F}$ and in the profile of cellular gene expression. Arrows indicate increase or decrease in biological activities or molecule expression.

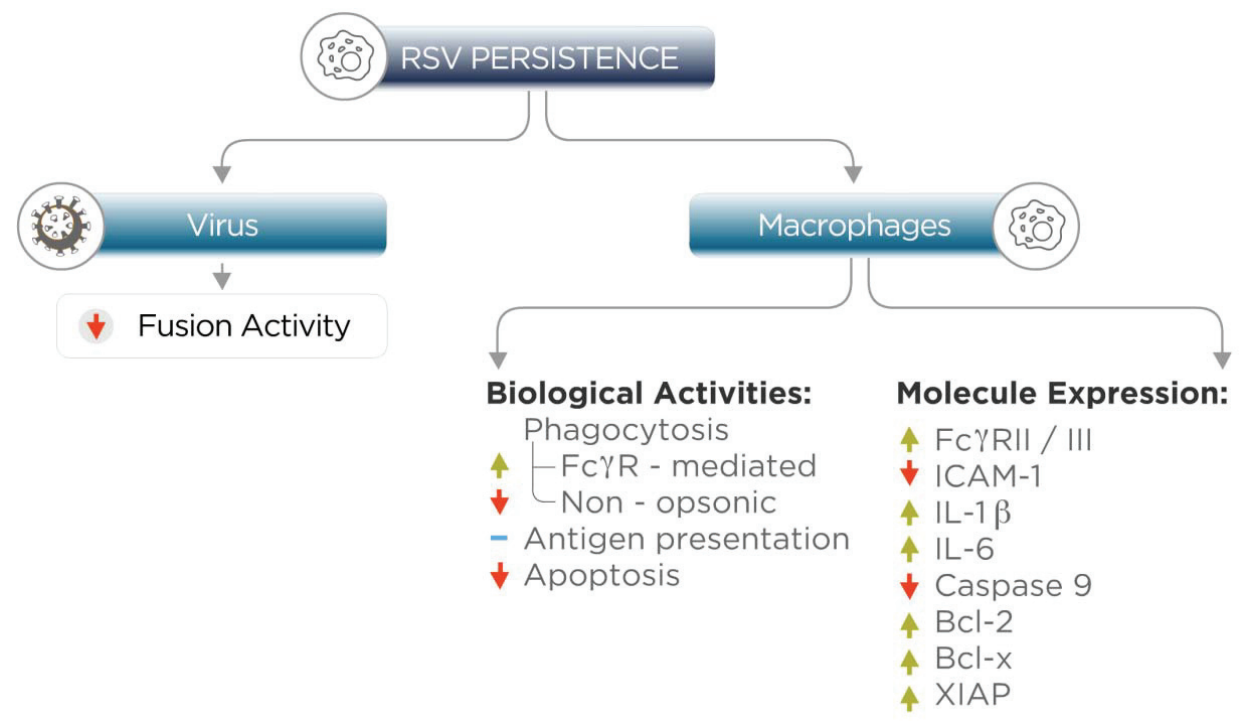

\section{Relevance of RSV Persistence in Macrophages and Epithelial Cells}

Understanding the virus-cell interactions during acute and persistent RSV infections is fundamental for the development of strategies to inhibit viral infection and to eliminate viral reservoirs. Models in vitro and in vivo have been useful tools in advancing comprehension both of the mechanisms by which RSV establishes persistence and of the pathology associated with chronic infection. Models in vitro with macrophages and epithelial cell lines have been particularly useful in determining, at the molecular level, alterations produced in the host cell by long-term RSV infection $[32,42,53,56]$. To date, in addition to $\mathrm{M} \varphi \mathrm{P}$, the only other cell model of persistent infection by RSV, in which changes in cellular gene expression have been studied, are persistently infected HEp-2 epithelial cells. Martínez et al. [32] reported that, as determined by microarray analysis, several genes with diverse functional categories were either up- or down-regulated in persistently RSV-infected HEp-2 cells. In particular, it was observed that some of the genes that were up-regulated were those involved in cell survival, such as those encoding for the anti-apoptotic molecules TRAF-1 and BIRC3, and that some of the genes that were down-regulated were pro-apoptotic genes, such as tnf- $\alpha$, bcl2111, and caspase 9 . In contrast to that in $\mathrm{M} \varphi \mathrm{P}$, persistent RSV infection in HEp-2 cells regulates caspase 9 expression at the translational level. The study also showed that, although the chemokines CCL3 and RANTES are up-regulated during acute and persistent RSV infection, the levels of these chemokines in persistently infected HEp-2 cells are up to two-fold greater than those in acutely infected HEp-2 cells. It has also 
been reported that, in a model of RSV persistence in human epithelial cells A549, the level of the cytokine IL-8, evaluated by ELISA in supernatants, is up to 2.6-fold greater than that in mock-infected cells [34]. Thus, when taken together, the findings (1) that RSV can establish persistent infection in macrophages and epithelial cell in vitro; (2) that alterations in gene expression lead to survival of persistently infected cells; and (3) that persistently infected cells produce excessive level of cytokines and chemokines that are associated with chronic inflammation lend strong support to the hypothesis that RSV persistence in patients may be a cause of chronic respiratory diseases. It is still to be determined whether altered expression of membrane molecules related to antigen uptake by macrophages occurs in models in vivo and, if so, whether such altered expression is relevant to pathogenesis.

\section{Conclusion}

RSV can productively infect macrophages in vivo and in vitro and can establish persistent infection in macrophage-like cells in vitro. The consequence of persistent RSV infection in macrophages is the altered expression of genes coding for pro-inflammatory cytokines, for trans-membrane proteins related to antigen uptake, and for those proteins related to cell survival. The evidence suggests that macrophages may be one of the cell populations that can serve as viral reservoirs for RSV in vivo. Understanding how RSV manipulates host cells during persistent infection may provide important insights into new approaches for rational drug design and vaccines.

\section{Acknowledgments}

The authors thank Enrique Graue Wiechers for his support, Andi Espinoza-Sánchez for his help in figure preparation and Veronica Yakoleff for revision of the original English version, editing of the manuscript, and helpful comments.

\section{Conflicts of Interest}

The authors declare no conflict of interest.

\section{References}

1. Cane, P.A. Molecular epidemiology of respiratory syncytial virus. Rev. Med. Virol. 2007, 11, 103-116.

2. Collins, P.L.; Graham, B.S. Viral and host factors in human respiratory syncytial virus pathogenesis. J. Virol. 2008, 82, 2040-2055.

3. Ogra, P.L. Respiratory syncytial virus: The virus, the disease and the immune response. Paediatr. Respir. Rev. 2004, 5, 119-126.

4. Hall, C.B.; Weinberg, G.A.; Iwane, M.K.; Blumkin, A.K.; Edwards, K.M.; Staat, M.A.; Auinger, P.; Griffin, M.R.; Poehling, K.A.; Erdman, D.; et al. The burden of respiratory syncytial virus infection in young children. N. Engl. J. Med. 2009, 360, 588-598. 
5. Law, B.J.; Carbonell-Estrany, X.; Simoes, E.A. An update on respiratory syncytial virus epidemiology: A developed country perspective. Respir. Med. 2002, 96, 1-7.

6. Hervás, D.; Reina, J.; Yañez, A.; del Valle, J.M.; Figuerola, J.; Hervás, J.A. Epidemiology of hospitalization for acute bronchiolitis in children: Differences between RSV and non-RSV bronchiolitis. Eur. J. Clin. Microbiol. Infect. Dis. 2012, 31, 1975-1981.

7. Langley, G.F.; Anderson, L.J. Epidemiology and prevention of respiratory syncytial virus infections among infants and young children. Pediatr. Infect. Dis. J. 2011, 30, 510-517.

8. Welliver, R.C. Review of epidemiology and clinical risk factors for severe respiratory syncytial virus (RSV) infection. J. Pediatr. 2003, 143, 112-117.

9. Mejías, A.; Chávez-Bueno, S.; Ramilo, O. Respiratory syncytial virus pneumonia: Mechanisms of inflammation and prolonged airway hyperresponsiveness. Curr. Opin. Infect. Dis. 2005, 18, 199-204.

10. Sigurs, N.; Gustafsson, P.M.; Bjarnason, R.; Lundberg, F.; Schmidt, S.; Sigurbergsson, F.; Kjellman, B. Severe respiratory syncytial virus bronchiolitis in infancy and asthma and allergy at age 13. Am. J. Respir. Crit. Care Med. 2005, 171, 137-141.

11. Staat, M.A. Respiratory syncytial virus infections in children. Semin. Respir. Infect. 2002, 17, 15-20.

12. Dowell, S.F.; Anderson, L.J.; Gary, H.E., Jr.; Erdman, D.D.; Plouffe, J.F.; File, T.M., Jr.; Marston, B.J.; Breiman, R.F. Respiratory syncytial virus is an important cause of community-acquired lower respiratory infection among hospitalized adults. J. Infect. Dis. 1996, 174, 456-462.

13. Falsey, A.R.; Hennessey, P.A.; Formica, M.A.; Cox, C.; Walsh, E.E. Respiratory syncytial virus infection in elderly and high-risk adults. N. Engl. J. Med. 2005, 352, 1749-1759.

14. Falsey, A.R.; Walsh, E.E. Respiratory syncytial virus infection in adults. Clin. Microbiol. Rev. 2000, 13, 371-384.

15. World Health Organization. Initiative for Vaccine Research: Respiratory Syncytial Virus. Available online: http://www.who.int/vaccine_research/diseases/ari/en/index3.html (accessed on 22 August 2012).

16. Bhatt, J.M.; Everard, M.L. Do environmental pollutants influence the onset of respiratory syncytial virus epidemics or disease severity? Paediatr. Respir. Rev. 2004, 5, 333-338.

17. Collins, P.L.; Melero, J.A. Progress in understanding and controlling respiratory syncytial virus: Still crazy after all these years. Virus Res. 2011, 162, 80-99.

18. Seemungal, T.; Harper-Owen, R.; Bhowmik, A.; Moric, I.; Sanderson, G.; Message, S.; Maccallum, P.; Meade, T.W.; Jeffries, D.J.; Johnston, S.L.; et al. Respiratory viruses, symptoms, and inflammatory markers in acute exacerbations and stable chronic obstructive pulmonary disease. Am. J. Respir. Crit. Care Med. 2001, 164, 1618-1623.

19. Sikkel, M.B.; Quint, J.K.; Mallia, P.; Wedzicha, J.A.; Johnston, S.L. Respiratory syncytial virus persistence in chronic obstructive pulmonary disease. Pediatr. Infect. Dis. J. 2008, 27, 63-70.

20. Kim, E.Y.; Battaile, J.T.; Patel, A.C.; You, Y.; Agapov, E.; Grayson, M.H.; Benoit, L.A.; Byers, D.E.; Alevy, Y.; Tucker, J.; et al. Persistent activation of an innate immune response translates respiratory viral infection into chronic lung disease. Nat. Med. 2008, 14, 633-640. 
21. Di Rosa, F.; Barnaba, V. Persisting viruses and chronic inflammation: Understanding their relation to autoimmunity. Immunol. Rev. 1998, 164, 17-27.

22. Wald, O.; Weiss, I.D.; Galun, E.; Peled, A. Chemokines in hepatitis C virus infection: Pathogenesis, prognosis and therapeutics. Cytokine 2007, 39, 50-62.

23. Culley, F.J.; Pennycook, A.M.; Tregoning, J.S.; Hussell, T.; Openshaw, P.J. Differential chemokine expression following respiratory virus infection reflects Th1- or Th2-biased immunopathology. J. Virol. 2006, 80, 4521-4527.

24. Krishnan, S.; Halonen, M.; Welliver, R.C. Innate immune responses in respiratory syncytial virus infections. Viral Immunol. 2004, 17, 220-233.

25. Mills, B.G.; Singer, F.R.; Weiner, L.P.; Holst, P.A. Immunohistological demonstration of respiratory syncytial virus antigens in Paget disease of bone. Proc. Natl. Acad. Sci. USA 1981, 78, 1209-1213.

26. Isaia G.; Teodosiu, O.; Popescu, G.; Athanasiu, P.; Sternberg, I.; Dumitriu, Z. Persistence of viruses in the nasopharynx of apparently healthy children aged $0-5$ years. Results of investigations performed in 1982-83. Virologie 1985, 36, 175-179.

27. Cubie, H.A.; Duncan, L.A.; Marshall, L.A.; Smith, N.M. Detection of respiratory syncytial virus nucleic acid in archival postmortem tissue from infants. Pediatr. Pathol. Lab. Med. 1997, 17, 927-938.

28. Rezaee, F.; Gibson, L.F.; Piktel, D.; Othumpangat, S.; Piedimonte, G. Respiratory syncytial virus infection in human bone marrow stromal cells. Am. J. Respir. Cell Mol. Biol. 2011, 45, 277-286.

29. Hegele, R.G.; Hayashi, S.; Bramley, A.M.; Hogg, J.C. Persistence of respiratory syncytial virus genome and protein after acute bronchiolitis in guinea pigs. Chest 1994, 105, 1848-1854.

30. Schwarze, J.; O’Donnell, D.R.; Rohwedder, A.; Openshaw, P.J. Latency and persistence of respiratory syncytial virus despite T cell immunity. Am. J. Respir. Crit. Care Med. 2004, 169, 801-805.

31. Sutton, T.C.; Tayyari, F.; Khan, M.A.; Manson, H.E.; Hegele, R.G. T helper 1 background protects against airway hyperresponsiveness and inflammation in guinea pigs with persistent respiratory syncytial virus infection. Pediatr. Res. 2007, 61, 525-529.

32. Martínez, I.; Lombardía, L.; Herranz, C.; García-Barreno, B.; Domínguez, O.; Melero, J.A. Cultures of HEp-2 cells persistently infected by human respiratory syncytial virus differ in chemokine expression and resistance to apoptosis as compared to lytic infections of the same cell type. Virology 2009, 388, 31-41.

33. Sarmiento, R.E.; Tirado, R.; Gómez, B. Characteristics of a respiratory syncytial virus persistently infected macrophage-like culture. Virus Res. 2002, 84, 45-58.

34. Tirado, R.; Ortega, A.; Sarmiento, R.E.; Gómez, B. Interleukin-8 mRNA synthesis and protein secretion are continuously up-regulated by respiratory syncytial virus persistently infected cells. Cell Immunol. 2005, 233, 61-71.

35. Valdovinos, M.R.; Gómez, B. Establishment of respiratory syncytial virus persistence in cell lines: Association with defective interfering particles. Intervirology 2003, 46, 190-198. 
36. Hobson, L.; Everard, M.L. Persistent of respiratory syncytial virus in human dendritic cells and influence of nitric oxide. Clin. Exp. Immunol. 2008, 151, 359-366.

37. Midulla, F.; Villani, A.; Panuska, J.R.; Dab, I.; Kolls, J.K.; Merolla, R.; Ronchetti, R. Respiratory syncytial virus lung infection in infants: Immunoregulatory role of infected alveolar macrophages. J. Infect. Dis. 1993, 168, 1515-1519.

38. Castleman, W.L.; Lay, J.C.; Dubovi, E.J.; Slauson, D.O. Experimental bovine respiratory syncytial virus infection in conventional calves: Light microscopic lesions, microbiology, and studies on lavaged lung cells. Am. J. Vet. Res. 1985, 46, 547-553.

39. Panuska, J.R.; Cirino, N.M.; Midulla, F.; Despot, J.E.; McFadden, E.R., Jr.; Huang, Y.T. Productive infection of isolated human alveolar macrophages by respiratory syncytial virus. J. Clin. Investig. 1990, 86, 113-119.

40. Oldstone, M.B. Viral persistence: Parameters, mechanisms and future predictions. Virology 2006, 344, 111-118.

41. Koren, H.S.; Handwerger, B.S.; Wunderlich, J.R. Identification of macrophage-like characteristics in a cultured murine tumor line. J. Immunol. 1975, 114, 894-897.

42. Arrevillaga, G.; Gaona, J.; Sánchez, C.; Rosales, V.; Gómez, B. Respiratory syncytial virus persistence in macrophages downregulates intercellular adhesion molecule-1 expression and reduces adhesion of non-typeable haemophilus influenzae. Intervirology 2012, 55, 442-450.

43. Sarmiento, R.E.; Arias, C.F.; Méndez, E.; Gómez, B. Characterization of a persistent respiratory syncytial virus showing a low-fusogenic activity associated to an impaired F protein. Virus Res. 2009, 139, 39-47.

44. Matsuyama, S.; Delos, S.E.; White, J.M. Sequential roles of receptor binding and low $\mathrm{pH}$ in forming prehairpin and hairpin conformations of a retroviral envelope glycoprotein. J. Virol. 2004, 78, 8201-8209.

45. Skehel, J.J.; Wiley, D.C. Receptor binding and membrane fusion in virus entry: The influenza hemagglutinin. Annu. Rev. Biochem. 2000, 69, 531-569.

46. Thoennes, S.; Li, Z.N.; Lee, B.J.; Langley, W.A.; Skehel, J.J.; Russell, R.J.; Steinhauer, D.A. Analysis of residues near the fusion peptide in the influenza hemagglutinin structure for roles in triggering membrane fusion. Virology 2008, 370, 403-414.

47. Hume, D.A. The mononuclear phagocyte system. Curr. Opin. Immunol. 2006, 18, 49-53.

48. Swanson, J.A.; Hoppe, A.D. The coordination of signaling during Fc receptor-mediated phagocytosis. J. Leukoc. Biol. 2004, 76, 1093-1103.

49. Van Lookeren Campagne, M.; Wiesmann, C.; Brown, E.J. Macrophage complement receptors and pathogen clearance. Cell. Microbiol. 2007, 9, 2095-2102.

50. Guerrero-Plata, A.; Ortega, E.; Gomez, B. Persistence of respiratory syncytial virus in macrophages alters phagocytosis and pro-inflammatory cytokine production. Viral Immunol. 2001, 14, 19-30.

51. Liu, K.; Nussenzweig, M.C. Origin and development of dendritic cells. Immunol. Rev. 2010, 234, $45-54$. 
52. Guth, A.M.; Janssen, W.J.; Bosio, C.M.; Crouch, E.C.; Henson, P.M.; Dow, S.W. Lung environment determines unique phenotype of alveolar macrophages. Am. J. Physiol. Lung Cell. Mol. Physiol. 2009, 296, 936-946.

53. Guerrero-Plata, A.; Ortega, E.; Ortíz-Navarrete, V.; Gómez, B. Antigen presentation by a macrophage-like cell line persistently infected with respiratory syncytial virus. Virus Res. 2004, 99, 95-100.

54. Lindemans, C.A.; Coffer, P.J.; Schellens, I.M.; de Graaff, P.M.; Kimpen, J.L.; Koenderman, L. Respiratory syncytial virus inhibits granulocyte apoptosis through a phosphatidylinositol 3-kinase and NF-kappaB-dependent mechanism. J. Immunol. 2006, 176, 5529-5537.

55. Monick, M.M.; Cameron, K.; Staber, J.; Powers, L.S.; Yarovinsky, T.O.; Koland, J.G.; Hunninghake, G.W. Activation of the epidermal growth factor receptor by respiratory syncytial virus results in increased inflammation and delayed apoptosis. J. Biol. Chem. 2005, 280, 2147-2158.

56. Nakamura-López, Y.; Villegas-Sepúlveda, N.; Sarmiento-Silva, R.E.; Gómez, B. Intrinsic apoptotic pathway is subverted in mouse macrophages persistently infected by RSV. Virus Res. 2011, 158, 98-107.

57. Nakamura-López, Y.; Sarmiento-Silva, R.E.; Moran-Andrade, J.; Gómez-García, B. Staurosporine-induced apoptosis in P388D1 macrophages involves both extrinsic and intrinsic pathways. Cell Biol. Int. 2009, 33, 1026-1031. 
Review

\title{
Th17 Lymphocytes in Respiratory Syncytial Virus Infection
}

\author{
Jonas Bystrom ${ }^{1, *}$, Nasra Al-Adhoubi ${ }^{2}$, Mohammed Al-Bogami ${ }^{1}$, Ali S. Jawad ${ }^{2}$ and \\ Rizgar A. Mageed ${ }^{1}$
}

1 Bone and Joint Research Unit, William Harvey Research Institute, Queen Mary University of London, London EC1M 6BQ, UK;

E-Mails: m.al-bogami@qmul.ac.uk (M.A.-B.); r.a.mageed@qmul.ac.uk (R.A.M.)

2 Department of Rheumatology, The Royal London Hospital, Mile End, Barts and The London, Queen Mary University of London, London EC1M 6BQ, UK;

E-Mails: nasrak2004@yahoo.com (N.A.-A.); alismjawad1@hotmail.com (A.S.J.)

* Author to whom correspondence should be addressed; E-Mail: j.bystrom@qmul.ac.uk; Tel.: +44-20-7882-2473; Fax: +44-20-7882-6121.

Received: 23 January 2013; in revised form: 22 February 2013 / Accepted: 25 February 2013 /

Published: 5 March 2013

\begin{abstract}
Infection by respiratory syncytial virus (RSV) affects approximately 33 million infants annually worldwide and is a major cause of hospitalizations. Helper T lymphocytes (Th) play a central role in the immune response during such infections. However, Th lymphocytes that produce interleukin 17 (IL-17), known as Th17 lymphocytes, in addition to been protective can also cause pathology that accompany this type of infection. The protective effects of Th17 is associated with better prognosis in most infected individuals but heightened Th17 responses cause inflammation and pathology in others. Studies employing animal models have shown that activated Th17 lymphocytes recruit neutrophils and facilitate tertiary lymphoid structure development in infected lungs. However, IL-17 also inhibits the ability of $\mathrm{CD}^{+}$lymphocytes to clear viral particles and acts synergistically with the innate immune system to exacerbate inflammation. Furthermore, IL-17 enhances IL-13 production which, in turn, promotes the activation of Th2 lymphocytes and excessive mucus production. Studies of animal models have also shown that a lack of, or inadequate, responses by the Th1 subset of $\mathrm{T}$ lymphocytes enhances Th17-mediated responses and that this is detrimental during RSV co-infection in experimental asthma. The available evidence, therefore, indicates that Th17 can play contradictory roles during RSV infections. The factors that determine the shift in the
\end{abstract}


balance between beneficial and adverse Th17 mediated effects during RSV infection remains to be determined.

Keywords: RSV; pneumovirus; mucus; interleukin 17; interleukin 23; interleukin 13; Th17

\section{Th17 Lymphocytes and IL-17 and the Immune System-Basic biology}

Th17 lymphocytes play a central role in host defences against a range of extracellular pathogens including bacteria, viruses and fungi [1-3]. This subset of helper T lymphocytes differs from the other subsets, Th1, Th2 and regulatory T lymphocytes (T-reg lymphocytes) both in their requirements for differentiation and expansion factors and in their targets pathogens. In addition, excess Th17 lymphocyte numbers have been associated with inflammatory autoimmune diseases [4-6]. In contrast to Th1 and Th2 lymphocytes which predominantly produce interferon gamma (IFN $\gamma$ ) and IL-4/IL-5/IL13, Th17 lymphocytes produce IL-17A, IL-17F, IL-21 and IL-22. IL-17A and IL-17F are members of IL-17 family of cytokines which includes six members; IL-17A-F. All members of this family are involved in inflammatory responses; however, only IL-17A, F and E (IL-25) are produced by haematopoietic cells. IL-17A and IL-17F show 50\% homology and both bind IL-17 receptor (IL-17R) which is a complex of IL-17RA and IL-17RC. IL-17A binds with higher affinity to the IL-17RA/C and induces stronger intracellular signalling than IL-17F. Both IL-17A and F are active as dimers; homodimers and heterodimers [7]. The studies reviewed in this article are mainly about IL-17A and this cytokine will thereafter be referred to as IL-17.

IL-17 acts on stromal cells to promote the production chemokines such as CXCL1, IL-8, CCL20 (MIP-3 $\alpha$ ) and IL-6 which then promote neutrophil recruitment to sites of infection (see Table 1) [8]. IL-17 itself is a weak inducer of these cytokines/chemokines but acts by stabilizing mRNA transcripts induced by other cytokines [9]. IL-17 also down-regulates micro-RNA 23b (miR-23b), which negatively regulates inflammatory responses [10]. Furthermore, IL-17 induces mucus production in the respiratory tract and increases the expression of polymeric Ig receptors that facilitate the release of IgA and IgM antibodies into the respiratory tract [11]. Of the other cytokines produced by Th17 lymphocytes, IL-21 promotes Th17 proliferation and antibody production by B lymphocytes [12]. Paradoxically, however, Il-21 also antagonizes some IL-17-mediated responses during RSV infection [13]. IL-22, in contrast, promotes mucosal homeostasis and induces the production of antibacterial peptides [14].

The production of low-levels of IL-17 by resident Th17 lymphocytes is necessary for maintaining immunological homeostasis in the gut. This occurs under the influence of IL-1 $\beta$ and transforming growth factor beta (TGF $\beta$ ) that are produced by gut epithelial cells [15]. During inflammation, IL-6 and prostaglandin $\mathrm{E}_{2}\left(\mathrm{PGE}_{2}\right)$ are produced and these induce IL-23 receptor expression which is necessary for the differentiation of naive $\mathrm{CD}^{+} \mathrm{T}$ lymphocytes to Th17 lymphocytes [16,17]. The differentiation of Th17 cells involves an intricate network of cytokines and transcription factors predominant among which is the retinoic orphan receptor gamma $\mathrm{t}(\mathrm{ROR} \gamma \mathrm{t})$ and retinoic acid receptor 
alpha $(\mathrm{RAR} \alpha)$ [18]. Interestingly, recent studies have revealed that low level CD3/TCR engagement, as compared with high level receptor engagement, preferentially promotes human Th17 differentiations and the effect is mediated through activating the NFAT-1 transcription factor $[19,20]$. Hypoxia also promotes Th17 differentiation through the hypoxia-inducible factor alpha (HIF-1 $\alpha$ ) transcription factor which binds to the promoter of ROR $\gamma$ t in naïve T lymphocytes [21,22]. The production of IL-23, in contrast, is associated with the expansion of Th17 lymphocytes in pathogenic settings such as in autoimmune disease [23]. In this respect, IL-23 production by DCs during RSV infection has been suggested to be responsible for Th17 propagation and exacerbated inflammation associated with the infection $[24,25]$.

\section{Th17 Lymphocytes in the Respiratory Tract}

Th17 lymphocytes are present in the respiratory tract and there is evidence that they play a key role in responses to fungal infections. These cells, however, also contribute to inflammatory disorders that afflict the respiratory tract, such as asthma and chronic obstructive pulmonary disease (COPD). Increased production of the Th17-related cytokines, such as IL-17A, IL-22 and IL-23 in COPD patients reflects the involvement of Th17 lymphocytes in initiating and driving the disease process $[26,27]$. In addition, excess IL-17 production has been reported in animal models and human patients has been associated with neutrophil dominated asthma and with cortisone-resistant severe airway and hyper-reactivity (AHR) [28,29]. Although both IL-17A and IL-17F have been shown to play a role in asthma, studies of gene knockout mice have suggested that IL-17F may in fact ameliorate the disease process [30]. Th17 lymphocytes have also been implicated in effector mechanisms triggered in response to RSV and other types of respiratory viral infections $[31,32]$.

\section{IL-17 and Th17 Lymphocytes in Human RSV Infection}

\subsection{The Immune Response at the Onset of RSV Infection}

Worldwide, infants are affected by lower respiratory tract infections caused by RSV. Although many such infections have a mild course, in certain infants the infection leads to bronchiolitis needing hospitalization and respiratory support in an intensive care unit [31,34,35]. Inhaled RSV particles bind glucose amino glycans on respiratory epithelial cells through their glycoproteins, major attachment protein $\mathrm{G}$ and fusion protein $\mathrm{F}$. The particles then fuse with the cells and initiate their propagation and spreading [36,37]. There is evidence that cells other than epithelial cells, including macrophages and dendritic cells (DCs), are also infected by the virus [38,39]. Infected epithelial cells initially respond either by releasing acute phase proteins or promoting their production, such as causing complement component $\mathrm{C} 3$ activation and the release of its pro-anaphylactic factor C3a [40]. The subsequent response to the infection is of innate immune-type resulting in the influx of neutrophils which become the dominant cells during the first four days of infection $[41,42]$. 
Figure 1. IL-17 mediated responses in the respiratory tract during RSV infections. RSV virus particles infect ciliated epithelial cells in the lower respiratory tract. C3a and other mediators of inflammation are then released from epithelial cells in response to the infection and this, in turn, induces IL-17 production (dark blue colour). During the early phase of the response to RSV infection, IL-17 is produced by CD11 $\mathrm{b}^{+}$innate immune cells. Subsequently, the production of IL-17 is predominantly by CD4 ${ }^{+}$Th17 lymphocytes. The production of IL-17 initiates a number of effects in the respiratory tract. Thus, IL-17 induces mild inflammation and exacerbates inflammatory responses triggered by other signals and cytokines. In this scenario, single stranded RNA in RSV particles bind to TLR3 and synergize with IL-17 to induce IL-6 (orange colour) and IL-8 (blue colour) by fibroblasts $(\mathrm{Fc})$. The binding of double stranded RNA to TLR7, however, is inhibitory to IL-17-mediated responses and, instead, promotes Th1-mediated responses. IL-17 co-operates with IL-1 $\beta$ and TNF $\alpha$ to induce the release of chemokines that mediate neutrophil recruitment. Furthermore, IL-17 induces mucus production from epithelial cells. IL-17 also binds receptors on $\mathrm{CD}^{+} \mathrm{T}$ lymphocytes and inhibits their ability to reduce viral load. Cytokines produced by Th1 and Th2 lymphocytes, IFN $\gamma$ and IL-13, in contrast, inhibit IL-17 production [25,33].

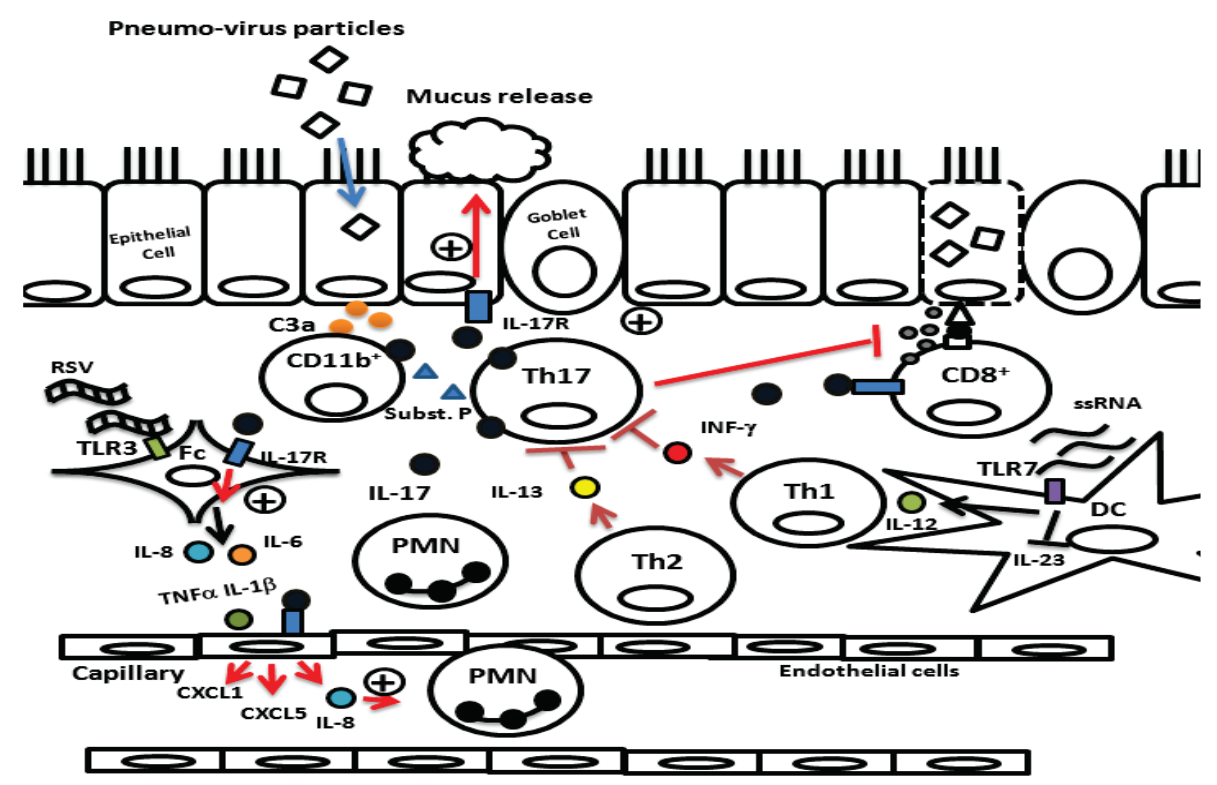

Figure: IL-17 responses in the respiratory tract during RSV infections.

\subsection{The Adaptive Immune Response to RSV Infection}

RSV infection activates $\mathrm{T}$ lymphocytes in lung draining lymph nodes with the help of DCs that migrate from sites of infection. This results in the induction and differentiation of $\mathrm{T}$ lymphocytes 
into viral-specific Th1 and Th2 lymphocytes in detectable numbers in the lung 6-8 days post RSV infection $[43,46] . \mathrm{CD}^{+} \mathrm{T}$ lymphocytes emerge after the initial inflammatory response that follows RSV infection to clear viral particles [42]. Th1 lymphocytes are also induced and these produce pro-inflammatory cytokines, such as IFN $\gamma$ and $\mathrm{TNF} \alpha$ and the combination of the two cellular responses efficiently clears RSV infections in most individuals [44]. However, humans and mice deficient in the transcription factor STAT1, which is activated following IFN $\gamma$ binding to its receptor, are prone to severe RSV infections [45,46]. In addition to cytotoxic and Th1 lymphocytes, the immune response also includes Th2 lymphocytes which cause key symptomatic features of RSV infections, such as excessive mucus production and wheezing that normally accompany asthma [47]. Measurement of cytokines in the bronchoalveolar lavage (BAL) of infected infants (1.5-6 months of age) has identified similar levels of Th1- and Th2-type cytokines [48,49]. In animal models of RSV infection, the lack of Th1 effects resulting from IFN $\gamma$ receptor deletion leads to a dominant Th2 response and worse pathology suggesting that Th1 responses ameliorate Th2-mediated effects during RSV infection in humans [50]. A recent study analysing the cytokine profile in the plasma of RSV infected infants (6 months or younger), however, revealed that infants with a moderate response to the virus had higher plasma levels of IL-17 than infants with a severe response to RSV. In this study IFN $\gamma$ and TNF $\alpha$ levels were shown to be lower in RSV infected infants than in control infants [51]. Furthermore, IL-17 levels were higher in BAL from paediatric patients (13 months and below) with non-ventilated RSV disease at admission and at discharge compared with BAL from more severe, ventilated cases. IFN $\gamma$ was undetectable in this study but IL-6 levels were 30 times higher in the ventilated cases [31]. A further study which examined tracheal aspirates reported increased IL-6 and IL-17 levels in severely ill ventilated infants compared with healthy infants (age not specified in this study) [32]. It is currently unknown what the function of IL-17 is in the respiratory tract and why higher levels are associated with better outcome in some but not all infected infants. One clue could be that the immune system of the newly born is immature with an impaired Th1 response [52]. DCs derived from infants' umbilical cord blood have, for example, been shown to produce low levels of IL-12 [53]. Furthermore, DCs from cord blood of newly born, but not DCs from the blood of adults, when infected with RSV induced IL-17 production when co-cultured with T lymphocytes [39]. DCs from infected infants were shown to produce TGF $\beta$, a cytokine known to promote Th17 lymphocyte differentiation [39]. Furthermore, co-culturing adult $\mathrm{T}$ lymphocytes with supernatants from human bronchial epithelial cells chronically-infected with the RSV A2 long strain promoted the differentiation of naïve $\mathrm{T}$ lymphocytes to Th2 and Th17 lymphocytes but not to Th1 lymphocytes [54]. These studies of $\mathrm{T}$ lymphocyte responses during RSV infection indicate that besides Th1 and Th2 responses, Th17 responses also occur. These studies, therefore, suggest that the Th17 response is beneficial in some cases of RSV infection. Th17 responses have, however, also been linked with pathology in the respiratory tract during severe neutrophil dominated asthma. More research is, therefore, needed to unravel the complex consequences of IL-17 production and when this is beneficial, when not and why. 


\section{The Role of Th17 Lymphocytes in the Patho-Physiology of RSV Infection: Some Insights from in Vitro and Animal Model Studies}

To better understand the role that Th17 lymphocytes play in the patho-physiology of RSV infection animal models and in vitro systems have been studied. Different strains of RSV were used to immunize mice and assess the immune response to RSV infection. In addition, cell lines were used to assess the direct effect viral particles have on immune cells. RSV strains "A2" and "A2 long" were used to immunize mice to define the nature of the response including immune cell infiltration during RSV infection. The RSV strain "line 19", by contrast, was used to study cellular and molecular mechanisms involved in excessive mucus secretion and IL-13 production. Studies using the three strains of RSV revealed that infection induces IL-17 production in mice. However, no studies have been carried out to compare and contrast the responses initiated by the three strains in the same experiment.

\subsection{Infection with RSV Induces IL-17 Production which Promotes Neutrophil Influx during the Early Response}

Immunization of wild-type mice with RSV strain A2 resulted in complement activation by infected epithelial cells leading to the production of $\mathrm{C} 3 \mathrm{a}$ which induced tachykinin and Substance $\mathrm{P}$ release. These mediators bound to their receptors on $\mathrm{T}$ lymphocytes, neutrophils and monocytes and lead to IL-17A production by $\mathrm{CD} 11 \mathrm{~b}^{+}$myeloid cells during the early phase of the response [55]. In addition, RSV RNA particles trigger innate immune system-mediated inflammatory responses by binding to TLRs. Studies on the interaction between viral particles and cell lines in vitro revealed that IL-17 acted synergistically with RSV RNA particles to induce IL-6 and IL-8 production by fibroblasts. Thus, RSV strain A2 RNA induced an innate immune-like response by binding to TLR3 and this response was enhanced by IL-17 [56]. Furthermore, IL-17 produced during the infection increased the influx of neutrophils. The influx of neutrophils was also observed following infection with the RSV strain A line 19. In this setting neutrophils were recruited by IL-8 which was induced by IL-17 [32] (Figure 1).

The impact of RSV infection on the cooperation between the innate system and IL-17/IL-17 producing cells is, however, complex. Thus, in addition to binding TLR3, single stranded RSV RNA particles bind TLR7 on DCs, plasmacytoid DCs, B lymphocytes and macrophages and induce IL-12 production, which promotes Th1-mediated responses (Figure 1). This can impact the balance between Th1 and Th17 responses and the patho-physiological response in vivo. For example, infection of TLR7 deficient mice with RSV Strain A line 19 resulted in increased numbers of Th17 lymphocytes due to an increase in IL-23 production by DCs. This response caused more pathology through the consequent increase in IL-13 and mucus production in TLR7 deficient mice compared with wild-type mice [24]. Furthermore, the inflammatory response to the mouse homologue of RSV, pneumo virus of mice (PVM), was diminished when TLR7 was missing [57]. This is somewhat analogous to the situation in newly born infants in whom effector Th17 lymphocytes are recruited when the activation of Th1 responses is inadequate. 


\subsection{Activation of Th17 and Th2 Cells in Response to RSV Infection}

An efficient immune response to RSV infection is dependent on antigen recognition and presentation by DCs in local lymph nodes. Viral antigen presentation by DCs results in the activation and migration of $\mathrm{CD}^{+}$and $\mathrm{CD}^{+}$lymphocytes to the lung. The process of DC migration is dependent on the chemokine CCR7. Kallal and colleagues noted that CCR7 deficient mice had impaired lymph node formation and, instead, responded by activating $\mathrm{T}$ lymphocytes in local ectopic lymphoid structures in response to infection with RSV Strain A, line 19. The T lymphocyte response to RSV infection in these structures was dominated by Th17 lymphocytes. These Th17 lymphocytes promoted pathology by inducing the production of IL-13 and IL-21 which induced excessive mucus production [58]. A similar response was observed in the absence of IFN $\gamma$ signaling in STAT1-deficient mice. Thus, infection of STAT1-deficient mice with RSV strain A2 resulted in elevated IL-13 and IL-17 levels, production of excess mucus and airway inflammation. Just as was the case in TLR $7^{-/}$mice, elevated IL-17 levels in STAT1-deficient mice were due to increased production of IL-23 [25]. In addition, to the inflammatory effects mediated by excess IL-17, it suppressed the ability of $\mathrm{CD}^{+}$to kill cells infected with RSV [59]. This latter study revealed that Th17-derived IL-17 bound to IL-17RA on $\mathrm{CD}^{+} \mathrm{T}$ lymphocytes and impaired their ability to reduce viral load and reduce the number of infected cells in the lung. The role of excess IL-17 in promoting pathology is further supported by the ability of neutralizing anti-IL-17 antibodies to reduce mucus and IL-13 production and increase viral clearance [36] (Table 1 and Figure 1). In vitro studies revealed that IL-17 enhanced mucus production by directly upregulating transcription of the mucus gene $M U C 5 B$ in human tracheal and bronchial epithelial cell lines $[60,61]$. This upregulation of the MUC5B gene was shown to be dependent on ERK signalling and the activation NF- $\kappa \mathrm{B}[60,61]$

A number of studies in which mice were infected with RSV particles have shown that the mice simultaneously produced IL-17 and IL-13 suggesting that the Th17 response is concomitant with the Th2 response $[25,32,54,58]$. The molecular mechanisms that underpin the co-production of IL-13 and IL-17 were further explored in STAT1-deficient mice. Newcomb et al. observed that IL-13 produced during infection of mice with RSV Strain A2 was capable, perhaps paradoxically, of suppressing IL-17 production [73]. Using double STAT1- and IL-13-deficient mice for immunization experiments, these investigators observed higher levels of IL-17 production than in mice deficient in STAT1 alone. Increased IL-17 production in STAT1/IL-13-deficient mice was explained by the fact that IL-10 production, which is induced by IL-13, reduces IL-17 production by Th17 lymphocytes. In addition, Th17 lymphocytes have been reported to express IL-13 receptor alpha (IL-13R $\alpha$, also known as IL-13RA) suggesting that these cells could be directly modulated by IL-13 [62]. As IL-17 has been shown to increase mucus production through enhancing IL-13 production, these findings may suggest that IL-13 can also negatively regulate Th17 lymphocytes through a negative feedback mechanism [33]. 


\subsection{IL-17 Causes RSV-Mediated Exacerbation of Asthma}

IL-13 and mucus production are not only associated with IL-17 production but a feature of virally-exacerbated asthma. In addition, a number of studies have indicated that IL-17 is involved in severe asthma [28,29]. Therefore, the involvement of IL-17 has been explored in animal models of RSV infection concomitant with experimental asthma. For example, infection of mice with RSV Strain A2 subsequent to immunization with ovalbumin (OVA) induced experimental asthma and increased the production of mucus-associated proteins, Muc5ac and Gob-5 [63]. Mice injected with OVA, or with RSV alone, also upregulated the expression of genes encoding the mucus-associated proteins but gene expression persisted for longer periods in the OVA and RSV-immunized mice compared with those immunized with either OVA or RSV alone. Importantly, the increase in mucus production was associated with increased levels of IL-17 in the lungs [63]. Another study in which investigators used cockroach allergen (CRA) with RSV Strain A line 19 to induce asthma in mice provided further evidence for Th17 lymphocytes involvement in experimental asthma. Thus, stimulated T lymphocytes from lymph nodes of RSV/CRA-immunized mice produced IL-17 while mice immunized with CRA alone did not. Furthermore, administration of anti-IL17 antibody intraperitoneally suppressed the expression of Muc5ac and Gob5 in the lung, and IL-13 production in lymph nodes but increased the number of $\mathrm{CD}^{+}$lymphocytes [32]. These observations are further evidence for a role for IL-17 in viral exacerbation of asthma.

Table 1. Responses and products released by the cells present in the respiratory tract when stimulated with IL-17.

\begin{tabular}{|c|c|c|c|}
\hline Cell type & Response & In vitro/in vivo & Reference \\
\hline CD8+ lymphocyte & Reduced RSV clearance & in vivo & {$[32]$} \\
\hline \multirow{5}{*}{ Epithelial cells } & IL-6, IL-8, PGE ${ }_{2}$ & in vitro & {$[64]$} \\
\hline & MUC5B, MUC5AC & in vitro & {$[60,61]$} \\
\hline & CCL20 & in vitro & {$[65]$} \\
\hline & beta defensin 2 & in vitro & {$[66]$} \\
\hline & IL-19 ** & in vitro & [67] \\
\hline Endothelial cells & IL-6, IL-8, PGE $_{2}$ & & \\
\hline $\begin{array}{l}\text { Lung microvascular } \\
\text { endothelial cells }\end{array}$ & CXCL1 (GRO $\alpha)$, CXCL5, and IL-8 * & in vitro & {$[68]$} \\
\hline Fibroblasts & IL-6, IL-8, PGE ${ }_{2}$ & in vitro & {$[64][69] *$} \\
\hline \multirow{2}{*}{ Smooth muscle } & AHR (OVA induced asthma) & in vivo & \multirow{2}{*}[70]{} \\
\hline & Contraction & in vitro & \\
\hline
\end{tabular}

* IL-17 potentiates the response by IL-1 beta and TNF $\alpha$ ** IL-17 potentiates the response by IL-13.

\section{Summary}

Th17 lymphocytes are important contributors to both protective immune responses and the pathology associated with RSV infection. The involvement of Th17 cells in the patho-physiology that 
accompanies RSV infections is of great topical interest. Measurements of IL-17 levels in plasma and BAL fluids from RSV-infected infants have indicated that the cytokine can be beneficial. These studies have also suggested that Th17 responses during RSV infections are independent of Th1 and Th2 responses and that they are, in some infants, supersede an immature/inadequate Th1 immune response in the newly born [39]. Studies of IL-17 in animal models and in vitro culture systems have revealed that the lack of INF $\gamma$-mediated response enhances Th17 response. Such a response is driven by IL-23 which is produced in preference to IL-12 [24,25]. In vitro systems and animal models have showed that IL-17 per se, or together with RSV RNA particles, can induce inflammatory cytokine and chemokine responses that promote the influx of neutrophils to sites of infection [32]. As have been shown in other models of inflammation, IL-17 can also orchestrate the development of tertiary lymphoid tissues in the lung $[58,71,72]$. These structures are termed inducible bronchus-associated lymphoid tissues and are similar to structures found in RA patients with pulmonary complications (an autoimmune disease associated with IL-17) [73]. In addition to enhancing the inflammatory response that accompanies RSV infections, IL-17 has also been shown to suppress the ability of CD8+ lymphocytes to kill virally-infected cells and reduce the viral load [32]. Furthermore, both in vitro and animal model studies have confirmed that IL-17 enhances mucus production by acting directly on epithelial cells. This mucus production was also shown to be accompanied by IL-13 production which acts synergistically with IL-17 to enhance mucus production. However, high levels of IL-13 were also reported to inhibit IL-17 production. The induction of IL-17 production by RSV infection exacerbates asthma through enhancing mucus production. It is intriguing that IL-17F was not upregulated concomitant with IL-17A in one model of RSV infection [32]. Further studies to determine if IL-17F has a distinct role in RSV infection are warranted. The involvement of other Th17 cytokines, such as IL-21 and IL-22 in RSV patho-physiology also remains to be determined.

Taken together, studies of infected individuals and animal models have revealed that IL-17 can have both beneficial and pathogenic effects during RSV infection. In animal models, just as in cases of patients with asthma, IL-17 induces pathology by enhancing neutrophil influx, mucus and IL-13 production. However, the beneficial effects of IL-17 continue to be debated. It is intriguing to note that although Th17 lymphocytes have evolved together with the rest of the adaptive immune system, the emergence of the IL-17 family of cytokines predates chordates [74]. Th17 lymphocytes differentiate in response to inflammatory cytokines, preferably with low level of TCR engagement and their expansion is favoured by low oxygen levels [21,22]. The cells might, therefore, emerge as a weak alternative to the more efficient antiviral response mediated by a missing, or immature/inadequate Th1 lymphocyte response. Under such circumstances, Th17 lymphocytes may provide a response that straddles adaptive and innate immune responses resulting in mucus release, neutrophil influx and augmentation of local tertiary lymphoid structures.

\section{Acknowledgments}

Studies of the role of IL-17 in arthritis carried out in our laboratory are generously supported by a research grant from Pfizer. 


\section{Conflicts of Interest}

The authors declare no conflicts of interest

\section{References}

1. Milner, J.D.; Brenchley, J.M.; Laurence, A.; Freeman, A.F.; Hill, B.J.; Elias, K.M.; Kanno, Y.; Spalding, C.; Elloumi, H.Z.; Paulson, M.L.; et al. Impaired T(H)17 cell differentiation in subjects with autosomal dominant hyper-IgE syndrome. Nature 2008, 452, 773-776.

2. Chen, Z.; O'Shea, J.J. Th17 cells: A new fate for differentiating helper T cells. Immunol. Res. 2008, 41, 87-102.

3. Sutton, C.; Brereton, C.; Keogh, B.; Mills, K.H.G.; Lavelle, E.C. A crucial role for interleukin (IL) -1 in the induction of IL-17-producing T cells that mediate autoimmune encephalomyelitis. J. Exp. Med. 2006, 203, 1685-1691.

4. Toussirot, E. The IL23/Th17 pathway as a therapeutic target in chronic inflammatory diseases. Inflamm. Allergy Drug Targets 2012, 11, 159-168.

5. Leonardi, C.; Matheson, R.; Zachariae, C.; Cameron, G.; Li, L.; Edson-Heredia, E.; Braun, D.; Banerjee, S. Anti-interleukin-17 monoclonal antibody ixekizumab in chronic plaque psoriasis. N. Engl. J. Med. 2012, 366, 1190-1199.

6. Papp, K.A.; Leonardi, C.; Menter, A.; Ortonne, J.-P.; Krueger, J.G.; Kricorian, G.; Aras, G.; Li, J.; Russell, C.B.; Thompson, E.H.Z.; et al. Brodalumab, an anti-interleukin-17-receptor antibody for psoriasis. N. Engl. J. Med. 2012, 366, 1181-1189.

7. Gaffen, S.L. Recent advances in the IL-17 cytokine family. Curr. Opin. Immunol. 2011, 23, 613-619.

8. Khader, S.A.; Gaffen, S.L.; Kolls, J.K. Th17 cells at the crossroads of innate and adaptive immunity against infectious diseases at the mucosa. Mucosal Immunol. 2009, 2, 403-411.

9. Hartupee, J.; Liu, C.; Novotny, M.; Li, X.; Hamilton, T. IL-17 enhances chemokine gene expression through mRNA stabilization. J. Immunol. 2007, 179, 4135-4141.

10. Zhu, S.; Pan, W.; Song, X.; Liu, Y.; Shao, X.; Tang, Y.; Liang, D.; He, D.; Wang, H.; Liu, W.; et al. The microRNA miR-23b suppresses IL-17-associated autoimmune inflammation by targeting TAB2, TAB3 and IKK-alpha. Nat. Med. 2012, 18, 1077-1086.

11. Jaffar, Z.; Ferrini, M.E.; Herritt, L.A.; Roberts, K. Cutting edge: Lung mucosal Th17-mediated responses induce polymeric Ig receptor expression by the airway epithelium and elevate secretory IgA levels. J. Immunol. 2009, 182, 4507-4511.

12. Mitsdoerffera, M.; Leea, Y.; Jägera, A.; Kimb, H.-J.; Kornc, T.; Koolsd, J.K.; Cantorb, H.; Bettellie, E.; Kuchrooa, V.K. Proinflammatory T helper type 17 cells are effective B-cell helpers. Proc. Natl. Acad. Sci. USA 2010, 107, 14292-14297.

13. Dodd, J.S.; Clark, D.; Muir, R.; Korpis, C.; Openshaw P.J. Endogenous IL-21 regulates pathogenic mucosal CD4 T-cell responses during enhanced RSV disease in mice. Mucosal Immunol. 2013, 6, $704-717$. 
14. Zheng, Y.; Valdez, P.A.; Danilenko, D.M.; Hu, Y.; Sa, S.M.; Gong, Q.; Abbas, A.R.; Modrusan, Z.; Ghilardi, N.; de Sauvage, F.J.; et al. Interleukin-22 mediates early host defense against attaching and effacing bacterial pathogens. Nat. Med. 2008, 14, 282-289.

15. Shaw, M.H.; Kamada, N.; Kim, Y.G.; Núñez, G. Microbiota-induced IL-1beta, but not IL-6, is critical for the development of steady-state TH17 cells in the intestine. J. Exp. Med. 2012, 209, 251-258.

16. Zhou, L.; Ivanov, I.I.; Spolski, R.; Min, R.; Shenderov, K.; Egawa, T.; Levy, D.E.; Leonard, W.J.; Littman, D.R. IL -6 programs $\mathrm{T}(\mathrm{H})-17$ cell differentiation by promoting sequential engagement of the IL-21 and IL-23 pathways. Nat. Immunol. 2007, 8, 967-974.

17. Boniface, K.; Bak-Jensen, K.S.; Li, Y.; Blumenschein, W.M.; McGeachy, M.J.; McClanahan, T.K.; McKenzie, B.S.; Kastelein, R.A.; Cua, D.J.; de Waal Malefyt, R. Prostaglandin E2 regulates Th17 cell differentiation and function through cyclic AMP and EP2/EP4 receptor signaling. J. Exp. Med. 2009, 206,535-548.

18. Ivanov, I.I.; McKenzie, B.S.; Zhou, L.; Tadokoro, C.E.; Lepelley, A.; Lafaille, J.J.; Cua, D.J.; Littman, D.R. The orphan nuclear receptor RORgammat directs the differentiation program of proinflammatory IL-17+ T helper cells. Cell 2006, 126, 1121-1133.

19. Purvis, H.A.; Stoop, J.N.; Mann, J.; Woods, S.; Kozijn, A.E.; Hambleton, S.; Robinson, J.H.; Isaacs, J.D.; Anderson, A.E.; Hilkens, C.M. Low-strength T-cell activation promotes Th17 responses. Blood 2010, 116, 4829-4837.

20. Liu, X.K.; Lin, X.; Gaffen, S.L. Crucial role for nuclear factor of activated T cells in T cell receptor-mediated regulation of human interleukin-17. J. Biol. Chem. 2004, 279, 52762-52771.

21. Dang, E.V.; Barbi, J.; Yang, H.Y.; Jinasena, D.; Yu, H.; Zheng, Y.; Bordman, Z.; Fu, J.; Kim, Y.; Yen, H.R.; et al. Control of T(H)17/T(reg) balance by hypoxia-inducible factor 1. Cell 2011, 146, 772-784.

22. Ikejiri, A.; Nagai, S.; Goda, N.; Kurebayashi, Y.; Osada-Oka, M.; Takubo, K.; Suda, T.; Koyasu, S. Dynamic regulation of Th17 differentiation by oxygen concentrations. Int. Immunol. 2012, 24 , $137-146$.

23. Hirota, K.; Duarte, J.H.; Veldhoen, M.; Hornsby, E.; Li, Y.; Cua, D.J.; Ahlfors, H.; Wilhelm, C.; Tolaini, M.; Menzel, U.; et al. Fate mapping of IL-17-producing T cells in inflammatory responses. Nat. Immunol. 2011, 12, 255-263.

24. Lukacs, N.W.; Smit, J.J.; Mukherjee, S.; Morris, S.B.; Nunez, G.; Lindell, D.M. Respiratory virus-induced TLR7 activation controls IL-17-associated increased mucus via IL-23 regulation. J. Immunol. 2010, 185, 2231-2239.

25. Hashimoto, K.; Durbin, J.E.; Zhou, W.; Collins, R.D.; Ho, S.B.; Kolls, J.K.; Dubin, P.J.; Sheller, J.R.; Goleniewska, K.; O’Neal, J.F.; et al. Respiratory syncytial virus infection in the absence of STAT 1 results in airway dysfunction, airway mucus, and augmented IL-17 levels. J. Allergy Clin. Immunol. 2005, 116, 550-557. 
26. Di Stefano, A.; Caramori, G.; Gnemmi, I.; Contoli, M.; Vicari, C.; Capelli, A.; Magno, F.; D'Anna, S.E.; Zanini, A.; Brun, P.; et al. T helper type 17-related cytokine expression is increased in the bronchial mucosa of stable chronic obstructive pulmonary disease patients. Clin. Exp. Immunol. 2009, 157, 316-324.

27. Vargas-Rojas, M.I.; Ramírez-Venegas, A.; Limón-Camacho, L.; Ochoa, L.; Hernández-Zenteno, R.; Sansores, R.H. Increase of Th17 cells in peripheral blood of patients with chronic obstructive pulmonary disease. Respir. Med. 2011, 105, 1648-1654.

28. Wilson, R.H.; Whitehead, G.S.; Nakano, H.; Free, M.E.; Kolls, J.K.; Cook, D.N. Allergic sensitization through the airway primes Th17-dependent neutrophilia and airway hyperresponsiveness. Am. J. Respir. Crit. Care Med. 2009, 180, 720-730.

29. Zhao, Y.; Yang, J.; Gao, Y.D.; Guo, W. Th17 immunity in patients with allergic asthma. Int. Arch. Allergy Immunol. 2010, 151, 297-307.

30. Yang, X.O.; Chang, S.H.; Park, H.; Nurieva, R.; Shah, B.; Acero, L.; Wang, Y.H.; Schluns, K.S.; Broaddus, R.R.; Zhu, Z.; et al. Regulation of inflammatory responses by IL-17F. J. Exp. Med. 2008, 205, 1063-1075.

31. Faber, T.E.; Groen, H.; Welfing, M.; Jansen, K.J.; Bont, L.J. Specific increase in local IL-17 production during recovery from primary RSV bronchiolitis. J. Med. Virol. 2012, 84, 1084-1088.

32. Mukherjee, S.; Lindell, D.M.; Berlin, A.A.; Morris, S.B.; Shanley, T.P.; Hershenson, M.B.; Lukacs, N.W. IL-17-induced pulmonary pathogenesis during respiratory viral infection and exacerbation of allergic disease. Am. J. Pathol. 2011, 179, 248-258.

33. Newcomb, D.C.; Boswell, M.G.; Huckabee, M.M.; Goleniewska, K.; Dulek, D.E.; Reiss, S.; Lukacs, N.W.; Kolls, J.K.; Peebles, R.S, Jr. IL-13 regulates Th17 secretion of IL-17A in an IL-10-dependent manner. J. Immunol. 2012,188, 1027-1035.

34. Polack, F.P.; Irusta, P.M.; Hoffman, S.J.; Schiatti, M.P.; Melendi, G.A.; Delgado, M.F.; Laham, F.R.; Thumar, B.; Hendry, R.M.; Melero, J.A.; et al. The cysteine-rich region of respiratory syncytial virus attachment protein inhibits innate immunity elicited by the virus and endotoxin. Proc. Natl. Acad. Sci. USA 2005, 102, 8996-9001.

35. Hall, C.B.; Weinberg, G.A.; Iwane, M.K.; Blumkin A.K.; Edwards, K.M.; Staat, M.A.; Auinger, P.; Griffin, M.R.; Poehling, K.A.; Erdma, D.; et al. The burden of respiratory syncytial virus infection in young children. N. Engl. J. Med. 2009, 360, 588-598.

36. Zhang, L.; Peeples, M.E.; Boucher, R.C.; Collins, P.L.; Pickles, R.J. Respiratory syncytial virus infection of human airway epithelial cells is polarized, specific to ciliated cells, and without obvious cytopathology. J. Virol. 2002, 76, 5654-5666.

37. Habibi, M.S.; Openshaw, P.J. Benefit and harm from immunity to respiratory syncytial virus: Implications for treatment. Curr. Opin. Infect. Dis. 2012, 25, 687-694.

38. Panuska, J.R.; Cirino, N.M.; Midulla, F.; Despot, J.E.; McFadden, E.R., Jr.; Huang, Y.T. Productive infection of isolated human alveolar macrophages by respiratory syncytial virus. J. Clin. Investig. 1990, 86, 113-119. 
39. Thornburg, N.J.; Shepherd, B.; Crowe, J.E., Jr. Transforming growth factor beta is a major regulator of human neonatal immune responses following respiratory syncytial virus infection. J. Virol. 2010, 84, 12895-12902.

40. Polack, F.P.; Teng, M.N.; Collins, P.L.; Prince, G.A.; Exner, M.; Regele, H.; Lirman, D.D.; Rabold, R.; Hoffman, S.J.; Karp, C.L.; et al. A role for immune complexes in enhanced respiratory syncytial virus disease. J. Exp. Med. 2002, 196, 859-865.

41. McNamara, P.S.; Ritson, P.; Selby, A.; Hart, C.A.; Smyth, R.L. Bronchoalveolar lavage cellularity in infants with severe respiratory syncytial virus bronchiolitis. Arch. Dis. Child. 2003, 88, 922-926.

42. Lukens, M.V.; van de Pol, A.C.; Coenjaerts, F.E.; Jansen, N.J.; Kamp, V.M.; Kimpen, J.L.; Rossen, J.W.; Ulfman, L.H.; Tacke, C.E.; Viveen, M.C.; et al. A systemic neutrophil response precedes robust $\mathrm{CD} 8(+) \mathrm{T}$-cell activation during natural respiratory syncytial virus infection in infants. J. Virol. 2010, 84, 2374-2383.

43. Lukens, M.V.; Kruijsen, D.; Coenjaerts, F.E.; Kimpen, J.L.L.; van Bleek, G.M. Respiratory syncytial virus-induced activation and migration of respiratory dendritic cells and subsequent antigen presentation in the lung-draining lymph node. J. Virol. 2009, 83, 7235-7243.

44. Graham, B.S.; Bunton, L.A.; Wright, P.F.; Karzon, D.T. Role of T lymphocyte subsets in the pathogenesis of primary infection and rechallenge with respiratory syncytial virus in mice. J. Clin. Investig. 1991, 88, 1026-1033.

45. Averbuch, D.; Chapgier, A.; Boisson-Dupuis, S.; Casanova, J.L.; Engelhard, D. The clinical spectrum of patients with deficiency of Signal Transducer and Activator of Transcription-1. Pediatr. Infect. Dis. J. 2011, 30, 352-355.

46. Durbin, J.E.; Johnson, T.R.; Durbin, R.K.; Mertz, S.E.; Morotti, R.A.; Peebles, R.S.; Graham, B.S. The role of IFN in respiratory syncytial virus pathogenesis. J. Immunol. 2002, 168, 2944-2952.

47. Welliver, R.C.; Wong, D.T.; Sun, M.; Middleton, E., Jr.; Vaughan, R.S.; Ogra, P.L. The development of respiratory syncytial virus-specific $\operatorname{IgE}$ and the release of histamine in nasopharyngeal secretions after infection. N. Engl. J. Med. 1981, 305, 841-846.

48. Mobbs, K.J.; Smyth, R.L.; O’Hea, U.; Ashby, D.; Ritson, P.; Hart, C.A. Cytokines in severe respiratory syncytial virus bronchiolitis. Pediatr. Pulmonol. 2002, 33, 449-452.

49. Hussell, T.; Spender, L.C.; Georgiou, A.; O’Garra, A.; Openshaw, P.J. Th1 and Th2 cytokine induction in pulmonary $\mathrm{T}$ cells during infection with respiratory syncytial virus. J. Gen. Virol. 1996, 77, 2447-2455.

50. Boelen, A.; Kwakkel, J.; Barends, M.; de Rond, L.; Dormans, J.; Kimman T. Effect of lack of Interleukin-4, Interleukin-12, Interleukin-18, or the Interferon-gamma receptor on virus replication, cytokine response, and lung pathology during respiratory syncytial virus infection in mice. J. Med. Virol. 2002, 66, 552-560.

51. Larranaga, C.L.; Ampuero, S.L.; Luchsinger, V.F.; Carrión, F.A.; Aguilar, N.V.; Morales, P.R.; Palomino, M.A.; Tapia, L.F.; Avendaño, L.F. Impaired immune response in severe human lower tract respiratory infection by respiratory syncytial virus. Pediatr. Infect. Dis. J. 2009, 28, 867-873. 
52. Tregoning, J.S.; Schwarze, J. Respiratory viral infections in infants: Causes, clinical symptoms, virology, and immunology. Clin. Microbiol. Rev. 2010, 23, 74-98.

53. Goriely, S.; Vincart, B.; Stordeur, P.; Vekemans, J.; Willems, F.; Goldman, M.; De Wit, D. Deficient IL-12(p35) gene expression by dendritic cells derived from neonatal monocytes. J. Immunol. 2001, 166, 2141-2146.

54. Qin, L.; Hu, C.P.; Feng, J.T.; Xia, Q. Activation of lymphocytes induced by bronchial epithelial cells with prolonged RSV infection. PLoS One 2011, 6, e27113.

55. Bera, M.M.; Lu, B.; Martin, T.R.; Cui, S.; Rhein, L.M.; Gerard, C.; Gerard, N.P. Th17 cytokines are critical for respiratory syncytial virus-associated airway hyperreponsiveness through regulation by complement C3a and tachykinins. J. Immunol. 2011, 187, 4245-4255.

56. Ryzhakov, G.; Lai, C.C.; Blazek, K.; To, K.W.; Hussell, T.; Udalova, I. IL-17 boosts proinflammatory outcome of antiviral response in human cells. J. Immunol. 2011, 187, 5357-5362.

57. Davidson, S.; Kaiko, G.; Loh, Z.; Lalwani, A.; Zhang, V.; Spann, K.; Foo, S.Y.; Hansbro, N.; Uematsu, S.; Akira, S.; et al. Plasmacytoid dendritic cells promote host defense against acute pneumovirus infection via the TLR7-MyD88-dependent signaling pathway. J. Immunol. 2011, 186, 5938-5948.

58. Kallal, L.E.; Hartigan, A.J.; Hogaboam, C.M.; Schaller, M.A.; Lukacs, N.W. Inefficient lymph node sensitization during respiratory viral infection promotes IL-17-mediated lung pathology. J. Immunol. 2010, 185, 4137-4147.

59. Cannon, M.J.; Openshaw, P.J.; Askonas, B.A. Cytotoxic T cells clear virus but augment lung pathology in mice infected with respiratory syncytial virus. J. Exp. Med. 1988, 168, 1163-1168.

60. Chen, Y.; Thai, P.; Zhao, Y.H.; Ho, Y.S.; DeSouza, M.M.; Wu, R. Stimulation of airway mucin gene expression by interleukin (IL)-17 through IL-6 paracrine/autocrine loop. J. Biol. Chem. 2003, 278, 17036-17043.

61. Fujisawa, T.; Chang, M.M.; Velichko, S.; Thai, P.; Hung, L.Y.; Huang, F.; Phuong, N.; Chen,Y.; Wu, R. NF-kappaB mediates IL-1beta- and IL-17A-induced MUC5B expression in airway epithelial cells. Am. J. Respir. Cell Mol. Biol. 2011, 45, 246-252.

62. Newcomb, D.C.; Boswell, M.G.; Zhou, W.; Huckabee, M.M.; Goleniewska, K.; Sevin, C.M.; Hershey, G.K.; Kolls, J.K.; Peebles, R.S., Jr. Human TH17 cells express a functional IL-13 receptor and IL-13 attenuates IL-17A production. J. Allergy Clin. Immunol. 2011, 127, 1006-1013.

63. Hashimoto, K.; Graham, B.S.; Ho, S.B.; Adler, K.B.; Collins, R.D.; Olson, S.J.; Zhou, W.; Suzutani, T.; Jones, P.W.; Goleniewska, K.; et al. Respiratory syncytial virus in allergic lung inflammation increases Muc5ac and gob-5. Am. J. Respir. Crit. Care Med. 2004, 170, 306-312.

64. Fossiez, F.; Djossou, O.; Chomarat, P.; Flores-Romo, L.; Ait-Yahia, S.; Maat, C.; Pin, J.J.; Garrone, P.; Garcia, E.; Saeland, S.; et al. T cell interleukin-17 induces stromal cells to produce proinflammatory and hematopoietic cytokines. J. Exp. Med. 1996, 183, 2593-2603.

65. Kao, C.Y.; Huang, F.; Chen, Y.; Thai, P.; Wachi, S.; Kim, C.; Tam, L.; Wu, R. Up-regulation of CC chemokine ligand 20 expression in human airway epithelium by IL-17 through a JAK-independent but MEK/NF-kappaB-dependent signaling pathway. J. Immunol. 2005, 175, 6676-6685. 
66. Kao, C.Y.; Chen, Y.; Thai, P.; Wachi, S.; Huang, F.; Kim, C.; Harper, R.W.; Wu, R. IL-17 markedly up-regulates beta-defensin-2 expression in human airway epithelium via JAK and NF-kappaB signaling pathways. J. Immunol. 2004, 173, 3482-3491.

67. Huang, F.; Wachi, S.; Thai, P.; Loukoianov, A.; Tan, K.H.; Forteza, R.M.; Wu, R. Potentiation of IL-19 expression in airway epithelia by IL-17A and IL-4/IL-13: Important implications in asthma. J. Allergy Clin. Immunol. 2008, 121, 1415-1421.

68. Fujie, H.; Niu, K.; Ohba, M.; Tomioka, Y.; Kitazawa, H.; Nagashima, K.; Ohrui, T.; Numasaki, M. A distinct regulatory role of Th17 cytokines IL-17A and IL-17F in chemokine secretion from lung microvascular endothelial cells. Inflammation 2012, 35, 1119-1131.

69. Katz, Y.; Nadiv, O.; Beer, Y. Interleukin-17 enhances tumor necrosis factor alpha-induced synthesis of interleukins 1,6, and 8 in skin and synovial fibroblasts: A possible role as a "fine-tuning cytokine" in inflammation processes. Arthritis Rheum. 2001, 44, 2176-2184.

70. Kudo, M.; Melton, A.C.; Chen, C.; Engler, M.B.; Huang, K.E.; Ren, X.; Wang, Y.; Bernstein, X.; Li, J.T.; Atabai, K.; et al. IL-17A produced by alphabeta T cells drives airway hyper-responsiveness in mice and enhances mouse and human airway smooth muscle contraction. Nat. Med. 2012, 18, 547-554.

71. Peters, A.; Pitcher, L.A.; Sullivan, J.M.; Mitsdoerffer, M.; Acton, S.E.; Franz, B.; Wucherpfennig, K.; Turley, S.; Carroll, M.C.; Sobel, R.A.; et al. Th17 cells induce ectopic lymphoid follicles in central nervous system tissue inflammation. Immunity 2011, 35, 986-996.

72. Rangel-Moreno, J.; Carragher, D.M.; de la Luz Garcia-Hernandez, M.; Hwang, J.Y.; Kusser, K.; Hartson, L.; Kolls, J.K.; Khader, S.A.; Randall, T.D. The development of inducible bronchus-associated lymphoid tissue depends on IL-17. Nat. Immunol. 2011, 12, 639-646.

73. Rangel-Moreno, J.; Hartson, L.; Navarro, C.; Gaxiola, M.; Selman, M.; Randall, T.D. Inducible bronchus-associated lymphoid tissue (iBALT) in patients with pulmonary complications of rheumatoid arthritis. J. Clin. Investig. 2006, 116, 3183-3194.

74. Chen, K.; McAleer, J.P.; Lin, Y.; Paterson, D.L.; Zheng, M.; Alcorn, J.F.; Weaver, C.T.; Kolls, J.K. Th17 cells mediate clade-specific, serotype-independent mucosal immunity. Immunity 2011, 35, 997-1009. 
Reprinted from Viruses. Cite as: Kolli, D.; Bao, X.; Casola, A. Human Metapneumovirus Antagonism of Innate Immune Responses. Viruses 2012, 4, 3551-3571.

Review

\title{
Human Metapneumovirus Antagonism of Innate Immune Responses
}

\author{
Deepthi Kolli ${ }^{1}$, Xiaoyong Bao ${ }^{1}$ and Antonella Casola ${ }^{1,2,3, *}$
}

1 Departments of Pediatrics, University of Texas Medical Branch, Galveston, TX 77550, USA; E-Mails: dekolli@utmb.edu (D.K.); xibao@utmb.edu (X.B.)

2 Microbiology and Immunology, University of Texas Medical Branch, Galveston, TX 77550, USA

3 Sealy Center for Molecular Medicine, University of Texas Medical Branch, Galveston, TX 77550, USA

* Author to whom correspondence should be addressed; E-Mail: ancasola@utmb.edu; Tel.: +1-409-747-0581; Fax: +1-409-772-1761.

Received: 1 November 2012; in revised form: 20 November 2012 / Accepted: 30 November 2012 / Published: 7 December 2012

\begin{abstract}
Human metapneumovirus (hMPV) is a recently identified RNA virus belonging to the Paramyxoviridae family, which includes several major human and animal pathogens. Epidemiological studies indicate that hMPV is a significant human respiratory pathogen with worldwide distribution. It is associated with respiratory illnesses in children, adults, and immunocompromised patients, ranging from upper respiratory tract infections to severe bronchiolitis and pneumonia. Interferon (IFN) represents a major line of defense against virus infection, and in response, viruses have evolved countermeasures to inhibit IFN production as well as IFN signaling. Although the strategies of IFN evasion are similar, the specific mechanisms by which paramyxoviruses inhibit IFN responses are quite diverse. In this review, we will present an overview of the strategies that hMPV uses to subvert cellular signaling in airway epithelial cells, the major target of infection, as well as in primary immune cells.
\end{abstract}

Keywords: metapneumovirus; viral proteins; innate immune system; interferon antagonism 


\section{Human Metapneumovirus (hMPV): A Recently Discovered Human Viral Pathogen}

The Paramyxoviridae family includes enveloped, negative-sense, single-stranded RNA viruses, which are major and ubiquitous disease causing pathogens of humans and animals [1]. Among them are important viruses that cause acute respiratory morbidity, particularly in infancy, elderly and in immunocompromised subjects of any age. The family is taxonomically divided into two subfamilies, the Paramyxovirinae, with five genera, and the Pneumovirinae, which includes two genera (Table 1). The classification of these viruses is based on their genome organization, morphological and biological characteristics, and sequence relationship of the encoded proteins. The pneumoviruses can be distinguished from the Paramyxovirinae members morphologically because they contain narrower nucleocapsids [1]. In addition, pneumoviruses have differences in genome organization, the number of encoded proteins and an attachment protein that is different from that of members of the subfamily Paramyxovirinae. There are two genera in the Pneumovirinae family, the Pneumovirus genus that includes human and bovine respiratory syncytial virus (RSV) and the Metapneumovirus genus that includes human metapneumovirus (hMPV) and avian metapneumovirus (APV) (Table 1). Human RSV encodes 11 separate proteins, while hMPV encodes nine proteins that generally correspond to those of RSV, except that hMPV lacks the non-structural proteins NS1 and NS2 and the gene order is different from that of pneumoviruses (Figure 1).

Table 1. Representative members of the Paramyxoviridae family.

\begin{tabular}{lll}
\hline Subfamily & \multicolumn{1}{c}{ Genus } & \multicolumn{1}{c}{ Virus } \\
\hline Paramyxovirinae & & Hendravirus \\
& Henipavirus & Nipah virus \\
& Morbillivirus & Measles virus (MeV) \\
& Respirovirus & Sendai virus (SeV) \\
& & Human parainfluenza virus type 1 (hPIV1) \\
& Human parainfluenza virus type 3 (hPIV3) \\
& Rovine parainfluenza virus type 3 (BPIV3) \\
& & Parainfluenza virus type 5 (PIV5) \\
& & Mumps virus (MuV) \\
& & Human parainfluenza virus type 2 (hPIV2) \\
\hline Pneumovirinae & & Human respiratory syncytial virus (hRSV) \\
& Pneumovirus & Bovine respiratory syncytial virus (BRSV) \\
& & Avian pneumovirus (APV) \\
& Metapneumovirus & Human metapneumovirus (hMPV) \\
\hline
\end{tabular}


Figure 1. Genomic organization of Pneumovirinae.

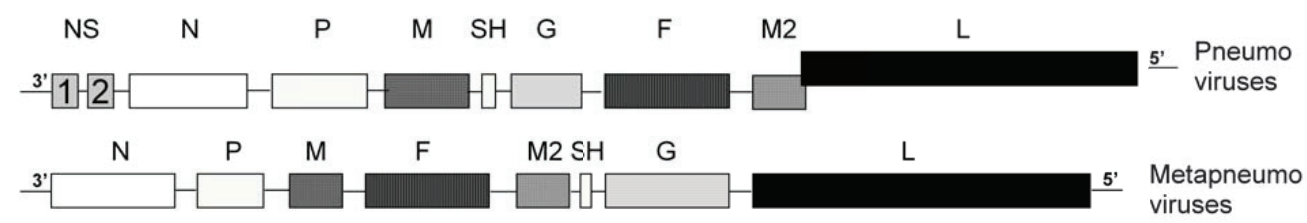

Since its first identification in 2001, hMPV has been isolated from individuals of all ages with acute respiratory tract infection worldwide [2]. Virtually, all children older than five years show 100\% serologic evidence of infection [3]. Around $12 \%$ of all respiratory tract infections in children are caused by hMPV, second only to RSV [2,4-6]. HMPV also accounts for $10 \%$ of all hospitalizations of elderly patients with respiratory tract infections and it has been isolated from respiratory samples of a single winter season as often as parainfluenza [7]. Phylogenetic analysis of strains from many countries demonstrates two distinct hMPV genotypes, A and B, which can be divided in two subgroups: A1, A2, B1 and B2 [2,4]. The clinical features associated with hMPV in children are similar to those of RSV. hMPV is associated with both upper and lower respiratory tract infections. Fever, cough, tachypnea, wheezing and hypoxia are frequently observed in infected children. Chest radiographs demonstrate focal infiltrates and peribronchial cuffing. Many children have a clinical syndrome consistent with bronchiolitis. A significant proportion of symptomatic children who tested positive for hMPV had co-morbidities such as a history of prematurity, chronic lung disease or complex congenital heart diseases [8]. These findings suggest that the populations of children prone to severe RSV disease may be also prone to hMPV disease. Although RSV and hMPV share similar clinic features, hMPV induces a different spectrum of immune mediators compared to RSV [9-11], suggesting that the host cell responses and likely the pathogenesis of lung disease are viral specific.

\section{Pattern Recognition Receptors (PRRs) in hMPV-Induced Signaling}

The innate immune response represents a critical component of the host defense against viruses and is coordinated at the cellular level by activation of transcription factors that regulate the expression of inducible gene products with antiviral and/or inflammatory activity. Viruses contain conserved structural moieties, known as pathogen associated molecular pattern (PAMPs), that are recognized by several families of PRRs, in particular Toll-Like Receptors (TLR) and RNA helicases. Their relative contribution in virus-triggered cellular signaling is stimulus- and cell-type-dependent (Reviewed in [12]). So far, 10 members of TLRs have been identified in humans, and 13 in mice. Among those, TLR3, 4, 7, 8 and 9 have been shown to be more commonly involved in the innate response to viral infections $[13,14]$. TLR3 recognizes double-stranded RNA (ds-RNA) that is produced during viral replication [12]. Recently, RSV has been shown to relocate TLR3 from endosomes to cytoplasmic membrane in infected airway epithelial cells, resulting in increased chemokine production after an initial phase that is dependent on RSV replication $[15,16]$. However, we did not find a similar role of 
TLR3 in hMPV-induced cellular signaling either in airway epithelial cells [17] or in primary immune cells, such as dendritic cells (DCs) [18].

Several viral envelop proteins, including RSV F protein and proteins of mammary tumor virus, murine leukemia virus, vesicular stomatitis virus [19-21], and more recently Ebola virus [22], have been shown to activate TLR4 in primary immune cells. Similar to LPS, the primary ligand of TLR4, RSV F protein requires the presence of CD14 and MD-2 for signaling [23,24]. TLR4 signaling has been shown to play an important role in controlling paramyxovirus infection. TLR4-deficient mice challenged with RSV exhibited impaired natural killer (NK) cell and CD14+ cell pulmonary trafficking, diminished NK cell function, and impaired IL-12 induction, in addition to impaired RSV clearance [20]. In a model of alveolar macrophage depletion of TLR4-defective C3H/HeJ mice, we have shown that the early NF- $\mathrm{BB}$ response that occurs in the lung after RSV infection, is dependent upon alveolar macrophages and TLR4 [25]. Furthermore, both TLR4 and the adaptor molecule MyD88 have been shown to be required for optimal protection against viral challenge in a mouse model of RSV infection [26]. Our recent investigations have shown that down regulation of TLR4 expression in human DCs or lack of functional TLR4 in mouse bone marrow-derived DCs result in significantly reduced expression of hMPV-induced cytokine, chemokine, and type I IFN secretion, indicating an important role of this TLR in the activation of cellular signaling following hMPV infection [18]. In addition, mice lacking TLR4 showed less clinical disease, significantly lower levels of cytokines and chemokines, compared to the wild type. Accordingly, inflammatory cell recruitment in the BAL, lungs, as well as in lymph nodes, was also significantly reduced [27]. These results indicate that TLR4 is important for activation of the innate immune response to hMPV infection, however, it also contributes to disease pathogenesis.

TLR7 and 8 share highest homology to each other among the TLR family and both of them recognize single-stranded RNA (ss-RNA) [28,29], while TLR9 recognize viral CpG DNA motif [30,31]. In case of hMPV, it has been shown that induction of cytokines and type I Interferon (IFN) is TLR7-dependent [32], using TLR7-deficient mice and TLR7 specific oligonucleotide-based inhibitor ISS661. While a similar requirement in RSV infection of pDCs has not been demonstrated yet, recently a critical role for TLR7 and MyD88 in the recognition and development of innate inflammatory responses necessary to limit pneumovirus infection has been reported [33]. No direct involvement of TLR9 in innate immune cellular signaling in response to either RSV or hMPV has been reported yet.

After recognition of their own PAMPs, following viral infection, TLRs trigger intracellular signaling pathways that are necessary to the induction of inflammatory cytokines, chemokines, as well as type I IFN. Three structural domains, i.e., a Leucine Rich Region (LRR) in the N-terminal ectodomain, a transmembrane region, and a Toll/IL-1R resistance (TIR) domain in the intracellular region, are structural hallmarks of all known Toll/TLRs. Differential utilization of four TIR-containing adapter molecules (i.e., MyD88, TIRAP, TRIF, and TRAM) by distinct TLRs leads to activation of downstream signaling pathways, findings based largely on studies in adapter knockout mice. Two major TLR signaling pathways have been identified, i.e., one that is MyD88-dependent, and 
gives rise to strong and early activation of the transcription factor NF- $\kappa \mathrm{B}$, and a TRIF-dependent, MyD88-independent pathway that primarily drives strong activation of IRF-3, with later activation of NF- $\mathrm{BB}$. The MyD88-dependent pathway results in induction of highly NF- $\kappa \mathrm{B}-$ dependent, proinflammatory genes (TNF- $\alpha$, IL-1 $\beta$, IL-6), while the MyD88-independent pathway leads to gene induction that is highly IRF-3-dependent (IFN- $\beta$, RANTES). TLR4 activates both pathways for gene expression, as it is the only TLR that uses both adapter proteins (Figure 2) [13,34,35].

Figure 2. Toll-Like Receptors (TLR) signaling pathway involved in hMPV-induced gene expression in primary immune cells. Upon binding of their specific viral PAMP, TLR4 and 7 lead to activation of NF- $\mathrm{BB}-$ and IRF-dependent gene expression by engaging the adaptor MyD88 alone (TLR7) or in combination with TRIF (TLR4). Ub indicates ubiquitination; $\mathrm{P}$ indicates phosphorylation.

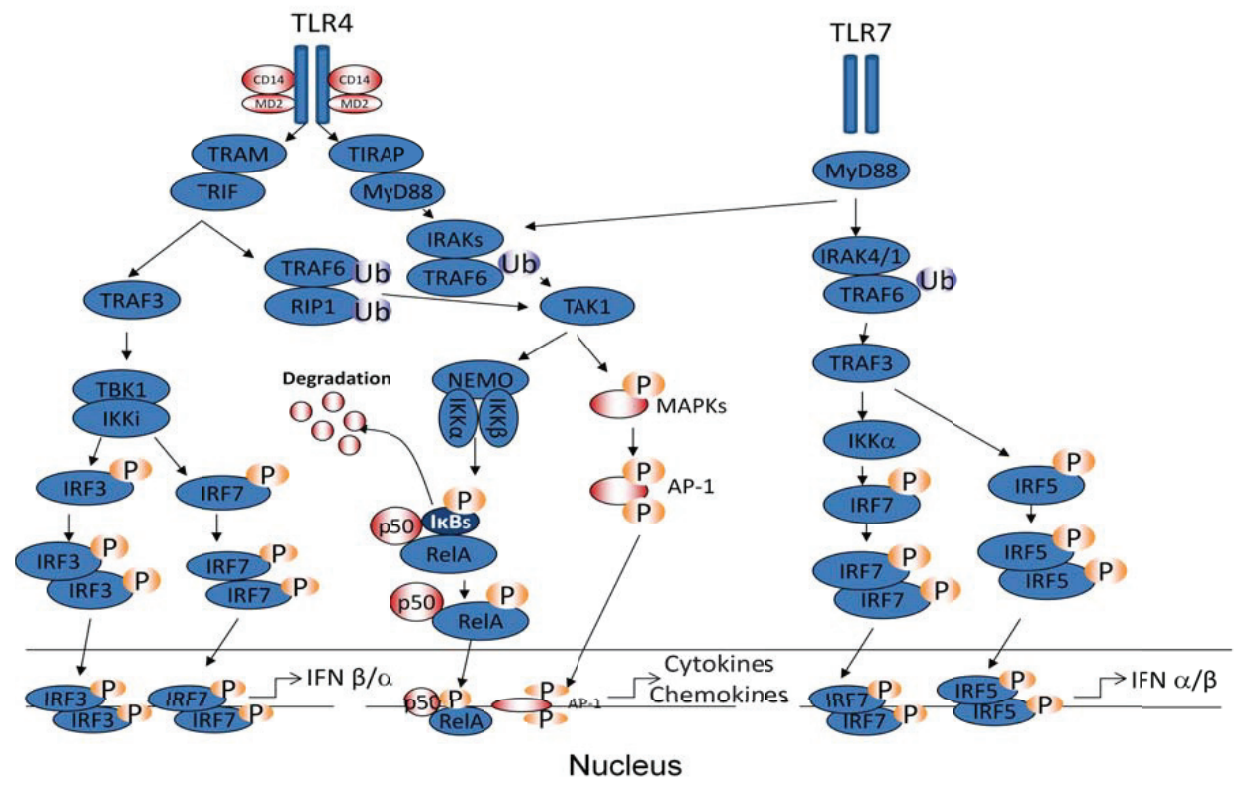

Aside from the recognition of viral RNA through the TLRs, two DExD/H box RNA helicases, retinoic acid inducible gene (RIG)-I and melanoma differentiation-associated gene (MDA)-5, have been identified to be essential for IFN induction by several viruses including Newcastle, Sendai, hepatitis C, influenza and RSV [16,36-39]. Both RIG-I and MDA-5 share two homologous CARD domains and a helicase domain that is required for its interaction with viral RNA [36,40]. The CARD domains of the RNA helicases mediate its interaction with the CARD domain of the mitochondrial protein MAVS (also known as IPS-1/VISA/Cardif), leading to subsequent activation of downstream signaling molecules, such as IRFs, NF- $\mathrm{B}$ and AP-1 [41,42-44]. Viral RNA signaling mediated by RIG-I is independent from TLRs, as dominant negative RIG-I does not block TLR-mediated signaling $[41,43]$. Generation of RIG-I and MDA-5 knock-out mice has identified a major antiviral 
role for RNA helicases in several cell types, including macrophages and conventional DCs, but not in plasmacytoid DCs (pDCs), which instead require TLR7 and/or TLR9 to mount an effective antiviral response (Reviewed in [44]). We have recently shown that hMPV infection of airway epithelial cells induces the expression RIG-I and MDA-5 and that RIG-I, but not MDA-5, plays a fundamental role in hMPV-induced cellular signaling, as inhibition of RIG-I expression significantly decreases activation of IRF and NF-кB transcription factors and production of type I IFN and proinflammatory cytokines and chemokines [17]. MAVS was also necessary for hMPV-induced cellular signaling, as expression of a dominant negative mutant MAVS significantly reduced IFN- $\beta$ and chemokine gene transcription, in response to hMPV infection. RIG-I-dependent signaling was necessary to induce a cellular antiviral state, as reduction of RIG-I expression resulted in enhanced hMPV replication (Figure 3) [17].

Figure 3. RIG-I/MAVS signaling pathway regulating hMPV-induced NF-кB and IRF activation in airway epithelial cells. Production of specific RNA moieties during viral replication leads to activation of the RIG-I-MAVs pathway, which subsequent activation of the IKK complex, which is upstream NF- $\kappa$ B activation, and of the TBK1/IKK $\beta$ complex, which regulates IRF-3 activation, leading to proinflammatory/immune gene expression. $\mathrm{P}$ indicates phosphorylation; ? indicates the unknown kinase that phosphorylates NF-кB in response to hMPV infection.

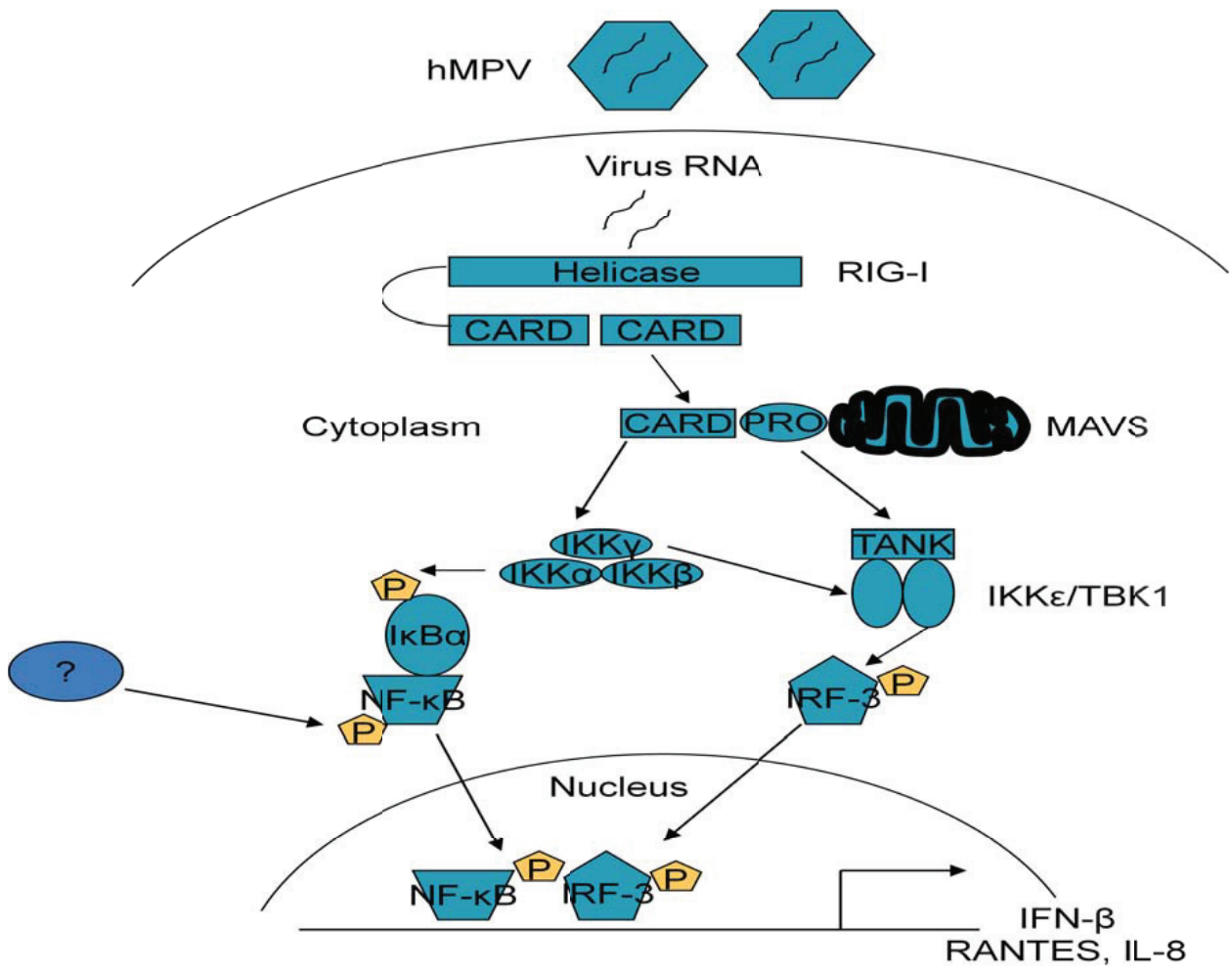




\section{Inhibition of TLR Signaling}

Dendritic cells are professional antigen-presenting cells that can be generally divided into myeloid CD11 $\mathrm{c}^{+}$"conventional” DCs (cDCs) or pDCs [45]. Both subsets specialize in detecting viruses and initiating innate and adaptive immune responses that lead to viral elimination or control [46]. Under baseline conditions, these cells reside in the peripheral tissue in an immature, resting state scattered throughout the respiratory mucosal wall [47] and express several receptors for recognizing viruses [48] such as TLRs. These DCs are often the first immune cells to come in contact with infecting viruses after infection, particularly after mucosal exposure, and can became primary targets of infection. After detection, uptake, and degradation of viruses, DCs initiate immune responses via the secretion of interferon, chemokines, and proinflammatory cytokines, and the upregulation of a variety of costimulatory molecules and receptors, a process globally known as cell maturation. The central role of DC in initiating and shaping the immune response, together with their presence at and recruitment to the site of infection and concomitant exposure to infectious virus, makes them obvious candidates for viral manipulation of the host immune response.

We and others have reported that hMPV can infect monocyte-derived DCs (moDCs), resulting in viral antigen expression [10,49,50] and maturation (increased expression of MHC II, CD86) [10,50]. HMPV can also infect pDCs, although less efficiently than moDCs [10]. In recent investigations we have shown that hMPV infection inhibits TLR-dependent signaling both in vitro and in vivo. Type I IFN production in isolated moDCs, following stimulation with TLR3 and 4 agonists, and in pDCs, in response to a TLR9 agonist, was significantly reduced by hMPV infection in a replication-dependent manner [10,18]. Furthermore, prior infection of BALB/c mice with hMPV completely suppressed IFN- $\alpha$ production induced by intranasal application of poly-ICLC (TLR3 ligand) or a synthetic CpG-ODN (TLR9 ligand) in mice lung [51], indicating that hMPV interferes with one or multiple signal transduction pathways activated in response to TLR stimulation in a variety of cell types.

\section{Interferon Signaling Antagonism}

IFNs are a group of cytokines that activate an array of cellular genes that are critical in restricting viral replication and modulating adaptive immunity. Production of IFNs is an important feature of the host response to viral infections. Type I IFNs (IFN- $\alpha$ and $-\beta$ ) are the key mediators produced by airway epithelial cells infected with paramyxoviruses [36,52,53] including hMPV [54]. Secreted IFN- $\alpha / \beta$ bind to IFN- $\alpha / \beta$ receptors (IFN $\alpha$ R) leading to dimerization of the two subunits, IFN $\alpha \mathrm{R} 1$ and IFN $\alpha \mathrm{R} 2$. IFN $\alpha \mathrm{R} 1$ and IFN $\alpha \mathrm{R} 2$ then undergo conformational changes resulting in the activation of the Janus tyrosine kinase (Jak)/signal transducer and activator of transcription protein (STAT) pathway [55]. Tyrosine kinase 2 (Tyk2), a kinase belonging to the Jak family, is constitutively bound to IFNAR1. Tyk2 phosphorylates IFNAR1 at tyrosine residue 466 (Y466) and creates a docking site for STAT2 [56]. Subsequently, Tyk2 phosphorylates STAT2 at tyrosine 690 (Y690). Phosphorylation of STAT2 Y690 creates a new docking site for the SH2 domain of STAT1 [57,58], which is subsequently phosphorylated at Y701 by IFNAR2 bound-Jak1 [59]. Phosphorylated STAT1 and STAT2 then 
dimerize and bind to IRF-9 [60]. This newly formed heterotrimer, known as IFN-stimulated gene factor 3 (ISGF3), translocates into the nucleus to bind ISG gene promoter and activate transcription. ISGs induced by type I IFN signaling typically contain either interferon stimulated response elements (ISRE) or a gamma activated sequence (GAS) elements within their promoters, although there is a clear preference for genes containing an ISRE. Examples of ISRE-containing ISGs are ISG15, Myxovirus (influenza virus) resistance (Mx)1, 2'-5'-oligoadenylate synthetase (OAS)1, IRF-7 and protein kinase R (PKR) [61], while GAS-containing genes are IRF-1, IRF-2, IRF-8 and IRF-9 [62,63]. In addition to activating this canonical Jak/STAT pathway described above, stimulation of the IFN $\alpha$ R also activates several non canonical signaling events such as recruitment and phosphorylation of other STATs [61-63] and tyrosine phosphorylation of and activation of insulin receptor substrates 1 (IRS1) and 2 (IRS2) [63].

As IFN response is critical for a robust innate immune response, almost all mammalian viruses have developed strategies to interfere with IFN production and signaling and to disrupt innate host antiviral factors. These include directly targeting the pathways required for the induction of IFN production, targeting of signaling molecules belonging to the Jak/STAT signaling pathway, and increasing the expression or activity of endogenous cellular key regulators, such as suppressor of cytokine signaling (SOCS) proteins, protein tyrosine phosphatases (PTPs) and protein inhibitor of activated STATs (PIAS) [64,65]. Several members of the Paramyxovirus family have been shown to directly target STAT signaling through distinct mechanisms which include proteasomal degradation [66-68], sequestration in high-molecular-weight complexes [69,70] and inhibition of nuclear localization of STAT proteins [71]. The first description of hMPV capacity to interfere with IFN signaling came from Harrod et al. who tested the ability of hMPV to subvert IFN- $\alpha$-dependent responses in airway epithelial cells using reporter gene assays. They showed that IFN- $\alpha$-mediated induction of ISRE-driven luciferase activity was completely abolished in hMPV-infected A549 cells, as well as induction of ISGs, such OAS1, Mx1, RIG-I and MDA-5 [72]. This observation was paralleled by the inhibition of IFN- $\alpha$-dependent tyrosine phosphorylation and subsequent nuclear translocation of STAT1 in hMPV-infected airway epithelial cells [72]. However, a mechanism responsible for the observed inhibition was not identified. Later, we have shown that hMPV infection affects several steps of the IFN signaling pathway, from inducing degradation of Jak 1 and Tyk2 via a ubiquitin-proteasome-dependent pathway, to reducing IFNAR1 surface expression in infected cells, possibly due to increased internalization of the receptor as a result of viral-induced degradation of Tyk2 (Figure 4) [73]. Both phenomena were independent of type I IFN expression, since inhibition was also observed in Vero cells which do not produce type I IFN, and required de novo viral gene expression and/or viral RNA replication [73].

\section{5. hMPV Proteins Identified as Antagonist of Host Innate Signaling Pathways}

Among hMPV encoded proteins, phosphoprotein $\mathrm{P}$, glycoprotein $\mathrm{G}$, small hydrophobic protein (SH) and M2-2 have been shown to modulate hMPV-induced innate immune response, the first line of 
host defense against invading pathogens [18,32,74-76]. A discussion of the inhibitory function for each of these proteins is presented below.

Small hydrophobic (SH) glycoprotein. hMPV SH protein is a type II transmembrane glycoprotein [77]. It is the largest among the pneumoviruses (179 aa for the hMPV isolate CAN97-83 versus 175 aa for APV, 81 aa for BRSV, and 64 aa for HRSV) [77,78]. Even though it is substantially longer than RSV, it has similar characteristics to the one of RSV, including a high percentage of threonine and serine residues and a similar hydrophilicity profile [77,78]. HMPV SH protein is more prone to frequent frameshift and point mutations in culture presumably to give a selective advantage of the clinical isolates in culture [79] and does not appear to be required for virus growth in vitro. In fact, a recombinant hMPV virus lacking the $\mathrm{SH}$ protein (rhMPV- $\Delta \mathrm{SH}$ ) is viable, grows as well as the wild-type virus in MK2 cells and A549 cells [74,80] and is not significantly attenuated in animal models of infection [74,80,81]. Similar to the SH protein of several members of the Paramyxoviridae family such PIV5 and RSV [82,83], and more recently J paramyxovirus (JPV) [84] and mumps virus $(\mathrm{MuV})$ [85], all of which have been shown to inhibit TNF- $\alpha$-mediated NF-кB signaling, we have also reported an inhibitory role of hMPV SH protein in NF- $\mathrm{BB}$ activation [74]. Infection of airway epithelial cells with rhMPV- $\triangle \mathrm{SH}$ led to increased interleukin 6 (IL-6), IL-8, and MCP-1 secretion, compared to rhMPV-WT [74]. Similarly, BALB/c mice infected with rhMPV- $\triangle \mathrm{SH}$ showed enhanced production of TNF- $\alpha$, IL-6, KC and MCP-1, compared to rhMPV-WT [74]. We observed significantly higher induction of IL-8 gene transcription in 293 cells infected with rhMPV- $\Delta \mathrm{SH}$ compared to rhMPV-WT, and SH protein expression lead to inhibition of TNF- $\alpha$ induced IL- 8 promoter activation, confirming a role of $\mathrm{SH}$ in inhibition of NF- $\mathrm{kB}-$ dependent gene transcription [74]. SH protein of hMPV affected NF- $\kappa B$-dependent gene transcription by modulating NF- $\kappa$ B transcriptional activity and not by inhibiting nuclear translocation, and therefore the canonical pathway leading to NF- $\kappa \mathrm{B}$ activation (Figure 4) [86]. Similar to Rep78 protein of adeno-associated virus type II, which targets PKA activation [87], hMPV SH might inhibit one of the kinases that phosphorylate NF- $\mathrm{BB}$ [74]. Our results indicate a possible novel mechanism by which paramyxovirus $\mathrm{SH}$ proteins can affect NF- $\mathrm{KB}$ activation, in addition to inhibiting TNF-induced NF- $\mathrm{B}$ nuclear translocation, as it has been shown for RSV and PIV5 SH proteins [82,83]. Whether RSV, PIV5 and JPV SH proteins can also affect viral-induced $\mathrm{NF}-\mathrm{\kappa B}$ post-translational modifications will require further investigation.

Glycoprotein G. hMPV G protein is a type II mucin-like glycosylated protein [77]. The membrane anchor of $\mathrm{G}$ protein is proximal to the $\mathrm{N}$ terminus and their $\mathrm{C}$ terminus is oriented externally. Although the postulated function of $\mathrm{G}$ protein is for attachment, it has been shown that hMPV F protein alone is sufficient to mediate attachment and fusion in the absence of other surface proteins including $G[80,81,88]$. In addition, the interaction of $F$ with cellular integrin receptors is independent of $G$ protein [89], suggesting that G protein plays a minimal role in hMPV attachment. Although lack of $\mathrm{G}$ protein does not decrease significantly the ability of hMPV to replicate in vitro, a recombinant virus in which the $\mathrm{G}$ protein is deleted (rhMPV- $\Delta \mathrm{G}$ ) exhibits reduced replication in the upper and lower respiratory tract of Syrian hamsters and African green monkeys [80,81]. 
In the past few years, our laboratory has been focusing on the molecular mechanism(s) underlying the attenuation of rhMPV- $\Delta \mathrm{G}$. We discovered that hMPV $\mathrm{G}$ is an important virulence factor which inhibits cellular signaling both in airway epithelial cells and in primary immune cells [75,90]. The respiratory epithelium represents the principal and primary target site of respiratory viruses, including $\mathrm{hMPV}$. We found that rhMPV $-\Delta \mathrm{G}$ induces significantly higher amounts of the cytokines, chemokines and type I IFN than hMPV-WT in airway epithelial cells, due to enhanced activation of transcription factors belonging to the NF- $\mathrm{BB}$ (e.g., p65 and p50) and IRF families (e.g., IRF-3), demonstrated by increased nuclear translocation and/or phosphorylation [75]. As RIG-I and MAVS are central regulators of hMPV-induced cellular signaling in airway epithelial cells, we investigated whether $\mathrm{G}$ targeted the RIG-I/MAVS pathway and, indeed, we found that G protein physically interacts with RIG-I and inhibits RIG-I-, but not MAVS-dependent IFN- $\beta$ gene transcription (Figure 4) [75].

Figure 4. Schematic diagram of sites of antagonism of cellular signaling and IFN production/responses by hMPV proteins. Major cellular targets of hMPV proteins in the TLR4, RIG-I/MAVS and type I IFN signaling pathways. P indicates phosphorylation.

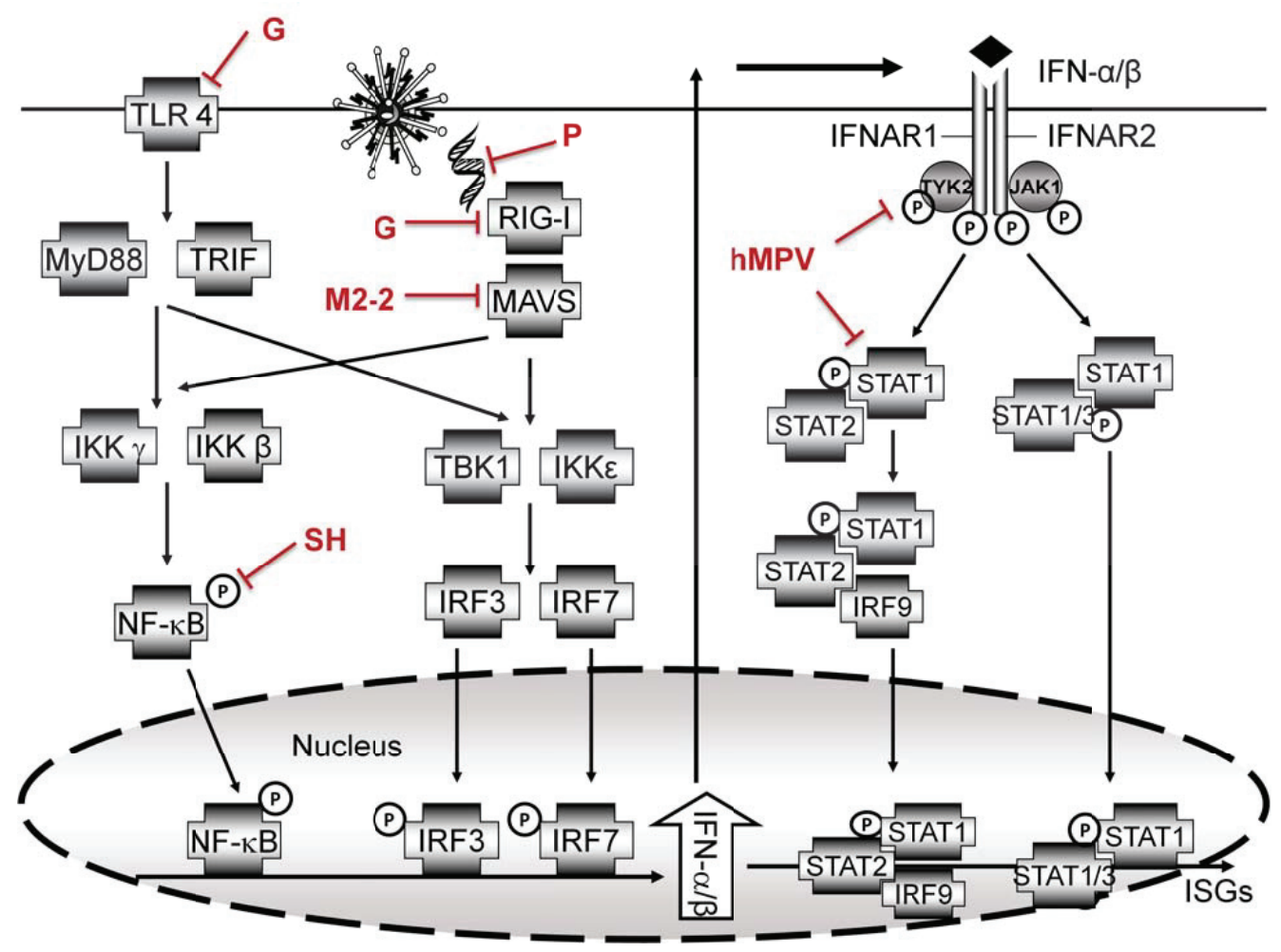

We have recently investigated the molecular mechanism by which $\mathrm{G}$ protein expression inhibits RIG-I activation. We found that the interaction of hMPV G with RIG-I occurs primarily through the 
CARD domains of its N-terminus, preventing RIG-I association with the adaptor protein MAVS and RIG-I recruitment to mitochondria. HMPV G expression also prevented the interaction between mitochondria and mitochondria-associated membrane (MAM) component of the endoplasmic reticulum (ER), which contains Stimulator of IFN genes (STINGS), an important part of the viral-induced RIG-I/MAVS signaling pathway, leading in the end to the inhibition of cytokine, chemokine and type I interferon (IFN) expression. Mutagenesis analysis showed that hMPV G protein cytoplasmic domain played a major role in the observed inhibitory activity, and recombinant viruses expressing a $G$ protein with amino acid substitution in position 2 and 3 recapitulated most of the phenotype observed with rhMPV- $\Delta \mathrm{G}$ mutant upon infection of airway epithelial cells [91].

DCs play a pivotal role in shaping antiviral immune responses in the respiratory tract. They can efficiently sense invading pathogens by TLRs and, because of their strategic localization at mucosal sites, are involved in the response to viral infections [92,93]. We have recently demonstrated that moDCs infected with rhMPV- $\Delta \mathrm{G}$ also produce higher levels of type I IFNs, cytokines and chemokines compared with cells infected with rhMPV-WT, suggesting that G protein plays an inhibitory role in viral-induced cellular responses of immune cells as well (Figure 4) [90]. As discussed above, TLR4 plays a major role in hMPV-induced activation of moDCs [90]. In our study, we found that G protein impaired TLR4-dependent signaling, as moDCs infection with rhMPV- $\Delta \mathrm{G}$ inhibited LPS-induced production of cytokine and chemokines significantly less than rhMPV-WT, and treatment of moDCs with purified G protein resulted in significant inhibition of LPS-dependent signaling. Taken together, our results demonstrate that hMPV G protein plays an important role in inhibiting host innate immune responses in dendritic cells, possibly affecting adaptive immune responses as well. This is an important finding, as inadequate TLR stimulation, with subsequent lack of antibody affinity maturation has been recently identified as an important cause of vaccine failure and enhanced disease following administration of the formaline-inactivated RSV vaccine [94].

Inhibition of cellular signaling by surface glycoproteins has been demonstrated for other viruses as well. RSV G protein inhibits cytokine and chemokine secretion, as infection with a recombinant RSV lacking the full-length $\mathrm{G}$ protein $(\mathrm{rRSV}-\Delta \mathrm{G})$ or the soluble part of $\mathrm{G}$ protein (rRSV- $\Delta \mathrm{sG}$ ) enhances production of IL-6 and IL-8 in monocytes [95], and IL-8 and RANTES secretion, and ICAM expression in airway epithelial cells [96]. RSV- $\Delta \mathrm{G}$ also induces more immune mediator production, compared to WT virus, in a mouse model of infection [95]. Similarly, the surface glycoproteins of hantaviruses, in particular, those associated with hemorrhagic pulmonary syndrome (HPS), have been shown to affect IRF-3 activation and IFN production via interaction with RIG-I and TBK-1, a kinase responsible for viral-induced IRF-3 phosphorylation, and to inhibit IFN-mediated cellular responses [97,98]. Taken together, it could be a common feature of surface glycoproteins of enveloped single strand, negative strand RNA viruses to be inhibitory to antiviral signaling, consequently, leading to host immune evasion.

M2-2 protein. HMPV M2 encodes two overlapping proteins: M2-1 and M2-2. The M2-1 open reading frame (ORF) of strain CAN 97-83 is assumed to start with the first AUG at nucleotide position 14, and encodes a protein of 187 amino acids. The M2-2 ORF possibly initiates with the AUGs at 
positions 525 and 537, overlapping the M2-1 ORF by 53 or 41 nucleotides, respectively [77,99]. M2-1 protein is not essential for hMPV recovery using the reverse genetic system in vitro, in contrast to RSV M2-1 protein, which is essential for full viral replication [99-101]. The role of hMPV M2-2 protein in regulating viral RNA synthesis has been confirmed by our group and several others $[81,99,102,103]$.

Similar to rhMPV- $\Delta$ G, recombinant rhMPV lacking M2-2 (rhMPV- $\Delta$ M2-2) is also listed as a live vaccine candidate, as it is attenuated, immunogenic and protective against hMPV challenge in both African green monkeys and hamsters $[81,99,102]$. Although the attenuation of rhMPV- $\triangle \mathrm{M} 2-2$ could be explained by decreased viral genome accumulation by M2-2 deletion [99,102,103], we found that other mechanism(s) could be also associated with rhMPV- $\triangle \mathrm{M} 2-2$ attenuation, as discussed below. We recently discovered that hMPV M2-2 is an important antagonist of host antiviral signaling, therefore, favoring hMPV replication. In fact, $\triangle \mathrm{M} 2$-2-infected airway epithelial cells produced higher levels of IFN- $\beta$ and other immune mediators, compared to rhMPV-WT-infected cells [76]. Although the expression of hMPV G protein was impaired due to a reduced ability of rhMPV- $\triangle \mathrm{M} 2-2$ to replicate, which might contribute indirectly to the enhanced cellular responses observed following infection with the M2-2 deleted mutant, ectopic expression of G protein at levels comparable or higher than the one observed in WT-infected cells only partially reversed the enhancement in cellular responses observed with rhMPV- $\triangle \mathrm{M} 2-2$, suggesting that M2-2 contributes to hMPV immune evasion as well. Indeed, in reporter gene assays, M2-2 protein, but not other soluble hMPV proteins, inhibited MAVS-induced IFN- $\beta$ gene transcription, but not the induction mediated by downstream signaling molecules of the RIG-I/MAVS pathway, suggesting that MAVS is the target of M2-2 (Figure 4). Coimmunoprecipitation studies, both in an overexpression system or in the context of viral infection, showed a clear association of M2-2 with MAVS, supporting this concept [76].

We have also identified the domains of M2-2 responsible for the regulation of viral gene transcription, viral replication, and RIG-I-mediated signaling. We found that the first 25 amino acids of M2-2 are critical to promote viral gene transcription, but not involved in the regulation of viral replication and hMPV-induced signaling. In contrast, the domains spanning from amino acid 26 to 69 are dispensable for the regulation of viral gene transcription, but responsible for RIG-I signaling inhibition and viral replication facilitation [76].

In summary, hMPV uses both $\mathrm{G}$ and M2-2 protein to target the RIG-I/MAVS pathway. The mechanism by which viruses use two distinct viral proteins to target molecules belonging to the same cellular signaling pathway is becoming recognized as a common strategy to evade host immune defenses. For example, influenza virus uses its NS1 protein to target RIG-I [104,105], and its PB1-F2 and PB2 proteins to inhibit MAVS [106,107]. In case of RSV, NS2 protein of RSV antagonizes the activation of IFN- $\beta$ transcription by interaction with RIG-I [108], and we recently found that NS1 protein inhibits IFN- $\beta$ synthesis by associating with RIG-I downstream transcription factor IRF-3 and its transcriptional co-activator CBP [109].

Phosphoprotein (P). The $\mathrm{P}$ protein gene of the pneumoviruses, different from the one of paramyxovirinae, is monocistronic, encoding a single polypeptide with a predicted mass of $32.5 \mathrm{kDa}$ [110], although production of multiple forms of phosphoprotein in infected cells has been 
reported [111]. Similar to other pneumoviruses, hMPV $\mathrm{P}$ protein is present within the helical ribonucleoprotein (RNP) complex. It has been shown that hMPV N and P proteins interact together and form cytoplasmic inclusion bodies [112].

Elegant studies conducted by Goutagny et al. [32], focusing on comparing the innate responses to hMPV A and B strains, showed that hMPV-B failed to elicit a type I IFN response in airway epithelial cells and in human monocytes, despite its ability to infect and replicate as efficiently as hMPV-A. Similar to our reports in epithelial cells [17], Goutagny and coworkers showed that hMPV A1 triggers RIG-I activation to induce type I IFN production while hMPV B1 did not [32]. They showed that in the context of the virus infection, the hMPV B viral RNA is prevented from being sensed by RIG-I and that the P protein is responsible for this inhibition (Figure 4). Since viral RNA is protected from free cellular RNases by RNP complex, proteins within the RNP, especially P protein in hMPV B1 probably could prevent the recognition of the viral RNA by the RIG-I pathway. This strain specific inhibition of RIG-I sensing by P protein has been ascribed to higher levels of expression of the P protein, as well as higher affinity for the RNA or other components of the RNP complex, during hMPV-B infection, compared to hMPV-A [32]. Moreover, the inhibitory effect of the hMPV-B P protein was restricted to the RIG-I pathway, because it did not prevent the induction of IFN- $\alpha / \beta$ in pDCs, which uses TLR7 pathway. In conclusion, this study suggests that in addition to other structural proteins such as $G$, SH and M2-2 proteins, RNP complex proteins might also play an important role in inhibition of viral- induced type I IFN expression.

In summary, hMPV antagonizes cellular responses and type I IFN secretion/signaling through a variety of mechanisms which involve the regulation of PRRs (both TLRs and RLR), as well as the key signaling molecules involved in the downstream signaling of IFN pathway such as STAT1, STAT2, Jak1 and Tyk2. The role of this inhibition of type I IFN signaling by hMPV in pathogenesis and severity of infection also needs to be investigated, as inhibition of IFN signaling may affect development of host adaptive immunity, leaving the host susceptible to reinfection. A better understanding of how hMPV inhibits cellular signaling and type I IFN pathway, and its consequences in regard to the innate and adaptive immune responses, is crucial for improving therapeutic approaches and the development of better vaccines against hMPV infection.

\section{Acknowledgments}

We would like to thank Allison Solari for her assistance in the manuscript submission.

\section{Conflicts of Interest}

The authors declare no conflict of interest. 


\section{References}

1. Lamb, R.A.; Kolakofsky, D. Paramyxoviridae: The Viruses and Their Replication. In Fundamental Virology, 4th ed.; Knipe, D.M., Howley, P.M., Eds; Lippincott, Williams and Wilkins: Philadelphia, PA, USA, 2001; pp. 689-724.

2. Principi, N.; Bosis, S.; Esposito, S. Human metapneumovirus in paediatric patients. Clin. Microbiol. Infect. 2006, 12, 301-308.

3. Van den Hoogen, B.G.; de Jong, J.C.; Groen, J.; Kuiken, T.; de Groot, R.; Fouchier, R.A.; Osterhaus, A.D. A newly discovered human pneumovirus isolated from young children with respiratory tract disease. Nat. Med. 2001, 7, 719-724.

4. Kahn, J.S. Epidemiology of human metapneumovirus. Clin. Microbiol. Rev. 2006, 19, 546-557.

5. Williams, J.V.; Harris, P.A.; Tollefson, S.J.; Halburnt-Rush, L.L.; Pingsterhaus, J.M.; Edwards, K.M.; Wright, P.F.; Crowe, J.E., Jr. Human metapneumovirus and lower respiratory tract disease in otherwise healthy infants and children. N. Engl. J. Med. 2004, 350, 443-450.

6. Crowe, J.E., Jr. Human metapneumovirus as a major cause of human respiratory tract disease. Pediatr. Infect. Dis. J. 2004, 23, 215-221.

7. Boivin, G.; Abed, L.; Pelletier, G.; Ruel, L.; Moisan, D.; Cote', S.; Peret, T.C.; Erdman, D.D., Anderson, L.J. Virological features and clinical manifestations associated with the human metapneumovirus, a newly discovered paramyxovirus. J. Infect. Dis. 2002, 186, 1330-1334.

8. Esper, F.; Boucher, D.; Weibel, C.; Martinello, R.A.; Kahn, J.S. Human metapneumovirus infection in the United States: Clinical manifestations associated with a newly emerging respiratory infection in children. Pediatrics 2003, 111, 1407-1410.

9. Guerrero-Plata, A.; Casola, A.; Garofalo, R.P. Human metapneumovirus induces a profile of lung cytokines distinct from that of respiratory syncytial virus. J. Virol. 2005, 79, 14992-14997.

10. Guerrero-Plata, A.; Casola, A.; Suarez, G.; Yu, X.; Spetch, L.; Peeples, M.E.; Garofalo, R.P. Differential response of dendritic cells to human metapneumovirus and respiratory syncytial virus. Am. J. Respir. Cell Mol. Biol. 2006, 34, 320-329.

11. Bao, X.; Liu, T.; Spetch, L.; Kolli, D.; Garofalo, R.P.; Casola, A. Airway epithelial cell response to human metapneumovirus infection. Virology 2007, 386, 91-101.

12. Seth, R.B.; Sun, L.; Chen, Z.J. Antiviral innate immunity pathways. Cell Res. 2006, 16, 141-147.

13. Kawai, T.; Akira, S. TLR signaling. Cell Death Differ. 2006, 13, 816-825.

14. Takeda, K.; Akira, S. Toll-like receptors in innate immunity. Int. Immunol. 2005, 17, 1-14.

15. Groskreutz, D.J.; Monick, M.M.; Powers, L.S.; Yarovinsky, T.O.; Look, D.C.; Hunninghake, G.W. Respiratory syncytial virus induces TLR3 protein and protein kinase $\mathrm{R}$, leading to increased double-stranded RNA responsiveness in airway epithelial cells. J. Immunol. 2006, 176, 1733-1740.

16. Liu, P.; Jamaluddin, M.; Li, K.; Garofalo, R.P.; Casola, A.; Brasier, A.R. Retinoic Acid-inducible gene I mediates early antiviral response and toll-like receptor 3 expression in respiratory syncytial virus-infected airway epithelial cells. J. Virol. 2007, 81, 1401-1411. 
17. Liao, S.; Bao, X.; Liu, T.; Lai, S.; Li, K.; Garofalo, R.P.; Casola, A. Role of retinoic acid inducible gene-I in human metapneumovirus-induced cellular signalling. J. Gen. Virol. 2008, 89, 1978-1986.

18. Kolli, D.; Bao, X.; Liu, T.; Hong, C.; Wang, T.; Garofalo, R.P.; Casola, A. Human metapneumovirus glycoprotein G inhibits TLR4-dependent signaling in monocyte-derived dendritic cells. J. Immunol. 2011, 187, 47-54.

19. Beutler, B.; Hoebe, K.; Georgel, P.; Tabeta, K.; Du, X. Genetic analysis of innate immunity: Identification and function of the TIR adapter proteins. Adv. Exp. Med. Biol. 2005, 560, 29-39.

20. Haynes, L.M.; Moore, D.D.; Kurt-Jones, E.A.; Finberg, R.W.; Anderson, L.J.; Tripp, R.A. Involvement of toll-like receptor 4 in innate immunity to respiratory syncytial virus. J. Virol. 2001, 75, 10730-10737.

21. Rassa, J.C.; Meyers, J.L.; Zhang, Y.; Kudaravalli, R.; Ross, S.R. Murine retroviruses activate B cells via interaction with toll-like receptor 4. Proc. Natl. Acad. Sci. USA 2002, 99, 2281-2286.

22. Okumura, A.; Pitha, P.M.; Yoshimura, A.; Harty, R.N. Interaction between Ebola virus glycoprotein and host toll-like receptor 4 leads to induction of proinflammatory cytokines and SOCS1. J. Virol. 2010, 84, 27-33.

23. Kurt-Jones, E.A.; Popova, L.; Kwinn, L.; Haynes, L.M.; Jones, L.P.; Tripp, R.A.; Walsh, E.E.; Freeman, M.W.; Golenbock, D.T.; Anderson, L.J.; et al. Pattern recognition receptors TLR4 and CD14 mediate response to respiratory syncytial virus. Nat. Immunol 2000, 1, 398-401.

24. Lizundia, R.; Sauter, K.S.; Taylor, G.; Werling, D. Host species-specific usage of the TLR4-LPS receptor complex. Innate Immun. 2008, 14, 223-231.

25. Haeberle, H.; Takizawa, R.; Casola, A.; Brasier, A.R.; Dieterich, H.-J.; van Rooijen, N.; Gatalica, Z.; Garofalo, R.P. Respiratory syncytial virus-induced activation of NF-kB in the lung involves alveolar macrophages and Toll-like receptor 4-dependent pathways. J. Infect. Dis. 2002, 186, 1199-1206.

26. Cyr, S.L.; Angers, I.; Guillot, L.; Stoica-Popescu, I.; Lussier, M.; Qureshi, S.; Burt, D.S.; Ward, B.J. TLR4 and MyD88 control protection and pulmonary granulocytic recruitment in a murine intranasal RSV immunization and challenge model. Vaccine 2009, 27, 421-430.

27. Velayutham, T.S.; Kolli, D.; Ivanciuc, D.; Garofalo, R.P.; Casola, A. Critical role of Toll-like receptor 4 in human metapneumovirus innate immune responses and disease pathogenesis. J. Infect. Dis. 2012, manuscript in submission.

28. Heil, F.; Hemmi, H.; Hochrein, H.; Ampenberger, F.; Kirschning, C.; Akira, S.; Lipford, G.; Wagner, H.; Bauer, S. Species-specific recognition of single-stranded RNA via toll-like receptor 7 and 8. Science 2004, 303, 1526-1529.

29. Jurk, M.; Heil, F.; Vollmer, J.; Schetter, C.; Krieg, A.M.; Wagner, H.; Lipford, G.; Bauer, S. Human TLR7 or TLR8 independently confer responsiveness to the antiviral compound R-848. Nat. Immunol. 2002, 3, 499. 
30. Krug, A.; French, A.R.; Barchet, W.; Fischer, J.A.; Dzionek, A.; Pingel, J.T.; Orihuela, M.M.; Akira, S.; Yokoyama, W.M.; Colonna, M. TLR9-dependent recognition of MCMV by IPC and DC generates coordinated cytokine responses that activate antiviral NK cell function. Immunity 2004, 21, 107-119.

31. Krug, A.; Luker, G.D.; Barchet, W.; Leib, D.A.; Akira, S.; Colonna, M. Herpes simplex virus type 1 activates murine natural interferon-producing cells through toll-like receptor 9. Blood 2004, 103, 1433-1437.

32. Goutagny, N.; Jiang, Z.; Tian, J.; Parroche, P.; Schickli, J.; Monks, B.G.; Ulbrandt, N.; Ji, H.; Kiener, P.A.; Coyle, A.J.; et al. Cell type-specific recognition of human metapneumoviruses (HMPVs) by retinoic acid-inducible gene I (RIG-I) and TLR7 and viral interference of RIG-I ligand recognition by HMPV-B1 phosphoprotein. J. Immunol. 2010, 184, 1168-1179.

33. Davidson, S.; Kaiko, G.; Loh, Z.; Lalwani, A.; Zhang, V.; Spann, K.; Foo, S.Y.; Hansbro, N.; Uematsu, S.; Akira, S.; et al. Plasmacytoid dendritic cells promote host defense against acute pneumovirus infection via the TLR7-MyD88-dependent signaling pathway. J. Immunol. 2011, 186, 5938-5948.

34. Akira, S.; Uematsu, S.; Takeuchi, O. Pathogen recognition and innate immunity. Cell 2006, 124, 783-801.

35. Yamamoto, M.; Sato, S.; Hemmi, H.; Hoshino, K.; Kaisho, T.; Sanjo, H.; Takeuchi, O.; Sugiyama, M.; Okabe, M.; Takeda, K.; et al. Role of adaptor TRIF in the MyD88-independent toll-like receptor signaling pathway. Science 2003, 301, 640-643.

36. Andrejeva, J.; Childs, K.S.; Young, D.F.; Carlos, T.S.; Stock, N.; Goodbourn, S.; Randall, R.E. The V proteins of paramyxoviruses bind the IFN-inducible RNA helicase, mda-5, and inhibit its activation of the IFN-beta promoter. Proc. Natl. Acad. Sci. USA 2004, 101, 17264-17269.

37. Breiman, A.; Grandvaux, N.; Lin, R.; Ottone, C.; Akira, S.; Yoneyama, M.; Fujita, T.; Hiscott, J.; Meurs, E.F. Inhibition of RIG-I-dependent signaling to the interferon pathway during hepatitis C virus expression and restoration of signaling by IKKepsilon. J. Virol. 2005, 79, 3969-3978.

38. tenOever, B.R.; Sharma, S.; Zou, W.; Sun, Q.; Grandvaux, N.; Julkunen, I.; Hemmi, H.; Yamamoto, M.; Akira, S.; Yeh, W.C.; et al. Activation of TBK1 and IKKvarepsilon kinases by vesicular stomatitis virus infection and the role of viral ribonucleoprotein in the development of interferon antiviral immunity. J. Virol. 2004, 78, 10636-10649.

39. Siren, J.; Imaizumi, T.; Sarkar, D.; Pietila, T.; Noah, D.L.; Lin, R.; Hiscott, J.; Krug, R.M.; Fisher, P.B.; Julkunen, I.; et al. Retinoic acid inducible gene-I and mda-5 are involved in influenza A virus-induced expression of antiviral cytokines. Microbes Infect. 2006, 8, 2013-2020.

40. Pichlmair, A.; Schulz, O.; Tan, C.P.; Naslund, T.I.; Liljestrom, P.; Weber, F.; Reis e Sousa, C. RIG-I-mediated antiviral responses to single-stranded RNA bearing 5'-phosphates. Science 2006, 314, 997-1001.

41. Hiscott, J.; Lin, R.; Nakhaei, P.; Paz, S. MasterCARD: A priceless link to innate immunity. Trends Mol. Med. 2006, 12, 53-56. 
42. Johnson, C.L.; Gale, M., Jr. CARD games between virus and host get a new player. Trends Immunol. 2006, 27, 1-4.

43. Kawai, T.; Takahashi, K.; Sato, S.; Coban, C.; Kumar, H.; Kato, H.; Ishii, K.J.; Takeuchi, O.; Akira, S. IPS-1, an adaptor triggering RIG-I- and Mda5-mediated type I interferon induction. Nat. Immunol. 2005, 6, 981-988.

44. Meylan, E.; Tschopp, J. Toll-like receptors and RNA helicases: Two parallel ways to trigger antiviral responses. Mol. Cell 2006, 22, 561-569.

45. Liu, Y.J.; Kanzler, H.; Soumelis, V.; Gilliet, M. Dendritic cell lineage, plasticity and cross-regulation. Nat. Immunol. 2001, 2, 585-589.

46. Rinaldo, C.R., Jr.; Piazza, P. Virus infection of dendritic cells: Portal for host invasion and host defense. Trends Microbiol. 2004, 12, 337-345.

47. Stumbles, P.A.; Upham, J.W.; Holt, P.G. Airway dendritic cells: Co-ordinators of immunological homeostasis and immunity in the respiratory tract. APMIS 2003, 111, 741-755.

48. Rescigno, M.; Borrow, P. The host-pathogen interaction: New themes from dendritic cell biology. Cell 2001, 106, 267-270.

49. Tan, M.C.; Battini, L.; Tuyama, A.C.; Macip, S.; Melendi, G.A.; Horga, M.A.; Gusella, G.L. Characterization of human metapneumovirus infection of myeloid dendritic cells. Virology 2007, 357, 1-9.

50. Le, N.C.; Munir, S.; Losq, S.; Winter, C.C.; McCarty, T.; Stephany, D.A.; Holmes, K.L.; Bukreyev, A.; Rabin, R.L.; Collins, P.L.; et al. Infection and maturation of monocyte-derived human dendritic cells by human respiratory syncytial virus, human metapneumovirus, and human parainfluenza virus type 3. Virology 2009, 385, 169-182.

51. Guerrero-Plata, A.; Baron, S.; Poast, J.S.; Adegboyega, P.A.; Casola, A.; Garofalo, R.P. Activity and regulation of alpha interferon in respiratory syncytial virus and human metapneumovirus experimental infections. J. Virol. 2005, 79, 10190-10199.

52. Horvath, C.M. Silencing STATs: Lessons from paramyxovirus interferon evasion. Cytokine Growth Factor Rev. 2004, 15, 117-127.

53. Horvath, C.M. Weapons of STAT destruction. Interferon evasion by paramyxovirus V protein. Eur. J. Biochem. 2004, 271, 4621-4628.

54. Bao, X.; Liu, T.; Spetch, L.; Kolli, D.; Garofalo, R.P.; Casola, A. Airway epithelial cell response to human metapneumovirus infection. Virology 2007, 368, 91-101.

55. Colamonici, O.R.; Uyttendaele, H.; Domanski, P.; Yan, H.; Krolewski, J.J. p135tyk2, an interferon-alpha-activated tyrosine kinase, is physically associated with an interferon-alpha receptor. J. Biol. Chem. 1994, 269, 3518-3522.

56. Colamonici, O.; Yan, H.; Domanski, P.; Handa, R.; Smalley, D.; Mullersman, J.; Witte, M.; Krishnan, K.; Krolewski, J. Direct binding to and tyrosine phosphorylation of the alpha subunit of the type I interferon receptor by p135tyk2 tyrosine kinase. Mol. Cell Biol. 1994, 14, 8133-8142. 
57. Leung, S.; Qureshi, S.A.; Kerr, I.M.; Darnell, J.E., Jr.; Stark, G.R. Role of STAT2 in the alpha interferon signaling pathway. Mol. Cell Biol. 1995, 15, 1312-1317.

58. Qureshi, S.A.; Salditt-Georgieff, M.; Darnell, J.E., Jr. Tyrosine-phosphorylated Stat1 and Stat2 plus a $48-\mathrm{kDa}$ protein all contact DNA in forming interferon-stimulated-gene factor 3 . Proc. Natl. Acad. Sci. USA 1995, 92, 3829-3833.

59. Uze, G.; Schreiber, G.; Piehler, J.; Pellegrini, S. The receptor of the type I interferon family. Curr. Top. Microbiol. Immunol. 2007, 316, 71-95.

60. Li, X.; Leung, S.; Burns, C.; Stark, G.R. Cooperative binding of Stat1-2 heterodimers and ISGF3 to tandem DNA elements. Biochimie 1998, 80, 703-710.

61. Hata, N.; Sato, M.; Takaoka, A.; Asagiri, M.; Tanaka, N.; Taniguchi, T. Constitutive IFN-alpha/beta signal for efficient IFN-alpha/beta gene induction by virus. Biochem. Biophys. Res. Commun. 2001, 285, 518-525.

62. Trinchieri, G. Type I interferon: Friend or foe? J. Exp. Med. 2010, 207, 2053-2063.

63. Hervas-Stubbs, S.; Perez-Gracia, J.L.; Rouzaut, A.; Sanmamed, M.F.; Le, B.A.; Melero, I. Direct effects of type I interferons on cells of the immune system. Clin. Cancer Res. 2011, 17, 2619-2627.

64. Christophi, G.P.; Hudson, C.A.; Panos, M.; Gruber, R.C.; Massa, P.T. Modulation of macrophage infiltration and inflammatory activity by the phosphatase SHP-1 in virus-induced demyelinating disease. J. Virol. 2009, 83, 522-539.

65. Yasukawa, H.; Misawa, H.; Sakamoto, H.; Masuhara, M.; Sasaki, A.; Wakioka, T.; Ohtsuka, S.; Imaizumi, T.; Matsuda, T.; Ihle, J.N.; et al. The JAK-binding protein JAB inhibits Janus tyrosine kinase activity through binding in the activation loop. EMBO J. 1999, 18, 1309-1320.

66. Ramaswamy, M.; Shi, L.; Monick, M.M.; Hunninghake, G.W.; Look, D.C. Specific inhibition of type I interferon signal transduction by respiratory syncytial virus. Am. J. Respir. Cell Mol. Biol. 2004, 30, 893-900.

67. Didcock, L.; Young, D.F.; Goodbourn, S.; Randall, R.E. The V protein of simian virus 5 inhibits interferon signalling by targeting STAT1 for proteasome-mediated degradation. J. Virol. 1999, 73, 9928-9933.

68. Kubota, T.; Yokosawa, N.; Yokota, S.; Fujii, N. C terminal CYS-RICH region of mumps virus structural $\mathrm{V}$ protein correlates with block of interferon alpha and gamma signal transduction pathway through decrease of STAT 1-alpha. Biochem. Biophys. Res. Commun. 2001, 283, 255-259.

69. Rodriguez, J.J.; Cruz, C.D.; Horvath, C.M. Identification of the nuclear export signal and STAT-binding domains of the Nipah virus $\mathrm{V}$ protein reveals mechanisms underlying interferon evasion. J. Virol. 2004, 78, 5358-5367.

70. Rodriguez, J.J.; Parisien, J.P.; Horvath, C.M. Nipah virus V protein evades alpha and gamma interferons by preventing STAT1 and STAT2 activation and nuclear accumulation. J. Virol. 2002, 76, 11476-11483.

71. Takeuchi, K.; Kadota, S.I.; Takeda, M.; Miyajima, N.; Nagata, K. Measles virus V protein blocks interferon (IFN)-alpha/beta but not IFN-gamma signaling by inhibiting STAT1 and STAT2 phosphorylation. FEBS Lett. 2003, 545, 177-182. 
72. Dinwiddie, D.L.; Harrod, K.S. Human Metapneumovirus Inhibits IFN-\{alpha\} Signaling Through Inhibition of STAT1 Phosphorylation. Am. J. Respir. Cell Mol. Biol. 2008, 38, 661-670.

73. Ren, J.; Kolli, D.; Liu, T.; Xu, R.; Garofalo, R.P.; Casola, A.; Bao, X. Human metapneumovirus inhibits IFN-beta signaling by downregulating Jak1 and Tyk2 cellular levels. PLoS One 2011, 6, e24496.

74. Bao, X.; Kolli, D.; Liu, T.; Shan, Y.; Garofalo, R.P.; Casola, A. Human metapneumovirus small hydrophobic protein inhibits NF-kappaB transcriptional activity. J. Virol. 2008, 82, 8224-8229.

75. Bao, X.; Liu, T.; Shan, Y.; Li, K.; Garofalo, R.P.; Casola, A. Human metapneumovirus glycoprotein G inhibits innate immune responses. PLoS. Pathog. 2008, 4, e1000077.

76. Ren, J.; Wang, Q.; Kolli, D.; Prusak, D.J.; Tseng, C.T.; Chen, Z.J.; Li, K.; Wood, T.G.; Bao, X. Human metapneumovirus M2-2 protein inhibits innate cellular signaling by targeting MAVS. J. Virol. 2012, 86, 13049-13061.

77. Van den Hoogen, B.G.; Bestebroer, T.M.; Osterhaus, A.D.; Fouchier, R.A. Analysis of the genomic sequence of a human metapneumovirus. Virology 2002, 295, 119-132.

78. Biacchesi, S.; Skiadopoulos, M.H.; Boivin, G.; Hanson, C.T.; Murphy, B.R.; Collins, P.L.; Buchholz, U.J. Genetic diversity between human metapneumovirus subgroups. Virology 2003, 315, 1-9.

79. Biacchesi, S.; Murphy, B.R.; Collins, P.L.; Buchholz, U.J. Frequent frameshift and point mutations in the SH gene of human metapneumovirus passaged in vitro. J. Virol. 2007, 81, 6057-6067.

80. Biacchesi, S.; Skiadopoulos, M.H.; Yang, L.; Lamirande, E.W.; Tran, K.C.; Murphy, B.R.; Collins, P.L.; Buchholz, U.J. Recombinant human Metapneumovirus lacking the small hydrophobic SH and/or attachment $\mathrm{G}$ glycoprotein: Deletion of $\mathrm{G}$ yields a promising vaccine candidate. J. Virol. 2004, 78, 12877-12887.

81. Biacchesi, S.; Pham, Q.N.; Skiadopoulos, M.H.; Murphy, B.R.; Collins, P.L.; Buchholz, U.J. Infection of nonhuman primates with recombinant human metapneumovirus lacking the $\mathrm{SH}, \mathrm{G}$, or M2-2 protein categorizes each as a nonessential accessory protein and identifies vaccine candidates. J. Virol. 2005, 79, 12608-12613.

82. Wilson, R.L.; Fuentes, S.M.; Wang, P.; Taddeo, E.C.; Klatt, A.; Henderson, A.J.; He, B. Function of small hydrophobic proteins of paramyxovirus. J. Virol. 2006, 80, 1700-1709.

83. Fuentes, S.; Tran, K.C.; Luthra, P.; Teng, M.N.; He, B. Function of the respiratory syncytial virus small hydrophobic protein. J. Virol. 2007, 81, 8361-8366.

84. Li, Z.; Xu, J.; Patel, J.; Fuentes, S.; Lin, Y.; Anderson, D.; Sakamoto, K.; Wang, L.F.; He, B. Function of the small hydrophobic protein of J paramyxovirus. J. Virol. 2011, 85, 32-42.

85. Xu, P.; Li, Z.; Sun, D.; Lin, Y.; Wu, J.; Rota, P.A.; He, B. Rescue of wild-type mumps virus from a strain associated with recent outbreaks helps to define the role of the SH ORF in the pathogenesis of mumps virus. Virology 2011, 417, 126-136.

86. Karin, M.; Delhase, M. The I kappa B kinase (IKK) and NF-kappa B: Key elements of proinflammatory signalling. Semin. Immunol. 2000, 12, 85-98. 
87. Schmidt, M.; Chiorini, J.A.; Afione, S.; Kotin, R. Adeno-associated virus type 2 Rep78 inhibition of PKA and PRKX: Fine mapping and analysis of mechanism. J. Virol. 2002, 76, 1033-1042.

88. Schowalter, R.M.; Smith, S.E.; Dutch, R.E. Characterization of human metapneumovirus F protein-promoted membrane fusion: Critical roles for proteolytic processing and low $\mathrm{pH}$. J. Virol. 2006, 80, 10931-10941.

89. Cox, R.G.; Livesay, S.B.; Johnson, M.; Ohi, M.D.; Williams, J.V. The human metapneumovirus fusion protein mediates entry via an interaction with RGD-binding integrins. J. Virol. 2012, 86, 12148-12160.

90. Kolli, D.; Bao, X.; Liu, T.; Hong, C.; Wang, T.; Garofalo, R.P.; Casola, A. Human metapneumovirus glycoprotein $\mathrm{G}$ inhibits TLR4-dependent signaling in monocyte-derived dendritic cells. J. Immunol. 2011, 187, 47-54.

91. Bao, X.; Kolli, D.; Ren, J.; Liu, T.; Garofalo, R.P.; Casola, A. Human metapneumovirus glycoprotein G targets RIG-I to inhibit airway epithelial cell responses. J. Virol. 2012, manuscript in submission.

92. Pulendran, B.; Palucka, K.; Banchereau, J. Sensing pathogens and tuning immune responses. Science 2001, 293, 253-256.

93. Mellman, I.; Steinman, R.M. Dendritic cells: Specialized and regulated antigen processing machines. Cell 2001, 106, 255-258.

94. Delgado, M.F.; Coviello, S.; Monsalvo, A.C.; Melendi, G.A.; Hernandez, J.Z.; Batalle, J.P.; Diaz, L.; Trento, A.; Chang, H.Y.; Mitzner, W.; et al. Lack of antibody affinity maturation due to poor Toll-like receptor stimulation leads to enhanced respiratory syncytial virus disease. Nat. Med. 2009, 15, 34-41.

95. Polack, F.P.; Irusta, P.M.; Hoffman, S.J.; Schiatti, M.P.; Melendi, G.A.; Delgado, M.F.; Laham, F.R.; Thumar, B.; Hendry, R.M.; Melero, J.A.; et al. The cysteine-rich region of respiratory syncytial virus attachment protein inhibits innate immunity elicited by the virus and endotoxin. Proc. Natl. Acad. Sci. USA 2005, 102, 8996-9001.

96. Arnold, R.; Konig, B.; Werchau, H.; Konig, W. Respiratory syncytial virus deficient in soluble G protein induced an increased proinflammatory response in human lung epithelial cells. Virology 2004, 330, 384-397.

97. Alff, P.J.; Gavrilovskaya, I.N.; Gorbunova, E.; Endriss, K.; Chong, Y.; Geimonen, E.; Sen, N.; Reich, N.C.; Mackow, E.R. The pathogenic NY-1 hantavirus G1 cytoplasmic tail inhibits RIG-I- and TBK-1-directed interferon responses. J. Virol. 2006, 80, 9676-9686.

98. Geimonen, E.; LaMonica, R.; Springer, K.; Farooqui, Y.; Gavrilovskaya, I.N.; Mackow, E.R. Hantavirus pulmonary syndrome-associated hantaviruses contain conserved and functional ITAM signaling elements. J. Virol. 2003, 77, 1638-1643. 
99. Buchholz, U.J.; Biacchesi, S.; Pham, Q.N.; Tran, K.C.; Yang, L.; Luongo, C.L.; Skiadopoulos, M.H.; Murphy, B.R.; Collins, P.L. Deletion of M2 gene open reading frames 1 and 2 of human metapneumovirus: Effects on RNA synthesis, attenuation, and immunogenicity. J. Virol. 2005, 79, 6588-6597.

100. Herfst, S.; de Graaf, M.; Schickli, J.H.; Tang, R.S.; Kaur, J.; Yang, C.F.; Spaete, R.R.; Haller, A.A.; van den Hoogen, B.G.; Osterhaus, A.D.; et al. Recovery of human metapneumovirus genetic lineages a and B from cloned cDNA. J. Virol. 2004, 78, 8264-8270.

101. Sutherland, K.A.; Collins, P.L.; Peeples, M.E. Synergistic effects of gene-end signal mutations and the M2-1 protein on transcription termination by respiratory syncytial virus. Virology 2001, 288, 295-307.

102. Schickli, J.H.; Kaur, J.; MacPhail, M.; Guzzetta, J.M.; Spaete, R.R.; Tang, R.S. Deletion of human metapneumovirus M2-2 increases mutation frequency and attenuates growth in hamsters. Virol. J. 2008, 3, 5-69.

103. Ren, J.; Wang, Q.; Kolli, D.; Prusak, D.J.; Tseng, C.T.; Li, K.; Wood, T.G.; Bao, X. Human metapneumovirus M2-2 protein inhibits the innate cellular signaling by targeting MAVS. J. Virol. 2012, 86, 13049-13061.

104. Guo, Z.; Chen, L.M.; Zeng, H.; Gomez, J.A.; Plowden, J.; Fujita, T.; Katz, J.M.; Donis, R.O.; Sambhara, S. NS1 protein of influenza A virus inhibits the function of intracytoplasmic pathogen sensor, RIG-I. Am. J. Respir. Cell Mol. Biol. 2007, 36, 263-269.

105. Opitz, B.; Rejaibi, A.; Dauber, B.; Eckhard, J.; Vinzing, M.; Schmeck, B.; Hippenstiel, S.; Suttorp, N.; Wolff, T. IFNbeta induction by influenza A virus is mediated by RIG-I which is regulated by the viral NS1 protein. Cell Microbiol. 2007, 9, 930-938.

106. Varga, Z.T.; Ramos, I.; Hai, R.; Schmolke, M.; Garcia-Sastre, A.; Fernandez-Sesma, A.; Palese, P. The influenza virus protein PB1-F2 inhibits the induction of type I interferon at the level of the MAVS adaptor protein. PLoS. Pathog. 2011, 7, e1002067.

107. Graef, K.M.; Vreede, F.T.; Lau, Y.F.; McCall, A.W.; Carr, S.M.; Subbarao, K.; Fodor, E. The PB2 subunit of the influenza virus RNA polymerase affects virulence by interacting with the mitochondrial antiviral signaling protein and inhibiting expression of beta interferon. J. Virol. 2010, 84, 8433-8445.

108. Ling, Z.; Tran, K.C.; Teng, M.N. Human respiratory syncytial virus nonstructural protein NS2 antagonizes the activation of beta interferon transcription by interacting with RIG-I. J. Virol. 2009, 83, 3734-3742.

109. Ren, J.; Liu, T.; Pang, L.; Li, K.; Garofalo, R.G.; Casola, A.; Bao, X. A novel mechanism for inhibition of IRF-3-dependent gene expression by human respiratory syncytial virus NS1 protein. J. Gen. Virol. 2011, 92, 2153-2159.

110. Bastien, N.; Normand, S.; Taylor, T.; Ward, D.; Peret, T.C.; Boivin, G.; Anderson, L.J.; Li, Y. Sequence analysis of the N, P, M and F genes of Canadian human metapneumovirus strains. Virus Res. 2003, 93, 51-62. 
111. Tedcastle, A.B.; Fenwick, F.; Ingram, R.E.; King, B.J.; Robinson, M.J.; Toms, G.L. The characterization of monoclonal antibodies to human metapneumovirus and the detection of multiple forms of the virus nucleoprotein and phosphoprotein. J. Med. Virolog. 2012, 84, 1061-1070.

112. Derdowski, A.; Peters, T.R.; Glover, N.; Qian, R.; Utley, T.J.; Burnett, A.; Williams, J.V.; Spearman, P.; Crowe, J.E., Jr. Human metapneumovirus nucleoprotein and phosphoprotein interact and provide the minimal requirements for inclusion body formation. J. Gen. Virol. 2008, 89, 2698-2708. 
Reprinted from Viruses. Cite as: Cox, R.G.; Williams, J.V. Breaking In: Human Metapneumovirus Fusion and Entry. Viruses 2013, 5, 192-210.

Review

\title{
Breaking In: Human Metapneumovirus Fusion and Entry
}

\author{
Reagan G. Cox ${ }^{1}$ and John V. Williams ${ }^{2, *}$
}

1 Department of Pathology, Microbiology and Immunology, Vanderbilt University School of Medicine, 1161 21st Ave. S., Nashville, TN 37232, USA; E-Mail: reagan.j.cox@vanderbilt.edu

2 Departments of Pediatrics and Pathology, Microbiology, and Immunology, Vanderbilt University School of Medicine, 1161 21st Ave. S., Nashville, TN 37232, USA

* Author to whom correspondence should be addressed; E-Mail: john.williams@vanderbilt.edu; Tel.: +1-615-322-2250; Fax: +1-615-343-7659.

Received: 14 December 2012; in revised form: 9 January 2013 / Accepted: 10 January 2013 /

Published: 16 January 2013

\begin{abstract}
Human metapneumovirus (HMPV) is a leading cause of respiratory infection that causes upper airway and severe lower respiratory tract infections. HMPV infection is initiated by viral surface glycoproteins that attach to cellular receptors and mediate virus membrane fusion with cellular membranes. Most paramyxoviruses use two viral glycoproteins to facilitate virus entry - an attachment protein and a fusion (F) protein. However, membrane fusion for the human paramyxoviruses in the Pneumovirus subfamily, HMPV and respiratory syncytial virus (hRSV), is unique in that the F protein drives fusion in the absence of a separate viral attachment protein. Thus, pneumovirus $\mathrm{F}$ proteins can perform the necessary functions for virus entry, i.e., attachment and fusion. In this review, we discuss recent advances in the understanding of how HMPV F mediates both attachment and fusion. We review the requirements for HMPV viral surface glycoproteins during entry and infection, and review the identification of cellular receptors for HMPV F. We also review our current understanding of how HMPV F mediates fusion, concentrating on structural regions of the protein that appear to be critical for membrane fusion activity. Finally, we illuminate key unanswered questions and suggest how further studies can elucidate how this clinically important paramyxovirus fusion protein may have evolved to initiate infection by a unique mechanism.
\end{abstract}

Keywords: metapneumovirus; fusion protein; paramyxovirus; integrin 


\section{Introduction}

Human metapneumovirus (HMPV) was first isolated in 2001 in the Netherlands [1]. Dutch investigators discovered an unknown virus in respiratory secretions collected from young children with lower respiratory illness. Virus-infected cell supernatants were examined by electron microscopy and found to contain pleomorphic virus particles measuring 150 to $600 \mathrm{~nm}$, with spike-like envelope projections of 13 to $17 \mathrm{~nm}$ [2]. PCR and sequence analysis revealed a single-stranded, negative-sense RNA genome with close resemblance to avian metapneumovirus (AMPV), an avian virus that causes serious respiratory disease in chickens and turkeys [3]. Based upon virion morphology and genome organization, HMPV was classified as the first human member of the Metapneumovirus genus, in the subfamily Pneumovirinae of the paramyxovirus family $[1,4]$.

HMPV is a ubiquitous respiratory pathogen that has been circulating in human populations undetected for decades. The original report detected HMPV-specific antibodies in archived human sera from the 1950s [1] and HMPV has been detected by RT-PCR in specimens from 1976 [5]. Phylogenetic analysis of multiple HMPV gene sequences suggests that HMPV diverged from AMPV between 200-300 years ago [6,7]. HMPV is a leading cause of lower respiratory infection in infants and children worldwide [5,8-18]. HMPV is also associated with severe disease in immunocompromized hosts or persons with underlying conditions [19-25]. HMPV causes a clinical spectrum of illness from upper airway infection to severe lower respiratory tract infections (e.g., bronchiolitis and pneumonia) [5,26]. HMPV pathogenesis is similar to hRSV and causes inflammation, sloughing and necrosis of the bronchiolar epithelium [27]. Experimental studies in nonhuman primates and small animal models (hamsters, cotton rats, and mice) indicate that HMPV replicates in the upper and lower respiratory tract epithelium and demonstrate no evidence of viral dissemination, indicating a distinct tissue tropism for HMPV which is consistent with clinical illness observed during human infection [28-30].

\section{HMPV F: A Dual Function Fusion Protein}

The HMPV virion is similar to other paramyxoviruses. The viral lipid bilayer is likely derived from the plasma membrane of infected cells during virus egress, as virions are thought to bud from the infected cell surface. The closely related pneumovirus, hRSV forms filaments at and buds from the apical surface of polarized epithelial cells [31,32]. Similar to hRSV [32], the HMPV matrix (M) protein has been shown to assemble into filaments on the surface of HMPV-infected cells [33]. The HMPV M protein lines the inner leaflet of the viral lipid bilayer, and virions contain three integral membrane surface glycoproteins, the fusion (F), glycoprotein $(\mathrm{G})$ and short hydrophobic $(\mathrm{SH})$ proteins (Figure 1). The envelope contains a helical ribonucleoprotein (RNAP) complex consisting of nucleoprotein $(\mathrm{N})$, phosphoprotein $(\mathrm{P})$, matrix 2 protein $(\mathrm{M} 2)$, large polymerase protein $(\mathrm{L})$, and the single-stranded, negative-sense RNA genome. 
Figure 1. Schematic representation of a human metapneumovirus (HMPV) virion. The fusion (orange), attachment (red), and short hydrophobic (black) glycoproteins are depicted at the virion surface. The matrix protein (gray ovals) lines the inner leaflet of the virus membrane. Encapsidated within the viral envelope is the ribonucleoprotein (RNAP) complex consisting of the helical, genomic RNA wrapped by the nucleoprotein $(\mathrm{N})$, the viral RNA-dependent, RNA polymerase (L), phosphoprotein (P), and matrix 2 protein (M2).

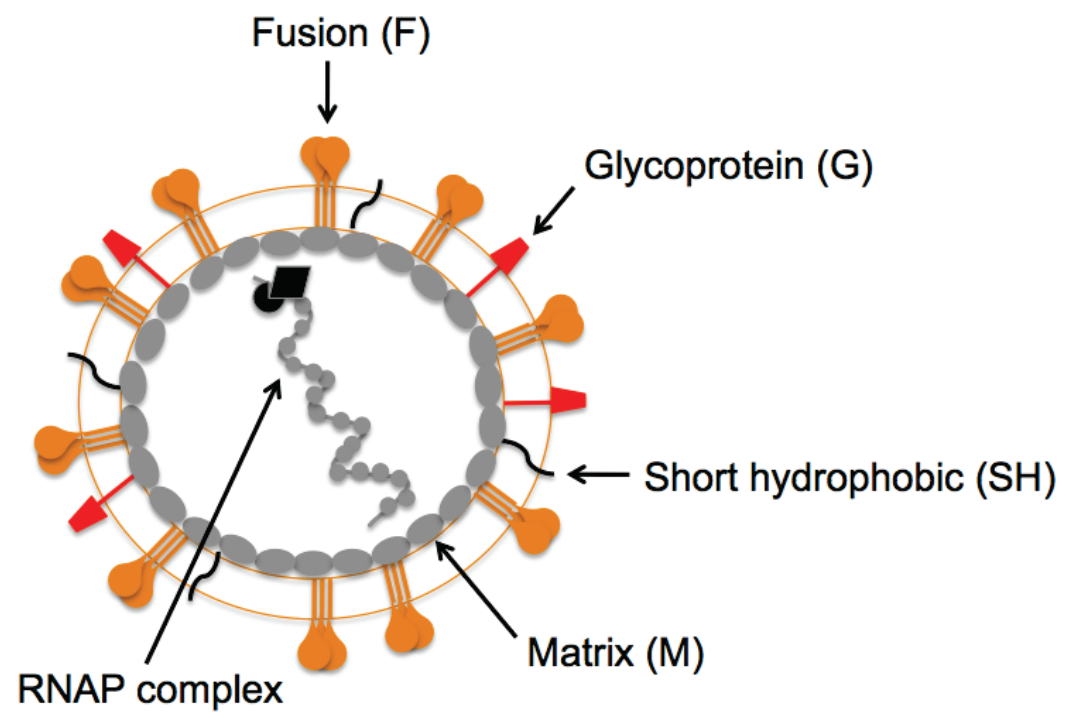

HMPV infection is initiated by viral surface glycoproteins that attach to cellular receptors and mediate virus membrane fusion with cellular membranes. Most paramyxoviruses use two viral glycoproteins to facilitate virus entry - an attachment protein, called $\mathrm{HN}, \mathrm{H}$ or $\mathrm{G}$ (depending on the virus), and a fusion (F) protein. Indeed, elegant studies on paramyxoviruses in the subfamily Paramyxovirinae have demonstrated that viral attachment and fusion are mediated by two individual viral proteins that act in concert during virus entry (reviewed in [34]). For the Paramyxovirinae, $\mathrm{F}$ is capable of catalyzing membrane fusion only in the presence of the attachment protein. The proposed model for paramyxovirus entry is a stepwise process where: (1) the attachment protein binds to cellular receptors; (2) the bound attachment protein directly interacts with and transmits a signal to the $\mathrm{F}$ protein; (3) the F protein becomes activated to undergo structural changes; (4) F refolds from a prefusion to postfusion structural conformation, resulting in the merging of the viral membrane with the plasma membrane; and (5) the genome is delivered into the cytoplasm from a fusion pore created at the cell surface.

However, membrane fusion for paramyxoviruses in the Pneumovirus subfamily is unique in that $\mathrm{F}$ drives fusion in the absence of a separate viral attachment protein. The pneumoviruses incorporate three surface glycoproteins that could potentially facilitate entry: F, SH, and G (termed the attachment protein based upon homology to other paramyxoviruses). The first indication that pneumovirus 
entry may not absolutely depend upon all surface glycoproteins was the identification of a live, cold-passaged hRSV strain (cp-52) that had a large deletion spanning most of the SH and G coding sequences but replicated efficiently in Vero cells [35]. Thus, in the absence of SH and G, hRSV was infectious and capable of replicating to high titer in vitro, although cp-52 replication was severely attenuated in vivo in both animals and humans [35]. Subsequent studies with hRSV engineered without the SH and G genes have confirmed that hRSV F is sufficient for virus entry in cell culture, but replication is attenuated in animal models of infection [36-42]. The invention of reverse genetics for HMPV has enabled researchers to rescue viruses engineered to express the F protein in the absence of $\mathrm{G}$ and/or SH, and define the individual contributions of each protein for virus entry. Deletion of the SH gene from HMPV did not alter viral growth in cell culture or animal models of infection [43,44], confirming that the SH protein does not serve a role in virus entry. HMPV lacking both $\mathrm{G}$ and $\mathrm{SH}$ replicated efficiently in cell culture [44] and in both the upper and lower respiratory tracts of hamsters after intranasal inoculation [44]. HMPV lacking G (HMPV $\Delta G)$ infected and was shed from both the upper and lower respiratory tracts of African green monkeys, a permissive nonhuman primate host. However, HMPV $\triangle \mathrm{G}$ replication was attenuated compared to wild type infection (viral titers were reduced six-fold in the upper respiratory tract and 3200-fold in the lower respiratory tract) [43].

The fact that both hRSV and HMPV viruses lacking the G and SH genes are infectious indicates that the $\mathrm{F}$ proteins from these pneumoviruses can perform the necessary functions for virus entry, i.e., attachment and fusion. However, the G protein is clearly important for virus fitness in vivo. Despite the fact that hRSV and HMPV G proteins share no homology and are very different in size [4], the G proteins are thought to serve similar functions in the pneumovirus lifecycle. Current evidence suggests that pneumovirus $\mathrm{G}$ proteins help tether virus particles to the cell surface, and are likely important for strengthening particle adhesion and concentrating virions on the cell surface. Techaarpornkul et al. demonstrated that hRSV G is required for optimal virus attachment to the cell surface, but not for virus fusion and entry as the F protein alone is sufficient for efficient virus fusion with cultured cells [39]. HRSV and HMPV G have been shown to bind adhesion molecules on the cell surface such as heparan sulfate or glycosaminoglycans (GAGs) [45-47]. Pretreatment of HMPV with soluble heparin inhibits infection, and recombinant $\mathrm{G}$ protein binds to heparin-agarose columns and cells in a GAG-dependent manner [47]. Thus, HMPV G is capable of binding cell surface GAGs and may contribute to virus attachment; however, because $\mathrm{G}$ is dispensable for viral entry, it is not absolutely required for the membrane fusion activity of $\mathrm{F}$ during the virus entry process.

Thus, HMPV F is necessary and sufficient for membrane fusion and capable of mediating entry without an additional attachment protein, in contrast to the firmly established model for most paramyxoviruses that absolutely require two viral proteins. To be sufficient for virus entry, HMPV F must be able to attach to cellular receptors and this attachment should activate F-mediated membrane fusion. How HMPV F mediates both attachment to cellular receptors and membrane fusion has been the focus of several recent studies and will be the topic of this review. 


\section{HMPV F: The Key to Intrusion}

All paramyxovirus $\mathrm{F}$ proteins are class I fusion proteins that due to the conservation of structural domains are thought to mediate fusion via the same global mechanism (reviewed in [48]). HMPV F is a trimeric, type I membrane glycoprotein. A schematic of the HMPV F protein structure is shown in Figure 2. Each F monomer is translated as an inactive precursor, F0, which is proteolytically cleaved at a monobasic consensus site by host cell proteases into two disulfide-linked subunits, F1 and F2. This cleavage reveals a hydrophobic fusion peptide (FP) at the N-terminus of the larger F1 subunit, which inserts into target cell membranes to initiate membrane fusion. Two heptad repeat (HR) regions are located in the F1 subunit; HRA is adjacent to the FP and HRB is adjacent to the transmembrane (TM) domain. During fusion a metastable, prefusion conformation of $\mathrm{F}$ refolds into a highly stable postfusion conformation. During the prefusion-to-postfusion transition, the HRA and HRB form trimeric coiled-coils that rearrange to fold into a highly stable six-helix bundle that drives the formation of a fusion pore between the virus and cell membranes. Formation of the six-helix bundle is essentially irreversible and is directly linked to membrane merging, as peptides mimicking the heptad repeats are capable of blocking membrane fusion [49-51].

Figure 2. Schematic representation of the cleaved HMPV fusion protein. The mature, proteolytically cleaved HMPV F protein contains 522 amino acids (without signal sequence), with 82 residues in the F2 subunit and 440 residues in the F1 subunit, which includes a large extracellular domain, an $~ 23$-amino acid transmembrane domain and a cytoplasmic tail of 25 residues. $\mathrm{FP}=$ fusion peptide; HRA $=$ heptad repeat $\mathrm{A}$; $\mathrm{HRB}=$ heptad repeat $\mathrm{B} ; \mathrm{TM}=$ transmembrane domain; $\mathrm{CT}=$ cytoplasmic tail. The approximate location of a conserved RGD motif (residues 329-331) is indicated as a magenta box. Arrows indicate the three N-linked glycosylation sites. The location of two disulfide bonds that connect the F1 and F2 protein subunits are shown. (Scale bar, amino acids.)

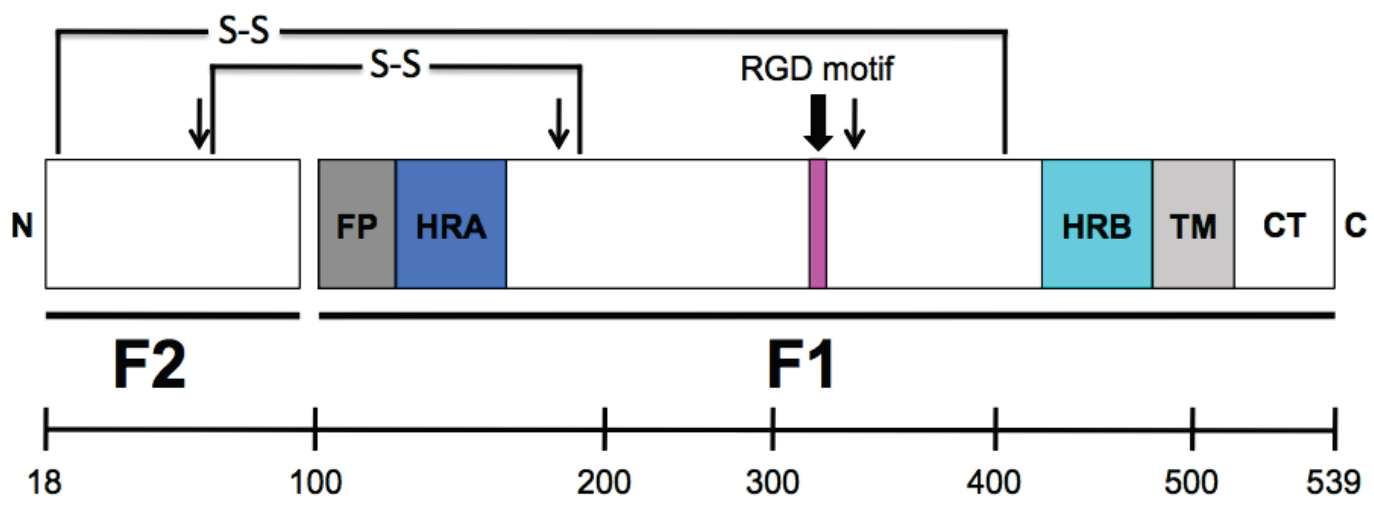


Figure 3. Structures of paramyxovirus F proteins. (A) The prefusion conformation of the PIV5 F trimer and the postfusion conformation of the hRSV F trimer. A schematic of the F protein color-coded to identify structural domains is shown below the structures. The DI domain is shown in orange, the DII domain is shown in red, and the DIII domain is shown in blue. HRA is shown in light blue, HRB is shown in cyan and the HRB linker domain is shown in gray. The fusion peptide is shown in green for prefusion PIV5 F, but was not solved in the hRSV F structure. The GCNt domain (shown in yellow) was added to the PIV5 F trimer for crystallization. (B) HMPV F in complex with a neutralizing antibody (DS7 Fab, shown as gray surface). DI, DII, and DIII coloring is as for the F trimers shown in (A). The conserved RGD motif is shown as magenta spheres. Residue 294 (Glu) is shown as green spheres. Conserved charged residues in DIII that impact fusion activity are indicated as spheres (yellow, acidic residues; brown, basic residues). (C) Side and top views of the prefusion PIV5 F trimer are shown with the homologous residues to HMPV F-RGD shown as magenta spheres. Each monomer is shown in blue, yellow, or green. (D) Side and top views of the postfusion hRSV F trimer are shown with the homologous residues to HMPV F-RGD shown as magenta spheres. Coloring is as for PIV5 F in (C). Structural coordinates were obtained from the Protein Data Bank [52] and figure constructed with PyMOL. PIV5 F, PDB ID: 2B9B [53]; hRSV F, PDB ID: 3RKI [54]; HMPV F complex, PDB ID: 4DAG [55].

A
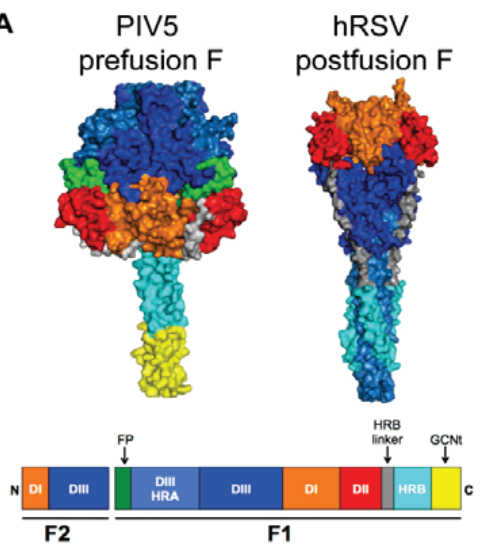

C

PIV5 prefusion $F$ with RGD location

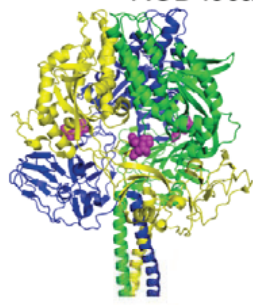

Side

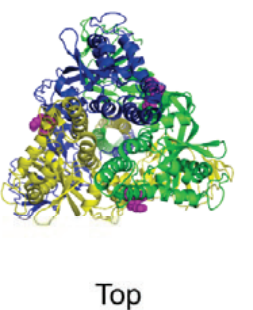

Top

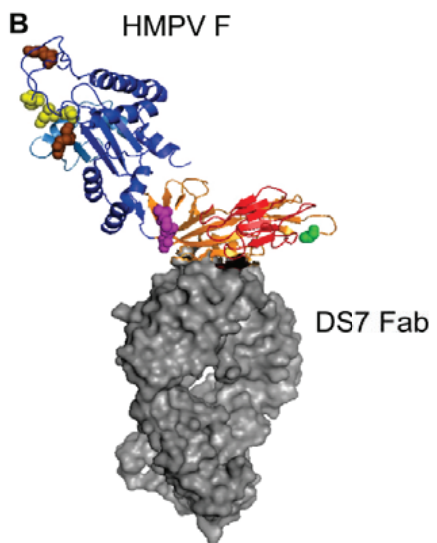

D hRSV postfusion $F$ with RGD location

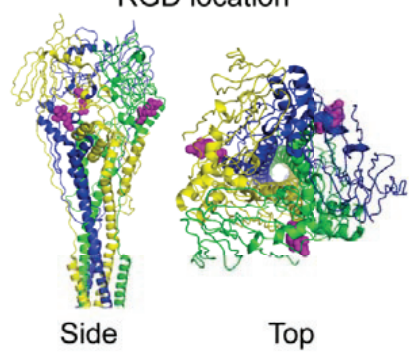


Crystal structures for the PIV5 prefusion $F$ [53] and the hRSV postfusion F [54] have been determined and are shown in Figure 3A. Three discrete domains, DI (orange), DII (red) and DIII (blue), have been colored to indicate how the linear sequence of amino acids is arranged in the tertiary structure of the prefusion and postfusion $\mathrm{F}$ proteins. The HRA domain (light blue) changes significantly in both secondary structure and location, and the F head domain becomes more compact, during the prefusion-to-postfusion F transition (reviewed in [48]). A crystal structure of HMPV F in complex with a neutralizing antibody (DS7 Fab) has recently been described [55]. The HMPV F structure was solved as a monomer, and is depicted in Figure 3B. The HMPV F DIII domain is similar to the prefusion PIV5 F protein but overall the HMPV F structure resembled the hRSV postfusion F [55].

HMPV F amino acid sequences are highly conserved, falling into two major lineages (A and B), each with two subgroups (1 and 2), which exhibit a mean of $\sim 96 \%$ identity [7,56]. A study in 2004 comparing 64 partial F gene sequences from the United States, Canada, Peru, France, Israel, Republic of South Africa, Australia and the Netherlands confirmed that HMPV isolates circulating around the world encode F proteins from the A1, A2, B1 and B2 genetic lineages [57]. Distinct canonical amino acid differences are present between major subgroups, and polymorphic variations tend to cluster in discrete regions [7,57]. Amino acid identity within and between subgroups is higher than nucleotide identity, suggesting structural or functional constraints on $\mathrm{F}$ protein diversity. A study comparing 85 full-length F gene sequences collected over a 20 -year period from the United States (all isolated in Tennessee), Canada, Japan and the Netherlands suggested that there was no progressive genetic drift over time, and the genetic lineages were stable over time in circulating viruses in a population of children with respiratory illnesses [7].

The HMPV F protein is glycosylated at three Asn residues (residues 57, 172 and 353) [58,59]. Mutating the $\mathrm{F} 2$ subunit glycosylation site at $\mathrm{N}_{57}$ results in an F protein that is expressed and processed at near wild type levels, and efficiently mediates both cell-cell fusion [58] and virus growth in a mouse model of infection [59]. Glycosylation of $\mathrm{N}_{172}$ appears to be critical for proper $\mathrm{F}$ protein folding, fusion activity, and virus growth in mice [58,59]. Glycosylation of $\mathrm{N}_{353}$ appears to be critical for proper $\mathrm{F}$ folding and cleavage [58], which results in the inability to recover viruses with an N353Q mutation [59].

An arginine-glycine-aspartate (RGD) motif at residues 329-331 in the HMPV F1 subunit, along with $5 \mathrm{~N}$ - and $16 \mathrm{C}$-terminal flanking residues, is strictly conserved in all gene sequences that have been studied to date, regardless of genetic lineage and despite diversity in other regions of the $\mathrm{F}$ gene [60]. This motif is unique to HMPV F among human paramyxovirus fusion proteins and absent in all published HRSV F sequences. The RGD motif is located in DI of the HMPV F protein and is shown in Figure 3B as magenta spheres. Based upon homology, the predicted location of the RGD motif is also shown on the PIV5 prefusion (Figure 3C, magenta spheres) and the hRSV postfusion (Figure 3D, magenta spheres) $\mathrm{F}$ structures. The presence of the invariant RGD motif led to the speculation that integrins function as receptors for HMPV, discussed in more detail in the next section. 


\section{HMPV Binding: Identifying the Door}

As noted, HMPV diverged from an avian pneumovirus (AMPV-C) between 200-300 years ago [6,7]. HMPV does not replicate in birds and evidence of AMPV infection has not been detected in humans [1]. The switch in virus tropism appears to be due to the attachment function of the F protein. In cell culture, HMPV expressing only $\mathrm{F}(\mathrm{HMPV} \Delta \mathrm{G} \Delta \mathrm{SH})$ binds to the cell surface with the same efficiency as wild type virus [61]. Moreover, HMPV F confers species specificity of infection, as F was found to be primarily responsible for the difference in the ability of AMPV-C and HMPV to infect quail fibroblast (QT6) cells [62]. Further, the F2 subunit (and not the G protein) of both hRSV [38] and HMPV [62] has been shown to confer species-specific infection of cells. This evidence strongly indicated that HMPV F interacts with cell surface receptors, and both protein and carbohydrate receptors for HMPV $\mathrm{F}$ have been discovered.

We identified a conserved RGD motif in the HMPV F1 subunit, and speculated that integrins might serve as receptors for HMPV. Integrins are heterodimeric integral membrane proteins composed of one $\alpha$ and one $\beta$ subunit. A subset of integrins $(\alpha \mathrm{V} \beta 1, \alpha \mathrm{V} \beta 3, \alpha \mathrm{V} \beta 5, \alpha \mathrm{V} \beta 6, \alpha \mathrm{V} \beta 8, \alpha 5 \beta 1, \alpha 8 \beta 1$ and $\alpha \mathrm{IIb} \beta 3$ ) bind proteins with RGD motifs, such as fibronectin and vitronectin. Integrins are adhesion receptors that bind extracellular proteins to modulate cell behavior and survival. Integrins associate with cytoskeletal proteins, adaptors, and kinases via the cytoplasmic tails of the $\alpha$ and $\beta$ subunits, allowing them to transduce bidirectional signals between the intra- and extra-cellular environments [63]. The intimate link between integrins and cell signaling cascades, as well as endosomal sorting pathways, makes them a desirable receptor for mammalian viruses. Several viruses, including adenovirus, hantavirus, herpesvirus, picornavirus, and reovirus, utilize integrins during entry, either as attachment or internalization receptors (reviewed in [64]).

Initial experiments in our laboratory found that the RGD-binding integrin $\alpha \mathrm{V} \beta 1$ promoted HMPV infection [60,65]. EDTA, which chelates divalent cations required for integrin function, exhibited a dose-dependent inhibition of HMPV infection. Synthetic RGD peptides blocked HMPV infection, while RGE peptides did not. Function-blocking monoclonal antibodies (mAbs) directed against RGD-binding integrins inhibited HMPV infection, with mAbs against $\alpha \mathrm{V}$ and $\beta 1$ exhibiting the most potent effect. Similarly, reduction of $\alpha \mathrm{V}$ and $\beta 1$ expression by siRNA reduced HMPV infection. Importantly, none of these loss-of-function experiments inhibited hRSV, which has a very similar F protein but lacks an RGD motif. Transfection of poorly permissive cells with $\alpha \mathrm{V}$ or $\beta 1$ cDNAs conferred HMPV infectivity. These data suggested that $\alpha \mathrm{V} \beta 1$ integrin is a receptor for HMPV.

Subsequent studies using both virus and virus-like particles showed that HMPV F is capable of binding multiple RGD-binding integrins in addition to $\alpha \mathrm{V} \beta 1$, specifically $\alpha 5 \beta 1$ and other $\alpha \mathrm{V}$-integrin heterodimers that may differ depending upon target cells [65]. Furthermore, recent data suggest that HMPV F utilizes RGD-binding integrins as attachment and entry receptors. HMPV F binds RGD-binding integrins during attachment, and this interaction is necessary for virus attachment and subsequent productive infection [65]. Blockade of integrin function with mAbs inhibits approximately half of virus binding, but $>90 \%$ of virus infectivity. Thus, while HMPV can bind to other cell surface molecules, integrin binding is required for efficient entry. Furthermore, mutating the conserved RGD 
motif significantly attenuated HMPV growth in vitro and altered the extent of virus-infected cell-cell fusion, suggesting a key role for the integrin-binding motif during F-mediated entry and fusion [65]. In addition to being necessary for efficient HMPV F-mediated attachment, our experiments suggest that integrin engagement is also required for postbinding events during HMPV entry, because blocking RGD-binding integrin attachment results in significantly lower levels of viral transcripts at eight hours post infection [65]. Thus, in the absence of integrin-mediated entry, virus transcription is impaired. These data suggest that RGD-binding integrin engagement is necessary but not sufficient for HMPV attachment (40\%-50\% of HMPV F-specific binding is mediated by integrins), and that integrin engagement is important for post-binding events that occur during HMPV entry [65].

Chang et al. recently identified heparan sulfate as a receptor for HMPV F, and suggested that the carbohydrate moiety was the first binding partner for HMPV F during virus attachment [61]. Experiments with Chinese hamster ovary (CHO-K1) cells and mutant CHO-K1-derived cell lines unable to synthesize GAGs demonstrated that heparan sulfate proteoglycans were required for HMPV binding and infection [61]. These authors concluded that $\beta 1$ integrin was not a direct cellular receptor for HMPV because virus still bound $\beta 1$ integrin-deficient murine embryonic fibroblasts [61]. However, when $\beta 1$-null fibroblasts were complemented with human $\beta 1$ integrin, HMPV infection was significantly enhanced, supporting a role for $\beta 1$ integrin in promoting HMPV infection [61]. In light of our recent findings that HMPV $F$ binds multiple integrins during attachment, HMPV $F$ would be expected to bind to other RGD-binding integrins such as $\alpha \mathrm{V} \beta 5, \alpha \mathrm{V} \beta 6$ and/or $\alpha \mathrm{V} \beta 8$ on the $\beta 1$-null cell surface. The $\beta 1$-null fibroblasts express several $\alpha \mathrm{V}$ heterodimers, including $\alpha \mathrm{V} \beta 3$ and $\alpha \mathrm{V} \beta 5$ [66,67]. These data show that an HMPV F interaction with heparan sulfate moieties on the cell surface contributes to HMPV attachment and infection, and heparan sulfate may be the first cell surface receptor that HMPV engages during virus attachment.

Interestingly, recent findings by Chang et al. suggest that efficient HMPV infection depends upon the expression of a proteinaceous receptor that is trypsin- and proteinase K-sensitive (integrins are reported to be resistant to both types of protease treatment) [61]. Thus, HMPV entry appears to involve more than one cell surface receptor: heparan sulfate, RGD-binding integrins, and other protease-sensitive surface proteins. However, further studies are needed to identify other putative proteinaceous receptors for HMPV.

Together, recent studies on the receptors involved in HMPV entry suggest that HMPV F interacts with multiple binding partners during attachment. We propose a multi-step model of HMPV attachment where $\mathrm{F}$ first binds heparan sulfate before engaging specific proteinaceous receptors including RGD-binding integrins, which mediate post-binding events that lead to productive HMPV infection.

\section{HMPV Fusion: Entering the Premises}

The mature HMPV F protein must be proteolytically cleaved to convert the F0 precursor into the fusion-competent, disulfide-linked F1/F2 heterodimer. The monobasic, consensus cleavage site (PRQSR) is a trypsin-like cleavage motif, which is cleaved after the final Arg residue. For most cell 
types, cell surface HMPV F is expressed in the F0 precursor form. In cell culture, F protein cleavage occurs when trypsin is added to the cell medium. Indeed, HMPV grows poorly (or not at all) unless trypsin is added to virus growth medium during virus propagation in cell culture $[1,44,68]$. Trypsin treatment of HMPV F-expressing cells is also required for cell-cell fusion activity [58]. In vivo, cleavage is likely mediated by extracellular proteases such as TMPRSS2 [69] or mini-plasmin [70] expressed in the respiratory tract of infected humans. HMPV F cleavage activates the protein by creating a new F1 N-terminus, the fusion peptide, which is inserted into target cell membranes during the fusion process.

Most paramyxovirus $\mathrm{F}$ proteins require an attachment protein to drive membrane fusion, both during entry (virus-cell fusion) and to induce syncytia (cell-cell fusion). Transient transfection of HMPV $F$ is sufficient to induce cell-cell fusion, and co-expression of the $G$ protein does not significantly enhance fusion [58]. Moreover, using virus-like particles containing only $\mathrm{F}$ or $\mathrm{F}+\mathrm{G}$, we have demonstrated that the $\mathrm{G}$ protein does not alter particle-cell hemifusion kinetics or the extent of fusion [65]. This evidence coupled with the observation that HMPV lacking the G protein is infectious suggests that the G protein is not a critical component of the HMPV fusion machinery.

To better understand how the HMPV F protein functions alone to mediate fusion, recent studies have explored the requirements for fusion and investigated the importance of specific residues within the extracellular domain of the protein for fusion activity. Paramyxovirus fusion proteins mediate fusion in a $\mathrm{pH}$-independent manner [71], and commonly induce fusion of cultured cells at neutral $\mathrm{pH}$ resulting in syncytium formation during virus infection. Surprisingly, the first report of HMPV F-mediated cell-cell fusion activity suggested that HMPV F required low pH for activation [58]. Schowalter et al. determined that HMPV F required both trypsin treatment, to mediate proteolytic processing, and low $\mathrm{pH}$ pulses for fusion activity of the A2 genotype strain CAN97-83 [58]. A follow-up study suggested that the low-pH-induced fusion phenotype of HMPV CAN97-83 may be strain-specific [72]. Herfst et al. used prototype F proteins from the four HMPV genetic lineages to demonstrate that fusion for lineage $\mathrm{B}$ strains was $\mathrm{pH}$-independent, but fusion for some lineage A strains (one A1 and one of two A2 strains tested) was enhanced by $\mathrm{pH} 5$ pulses [72]. Mutagenesis studies determined that a Gly residue at position 294 was a key determinant of the low-pH-induced fusion phenotype for HMPV A strains, but inserting the Gly 294 residue into HMPV B F proteins did not confer $\mathrm{pH}$ sensitivity [72]. We compared the sequences of more than 1000 published full-length and partial HMPV $\mathrm{F}$ sequences from around the world to determine the frequency of the residue present at position 294 (see Figure 3B, green spheres). Most HMPV strains encode a Glu residue at position 294, 6\% encode a Gly (59 of 1005), and rarely a Lys residue is found at this position in the sequence (15 of 1005). The lack of HMPV F proteins with the low-pH sensitive Gly 294 suggests that exposure to low $\mathrm{pH}$ is not a general requirement for fusion activity. Thus, the general trigger that activates HMPV F to drive the membrane fusion process is still not known.

The finding that some HMPV F strains may require low $\mathrm{pH}$ for fusogenicity has led to follow-up studies that have elucidated the importance of other residues in the HMPV F protein that serve a role in protein stability and fusion triggering. Mutagenesis studies of CAN97-83 suggest that protonation of 
a conserved His residue at position 435 in the HRB linker domain serves a critical role in the low $\mathrm{pH}$ activation of the $\mathrm{F}$ protein $[73,74]$. A predicted cluster of basic residues $\mathrm{Lys}_{295}, \mathrm{Arg}_{396}$, and $\mathrm{Arg}_{438}$ are also important for the low $\mathrm{pH}$ fusion phenotype [73]. Interestingly, a predicted cluster of positively charged residues in this same region of the AMPV-A F protein (a distantly related pneumovirus) are important for fusion activity at neutral $\mathrm{pH}$ [75]. These studies suggest that the His residue at position 435 may serve as a low-pH sensor, and one model proposed that protonation of the His residue at low $\mathrm{pH}$ induces localized electrostatic repulsion that leads to the destabilization of $\mathrm{F}$ and fusion triggering $[73,76]$. Alternatively, electrostatic repulsion among the basic residues induced by receptor binding could destabilize the HRB linker domain and trigger fusion [75]. In any case, mutational analyses suggest that specific residues in domain II of HMPV F (Figure 3B, red residues) impact the initiation of membrane fusion. It is interesting that the cluster of basic residues is located in close proximity to a recently identified epitope that is recognized by a potently neutralizing mAb $[55,77]$. Thus, structural and functional studies support a significant role for domain II in the fusion activity of HMPV F.

Another charged region in the HMPV F extracellular head domain has recently been implicated in the low-pH sensitive fusion phenotype. Acidic residues $\mathrm{Glu}_{51}, \mathrm{Asp}_{54}$, and $\mathrm{Glu}_{56}$ (Figure 3B, yellow spheres) are important for low-pH triggered F-mediated cell-cell fusion, but also appear to be critical for protein stability [76]. These residues are clustered in a charged region present in the F2 subunit of $\mathrm{F}$ in close proximity to two basic residues (Figure 3B, brown spheres) [76]. The specific acidic residues in the F2 subunit and basic residues in the HRA domain are highly conserved in all HMPV strains; therefore, others have predicted that salt bridges and/or electrostatic interactions in this region may contribute to F triggering during HMPV fusion [76]. This hypothesis is supported by another study that indicated that the F2 subunit from AMPV-C could confer a hyperfusogenic phenotype to HMPV F1 [62].

Taking these studies together, it is clear that multiple regions in the HMPV $F$ head domain are critical for fusion activity. Interestingly, two distal regions with predicted charged residue clusters have been implicated in fusion triggering (located in domains II and III). The idea that electrostatic repulsions can trigger the HMPV F prefusion protein to spring into the postfusion conformation during the fusion process is logical. The HRA region of $\mathrm{F}$ must undergo a significant structural rearrangement during fusion, extending to form a long coiled-coil and moving a long distance to refold into a six-helix bundle with HRB coils near the TM domain [48]. This structural change must also occur at the right time and place, as refolding of class I fusion proteins is irreversible under physiological conditions. Thus, sensor residues could act as molecular switches that modulate electrostatic interactions, leading to repulsive forces that drive movement of structural elements within the $\mathrm{F}$ protein. While histidine protonation at residue 435 appears to serve as one sensor in HMPV strains that exhibit enhanced fusogenicity at low $\mathrm{pH}$, it remains unclear how $\mathrm{pH}$-independent HMPV $\mathrm{F}$ fusion is triggered. Perhaps receptor interactions serve to augment local protein structure, driving the same electrostatic repulsion that can be triggered by exposure to low $\mathrm{pH}$ in some HMPV strains. 
It is tempting to speculate that a direct interaction between HMPV F and RGD-binding integrins serves to initiate F-mediated fusion. However, current evidence does not support this hypothesis. We recently found that blocking HMPV F interaction with RGD-binding integrins did not alter virus-cell hemifusion kinetics, and virus-like particles bearing an F-RAE mutation fused with the same hemifusion kinetics as wild type F particles [65]. Moreover, Chang et al. showed that an HMPV F-RGA mutant promoted cell-cell fusion at levels approximately $80 \%$ of the wild type F protein [61], indicating that the RGD motif was not absolutely required for fusion activity. However, our results support a post-binding role for RGD-binding integrins during HMPV entry, as integrin blockade reduced HMPV transcription at 8 hours by $50 \%$, in addition to a $40-50 \%$ block during virus attachment [65]. Further, when we introduced the F-RAE mutation into a virus, the F-RAE virus was severely attenuated and exhibited a small plaque phenotype that lacked the characteristic syncytia (indicative of F-mediated cell-cell fusion) of wild type virus [65]. Thus, while it appears that an F-integrin interaction is not required for efficient HMPV hemifusion, it remains possible that this interaction promotes fusion pore opening during virus-cell fusion.

\section{HMPV Entry: Unlocking the Mystery}

The solved crystal structures of postfusion PIV3, NDV, and hRSV F proteins are similar $[54,78,79]$, supporting the model that all paramyxovirus $\mathrm{F}$ proteins mediate fusion via a highly conserved mechanism. Only one paramyxovirus F protein, PIV5, has been crystallized in the prefusion structural conformation (shown in Figure 3A, [53]). Comparison of this structure to the postfusion conformation has facilitated our understanding of the paramyxovirus fusion process (reviewed in [48]). The unique activity of pneumovirus fusion proteins to mediate fusion in the absence of an attachment protein suggests that hRSV and HMPV F may have unique prefusion structures compared to the Paramyxovirinae subfamily F proteins. Unfortunately, crystal structures of hRSV and HMPV prefusion trimers have not been published. The recently described structure of HMPV F in complex with a neutralizing antibody [55] provides insight into the structure of the protein, but HMPV F may trimerize slightly differently than PIV5 F [53]. Furthermore, while the HMPV F DIII domain had a similar fold to the PIV5 prefusion protein, the overall structural fold more closely resembled the postfusion hRSV F protein [55]. Thus, a crystal structure of a prefusion pneumovirus F trimer will be required to understand how these proteins have evolved to mediate fusion in the absence of an attachment protein. Further, because hRSV and HMPV F proteins utilize distinct proteinaceous cellular receptors $[65,80]$, these proteins may also have unique prefusion structures. Such structures could provide insight into how pneumovirus F proteins couple receptor-binding and fusion activities.

The proverbial "black box" with respect to paramyxovirus entry is a lack of understanding of the fusion protein triggering process. For all paramyxoviruses, the process by which receptor binding triggers conformational changes in F remains unclear. HMPV F interacts with at least two different cell surface receptors during entry, heparan sulfate and RGD-binding integrins. How binding to receptor(s) results in structural changes in $\mathrm{F}$ that drive fusion is not clear. Furthermore, whether HMPV binds receptors simultaneously or sequentially is not known. Simultaneously binding to 
multiple receptors may induce conformational changes in multiple sites of the HMPV F extracellular domain, thereby promoting destabilization, protein refolding, and fusion. Alternatively, HMPV F binding to one receptor may expose the receptor binding site for another co-receptor, similar to the strategy utilized by HIV gp120/gp41 during fusion [81]. Our understanding of HMPV F triggering is further complicated by observations that some $\mathrm{F}$ proteins are more fusogenic at low $\mathrm{pH}$. Typically, enveloped viruses either fuse at neutral $\mathrm{pH}$ or require exposure to low $\mathrm{pH}$ for fusion activity. We speculate that low $\mathrm{pH}$ treatment could result in histidine protonation events that serve to destabilize HMPV $F$ in a similar manner to receptor engagement, although this hypothesis requires further investigation. This could explain why not all strains require low-pH exposure, but highly conserved charged regions of $\mathrm{F}$ are critical for function of all HMPV strains.

The role of low $\mathrm{pH}$ in HMPV entry is still unclear. Inhibitors of endosomal acidification such as bafilomycin A1 and concanamycin A have been shown to partially reduce HMPV infection of the low-pH sensitive CAN97-83 virus strain [73]. Intriguingly, bafilomycin A1 and concanamycin A also partially inhibit infection by the pH-independent NL199 (B1) strain of HMPV, but not the NL1-00 (A1) strain which has been shown to be triggered by low $\mathrm{pH}$ exposure in syncytium assays [74]. Because the low-pH-sensitive phenotype observed in cell-cell fusion assays does not necessarily correlate with inhibition of HMPV infection by endosomal acidification inhibitors, it is not clear that the ability to enhance fusogenicity with low $\mathrm{pH}$ pulses is correlated with a requirement for endosomal acidification during entry. Furthermore, whether particle-cell fusion requires exposure to low $\mathrm{pH}$ requires further investigation to determine whether low $\mathrm{pH}$ is an absolute requirement for triggering of F proteins from some HMPV strains. The site of HMPV fusion also requires further investigation. Schowalter et al. reported that infection by the low-pH sensitive HMPV strain CAN97-83 was significantly impaired by chemical inhibitors of endocytosis pathways, e.g., chlorpromazine and dynasore [73]. Thus, one study suggests that HMPV entry may involve virus internalization, although this speculation requires further studies with other HMPV lineage strains and more specific inhibitors of various endocytosis pathways.

\section{Conclusions}

HMPV is a leading cause of lower respiratory illness in adults and children. Although the virus was only discovered in 2001, remarkable progress has been made in elucidating the biology of HMPV. The F protein serves both to bind cellular receptors and to mediate fusion, although $\mathrm{G}$ protein is required for full virulence. HMPV $\mathrm{F}$ binds to heparan sulfate, and uniquely among human paramyxoviruses uses multiple RGD-binding integrins as attachment and entry receptors. Some HMPV F molecules exhibit sensitivity to low $\mathrm{pH}$, though the function of this low $\mathrm{pH}$ triggering during the context of infection is not clear. While HMPV F shares many features of other paramyxovirus fusion proteins, there are distinct aspects of the attachment, entry, and fusion mechanisms of this recently discovered virus. 


\section{Acknowledgments}

This work was supported by Public Health Service grants AI-73697 and AI-85062 (JVW) and T32 AI-7611 (RGC), from the National Institute of Allergy and Infectious Diseases. We thank current and past members of the Williams laboratory for helpful discussion.

\section{Conflicts of Interest}

JVW serves on the Scientific Advisory Board of Quidel. RGC declares no conflict of interest.

\section{References}

1. Van den Hoogen, B.G.; de Jong, J.C.; Groen, J.; Kuiken, T.; de Groot, R.; Fouchier, R.A.; Osterhaus, A.D. A newly discovered human pneumovirus isolated from young children with respiratory tract disease. Nat. Med. 2001, 7, 719-724.

2. Schildgen, V.; van den Hoogen, B.; Fouchier, R.; Tripp, R.A.; Alvarez, R.; Manoha, C.; Williams, J.; Schildgen, O. Human metapneumovirus: Lessons learned over the first decade. Clin. Microbiol. Rev. 2011, 24, 734-754.

3. Cook, J.K. Avian pneumovirus infections of turkeys and chickens. Vet. J. 2000, 160, 118-125.

4. Van den Hoogen, B.G.; Bestebroer, T.M.; Osterhaus, A.D.; Fouchier, R.A. Analysis of the genomic sequence of a human metapneumovirus. Virology 2002, 295, 119-132.

5. Williams, J.V.; Harris, P.A.; Tollefson, S.J.; Halburnt-Rush, L.L.; Pingsterhaus, J.M.; Edwards, K.M.; Wright, P.F.; Crowe, J.E., Jr. Human metapneumovirus and lower respiratory tract disease in otherwise healthy infants and children. N. Engl. J. Med. 2004, 350, 443-450.

6. De Graaf, M.; Osterhaus, A.D.; Fouchier, R.A.; Holmes, E.C. Evolutionary dynamics of human and avian metapneumoviruses. J. Gen. Virol. 2008, 89, 2933-2942.

7. Yang, C.F.; Wang, C.K.; Tollefson, S.J.; Piyaratna, R.; Lintao, L.D.; Chu, M.; Liem, A.; Mark, M.; Spaete, R.R.; Crowe, J.E., Jr.; et al. Genetic diversity and evolution of human metapneumovirus fusion protein over twenty years. Virol. J. 2009, 6, 138.

8. Williams, J.V.; Wang, C.K.; Yang, C.F.; Tollefson, S.J.; House, F.S.; Heck, J.M.; Chu, M.; Brown, J.B.; Lintao, L.D.; Quinto, J.D.; et al. The role of human metapneumovirus in upper respiratory tract infections in children: A 20-year experience. J. Infect. Dis. 2006, 193, 387-395.

9. Van den Hoogen, B.G.; van Doornum, G.J.; Fockens, J.C.; Cornelissen, J.J.; Beyer, W.E.; de Groot, R.; Osterhaus, A.D.; Fouchier, R.A. Prevalence and clinical symptoms of human metapneumovirus infection in hospitalized patients. J. Infect. Dis. 2003, 188, 1571-1577.

10. Peiris, J.S.; Tang, W.H.; Chan, K.H.; Khong, P.L.; Guan, Y.; Lau, Y.L.; Chiu, S.S. Children with respiratory disease associated with metapneumovirus in hong kong. Emerg. Infect. Dis. 2003, 9, 628-633.

11. Mullins, J.A.; Erdman, D.D.; Weinberg, G.A.; Edwards, K.; Hall, C.B.; Walker, F.J.; Iwane, M.; Anderson, L.J. Human metapneumovirus infection among children hospitalized with acute respiratory illness. Emerg. Infect. Dis. 2004, 10, 700-705. 
12. McAdam, A.J.; Hasenbein, M.E.; Feldman, H.A.; Cole, S.E.; Offermann, J.T.; Riley, A.M.; Lieu, T.A. Human metapneumovirus in children tested at a tertiary-care hospital. J. Infect. Dis. 2004, 190, 20-26.

13. Mackay, I.M.; Bialasiewicz, S.; Jacob, K.C.; McQueen, E.; Arden, K.E.; Nissen, M.D.; Sloots, T.P. Genetic diversity of human metapneumovirus over 4 consecutive years in australia. J. Infect. Dis. 2006, 193, 1630-1633.

14. Foulongne, V.; Guyon, G.; Rodiere, M.; Segondy, M. Human metapneumovirus infection in young children hospitalized with respiratory tract disease. Pediatr. Infect. Dis. J. 2006, 25 , 354-359.

15. Esper, F.; Martinello, R.A.; Boucher, D.; Weibel, C.; Ferguson, D.; Landry, M.L.; Kahn, J.S. A 1-year experience with human metapneumovirus in children aged $<5$ years. J. Infect. Dis. 2004, 189, 1388-1396.

16. Ebihara, T.; Endo, R.; Kikuta, H.; Ishiguro, N.; Ishiko, H.; Hara, M.; Takahashi, Y.; Kobayashi, K. Human metapneumovirus infection in japanese children. J. Clin. Microbiol. 2004, 42, 126-132.

17. Dollner, H.; Risnes, K.; Radtke, A.; Nordbo, S.A. Outbreak of human metapneumovirus infection in norwegian children. Pediatr. Infect. Dis. J. 2004, 23, 436-440.

18. Boivin, G.; de Serres, G.; Cote, S.; Gilca, R.; Abed, Y.; Rochette, L.; Bergeron, M.G.; Dery, P. Human metapneumovirus infections in hospitalized children. Emerg. Infect. Dis. 2003, 9, 634-640.

19. Williams, J.V.; Martino, R.; Rabella, N.; Otegui, M.; Parody, R.; Heck, J.M.; Crowe, J.E., Jr. A prospective study comparing human metapneumovirus with other respiratory viruses in adults with hematologic malignancies and respiratory tract infections. J. Infect. Dis. 2005, 192, 1061-1065.

20. Williams, J.V.; Crowe, J.E., Jr.; Enriquez, R.; Minton, P.; Peebles, R.S., Jr.; Hamilton, R.G.; Higgins, S.; Griffin, M.; Hartert, T.V. Human metapneumovirus infection plays an etiologic role in acute asthma exacerbations requiring hospitalization in adults. J. Infect. Dis. 2005, 192, 1149-1153.

21. Vicente, D.; Montes, M.; Cilla, G.; Perez-Trallero, E. Human metapneumovirus and chronic obstructive pulmonary disease. Emerg. Infect. Dis. 2004, 10, 1338-1339.

22. Pelletier, G.; Dery, P.; Abed, Y.; Boivin, G. Respiratory tract reinfections by the new human metapneumovirus in an immunocompromised child. Emerg. Infect. Dis. 2002, 8, 976-978.

23. Madhi, S.A.; Ludewick, H.; Abed, Y.; Klugman, K.P.; Boivin, G. Human metapneumovirus-associated lower respiratory tract infections among hospitalized human immunodeficiency virus type 1 (hiv-1)-infected and hiv-1-uninfected african infants. Clin. Infect. Dis. 2003, 37, 1705-1710.

24. Larcher, C.; Geltner, C.; Fischer, H.; Nachbaur, D.; Muller, L.C.; Huemer, H.P. Human metapneumovirus infection in lung transplant recipients: Clinical presentation and epidemiology. J. Heart Lung Transplant. 2005, 24, 1891-1901. 
25. Englund, J.A.; Boeckh, M.; Kuypers, J.; Nichols, W.G.; Hackman, R.C.; Morrow, R.A.; Fredricks, D.N.; Corey, L. Brief communication: Fatal human metapneumovirus infection in stem-cell transplant recipients. Ann. Intern. Med. 2006, 144, 344-349.

26. Brodzinski, H.; Ruddy, R.M. Review of new and newly discovered respiratory tract viruses in children. Pediatr. Emerg. Care 2009, 25, 352-360.

27. Loughlin, G.M.; Moscona, A. The cell biology of acute childhood respiratory disease: Therapeutic implications. Pediatr. Clin. North Am. 2006, 53, 929-959.

28. Kuiken, T.; van den Hoogen, B.G.; van Riel, D.A.; Laman, J.D.; van Amerongen, G.; Sprong, L.; Fouchier, R.A.; Osterhaus, A.D. Experimental human metapneumovirus infection of cynomolgus macaques (macaca fascicularis) results in virus replication in ciliated epithelial cells and pneumocytes with associated lesions throughout the respiratory tract. Am. J. Pathol. 2004, 164, 1893-1900.

29. Williams, J.V.; Tollefson, S.J.; Johnson, J.E.; Crowe, J.E., Jr. The cotton rat (sigmodon hispidus) is a permissive small animal model of human metapneumovirus infection, pathogenesis, and protective immunity. J. Virol. 2005, 79, 10944-10951.

30. Hamelin, M.E.; Prince, G.A.; Gomez, A.M.; Kinkead, R.; Boivin, G. Human metapneumovirus infection induces long-term pulmonary inflammation associated with airway obstruction and hyperresponsiveness in mice. J. Infect. Dis. 2006, 193, 1634-1642.

31. Roberts, S.R.; Compans, R.W.; Wertz, G.W. Respiratory syncytial virus matures at the apical surfaces of polarized epithelial cells. J. Virol. 1995, 69, 2667-2673.

32. Shaikh, F.Y.; Cox, R.G.; Lifland, A.W.; Hotard, A.L.; Williams, J.V.; Moore, M.L.; Santangelo, P.J.; Crowe, J.E., Jr. A critical phenylalanine residue in the respiratory syncytial virus fusion protein cytoplasmic tail mediates assembly of internal viral proteins into viral filaments and particles. MBio 2012, 3, doi: 10.1128/mBio.00270-11.

33. Sabo, Y.; Ehrlich, M.; Bacharach, E. The conserved yagl motif in human metapneumovirus is required for higher-order cellular assemblies of the matrix protein and for virion production. J. Virol. 2011, 85, 6594-6609.

34. Lamb, R.A. Paramyxovirus fusion: A hypothesis for changes. Virology 1993, 197, 1-11.

35. Karron, R.A.; Buonagurio, D.A.; Georgiu, A.F.; Whitehead, S.S.; Adamus, J.E.; Clements-Mann, M.L.; Harris, D.O.; Randolph, V.B.; Udem, S.A.; Murphy, B.R.; et al. Respiratory syncytial virus (rsv) sh and g proteins are not essential for viral replication in vitro: Clinical evaluation and molecular characterization of a cold-passaged, attenuated rsv subgroup b mutant. Proc. Natl. Acad. Sci. USA 1997, 94, 13961-13966.

36. Feldman, S.A.; Audet, S.; Beeler, J.A. The fusion glycoprotein of human respiratory syncytial virus facilitates virus attachment and infectivity via an interaction with cellular heparan sulfate. J. Virol. 2000, 74, 6442-6447.

37. Kahn, J.S.; Schnell, M.J.; Buonocore, L.; Rose, J.K. Recombinant vesicular stomatitis virus expressing respiratory syncytial virus (rsv) glycoproteins: Rsv fusion protein can mediate infection and cell fusion. Virology 1999, 254, 81-91. 
38. Schlender, J.; Zimmer, G.; Herrler, G.; Conzelmann, K.K. Respiratory syncytial virus (rsv) fusion protein subunit $\mathrm{f} 2$, not attachment protein $\mathrm{g}$, determines the specificity of rsv infection. J. Virol. 2003, 77, 4609-4616.

39. Techaarpornkul, S.; Barretto, N.; Peeples, M.E. Functional analysis of recombinant respiratory syncytial virus deletion mutants lacking the small hydrophobic and/or attachment glycoprotein gene. J. Virol. 2001, 75, 6825-6834.

40. Techaarpornkul, S.; Collins, P.L.; Peeples, M.E. Respiratory syncytial virus with the fusion protein as its only viral glycoprotein is less dependent on cellular glycosaminoglycans for attachment than complete virus. Virology 2002, 294, 296-304.

41. Widjojoatmodjo, M.N.; Boes, J.; van Bers, M.; van Remmerden, Y.; Roholl, P.J.; Luytjes, W. A highly attenuated recombinant human respiratory syncytial virus lacking the g protein induces long-lasting protection in cotton rats. Virol. J. 2010, 7, 114.

42. Teng, M.N.; Whitehead, S.S.; Collins, P.L. Contribution of the respiratory syncytial virus $g$ glycoprotein and its secreted and membrane-bound forms to virus replication in vitro and in vivo. Virology 2001, 289, 283-296.

43. Biacchesi, S.; Pham, Q.N.; Skiadopoulos, M.H.; Murphy, B.R.; Collins, P.L.; Buchholz, U.J. Infection of nonhuman primates with recombinant human metapneumovirus lacking the sh, $\mathrm{g}$, or $\mathrm{m} 2-2$ protein categorizes each as a nonessential accessory protein and identifies vaccine candidates. J. Virol. 2005, 79, 12608-12613.

44. Biacchesi, S.; Skiadopoulos, M.H.; Yang, L.; Lamirande, E.W.; Tran, K.C.; Murphy, B.R.; Collins, P.L.; Buchholz, U.J. Recombinant human metapneumovirus lacking the small hydrophobic sh and/or attachment $\mathrm{g}$ glycoprotein: Deletion of $\mathrm{g}$ yields a promising vaccine candidate. J. Virol. 2004, 78, 12877-12887.

45. Hallak, L.K.; Collins, P.L.; Knudson, W.; Peeples, M.E. Iduronic acid-containing glycosaminoglycans on target cells are required for efficient respiratory syncytial virus infection. Virology 2000, 271, 264-275.

46. Krusat, T.; Streckert, H.J. Heparin-dependent attachment of respiratory syncytial virus (rsv) to host cells. Arch. Virol. 1997, 142, 1247-1254.

47. Thammawat, S.; Sadlon, T.A.; Hallsworth, P.G.; Gordon, D.L. Role of cellular glycosaminoglycans and charged regions of viral g protein in human metapneumovirus infection. J. Virol. 2008, 82, 11767-11774.

48. Lamb, R.A.; Jardetzky, T.S. Structural basis of viral invasion: Lessons from paramyxovirus f. Curr. Opin. Struct. Biol. 2007, 17, 427-436.

49. Russell, C.J.; Jardetzky, T.S.; Lamb, R.A. Membrane fusion machines of paramyxoviruses: Capture of intermediates of fusion. EMBO J. 2001, 20, 4024-4034.

50. Miller, S.A.; Tollefson, S.; Crowe, J.E., Jr.; Williams, J.V.; Wright, D.W. Examination of a fusogenic hexameric core from human metapneumovirus and identification of a potent synthetic peptide inhibitor from the heptad repeat 1 region. J. Virol. 2007, 81, 141-149. 
51. Deffrasnes, C.; Hamelin, M.E.; Prince, G.A.; Boivin, G. Identification and evaluation of a highly effective fusion inhibitor for human metapneumovirus. Antimicrob. Agents Chemother. 2008, 52, 279-287.

52. Berman, H.M.; Westbrook, J.; Feng, Z.; Gilliland, G.; Bhat, T.N.; Weissig, H.; Shindyalov, I.N.; Bourne, P.E. The protein data bank. Nucleic Acids Res. 2000, 28, 235-242.

53. Yin, H.S.; Wen, X.; Paterson, R.G.; Lamb, R.A.; Jardetzky, T.S. Structure of the parainfluenza virus $5 \mathrm{f}$ protein in its metastable, prefusion conformation. Nature 2006, 439, 38-44.

54. Swanson, K.A.; Settembre, E.C.; Shaw, C.A.; Dey, A.K.; Rappuoli, R.; Mandl, C.W.; Dormitzer, P.R.; Carfi, A. Structural basis for immunization with postfusion respiratory syncytial virus fusion $\mathrm{f}$ glycoprotein (rsv f) to elicit high neutralizing antibody titers. Proc. Natl. Acad. Sci. USA 2011, 108, 9619-9624.

55. Wen, X.; Krause, J.C.; Leser, G.P.; Cox, R.G.; Lamb, R.A.; Williams, J.V.; Crowe, J.E., Jr.; Jardetzky, T.S. Structure of the human metapneumovirus fusion protein with neutralizing antibody identifies a pneumovirus antigenic site. Nat. Struct. Mol. Biol. 2012, 19, 461-463.

56. Van den Hoogen, B.G.; Herfst, S.; Sprong, L.; Cane, P.A.; Forleo-Neto, E.; de Swart, R.L.; Osterhaus, A.D.; Fouchier, R.A. Antigenic and genetic variability of human metapneumoviruses. Emerg. Infect. Dis. 2004, 10, 658-666.

57. Boivin, G.; Mackay, I.; Sloots, T.P.; Madhi, S.; Freymuth, F.; Wolf, D.; Shemer-Avni, Y.; Ludewick, H.; Gray, G.C.; LeBlanc, E. Global genetic diversity of human metapneumovirus fusion gene. Emerg. Infect. Dis. 2004, 10, 1154-1157.

58. Schowalter, R.M.; Smith, S.E.; Dutch, R.E. Characterization of human metapneumovirus $\mathrm{f}$ protein-promoted membrane fusion: Critical roles for proteolytic processing and low ph. J. Virol. 2006, 80, 10931-10941.

59. Zhang, J.; Dou, Y.; Wu, J.; She, W.; Luo, L.; Zhao, Y.; Liu, P.; Zhao, X. Effects of n-linked glycosylation of the fusion protein on replication of human metapneumovirus in vitro and in mouse lungs. J. Gen. Virol. 2011, 92, 1666-1675.

60. Cseke, G.; Maginnis, M.S.; Cox, R.G.; Tollefson, S.J.; Podsiad, A.B.; Wright, D.W.; Dermody, T.S.; Williams, J.V. Integrin alphavbetal promotes infection by human metapneumovirus. Proc. Natl. Acad. Sci. USA 2009, 106, 1566-1571.

61. Chang, A.; Masante, C.; Buchholz, U.J.; Dutch, R.E. Human metapneumovirus (hmpv) binding and infection are mediated by interactions between the hmpv fusion protein and heparan sulfate. J. Virol. 2012, 86, 3230-3243.

62. De Graaf, M.; Schrauwen, E.J.; Herfst, S.; van Amerongen, G.; Osterhaus, A.D.; Fouchier, R.A. Fusion protein is the main determinant of metapneumovirus host tropism. J. Gen. Virol. 2009, 90, 1408-1416.

63. Hynes, R.O. Integrins: Bidirectional, allosteric signaling machines. Cell 2002, 110, 673-687.

64. Stewart, P.L.; Nemerow, G.R. Cell integrins: Commonly used receptors for diverse viral pathogens. Trends Microbiol. 2007, 15, 500-507. 
65. Cox, R.G.; Livesay, S.B.; Johnson, M.; Ohi, M.D.; Williams, J.V. The human metapneumovirus fusion protein mediates entry via an interaction with rgd-binding integrins. J. Virol. 2012, 86, $12148-12160$.

66. Wennerberg, K.; Lohikangas, L.; Gullberg, D.; Pfaff, M.; Johansson, S.; Fassler, R. Beta 1 integrin-dependent and -independent polymerization of fibronectin. J. Cell. Biol. 1996, 132, 227-238.

67. Fassler, R.; Pfaff, M.; Murphy, J.; Noegel, A.A.; Johansson, S.; Timpl, R.; Albrecht, R. Lack of beta 1 integrin gene in embryonic stem cells affects morphology, adhesion, and migration but not integration into the inner cell mass of blastocysts. J. Cell. Biol. 1995, 128, 979-988.

68. Tollefson, S.J.; Cox, R.G.; Williams, J.V. Studies of culture conditions and environmental stability of human metapneumovirus. Virus Res. 2010, 151, 54-59.

69. Shirogane, Y.; Takeda, M.; Iwasaki, M.; Ishiguro, N.; Takeuchi, H.; Nakatsu, Y.; Tahara, M.; Kikuta, H.; Yanagi, Y. Efficient multiplication of human metapneumovirus in vero cells expressing the transmembrane serine protease tmprss2. J. Virol. 2008, 82, 8942-8946.

70. Murakami, M.; Towatari, T.; Ohuchi, M.; Shiota, M.; Akao, M.; Okumura, Y.; Parry, M.A.; Kido, H. Mini-plasmin found in the epithelial cells of bronchioles triggers infection by broad-spectrum influenza a viruses and sendai virus. Eur. J. Biochem. 2001, 268, 2847-2855.

71. Lamb, R.A.; Parks, G.D. Paramyxoviridae: The Viruses and their Replication. In Fields virology, 5th ed.; Knipe, D.M., Howley, P.M., Eds.; Lippincott Williams \& Wilkins: Philadelphia, PA, USA, 2007; Volume 1, pp. 1449-1646.

72. Herfst, S.; Mas, V.; Ver, L.S.; Wierda, R.J.; Osterhaus, A.D.; Fouchier, R.A.; Melero, J.A. Low-ph-induced membrane fusion mediated by human metapneumovirus $f$ protein is a rare, strain-dependent phenomenon. J. Virol. 2008, 82, 8891-8895.

73. Schowalter, R.M.; Chang, A.; Robach, J.G.; Buchholz, U.J.; Dutch, R.E. Low-ph triggering of human metapneumovirus fusion: Essential residues and importance in entry. J. Virol. 2009, 83, 1511-1522.

74. Mas, V.; Herfst, S.; Osterhaus, A.D.; Fouchier, R.A.; Melero, J.A. Residues of the human metapneumovirus fusion (f) protein critical for its strain-related fusion phenotype: Implications for the virus replication cycle. J. Virol. 2011, 85, 12650-12661.

75. Wei, Y.; Feng, K.; Yao, X.; Cai, H.; Li, J.; Mirza, A.M.; Iorio, R.M. Localization of a region in the fusion protein of avian metapneumovirus that modulates cell-cell fusion. J. Virol. 2012, 86, 11800-11814.

76. Chang, A.; Hackett, B.; Winter, C.C.; Buchholz, U.J.; Dutch, R.E. Potential electrostatic interactions in multiple regions affect hmpv f-mediated membrane fusion. J. Virol. 2012, 86, 9843-9853.

77. Williams, J.V.; Chen, Z.; Cseke, G.; Wright, D.W.; Keefer, C.J.; Tollefson, S.J.; Hessell, A.; Podsiad, A.; Shepherd, B.E.; Sanna, P.P.; et al. A recombinant human monoclonal antibody to human metapneumovirus fusion protein that neutralizes virus in vitro and is effective therapeutically in vivo. J. Virol. 2007, 81, 8315-8324. 
78. Swanson, K.; Wen, X.; Leser, G.P.; Paterson, R.G.; Lamb, R.A.; Jardetzky, T.S. Structure of the newcastle disease virus f protein in the post-fusion conformation. Virology 2010, 402, 372-379.

79. Yin, H.S.; Paterson, R.G.; Wen, X.; Lamb, R.A.; Jardetzky, T.S. Structure of the uncleaved ectodomain of the paramyxovirus (hpiv3) fusion protein. Proc. Natl. Acad. Sci. USA 2005, 102, 9288-9293.

80. Tayyari, F.; Marchant, D.; Moraes, T.J.; Duan, W.; Mastrangelo, P.; Hegele, R.G. Identification of nucleolin as a cellular receptor for human respiratory syncytial virus. Nat. Med. 2011, 17, 1132-1135.

81. Wilen, C.B.; Tilton, J.C.; Doms, R.W. Molecular mechanisms of hiv entry. Adv. Exp. Med. Biol. 2012, 726, 223-242. 
Reprinted from Viruses. Cite as: Watkiss, E.R.T.; Shrivastava, P.; Arsic, N.; Gomis, S.; van Drunen Littel-van den Hurk, S. Innate and Adaptive Immune Response to Pneumonia Virus of Mice in a Resistant and a Susceptible Mouse Strain. Viruses 2013, 5, 295-320.

Article

\title{
Innate and Adaptive Immune Response to Pneumonia Virus of Mice in a Resistant and a Susceptible Mouse Strain
}

\author{
Ellen R. T. Watkiss ${ }^{1,2}$, Pratima Shrivastava ${ }^{1}$, Natasa Arsic ${ }^{1}$, Susantha Gomis ${ }^{3}$ \\ and Sylvia van Drunen Littel-van den Hurk ${ }^{1,4, *}$
}

1 VIDO-Intervac, University of Saskatchewan, 120 Veterinary Road, Saskatoon, Saskatchewan, S7N 5E3, Canada; E-Mails: erw847@mail.usask.ca (E.R.T.W.); prs592@mail.usask.ca (P.S.); natasa.arsic@usask.ca (N.A.)

2 Veterinary Microbiology, University of Saskatchewan, 52 Campus Drive, Saskatoon, Saskatchewan, S7N 5B4, Canada

3 Veterinary Pathology, University of Saskatchewan, 52 Campus Drive, Saskatoon, Saskatchewan, S7N 5B4, Canada; E-Mail: susantha.gomis@usask.ca (S.G.)

4 Microbiology and Immunology, University of Saskatchewan, 107 Wiggins Road, Saskatoon, Saskatchewan, S7N 5E5, Canada

* Author to whom correspondence should be addressed; E-Mail: sylvia.vandenhurk@usask.ca; Tel.: +1-306-966-1559; Fax: +1-306-966-7478.

Received: 31 December 2012; in revised form: 13 January 2013 / Accepted: 15 January 2013 / Published: 21 January 2013

\begin{abstract}
Respiratory syncytial virus (RSV) is the leading cause of infant bronchiolitis. The closely related pneumonia virus of mice (PVM) causes a similar immune-mediated disease in mice, which allows an analysis of host factors that lead to severe illness. This project was designed to compare the immune responses to lethal and sublethal doses of PVM strain 15 in Balb/c and C57B1/6 mice. Balb/c mice responded to PVM infection with an earlier and stronger innate response that failed to control viral replication. Production of inflammatory cyto- and chemokines, as well as infiltration of neutrophils and IFN- $\gamma$ secreting natural killer cells into the lungs, was more predominant in Balb/c mice. In contrast, $\mathrm{C} 57 \mathrm{Bl} / 6$ mice were capable of suppressing both viral replication and innate inflammatory responses. After a sublethal infection, PVM-induced IFN- $\gamma$ production by splenocytes was stronger early during infection and weaker at late time points in C57Bl/6 mice when compared to Balb/c mice. Furthermore, although the IgG levels were similar
\end{abstract}


and the mucosal IgA titres lower, the virus neutralizing antibody titres were higher in $\mathrm{C} 57 \mathrm{~B} 1 / 6$ mice than in Balb/c mice. Overall, the difference in susceptibility of these two strains appeared to be related not to an inherent $\mathrm{T}$ helper bias, but to the capacity of the C57B1/6 mice to control both viral replication and the immune response elicited by PVM.

Keywords: PVM; Balb/c and C57Bl/6 mice; innate and adaptive immunity; immunopathogenesis

\section{Introduction}

Pneumonia virus of mice (PVM) is a natural pathogen in rodents that induces severe respiratory illness which is in many ways similar to severe respiratory syncytial virus (RSV) infection in infants [1,2]. RSV is one of the most important causes of infant mortality and hospitalization worldwide and causes more cases of viral pneumonia in infants than any other virus [3-5]. It is highly transmissible, spreading through communities rapidly and infecting most of the population by the age of one [6]. RSV can infect and reinfect individuals throughout life and causes mild cold-like symptoms in most healthy adults and children [6,7]. In infants, young children, and immune-compromised individuals, however, the virus is more likely to progress to the lower respiratory tract, often causing an infection severe enough to require lengthy hospitalization and sometimes causing death [8]. The factors involved in the development of severe RSV are largely unknown, for a number of reasons. RSV pathogenesis involves the complex interplay of immune cells in the lungs, resulting in an inadequate or misdirected response that damages lung tissue [9]. In addition, studies on very young and severely ill infants are difficult, especially those involving the lower respiratory tract. The use of animal models can overcome many of the hindrances associated with human studies by providing access to the site of replication in the lower respiratory tract. However, the pneumoviruses are highly species-specific, so natural host-pathogen pairs are preferred or, arguably, necessary to examine the immune-mediated mechanisms of severe pneumovirus infection [10,11].

In the past three decades, PVM has been evaluated as a potential murine model of natural severe RSV infection. PVM causes respiratory illness that varies in severity depending on the virus and mouse strain used and can replicate to a high titre from a low inoculum [12,13]. To date two strains of PVM have been characterized, PVM J3666 and PVM 15. These two strains differ significantly in select regions of the $\mathrm{G}$ protein and throughout the sequence of the SH glycoprotein [13,14]. PVM 15 has a genome of $14.8 \mathrm{~KB}$, containing 10 genes which encode 12 proteins. The genome sequence is 99.7\% identical to that of PVM J366. With exception of a 137-amino acid protein encoded by a second ORF in the P mRNA, all PVM proteins have a respective counterpart in RSV [14]. An earlier study indicated that the PVM strain 15 is replication-competent, but non-pathogenic in mice [13,15]. However, more recently Krempl et al. concluded that a PVM 15 strain available from ATCC is pathogenic in Balb/c mice to a similar degree as the J3666 strain and lacks the same mutation seen in 
the PVM 15 strain variant used earlier [14,16], thus mimicking the original PVM strain 15 isolated by Horsfall and Hahn [17]. They also clarify that the earlier reported attenuation of the PVM strain 15 in mice was specific for that particular preparation and that the attenuation was not indigenous $[14,16]$. The susceptibility of different inbred mouse strains to infection with PVM J3666 has been studied extensively [12]. However, this does not apply to PVM 15, so the present study is focused on the direct comparison of the pathogenesis of PVM 15 in Balb/c and C57Bl/6 mice.

Balb/c mice show classic Th2-biased responses to several intracellular pathogens, making them more susceptible to severe infections compared to $\mathrm{C} 57 \mathrm{Bl} / 6$ mice, which tend to show protective Th1-biased responses [18-21]. Balb/c mice are also more susceptible to RSV-induced eosinophilia following priming with the RSV G protein [22,23]. The basis of this susceptibility to eosinophilia is dependent on genetic background, rather than the MHC haplotypes expressed in these strains, as $\mathrm{Balb} / \mathrm{b}$ mice, which express the same MHC haplotypes as $\mathrm{C} 57 \mathrm{Bl} / 6$ mice in the context of the Balb/c genetic background, were as susceptible to eosinophilia as the Balb/c strain [22]. Thus, genetic differences in Balb/c and $\mathrm{C} 57 \mathrm{Bl} / 6$ mice could lead to very different responses following natural pneumovirus infection in vivo.

The goal of this study was to understand the basis of the difference in susceptibility between Balb/c and $\mathrm{C} 57 \mathrm{Bl} / 6$ mice to PVM infection by comparing their immune response to PVM 15 in vivo. To investigate the immune response and pathogenesis of PVM in Balb/c and C57Bl/6 mice, we inoculated mice with increasing doses of PVM 15 and compared the level of weight loss and viral replication, the expression of key inflammatory mediators in, and recruitment of cells to the lungs, and the adaptive immune responses following infection. Clinical disease and viral replication was enhanced in the Balb/c strain, which correlated to earlier production of inflammatory mediators and influx of immune cells, particularly neutrophils and natural killer (NK) cells, when compared to the C57B1/6 strain. PVM 15 induced virtually no Th2-biased cytokines or CCL11 (eotaxin) in either strain, and eosinophils were not detected in the lungs of infected mice. The adaptive immune responses, however, developed more rapidly and appeared to be more protective in C57B1/6 mice.

\section{Results and Discussion}

\subsection{Balb/c Mice Show Earlier and More Weight Loss, and Enhanced Virus Replication in the Lungs after PVM 15 Infection in Comparison with C57Bl/6 Mice}

As the first sign and best measure of clinical illness, the animals' weights were recorded daily from day 0 to day 7 post infection (p.i.) (Figure 1A,B). Overall, Balb/c mice lost weight earlier than $\mathrm{C} 57 \mathrm{Bl} / 6$ mice and succumbed to the PVM infection at a lower dose than the latter strain. No weight loss was observed in control mice, in Balb/c mice inoculated with $30 \mathrm{pfu}$ of PVM, or in C57Bl/6 mice given 300 pfu or 30 pfu (data not shown) during the 7-day period after PVM challenge. Balb/c mice, however, lost weight as early as day 5 following inoculation with 300 pfu of PVM 15 and dropped to a median of $93 \%$ and $85 \%$ of their starting weight on days 6 and 7 p.i., respectively. At a dose of $3000 \mathrm{pfu}$, Balb/c mice began losing weight one day earlier, dropping to a significantly lower weight of 
$96 \%$ on day 5 and $89 \%$ on day 6 p.i.. On days 5 and 6 there was a significant difference in weight loss between Balb/c mice infected with 3000 pfu and those given $30 \mathrm{pfu}$, while the difference between the Balb/c mice given 300 and 30 pfu doses was significant on days 6 and 7 . When given 3000 pfu, the $\mathrm{C} 57 \mathrm{Bl} / 6$ mice showed the same degree of weight loss as Balb/c mice one day later, dropping to a median of $96 \%$ on day 6 and $89 \%$ on day 7 p.i. of their starting weight. The C57B1/6 mice infected with 3000 pfu lost significantly more weight than those given 300 pfu on days 6 and 7 p.i.. Among the Balb/c mice challenged with 3000 pfu of PVM 15 all survived through day 5 p.i., while by day 6, 25\% had died and by day 7 all had died. The C57B1/6 mice challenged with 3000 pfu all survived through day 6 , but $25 \%$ had died by day 7 . All other mice survived.

Figure 1. Weight loss and accumulation of virus in the lungs following infection with PVM 15 in Balb/c and C57Bl/6 mice. Five to six week-old Balb/c and C57B1/6 mice were inoculated with medium, $30 \mathrm{pfu}, 300 \mathrm{pfu}$, or $3000 \mathrm{pfu}$ of PVM 15 and weighed daily for 7 days following infection, and 4 mice per group were sacrificed daily. (A-B) median weight for each group, expressed as a percentage of the starting weight, with error bars indicating the interquartile range. (C-D) median viral load for each group with error bars indicating the interquartile range. a: $p<0.05$ compared to control group; b. $p<0.05$ between 3000 and 300 pfu; c: $p<0.05$ between 3000 and 30 pfu; $\mathrm{d}: p<0.05$ between 300 and $30 \mathrm{pfu}$. Differences in virus replication with the control groups are not shown.
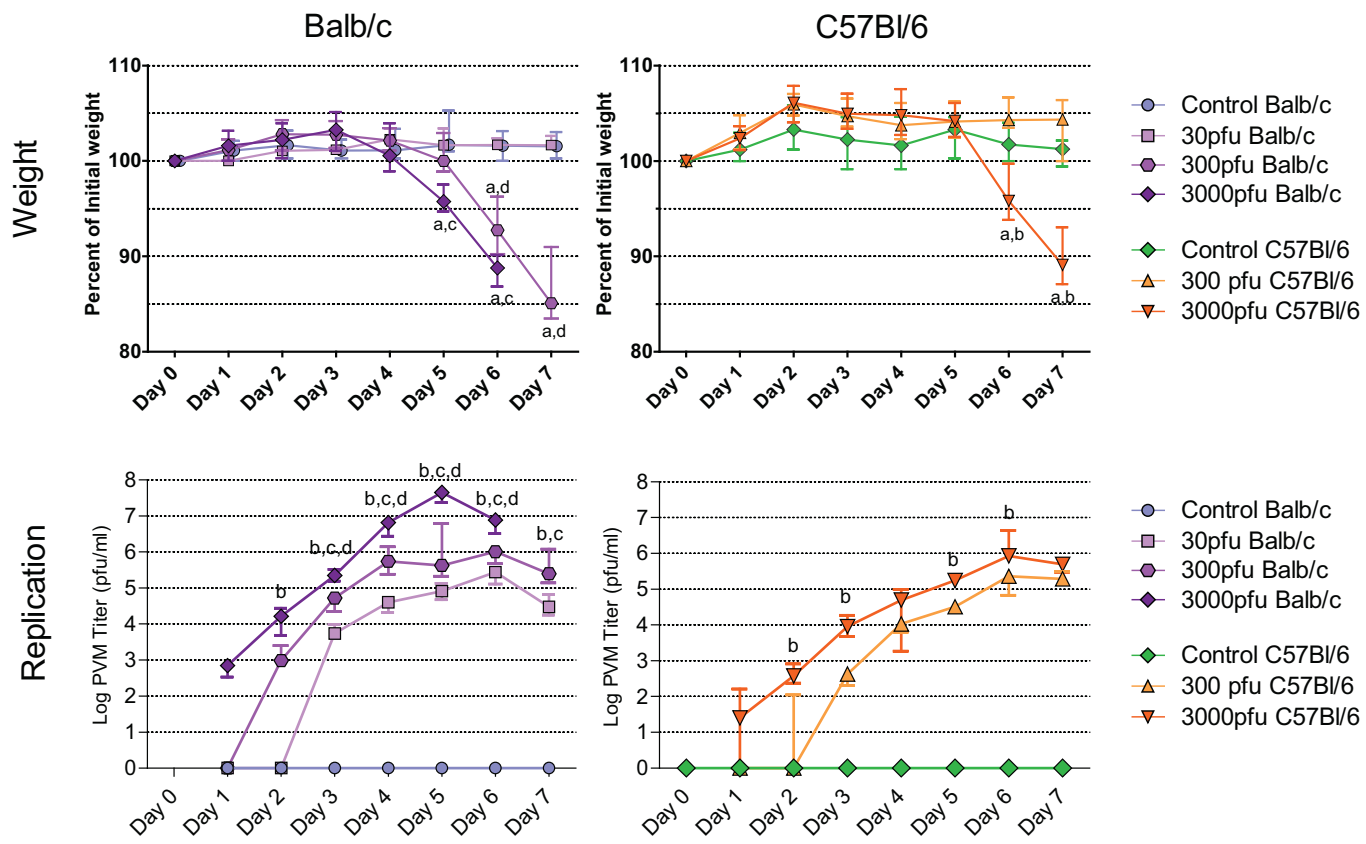

- Control Balb/c

- 30pfu Balb/c

- o 300pfu Balb/c

$\checkmark$ 3000pfu Balb/c

$\diamond$ Control C57BI/6

$\triangle 300 \mathrm{pfu}$ C57BI/6

$\rightarrow$ 3000pfu C57BI/6

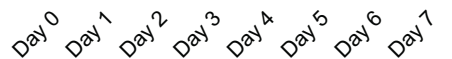


The susceptibility of Balb/c and $\mathrm{C} 57 \mathrm{Bl} / 6$ mice was further evaluated based on the level of viral replication in the lungs. At all three doses, Balb/c mice had a more rapid accumulation of virus in the lungs that reached an earlier peak followed by a clear decline (Figure 1C), while the C57Bl/6 strain displayed a slower increase in viral replication that reached a plateau on day 6 at both doses (Figure 1D). Indeed, PVM replication was so rapid in Balb/c mice that by days 3 and 4 p.i., the 30 pfu dose had replicated to similar titres as the $3000 \mathrm{pfu}$ dose in $\mathrm{C} 57 \mathrm{Bl} / 6 \mathrm{mice}$, and by day 6 p.i. reached a peak that was equal to that of the $300 \mathrm{pfu} \mathrm{C} 57 \mathrm{Bl} / 6$ mice. The amount of virus recovered from the lungs of infected mice was dose-dependent in the Balb/c strain at all times with the $3000 \mathrm{pfu}$ dose causing more virus replication than the 300 and 30 pfu doses from days 2 through 7, and the 300 pfu dose more than the 30 pfu dose from days 3 through 6 p.i.. The 3000 pfu group of C57B1/6 mice reached a significantly higher median viral titre than the $300 \mathrm{pfu}$ group, except on days 4 and 7 , when the C57Bl/6 mice infected with 300 or 3000 pfu had similar titres. A comparison of equivalent PVM 15 challenge doses in Balb/c and $\mathrm{C} 47 \mathrm{Bl} / 6$ mice showed that 3000 pfu or 300 pfu produced a significantly higher viral load in the Balb/c mice than in the C57B1/6 mice from days 2 through 6 p.i. $(p<0.05)$. Based on this direct comparison, PVM 15 causes more severe disease in Balb/c mice than in C57B1/6 mice.

Interestingly, Anh et al. [12] have characterized different strains of mice with respect to resistance to disease induced by $\sim 1000$ pfu of PVM J3666. Based on a combination of clinical, histological and virological parameters, the SJL mouse strain was most resistant followed by $\mathrm{C} 57 \mathrm{BL} / 6, \mathrm{BALB} / \mathrm{c}$, $\mathrm{C} 3 \mathrm{HeN}, \mathrm{DBA} / 2$ and129/Sv strains. This is in agreement with the observations we made for PVM 15 in $\mathrm{C} 57 \mathrm{Bl} / 6$ and $\mathrm{Balb} / \mathrm{c}$ mice.

\subsection{Comparison of Lung Pathology in PVM-Infected Balb/c and C57Bl/6 Mice}

To assess the level of lung pathology induced by different doses of PVM in Balb/c and C57B1/6 mice, lungs were processed for histopathological analysis on day 6 p.i. (Figure 2). Mice in the control groups had a score of zero, or normal lungs (Figure 2A, upper left panel), had few cells dispersed throughout the alveolar space, and the airway epithelium was intact and free of fluid and infiltrating immune cells. Although there was no significant difference in lung score between PVM-infected groups, there was a trend towards a dose-dependent increase in lung score for both strains of mice (Figure 2B). The C57B1/6 mice given 300 pfu had a score close to 1, which indicates a localized, mild inflammation of the peribronchiolar and perivascular space involving fluid accumulation with few infiltrating immune cells (Figure 2A, upper right panel). The Balb/c mice inoculated with 30 or 300 pfu scored 1.5 to 2 , which indicates multiple lesions or a single extensive lesion, the lesions being more severe, with higher numbers of infiltrating cells in the inflamed tissue and the alveolar space (Figure 2A, lower left panel). The $3000 \mathrm{pfu}$ C57Bl/6 group had a median score of 2.5, while the $3000 \mathrm{pfu}$ Balb/c group was scored as 3 , indicating broadly dispersed lesions with cellular infiltrates in the alveolar space and surrounding tissues. The severity of the lesion is evident by the presence of inflammatory cells in the alveolar space and surrounding the blood vessel and bronchiole. There is little air space left in the lung, as much cell debris has accumulated in the alveoli (Figure 2A, lower 
right panel). These data show that overall, the level of lung pathology tended to be lower in the $\mathrm{C} 57 \mathrm{Bl} / 6$ mice than in the Balb/c mice when infected with the same dose of PVM.

Figure 2. Histopathological analysis of PVM-infected mice. Five to six week-old Balb/c and $\mathrm{C} 57 \mathrm{Bl} / 6$ mice were inoculated with medium, $30 \mathrm{pfu}, 300 \mathrm{pfu}$, or $3000 \mathrm{pfu}$ of PVM 15 and lungs were collected from four mice on day 6 p.i. for histopathological analysis. In (A), representative lung sections for animals scoring $\mathbf{0 , 1}, \mathbf{2}$, and $\mathbf{3}$ are shown, with the upward arrows $(\uparrow)$ indicating infiltrating inflammatory cells and the downward arrows $(\downarrow)$ indicating oedema in the tissue. The bronchiole is labeled with the letter B and the blood vessel with V. Scores were given on the basis of the severity and dissemination of the lesions visible in duplicate lung sections, and median values are shown for each group (B).
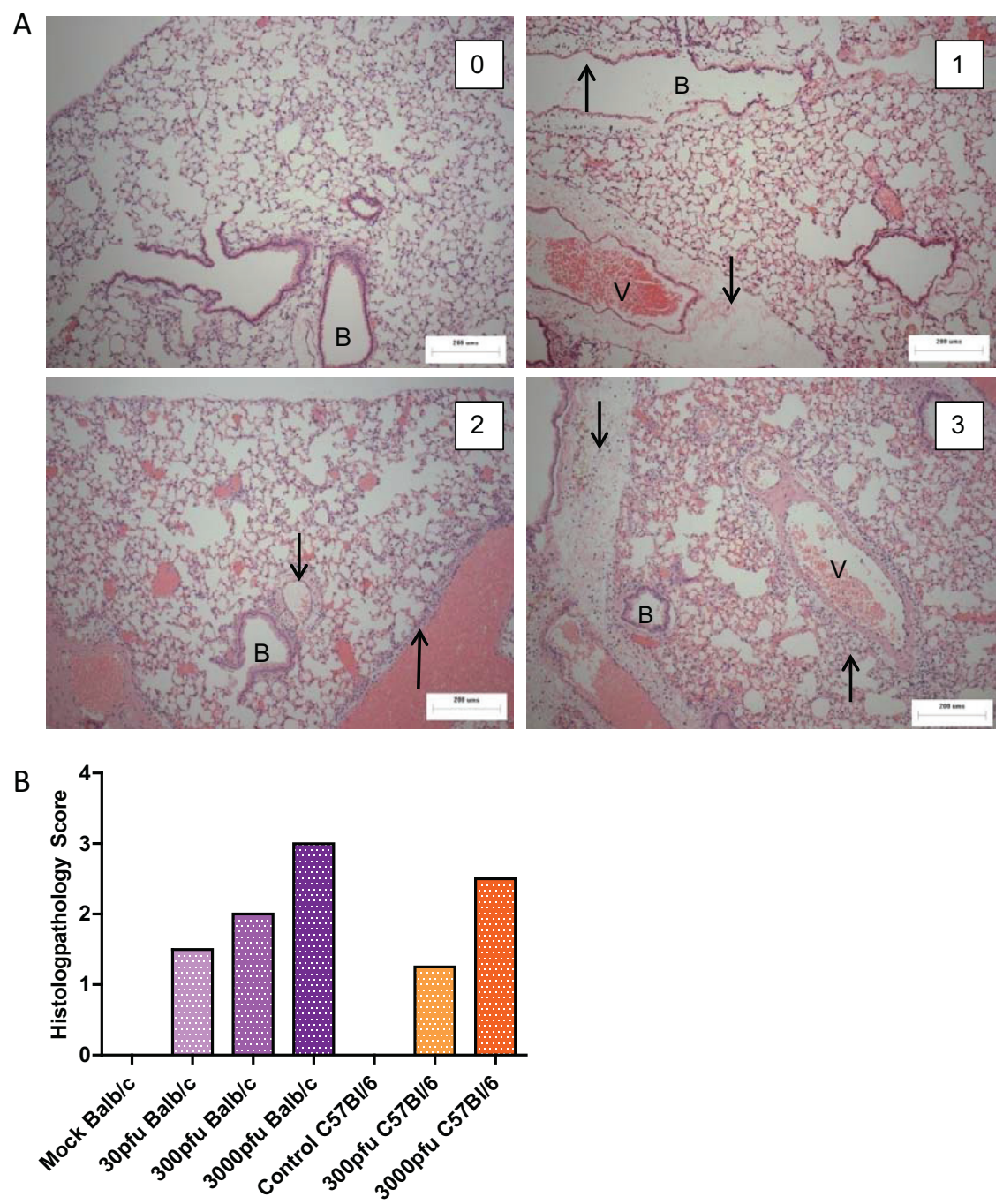


\subsection{PVM 15 Induces Earlier Transcription of Chemokines and Cytokines in Balb/c Mice When}

Compared to $\mathrm{C} 57 \mathrm{Bl} / 6$ Mice

As there was a significant difference between PVM-infected Balb/c and C57Bl/6 mice in clinical disease, virus replication and lung pathology, the innate responses, in particular the production of key inflammatory cytokines and chemokines, were investigated. There was little to no upregulation of IFN- $\beta$, TNF- $\alpha$, IL-4, IL-10, CCL5, or CCL11 upon infection with PVM (data not shown). In contrast, expression of IFN- $\alpha$ and IFN- $\gamma$ (Figure 3), as well as CXCL8, CXCL10, CCL3, and CCL2 (Figure 4) changed significantly upon infection with PVM.

Figure 3. Cytokine expression by $\mathrm{Balb} / \mathrm{c}$ and $\mathrm{C} 57 \mathrm{Bl} / 6$ mice following infection with PVM 15. Five to six week-old Balb/c and C57B1/6 mice were inoculated with medium, $30 \mathrm{pfu}, 300 \mathrm{pfu}$, or $3000 \mathrm{pfu}$ of PVM 15 and lungs were collected from four mice per group on days 3 (A and $\mathbf{C}$ ) and 5 p.i. (B and D). Expression levels of IFN- $\alpha$ (A and B) and IFN- $\gamma(\mathrm{C}$ and $\mathrm{D})$ transcripts were calculated using the Bio-Rad analysis software (Bio-Rad CFX Manager Version 2.0), normalized against the expression of both $\beta$-actin and GAPDH housekeeping genes, and expressed as the normalized fold-change over mock-infected control animals euthanized on the same day p.i.. Each data point represents a single animal and the line represents the group median. *, $p<0.05$.

A

Day 3

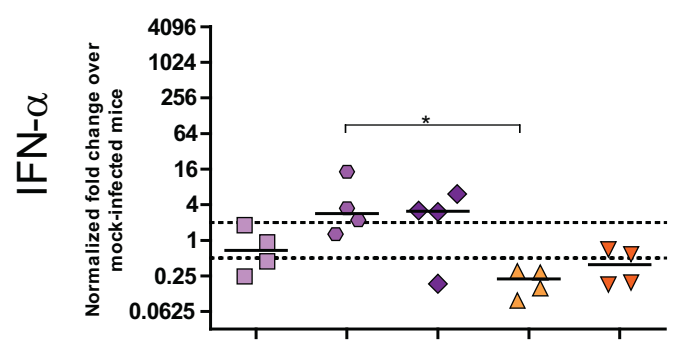

C

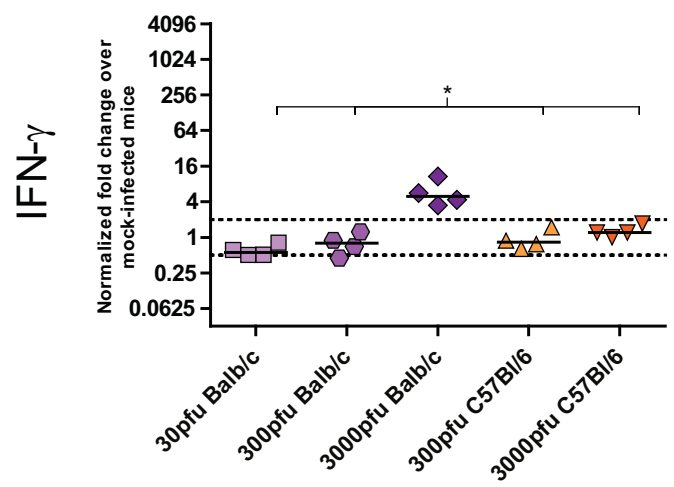

B
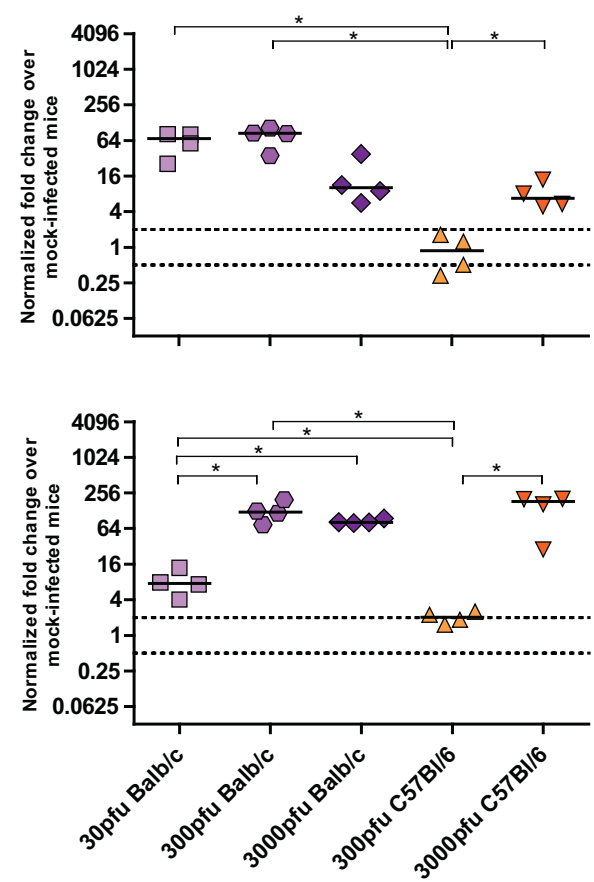
Figure 4. Chemokine expression by Balb/c and $\mathrm{C} 57 \mathrm{~B} 1 / 6$ mice following infection with PVM 15. Five to six week-old Balb/c and C57Bl/6 mice were inoculated with medium, 30 pfu, 300 pfu, or 3000 pfu of PVM 15 and lungs were collected from four mice per group on days 3 (A-D) and 5 p.i. (E-H). The expression levels of CXCL8 (A and E), CXCL10 (B and F), CCL3 (C and G), and CCL2 (D and H) were calculated using the Bio-Rad analysis software (Bio-Rad CFX Manager Version 2.0), normalized against the expression of both $\beta$-actin and GAPDH housekeeping genes, and expressed as the normalized fold-change over mock-infected control animals euthanized on the same day p.i.. Each data point represents a single animal and the line represents the group median. ${ }^{*}, p<0.05$.

Day 3

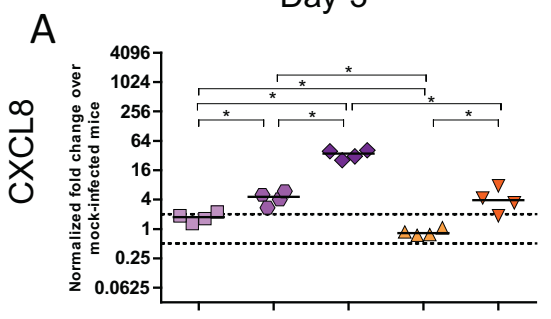

$$
\text { B }
$$

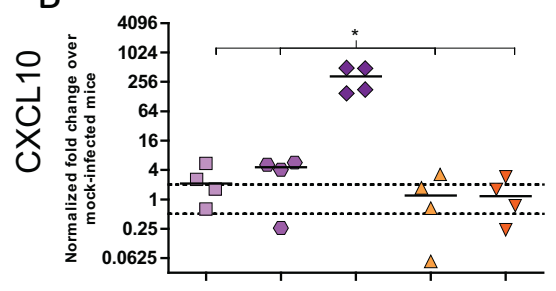

C

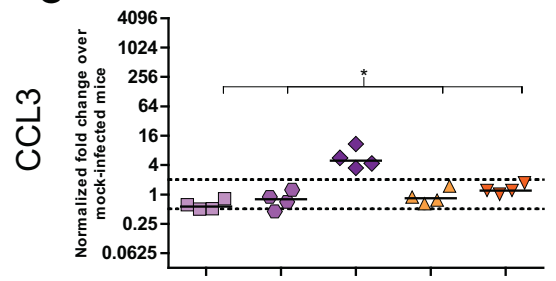

D

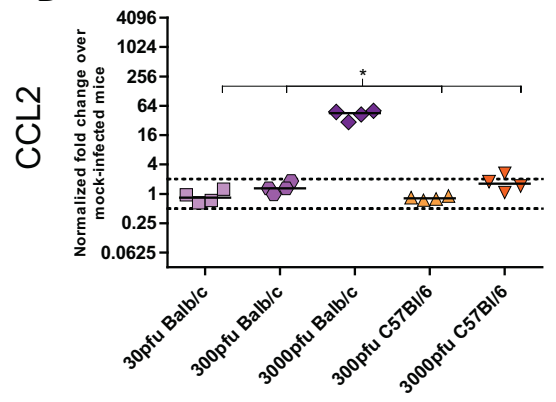

Day 5

E

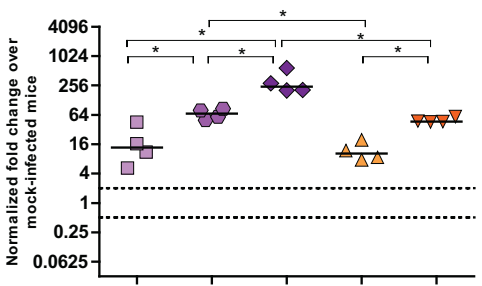

$\mathrm{F}$

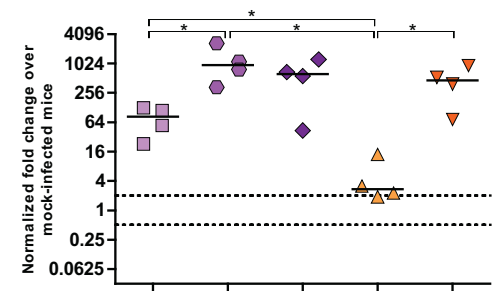

G

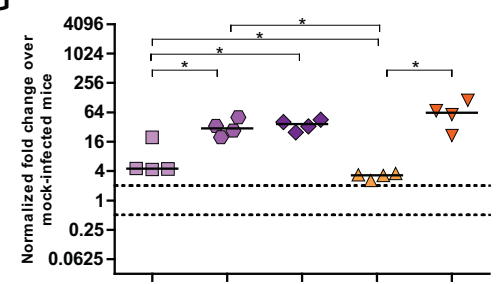

$\mathrm{H}$

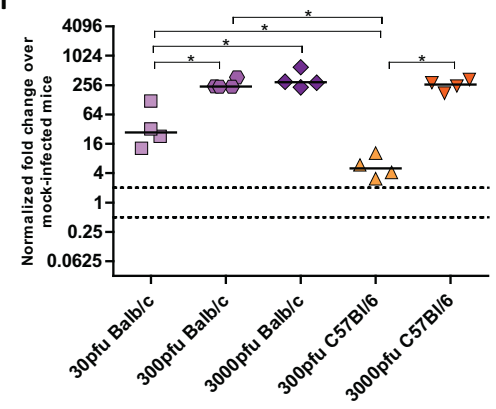


There were three distinct patterns of mRNA expression following infection with PVM. IFN- $\alpha$ and CXCL8 showed unique profiles, while IFN- $\gamma$ and the remaining chemokines had virtually identical patterns of upregulation. Strikingly, the C57B1/6 mice showed virtually no upregulation of any chemokine or cytokine other than CXCL8 following infection with $300 \mathrm{pfu}$, in contrast to Balb/c mice which showed higher levels of all transcripts following inoculation with 300 pfu and even as little as 30 pfu of PVM 15.

On day 3 p.i., IFN- $\alpha$ mRNA expression was not increased in any group, and, indeed, the C57Bl/6 mice appeared to have downregulated this cytokine. By day 5, however, the $3000 \mathrm{pfu}$ dose of PVM 15 induced similar levels of IFN- $\alpha$ in the two mouse strains, while 30 and 300 pfu induced IFN- $\alpha$ upregulation only in Balb/c mice. Direct analysis of the role of type I IFN in PVM infection has only been carried out in $\mathrm{C} 57 \mathrm{Bl} / 6$ mice with a deletion in the IFN- $\alpha / \beta$ receptor gene. Abrogation of type I IFN signalling during PVM J3666 infection led to decreased expression of IFN response genes, lower levels of IFN- $\alpha$ and IFN- $\beta$, and, intriguingly, a slightly enhanced survival time in C57Bl/6 mice given a lethal dose of $60 \mathrm{pfu}$ [24]. This would agree with the lower degree of IFN- $\alpha$ expression and better survival we observed in PVM 15 infected C57B1/6 mice in comparison to Balb/c mice.

The CXC chemokine, CXCL8, was upregulated in a dose-dependent manner on both days 3 and 5 p.i., such that a 10-fold higher dose of PVM induced between 3 and 5-fold higher levels of mRNA upregulation in the Balb/c strain, and $\sim 7$-fold higher upregulation in the C57B1/6 strain. IFN- $\gamma$ and the remaining chemokines were upregulated in the 3000 pfu Balb/c group on day 3 p.i., and by day 5 they were expressed by all groups other than the $300 \mathrm{pfu}$ C57Bl/ 6 group. The lethal doses of PVM induced similar mRNA upregulation of these proinflammatory mediators in Balb/c and C57B1/6 mice by day 5 , which was significantly higher than that in the $30 \mathrm{pfu} \mathrm{Balb} / \mathrm{c}$ or the $300 \mathrm{pfu} \mathrm{C} 57 \mathrm{Bl} / 6$ groups. Expression of CXCL10 was induced upon infection with PVM in a similar pattern to IFN- $\gamma$, which would be expected based on its role as an interferon response gene. Its mRNA upregulation was also similar to that of the CC chemokines, CCL2 and CCL3, with the three lethal doses showing a similar level of gene upregulation on day 5 p.i..

PVM J3666 pathogenesis is strongly governed by CCL3 production in vivo. Transgenic mice in the $\mathrm{C} 57 \mathrm{Bl} / 6$ background that lack functional CCL3 or CCR1 were more susceptible to infection with PVM J3666, and had drastically reduced levels of neutrophil infiltration, with no eosinophils and very low levels of lymphocytes in the lungs upon infection $[25,26]$. The virus also replicated to a higher titre in these mice and they succumbed to infection earlier than non-transgenic littermates. However, blockade of CCL3 signalling by the CCR1/CCR5 receptor antagonist, Met-RANTES, resulted in decreased lung inflammation without affecting PVM replication in the lungs [25]. This is in agreement with our observation that $\mathrm{C} 57 \mathrm{Bl} / 6$ mice, which responded to PVM 15 infection with delayed and reduced CCL3 expression as well as less neutrophil infiltration, also showed less disease and lung pathology when compared to Balb/c mice.

Despite reports that CCL2 is one of the predominant chemokines upregulated during PVM infection, its exact role in PVM pathogenesis has not been defined yet [1]. CCL2 was significantly decreased by use of the antiviral ribavirin, suggesting that PVM replication stimulates its production 
in vivo [27]. Interestingly, in another study on age-related changes in susceptibility older mice showed decreased CCL2 production in response to PVM, which was associated with reduced disease presentation, despite similar viral replication [28]. This would support a role for the increased CCL2 expression in the enhanced pathogenesis in PVM 15-infected Balb/c mice.

\subsection{Balb/c Mice Experience Earlier and Enhanced Infiltration of Immune Cells Compared to C57Bl/6 Mice in Response to PVM Infection}

In order to determine the biological effects of the proinflammatory mediators reported above, we examined cell populations infiltrating the lungs of the infected mice (Figure 5). As expected from the earlier upregulation of proinflammatory mediators seen in Balb/c mice, this strain showed an earlier and stronger influx of cells into the lungs that was dominated by neutrophils. The degree of neutrophilia appeared to be dose-dependent in the Balb/c strain on day 5 p.i., while the lethal doses showed similar levels of lymphocyte infiltration. The C57Bl/6 mice, on the other hand, had little influx of cells by day 5 p.i., even at the highest dose of 3000 pfu. Comparing the two sublethal doses, the $30 \mathrm{pfu}$ Balb/c group showed a low level of inflammation by day 5 p.i., while the 300 pfu C57Bl/6 group had essentially normal lung washes. There were few to no eosinophils in response to PVM 15 in either strain at any dose. In contrast, Domachowski et al. demonstrated that in response to PVM J3666, Balb/c mice showed a prominent eosinophilia, as early as 3 days after inoculation, while at later time points cell infiltration was dominated by neutrophils. In their study they did not find any up regulation of eosinophilic chemoattractant IL-5, CCL2, CCL11 or CCL5, but CCL3 was up regulated. Thus, pulmonary eosinophilia and production of CCL3 are prominent responses to infection with PVM J3666 [29].

Macrophages were the predominant population in the lungs at homeostasis. The number of macrophages tended to increase in the Balb/c mice on days 3 respectively 5 for the 3000 and 300 pfu doses of PVM, but remained stable in the $\mathrm{C} 57 \mathrm{Bl} / 6$ mice. Rigaux et al. determined that depletion of alveolar macrophages in PVM J366-infected Balb/c mice resulted in prolonged survival, despite a slight but significant increase in viral replication [30]. This suggests that macrophages may also play a role in the enhanced pathology observed in PVM 15-infected Balb/c mice, although this would need to be further investigated.

CXCL8 is produced in response to RSV infection of infants and is associated with disease severity and neutrophil degranulation products [31-34]. Although CXCL8 also is an important mediator of neutrophil recruitment following PVM infection, recent work on the contribution of CCL3 and IFN- $\gamma$ to PVM pathogenesis suggests that these latter proinflammatory mediators may be as important, if not more so, than CXCL8 in recruiting neutrophils to the lungs of PVM-infected mice. Abrogation of IFN- $\gamma$ or CCL3 signalling drastically reduced the number of neutrophils recruited to the lungs of PVM-infected C57Bl/6 mice [35]. In an IFN- $\gamma^{-1-}$ Balb/c background, overexpression of CCL3 in the lungs was not sufficient to induce neutrophil recruitment to the lungs, despite the expression of its receptor on neutrophils in these mice [35]. When IFN- $\gamma$ was administered to mice that overexpress CCL3 in the lungs, however, they rapidly developed neutrophilic inflammation and showed signs of 
clinical illness and weight loss similar to that experienced by PVM-infected mice. In our study, CCL3 and IFN- $\gamma$ were (highly) upregulated by Balb/c mice upon infection with 30 or 300 pfu, while these chemokines were not expressed in C57Bl/6 mice given $300 \mathrm{pfu}$; furthermore, C57BL/6 mice had lower numbers of inflammatory cells, in particular neutrophils, in the lungs than Balb/c mice. These data agree with a role of CCL3 and IFN- $\gamma$ in neutrophil recruitment, as well as the enhanced disease symptoms in PVM15-infected Balb/c mice.

Figure 5. Infiltration of immune cells in $\mathrm{Balb} / \mathrm{c}$ and $\mathrm{C} 57 \mathrm{Bl} / 6$ mice in response to $\mathrm{PVM}$ infection. Five to six week-old Balb/c and $\mathrm{C} 57 \mathrm{~B} 1 / 6$ mice were inoculated with medium, $30 \mathrm{pfu}$, or $300 \mathrm{pfu}$ of PVM 15 and sacrificed on days 3 and 5 p.i.. Cells collected from the pooled lung washes of 4-6 animals were stained and analyzed for the presence of macrophages, neutrophils, lymphocytes, and eosinophils. The average number of these cells is shown, calculated based on the number of total cells collected from the group and the proportion of these cell populations in the lungs.

A

Day 3

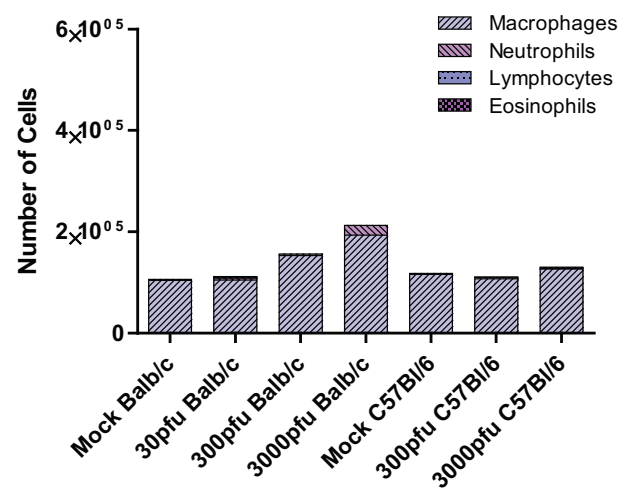

B

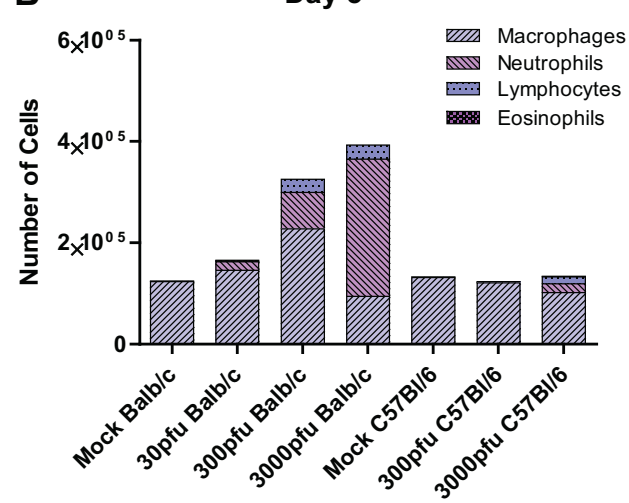

\subsection{Balb/c Mice Show an Increase in IFN- $\gamma$ Producing NK Cells in the Lung Compared to C57Bl/6} Mice in Response to PVM Infection

As by day 5 p.i. IFN- $\gamma$ and CXCL10 were significantly upregulated in the lungs, the population responsible for IFN- $\gamma$ production was further characterized by dual staining and flow cytometry on day 6 p.i. While no IFN- $\gamma$ secreting $\mathrm{CD}^{+}$or $\mathrm{CD}^{+} \mathrm{T}$ cells were found, a significant increase in IFN- $\gamma$ secreting NK cells was observed in Balb/c mice, and a moderate increase in $\mathrm{C} 57 \mathrm{Bl} / 6$ mice (Figure 6). The difference in IFN- $\gamma$ producing NK cell in Balb/c and C57Bl/6 mice was significant, suggesting that NK cells contribute to lung pathology observed in Balb/c mice. Our results with respect to the absence of IFN- $\gamma$ secreting T cell populations in lungs of PVM 15 infected mice agrees with previous reports by Claassen et al. using PVM J3666, in which authors demonstrated that during primary and secondary infection CD8 $\mathrm{T}$ cells are functionally restricted in IFN- $\gamma$ production. They speculated that PVM infection results in effector T cell inactivation as reported in case of RSV infection [36]. 
Figure 6. Percentage of IFN- $\gamma$ secreting NK cells in lungs of Balb/c and C57Bl/6 mice in response to PVM infection. Five to six week old-mice were inoculated with medium (control), or $30 \mathrm{pfu}(\mathrm{Balb} / \mathrm{c})$, or $300 \mathrm{pfu}(\mathrm{C} 57 \mathrm{Bl} / 6)$ of PVM 15 and sacrificed on day 6 p.i.. Cells collected from pooled lungs of five animals were stained with anti-CD8a-FITC, anti-CD3-PE, anti-CD4-FITC, anti-CD335(NKp46)PerCP-Cy ${ }^{\mathrm{TM}} 5.5$ for surface markers, followed by permeabilization and fixation with $\mathrm{BD}$ Cytofix/Cytoperm ${ }^{\mathrm{TM}}$ Plus and staining with anti-IFN- $\gamma$-APC. Flow cytometry was performed using a FACS Calibur (BD Biosciences), and data analysis was performed using Kaluza software. Median values with interquartile range are shown. $* *, p<0.01 ; * * *, p<0.001$.
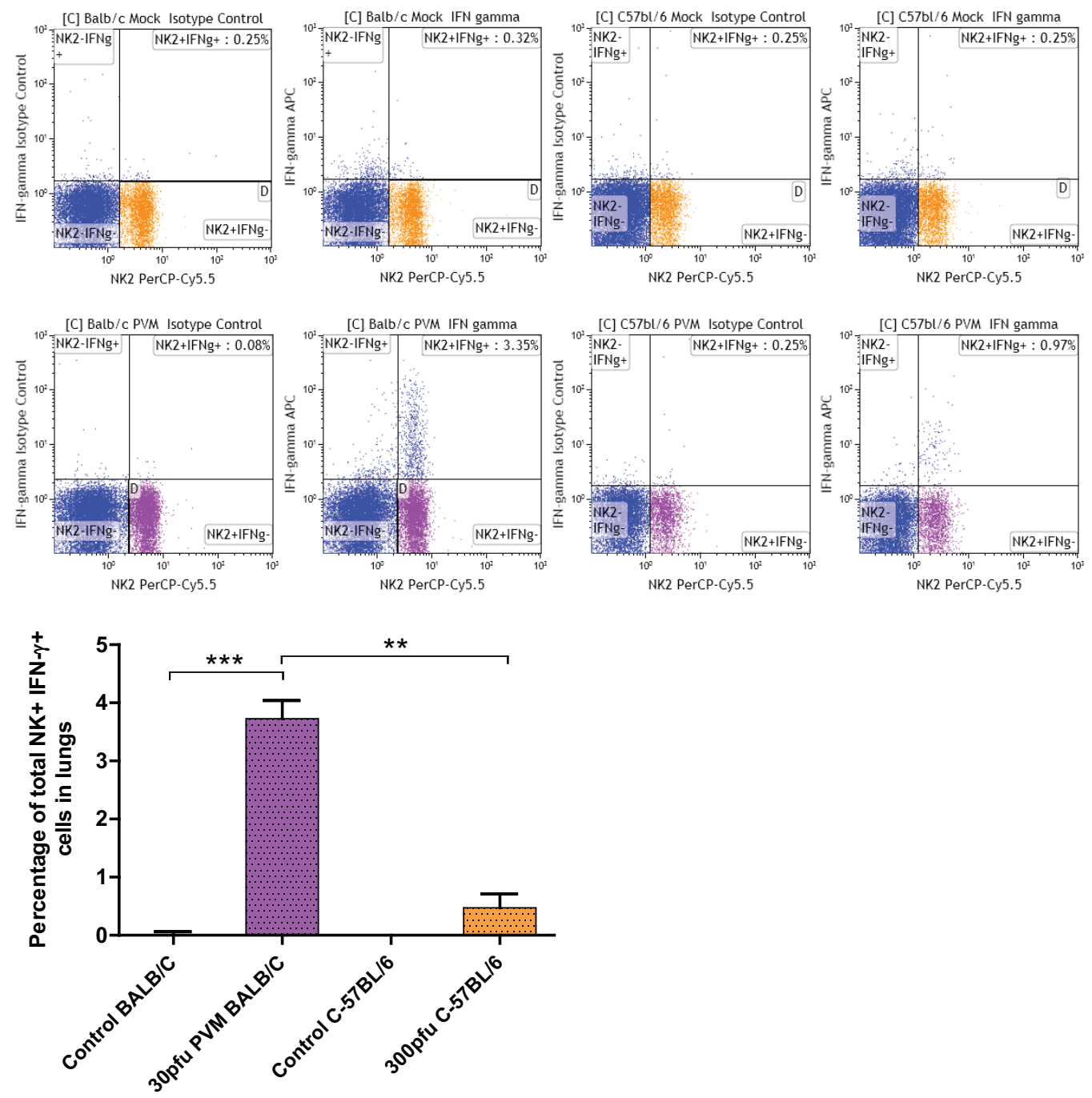
Several chemokines are reported to act as chemoattractants to NK cells, including CCL3, CCL2, CXCL8, CCL5, and CXCL10 [37-39]. NK cells have been found to accumulate both after RSV [40] and PVM [41] infection of Balb/c mice, and to contribute to lung immune injury after RSV infection [40]. In our study, CCL3, CCL2, CXCL8 and CXCL10 were all upregulated after PVM infection specifically in Balb/c mice, CXCL8 as early as three days after infection, which agrees with the greater number of NK cells in Balb/c mice. The increased numbers of IFN- $\gamma$ secreting NK cells in $\mathrm{Balb} / \mathrm{c}$ mice when compared to C57B1/6 mice further support a role for NK cells and IFN- $\gamma$ in the enhanced disease observed in PVM-infected Balb/c mice.

\subsection{Balb/c and C57Bl/6 Mice Develop Similar, Th1-Biased Adaptive Immune Responses to a Sublethal Dose of PVM}

To determine the magnitude and quality of the adaptive immune responses Balb/c and $\mathrm{C} 57 \mathrm{Bl} / 6$ mice were inoculated intranasally with $50 \mu \mathrm{L}$ of medium or a sub-lethal dose of PVM (30 pfu in $\mathrm{Balb} / \mathrm{c}$ and $300 \mathrm{pfu}$ in $\mathrm{C} 57 \mathrm{Bl} / 6$ ), and followed for a longer period p.i.. To examine the PVM-specific humoral response, production of $\mathrm{IgA}, \mathrm{IgE}$, and $\mathrm{IgG}$ in the lungs and sera of Balb/c and $\mathrm{C} 57 \mathrm{Bl} / 6$ mice was measured by ELISA. Neither strain produced IgE in the sera or lungs. IgA was detected in the lungs and IgG was present in the lungs and sera of both strains by day 14 p.i.. Balb/c mice showed increasing levels of IgG and IgA in the lungs, as well increasing IgG in the serum between days 14 and 28 p.i. (Figure 7A-C). Although they generally had the same or slightly lower levels of antibodies than $\mathrm{Balb} / \mathrm{c}$ mice, the $\mathrm{C} 57 \mathrm{~B} 1 / 6$ mice had already developed maximal antibody titres by day 14 p.i., which were maintained until day 28. To determine the biological relevance of the antibodies produced in these two strains of mice, a virus neutralization (VN) assay was performed (Figure 7D,E) showing that all PVM-infected mice contained VN antibodies in the serum and lungs on day 28 p.i.. Despite having similar levels of PVM-specific IgG in the serum and lower IgA levels in the lung, the C57Bl/6 mice had significantly higher VN antibody titres compared to the Balb/c mice.

To determine the T-helper bias of the immune response elicited by PVM in Balb/c and C57B1/6 mice, we performed IFN- $\gamma$ and IL-5 ELISPOT assays. All infected mice generated significantly higher numbers of PVM-specific IFN- $\gamma$-secreting cells compared to mock-infected animals of the same strain (Figure 8), while IL-5-secreting cells numbers remained low at all time points, suggesting a Th1-biased response to PVM in both strains of mice. However, the two strains showed a difference in the kinetics of the T-cell response. The $\mathrm{C} 57 \mathrm{Bl} / 6$ mice showed a strong PVM-induced IFN- $\gamma$ response as early as day 14, significantly higher than that of Balb/c mice; however, these responses were reduced between days 14 to 28 p.i., at which time they became lower than those in Balb/c mice.

These data show that the adaptive immune response in both strains was Th1-biased. However, while the IgG and IgA titres in the lungs were overall similar between Balb/c and C57B1/6 mice, the $\mathrm{C} 57 \mathrm{Bl} / 6$ mice appeared to develop a more rapid systemic response based on higher serum IgG levels and IFN- $\gamma$ secreting splenocytes on day 14 after infection. In addition, the C57B1/6 mice had higher $\mathrm{VN}$ antibody levels in the lung and serum four weeks after infection. 
Figure 7. PVM-specific antibody response in the serum and lungs. Five to six week-old $\mathrm{Balb} / \mathrm{c}$ and $\mathrm{C} 57 \mathrm{Bl} / 6$ mice were inoculated with medium, $30 \mathrm{pfu}(\mathrm{Balb} / \mathrm{c})$ or $300 \mathrm{pfu}$ (C57B1/6) of PVM 15 and sacrificed on days 14, 28, and 42 p.i.. Time course of (A) lung IgA, (B) lung IgG, and (C) serum IgG, represented as the group median with vertical lines indicating the interquartile range. $\mathrm{VN}$ titres on day 28 in lung (D) and serum (E). Each data point represents a single animal and the line represents the group median. ${ }^{*}, p<0.05$; $* *, p<0.01$.
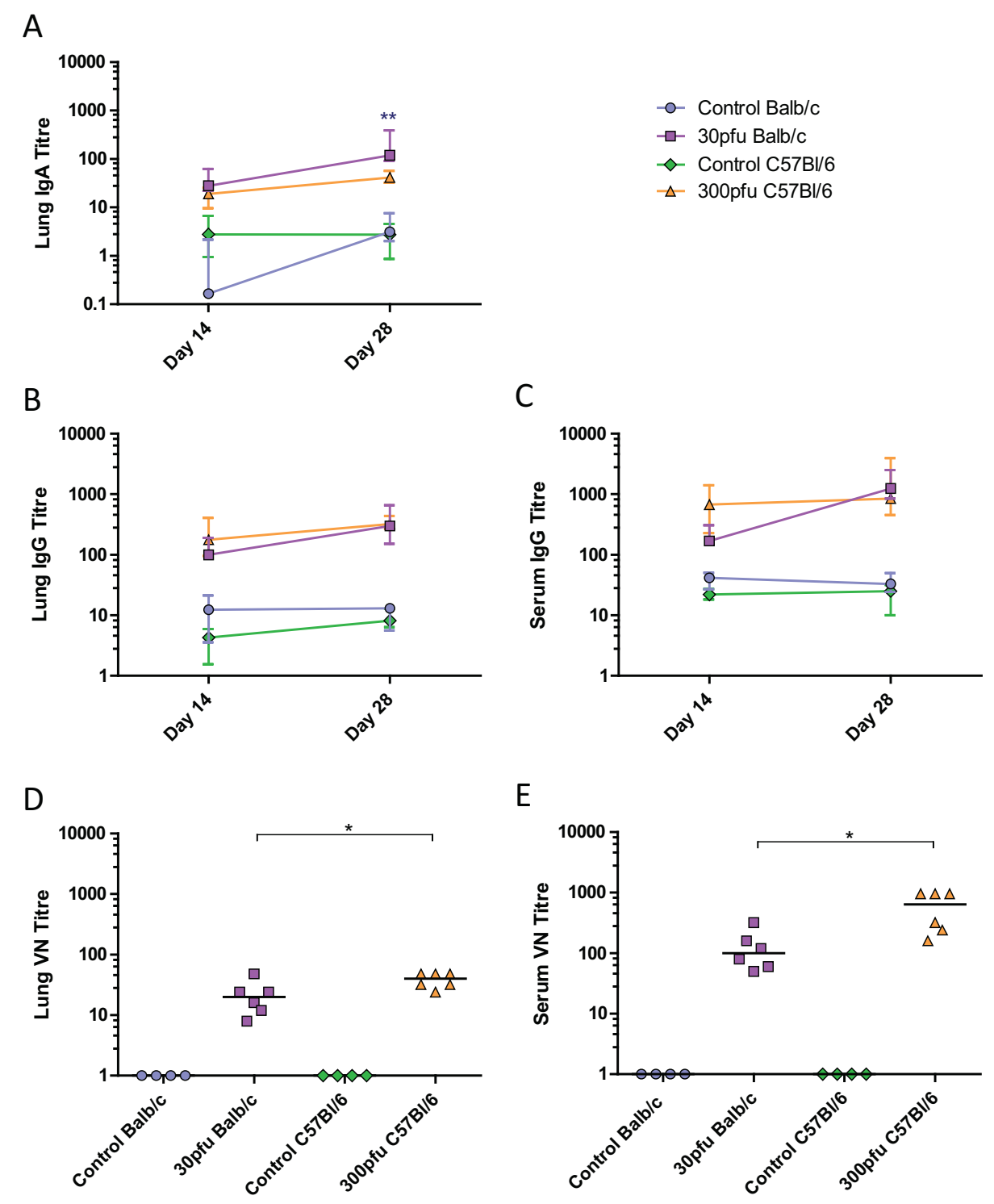
Figure 8. PVM-specific IFN- $\gamma$-secreting (A) and IL-5-secreting (B) splenocytes following in vitro restimulation with PVM-infected cell lysates. Five to six week-old Balb/c and $\mathrm{C} 57 \mathrm{Bl} / 6$ mice were inoculated with medium, $30 \mathrm{pfu}(\mathrm{Balb} / \mathrm{c})$ or $300 \mathrm{pfu}(\mathrm{C} 57 \mathrm{Bl} / 6)$ and sacrificed on days 14, 28, and 42 p.i.. The PVM-specific response is calculated by subtracting the number of cytokine-secreting cells restimulated with mock-infected cell lysates from those restimulated with PVM-infected lysates. The data points represent individual animals, with the median indicated by a line. ${ }^{* *}, p<0.01$.

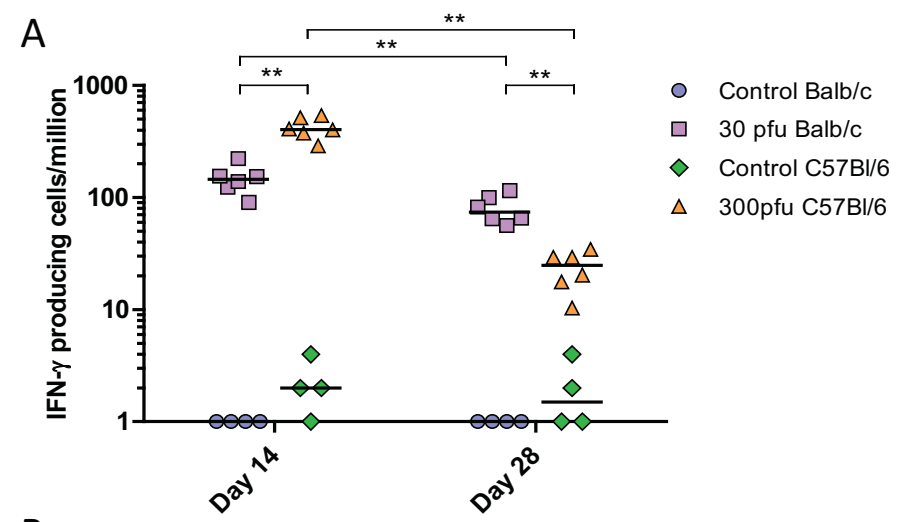

B

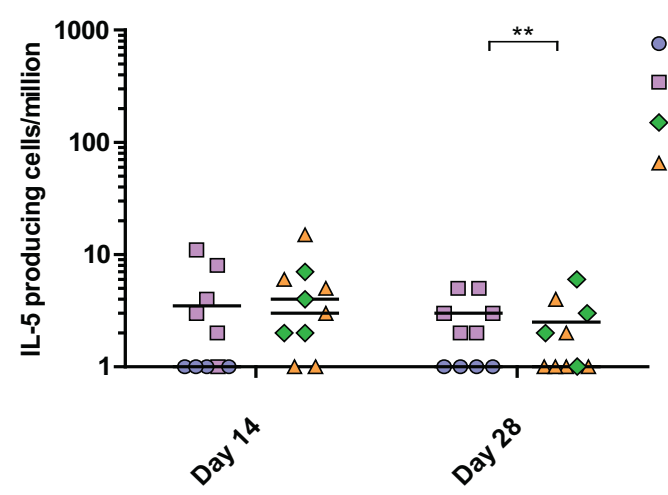

\section{Experimental Section}

\subsection{Cell Lines and Virus}

PVM 15 (American Type Culture Collection (ATCC)) was propagated in Baby Hamster Kidney (BHK)-21 cells (ATCC) in growth medium consisting of Dulbecco's Modified Eagle Medium (DMEM; Sigma) supplemented with $2 \%$ fetal bovine serum (FBS, PAA Laboratories Inc), $0.1 \mathrm{mM}$ non-essential amino acids (Invitrogen), $10 \mathrm{mM}$ HEPES (Invitrogen), and $50 \mu \mathrm{g} / \mathrm{mL}$ gentamicin (Invitrogen). 


\subsection{Challenge of Mice with PVM}

Five to six week-old female Balb/c and C57Bl/6 mice (Charles River Laboratories) were housed in groups of four to six animals and acclimatized for one week. Mice were placed under light anaesthesia and inoculated intranasally with $50 \mu \mathrm{L}$ of medium or with 30,300 , or 3000 pfu of PVM 15 in $50 \mu \mathrm{L}$. Mice were weighed daily and scored for clinical illness according to a modified version of Morton and Griffiths [42]. Sera were collected at regular intervals for detection of IgG. Mice were euthanized by an overdose of isoflurane and bled out by cardiac puncture. Spleens were collected for cytokine enzyme-linked immunospot (ELISPOT) assays. Lungs were collected for detection of cytokines/chemokines, virus, infiltrating cell populations, production of IgG and IgA, or histology. All experiments were performed within the guidelines of the Canadian Council for Animal Care.

\subsection{Lung Samples}

To evaluate virus replication and innate responses, lungs were collected daily from PVM-infected or mock-infected mice. The single-lobed, left lung was clamped off and removed into a $2 \mathrm{~mL}$ screwcap tube (VWR International) containing a $\sim 1 \mathrm{~mL}$ volume of $2.4 \mathrm{~mm}$ zirconia beads (Biospec Products, Inc.) and $1.5 \mathrm{~mL}$ of DMEM supplemented as above, but with $1 \times$ antibiotic/antimycotic (Invitrogen) and without FBS. The multi-lobed, right lung was removed into a tube containing $1 \mathrm{~mL}$ of Trizol $^{\circledR}$ reagent (Invitrogen) and $2.4 \mathrm{~mm}$ zirconia beads. The lungs were homogenized for $10 \mathrm{~s}$ at $4800 \mathrm{rpm}$ using a Mini-beadbeater (Biospec Products, Inc.) and centrifuged at $4{ }^{\circ} \mathrm{C}$ for $1 \mathrm{~min}$ at $10,000 \times \mathrm{g}$ to remove the gross debris. All samples were flash-frozen in liquid nitrogen immediately following centrifugation, and stored at $-80{ }^{\circ} \mathrm{C}$. Prior to removal, the right lung was washed with $500 \mu \mathrm{L}$ of phosphate buffered saline (PBS), pH 7.2 (Invitrogen) supplemented with $2 \%$ FBS and $50 \mu \mathrm{M}$ EDTA, and the fluids from groups of four to six mice were pooled. Cytospin slides were prepared with $5 \times 10^{4}$ and $1 \times 10^{5}$ brochoalveolar lavage (BAL) cells per slide using a Cytospin 4 (Thermo Shandon). The slides were stained with a Giemsa-Wright stain (Bayer HealthCare) using an automated slide stainer, and differential analysis of the cell populations was performed by counting at least 200 cells in a blinded manner.

\subsection{Histology}

For histology the multi-lobed right lung was collected on day 6 p.i. and perfused with $1 \mathrm{~mL}$ of $10 \%$ neutral buffered formalin (VWR) and placed in a cassette, which was then immersed in formalin. The perfused lungs were embedded in paraffin wax and sectioned. Duplicate $5 \mu \mathrm{m}$ sections were stained with hematoxylin and eosin, and scored in a blinded manner by a veterinary pathologist. Scores were given based on the presence and severity, as well as the dissemination of lesions characterized by cellular infiltrates and edema in the tissue surrounding the bronchioles and blood vessels visible in lung sections. A score of 0 denotes a normal lung, 1 indicates signs of perivascular edema and mild perivasculitis, limited to small foci that were not widespread, while a score of 2 denotes moderate levels of perivasculitis, vasculitis, and multifocal perivascular edema. Finally, a 
score of 3 represents animals with extensive, severe, multifocal perivasculitis, vasculitis, and vascular edema, and/or necrotic and fibrinous areas.

\subsection{Lung Fragment Cultures}

To measure local adaptive responses the multilobed lungs were collected and placed into a tube containing $5 \mathrm{~mL}$ of RPMI 1640 medium (Invitrogen) supplemented with $0.1 \mathrm{mM}$ non-essential amino acids, $10 \mathrm{mM}$ HEPES buffer, $1 \mathrm{mM}$ sodium pyruvate (Invitrogen), $2 \mathrm{mM}$ L-glutamine (Invitrogen), $1 \times$ antibiotic/antimycotic (Invitrogen), $50 \mu \mathrm{g} / \mathrm{mL}$ gentamicin (Invitrogen), and 10\% FBS (lung fragment culture (LFC) medium). The lungs were cut into four roughly equal-sized pieces, placed into four wells of a 48-well plate (Corning Inc.) containing LFC medium, and minced into pieces $\sim 1 \mathrm{~mm}$ in diameter. The lung fragments were cultured for 5 days at $37^{\circ} \mathrm{C}$, at which point the culture medium was collected (BD Biosciences) and centrifuged at $4{ }^{\circ} \mathrm{C}$ and $311 \times \mathrm{g}$, for $10 \mathrm{~min}$. The supernatants were stored at $-80{ }^{\circ} \mathrm{C}$.

\subsection{Lung Cell Isolation and Flow Cytometry}

The lungs were gently perfused via the right ventricle with $5 \mathrm{~mL}$ of Hanks balanced salt solution (HBSS) (Gibco) containing $0.5 \mathrm{mM}$ EDTA. Lungs were then mechanically disrupted using a gentle MACS dissociator (Miltenyi Biotec) according to the manufacturer's instructions and incubated with collagenase type IA $(0.5 \mathrm{mg} / \mathrm{mL}$; Sigma-Aldrich) and type IV bovine pancreatic DNase $(20 \mu \mathrm{g} / \mathrm{mL}$; Sigma-Aldrich) for $30 \mathrm{~min}$ at $37{ }^{\circ} \mathrm{C}$ in HBSS containing 5\% FBS. After digestion, lung cells were filtered through a $100 \mu \mathrm{m}$ nylon screen cell strainer. Single cell suspensions were washed, contaminating erythrocytes were lysed using ACK lysis buffer (Invitrogen), and viable cells were counted by trypan blue exclusion. To detect intracellular IFN- $\gamma$ production in lung cells, cells were incubated for $6-8 \mathrm{~h}$ at $37{ }^{\circ} \mathrm{C}$ in complete RPMI containing $1 \mu \mathrm{L}$ Golgi plug per mL. Subsequently, the cells were stained with the following antibodies from BD Biosciences: anti-CD8a FITC (cat no.553031), anti-CD3 PE (cat no.555275), anti-CD4 FITC (cat no.553729), anti-CD335(NKp46) PerCP-Cy ${ }^{\mathrm{TM}} 5.5$ (cat no.560800) at $4{ }^{\circ} \mathrm{C}$ for $30 \mathrm{~min}$, followed by permeabilization and fixation with Cytofix/Cytoperm ${ }^{\mathrm{TM}}$ Plus (BD Biosciences) at $4{ }^{\circ} \mathrm{C}$ for $20-30 \mathrm{~min}$. Finally, cells were stained with anti-IFN- $\gamma$-APC (cat no.554413) from BD Biosciences at $4{ }^{\circ} \mathrm{C}$ for $30 \mathrm{~min}$. Flow cytometry was performed using a FACS Calibur (BD Biosciences). Analysis of flow cytometry data was performed using Kaluza software.

\subsection{PVM Quantification}

PVM was quantified using a standard immunofluorescent plaque assay. Ten-fold serial dilutions of mouse lung homogenates were made in sterile 96-well dilution plates (Nalgen Nunc International) and $100 \mu \mathrm{L}$ was transferred in duplicate onto $80 \%$ confluent BHK-21 cell monolayers. After $72 \mathrm{~h}$ incubation at $37{ }^{\circ} \mathrm{C}$ and $5 \% \mathrm{CO}_{2}$ the cells were fixed with an ice-cold solution of 4 parts acetone to 1 part methanol, and dried at RT. The staining procedure was carried out at RT, and the plates were 
washed three times before blocking, and between each incubation in PBS. Plates were blocked for $30 \mathrm{~min}$ in PBS containing 5\% goat serum (Invitrogen), followed by a 2-3 h incubation with a 1:500 dilution of a PVM nucleoprotein ( $\mathrm{N}$ protein)-specific polyclonal rabbit antibody in PBS (made in-house) containing $1 \%$ goat serum, and a $1 \mathrm{~h}$ incubation in darkness with an Alexafluor488-congujated goat-anti-rabbit antibody (Invitrogen). The N-specific antibody was raised by immunization of rabbits with alternately KLH- and BSA-conjugated peptide (VVAKELKTGARLPDNQRHTAPDCGV) as described previously [43], and the specificity was confirmed by Western blotting (Supplementary Figure 1). Plaques were visualized ad counted using a fluorescent microscope (Zeiss).

\subsection{Analysis of Chemokine and Cytokine mRNA Expression}

Following RNA isolation according to the manufacturer's instructions (Invitrogen) and cDNA synthesis using a QuantiTect Reverse Transcription Kit (Qiagen), semi-quantitative real-time PCR (qPCR) was carried out using Platinum SYBR Green qPCR Supermix-UDG (Invitrogen) as per manufacturer's instructions. The primers and annealing temperatures used in the qPCR experiments are listed in Table 1. Some primers were designed in house using NCBI PrimerBlast (http://www.ncbi.nlm.nih.gov/tools/primer-blast/) and primer design software (CloneManager Version 9.0). The qPCR reaction was carried out according to the following parameters: 40 cycles of denaturation at $95^{\circ} \mathrm{C}$ for $30 \mathrm{~s}$, followed by $30 \mathrm{~s}$ annealing and extension. Expression levels of cytokine and chemokine transcripts were calculated using the Bio-Rad analysis software (Bio-Rad CFX Manager Version 2.0), normalized against both $\beta$-actin and GAPDH, and expressed as the normalized fold-change over mock-infected control animals euthanized on the same day.

\subsection{PVM-Specific ELISA}

Immulon II plates (Thermo Electron) were coated with PVM-infected and mock-infected cell lysates. Plates were washed, and blocked with 5\% gelatin in PBS (Sigma-Aldrich). Four-fold serial dilutions of serum or LFC supernatant were prepared in 96-well non-sterile dilution plates (Nalgen Nunc International), starting at a 1:40 dilution for serum and 1:10 for LFC supernatants. The diluted sera and LFC supernatants were added to the coated ELISA plates and incubated overnight at $4{ }^{\circ} \mathrm{C}$. The plates were washed and either 1:5000 diluted AP-conjugated IgG (Kirkegaard \& Perry Laboratories) or 1:2000 diluted biotinylated-anti-mouse IgA (Invitrogen) was added to the plates and allowed to react for 1-2 $\mathrm{h}$. For the IgA ELISA, an additional $1 \mathrm{~h}$ incubation with a 1:10,000 dilution of AP-conjugated streptavidin (Jackson ImmunoResearch Laboratories) was carried out at RT. All washes were performed with PBS with $0.05 \%$ Tween 20 (Sigma-Aldrich) (PBST) and $\mathrm{ddH}_{2} \mathrm{O}$. The plates were developed with p-nitrophenyl phosphate (Sigma-Aldrich) and the absorbances read at $405 \mathrm{~nm}$ with a reference wavelength of $490 \mathrm{~nm}$. Titres were calculated based on the dilution at which the ELISA read-out for the PVM-positive antigen was equal to the highest reading from the same animal's serum tested with the PVM-negative antigen. 
Table 1. Cytokine and Chemokine Primers and their Optimal Annealing Temperatures.

\begin{tabular}{|c|c|c|c|c|}
\hline $\begin{array}{l}\text { Target } \\
\text { gene }\end{array}$ & Direction & Sequence & Source & $\begin{array}{l}\text { Annealing } \\
\text { Temp }\left({ }^{\circ} \mathrm{C}\right)\end{array}$ \\
\hline \multirow{2}{*}{$\beta$-actin } & Forward & ACTGGGACGACATGGAG & \multirow{2}{*}{ [44] } & \multirow{2}{*}{57.5} \\
\hline & Reverse & GTAGATGGGCACAGTGTGGG & & \\
\hline \multirow{2}{*}{ GAPDH } & Forward & AACTTTGGCATTGTGGAAGG & \multirow{2}{*}{ [45] } & \multirow{2}{*}{57.5} \\
\hline & Reverse & ACACATTGGGGGTAGGAACA & & \\
\hline \multirow{2}{*}{$\begin{array}{l}\text { CCL3/ } \\
\text { MIP-1 } \alpha\end{array}$} & Forward & CTTCTCTGTACCATGACACTC & \multirow{2}{*}{ [44] } & \multirow{2}{*}{57.5} \\
\hline & Reverse & AGGTCTCTTTGGAGTCAGCG & & \\
\hline \multirow{2}{*}{$\begin{array}{l}\text { CXCL8/ } \\
\text { MIP-2 }\end{array}$} & Forward & TGCGCCCAGACAGAAGTCATAGC & \multirow{2}{*}{$\begin{array}{l}\text { designed } \\
\text { in house }\end{array}$} & \multirow{2}{*}{63.9} \\
\hline & Reverse & GCTCTAGAGTCAGTTAGCCTTGCCTTTG & & \\
\hline \multirow{2}{*}{$\begin{array}{l}\text { CCL2/ } \\
\text { MCP-1 }\end{array}$} & Forward & CTTCTGGGCCTGCTGTTCA & \multirow{2}{*}[46]{} & \multirow{2}{*}{57.5} \\
\hline & Reverse & CCAGCCTACTCATTGGGATCA & & \\
\hline \multirow{2}{*}{ IFN- $\gamma$} & Forward & TCAAGTGGCATAGATGTGGAAGAA & \multirow{2}{*}{ [46] } & \multirow{2}{*}{57.5} \\
\hline & Reverse & TGGCTCTGCAGGATTTTCATG & & \\
\hline \multirow{2}{*}{ IL-4 } & Forward & GGAGATGGATGTGCCAAACG & \multirow{2}{*}{$\begin{array}{l}\text { designed } \\
\text { in house }\end{array}$} & \multirow{2}{*}{63.9} \\
\hline & Reverse & ACCTTGGAAGCCCTACAGAC & & \\
\hline \multirow{2}{*}{ IFN- $\alpha$} & Forward & CCTGTGTGATGCAACAGGTC & \multirow{2}{*}{ [47] } & \multirow{2}{*}{59.3} \\
\hline & Reverse & TCACTCCTCCTTGCTCAATC & & \\
\hline \multirow{2}{*}{ IFN- $\beta$} & Forward & ATCATGAACAACAGGTGGATCCTCC & \multirow{2}{*}{ [47] } & \multirow{2}{*}{63.9} \\
\hline & Reverse & TTCAAGTGGAGAGCAGTTGAG & & \\
\hline \multirow{2}{*}{ TNF- $\alpha$} & Forward & GAACTGGCAGAAGAGGCACT & \multirow{2}{*}{ [47] } & \multirow{2}{*}{68.9} \\
\hline & Reverse & AGGGTCTGGGCCATAGAACT & & \\
\hline \multirow{2}{*}{$\begin{array}{l}\text { CXCL10/ } \\
\text { IP-10 }\end{array}$} & Forward & GAGATCATTGCCACGATGAA & \multirow{2}{*}{$\begin{array}{l}\text { designed } \\
\text { in house }\end{array}$} & \multirow{2}{*}{63.9} \\
\hline & Reverse & CACTGGGTAAAGGGGAGTGA & & \\
\hline CCL5/ & Forward & CTCACTGCAGCCGCCCTCTG & designed & 7 \\
\hline RANTES & Reverse & CCTTGACGTGGGCACGAGGC & in house & 57.5 \\
\hline CCL11/ & Forward & AGAGGCTGAGATCCAAGCAG & designed & 639 \\
\hline Eotaxin & Reverse & CAGATCTCTTTGCCCAACCT & in house & 63.9 \\
\hline
\end{tabular}

\subsection{Virus Neutralization Assay}

Sera and LFC supernatants were serially diluted in in sterile flat-bottom 96-well tissue culture plates (Nalgen Nunc International) starting at 1:10 and 1:2, respectively, and continuing in 2-fold dilutions down the plate. An equal volume of PVM 15 at a concentration of $500 \mathrm{pfu} / \mathrm{well}$ was added to the dilution plate, such that the resulting dilution of the serum or supernatant was 1:20 and 1:4, respectively. After $1 \mathrm{~h}$ incubation at $37{ }^{\circ} \mathrm{C}$ and $5 \% \mathrm{CO}_{2}$, the virus mixture was transferred in duplicate onto BHK-21 cell monolayers grown to $80 \%$ confluency in flat-bottom 96 -well tissue culture plates. Following a further $72 \mathrm{~h}$ incubation at $37{ }^{\circ} \mathrm{C}$ and $5 \% \mathrm{CO}_{2}$, the cells were fixed and stained according to the PVM plaque assay protocol. 


\subsection{IFN- $\gamma$ and IL-5 ELISPOT Assays}

Splenocytes were isolated as previously described [48]. Multiscreen-HA ELISPOT plates (Millipore) were coated with murine IFN- $\gamma$ - or IL-5-specific monoclonal antibodies (BD PharMingen) at $2 \mu \mathrm{g} / \mathrm{mL}$ in sterile coating buffer. After overnight incubation at $4{ }^{\circ} \mathrm{C}$, the plates were washed four times with sterile PBS, pH 7.2 (Invitrogen) and blocked for $1 \mathrm{~h}$ with $1 \%$ BSA (Sigma-Aldrich) at $37{ }^{\circ} \mathrm{C}$. Splenocytes were restimulated with medium alone, mock- or PVM-infected cell lysates at $25 \mu \mathrm{g} / \mathrm{mL}$ or Concanavalin A (Con A). Each treatment was assayed in triplicate for each animal. After $\sim 40 \mathrm{~h}$ of incubation at $37{ }^{\circ} \mathrm{C}$ in a $5 \% \mathrm{CO}_{2}$ incubator, the medium was removed and the cells were lysed in $\mathrm{ddH}_{2} \mathrm{O}$. Plates were washed three times in PBST and twice with $\mathrm{ddH}_{2} \mathrm{O}$, and then biotinylated anti-mouse IFN- $\gamma$ or IL-5 monoclonal antibodies (BD Biosciences) were added to the wells at a concentration of $2 \mu \mathrm{g} / \mathrm{mL}$ in PBS with $1 \%$ BSA. After $1-2 \mathrm{~h}$ incubation at RT, the plates were washed, and alkaline phosphatase (AP)-conjugated streptavidin was added at a dilution of 1:1000 in PBS with $1 \%$ BSA (Sigma-Aldrich) for 1-2 h at RT. After a final wash, cytokine-secreting cells were visualized by the addition of 5-bromo-4-chloro-3-indolylphosphate and nitroblue substrate (Sigma-Aldrich). Plates were washed in $\mathrm{ddH}_{2} \mathrm{O}$ and spots were counted under an inverted microscope (Olympus SZ71). The results were expressed as the number of cytokine-secreting cells per million splenocytes stimulated with mock-infected cell lysates subtracted from the number of cytokine-secreting cells stimulated with PVM-infected cell lysates.

\subsection{Statistical Analysis}

Statistical software (GraphPad Prism Version 5.00) was used to analyze all data. Since there were few animals and the variability in biological systems makes normal distribution unlikely, the data were analyzed using non-parametric tests. The Kruskall-Wallis test was used to determine whether there was a difference between all groups. If the test indicated significant differences between the groups, Mann-Whitney $U$ tests were used to compare the median of individual groups. Differences were considered significant if $p<0.05$.

\section{Conclusions}

The goal of this study was to compare the immune response of Balb/c and C57Bl/6 mice to PVM 15 in order to understand the relative susceptibility of these strains to infection. To our knowledge, this is the first direct comparison of the pathogenesis of PVM 15 in Balb/c and C57B1/6 mice. Based on our results, it appears that a suppression or delay in the innate immune response, as well as decreased neutrophil and NK cell infiltration, in the $\mathrm{C} 57 \mathrm{Bl} / 6$ strain could be a major factor in protecting these mice from severe illness. Furthermore, while both mouse strains developed a Th1-biased response to PVM infection, the VN antibody levels were higher in $\mathrm{C} 57 \mathrm{Bl} / 6$ mice than in $\mathrm{Balb} / \mathrm{c}$ mice, which correlated to an earlier increase in PVM-induced IFN- $\gamma$ secreting cells. Surprisingly, neither strain developed eosinophilia following PVM 15 infection. During infection with PVM J3666, the inflammatory cell recruitment is of typically eosinophilic nature with early and prominent recruitment 
of eosinophils. Amongst the mouse strains documented by Anh and colleagues in their study all, except SJL, showed eosinophilic infiltration [12]. Furthermore, PVM J3666 infects murine eosinophils and elicits cytokine production further supporting the involvement of eosinophils in PVM J3666 pathogenesis [49]. In this respect strain PVM strain 15 differs from the J366 strain at least in Balb/c and C57Bl/6 mice, although it is not known whether PVM 15 infects eosinophils.

These results suggest that in contrast to J3666, the PVM 15 strain has a distinct mode of pathogenesis in these two mouse strains. Balb/c mice responded to PVM 15 infection with earlier and higher expression of cytokines and chemokines, and earlier and enhanced lung inflammation dominated by neutrophils. These mice also showed earlier and higher levels of viral replication compared to $\mathrm{C} 57 \mathrm{~B} 1 / 6$ mice given the same dose. Surprisingly, the IFN- $\alpha$ response was stronger in $\mathrm{Balb} / \mathrm{c}$ mice than in $\mathrm{C} 47 \mathrm{Bl} / 6$ mice. Although IFN- $\alpha$ usually suppresses viral replication, this was not the case in the PVM-infected Balb/c mice. This could be related to the fact that PVM, like RSV, suppresses the type-I IFN response [50], which might have been more efficient during production of high levels of de novo synthesized PVM proteins during viral replication. Alternatively, viral entry and replication of PVM in epithelial cells may be more efficient in Balb/c mice. The lungs can be partially protected by the immunomodulatory effects of surfactant proteins (SP) secreted by type II pneumocytes in the lower airways. The basal levels of surfactant A and D proteins are different in the lungs of naive Balb/c and $\mathrm{C} 57 \mathrm{~B} 1 / 6$ mice, and are differentially upregulated upon sensitization with antigens [51], and as such may play a role in the different susceptibility of these two strains to PVM. Furthermore, naïve Balb/c mice have higher TLR4 mRNA expression in the lungs than C57B1/6 mice, which may indicate a difference in TLR surface expression on resident macrophages or the respiratory epithelium [52] facilitating PVM entry. If Balb/c macrophages have higher levels of surface TLR4, this could trigger a stronger and earlier immune response to PVM infection than in C57B1/6 mice. In a recent study by Glineur et al. on the potential mechanisms of the PVM resistance of SJL/J mice a role for innate, but not adaptive, immune responses was proposed, which would agree with our observations for PVM 15 in Balb/c and C57B1/6 mice. They also concluded that PVM resistance of $\mathrm{SJL} / \mathrm{J}$ mice is polygenic, that the resistance genes are recessive, and that radioresistant lung epithelial cells and macrophages may control the severity of lung disease caused by PVM [53]. A study of the contribution of epithelial cells and macrophages may provide further information on the pathogenesis of PVM 15 in Balb/c and C57B1/6 strains.

The Balb/c strain had a slower but ultimately stronger cell-mediated response to PVM, along with enhanced production of mucosal IgA by day 28; however, the antibody response was functionally less effective than that in the C57Bl/6 strain. Since RSV can cause a spectrum of disease depending on the age and immune history of the child in question, both mouse strains may be useful in examining specific aspects of pneumovirus pathogenesis. In terms of comparing their usefulness as a model of $\mathrm{RSV}$, the next critical step will be to determine the protective efficacy of these responses upon reinfection. The $\mathrm{C} 57 \mathrm{Bl} / 6$ strain infected with $300 \mathrm{pfu}$ showed a rapid decline in activated peripheral $\mathrm{T}$ cells similar to that reported in infants monitored at the height of severe infection and four weeks later. Since humoral responses in children are also known to decline following infection with RSV, 
an analysis of the memory response to PVM in these two strains of mice will provide further insight into their use as a model of RSV.

\section{Acknowledgments}

The authors would like to thank Laura Latimer, Marlene Snider and Sheryl Maher for technical assistance, as well as Barry Carroll, Sherry Tetland and Jan Erickson for the handling and care of the animals. This work was supported by grant MOP 42436 from the Canadian Institutes of Health Research (CIHR). Published as VIDO manuscript number 657.

\section{Conflicts of Interest}

The authors declare no conflict of interest.

\section{References}

1. Bonville, C.A.; Bennett, N.J.; Koehnlein, M.; Haines, D.M.; Ellis, J.A.; DelVecchio, A.M; Rosenberg, H.F.; Domachowske, J.B. Respiratory dysfunction and proinflammatory chemokines in the pneumonia virus of mice (PVM) model of viral bronchiolitis. Virology 2006, 349, 87-95.

2. Rosenberg, H.F.; Bonville, C.A.; Easton, A.J.; Domachowske, J.B. The pneumonia virus of mice infection model for severe respiratory syncytial virus infection: Identifying novel targets for therapeutic intervention. Pharmacol. Ther. 2005, 105, 1-6.

3. El-Hajje, M.J.; Lambe, C.; Moulin, F.; Suremain, N.; Pons-Catalano, C.; Chalumeau, M.; Raymond, J.; Lebon, P.; Gendrel, D. The burden of respiratory viral disease in hospitalized children in Paris. Eur. J. Pediatr. 2008, 167, 435-436.

4. Deshpande, S.A.; Northern, V. The clinical and health economic burden of respiratory syncytial virus disease among children under 2 years of age in a defined geographical area. Arch. Dis. Child. 2003, 88, 1065-1069.

5. Iwane, M.K.; Edwards, K.M.; Szilagyi, P.G.; Walker, F.J.; Griffin, M.R.; Weinberg, G.A.; Coulen, C.; Poehling, K.A.; Shone, L.P.; Balter, S.; et al. Population-based surveillance for hospitalizations associated with respiratory syncytial virus, influenza virus, and parainfluenza viruses among young children. Pediatrics 2004, 113, 1758-1764.

6. Glezen, W.P.; Taber, L.H.; Frank, A.L.; Kasel, J.A. Risk of primary infection and reinfection with respiratory syncytial virus. Am. J. Dis. Child. 1986,140, 543-546.

7. Hall, C.B. Respiratory syncytial virus: Its transmission in the hospital environment. Yale $J$. Biol. Med. 1982, 55, 219-223.

8. Welliver, R.C. Review of epidemiology and clinical risk factors for severe respiratory syncytial virus (RSV) infection. J. Pediatr. 2003, 143, 112-117.

9. Openshaw, P.J.; Tregoning, J.S. Immune responses and disease enhancement during respiratory syncytial virus infection. Clin. Microbiol. Rev. 2005, 18, 541-555. 
10. Byrd, L.G.; Prince, G.A. Animal models of respiratory syncytial virus infection. Clin. Infect. Dis. 1997, 25, 1363-1368.

11. Easton, A.J.; Domachowske, J.B.; Rosenberg, H.F. Animal pneumoviruses: Molecular genetics and pathogenesis. Clin. Microbiol. Rev 2004, 17, 390-412.

12. Anh, D.B.; Faisca, P.; Desmecht, D.J. Differential resistance/susceptibility patterns to pneumovirus infection among inbred mouse strains. Am. J. Physiol. Lung Cell. Mol. Physiol. 2006, 291, L426-435.

13. Rosenberg, H.F.; Domachowske, J.B. Pneumonia virus of mice: Severe respiratory infection in a natural host. Immunol. Lett. 2008, 118, 6-12.

14. Krempl, C.D.; Lamirande, E.W.; Collins, P.L. Complete sequence of the RNA genome of pneumonia virus of mice (PVM). Virus Gene. 2005, 30, 237-249.

15. Domachowske, J.B.; Bonville, C.A.; Easton, A.J.; Rosenberg, H.F. Differential expression of proinflammatory cytokine genes in vivo in response to pathogenic and nonpathogenic pneumovirus infections. J. Infect. Dis. 2002, 186, 8-14.

16. Krempl, C.D.; Collins, P.L. Reevaluation of the virulence of prototypic strain 15 of pneumonia virus of mice. J. Virol. 2004, 78, 13362-13365.

17. Horsfall, F.L.; Hahn, R.G. A latent virus in normal mice capable of producing pneumonia in its natural host. J. Exp. Med. 1940, 71, 391-408.

18. Kuroda, E.; Kito, T.; Yamashita, U. Reduced expression of STAT4 and IFN-gamma in macrophages from BALB/c mice. J. Immunol. 2002, 168, 5477-5482.

19. Launois, P.; Swihart, K.G.; Milon, G.; Louis, J.A. Early production of IL-4 in susceptible mice infected with Leishmania major rapidly induces IL-12 unresponsiveness. J. Immunol. 1997, 158, 3317-3324.

20. Liu, T.; Matsuguchi, T.; Tsuboi, N.; Yajima, T.; Yoshikai, Y. Differences in expression of toll-like receptors and their reactivities in dendritic cells in BALB/c and $\mathrm{C} 57 \mathrm{BL} / 6$ mice. Infect. Immun. 2002, 70, 6638-6645.

21. Scharton-Kersten, T.; Afonso, L.C.; Wysocka, M.; Trinchieri, G.; Scott, P. IL-12 is required for natural killer cell activation and subsequent $\mathrm{T}$ helper 1 cell development in experimental leishmaniasis. J. Immunol. 1995, 154, 5320-5330.

22. Srikiatkhachorn, A.; Chang, W.; Braciale, T.J. Induction of Th-1 and Th-2 responses by respiratory syncytial virus attachment glycoprotein is epitope and major histocompatibility complex independent. J. Virol. 1999, 73, 6590-6597.

23. Srikiatkhachorn, A.; Braciale, T.J. Virus-specific CD8+ T lymphocytes downregulate T helper cell type 2 cytokine secretion and pulmonary eosinophilia during experimental murine respiratory syncytial virus infection. J. Exp. Med. 1997, 186, 421-432.

24. Garvey, T.L.; Dyer, K.D.; Ellis, J.A.; Bonville, C.A.; Foster, B.; Prussin, C.; Easton, A.J.; Domachowske, J.B.; Rosenberg, H.F. Inflammatory responses to pneumovirus infection in IFN-alpha beta R gene-deleted mice. J. Immunol. 2005, 175, 4735-4744. 
25. Bonville, C.A.; Lau, V.K.; DeLeon, J.M.; Gao, J.L.; Easton, A.J.; Rosenberg, H.F.; Domachowske, J.B. Functional antagonism of chemokine receptor CCR1 reduces mortality in acute pneumovirus infection in vivo. J. Virol. 2004, 78, 7984-7089.

26. Domachowske, J.B.; Bonville, C.A.; Gao, J.L.; Murphy, P.M.; Easton, A.J.; Rosenberg, H.F. The chemokine macrophage-inflammatory protein-1 alpha and its receptor CCR1 control pulmonary inflammation and antiviral host defense in paramyxovirus infection. J. Immunol. 2000, 165, 2677-2682.

27. Bonville, C.A.; Easton, A.J.; Rosenberg, H.F.; Domachowske, J.B. Altered pathogenesis of severe pneumovirus infection in response to combined antiviral and specific immunomodulatory agents. J. Virol. 2003, 77, 1237-1244.

28. Bonville, C.A.; Bennett, N.J.; Percopo, C.M.; Branigan, P.J.; Del Vecchio, A.M.; Rosenberg, H.F.; Domachowske, J.B. Diminished inflammatory responses to natural pneumovirus infection among older mice. Virology 2007, 368, 182-190.

29. Domachowske, J.B.; Bonville, C.A.; Dyer, K.D.; Easton, A.J.; Rosenberg, H.F. Pulmonary Eosinophilia and Production of MIP-1[alpha] Are Prominent Responses to Infection with Pneumonia Virus of Mice. Cellular Immunol. 2000, 200, 98-104.

30. Rigaux, P.; Killoran, K.E.; Qiu, Z.; Rosenberg, H.F. Depletion of alveolar macrophages prolongs survival in response to acute pneumovirus infection. Virology 2012, 422, 338-345.

31. Noah, T.L.; Becker, S. Chemokines in nasal secretions of normal adults experimentally infected with respiratory syncytial virus. Clin. Immunol. 2000, 97, 43-9.

32. Sheeran, P.; Jafri, H.; Carubelli, C.; Saavedra, J.; Johnson, C.; Krisher, K.; Sánchez, P.J.; Ramilo, O. Elevated cytokine concentrations in the nasopharyngeal and tracheal secretions of children with respiratory syncytial virus disease. Pediatr. Infect. Dis. J. 1999, 18, 115-122.

33. Abu-Harb, M.; Bell, F.; Finn, A.; Rao, W.H.; Nixon, L.; Shale, D.; Everard, M.L. IL-8 and neutrophil elastase levels in the respiratory tract of infants with RSV bronchiolitis. Eur. Resp. J. 1999, 14, 139-143.

34. Bont, L.; Heijnen, C.J.; Kavelaars, A.; van Aalderen, W.M.; Brus, F.; Draaisma, J.T.; Geelen, S.M.; van Vught, H.J. Peripheral blood cytokine responses and disease severity in respiratory syncytial virus bronchiolitis. Eur. Respir. J. 1999, 14, 144-149.

35. Bonville, C.A.; Percopo, C.M.; Dyer, K.D.; Gao, J.; Prussin, C.; Foster, B.; Rosenberg, H.F.; Domachowske, J.B. Interferon-gamma coordinates CCL3-mediated neutrophil recruitment in vivo. BMC Immunol. 2009, 10, 14.

36. Claassen, E.A.W.; van der Kant, P.A.A.; Rychnavska, Z.S.; van Bleek, G.M.; Easton, A.J.; van der Most, R.G. Activation and Inactivation of Antiviral CD8 T Cell Responses during Murine Pneumovirus Infection. J. Immunol. 2005, 175, 6597-604.

37. Biron, C.A.; Nguyen, K.B.; Pien, G.C.; Cousens, L.P.; Salazar-Mather, T.P. Natural killer cells in antiviral defense: Function and regulation by innate cytokines. Annu. Rev. Immunol. 1999, 17, 189-220. 
38. Campbell, J.J.; Qin, S.; Unutmaz, D.; Soler, D.; Murphy, K.E.; Hodge, M.R; Wu, L.; Butcher, EC. Unique subpopulations of CD56+ NK and NK-T peripheral blood lymphocytes identified by chemokine receptor expression repertoire. J. Immunol. 2001, 166, 6477-6482.

39. Moretta, A. Natural killer cells and dendritic cells: Rendezvous in abused tissues. Nat. Rev. Immunol. 2002, 2, 957-964.

40. Li, F.; Zhu, H.; Sun, R.; Wei, H.; Tian, Z. Natural killer cells are involved in acute lung immune injury caused by respiratory syncytial virus infection. J. Virol. 2012, 86, 2251-2258.

41. Van Helden, M.J.; van Kooten, P.J.; Bekker, C.P; Grone, A.; Topham, D.J.; Easton, A.J.; Boog, C.J.; Busch, D.H.; Zaiss, D.M.; Sijts, A.J. Pre-existing virus-specific CD8(+) T-cells provide protection against pneumovirus-induced disease in mice. Vaccine 2012, 30, 6382-6388.

42. Morton, D.B.; Griffiths, P.H. Guidelines on the recognition of pain, distress and discomfort in experimental animals and an hypothesis for assessment. Vet. Rec. 1985, 116, 431-436.

43. Labiuk, S.L.; Babiuk, L.A.; van Drunen Littel-van den Hurk, S. Major tegument protein VP8 of bovine herpesvirus 1 is phosphorylated by viral US3 and cellular CK2 protein kinases. J. Gen. Virol. 2009, 90, 2829-2839.

44. Park, J.K.; Cho, K.; Johnson, J.; Perez, R.V. Induction of MIP-1alpha in Kupffer cell by portal venous transfusion. Transplant. Immunol. 2004, 13, 33-38.

45. Vogel, C.F.; Nishimura, N.; Sciullo, E.; Wong, P.; Li, W.; Matsumura, F. Modulation of the chemokines $\mathrm{KC}$ and MCP-1 by 2,3,7,8-tetrachlorodibenzo-p-dioxin (TCDD) in mice. Arch. Biochem. Biophys.2007, 461, 169-175.

46. Overbergh, L.; Giulietti, A.; Valckx, D.; Decallonne, R.; Bouillon, R.; Mathieu, C. The use of real-time reverse transcriptase PCR for the quantification of cytokine gene expression. J. Biomol. Tech. 2003, 14, 33-43.

47. Pascual, M.; Fernandez-Lizarbe, S.; Guerri, C. Role of TLR4 in ethanol effects on innate and adaptive immune responses in peritoneal macrophages. Immunol. Cell Biol. 2011, 89, 716-727.

48. Mapletoft, J.W.; Latimer, L.; Babiuk, L.A.; van Drunen Littel-van den Hurk, S. Intranasal immunization of mice with a bovine respiratory syncytial virus vaccine induces superior immunity and protection compared to those by subcutaneous delivery or combinations of intranasal and subcutaneous prime-boost strategies. Clin. Vaccine Immunol. 2010, 17, 23-35.

49. Dyer, K.D.; Percopo, C.M.; Fischer, E.R.; Gabryszewski, S.J.; Rosenberg, H.F. Pneumoviruses infect eosinophils and elicit MyD88-dependent release of chemoattractant cytokines and interleukin-6. Blood 2009, 114, 2649-2656.

50. Heinze, B.; Frey, S.; Mordstein, M.; Schmitt-Graff, A.; Ehl, S.; Buchholz, U.J.; Collins, P.L.; Staeheli, P.; Krempl, C.D. Both nonstructural proteins NS1 and NS2 of pneumonia virus of mice are inhibitors of the interferon type I and type III responses in vivo. J. Virol. 2011, 85, 4071-4084.

51. Atochina, E.N.; Beers, M.F.; Tomer, Y.; Scanlon, S.T.; Russo, S.J.; Panettieri, R.A., Jr.; Haczku, A. Attenuated allergic airway hyperresponsiveness in C57BL/6 mice is associated with enhanced surfactant protein (SP)-D production following allergic sensitization. Respir. Res. 2003, 4,15 . 
52. Rodriguez-Martinez, S.; Cancino-Diaz, M.E.; Jimenez-Zamudio, L.; Garcia-Latorre, E.; Cancino-Diaz, J.C. TLRs and NODs mRNA expression pattern in healthy mouse eye. $\mathrm{Br} . \mathrm{J}$. Ophthalmol. 2005, 89, 904-910.

53. Glineur, S.; Tran Anh, D.B.; Sarlet, M.; Michaux, C.; Desmecht, D. Characterization of the resistance of SJL/J mice to pneumonia virus of mice, a model for infantile bronchiolitis due to a respiratory syncytial virus. PLoS One 2012, 7, e44581. 
Reprinted from Viruses. Cite as: van den Berg, E.; van Woensel, J.B.; Bem, R.A. Apoptosis in Pneumovirus Infection. Viruses 2013, 5, 406-422.

Review

\title{
Apoptosis in Pneumovirus Infection
}

\section{Elske van den Berg, Job B.M. van Woensel and Reinout A. Bem *}

Pediatric Intensive Care Unit, Emma Children's Hospital, Academic Medical Center, Meibergdreef 9, Amsterdam 1105 AZ, The Netherlands;

E-Mails: elske.vandenberg@amc.nl (E.B.); j.b.vanwoensel@amc.nl (J.B.M.W.)

* Author to whom correspondence should be addressed; E-Mail: r.a.bem@amc.nl; Tel.: +31-20-5665769, Fax: +31-20-6919338.

Received: 30 November 2012; in revised form: 15 January 2013 / Accepted: 18 January 2013 /

Published: 23 January 2013

\begin{abstract}
Pneumovirus infections cause a wide spectrum of respiratory disease in humans and animals. The airway epithelium is the major site of pneumovirus replication. Apoptosis or regulated cell death, may contribute to the host anti-viral response by limiting viral replication. However, apoptosis of lung epithelial cells may also exacerbate lung injury, depending on the extent, the timing and specific location in the lungs. Differential apoptotic responses of epithelial cells versus innate immune cells (e.g., neutrophils, macrophages) during pneumovirus infection can further contribute to the complex and delicate balance between host defense and disease pathogenesis. The purpose of this manuscript is to give an overview of the role of apoptosis in pneumovirus infection. We will examine clinical and experimental data concerning the various pro-apoptotic stimuli and the roles of apoptotic epithelial and innate immune cells during pneumovirus disease. Finally, we will discuss potential therapeutic interventions targeting apoptosis in the lungs.
\end{abstract}

Keywords: respiratory syncytial virus; cell death; host defense; acute lung injury

\section{Introduction}

Pneumoviruses are single-stranded, negative-sense, enveloped RNA viruses belonging to the family Paramyxoviridae, subfamily Pneumovirinae, and include several closely related, but species-limited, members (reviewed by Easton et al. [1]). The human pneumovirus respiratory syncytial virus (hRSV) 
is a leading respiratory pathogen in young children and the elderly worldwide and is associated with considerable morbidity and mortality and high health care costs [2,3]. Likewise, bovine RSV (bRSV) causes outbreaks of respiratory disease in young beef and dairy cattle. Both bRSV infection in cattle and infection of mice by the rodent-specific pneumovirus pneumonia virus of mice (PVM) have been studied extensively as a model for hRSV disease in humans [4]. Pneumovirus infections in humans and animals cause a wide spectrum of respiratory disease symptoms, ranging from mild upper airway illness, such as coryza and cough, to lower respiratory tract disease (e.g., bronchiolitis and bronchopneumonia), which may eventually lead to impaired gas-exchange and life threatening respiratory failure. Human infants with hRSV infection are prone to develop acute respiratory distress syndrome (ARDS), an acute-onset life threatening inflammatory lung condition associated with widespread lung injury [5,6]. Currently, as no specific treatment options for severe hRSV disease in humans exist, there is an ongoing research effort focusing on pneumovirus biology and host-interaction, ultimately to find novel therapies.

Apoptosis, a highly regulated energy-dependent type of cell death with distinct morphological and biochemical characteristics [7], is a basic biological response of cells to virus entry and replication [8]. While apoptosis of virus-infected cells may be an important first line host defense mechanism to limit pathogen replication and spread, many viruses have evolved strategies to evade and modulate intracellular pro-apoptotic signaling in the early replication phase $[9,10]$. Conversely, it has become clear that viruses may also exploit the cellular pro-apoptotic machinery in the formation and spread of infectious progeny virions in the late phase or in the elimination of immune cells, thereby evading host defense [10,11]. At the same time, from the host's perspective, extensive pro-apoptotic signaling may be beneficial in attacking a virus, but may become devastating upon the occurrence of an overshoot in apoptosis, leading to widespread loss of infected and/or uninfected bystander structural cells. Such an unbalanced extensive apoptotic response is implicated in the pathogenesis of a wide variety of diseases, including the development of diffuse lung epithelial injury in ARDS [12]. Taken together, the outcome of apoptosis during viral infection for the host may depend on its extent, timing and cell-specificity.

The occurrence and potential role of apoptosis in pneumovirus infections have been investigated in both in vivo (human and animal) and in vitro studies. The main goal of this manuscript is to provide an overview of the existing literature on pro- and anti-apoptotic signaling in pneumovirus infections with a focus on lung (airway and alveolar) epithelial cells, neutrophils and macrophages as first line cellular responders to acute pneumovirus infection. Furthermore, we will speculate on future apoptosis-based pharmacological therapies in hRSV disease.

\section{Apoptotic Signaling Pathways}

Cell death in multicellular organisms occurs either by necrosis or apoptosis, however their strict distinction is somewhat artificial, because overlap of their characteristics and cellular pathways may occur [13-15]. Apoptosis is associated with membrane blebbing, cell breakdown into apoptotic bodies and fragmentation of DNA. "Classical" apoptosis refers to the activation of the caspase cascade, a 
family of intracellular substrate specific proteases of which the final executioner, caspase-3, is a commonly used marker for apoptosis. Non-classical apoptosis occurs independent of caspase activation and involves release of the flavoprotein apoptosis-inducing factor (AIF) from mitochondria. Galluzzi el al. have provided an extensive and detailed review on the use and interpretation of caspase-(in)dependent apoptosis assays in laboratory research [16].

Figure 1. Schematic overview of three pathways of caspase-dependent apoptosis. First, the death receptor (extrinsic) pathway is activated upon tumor necrosis factor (TNF) death receptor family ligation by membrane-bound or soluble ligands, such as Fas ligand (FasL) and TNF-related apoptosis-inducing ligand (TRAIL), presented or secreted by local immune cells, including effector lymphocytes, neutrophils (PMN) and/or macrophages. Intracellular adaptor protein interactions through death domain modules follow the death receptor ligation and subsequently lead to activation of initiator caspase- 8 and the downstream caspase cascade resulting in apoptosis. The inhibitor of apoptosis proteins (IAPs) can block several caspases, thereby inhibiting cell death. Second, granzymes delivered into the cytosol by effector lymphocytes can interact with several caspases and Bid to induce apoptosis. Third, members of the Bcl-2 family, including Bcl-2, Bax and $\mathrm{Bcl}-\mathrm{XL}$ and $\mathrm{p} 53$, regulate cytochrome $\mathrm{c}$ release from the mitochondria (intrinsic pathway) in response to stimuli, such as DNA damage, infection and formation of reactive oxygen species (ROS). Cytochrome $\mathrm{c}$ in the cytosol assembles with apoptotic peptidase activating factor 1 (Apaf 1) to activate initiator caspase-9 with subsequent activation of the caspasecascade and apoptosis. The mitochondrial and death receptor pathway can interact through BH3-interacting domain death agonist (Bid).

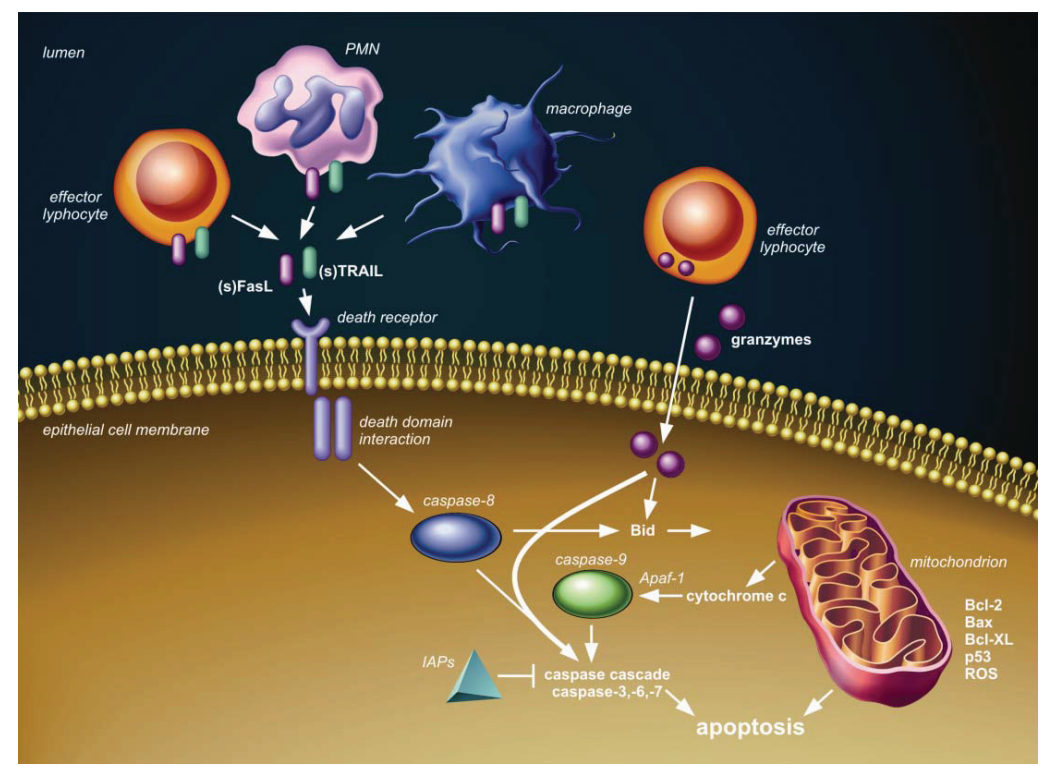


Figure 1 shows the conceptual framework of the major (caspase-dependent) apoptotic pathways, which are relevant to discuss in the context of pneumovirus infection. The intrinsic or mitochondrial pathway involves the release of cytochrome $\mathrm{c}$ from mitochondria into the cytoplasm and is regulated by Bcl-2 protein family members. The extrinsic or death receptor pathway is triggered by ligation of transmembrane death receptors that belong to the tumor necrosis factor (TNF) death receptor family, which include TNF receptor (TNFR), Fas (CD95) and TNF-related apoptosis-inducing ligand (TRAIL) death receptors (TRAIL-R1 and -R2). Expression and activation of proteins in both these pathways can be modulated by p53, a major cell stress sensor protein. Finally, the granule-mediated cytotoxic pathway is exploited by effector lymphocytes, which can release serine proteases, known as granzymes, into the cytosol of target cells, which subsequently results in caspase-(in)dependent apoptosis. Activation and modulation of these three apoptotic pathways is associated with pneumovirus infection in humans and animals, as will be discussed below.

\section{Lung (Airway and Alveolar) Epithelial Cell Apoptosis}

In the lower airways, the bronchiolar epithelium is the primary site of human and animal pneumovirus infection [17-20]. In addition, viral antigen can be detected in alveolar epithelial cells in severe hRSV-, bRSV- and PVM-induced bronchopneumonia [18-21]. An interesting and consistently reported feature of severe pneumovirus disease is the sloughing of dead airway epithelial cells, which form dense plugs with mucus, fibrin and leukocytes, resulting in air trapping and ventilatory failure [18,20-23]. The loss of these epithelial cells has always been thought to occur primarily through necrosis, however Welliver et al. observed marked active caspase-3 immunostaining in bronchiolar epithelium of children with fatal hRSV disease, suggesting that apoptosis is an important mechanism of cell death as well (Figure 2A) [20,24]. Likewise, lung epithelial cells show enhanced DNA fragmentation as detected by terminal dUTP nick-end labeling (TUNEL) in bRSV infected calves and increased caspase-3 activation in PVM infected mice [19,25] (Figure 2B). The relative importance of apoptosis in cell decay in pneumovirus infected lungs is further supported by the finding of a strong correlation between caspase-3 and -7 activity and LDH concentration in the airways in children with hRSV-induced bronchiolitis; however, from this study, the exact cellular source of these markers is not clear [26].

The occurrence of apoptosis in the lung epithelium during the course of pneumovirus infection may serve to limit viral replication. However, the widespread and extensive scale on which this takes place, involving the whole pulmonary system, including the alveoli, may also suggests an overshoot and/or inefficiency of pro-apoptotic signaling during the late and severe phase of pneumovirus infection. This may lead to enhanced respiratory disease, e.g., diffuse alveolar epithelial injury, as seen in human ARDS [12] and influenza virus animal models [27]. We believe three scenarios regarding lung epithelial cell apoptosis during pneumovirus infection may co-exist as described below (Figure 3), and the balance between them is likely to be critical for the development and outcome of host disease. 
Figure 2. (A) Positive immunohistochemical staining for the apoptosis marker caspase-3 (brown) in bronchiolar epithelial cells in lung tissue from a child with fatal human pneumovirus respiratory syncytial virus (hRSV) disease. From Welliver et al. [24], by permission of Oxford University Press. (B) Positive immunohistochemical staining for caspase-3 (brown, arrows) in alveolar epithelial cells in lung tissue from a mouse (C57B1/6 background) with severe pneumonia virus of mice (PVM) disease. From Bem et al. [25], Copyright 2010, The American Association of Immunologists, Inc.

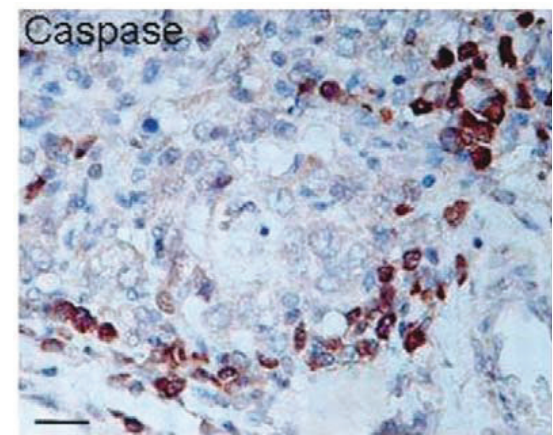

(A)

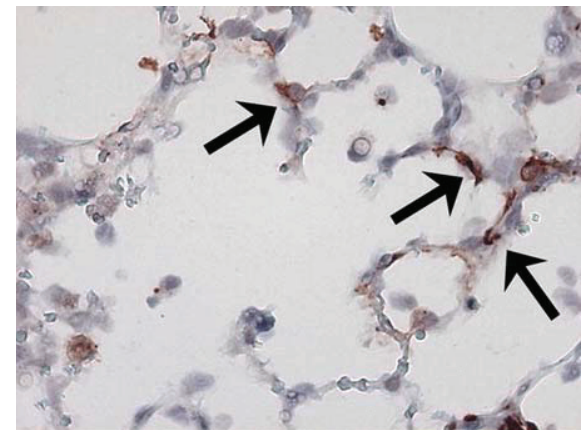

(B)

\subsection{Pneumovirus Infected Cells May Undergo Apoptosis as a Result of Direct Activation of} Intrinsic Pathways.

Based on the results of a number of in vitro studies in cultured primary airway epithelial cells and A549 cells (an adenocarcinomic human alveolar basal epithelial cell line) it appears that, although in the early phase of hRSV infection apoptosis does not occur, this type of cell death becomes an important response in the later phase of viral replication [28-39]. It should be noted however that several of these studies show some conflicting results with regard to the time point at which apoptosis is detected, possibly related to the use of different cell lines/cultures, multiplicity of infection, hRSV strains, early versus late apoptosis markers and assays used.

The relative paucity of apoptosis during the early phase of hRSV infection may be explained by viral-induced anti-apoptotic intrinsic pathway signaling, which ensures more time for viral replication and assembly of virions. Mechanisms of early hRSV-induced alteration of the intrinsic pathway towards an anti-apoptotic balance in Bcl-2 family proteins include post-transcriptional regulation of p53 [32], microRNA modulation [40] and three important basic cell survival/proliferation pathways involving nerve growth factor/tyrosine kinase receptor (NGF/Trk) [36], epidermal growth factor receptor (EGFR) [35] and phosphoinositide 3-kinase(PI3K)/Akt signaling [38]. Interestingly, by using recombinant and RNA silencing techniques, it was shown that both the hRSV-encoded small hydrophobic $(\mathrm{SH})$ protein and nonstructural (NS) proteins are critical regulators of the early anti-apoptotic effects [29,31]. The NS proteins have been found to interfere with interferon (IFN) signaling [41], which may affect apoptotic pathways in hRSV infected cells. However, Bitko 
et al. [29] suggested that the early NS protein associated anti-apoptotic effect was mediated through $\mathrm{NF}-\kappa \mathrm{B}$ and PI3K/Akt signaling, independent of the IFN pathway.

Figure 3. Three theoretical scenarios regarding lung epithelial cell apoptosis during pneumovirus infection, which may co-exist. First (A), viral infection triggers the mitochondrial (intrinsic) apoptotic pathway via interaction with $\mathrm{Bcl}-2$ family proteins. Second (B), death receptor ligands (either membrane-bound or soluble) presented or secreted by local immune cells activate the death receptor (extrinsic) apoptotic pathway in viral-infected cells. Similarly, granzymes released from effector lymphocytes into the cytosol of target cells induce apoptosis. Viral infection may modulate the susceptibility to death receptor ligands or granzymes by altering the expression of and interaction with the protein machinery, such as surface death receptors, involved in these pathways. Third (C), bystander (uninfected) epithelial cells undergo apoptosis as a result of extensive, non-specific signaling via the death receptor (extrinsic) and/or granzyme apoptotic pathway.

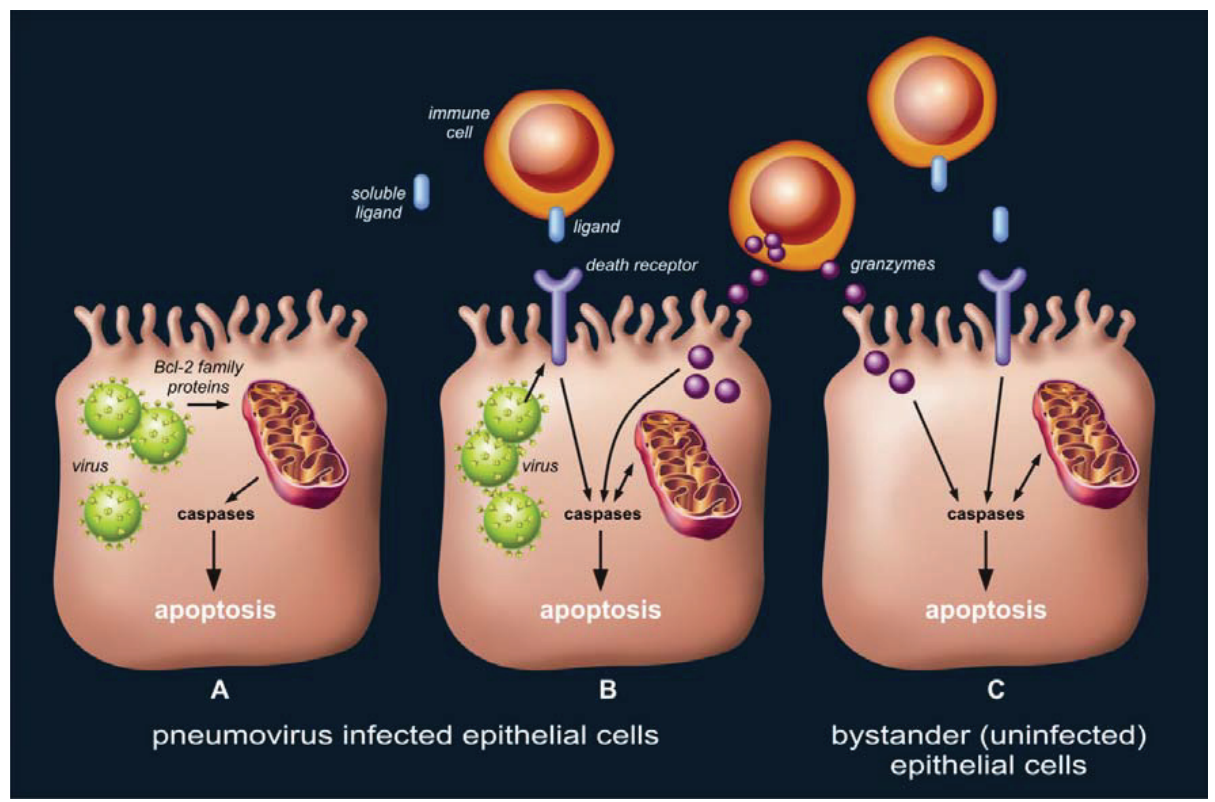

Pro-apoptotic signaling appears to predominate at a later phase of hRSV infection [29,30,34,39]. The hRSV-encoded fusion (F) protein, which is expressed at a later time point as compared to the NS proteins [29], has been found to be critical in the activation of apoptosis by p53-mediated activation of pro-apoptotic Bcl-2 family proteins [30]. Apoptosis markers in hRSV-infected A549 and human primary airway epithelial cells further include caspase activation, detection of phosphatidylserine exposition by Annexin V and TUNEL staining. However, the susceptibility of epithelial cells to pneumovirus-associated apoptosis may vary depending on the specific localization (proximal versus distal) in the lungs [34]. Importantly, in the recent study by Villenave et al. well-differentiated primary 
bronchial epithelial cells of children cultured in air-liquid interface showed TUNEL-positive staining in detached cells six days after hRSV infection [39]. This supports the hypothesis that apoptosis contributes to the characteristic sloughing of epithelial cells in the airways of children during the late phase of pneumovirus infection.

\subsection{Pneumovirus Infected Cells May Undergo Apoptosis by Activation of Extrinsic and} Granule-Mediated Pathways

First, death receptor ligands may trigger apoptosis of virus infected epithelial cells. Several studies have shown enhanced local production of TNFa, FasL and TRAIL by recruited immune cells or neighbor epithelial cells upon pneumovirus infection [4,28,34,39,42,43]. Interestingly, in vitro studies have shown that, whereas hRSV infection results in decreased susceptibility to TNF $\alpha$-induced apoptosis [44], it sensitizes lung epithelial cells to the pro-apoptotic effects of TRAIL [34], which likely occurs as a result of increased expression of death receptors TRAIL-R1 and -R2 [34,42]. Similarly, enhanced epithelial surface expression of Fas in hRSV infection has been found in both in vitro and human histopathology studies $[20,28,45]$. Interestingly, mice with a non-functional FasL ( $g l d$ strain) have been reported to have delayed hRSV clearance [46], but van den Berg et al. [47] did not detect differences in lung viral loads in PVM-infected mice with a defective Fas (lpr strain) as compared to wild type mice. This differential effect of a dysfunctional Fas/FasL system on viral clearance in these different pneumovirus models may be explained by specific pneumovirus-host interactions, although may potentially also be related to a leaky, incomplete Fas defect in lpr mice.

Second, granzymes released from the granules of natural killer cells and cytotoxic T-lymphocytes into virus infected target cells may induce apoptosis. In children with severe hRSV disease, we have found strong release of granzymes in the lungs [48]. Similarly, PVM infected mice show enhanced granzyme expression, however this appears not to affect local viral titers [25], suggesting that the granule-mediated apoptotic pathway does not have an important role in clearance of pneumovirus infected epithelial cells.

\subsection{Uninfected Bystander Epithelial Cells May Undergo Apoptosis as a Result of Extensive and Non-Specific Activation of Extrinsic and Granule-Mediated Pathways}

Although pneumovirus antigen can be detected in alveolar epithelium in the late and severe phase of human and animal pneumovirus disease, the primary site of replication are (small) airway epithelial cells [17-21]. Interestingly, histopathology studies in humans with fatal hRSV disease and animal models show epithelial cell apoptosis may occur in the entire pulmonary system, including the alveolar compartment $[4,19,24,25]$. In fact, we have previously detected active caspase-3 immunostaining primarily in alveolar epithelial cells in mice with severe PVM disease, while this apoptosis marker was scarce in bronchial epithelium [25]. Such a potential disparity in the localization between pneumovirus presence and apoptosis may suggest uninfected bystander cells in the lungs are victim of an overshoot and/or inefficiency in pro-apoptotic signaling, and this may lead to enhanced pneumovirus disease. 
The bystander injury hypothesis is supported by two in vivo studies in mice: Rutigliano et al. [46] showed that while a dysfunctional FasL results in delayed hRSV clearance, at the same time it significantly diminishes clinical illness; and, our own group showed that while a deficiency in granzymes did not affect PVM clearance, it resulted in delayed clinical disease in association with a marked decrease in lung caspase-3 activity [25]. In addition, we have previously shown that soluble TRAIL is present in increased levels in bronchoalveolar lavage fluid of children with severe hRSV disease, and this death receptor ligand induced cell death in uninfected primary pediatric airway epithelial cells in vitro [42]. Interestingly, similar findings for soluble FasL were found in relation to epithelial injury in human ARDS [49]. When considering the bystander epithelial injury hypothesis, soluble death receptor ligands present in the lung microenvironment during pneumovirus disease are of particular interest due to the potential increased likelihood of non-specific signaling. They may be released into the extracellular space from recruited immune cells, such as macrophages [42] and neutrophils [50] or from (infected) neighbor epithelial cells (paracrine pathway) [51], although in vitro studies by Bitko et al. [28] suggested a minor role for the latter mechanism.

\section{Neutrophil Apoptosis}

Neutrophils, recruited by local production of chemoattractants, are the predominant inflammatory cells in the pulmonary compartment during acute pneumovirus infection [4,52-54]. They possess a wide arsenal of defensive strategies against invading microorganisms, including phagocytosis, production of toxic reactive oxygen species and release of immune defense proteins. Although the role of neutrophils in anti-viral immunity remains relatively unclarified, several studies have shown a protective effect of neutrophils against influenza virus [55,56]. Both animal and human studies demonstrated that during pneumovirus infection, recruited neutrophils contain virus, suggesting a contributive role to viral clearance [19,57]. This is further supported by in vitro studies that showed neutrophil-mediated injury and detachment of hRSV infected lung epithelial cells [58]. On the other hand, it is well recognized that uncontrolled or prolonged neutrophil activity may cause collateral tissue injury [59], such as diffuse alveolar damage in human ARDS [60-62]. Although direct evidence of neutrophil-mediated respiratory disease in pneumovirus infection is lacking, the acute and strong neutrophil influx in the lungs coincides with peak disease severity in animal models [19,25].

To prevent their potential inadvertent harmful effect, the life span of neutrophils is limited by apoptosis. Interestingly, incubation of human neutrophils with hRSV in vitro results in activation of anti-apoptotic signaling through the Bcl-2 family proteins [63]. Although this inhibition of neutrophil cell death by hRSV does not require live virus, it does require cellular uptake. Lindemans et al. [63] further suggested this effect depends on auto- or paracrine-signaling via the release of IL-6. Others, however, have suggested that the anti-apoptotic effect of hRSV on human neutrophils is primarily mediated by soluble factors via monocyte interaction and, thus, is a secondary response [64]. Regardless of the precise mechanism involved, these in vitro studies suggest prolonged neutrophil survival in the setting of hRSV infection and, as such, this may be an important event in the development of lung injury during pneumovirus disease. In contrast, Wang et al. [65] reported an 
increased number of Annexin $\mathrm{V}$ positive neutrophils in nasopharyngeal aspirates and the peripheral blood of infants with hRSV disease, as compared to peripheral blood neutrophils of healthy children. This would imply that despite the pro-survival effect of hRSV on neutrophils as observed in vitro, pro-apoptotic signaling by mediators (e.g., cytokines) in the lung microenvironment during hRSV disease prevails. Whether this truly would protect against harmful prolonged neutrophil survival remains speculative. In particular, it is possible that different neutrophil apoptotic responses in functionally heterogeneous neutrophil subsets co-exist and change over time [66,67]. As such, it is clear that further research on the precise role of neutrophil apoptosis during pneumovirus infections is highly needed.

\section{Macrophage Apoptosis}

Macrophages are observed in high numbers in the airways and lungs of humans and animals with pneumovirus disease [4,18-20,22,23,52,54]. Macrophages are key innate immune cells acting in pathogen surveillance and initiation and resolution of inflammation through mechanisms involving antigen processing/presentation, phagocytosis and cytokine production. Macrophage depletion in wild-type mice infected by hRSV or PVM results in increased lung viral titers and greatly affects the local cytokine production $[23,68,69]$. However, paradoxically in PVM-infected mice, this results in prolonged survival, whereas in hRSV challenged mice, this is associated with enhanced airway occlusion on histopathological examination [23,69], suggesting that the role of macrophages may depend on specific host-pneumovirus interactions. Nevertheless, these findings underline the importance of macrophage biology in pneumovirus infection. As such, dysregulation of (apoptotic) cell death pathways in resident and migrated macrophages in the lungs may have profound consequences for the outcome of pneumovirus infection.

Unfortunately, very few studies have focused on macrophage apoptosis in pneumovirus disease. In the histopathological studies from Welliver et al., fatal hRSV disease in children was associated with relatively low numbers of caspase-3 immunoreactive inflammatory cells in the lungs, as compared to children with fatal influenza virus disease [24]. In mice challenged with PVM, we observed cleaved caspase-3 positive macrophages in the alveolar spaces (unpublished observations, [70]); however, the exact magnitude and role of apoptosis of these cells in this model is not yet clear.

Ex vivo and autopsy studies have shown that virus antigen is detected in macrophages in the airways and lungs of hRSV infected patients [18,71]. In addition, isolated peripheral and cord blood monocytes, as well as alveolar macrophages, are susceptible to hRSV infection in vitro [72,73], and PVM was recently shown to replicate in primary mouse macrophage culture [69]. Interestingly, hRSV presence in human monocytes and mouse macrophages results in decreased (susceptibility of) apoptosis, associated with decreased caspase-3 activity and enhanced expression of anti-apoptotic members of the Bcl-2 protein family and inhibitor of apoptosis protein (IAP) family [74,75]. These observations suggest pneumoviruses exploit strategies to escape apoptosis in macrophages and could explain the apparent paucity of evidence for macrophage apoptosis in the aforementioned histopathology studies. 


\section{Apoptosis-Based Pharmacological Intervention}

During the last decades, much progress has been made in the development of apoptosis-based therapeutic agents, including antisense oligodeoxynucleotides, small interfering (si)RNA, peptides/proteins and antibodies (reviewed by Fischer et al. [76]). Much attention in this field has been directed towards cancer treatment, however inflammatory and infectious diseases are of emerging focus. The increasing knowledge in the regulation and molecular machinery of apoptotic cell death pathways involving many different target genes and proteins has set the stage for highly promising research in pharmacological intervention. Death receptors, caspases and IAP and Bcl-2 protein family members all belong to prominent targets in current drug development [76]. However, despite the ongoing success of pre-clinical and even clinical studies exploiting the use of apoptosis-based drugs in a wide variety of diseases, many obstacles still have to be overcome, including cell-specificity and permeability, timing of intervention and potential toxicity or interference with other biological processes, such as inflammation.

To our knowledge, up to date, no published studies have directly examined the effects of apoptosis-based pharmacological treatments in pneumovirus disease in vivo. Although originally designed to study macrophage depletion rather than directly focusing on apoptosis intervention, Rigaux et al. [69] found that intratracheal treatment with clodronate-liposomes, which induces apoptosis upon specific uptake by macrophages [77], prolongs the survival of PVM-infected mice. This suggests that enhancing macrophage apoptosis in pneumovirus disease is of clinical interest. Currently, our own group is studying the use of the irreversible pan-caspase inhibitor, z-VAD-fmk, administrated by a systemic route in mice challenged with PVM (study in progress). In addition, we have shown that treatment with DR5-Fc fusion protein, which inhibits TRAIL death receptor signaling, partly attenuates cell death of primary pediatric bronchial airway cells exposed to bronchoalveolar lavage fluid from hRSV-infected children in vitro [42]. However the effects of this compound in vivo are yet unclear. Given the strong evidence of activation of pro- and anti-apoptotic pathways during pneumovirus infection as described in the above paragraphs, more attention towards this field is highly needed.

Several critical points need to be considered in potential apoptosis-based interventions in pneumovirus disease. First is the cell-specificity. From the present overview, it becomes clear that different cell types in the lungs may show differential apoptotic responses during pneumovirus disease. Interestingly, even within a lung cell population (e.g., epithelial cells), differential responses to a single apoptotic mediator may exist [78,79], including in the setting of pneumovirus infection [34], which further increases the complexity of intervention. This stresses the need for insight in cell-specific pro- and anti-apoptotic targets and for development of small molecule compounds and vehicles for specific local intervention. On the other hand, even without specific target cell delivery systems, animal studies modeling ARDS have shown promising beneficial effects on survival and histopathological changes by using a number of systemic or intratracheal apoptosis-based treatments, including blockade of FasL by decoy receptor-3 [80], Fas:Ig fusion protein [81] or Fas-siRNA [82] and pan-caspase inhibition by z-VAD-fmk [83]. These studies successfully aimed to inhibit lung 
epithelial cell apoptosis during the process of lung injury. However, it remains to be elucidated whether such strategies also effectively reduce lung epithelial cell death in pneumovirus disease and, subsequently, to what extent this affects viral replication and, most importantly, clinical outcome. Second, given the dynamic course of anti- and pro-apoptotic signaling in lung epithelial cells, timing of apoptotic interventions may be critical, as, for example, inhibitory strategies may be beneficial in the late severe phase of disease, but detrimental in the early stages of viral replication. Third, concurrent iatrogenic treatments with pro-apoptotic effects, such as mechanical ventilation and/or oxygen therapy [70,84], may interact with apoptosis-based interventions in the lungs. Fourth and finally, the effect of modulating apoptosis in the lungs needs to be considered with special focus on age, as apoptosis is an important event in lung development and maturation [85].

\section{Conclusion}

Pneumovirus infection is associated with both pro- and anti-apoptotic signaling in the lungs, depending on the cell-type, involvement of specific cell death pathway and timing during the course of infection. The balance between these events is likely to be critical for the development and outcome of pneumovirus disease. However, more research is needed to fully understand the dynamic character of apoptosis during this important respiratory illness. In particular, we need to address which factors favor the viral propagation versus host defense. Here, we have reviewed the existing literature on this topic and have speculated on future apoptosis-based pharmacological treatments.

\section{Acknowledgments}

The authors thank Inge Kos-Oosterling from the AMC Medical Illustration Service for help with the figure drawings. No specific financial support or grants are reported.

\section{Conflicts of Interest}

The authors declare no conflict of interest.

\section{References}

1. Easton, A.J.; Domachowske, J.B.; Rosenberg, H.F. Animal pneumoviruses: Molecular genetics and pathogenesis. Clin. Microbiol. Rev. 2004, 17, 390-412.

2. Falsey, A.R.; Hennessey, P.A.; Formica, M.A.; Cox, C.; Walsh, E.E. Respiratory syncytial virus infection in elderly and high-risk adults. N. Engl. J. Med. 2005, 352, 1749-1759.

3. Nair, H.; Nokes, D.J.; Gessner, B.D.; Dherani, M.; Madhi, S.A.; Singleton, R.J.; O’Brien, K.L.; Roca, A.; Wright, P.F.; Bruce, N.; et al. Global burden of acute lower respiratory infections due to respiratory syncytial virus in young children: A systematic review and meta-analysis. Lancet 2010, 375, 1545-1555.

4. Bem, R.A.; Domachowske, J.B.; Rosenberg, H.F. Animal models of human respiratory syncytial virus disease. Am. J. Physiol. Lung Cell. Mol. Physiol. 2011, 301, 148-156. 
5. ARDS definition task force Acute respiratory distress syndrome-The Berlin definition. JAMA 2012, 307, 256-2533.

6. Dahlem, P.; van Aalderen, W.M.; Hamaker, M.E.; Dijkgraaf, M.G.; Bos, A.P. Incidence and short-term outcome of acute lung injury in mechanically ventilated children. Eur. Respir. J. 2003, 22, 980-985.

7. Kerr, J.F.; Wyllie, A.H.; Currie, A.R. Apoptosis: A basic biological phenomenon with wide-ranging implications in tissue kinetics. Br. J. Cancer 1972, 26, 239-257.

8. Barber, G.N. Host defense, viruses and apoptosis. Cell Death Differ. 2001, 8, 113-126.

9. Benedict, C.A.; Norris, P.S.; Ware, C.F. To kill or be killed: Viral evasion of apoptosis. Nat. Immunol. 2002, 3, 1013-1018.

10. Galluzzi, L.; Brenner, C.; Morselli, E.; Touat, Z.; Kroemer, G. Viral control of mitochondrial apoptosis. PLoS Pathog. 2008, 4, e1000018.

11. Herold, S.; Ludwig, S.; Pleschka, S.; Wolff, T. Apoptosis signaling in influenza virus propagation, innate host defense and lung injury. J. Leukoc. Biol. 2012, 92, 75-82.

12. Martin, T.R.; Hagimoto, N.; Nakamura, M.; Matute-Bello, G. Apoptosis and epithelial injury in the lungs. Proc. Am. Thorac. Soc. 2005, 2, 214-220.

13. Denecker, G.; Vercammen, D.; Declercq, W.; Vandenabeele, P. Apoptotic and necrotic cell death induced by death domain receptors. Cell Mol. Life Sci. 2001, 58, 356-370.

14. Leist, M.; Jaattela, M. Four deaths and a funeral: From caspases to alternative mechanisms. Nat. Rev. Mol. Cell Biol. 2001, 2, 589-598.

15. Wang, X.; Ryter, S.W.; Dai, C.; Tang, Z.L.; Watkins, S.C.; Yin, X.M.; Song, R.; Choi, A.M. Necrotic cell death in response to oxidant stress involves the activation of the apoptogenic caspase-8/bid pathway. J. Biol. Chem. 2003, 278, 29184-29191.

16. Galluzzi, L.; Aaronson, S.A.; Abrams, J.; Alnemri, E.S.; Andrews, D.W.; Baehrecke, E.H.; Bazan, N.G.; Blagosklonny, M.V.; Blomgren, K.; Borner, C.; et al. Guidelines for the use and interpretation of assays for monitoring cell death in higher eukaryotes. Cell Death Differ. 2009, 16, 1093-1107.

17. Bonville, C.A.; Bennett, N.J.; Koehnlein, M.; Haines, D.M.; Ellis, J.A.; DelVecchio, A.M.; Rosenberg, H.F.; Domachowske, J.B. Respiratory dysfunction and proinflammatory chemokines in the pneumonia virus of mice (PVM) model of viral bronchiolitis. Virology 2006, 349, 87-95.

18. Johnson, J.E.; Gonzales, R.A.; Olson, S.J.; Wright, P.F.; Graham, B.S. The histopathology of fatal untreated human respiratory syncytial virus infection. Mod. Pathol. 2007, 20, 108-119.

19. Viuff, B.; Tjornehoj, K.; Larsen, L.E.; Rontved, C.M.; Uttenthal, A.; Ronsholt, L.; Alexandersen, S. Replication and clearance of respiratory syncytial virus: Apoptosis is an important pathway of virus clearance after experimental infection with bovine respiratory syncytial virus. Am. J. Pathol. 2002, 161, 2195-2207.

20. Welliver, T.P.; Reed, J.L.; Welliver, R.C., Sr. Respiratory syncytial virus and influenza virus infections: Observations from tissues of fatal infant cases. Pediatr. Infect. Dis. J. 2008, 27, 92-96. 
21. Cook, P.M.; Eglin, R.P.; Easton, A.J. Pathogenesis of pneumovirus infections in mice: Detection of pneumonia virus of mice and human respiratory syncytial virus mRNA in lungs of infected mice by in situ hybridization. J. Gen. Virol. 1998, 79, 2411-2417.

22. Aherne, W.; Bird, T.; Court SD; Gardner, P.S.; McQuillin, J. Pathological changes in virus infections of the lower respiratory tract in children. J. Clin. Pathol. 1970, 23, 7-18.

23. Reed, J.L.; Brewah, Y.A.; Delaney, T.; Welliver, T.; Burwell, T.; Benjamin, E.; Kuta, E.; Kozhich, A.; McKinney, L.; Suzich, J.; et al. Macrophage impairment underlies airway occlusion in primary respiratory syncytial virus bronchiolitis. J. Infect. Dis. 2008, 198, 1783-1793.

24. Welliver, T.P.; Garofalo, R.P.; Hosakote, Y.; Hintz, K.H.; Avendano, L.; Sanchez, K.; Velozo, L.; Jafri, H.; Chavez-Bueno, S.; Ogra, P.L.; et al. Severe human lower respiratory tract illness caused by respiratory syncytial virus and influenza virus is characterized by the absence of pulmonary cytotoxic lymphocyte responses. J. Infect. Dis. 2007, 195, 1126-1136.

25. Bem, R.A.; van Woensel, J.B.; Lutter, R.; Domachowske, J.B.; Medema, J.P.; Rosenberg, H.F.; Bos, A.P. Granzyme A- and B-cluster deficiency delays acute lung injury in pneumovirus-infected mice. J. Immunol. 2010, 184, 931-938.

26. Laham, F.R.; Trott, A.A.; Bennett, B.L.; Kozinetz, C.A.; Jewell, A.M.; Garofalo, R.P.; Piedra, P.A. LDH concentration in nasal-wash fluid as a biochemical predictor of bronchiolitis severity. Pediatrics 2010, 125, 225-233.

27. Herold, S.; Steinmueller, M.; von, W.W.; Cakarova, L.; Pinto, R.; Pleschka, S.; Mack, M.; Kuziel, W.A.; Corazza, N.; Brunner, T.; et al. Lung epithelial apoptosis in influenza virus pneumonia: The role of macrophage-expressed TNF-related apoptosis-inducing ligand. J. Exp. Med. 2008, 205, 3065-3077.

28. Bitko, V.; Barik, S. An endoplasmic reticulum-specific stress-activated caspase (caspase-12) is implicated in the apoptosis of A549 epithelial cells by respiratory syncytial virus. J. Cell Biochem. 2001, 80, 441-454.

29. Bitko, V.; Shulyayeva, O.; Mazumder, B.; Musiyenko, A.; Ramaswamy, M.; Look, D.C.; Barik, S. Nonstructural proteins of respiratory syncytial virus suppress premature apoptosis by an NF-kappaB-dependent, interferon-independent mechanism and facilitate virus growth. J. Virol. 2007, 81, 1786-1795.

30. Eckardt-Michel, J.; Lorek, M.; Baxmann, D.; Grunwald, T.; Keil, G.M.; Zimmer, G. The fusion protein of respiratory syncytial virus triggers p53-dependent apoptosis. J. Virol. 2008, 82, 3236-3249.

31. Fuentes, S.; Tran, K.C.; Luthra, P.; Teng, M.N.; He, B. Function of the respiratory syncytial virus small hydrophobic protein. J. Virol. 2007, 81, 8361-8366.

32. Groskreutz, D.J.; Monick, M.M.; Yarovinsky, T.O.; Powers, L.S.; Quelle, D.E.; Varga, S.M.; Look, D.C.; Hunninghake, G.W. Respiratory syncytial virus decreases p53 protein to prolong survival of airway epithelial cells. J. Immunol. 2007, 179, 2741-2747. 
33. Groskreutz, D.J.; Monick, M.M.; Babor, E.C.; Nyunoya, T.; Varga, S.M.; Look, D.C.; Hunninghake, G.W. Cigarette smoke alters respiratory syncytial virus-induced apoptosis and replication. Am. J. Respir. Cell Mol. Biol. 2009, 41, 189-198.

34. Kotelkin, A.; Prikhod'ko, E.A.; Cohen, J.I.; Collins, P.L.; Bukreyev, A. Respiratory syncytial virus infection sensitizes cells to apoptosis mediated by tumor necrosis factor-related apoptosis-inducing ligand. J. Virol. 2003, 77, 9156-9172.

35. Monick, M.M.; Cameron, K.; Staber, J.; Powers, L.S.; Yarovinsky, T.O.; Koland, J.G.; Hunninghake, G.W. Activation of the epidermal growth factor receptor by respiratory syncytial virus results in increased inflammation and delayed apoptosis. J. Biol. Chem. 2005, 280, 2147-2158.

36. Othumpangat, S.; Gibson, L.F.; Samsell, L.; Piedimonte, G. NGF is an essential survival factor for bronchial epithelial cells during respiratory syncytial virus infection. PLoS One 2009, 4, e6444.

37. Takeuchi, R.; Tsutsumi, H.; Osaki, M.; Haseyama, K.; Mizue, N.; Chiba, S. Respiratory syncytial virus infection of human alveolar epithelial cells enhances interferon regulatory factor 1 and interleukin-1beta-converting enzyme gene expression, but does not cause apoptosis. J. Virol. 1998, 72, 4498-4502.

38. Thomas, K.W.; Monick, M.M.; Staber, J.M.; Yarovinsky, T.; Carter, A.B.; Hunninghake, G.W. Respiratory syncytial virus inhibits apoptosis and induces NF-kappa B activity through a phosphatidylinositol 3-kinase-dependent pathway. J. Biol. Chem. 2002, 277, 492-501.

39. Villenave, R.; Thavagnanam, S.; Sarlang, S.; Parker, J.; Douglas, I.; Skibinski, G.; Heaney, L.G.; McKaigue, J.P.; Coyle, P.V.; Shields, M.D.; et al. In vitro modeling of respiratory syncytial virus infection of pediatric bronchial epithelium, the primary target of infection in vivo. Proc. Natl. Acad. Sci. USA 2012, 109, 5040-5045.

40. Othumpangat, S.; Walton, C.; Piedimonte, G. MicroRNA-221 modulates RSV replication in human bronchial epithelium by targeting NGF expression. PLoS One 2012, 7, e30030.

41. Spann, K.M.; Tran, K.C.; Chi, B.; Rabin, R.L.; Collins, P.L. Suppression of the induction of alpha, beta and lambda interferons by the NS1 and NS2 proteins of human respiratory syncytial virus in human epithelial cells and macrophages. J. Virol. 2004, 78, 4363-4369.

42. Bem, R.A.; Bos, A.P.; Wosten-van Asperen, R.M.; Bruijn, M.; Lutter, R.; Sprick, M.R.; van Woensel, J.B. Potential Role of Soluble TRAIL in Epithelial Injury in Children with Severe RSV Infection. Am. J. Respir. Cell Mol. Biol. 2009, 42, 697-705.

43. Aung, S.; Rutigliano, J.A.; Graham, B.S. Alternative mechanisms of respiratory syncytial virus clearance in perforin knockout mice lead to enhanced disease. J. Virol. 2001, 75, 9918-9924.

44. Domachowske, J.B.; Bonville, C.A.; Mortelliti, A.J.; Colella, C.B.; Kim, U.; Rosenberg, H.F. Respiratory syncytial virus infection induces expression of the anti-apoptosis gene IEX-1L in human respiratory epithelial cells. J. Infect. Dis. 2000, 181, 824-830.

45. O'donnell, D.R.; Milligan, L.; Stark, J.M. Induction of CD95 (Fas) and apoptosis in respiratory epithelial cell cultures following respiratory syncytial virus infection. Virology 1999, 257, 198-207. 
46. Rutigliano, J.A.; Graham, B.S. Prolonged production of TNF-alpha exacerbates illness during respiratory syncytial virus infection. J. Immunol. 2004, 173, 3408-3417.

47. Van den Berg E.; van Woensel, J.B.; Bos, A.P.; Bem, R.A.; Altemeier, W.A.; Gill, S.E.; Martin, T.R.; Matute-Bello, G. Role of the Fas/FasL system in a model of RSV infection in mechanically ventilated mice. Am. J. Physiol. Lung Cell Mol. Physiol. 2011, 301, 451-460.

48. Bem, R.A.; Bos, A.P.; Bots, M.; Wolbink, A.M.; van Ham, S.M.; Medema, J.P.; Lutter, R.; van Woensel, J.B. Activation of the granzyme pathway in children with severe respiratory syncytial virus infection. Pediatr. Res. 2008, 63, 650-655.

49. Matute-Bello, G.; Liles, W.C.; Steinberg, K.P.; Kiener, P.A.; Mongovin, S.; Chi, E.Y.; Jonas, M.; Martin, T.R. Soluble Fas ligand induces epithelial cell apoptosis in humans with acute lung injury (ARDS). J. Immunol. 1999, 163, 2217-2225.

50. Serrao, K.L.; Fortenberry, J.D.; Owens, M.L.; Harris, F.L.; Brown, L.A. Neutrophils induce apoptosis of lung epithelial cells via release of soluble Fas ligand. Am. J. Physiol. Lung Cell Mol. Physiol. 2001, 280, 298-305.

51. Powell, W.C.; Fingleton, B.; Wilson, C.L.; Boothby, M.; Matrisian, L.M. The metalloproteinase matrilysin proteolytically generates active soluble Fas ligand and potentiates epithelial cell apoptosis. Curr. Biol. 1999, 9, 1441-1447.

52. Everard, M.L.; Swarbrick, A.; Wrightham, M.; McIntyre, J.; Dunkley, C.; James, P.D.; Sewell, H.F.; Milner, A.D. Analysis of cells obtained by bronchial lavage of infants with respiratory syncytial virus infection. Arch. Dis. Child. 1994, 71, 428-432.

53. Van Woensel, J.B.; Lutter, R.; Biezeveld, M.H.; Dekker, T.; Nijhuis, M.; van Aalderen, W.M.; Kuijpers, T.W. Effect of dexamethasone on tracheal viral load and interleukin- 8 tracheal concentration in children with respiratory syncytial virus infection. Pediatr. Infect. Dis. J. 2003, 22, 721-726.

54. McNamara, P.S.; Ritson, P.; Selby, A.; Hart, C.A.; Smyth, R.L. Bronchoalveolar lavage cellularity in infants with severe respiratory syncytial virus bronchiolitis. Arch. Dis. Child. 2003, 88, 922-926.

55. Tate, M.D.; Deng, Y.M.; Jones, J.E.; Anderson, G.P.; Brooks, A.G.; Reading, P.C. Neutrophils ameliorate lung injury and the development of severe disease during influenza infection. J. Immunol. 2009, 183, 7441-7450.

56. Tumpey, T.M.; Garcia-Sastre, A.; Taubenberger, J.K.; Palese, P.; Swayne, D.E.; Pantin-Jackwood, M.J.; Schultz-Cherry, S.; Solorzano, A.; van, R.N.; Katz, J.M.; et al. Pathogenicity of influenza viruses with genes from the 1918 pandemic virus: Functional roles of alveolar macrophages and neutrophils in limiting virus replication and mortality in mice. J. Virol. 2005, 79, 14933-14944.

57. Halfhide, C.P.; Flanagan, B.F.; Brearey, S.P.; Hunt, J.A.; Fonceca, A.M.; McNamara, P.S.; Howarth, D.; Edwards, S.; Smyth, R.L. Respiratory syncytial virus binds and undergoes transcription in neutrophils from the blood and airways of infants with severe bronchiolitis. J. Infect. Dis. 2011, 204, 451-458. 
58. Wang, S.Z.; Forsyth, K.D. The interaction of neutrophils with respiratory epithelial cells in viral infection. Respirology 2000, 5, 1-10.

59. Segel, G.B.; Halterman, M.W.; Lichtman, M.A. The paradox of the neutrophil's role in tissue injury. J. Leukoc. Biol. 2011, 89, 359-372.

60. Martin, T.R. Neutrophils and lung injury: Getting it right. J. Clin. Investig. 2002, 110, 1603-1605.

61. Perl, M.; Lomas-Neira, J.; Chung, C.S.; Ayala, A. Epithelial cell apoptosis and neutrophil recruitment in acute lung injury-a unifying hypothesis? What we have learned from small interfering RNAs. Mol. Med. 2008, 14, 465-475.

62. Ware, L.B.; Matthay, M.A. The acute respiratory distress syndrome. N. Engl. J. Med. 2000, 342, 1334-1349.

63. Lindemans, C.A.; Coffer, P.J.; Schellens, I.M.; de Graaff, P.M.; Kimpen, J.L.; Koenderman, L. Respiratory syncytial virus inhibits granulocyte apoptosis through a phosphatidylinositol 3-kinase and NF-kappaB-dependent mechanism. J. Immunol. 2006, 176, 5529-5537.

64. Coleman, C.M.; Plant, K.; Newton, S.; Hobson, L.; Whyte, M.K.; Everard, M.L. The anti-apoptotic effect of respiratory syncytial virus on human peripheral blood neutrophils is mediated by a monocyte derived soluble factor. Open Virol. J. 2011, 5, 114-123.

65. Wang, S.Z.; Smith, P.K.; Lovejoy, M.; Bowden, J.J.; Alpers, J.H.; Forsyth, K.D. The apoptosis of neutrophils is accelerated in respiratory syncytial virus (RSV)-induced bronchiolitis. Clin. Exp. Immunol. 1998, 114, 49-54.

66. Pillay, J.; Ramakers, B.P.; Kamp, V.M.; Loi, A.L.; Lam, S.W.; Hietbrink, F.; Leenen, L.P.; Tool, A.T.; Pickkers, P.; Koenderman, L. Functional heterogeneity and differential priming of circulating neutrophils in human experimental endotoxemia. J. Leukoc. Biol. 2010, 88, 211-220.

67. Simon, H.U. Neutrophil apoptosis pathways and their modifications in inflammation. Immunol. Rev. 2003, 193, 101-110.

68. Pribul, P.K.; Harker, J.; Wang, B.; Wang, H.; Tregoning, J.S.; Schwarze, J.; Openshaw, P.J. Alveolar macrophages are a major determinant of early responses to viral lung infection, but do not influence subsequent disease development. J. Virol. 2008, 82, 4441-4448.

69. Rigaux, P.; Killoran, K.E.; Qiu, Z.; Rosenberg, H.F. Depletion of alveolar macrophages prolongs survival in response to acute pneumovirus infection. Virology 2012, 422, 338-345.

70. Bem, R.A.; van Woensel, J.B.; Bos, A.P.; Koski, A.; Farnand, A.W.; Domachowske, J.B.; Rosenberg, H.F.; Martin, T.R.; Matute-Bello, G. Mechanical ventilation enhances lung inflammation and caspase activity in a model of mouse pneumovirus infection. Am. J. Physiol. Lung Cell Mol. Physiol. 2009, 296, 46-56.

71. Midulla, F.; Villani, A.; Panuska, J.R.; Dab, I.; Kolls, J.K.; Merolla, R.; Ronchetti, R. Respiratory syncytial virus lung infection in infants: Immunoregulatory role of infected alveolar macrophages. J. Infect. Dis. 1993, 168, 1515-1519.

72. Midulla, F.; Huang, Y.T.; Gilbert, I.A.; Cirino, N.M.; McFadden, E.R., Jr.; Panuska, J.R. Respiratory syncytial virus infection of human cord and adult blood monocytes and alveolar macrophages. Am. Rev. Respir. Dis. 1989, 140, 771-777. 
73. Panuska, J.R.; Cirino, N.M.; Midulla, F.; Despot, J.E.; McFadden, E.R., Jr.; Huang, Y.T. Productive infection of isolated human alveolar macrophages by respiratory syncytial virus. J. Clin. Investig. 1990, 86, 113-119.

74. Krilov, L.R.; McCloskey, T.W.; Harkness, S.H.; Pontrelli, L.; Pahwa, S. Alterations in apoptosis of cord and adult peripheral blood mononuclear cells induced by in vitro infection with respiratory syncytial virus. J. Infect. Dis. 2000, 181, 349-353.

75. Nakamura-Lopez, Y.; Villegas-Sepulveda, N.; Sarmiento-Silva, R.E.; Gomez, B. Intrinsic apoptotic pathway is subverted in mouse macrophages persistently infected by RSV. Virus Res. 2011, 158, 98-107.

76. Fischer, U.; Schulze-Osthoff, K. Apoptosis-based therapies and drug targets. Cell Death Differ. 2005, 12, 942-961.

77. Van Rooijen N.; Sanders, A. Liposome mediated depletion of macrophages: Mechanism of action, preparation of liposomes and applications. J. Immunol. Methods 1994, 174, 83-93.

78. Janssen, W.J.; Barthel, L.; Muldrow, A.; Oberley-Deegan, R.E.; Kearns, M.T.; Jakubzick, C.; Henson, P.M. Fas determines differential fates of resident and recruited macrophages during resolution of acute lung injury. Am. J. Respir. Crit. Care Med. 2011, 184, 547-560.

79. Nakamura, M.; Matute-Bello, G.; Liles, W.C.; Hayashi, S.; Kajikawa, O.; Lin, S.M.; Frevert, C.W.; Martin, T.R. Differential response of human lung epithelial cells to fas-induced apoptosis. Am. J. Pathol. 2004, 164, 1949-1958.

80. Matute-Bello, G.; Liles, W.C.; Frevert, C.W.; Dhanireddy, S.; Ballman, K.; Wong, V.; Green, R.R.; Song, H.Y.; Witcher, D.R.; Jakubowski, J.A.; et al. Blockade of the Fas/FasL system improves pneumococcal clearance from the lungs without preventing dissemination of bacteria to the spleen. J. Infect. Dis. 2005, 191, 596-606.

81. Matute-Bello, G.; Liles, W.C.; Frevert, C.W.; Nakamura, M.; Ballman, K.; Vathanaprida, C.; Kiener, P.A.; Martin, T.R. Recombinant human Fas ligand induces alveolar epithelial cell apoptosis and lung injury in rabbits. Am. J. Physiol. Lung Cell Mol. Physiol. 2001, 281, 328-335.

82. Perl, M.; Chung, C.S.; Lomas-Neira, J.; Rachel, T.M.; Biffl, W.L.; Cioffi, W.G.; Ayala, A. Silencing of Fas, but not caspase-8, in lung epithelial cells ameliorates pulmonary apoptosis, inflammation and neutrophil influx after hemorrhagic shock and sepsis. Am. J. Pathol. 2005, 167, 1545-1559.

83. Kawasaki, M.; Kuwano, K.; Hagimoto, N.; Matsuba, T.; Kunitake, R.; Tanaka, T.; Maeyama, T.; Hara, N. Protection from lethal apoptosis in lipopolysaccharide-induced acute lung injury in mice by a caspase inhibitor. Am. J. Pathol. 2000, 157, 597-603.

84. Altemeier, W.A.; Sinclair, S.E. Hyperoxia in the intensive care unit: Why more is not always better. Curr. Opin. Crit. Care 2007, 13, 73-78.

85. Bem, R.A.; Bos, A.P.; Matute-Bello, G.; van, T.M.; van Woensel, J.B. Lung epithelial cell apoptosis during acute lung injury in infancy. Pediatr. Crit. Care Med. 2007, 8, 132-137. 
Reprinted from Viruses. Cite as: Guerrero-Plata, A. Dendritic Cells in Human Pneumovirus and Metapneumovirus Infections. Viruses 2013, 5, 1553-1570.

Review

\title{
Dendritic Cells in Human Pneumovirus and Metapneumovirus Infections
}

\section{Antonieta Guerrero-Plata ${ }^{1,2}$}

1 Department of Pathobiological Sciences, Louisiana State University, Baton Rouge, LA 70803, USA; E-Mail: aguerrp@1su.edu; Tel.: +1-225-578-9678; Fax: +1-225-578-9701

2 Center for Experimental Infectious Disease Research, Louisiana State University, Baton Rouge, LA 70803, USA

Received: 1 May 2013; in revised form: 24 May 2013 / Accepted: 6 June 2013 /

Published: 20 June 2013

\begin{abstract}
Lung dendritic cells (DC) play a fundamental role in sensing invading pathogens, as well as in the control of tolerogenic responses in the respiratory tract. Their strategic localization at the site of pathogen entry makes them particularly susceptible to initial viral invasion. Human respiratory syncytial virus (hRSV) and human metapneumovirus (hMPV) belong to the Paramyxoviridae family, within the Pneumovirus and Metapneumovirus genera, respectively. hRSV and hMPV are significant human respiratory pathogens that cause similar clinical manifestations and affect many of the same subpopulations. However, they differentially activate the host immune response, including DC, which represents a fundamental link between the innate and adaptive immune response. In this review, the role of DC in the immune response against hRSV and hMPV infections, as well as the inhibitory effects of these paramyxoviruses on the DC immunity will be discussed.
\end{abstract}

Keywords: dendritic cells; human metapneumovirus; respiratory syncytial virus; lung; paramyxovirus

\section{Introduction}

Human respiratory syncytial virus (hRSV) and human metapneumovirus (hMPV) are classified within the Paramyxoviridae family, Pneumovirinae subfamily, which is divided into the Pneumovirus 
and the Metapneumovirus genera. Both viruses belong to the order Mononegavirales and contain a nonsegmented, negative-sense RNA with genomic organization, which is similar, but not identical [1-4]. Metapneumoviruses lack the nonstructural proteins NS1 and NS2, and the gene order is different from that of pneumoviruses. hRSV is the type species of the Pneumovirus genus, while (based on the biological properties and genomic sequence) hMPV has been assigned to the Metapneumovirus genus. hRSV encodes 11 proteins (nonstructural (NS) protein1, NS2, nucleocapsid (N), phosphoprotein (P), matrix (M)1, small hydrophobic (SH), attachment (G), fusion (F), M2-1, M2-2 and polymerase (L), Figure 1), while hMPV encodes nine proteins (N, P, M1, F, M2-1, M2-2, SH, G and L, Figure 1).

Figure 1. Schematic representation of human Pneumovirus and Metapneumovirus. Gene maps and encoded proteins of members of the subfamily Pneumovirinae: human respiratory syncytial virus (hRSV) and human metapneumovirus (hMPV), which belong to the genera Pneumovirus and Metapneumovirus, respectively. Genes are represented as boxes with the corresponding encoded protein.

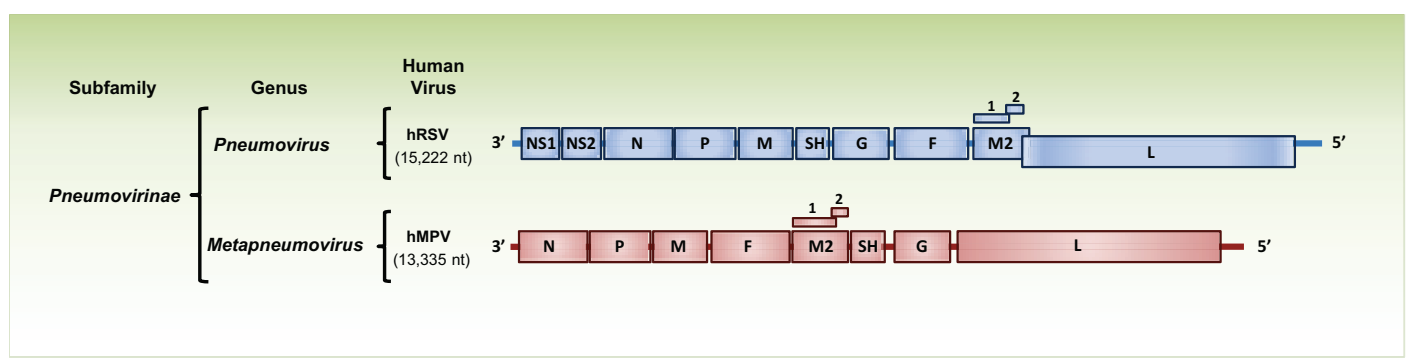

hRSV was first isolated in 1956 from an infected chimpanzee [5] and represents the most important cause of bronchiolitis and pneumonia in infants and young children worldwide. More than $95 \%$ of children are infected with hRSV by two years of age [1]. hMPV was first identified in 2001 following its isolation from infants and children with lower respiratory tract infections (LTRI) of unknown etiology [2]. Serologic evidence indicated that hMPV seropositivity is almost universal by the age of five years. The clinical manifestations of hMPV infection in young children are indistinguishable from those of hRSV infection. LRTI associated with hMPV in infants and young children is a frequent cause of hospitalization. Several studies indicate that hMPV likely accounts for 5\% to $15 \%$ of LRTI hospitalizations in infants and young children and is second only to hRSV as a cause of bronchiolitis in early childhood [6-10]. Both viruses can be transmitted mainly by large droplets from infected individuals [11,12], and the incubation period could last between three and seven days for hRSV and four to six days for hMPV [13]. hRSV and hMPV are present year-round, but their incidence increases in the fall, peaks in the winter and goes down in early spring $[1,14,15]$. Host risk factors for these infections include premature birth, congenital heart disease, immunodeficiency, elderly individuals, gender and Down's syndrome, among others [16-18]. Adults and older children are commonly reinfected without complications by these paramyxoviruses, because the natural infection does not induce lifelong immunological protection. However, it is in infants, elderly and immunosuppressed 
individuals that severe disease can result [19-21]. Currently, there are no vaccines available against hRSV or hMPV. However, infected individuals can be treated with ribavirin and immunoglobulins [22].

Knowledge of the critical aspects of the host immune response to these infections has been crucial to understanding the pathology associated with hRSV and hMPV infections. In that regard, dendritic cells (DC) play a pivotal role in shaping antiviral immune response in the respiratory tract, as they represent the perfect link between innate and adaptive immune response [23-25]. Although the mechanisms underlying the activation of these cells by paramyxovirus infections still is largely unknown, substantial progress towards our understanding of the DC response to hRSV has been made, and the role of these cells in hMPV infection has also been explored.

\section{Dendritic Cells in the Respiratory Tract}

Dendritic cells are professional antigen-presenting cells within the immune system. They arise from both myeloid and lymphoid progenitors within the bone marrow and are widely distributed (as immature DC) into both lymphoid and nonlymphoid tissues [26-28]. Respiratory tract dendritic cells are present within airway epithelium, submucosa and associated lung parenchymal tissue under resting conditions [29]. In the absence of inflammation, lung DC are present at an average density of several hundred cells per square millimeter in the large airways, decreasing to less than a hundred DC per square millimeter within smaller intrapulmonary airways [30]. Pulmonary DC have a rapid turnover, with a half-life of $\leq 2$ days $[30,31]$. Their strategic localization at the site of pathogen entry makes them particularly susceptible to initial viral invasion. After detection, uptake and degradation of viruses, DC initiate immune responses via the secretion of interferon, chemokines and proinflammatory cytokines, as well as the upregulation of a variety of costimulatory molecules and receptors, a process globally known as cell maturation. After maturation, DC efficiently present antigens and initiate adaptive immune response by migrating into lymph nodes (LN) to activate the virus-specific T-cell response [32].

\section{Activation and Inhibition of DC Infected with hRSV and hMPV in Vitro}

\subsection{Maturation and Cytokine Production}

DC maturation is necessary for the transition from innate to adaptive immunity. In human monocyte-derived DC (moDC), hRSV and hMPV are able to induce cell maturation, indicated by an upregulation of MHC class I and class II molecules, as well as the overexpression of CD80, CD86, CD38 and CD83 [33-37]. Cytokine production by these cells is differentially induced by hRSV and hMPV, hRSV being a more potent inducer of IL-10, TNF- $\alpha$, IL-1 $\beta$ and IL-12p70, while hMPV induces a more robust response of type I IFN than hRSV. IFN induction is dependent of viral replication, as UV-inactivated virus failed to induce IFN in hRSV-or hMPV-infected cells [34-36,38]. The response of human primary blood myeloid DC to hRSV has also been characterized, and it resembles that of moDC [39]. hRSV also induces maturation in mouse DC. Bone marrow-derived DC (BMDC) exhibited an increased expression of CD40, CD80, CD86, MHC class I and class II and higher production of IL-6, IL-10 and IL-12p70. Consistent with reports in human cells, hRSV 
replication was required also for DC maturation [40]. However, DC are not highly permissive to hRSV or hMPV infections, regardless of their source (PBMCs, cord-blood or primary DC), as demonstrated by the low percentage ( $4 \%$ to $25 \%$ ) of antigen positive cells by flow cytometry analysis [33-37,39,41]. This suggests a critical role of the virus-induced cytokines, such as type I IFN, during the maturation process, as reported in BMDC [42]. Infection of plasmacytoid dendritic cells (pDC) by hRSV and hMPV induce differential expression of cytokines and chemokines. As in moDC, hRSV is a more potent inducer of IL-6, IL-10, GM-CSF, TNF- $\alpha$, IL-1 $\beta$, IL-12p70 and G-CSF than hMPV. However, both viruses induce a similar response of IFN- $\alpha$ release in infected pDC [33].

In understanding the mechanisms of activation and maturation by hRSV, Munir et al. [43] have reported that the NS proteins of hRSV suppress the expression of costimulatory molecules, as well as the secretion of cytokines and chemokines in human moDC in a type I IFN-dependent process. The inhibitory effect was mediated mostly by NS1 protein, but was enhanced by the combined deletion of NS2 in the same recombinant hRSV virus. More recently, Johnson et al. [44] reported that the interaction of hRSV G protein with the DC/L-SIGN (C-type lectins commonly found in DC) inhibits maturation of primary human DC. They found that when this interaction was neutralized with specific antibodies, RSV-infected myeloid DC and pDC increased both maturation and cytokine/chemokine production [44]. The role of cellular mechanisms critical for DC function has also been explored. Morris et al. [45] investigated the role of autophagy, a cellular mechanism that involves cell degradation of unnecessary or dysfunctional cellular components [46]. They found that cytokine production and the expression of surface markers (MHC class II, CD40, CD80 and CD86) was inhibited when autophagy was blocked in hRSV-infected BMDC, indicating that autophagy is a critical cellular process for DC maturation during hRSV infection [45].

\subsection{Activation and Inhibition of IFN Responses}

Interferons (IFNs) are a heterogeneous family of cytokines with demonstrated antiviral, antitumor and immunomodulatory activities. The IFN family includes type I (IFN- $\alpha,-\beta,-\varepsilon,-\kappa$ and $-\omega)$, type II (IFN- $\gamma$ ) and type III (IFN- $\lambda 1,-\lambda 2$ and $-\lambda 3$ ) IFNs $[47,48]$. Local production of IFNs plays an important defensive role in many respiratory virus infections by limiting viral replication until virus-specific host defense mechanisms develop [49]. It is known that hRSV and hMPV are able to induce and inhibit the IFN response in human and mouse DC. Moreover, they use different molecular mechanisms depending of the DC subset infected (Table 1). The induction of IFN production by RNA viruses is triggered by the activation by several pattern recognition receptors that recognize different viral components. Among them, the cytosolic RNA helicases, retinoic acid-inducible gene (RIG-I) and melanoma differentiation-associated gene 5 (MDA5) play a role in recognizing short or long dsRNA, respectively [50]. Other receptors include Toll-like receptors (TLR3 and TLR7) found in the endosomal compartment, which recognize dsRNA and ssRNA, respectively [51].

As previously reported, both hRSV and hMPV induce a robust response of IFN- $\alpha$ in human pDC, and this production is dependent on viral replication [33,38,52-54]. Using splenic mouse pDC, it has been further demonstrated that IFN production by hMPV is dependent on TLR7 expression [54]. 
Unlike in pDC, moDC produce IFN- $\alpha$ mostly after infection with hMPV, not with hRSV $[33,38]$. Using human moDC, previous results from my laboratory have demonstrated that the cytosolic helicase, MDA5, contributes to the production of type I and type III IFNs after hMPV infection. Those observations were further confirmed in an in vivo model of infection using MDA5 $5^{-/}$mice [55]. Activation of TLR4 has also been reported to contribute to the production of IFN- $\beta$ in moDC after hMPV infection [56].

Although the mechanisms underlying the differential activation of IFN response by hRSV and hMPV in moDC have not been fully elucidated, some experimental evidence indicates the involvement of several viral proteins. Munir et al. [43] demonstrated that the hRSV NS1 and, to a lesser extent, NS2 protein suppress the expression of IFN- $\alpha / \beta$ by using recombinant RSVs bearing deletions of the $\mathrm{NS} 1$ and/or NS2 protein [43]. In the case of hMPV, it is the expression of the protein $\mathrm{G}$ that is responsible for the reduced production of IFN- $\alpha / \beta$ in infected moDC [56].

hRSV and hMPV infections are also known to subvert the immune responses by interfering with DC functions. The modulating mechanisms of DC immunity by these viruses have been investigated in several in vitro systems, including human DC. It has been shown that hRSV and hMPV inhibit the production of IFN- $\alpha$ in human pDC in response to TLR7 and TL9 agonists [33,52] and in moDC after TLR3 activation [33]. In addition, the soluble form of the G protein of hRSV and hMPV has also been reported to block the IFN response after TLR activation in moDC [56,57].

Overall, these data indicate that hRSV and hMPV activate and inhibit the IFN responses in DC, most possibly through different mechanisms. This critical knowledge contributes to our understanding of the molecular mechanisms of hRSV and hMPV immunopathogenesis and may help to explain the lack of protective immunity after natural infection and the multiple reinfections by these viruses.

Table 1. Activation and inhibition of IFN responses by human respiratory syncytial virus (hRSV) and human metapneumovirus (hMPV) in dendritic cells (DC). pDC, plasmacytoid dendritic cells; moDC, monocyte-derived DC.

\begin{tabular}{cccc}
\hline Virus & Effect & DC Subset & References \\
\hline hRSV & Induce IFN- $\alpha$ & $\mathrm{pDC}$ & {$[33,38,52,53]$} \\
hRSV & Blocks TLR7 and TLR9 activation & $\mathrm{pDC}$ & {$[33,52]$} \\
hRSV & Blocks TLR3 activation & $\operatorname{moDC}$ & {$[33]$} \\
hRSV NS1 and NS2 & Suppress IFN- $\alpha / \beta$ production & $\operatorname{moDC}$ & {$[43]$} \\
hRSV soluble G & Blocks TLR3 and TLR4 activation & $\operatorname{moDC}$ & {$[57]$} \\
hMPV & Induce IFN- $\alpha$ & $\mathrm{pDC}$ & {$[33,54]$} \\
hMPV & Induce IFN- $\alpha, \beta, \lambda$ & $\operatorname{moDC}$ & {$[33,55]$} \\
hMPV & Activates MDA5 & moDC & {$[55]$} \\
hMPV & Activates TLR4 & moDC & {$[56]$} \\
hMPV & Activates TLR7 & pDC & {$[54]$} \\
hMPV & Blocks TLR7 activation & pDC & {$[33]$} \\
hMPV & Blocks TLR3 activation & moDC & {$[33]$} \\
hMPV G & Suppresses IFN- $\alpha / \beta$ production & moDC & {$[56]$} \\
\hline
\end{tabular}




\subsection{Regulation of T-Cell Responses}

Another aspect of the biology of hRSV and hMPV is that they reinfect throughout life, suggesting incomplete or transient immunity [19,58-60]. Several pieces of evidence indicate that hRSV and hMPV interact with dendritic cells and that the primary T-cell response to these viruses is altered significantly by this interaction. Human DC infected with hRSV have shown a severely impaired capacity to stimulate naive $\mathrm{CD}^{+} \mathrm{T}$-cell proliferation $[33,36,61]$. The possible mechanisms of this inhibition has been attributed to soluble factors in the supernatant of hRSV-infected dendritic cells [36], as well as to direct contact with RSV-infected cells to inhibit proliferation of T-cells [52,61]. In fact, Gonzalez et al. [40] demonstrated, in bone marrow-derived DC (BMDC), that immunological synapse assembly between hRSV-infected DC and T-cells was impaired, supporting the notion that contact is necessary for the inhibition of T-cell activation by hRSV infection [40]. Inhibition of T-cell proliferation by hMPV-infected DC, however, has also been attributed to soluble factors secreted by hMPV-infected DC, but not by interference with DC-T-cell immunological synapse formation [62]. Others have not observed this inhibitory effect using hRSV-infected cord blood-derived DC or moDC cultured with naive T-cells and superantigens $[37,63]$. Whether this discrepancy in the activation of the T-cells by hRSV-infected cells is related to the different experimental conditions remains to be determined. Further characterization of the interaction between T-cells and hRSV- and hMPV-infected $\mathrm{DC}$ is warranted.

\section{Response of Human Dendritic Cells to hRSV Infection in Vivo}

Current characterization of human lung DC populations includes three types of DC: two myeloid $\mathrm{DC}(\mathrm{mDC})$ in the lung parenchyma $\left(\mathrm{mDC} 1\left(\mathrm{BDCA}-1^{+} / \mathrm{HLA}-\mathrm{DR}{ }^{+}\right)\right.$and $\left.\mathrm{mDC} 2\left(\mathrm{CD} 11 \mathrm{c}^{+} / \mathrm{BDCA}^{-} 3^{+}\right)\right)$ and one pDC subset (CD11 ${ }^{-} / \mathrm{BDCA}^{-} 2^{+} / \mathrm{CD} 123^{+} / \mathrm{CD}^{-} 4^{-} / \mathrm{HLA}^{-D R^{+}}$) [64-66]. Additional reports have confirmed the presence of these lung DC subsets in human bronchoalveolar lavage samples [67,68]. However, further characterization of human lung DC populations, under the steady state and in response to stimulus, is needed in order to define the composition of human DC in the lung.

There have been a limited number of studies focused on the DC response to hRSV or hMPV infections in humans. In fact, the response of pulmonary DC in hMPV-infected individuals has not yet been reported. However, despite the human sample limitations, analyses of nasal washes from young children with acute hRSV infection have revealed that hRSV attracts both $\mathrm{mDC}$ and pDC to the site of viral entry. The number of $\mathrm{pDC}$, and, to a lesser extent, that of $\mathrm{mDC}$, positively correlates with the viral load in the infected individuals [69]. However, hRSV recruits DC to a lesser extent relative to other relevant respiratory viruses, such as influenza virus [70]. On the other hand, the numbers of $\mathrm{mDC}$ and $\mathrm{pDC}$ decreased in peripheral blood, suggesting that the increase of $\mathrm{mDC}$ and $\mathrm{pDC}$ in nasal mucosa results from their migration from the blood [69,70]. In line with those data, Silver E. et al. [71] has found that the lower levels of $\mathrm{pDC}$ in peripheral blood have been associated with asthma after severe hRSV bronchiolitis [71]. Overall, these data indicate a relevant role of DC in hRSV infection in humans. However, the better understanding of the response of DC to hRSV or hMPV has been 
revealed from in vitro experiments using human and mouse cells and from experimental animal models, specifically, the mouse model.

\section{Response of Pulmonary DC in Experimental Mouse Models after hRSV and hMPV Infection}

Because the study of the human DC at multiple stages of respiratory viral infections is technically and ethically difficult, the experimental mouse model has provided an excellent opportunity to investigate the response of DC in vivo. To date, there have been at least three major subsets of murine lung DC described. These include plasmacytoid DC (pDC), the myeloid DC (also known as conventional DC (cDC)) and the interferon-producing killer dendritic cells (IKDC) (Figure 2). All three DC populations have been reported to participate in the innate and adaptive immune response to hRSV and hMPV infections, indicating their critical role in the antiviral immunity to these viruses.

Figure 2. Mouse lung DC activated during hRSV and hMPV infection.

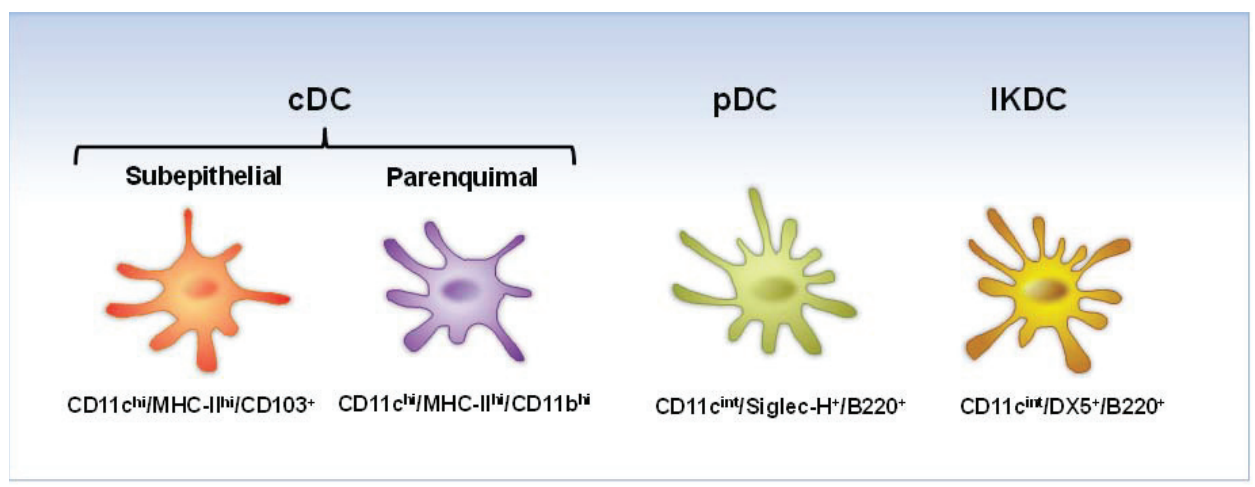

$\mathrm{pDC}$ are identified by the expression of $\mathrm{CD} 11 \mathrm{c}^{\mathrm{int}} / \mathrm{B} 220^{+} / \mathrm{Gr}-1^{+} / \mathrm{Siglec}-\mathrm{H}^{+} / \mathrm{mPDCA} 1^{+}$. They are best known for secreting large amounts of type I interferons (IFN) in response to viral infections [72]. Type I IFN confer resistance to viral infections and promote apoptosis of virally infected cells [73]. Also, type I IFN promote myeloid DC, B-cells, T-cells and natural killer (NK) cell functions [74,75]. Therefore, $\mathrm{pDC}$ regulate both innate and adaptive immune response in viral infections. $\mathrm{pDC}$ can also present endogenous viral antigens in their activated state to $\mathrm{CD} 4^{+} \mathrm{T}$-cells, but they are less efficient antigen-presenting cells, as compared to cDC [76]. Additionally, pDC are able to produce a variety of cytokines and chemokines that are important for the activation and trafficking of $\mathrm{CD} 4^{+}$and $\mathrm{CD} 8^{+}$ T-cells to the site of infection [77,78].

cDC are identified as major histocompatibility complex class II (MHC-II) $)^{\mathrm{hi}} / \mathrm{CD} 11 \mathrm{c}^{\mathrm{hi}}$. They are considered the main antigen-presenting cells of the immune system [26,79]. In mice, lung cDC comprise two major $\mathrm{CDC}$ subpopulations based on the expression of the integrin marker, CD103, and myeloid marker, CD11b, to give place to $\mathrm{MHC}-\mathrm{II}^{\mathrm{hi}} / \mathrm{CD} 11 \mathrm{c}^{\mathrm{hi}} / \mathrm{CD} 11 \mathrm{~b}^{-} / \mathrm{CD} 103^{+}$in the intraepithelial network and the parenchymal MHC-II ${ }^{\mathrm{hi}} / \mathrm{CD} 11 \mathrm{c}^{\mathrm{hi}} / \mathrm{CD} 11 \mathrm{~b}^{+} / \mathrm{CD} 103^{-} \mathrm{DC}$ [79]. These two cDC subsets differ in their relative abilities to prime $\mathrm{CD} 4^{+}$and $\mathrm{CD}^{+} \mathrm{T}$-cells [80], produce proinflammatory 
cytokines and generate Foxp3-mediated regulatory function of naive T-cells $[81,82]$. CD103 $\left(\alpha_{\mathrm{E}}\right)$ is the $\alpha$-chain of the $\alpha_{E} \beta_{7}$ integrin that mediates human and mouse T lymphocyte adhesion to epithelial cells through its binding to E-cadherin, which is selectively expressed on the basolateral side of epithelial cells [83]. The MHC-II ${ }^{\mathrm{hi}} / \mathrm{CD} 11 \mathrm{c}^{\mathrm{hi}} / \mathrm{CD} 11 \mathrm{~b}^{-} / \mathrm{CD} 103^{+} \mathrm{DC}$ are also known as migratory DC. $\mathrm{CD}_{103}{ }^{+} \mathrm{DC}$ can migrate to the draining lymph nodes (LN), produce IL-12 and are specialized in cross-presentation $[80,82]$. MHC-II ${ }^{\mathrm{hi}} / \mathrm{CD} 11 \mathrm{c}^{\mathrm{hi}} / \mathrm{CD} 11 \mathrm{~b}^{+} / \mathrm{CD} 103^{-} \mathrm{DC}$ are more efficient at presenting antigens on MHC II [84].

IKDC are characterized by the expression of CD $11 \mathrm{c}^{\mathrm{int}} / \mathrm{Gr}-1^{+} / \mathrm{DX} 5^{+}$or NK $1.1^{+}$. They are present in the lung and express cell surface markers of DC, as well as NK cell markers $[85,86]$. IKDC could be considered as NK-like DC or DC-like NK cells, playing a major role as a distinct population of innate effectors against viral pathogens [85,87,88]. However, their classification [85,86,88-90], origin [91] and physiological roles $[85,87,92,93]$ remain controversial.

More recently, the TNF- $\alpha$ /inducible nitric oxide synthase (iNOS)-producing DC (tipDC) have been identified and found to control viral infections in the lung [94]. However, their role in hRSV or hMPV has not yet been described. TipDC, also known as inflammatory DC or activated macrophages, contribute to the control of the antimicrobial defense and are responsible for severe tissue damage in several models of infection [94-97]. However, based on the overlapping phenotypes of myeloid cells, further characterization of lung tipDC is needed in order to eliminate the possibility that they represent myeloid cells in a transient maturation stage, in response to infection.

\subsection{Lung DC Trafficking}

Several studies have reported the trafficking and function of respiratory DC in response to hRSV or hMPV infection. Experimental evidence indicates that hRSV induces the recruitment of DC into the lungs and LN of BALB/c [98-100] and C57BL/6 [101] mice. In previous studies, I have observed that IKDC is the smallest DC subset recruited to the airways upon hRSV infection (two-fold) [98], followed by $\mathrm{pDC}$ (four-fold) and is the $\mathrm{CDC}$ the predominant $\mathrm{DC}$ population recruited to the lung after hRSV infection (20-fold) [98]. Some differences in the kinetics of pDC and IKDC into the lung of Hmpv-infected mice have been observed when compared to hRSV, as the recruitment of pDC and IKDC peaked by day eight after hMPV infection versus day three in hRSV-infected mice [98]. On the other hand, a similar trafficking pattern of cDC has been observed after hMPV infection when compared to hRSV [98], including a sustained recruitment of this cell population for about 18-21 days beyond the acute phase of infection [98,99]. It has also been found that $\mathrm{CD}_{103}{ }^{+} \mathrm{cDC}$ are substantially decreased after hRSV infection [98,101], and the same effect has been observed in the lungs of hMPV-infected mice in which $\mathrm{CD}_{103^{+}} \mathrm{cDC}$ decreased even after three weeks of infection and returned to basal levels by week eight [98]. By contrast, after either hRSV or hMPV infection, CD11b ${ }^{\text {hi }}$ cDC increased about four-fold in the lung of infected mice [98,101]. Furthermore, hRSV infection stimulates the mobilization of both populations of $\mathrm{CDC}$, as the numbers of $\mathrm{CD}_{103^{+}}$and $\mathrm{CD} 11 \mathrm{~b}^{\mathrm{hi}}$ are increased in the lung-draining mediastinal LN [101]. 


\subsection{Lung DC Activation}

Upon viral challenge, lung cDC are activated and acquire a mature phenotype [23,26,28,102]. In that regard, previous experimental observations indicate that hRSV or hMPV induce the overexpression of surface molecules, including CD40, CD80, CD86, PD-L1, OX-40L and MHC-II, in pulmonary $\mathrm{cDC}$ as early as day one after infection and remained activated until three weeks after infection [98,101,103]. Moreover, the profile of cytokines produced by pulmonary pDC and cDC, infected with hRSV or hMPV, differ substantially. cDC produce IL-10, IL-1 $\alpha$, IL-6, CXCL1 and CCL11, while pulmonary pDC do not. However, both lung cDC and pDC produce IFN- $\alpha$ after hMPV infection ex vivo, while infection with hRSV did not stimulate the release of the antiviral cytokine [98]. hRSV also induces maturation of lung pDC, as indicated by the overexpression of CD80, CD86 and MHC class II molecules [104].

\subsection{Lung pDC Function in hRSV Infection}

It has been demonstrated that the balance between the numbers of $\mathrm{pDC}$ and $\mathrm{cDC}$ in the lung is important for the regulation of the immune response against hRSV. Smit et al. [105] demonstrated that when both $\mathrm{pDC}$ and $\mathrm{cDC}$ populations were expanded in hRSV-infected mice, that resulted in a decreased $\mathrm{Th} 2$ cell response, but an increased Th1 response and lower immunopathology. However, by depleting $\mathrm{pDC}$ and expanding $\mathrm{cDC}$, the T-cell response was skewed towards Th2, resulting in an exacerbated inflammatory response.

The specific role of pulmonary pDC in hRSV infection has also been explored by several other groups $[100,104,106]$. To this end, pDC have also been depleted with the monoclonal antibody (mAb), 120G8, that recognizes the murine surface antigen, CD317 (BST-2; mPDCA1), in several mouse strains [107]. Some studies have reported that the inflammatory response and the airway resistance was substantially exacerbated, and the lung viral titer was increased $[100,104]$, suggesting that pDC play a protective role during hRSV infection. However, it is important to consider that, despite BST-2 antigen being expressed predominantly on $\mathrm{pDC}$ in naive mice, it is known that after viral infection or stimulation with type I or type II IFN, BST-2 is induced on most cell types [108]. Therefore, this fact should be taken into consideration for the interpretation of these studies. On the hand, it seems that pDC do not contribute significantly to the production of type I IFN in vivo in hRSV infection, as the levels of IFN- $\alpha$ and IFN- $\beta$ remained unchanged in hRSV-infected mice after successful depletion of pDC [106]. In fact, it has been reported that alveolar macrophages are the primary IFN- $\alpha$ producer in lung infections by RNA viruses [109]. In support to that, Pribul et al. [110] has demonstrated that alveolar macrophages significantly contribute to the production of IFN- $\alpha$ in hRSV infection. As for the role of DC subsets in hMPV infection, there are no reports exploring the contribution of these cells in the hMPV-induced immune response. Therefore, future experiments aimed to determine the role of DC subpopulations in hMPV-infected mice are needed to understand the contribution of these cells in hMPV-induced immune response. 


\subsection{Impairment of Mouse Lung DC Response in hRSV and hMPV}

Infection by hRSV and hMPV is characterized by short-lasting virus specific immunity and, often, long-term airway morbidity. Previous studies have revealed that hRSV or hMPV impair the capacity of human DC to present antigens to T-cells in vitro [33,101,111]. That detrimental effect has also been observed in experiments in vivo using the mouse model [98]. In that system, I have previously observed that, when compared with $\mathrm{cDC}$ from mock-infected mice, lung cDC from mice infected with hRSV or hMPV have an impaired capacity to present antigens to $\mathrm{CD} 4{ }^{+} \mathrm{T}$-cells that lasted beyond the acute phase of infection, suggesting that acute pneumovirus and metapneumovirus infections can alter the long-term immune function of pulmonary DC. Moreover, that inhibitory effect seems to be selective for lung cells, since that inhibitory effect was not observed when spleen cDC from the same infected mice were used [98]. The mechanisms by which hRSV and hMPV impair cDC function are largely unknown. However, one of the surface molecules that was upregulated after viral infection in lung cDC was programmed death-1 ligand (PD-L1) [98], which is known to inhibit some T-cell functions, including T-cell proliferation [112-114], suggesting that this molecule may play a role in the impaired capacity of cDC to present antigens to T-cells after hRSV and hMPV infection.

The production of type I IFN and other cytokines by lung DC is also altered by hRSV and hMPV infection in vivo [98]. I have previously observed that lung $\mathrm{pDC}$ isolated from infected mice produced significantly lower levels of IFN- $\alpha$, IL-6, TNF- $\alpha$, CCL3, CCL4 and CCL5 in response to CpG ODN [98], indicating that both viruses are able to interfere with the capacity of pulmonary pDC to mount an antiviral response in response to a secondary stimulus. Age is also a factor that negatively impacts DC response in the lung. The recruitment of DC after hRSV infection can be impaired by the age of the infected individuals, as shown by Zhao et al. in aged mice (6-22 months-old) where the ability of lung DC to migrate to LN was compromised in hRSV-infected aged mice, with a decline in migration occurring as early as six months of age [115].

\subsection{Contribution Lung DC in Vaccine Development}

Data in vivo using the C57BL/6 mouse model of hRSV infection have indicated that after $\mathrm{hRSV}$ intranasal challenge to formalin-inactivated RSV (FI-RSV)-immunized mice, the numbers of CD $11 \mathrm{~b}^{+}$ and $\mathrm{CD}_{103}{ }^{+} \mathrm{cDC}$ recruited into the lung are increased [116]. Considering that differences between these DC populations in priming the T-cell responses exist [80] and that the balance between the numbers of DC subsets influences the $\mathrm{CD}^{+} \mathrm{Th} 1 / \mathrm{Th} 2$ responses in the lung [105], the development of hRSV and hMPV vaccines should consider the characterization of the lung DC subsets response and their contribution to prime an immune response against these viral infections, which will contribute toward the better design of an effective vaccine against these respiratory viral infections.

\section{Conclusions}

Lung DC participate in the innate and adaptive immune response to hRSV and hMPV infections, indicating their critical role in the antiviral immunity to these paramyxoviruses. hRSV and hMPV can 
induce similar DC responses, as DC can be activated by both hRSV and hMPV infections in vivo. In addition, both viruses can induce the maturation and trafficking of the different DC populations from lung to LN. Moreover, they interfere with the T-cell response, as the antigen-presenting capacity of pulmonary DC to T-cells is impaired after hRSV or hMPV infection, which may contribute to the lack of protection and multiple reinfections by these viruses. On the other hand, hRSV and hMPV differentially induce the production of type I IFN in lung DC, as hMPV is a more potent inducer of the antiviral cytokine.

Although many aspects of the immune mechanisms involving DC in hRSV infection have not been elucidated, considerable progress has been made with respect to our understanding of the role of pulmonary DC in hRSV infection. However, less is known regarding the interaction of hMPV with the lung DC, and in general, the mechanisms that regulate the host immune response to hMPV infection remain largely unknown. Additional studies are necessary to better understand the mechanisms that regulate the DC response in hRSV and hMPV infections.

\section{Acknowledgments}

This work was supported by Grants from the National Institute of Allergy and Infectious Diseases AI081171, the National Center for Research Resources 5P20RR020159-09 and the National Institute of General Medical Sciences 8P20GM103458-09 from the National Institutes of Health and the Flight Attendant Medical Research Institute YCSA grant. The author thanks Dawn Simms for critical reading of the manuscript.

\section{Conflicts of Interest}

The author declares no conflict of interest.

\section{References}

1. Collins PL; Crowe, J. Respiratory Syncytial Virus and Metapneumovirus. In Fileds Virology, 5th ed.; Knipe, D.M., Howley, P.M., Eds.; Wolters Kluwer: Philadelphia, PA, USA, 2007; Volume 2, pp. 1601-1646.

2. Van den Hoogen, B.G.; de Jong, J.C.; Groen, J.; Kuiken, T.; de Groot, R.; Fouchier, R.A.; Osterhaus, A.D. A newly discovered human pneumovirus isolated from young children with respiratory tract disease. Nat. Med. 2001, 7, 719-724.

3. Domachowske, J.B.; Rosenberg, H.F. Respiratory syncytial virus infection: Immune response, immunopathogenesis, and treatment. Clin. Microbiol. Rev. 1999, 12, 298-309.

4. Easton, A.J.; Domachowske, J.B.; Rosenberg, H.F. Animal pneumoviruses: Molecular genetics and pathogenesis. Clin. Microbiol. Rev. 2004, 17, 390-412.

5. Blount, R.E., Jr.; Morris, J.A.; Savage, R.E. Recovery of cytopathogenic agent from chimpanzees with coryza. Proc. Soc. Exp. Biol. Med. 1956, 92, 544-549. 
6. Boivin, G.; de Serres, G.; Cote, S.; Gilca, R.; Abed, Y.; Rochette, L.; Bergeron, M.G.; Dery, P. Human metapneumovirus infections in hospitalized children. Emerg. Infect. Dis. 2003, 9, 634-640.

7. Mullins, J.A.; Erdman, D.D.; Weinberg, G.A.; Edwards, K.; Hall, C.B.; Walker, F.J.; Iwane, M.; Anderson, L.J. Human metapneumovirus infection among children hospitalized with acute respiratory illness. Emerg. Infect. Dis. 2004, 10, 700-705.

8. Van den Hoogen, B.G.; van Doornum, G.J.; Fockens, J.C.; Cornelissen, J.J.; Beyer, W.E.; de Groot, R.; Osterhaus, A.D.; Fouchier, R.A. Prevalence and clinical symptoms of human metapneumovirus infection in hospitalized patients. J. Infect. Dis. 2003, 188, 1571-1577.

9. Williams, J.V.; Harris, P.A.; Tollefson, S.J.; Halburnt-Rush, L.L.; Pingsterhaus, J.M.; Edwards, K.M.; Wright, P.F.; Crowe, J.E., Jr. Human metapneumovirus and lower respiratory tract disease in otherwise healthy infants and children. N. Engl. J. Med. 2004, 350, 443-450.

10. Kahn, J.S. Epidemiology of human metapneumovirus. Clin. Microbiol. Rev. 2006, 19, 546-557.

11. Hall, C.B.; McCarthy, C.A. Respiratory Syncytial Virus. In Principles and Practice of Infectious Diseases; Mandel, G.L., Bennett, J.E., Dolin, R., Eds.; Churchill Livingston: New York, NY, USA, 1995; Volume 4, p. 1501.

12. Hall, C.B.; Douglas, R.G., Jr. Modes of transmission of respiratory syncytial virus. J. Pediatr. 1981, 99, 100-103.

13. Lessler, J.; Reich, N.G.; Brookmeyer, R.; Perl, T.M.; Nelson, K.E.; Cummings, D.A. Incubation periods of acute respiratory viral infections: A systematic review. Lancet Infect. Dis. 2009, 9, 291-300.

14. Welliver, R.C. Respiratory syncytial virus and other respiratory viruses. Pediatr. Infect. Dis. J. 2003, 22, S6-S10; discussion S10-S12.

15. Hermos, C.R.; Vargas, S.O.; McAdam, A.J. Human metapneumovirus. Clin. Lab. Med. 2010, 30, 131-148.

16. Papenburg, J.; Hamelin, M.E.; Ouhoummane, N.; Carbonneau, J.; Ouakki, M.; Raymond, F.; Robitaille, L.; Corbeil, J.; Caouette, G.; Frenette, L.; et al. Comparison of risk factors for human metapneumovirus and respiratory syncytial virus disease severity in young children. J. Infect. Dis. 2012, 206, 178-189.

17. Papenburg, J.; Boivin, G. The distinguishing features of human metapneumovirus and respiratory syncytial virus. Rev. Med. Virol. 2010, 20, 245-260.

18. Van Drunen Littel-van den Hurk, S.; Watkiss, E.R. Pathogenesis of respiratory syncytial virus. Curr. Opin. Virol. 2012, 2, 300-305.

19. Pavlin, J.A.; Hickey, A.C.; Ulbrandt, N.; Chan, Y.P.; Endy, T.P.; Boukhvalova, M.S.; Chunsuttiwat, S.; Nisalak, A.; Libraty, D.H.; Green, S.; et al. Human metapneumovirus reinfection among children in Thailand determined by ELISA using purified soluble fusion protein. J. Infect. Dis. 2008, 198, 836-842.

20. Ebihara, T.; Endo, R.; Ishiguro, N.; Nakayama, T.; Sawada, H.; Kikuta, H. Early reinfection with human metapneumovirus in an infant. J. Clin. Microbiol. 2004, 42, 5944-5946. 
21. Ohuma, E.O.; Okiro, E.A.; Ochola, R.; Sande, C.J.; Cane, P.A.; Medley, G.F.; Bottomley, C.; Nokes, D.J. The natural history of respiratory syncytial virus in a birth cohort: The influence of age and previous infection on reinfection and disease. Am. J. Epidemiol. 2012, 176, 794-802.

22. Graham, B.S. Biological challenges and technological opportunities for respiratory syncytial virus vaccine development. Immunol. Rev. 2011, 239, 149-166.

23. Steinman, R.M. Decisions about dendritic cells: Past, present, and future. Annu. Rev. Immunol. 2012, 30, 1-22.

24. Grayson, M.H.; Holtzman, M.J. Emerging role of dendritic cells in respiratory viral infection. J. Mol. Med. 2007, 85, 1057-1068.

25. Reis e Sousa, C. Activation of dendritic cells: Translating innate into adaptive immunity. Curr. Opin. Immunol. 2004, 16, 21-25.

26. Banchereau, J.; Briere, F.; Caux, C.; Davoust, J.; Lebecque, S.; Liu, Y.T.; Pulendran, B.; Palucka, K. Immunobiology of dendritic cells. Ann. Rev. Immunol. 2000, 18, 767-811.

27. Pulendran, B.; Palucka, K.; Banchereau, J. Sensing pathogens and tuning immune responses. Science 2001, 293, 253-256.

28. Steinman, R.M.; Cohn, Z.A. Identification of a novel cell type in peripheral lymphoid organs of mice. I. Morphology, quantitation, tissue distribution. J. Exp. Med. 1973, 137, 1142-1162.

29. Stumbles, P.A.; Upham, J.W.; Holt, P.G. Airway dendritic cells: Co-ordinators of immunological homeostasis and immunity in the respiratory tract. APMIS 2003, 111, 741-755.

30. Schon-Hegrad, M.A.; Oliver, J.; McMenamin, P.G.; Holt, P.G. Studies on the density, distribution, and surface phenotype of intraepithelial class II major histocompatibility complex antigen (Ia)-bearing dendritic cells (DC) in the conducting airways. J. Exp. Med. 1991, 173, 1345-1356.

31. McWilliam, A.S.; Napoli, S.; Marsh, A.M.; Pemper, F.L.; Nelson, D.J.; Pimm, C.L.; Stumbles, P.A.; Wells, T.N.; Holt, P.G. Dendritic cells are recruited into the airway epithelium during the inflammatory response to a broad spectrum of stimuli. J. Exp. Med. 1996, 184, 2429-2432.

32. Manicassamy, S.; Pulendran, B. Dendritic cell control of tolerogenic responses. Immunol. Rev. 2011, 241, 206-227.

33. Guerrero-Plata, A.; Casola, A.; Suarez, G.; Yu, X.; Spetch, L.; Peeples, M.E.; Garofalo, R.P. Differential response of dendritic cells to human metapneumovirus and respiratory syncytial virus. Am. J. Respir. Cell Mol. Biol. 2006, 34, 320-329.

34. Le Nouen, C.; Munir, S.; Losq, S.; Winter, C.C.; McCarty, T.; Stephany, D.A.; Holmes, K.L.; Bukreyev, A.; Rabin, R.L.; Collins, P.L.; et al. Infection and maturation of monocyte-derived human dendritic cells by human respiratory syncytial virus, human metapneumovirus, and human parainfluenza virus type 3. Virology 2009, 385, 169-182.

35. Jones, A.; Morton, I.; Hobson, L.; Evans, G.S.; Everard, M.L. Differentiation and immune function of human dendritic cells following infection by respiratory syncytial virus. Clin. Exp. Immunol. 2006, 143, 513-522. 
36. De Graaff, P.M.; de Jong, E.C.; van Capel, T.M.; van Dijk, M.E.; Roholl, P.J.; Boes, J.; Luytjes, W.; Kimpen, J.L.; van Bleek, G.M. Respiratory syncytial virus infection of monocyte-derived dendritic cells decreases their capacity to activate CD4 T cells. J. Immunol. 2005, 175, 5904-5911.

37. Bartz, H.; Turkel, O.; Hoffjan, S.; Rothoeft, T.; Gonschorek, A.; Schauer, U. Respiratory syncytial virus decreases the capacity of myeloid dendritic cells to induce interferon-gamma in naive T cells. Immunology 2003, 109, 49-57.

38. Hornung, V.; Schlender, J.; Guenthner-Biller, M.; Rothenfusser, S.; Endres, S.; Conzelmann, K.K.; Hartmann, G. Replication-dependent potent IFN-alpha induction in human plasmacytoid dendritic cells by a single-stranded RNA virus. J. Immunol. 2004, 173, 5935-5943.

39. Johnson, T.R.; Johnson, C.N.; Corbett, K.S.; Edwards, G.C.; Graham, B.S. Primary human $\mathrm{mDC} 1, \mathrm{mDC} 2$, and $\mathrm{pDC}$ dendritic cells are differentially infected and activated by respiratory syncytial virus. PLoS One 2011, 6, e16458.

40. Gonzalez, P.A.; Prado, C.E.; Leiva, E.D.; Carreno, L.J.; Bueno, S.M.; Riedel, C.A.; Kalergis, A.M. Respiratory syncytial virus impairs $\mathrm{T}$ cell activation by preventing synapse assembly with dendritic cells. Proc. Natl. Acad. Sci. USA 2008, 105, 14999-15004.

41. Bartz, H.; Buning-Pfaue, F.; Turkel, O.; Schauer, U. Respiratory syncytial virus induces prostaglandin E2, IL-10 and IL-11 generation in antigen presenting cells. Clin. Exp. Immunol. 2002, 129, 438-445.

42. Rudd, B.D.; Luker, G.D.; Luker, K.E.; Peebles, R.S.; Lukacs, N.W. Type I interferon regulates respiratory virus infected dendritic cell maturation and cytokine production. Viral Immunol. 2007, 20, 531-540.

43. Munir, S.; Le, N.C.; Luongo, C.; Buchholz, U.J.; Collins, P.L.; Bukreyev, A. Nonstructural proteins 1 and 2 of respiratory syncytial virus suppress maturation of human dendritic cells. J. Virol. 2008, 82, 8780-8796.

44. Johnson, T.R.; McLellan, J.S.; Graham, B.S. Respiratory syncytial virus glycoprotein G interacts with DC-SIGN and L-SIGN to activate ERK1 and ERK2. J. Virol. 2012, 86, 1339-1347.

45. Morris, S.; Swanson, M.S.; Lieberman, A.; Reed, M.; Yue, Z.; Lindell, D.M.; Lukacs, N.W. Autophagy-mediated dendritic cell activation is essential for innate cytokine production and APC function with respiratory syncytial virus responses. J. Immunol. 2011, 187, 3953-3961.

46. Kundu, M.; Thompson, C.B. Autophagy: Basic principles and relevance to disease. Ann. Rev. Pathol. 2008, 3, 427-455.

47. Ank, N.; Paludan, S.R. Type III IFNs: New layers of complexity in innate antiviral immunity. Biofactors 2009, 35, 82-87.

48. Piehler, J.; Thomas, C.; Garcia, K.C.; Schreiber, G. Structural and dynamic determinants of type I interferon receptor assembly and their functional interpretation. Immunol. Rev. 2012, 250, 317-334.

49. Garcia-Sastre, A.; Biron, C.A. Type 1 interferons and the virus-host relationship: A lesson in detente. Science 2006, 312, 879-882. 
50. Takeuchi, O.; Akira, S. MDA5/RIG-I and virus recognition. Curr. Opin. Immunol. 2008, 20, 17-22.

51. Baum, A.; Garcia-Sastre, A. Induction of type I interferon by RNA viruses: Cellular receptors and their substrates. Amino Acids 2009, 38, 1283-1299.

52. Schlender, J.; Hornung, V.; Finke, S.; Gunthner-Biller, M.; Marozin, S.; Brzozka, K.; Moghim, S.; Endres, S.; Hartmann, G.; Conzelmann, K.K. Inhibition of toll-like receptor 7- and 9-mediated alpha/beta interferon production in human plasmacytoid dendritic cells by respiratory syncytial virus and measles virus. J. Virol. 2005, 79, 5507-5515.

53. Castro, S.M.; Chakraborty, K.; Guerrero-Plata, A. Cigarette smoke suppresses TLR-7 stimulation in response to virus infection in plasmacytoid dendritic cells. Toxicol. Vitro 2011, 25, 1106-1113.

54. Goutagny, N.; Jiang, Z.; Tian, J.; Parroche, P.; Schickli, J.; Monks, B.G.; Ulbrandt, N.; Ji, H.; Kiener, P.A.; Coyle, A.J.; et al. Cell type-specific recognition of human metapneumoviruses (HMPVs) by retinoic acid-inducible gene I (RIG-I) and TLR7 and viral interference of RIG-I ligand recognition by HMPV-B1 phosphoprotein. J. Immunol. 2010, 184, 1168-1179.

55. Banos-Lara Mdel, R.; Ghosh, A.; Guerrero-Plata, A. Critical role of MDA5 in the interferon response induced by human metapneumovirus infection in dendritic cells and in vivo. J. Virol. 2013, 87, 1242-1251.

56. Kolli, D.; Bao, X.; Liu, T.; Hong, C.; Wang, T.; Garofalo, R.P.; Casola, A. Human metapneumovirus glycoprotein G inhibits TLR4-dependent signaling in monocyte-derived dendritic cells. J. Immunol. 2011, 187, 47-54.

57. Shingai, M.; Azuma, M.; Ebihara, T.; Sasai, M.; Funami, K.; Ayata, M.; Ogura, H.; Tsutsumi, H.; Matsumoto, M.; Seya, T. Soluble G protein of respiratory syncytial virus inhibits Toll-like receptor 3/4-mediated IFN-beta induction. Int. Immunol. 2008, 20, 1169-1180.

58. Henderson, F.W.; Collier, A.M.; Clyde, W.A., Jr.; Denny, F.W. Respiratory-syncytial-virus infections, reinfections and immunity. A prospective, longitudinal study in young children. N. Engl. J. Med. 1979, 300, 530-534.

59. Falsey, A.R.; Walsh, E.E. Viral pneumonia in older adults. Clin. Infect. Dis. 2006, 42, 518-524.

60. Hall, C.B.; Walsh, E.E.; Long, C.E.; Schnabel, K.C. Immunity to and frequency of reinfection with respiratory syncytial virus. J. Infect. Dis. 1991, 163, 693-698.

61. Rothoeft, T.; Fischer, K.; Zawatzki, S.; Schulz, V.; Schauer, U.; Korner Rettberg, C. Differential response of human naive and memory/effector $\mathrm{T}$ cells to dendritic cells infected by respiratory syncytial virus. Clin. Exp. Immunol. 2007, 150, 263-273.

62. Cespedes, P.F.; Gonzalez, P.A.; Kalergis, A.M. Human metapneumovirus keeps dendritic cells from priming antigen-specific naive T cells. Immunology 2013, 139, 366-376.

63. Le Nouen, C.; Hillyer, P.; Munir, S.; Winter, C.C.; McCarty, T.; Bukreyev, A.; Collins, P.L.; Rabin, R.L.; Buchholz, U.J. Effects of human respiratory syncytial virus, metapneumovirus, parainfluenza virus 3 and influenza virus on CD4+ T cell activation by dendritic cells. PLoS One 2010, 5, e15017. 
64. Demedts, I.K.; Brusselle, G.G.; Vermaelen, K.Y.; Pauwels, R.A. Identification and characterization of human pulmonary dendritic cells. Am. J. Respir. Cell Mol. Biol. 2005, 32, 177-184.

65. Masten, B.J.; Olson, G.K.; Tarleton, C.A.; Rund, C.; Schuyler, M.; Mehran, R.; Archibeque, T.; Lipscomb, M.F. Characterization of myeloid and plasmacytoid dendritic cells in human lung. J. Immunol. 2006, 177, 7784-7793.

66. Condon, T.V.; Sawyer, R.T.; Fenton, M.J.; Riches, D.W. Lung dendritic cells at the innate-adaptive immune interface. J. Leukoc. Biol. 2011, 90, 883-895.

67. Bratke, K.; Lommatzsch, M.; Julius, P.; Kuepper, M.; Kleine, H.D.; Luttmann, W.; Christian Virchow, J. Dendritic cell subsets in human bronchoalveolar lavage fluid after segmental allergen challenge. Thorax 2007, 62, 168-175.

68. Lommatzsch, M.; Bratke, K.; Bier, A.; Julius, P.; Kuepper, M.; Luttmann, W.; Virchow, J.C. Airway dendritic cell phenotypes in inflammatory diseases of the human lung. Eur. Respir. J. 2007, 30, 878-886.

69. Gill, M.A.; Palucka, A.K.; Barton, T.; Ghaffar, F.; Jafri, H.; Banchereau, J.; Ramilo, O. Mobilization of plasmacytoid and myeloid dendritic cells to mucosal sites in children with respiratory syncytial virus and other viral respiratory infections. J. Infect. Dis. 2005, 191, 1105-1115.

70. Gill, M.A.; Long, K.; Kwon, T.; Muniz, L.; Mejias, A.; Connolly, J.; Roy, L.; Banchereau, J.; Ramilo, O. Differential recruitment of dendritic cells and monocytes to respiratory mucosal sites in children with influenza virus or respiratory syncytial virus infection. J. Infect. Dis. 2008, 198, 1667-1676.

71. Silver, E.; Yin-DeClue, H.; Schechtman, K.B.; Grayson, M.H.; Bacharier, L.B.; Castro, M. Lower levels of plasmacytoid dendritic cells in peripheral blood are associated with a diagnosis of asthma $6 \mathrm{yr}$ after severe respiratory syncytial virus bronchiolitis. Pediatr. Allergy Immunol. 2009, 20, 471-476.

72. Gilliet, M.; Cao, W.; Liu, Y.J. Plasmacytoid dendritic cells: Sensing nucleic acids in viral infection and autoimmune diseases. Nat. Rev. Immunol. 2008, 8, 594-606.

73. Honda, K.; Yanai, H.; Takaoka, A.; Taniguchi, T. Regulation of the type I IFN induction: A current view. Int. Immunol. 2005, 17, 1367-1378.

74. Colonna, M.; Trinchieri, G.; Liu, Y.J. Plasmacytoid dendritic cells in immunity. Nat. Immunol. 2004, 5, 1219-1226.

75. Swiecki, M.; Colonna, M. Unraveling the functions of plasmacytoid dendritic cells during viral infections, autoimmunity, and tolerance. Immunol. Rev. 2010, 234, 142-162.

76. Young, L.J.; Wilson, N.S.; Schnorrer, P.; Proietto, A.; ten Broeke, T.; Matsuki, Y.; Mount, A.M.; Belz, G.T.; O'Keeffe, M.; Ohmura-Hoshino, M.; et al. Differential MHC class II synthesis and ubiquitination confers distinct antigen-presenting properties on conventional and plasmacytoid dendritic cells. Nat. Immunol. 2008, 9, 1244-1252.

77. Sozzani, S.; Vermi, W.; Del Prete, A.; Facchetti, F. Trafficking properties of plasmacytoid dendritic cells in health and disease. Trends Immunol. 2010, 31, 270-277. 
78. Villadangos, J.A.; Young, L. Antigen-presentation properties of plasmacytoid dendritic cells. Immunity 2008, 29, 352-361.

79. Lambrecht, B.N.; Hammad, H. Biology of lung dendritic cells at the origin of asthma. Immunity 2009, 31, 412-424.

80. Del Rio, M.L.; Rodriguez-Barbosa, J.I.; Kremmer, E.; Forster, R. CD103- and CD103+ bronchial lymph node dendritic cells are specialized in presenting and cross-presenting innocuous antigen to CD4+ and CD8+ T cells. J. Immunol. 2007, 178, 6861-6866.

81. Coombes, J.L.; Siddiqui, K.R.; Arancibia-Carcamo, C.V.; Hall, J.; Sun, C.M.; Belkaid, Y.; Powrie, F. A functionally specialized population of mucosal CD103+ DCs induces Foxp3+ regulatory $\mathrm{T}$ cells via a TGF-beta and retinoic acid-dependent mechanism. J. Exp. Med. 2007, 204, 1757-1764.

82. Del Rio, M.L.; Bernhardt, G.; Rodriguez-Barbosa, J.I.; Forster, R. Development and functional specialization of CD103+ dendritic cells. Immunol. Rev. 2010, 234, 268-281.

83. Cepek, K.L.; Shaw, S.K.; Parker, C.M.; Russell, G.J.; Morrow, J.S.; Rimm, D.L.; Brenner, M.B. Adhesion between epithelial cells and T lymphocytes mediated by E-cadherin and the alpha $\mathrm{E}$ beta 7 integrin. Nature 1994, 372, 190-193.

84. Dudziak, D.; Kamphorst, A.O.; Heidkamp, G.F.; Buchholz, V.R.; Trumpfheller, C.; Yamazaki, S.; Cheong, C.; Liu, K.; Lee, H.W.; Park, C.G.; et al. Differential antigen processing by dendritic cell subsets in vivo. Science 2007, 315, 107-111.

85. Chan, C.W.; Crafton, E.; Fan, H.N.; Flook, J.; Yoshimura, K.; Skarica, M.; Brockstedt, D.; Dubensky, T.W.; Stins, M.F.; Lanier, L.L.; et al. Interferon-producing killer dendritic cells provide a link between innate and adaptive immunity. Nat. Med. 2006, 12, 207-213.

86. Taieb, J.; Chaput, N.; Menard, C.; Apetoh, L.; Ullrich, E.; Bonmort, M.; Pequignot, M.; Casares, N.; Terme, M.; Flament, C.; et al. A novel dendritic cell subset involved in tumor immunosurveillance. Nat. Med. 2006, 12, 214-219.

87. Bonmort, M.; Dalod, M.; Mignot, G.; Ullrich, E.; Chaput, N.; Zitvogel, L. Killer dendritic cells: IKDC and the others. Curr. Opin. Immunol. 2008, 20, 558-565.

88. Chauvin, C.; Josien, R. Dendritic cells as killers: Mechanistic aspects and potential roles. J. Immunol. 2008, 181, 11-16.

89. Blasius, A.L.; Barchet, W.; Cella, M.; Colonna, M. Development and function of murine B220+CD11c+NK1.1+ cells identify them as a subset of NK cells. J. Exp. Med. 2007, 204, 2561-2568.

90. Vosshenrich, C.A.; Lesjean-Pottier, S.; Hasan, M.; Richard-Le Goff, O.; Corcuff, E.; Mandelboim, O.; di Santo, J.P. CD11cloB220+ interferon-producing killer dendritic cells are activated natural killer cells. J. Exp. Med. 2007, 204, 2569-2578.

91. Welner, R.S.; Pelayo, R.; Garrett, K.P.; Chen, X.; Perry, S.S.; Sun, X.H.; Kee, B.L.; Kincade, P.W. Interferon-producing killer dendritic cells (IKDCs) arise via a unique differentiation pathway from primitive c-kitHiCD62L+ lymphoid progenitors. Blood 2007, 109, 4825-4931. 
92. Caminschi, I.; Ahmet, F.; Heger, K.; Brady, J.; Nutt, S.L.; Vremec, D.; Pietersz, S.; Lahoud, M.H.; Schofield, L.; Hansen, D.S.; et al. Putative IKDCs are functionally and developmentally similar to natural killer cells, but not to dendritic cells. J. Exp. Med. 2007, 204, 2579-2590.

93. Spits, H.; Lanier, L.L. Natural killer or dendritic: What's in a name? Immunity 2007, 26, 11-16.

94. Aldridge, J.R., Jr.; Moseley, C.E.; Boltz, D.A.; Negovetich, N.J.; Reynolds, C.; Franks, J.; Brown, S.A.; Doherty, P.C.; Webster, R.G.; Thomas, P.G. TNF/iNOS-producing dendritic cells are the necessary evil of lethal influenza virus infection. Proc. Natl. Acad. Sci. USA 2009, 106, 5306-5311.

95. Serbina, N.V.; Salazar-Mather, T.P.; Biron, C.A.; Kuziel, W.A.; Pamer, E.G. TNF/iNOS-producing dendritic cells mediate innate immune defense against bacterial infection. Immunity 2003, 19, 59-70.

96. Bosschaerts, T.; Guilliams, M.; Stijlemans, B.; Morias, Y.; Engel, D.; Tacke, F.; Herin, M.; de Baetselier, P.; Beschin, A. Tip-DC development during parasitic infection is regulated by IL-10 and requires CCL2/CCR2, IFN-gamma and MyD88 signaling. PLoS Pathog. 2010, 6, e1001045.

97. Hespel, C.; Moser, M. Role of inflammatory dendritic cells in innate and adaptive immunity. Eur. J. Immunol. 2012, 42, 2535-2543.

98. Guerrero-Plata, A.; Kolli, D.; Hong, C.; Casola, A.; Garofalo, R.P. Subversion of pulmonary dendritic cell function by paramyxovirus infections. J. Immunol. 2009, 182, 3072-3083.

99. Beyer, M.; Bartz, H.; Horner, K.; Doths, S.; Koerner-Rettberg, C.; Schwarze, J. Sustained increases in numbers of pulmonary dendritic cells after respiratory syncytial virus infection. J. Allergy Clin. Immunol. 2004, 113, 127-133.

100. Smit, J.J.; Rudd, B.D.; Lukacs, N.W. Plasmacytoid dendritic cells inhibit pulmonary immunopathology and promote clearance of respiratory syncytial virus. J. Exp. Med. 2006, 203, 1153-1159.

101. Lukens, M.V.; Kruijsen, D.; Coenjaerts, F.E.; Kimpen, J.L.; van Bleek, G.M. Respiratory syncytial virus-induced activation and migration of respiratory dendritic cells and subsequent antigen presentation in the lung-draining lymph node. J. Virol. 2009, 83, 7235-7243.

102. Mellman, I.; Steinman, R.M. Dendritic cells: Specialized and regulated antigen processing machines. Cell 2001, 106, 255-258.

103. Wythe, S.E.; Dodd, J.S.; Openshaw, P.J.; Schwarze, J. OX40 ligand and programmed cell death 1 ligand 2 expression on inflammatory dendritic cells regulates CD4 T cell cytokine production in the lung during viral disease. J. Immunol. 2012, 188, 1647-1655.

104. Wang, H.; Peters, N.; Schwarze, J. Plasmacytoid dendritic cells limit viral replication, pulmonary inflammation, and airway hyperresponsiveness in respiratory syncytial virus infection. J. Immunol. 2006, 177, 6263-6270.

105. Smit, J.J.; Lindell, D.M.; Boon, L.; Kool, M.; Lambrecht, B.N.; Lukacs, N.W. The balance between plasmacytoid DC versus conventional DC determines pulmonary immunity to virus infections. PLoS One 2008, 3, e1720. 
106. Jewell, N.A.; Vaghefi, N.; Mertz, S.E.; Akter, P.; Peebles, R.S., Jr.; Bakaletz, L.O.; Durbin, R.K.; Flano, E.; Durbin, J.E. Differential type I interferon induction by respiratory syncytial virus and influenza a virus in vivo. J. Virol. 2007, 81, 9790-9800.

107. Asselin-Paturel, C.; Brizard, G.; Pin, J.J.; Briere, F.; Trinchieri, G. Mouse strain differences in plasmacytoid dendritic cell frequency and function revealed by a novel monoclonal antibody. J. Immunol. 2003, 171, 6466-6477.

108. Blasius, A.L.; Giurisato, E.; Cella, M.; Schreiber, R.D.; Shaw, A.S.; Colonna, M. Bone marrow stromal cell antigen 2 is a specific marker of type I IFN-producing cells in the naive mouse, but a promiscuous cell surface antigen following IFN stimulation. J. Immunol. 2006, 177, 3260-3265.

109. Kumagai, Y.; Takeuchi, O.; Kato, H.; Kumar, H.; Matsui, K.; Morii, E.; Aozasa, K.; Kawai, T.; Akira, S. Alveolar macrophages are the primary interferon-alpha producer in pulmonary infection with RNA viruses. Immunity 2007, 27, 240-252.

110. Pribul, P.K.; Harker, J.; Wang, B.; Wang, H.; Tregoning, J.S.; Schwarze, J.; Openshaw, P.J. Alveolar macrophages are a major determinant of early responses to viral lung infection but do not influence subsequent disease development. J. Virol. 2008, 82, 4441-4448.

111. Biacchesi, S.; Skiadopoulos, M.H.; Boivin, G.; Hanson, C.T.; Murphy, B.R.; Collins, P.L.; Buchholz, U.J. Genetic diversity between human metapneumovirus subgroups. Virology 2003, 315, 1-9.

112. Erickson, J.J.; Gilchuk, P.; Hastings, A.K.; Tollefson, S.J.; Johnson, M.; Downing, M.B.; Boyd, K.L.; Johnson, J.E.; Kim, A.S.; Joyce, S.; et al. Viral acute lower respiratory infections impair CD8+ T cells through PD-1. J. Clin. Investig. 2012, 122, 2967-2982.

113. Carter, L.; Fouser, L.A.; Jussif, J.; Fitz, L.; Deng, B.; Wood, C.R.; Collins, M.; Honjo, T.; Freeman, G.J.; Carreno, B.M. PD-1:PD-L inhibitory pathway affects both CD4(+) and CD8(+) T cells and is overcome by IL-2. Eur. J. Immunol. 2002, 32, 634-643.

114. Freeman, G.J.; Long, A.J.; Iwai, Y.; Bourque, K.; Chernova, T.; Nishimura, H.; Fitz, L.J.; Malenkovich, N.; Okazaki, T.; Byrne, M.C.; et al. Engagement of the PD-1 immunoinhibitory receptor by a novel $\mathrm{B} 7$ family member leads to negative regulation of lymphocyte activation. J. Exp. Med. 2000, 192, 1027-1034.

115. Zhao, J.; Legge, K.; Perlman, S. Age-related increases in PGD(2) expression impair respiratory $\mathrm{DC}$ migration, resulting in diminished $\mathrm{T}$ cell responses upon respiratory virus infection in mice. J. Clin. Investig. 2011, 121, 4921-4930.

116. Kruijsen, D.; Schijf, M.A.; Lukens, M.V.; van Uden, N.O.; Kimpen, J.L.; Coenjaerts, F.E.; van Bleek, G.M. Local innate and adaptive immune responses regulate inflammatory cell influx into the lungs after vaccination with formalin inactivated RSV. Vaccine 2011, 29, 2730-2741. 
Reprinted from Viruses. Cite as: Rudraraju, R.; Jones, B.G.; Sealy, R.; Surman, S.L.; Hurwitz, J.L. Respiratory Syncytial Virus: Current Progress in Vaccine Development. Viruses 2013, 5, 577-594.

Review

\title{
Respiratory Syncytial Virus: Current Progress in Vaccine Development
}

\author{
Rajeev Rudraraju ${ }^{1}$, Bart G. Jones ${ }^{1}$, Robert Sealy ${ }^{1}$, Sherri L. Surman ${ }^{1}$ \\ and Julia L. Hurwitz ${ }^{1,2, *}$
}

1 Department of Infectious Diseases, St. Jude Children's Research Hospital, 262 Danny Thomas Place, Memphis, TN 38105, USA;

E-Mails: Rajeev.rudraraju@stjude.org (R.R.); bart.jones@stjude.org (B.G.J.); robert.sealy@stjude.org (R.S.); sherri.surman@stjude.org (S.L.S.)

2 Department of Microbiology, Immunology and Biochemistry, University of Tennessee Health Science Center, 858 Madison Avenue, Memphis, TN 38163, USA

* Author to whom correspondence should be addressed; E-Mail: julia.hurwitz@stjude.org; Tel.: +1-901-595-2464; Fax: +1-901-595-3099.

Received: 16 January 2013; in revised form: 1 February 2013 / Accepted: 4 February 2013 /

Published: 5 February 2013

\begin{abstract}
Respiratory syncytial virus (RSV) is the etiological agent for a serious lower respiratory tract disease responsible for close to 200,000 annual deaths worldwide. The first infection is generally most severe, while re-infections usually associate with a milder disease. This observation and the finding that re-infection risks are inversely associated with neutralizing antibody titers suggest that immune responses generated toward a first RSV exposure can significantly reduce morbidity and mortality throughout life. For more than half a century, researchers have endeavored to design a vaccine for RSV that can mimic or improve upon natural protective immunity without adverse events. The virus is herein described together with the hurdles that must be overcome to develop a vaccine and some current vaccine development approaches.
\end{abstract}

Keywords: respiratory syncytial virus; candidate vaccines; protective immunity 


\section{RSV, the Virus}

Respiratory syncytial virus is a negative strand RNA virus in the family Paramyxoviridae, the subfamily Pneumovirinae and the genus Pneumovirus. RSV was first discovered in 1952 as the cause of a serious lower respiratory tract disease, most pronounced among children in their first year of life [1]. Other individuals who are susceptible to severe RSV disease include patients with cardiac and pulmonary disorders, patients with immunodeficiencies, and the elderly [2-6]. RSV infection generally presents as an upper respiratory tract (URT) infection which progresses for several days before virus traffics to the lung [1,7]. Most infants are able to clear virus without extreme adverse events, but in the United States approximately 2\% require hospitalization [8,9]. Globally, RSV infections are estimated to result in up to 199,000 deaths annually in children younger than 5 years of age [10] and treatment options remain controversial. Re-infections can occur, but generally result in milder disease; risks of infection are inversely associated with RSV-specific serum neutralizing antibody titers $[11,12]$. For infants at risk for first infections, the passive transfer of monoclonal RSV F-specific antibodies is recommended, but this type of prophylaxis is expensive and unavailable for most individuals who need it [13]. A licensed vaccine, the single best health care solution to infectious disease, is unavailable in the RSV field.

The RSV genome consists of 11 coding sequences including NS1, NS2, N, P, M, SH, G, F, M2-1, M2-2 and L [1,14]. The predominant proteins on the outer membrane of the mature virus are G, F and $\mathrm{SH}$. Initiation of infection is usually mediated by the interaction between RSV G, the major attachment glycoprotein, and the host cell membrane. The viral receptors for $\mathrm{G}$ (and F) proteins on mammalian cells are not fully defined, but $\mathrm{G}$ can bind highly sulfated heparin-like glycosaminoglycans [15] as well as the CX3CR1 fracktalkine chemokine receptor [16]. G attachment facilitates F-mediated membrane fusion, although $\mathrm{F}$ protein can bind cell membranes independently (e.g., via binding to cellular heparan sulfate and nucleolin [16,17]) and mediate virus infection in the absence of G $[1,18]$. Fusion occurs when the F1 subunit of F protein is inserted into the target membrane and the protein refolds into a hairpin structure bringing virus and cell membranes into close proximity [19]. Upon membrane fusion, virus material including a single negative strand RNA genome is released into the cell cytoplasm [19]. Viral RNA is then transcribed and replicated yielding viral mRNA and new virus genome. New virions are assembled at the cell membrane, bud using cell membranes as their outer coat, and then target new cells for additional rounds of infection. Non-structural proteins support virus production while down-regulating host cell growth and defense mechanisms [1].

\section{Hurdles to Vaccine Development}

A main hurdle for RSV vaccine development was encountered in the 1960s during the testing of a formalin-inactivated RSV vaccine (FI-RSV). Unfortunately, the vaccinated infants were not protected from RSV infection. Instead, an unusually large percentage of the vaccinated children, when subsequently exposed to RSV by natural causes, developed disease severe enough to require hospitalization and there were two vaccine-related deaths [20]. It is essential that such a scenario never 
be repeated, and because the reason for the vaccine-related adverse events has not been confirmed, scientists are unsure precisely how to proceed. There remains no licensed vaccine product after one-half century of additional research.

The cause of vaccine-related deaths remains a point of discussion. One potential explanation is as follows: The formalin treatment of RSV altered membrane proteins on the virus and in so doing, rendered a vaccine that induced antibody responses that were non-neutralizing $[21,22]$. The inactivated vaccine also failed to elicit robust CD8+ T cells, because these classical killers of virus-infected targets are best induced by endogenously expressed viral antigens. In the absence of robust neutralizing antibodies and CD8 $+\mathrm{T}$ cells, RSV persisted and induced an aggressive CD4 $+\mathrm{T}$ cell and cytokine response in the lower respiratory tract [23,24]. Uncontrolled RSV replication and persistent inflammation led to blockage of the small airways in infants, leading to substantial morbidity and mortality $[1,4,14,25]$.

There is now debate as to which immune cell populations or effector molecules were responsible for disease [21,26-33]. In a murine model designed to recapitulate the clinical outcome with FI-RSV, it was demonstrated that a subset of RSV G-specific CD4+ Th2 cells and eosinophils associated with disease. [23,24,31,32,34,35]. Additional mouse experiments showed that inhibition of IL-4 and IL-10 Th2 cytokines abrogated pulmonary histopatholgy [24]. Some researchers argue that the induction of Th2 cells, or perhaps the induction of any RSV-specific T cells should be avoided [36], even though $\mathrm{T}$ cells may be key providers of help for B cells and cytotoxic $\mathrm{T}$ lymphocyte functions at the time of RSV exposure. Others argue that the large majority of granulocytes described in original clinical autopsy reports were neutrophils rather than eosinophils [37], questioning the absolute comparison between mouse and human responses to FI-RSV. The potential benefits provided under the appropriate conditions by Th2 cells, other CD4+ and CD8+ T cell subsets, and eosinophil populations are contemplated [37-42]. For example, it is noted that individuals who lack $\mathrm{T}$ cells can experience great difficulty in RSV clearance and suffer worse outcomes than their immunocompetent counterparts $[4,14,43,44]$. It is further noted that eosinophils can be beneficial in that their transfer to the lungs of RSV-infected mice can enhance RSV clearance and inhibit airway hyper-reactivity [45]. Until debates are resolved, researchers and regulatory boards struggle to define 'go' and 'no go' criteria for the advancement of candidate vaccines, particularly when clinical studies target the pediatric arena. It is possible that past RSV vaccine candidates may have proved safe and efficacious in children, but were never tested in seronegative, pediatric populations. Perhaps a solution resides in comprehensive data analyses, revealing the complexities of the immune system and that each lymphocyte subset need not be categorically designated as beneficial or injurious [46]. Rather, as is the case in most RSV-experienced adults, a variety of adaptive and innate immune effectors including B cells, CD4+ T helper cells and CD8+ cytotoxic T lymphocytes function synergistically to mediate safe elimination of virus and virus-infected cells.

Another issue pertinent to RSV vaccine development concerns the selection of an appropriate human test population. Vaccine studies in young infants are performed with hesitancy due to the devastating experience with the FI-RSV vaccine. One suggested possibility is that vaccines might be 
tested in seropositive adults including the elderly rather than in infants. A number of strategies are considered including: (i) standard vaccination of placebo and control groups with subsequent assessment of disease caused by natural RSV exposure; (ii) the use of an experimental RSV challenge virus [47]; and (iii) the vaccination of pregnant females to measure protection afforded to the infant at birth. One or more of these strategies may prove fruitful provided that attention is paid to a number of confounding variables. First, due to varying degrees of seropositivity in older individuals, immune responsiveness toward vaccination may be difficult to interpret. Individuals with high pre-existing immunity might be expected to show a boost in antibody titers following vaccination, but may instead clear vectors and antigens so rapidly that there is little opportunity for immune cells to re-activate. Second, vaccine safety data may be difficult to interpret due to frequent, unrelated disease complications in the oldest adults. One additional variable to be considered (upon design of either pediatric or adult vaccine protocols) is that individuals with poor diets or poor metabolism (e.g., vitamin deficiency) may exhibit general defects in immune responses toward respiratory virus vaccines $[48,49]$. With these considerations in mind, it has been proposed that RSV vaccine studies in the elderly are feasible, but would require the recruitment of thousands of participants to ensure fair vaccine assessment [50]. Another recent proposal has been that RSV vaccine studies might best be conducted not in the youngest infants or in adults, but in seronegative children who are at least 6 months of age [51]. Perhaps efficacy studies in older, seronegative children could assist vaccine licensure for a restricted age group while prompting additional clinical studies in the youngest infants.

\section{Current RSV Vaccine Strategies}

The first formal vaccine development effort occurred two centuries ago when Edward Jenner demonstrated that material from a cowpox lesion could serve as a vaccine for smallpox [52]. Successful vaccination, both then and now, relies on the safe introduction of a pathogen's antigenic determinants to the immune system. If the vaccine's antigenic determinants are well matched to those of the native pathogen (as was the case for cowpox and smallpox), the antigens will activate pathogen-specific lymphocytes. In the case of virus-specific B cells, some effectors will mature to the plasma cell stage and constitutively secrete antibodies into blood, lymph and mucosal secretions, while others will maintain memory status, capable of immediate re-activation upon pathogen exposure. Activated CD8 $+\mathrm{T}$ cells are classically known for their killing of virus infected cells, while CD4+ T cells, (including Th1, Th2, Th17, TFh, Treg, and other subsets) are known for their support and regulation of CD8+ and B cell activities [53-57]. The heightened or "primed" state of immune surveillance may last months or years to inhibit pathogen entry and pathogen-mediated damage to the host [58]. Generally, vaccines are developed by: (i) inactivating the virus; (ii) identifying a related virus in another species that is safe in humans (the Jennerian approach); (iii) attenuating the virus; or (iv) using recombinant technology to present viral antigens, often in the context of a replication competent or replication-incompetent vector. In the RSV vaccine field, because of the outcome of the FI-RSV vaccine study, the first approach is generally discouraged, even if candidate vaccines retain RSV neutralizing determinants. The second strategy, the Jennerian approach, has not been advanced 
due to an insufficient antigenic match between bovine and human RSV [59]. The third and fourth strategies are the topics of most current vaccine research, described in greater detail below. Preclinical testing of vaccine candidates is generally accomplished first in small animals and then in non-human primates. Most small animal studies use hamsters, BALB/c mice, or cotton rats [60-63] while non-human primate studies generally use African green monkeys, rhesus macaques and chimpanzees [64-66]. Each animal is at least semi-permissive for RSV infection and is therefore advantageous in that immune responses and immunopathological events can be measured, but no animal model fully predicts the course of immune responses and disease in humans. In one instance, for example, an attenuated RSV vaccine appeared to be safe in non-human primates, but proved unsafe when tested in seronegative children [67]. Again, the debates described above concerning: (i) interpretation of data from animal experiments; and (ii) selection of clinical trial target populations, must be considered to determine when and how to advance vaccine candidates from pre-clinical to clinical trials.

\subsection{Attenuated Virus Vaccines: Testing Variant Mutations}

One strategy for RSV vaccine development has been to attenuate virus by cold adaption $[68,69]$. An early product of this research was cpts-248/404 [70,71]. However, in the youngest infants, the vaccine caused URT congestion associated with peak virus recovery and was deemed unacceptable for further development. More recently the cpts-248/404/1030/ $\Delta \mathrm{SH}$ vaccine was developed by introducing further mutations and deleting the SH gene, resulting in a more satisfactory product [72]. In a recent clinical study with this candidate, post-vaccination nasal washes revealed viruses with partial loss of the temperature sensitive phenotype, often due to a tyrosine/asparagine substitution in the $\mathrm{L}$ gene at position 1321 [73]. Researchers corrected the problem by creating a reversion-resistant virus with an alternative attenuating codon at position 1321. However, they then discovered a compensatory mutation at position 1313 , forcing a deletion of that position $(\Delta 1313)$ to yield a safer vaccine. When the $\Delta 1313$ deletion was paired with an NS2 gene deletion and tested at incrementally increasing temperatures, another compensatory mutation was discovered, I1314T. Finally, a vaccine with a new combination of mutations $(\Delta \mathrm{NS} 2 / \Delta 1313 / 1314 \mathrm{~L})$ is being developed for evaluation in phase I clinical trials [74]. Reverse genetics, a powerful technology that allows the manipulation of viral genomes and recovery of infectious, recombinant virus particles, has assisted the progress described above [75].

A number of attenuated RSV vaccines have now been tested clinically. Currently, a phase I clinical study in adults, seropositive children and seronegative children is in progress to test safety and immunogenicity of an intranasally (I.N.) delivered RSV M2-2 deletion mutant $[73,76,77]$.

The development of live-attenuated vaccines presents significant challenges, particularly when vaccines are delivered by the respiratory route to neonates. A concern is that viruses with compensatory mutations in the live-attenuated vaccines may associate with reversion to pathogenic phenotypes and lead to increased frequencies of adverse reactions in vivo. There is also a difficulty related to manufacturing and distribution, as RSV is naturally sensitive to changes in temperature, and attenuated strains by definition are difficult to propagate to high titers. 


\subsection{Recombinant Protein Vaccines}

Recombinant technology provides great flexibility both in terms of the RSV antigen(s) and the vector(s) with which the antigen is expressed. The major target antigens of recombinant vaccine technology are RSV G and F, as these are each capable of eliciting neutralizing antibodies as well as T cell responses. $\mathrm{F}$ is particularly attractive due to its considerable conservation among RSV isolates. Another antigen of recent interest is the small hydrophobic protein, $\mathrm{SH}$ [78].

As an example of progress in recombinant vaccine technology, Novartis is developing a postfusion RSV F trimer that elicits neutralizing antibodies and protection against RSV challenge in cotton rats [79]. Most researchers strive to match vaccine protein with pathogen protein to take advantage of polyclonal $\mathrm{B}$ and $\mathrm{T}$ cell responses that can work in unison to recognize and combat pathogen. Other researchers target particular epitopes such as a central conserved region of the $\mathrm{G}$ protein that induces antibodies to block the CX3C-CX3CR1 interaction. Mice vaccinated with fragments containing the $\mathrm{CX} 3 \mathrm{C}$ motif have been shown to generate immune responses that can reduce lung virus titers and pulmonary inflammation following RSV challenge [80]. Still other researchers, as described above, propose that all $\mathrm{T}$ cell epitopes (and many $\mathrm{B}$ cell epitopes) should be removed from RSV vaccines [36,42]. Using computational design, epitope-scaffold vaccines have been developed to mimic an individual epitope on the $\mathrm{F}$ protein known to correspond with a neutralizing monoclonal antibody activity (motavizumab, [13]). In one case, several scaffolds were developed based on 13 discontinuous RSV F contact residues (xSxxLSxINDxxxxNDxKKLxSNx) for motavizumab. An automated search of protein structures supported the selection of three proteins as scaffolds: protein Z, a domain of protein A from Staphylococcus aureus, Cag-Z from Helicobacter pylori and the p26 capsid protein from equine infectious anemia virus. Amino acids outside of the motavizumab epitope were then modified or removed to optimize stability, solubility and motavizumab binding affinity. Critical to decisions concerning scaffold design are demonstrations that $\mathrm{B}$ cell and $\mathrm{T}$ cell determinants on viruses are often dependent on structural and spatial context, as regions outside the epitope affect 3-dimensional folding, post-translational modifications, and antigen processing [81-84]. For this reason, immune cells that respond to a protein fragment in one context (e.g., vaccine) do not necessarily recognize the same fragment when the context is changed (e.g., virus). When the Stapylococcus aureus protein A scaffold was fused to a pan-HLA DR binding epitope and tested for immunogenicity in mice, it induced RSV-binding antibodies, but these antibodies failed to neutralize RSV [36]. A separate study of a motavizumab-based scaffold in non-human primates illustrated neutralizing antibodies in a fraction of animals [85].

Most protein vaccines, whether designed to match unmanipulated viral proteins or targeted determinants, are combined with adjuvants. A plethora of adjuvants now exist including $\mathrm{W}_{80} 5 \mathrm{EC}$ [86], alum, 3-O-desacyl-4'-monophosphoryl lipid A (MPL), muramyl dipeptide (MDP), natural host defense peptides, $\mathrm{CpG}$ oligodeoxynucleotides (ODN) and polyphosphazenes. Polyphosphazenes are synthetic water-soluble polymers containing an inorganic backbone of alternating phosphorus and nitrogen atoms. Adjuvants are in some cases known to trigger cell molecules (e.g., toll-like receptors, TLR) to activate innate and adaptive immune responses. For example, MPL, CpG ODN, and MDP are 
ligands for TLR-4, TLR-9, and NOD2, respectively. The $\mathrm{W}_{80} 5 \mathrm{EC}$ product can serve both as an adjuvant and as a virus-inactivation method [86]. While adjuvant choices are many, U.S. Food and Drug Administration (FDA)-approved and licensed adjuvants are limited (alum and MPL). There is also a large variety of combinations for formulations of liposomes, nanoparticles or microparticles (synthetic particles and/or particles encompassing bacterial or viral components [87]) for the delivery of RSV proteins, peptides, and/or adjuvants. As an example, a truncated, secreted, trimeric F protein has been formulated for I.N. delivery with combinations of a TLR agonist (CpG ODN), an innate defense regulator peptide (IDR1002-VQRWLIVWRIRK), and polyphosphazene as nano- or microparticles, to induce RSV protective immunity [88,89]. Yet another example is Novavax's near-full length F glycoprotein formulated as a nanoparticle vaccine. This vaccine has been tested in healthy adults and has been shown to induce significant increases in the anti-F antibody response, including micro-neutralizing activities and competitive activity against the neutralizing monoclonal antibody Palivizumab [13,90-92]. An additional use of adjuvant has been with MPL combined not with a recombinant protein or particle, but with virosomes comprising membranes from RSV [93].

\subsection{Replication Competent, Recombinant Viral Vaccines}

Reverse genetics has assisted the development of recombinant viral vaccines that can serve as delivery systems for RSV antigens. One such vaccine, which has been well advanced in clinical trials, is MedImmune's MEDI-534 [94,95]. This vaccine is a replication-competent vaccine that expresses RSV F $[75,96]$. The backbone is based on a bovine parainfluenza virus type 3 with substituted human PIV3 F and HN glycoproteins. MEDI-534 was tested in non-human primates and also in a phase I study in young children between the ages of 6 months and $<24$ months. Doses of 10,000, 100,000 and $1,000,000 \mathrm{TCID}_{50}$ were tested in the clinical trial and RSV specific antibody responses were noted in $50 \%$ of vaccinees administered three $1,000,000 \mathrm{TCID}_{50}$ doses of vaccine with two month intervals. Virus that was shed from study participants revealed genetic changes that were associated with reduced RSV F protein expression. A close analysis of the MEDI-534 vaccine then demonstrated that some of the same genetic variants were minor components of the administered vaccine [97]. The implication of these sequence variants is a current topic of discussion.

Another promising candidate is St. Jude's recombinant, replication competent vaccine (SeVRSV), also developed with reverse genetics technology [75]. Sendai virus (SeV), a mouse parainfluenza virus type I with a high sequence and antigenic similarity to human parainfluenza virus type I (hPIV-1), was used as the vaccine's backbone [98-102]. $\mathrm{SeV}$ is an attractive vaccine candidate and vaccine backbone, because there has never been a confirmed case of $\mathrm{SeV}$-associated disease in humans. The species specificity of Sendai virus is attributed in part to its unique sensitivity to human type I interferon [103]. In small animals a single I.N. dose of SeV induced B and T cell responses within days after immunization that lasted for the animal's lifetime without need for a booster [62]. The SeVRSV recombinant carries the RSV F gene and thereby instructs its expression in infected cells [104]. When tested in cotton rats, SeVRSV protected animals from challenge with both A and B RSV isolates. SeVRSV could also be mixed with two additional SeV-based vaccines in a single I.N. 
inoculation to protect against four different challenge viruses: RSV, hPIV-1, hPIV-2 and hPIV-3 [62]. When SeVRSV was tested as a vaccine in African green monkeys with non-recombinant $\mathrm{SeV}$ as a control, it safely and fully prevented infection of the lower airways following RSV challenge [66]. The non-recombinant $\mathrm{SeV}$ has already entered clinical trials as a hPIV-1 vaccine and has been well tolerated in adults [105] and 3-6 year old children. SeVRSV is now being manufactured for testing in an age de-escalation clinical trial. Previous pre-clinical and clinical data suggest that SeVRSV will safely protect children from both RSV and hPIV-1 infections. When compared to the live-attenuated RSV vaccines, $\mathrm{SeV}$ based vaccines benefit from their relative stability to temperature changes and ease of growth to high titers in chicken eggs and cell cultures, a boon for manufacturing and vaccine distribution.

\subsection{A Variety of New Vectors and Concepts in the RSV Vaccine Field}

A great number of additional recombinant vaccines are in stages of pre-clinical testing. Vectors include Semliki Forest virus [106], Venezuelan equine encephalitis virus [107,108], adenovirus from humans or non-human primates [109,110], influenza virus [111], measles virus [112], Newcastle disease virus like particles (VLPs [113]) and plasmid DNA [114,115]. Other vaccine delivery systems are based not on viruses, but bacteria, yeast or plants [116], such as the Mucosis SynGEM ${ }^{\circledR}$ vaccine, an I.N. vaccine that presents native trimeric F protein formulated in a non-living bacterium-like particle (BLP) [117]. There have also been combination prime-boost strategies using one form of recombinant vaccine followed by another. For example a recombinant vaccine prime based on replication-defective chimpanzee adenovirus can be followed with a boost based on modified vaccinia Ankara (MVA) [118], or a recombinant DNA prime can be followed by recombinant adenoviral vector boosts [119]. This article describes a portion, but not all of the vaccine candidates that are currently under investigation. Table 1 provides a short list as a sampling of vaccine strategies with references and review articles. It is quite likely that one or more than one of the current vaccine candidates will prove successful. The advanced development and licensure of a safe and effective RSV vaccine will indeed be momentous, a long-awaited milestone for the prevention of the significant sickness and death caused by RSV infections.

Table 1. Sample respiratory syncytial virus (RSV) vaccine references and review articles.

\begin{tabular}{lc}
\hline \multicolumn{1}{c}{ Vaccine Type } & Sample references \\
\hline Attenuated RSV & {$[73,74]$} \\
Inactivated RSV & {$[86,120]$} \\
RSV protein(s) adjuvanted and/or as micro/nano-particles & {$[79,89]$} \\
Epitope scaffold & {$[36]$} \\
Virosome & {$[93,121]$} \\
Virus like particle (VLP) & {$[113,122]$} \\
Replication competent virus-based vector & {$[66,94,95,97]$} \\
Bacteria-based vector & {$[123-125]$} \\
Plant-based vector & {$[116]$} \\
Prime-boost with heterologous vectors & {$[118,119]$.} \\
Related review articles & {$[\mathbf{1 , 4 , 1 4 , 2 5 , 1 1 5 , 1 2 6}]$} \\
\hline
\end{tabular}




\section{Acknowledgments}

This work was supported in part by NIH, NIAID grants P01-AI-054955, R01-AI088729, and R01-AI78819, NCI grant P30-CA21765 and the American Lebanese Syrian Associated Charities. Acknowledgement is given to S. Varga, N. Lukacs and K. Harrod for organizing the 8th International Respiratory Syncytial Virus Symposium in 2012.

\section{Conflicts of Interest}

JLH is a co-author of a patent application relevant to SeVRSV vaccine development.

\section{References}

1. Collins, P.L.; Crowe, J.E. Respiratory Syncytial Virus and Metapneumovirus. In Fields Virology, 5th ed.; Knipe, D.M., Howley, P.M, Griffin, D.E., Lamb, R.A., Martin, M.A., Roizman B., et al., Eds.; Lippincott Williams\&Wilkins: Philadelphia, PA, USA, 2007; pp. 1601-1646.

2. Groothuis, J.R.; Gutierrez, K.M.; Lauer, B.A. Respiratory syncytial virus infection in children with bronchopulmonary dysplasia. Pediatrics 1988, 82, 199-203.

3. Falsey, A.R.; Hennessey, P.A.; Formica, M.A.; Cox, C.; Walsh, E.E. Respiratory syncytial virus infection in elderly and high-risk adults. N. Engl. J. Med. 2005, 352, 1749-1759.

4. Collins, P.L.; Melero, J.A. Progress in understanding and controlling respiratory syncytial virus: Still crazy after all these years. Virus Res. 2011, 162, 80-99.

5. El Saleeby, C.M.; Somes, G.W.; DeVincenzo, J.P.; Gaur, A.H. Risk factors for severe respiratory syncytial virus disease in children with cancer: The importance of lymphopenia and young age. Pediatrics 2008, 121, 235-243.

6. Madhi, S.A.; Schoub, B.; Simmank, K.; Blackburn, N.; Klugman, K.P. Increased burden of respiratory viral associated severe lower respiratory tract infections in children infected with human immunodeficiency virus type-1. J. Pediatr. 2000, 137, 78-84.

7. Wright, P.F.; Gruber, W.C.; Peters, M.; Reed, G.; Zhu, Y.; Robinson, F.; Coleman-Dockery, S.; Graham, B.S. Illness severity, viral shedding, and antibody responses in infants hospitalized with bronchiolitis caused by respiratory syncytial virus. J. Infect. Dis. 2002, 185, 1011-1018.

8. Zhou, H.; Thompson, W.W.; Viboud, C.G.; Ringholz, C.M.; Cheng, P.Y.; Steiner, C.; Abedi, G.R.; Anderson, L.J.; Brammer, L.; Shay, D.K. Hospitalizations associated with influenza and respiratory syncytial virus in the United States, 1993-2008. Clin. Infect. Dis. 2012, 54, 1427-1436.

9. Thompson, W.W.; Shay, D.K.; Weintraub, E.; Brammer, L.; Cox, N.; Anderson, L.J.; Fukuda, K. Mortality associated with influenza and respiratory syncytial virus in the United States. JAMA 2003, 289, 179-186.

10. Nair, H.; Nokes, D.J.; Gessner, B.D.; Dherani, M.; Madhi, S.A.; Singleton, R.J.; O’Brien, K.L.; Roca, A.; Wright, P.F.; Bruce, N.; et al. Global burden of acute lower respiratory infections due to respiratory syncytial virus in young children: A systematic review and meta-analysis. Lancet 2010, 375, 1545-1555. 
11. Glezen, W.P.; Taber, L.H.; Frank, A.L.; Kasel, J.A. Risk of primary infection and reinfection with respiratory syncytial virus. Am. J. Dis. Child. 1986, 140, 543-546.

12. Shinoff, J.J.; O’Brien, K.L.; Thumar, B.; Shaw, J.B.; Reid, R.; Hua, W.; Santosham, M.; Karron, R.A. Young infants can develop protective levels of neutralizing antibody after infection with respiratory syncytial virus. J. Infect. Dis. 2008, 198, 1007-1015.

13. Fernandez, P.; Trenholme, A.; Abarca, K.; Griffin, M.P.; Hultquist, M.; Harris, B.; Losonsky, G.A. A phase 2, randomized, double-blind safety and pharmacokinetic assessment of respiratory syncytial virus (RSV) prophylaxis with motavizumab and palivizumab administered in the same season. BMC. Pediatr. 2010, 10, 38.

14. Collins, P.L.; Graham, B.S. Viral and host factors in human respiratory syncytial virus pathogenesis. J. Virol. 2008, 82, 2040-2055.

15. Feldman, S.A.; Hendry, R.M.; Beeler, J.A. Identification of a linear heparin binding domain for human respiratory syncytial virus attachment glycoprotein G. J. Virol. 1999, 73, 6610-6617.

16. Harcourt, J.L.; Karron, R.A.; Tripp, R.A. Anti-G protein antibody responses to respiratory syncytial virus infection or vaccination are associated with inhibition of $G$ protein CX3C-CX3CR1 binding and leukocyte chemotaxis. J. Infect. Dis. 2004, 190, 1936-1940.

17. Tayyari, F.; Marchant, D.; Moraes, T.J.; Duan, W.; Mastrangelo, P.; Hegele, R.G. Identification of nucleolin as a cellular receptor for human respiratory syncytial virus. Nat. Med. 2011, 17, 1132-1135.

18. Techaarpornkul, S.; Collins, P.L.; Peeples, M.E. Respiratory syncytial virus with the fusion protein as its only viral glycoprotein is less dependent on cellular glycosaminoglycans for attachment than complete virus. Virology 2002, 294, 296-304.

19. Russell, C.J.; Luque, L.E. The structural basis of paramyxovirus invasion. Trends Microbiol. 2006, 14, 243-246.

20. Chin, J.; Magoffin, R.L.; Shearer, L.A.; Schieble, J.H.; Lennette, E.H. Field evaluation of a respiratory syncytial virus vaccine and a trivalent parainfluenza virus vaccine in a pediatric population. Am. J. Epidemiol. 1969, 89, 449-463.

21. Murphy, B.R.; Prince, G.A.; Walsh, E.E.; Kim, H.W.; Parrott, R.H.; Hemming, V.G.; Rodriguez, W.J.; Chanock, R.M. Dissociation between serum neutralizing and glycoprotein antibody responses of infants and children who received inactivated respiratory syncytial virus vaccine. J. Clin. Microbiol. 1986, 24, 197-202.

22. Murphy, B.R.; Walsh, E.E. Formalin-inactivated respiratory syncytial virus vaccine induces antibodies to the fusion glycoprotein that are deficient in fusion-inhibiting activity. J. Clin. Microbiol. 1988, 26, 1595-1597.

23. Varga, S.M.; Wang, X.; Welsh, R.M.; Braciale, T.J. Immunopathology in RSV infection is mediated by a discrete oligoclonal subset of antigen-specific CD4(+) T cells. Immunity 2001, 15, 637-646. 
24. Connors, M.; Giese, N.A.; Kulkarni, A.B.; Firestone, C.Y.; Morse, H.C., III; Murphy, B.R. Enhanced pulmonary histopathology induced by respiratory syncytial virus (RSV) challenge of formalin-inactivated RSV-immunized BALB/c mice is abrogated by depletion of interleukin-4 (IL-4) and IL-10. J. Virol. 1994, 68, 5321-5325.

25. Blanco, J.C.; Boukhvalova, M.S.; Shirey, K.A.; Prince, G.A.; Vogel, S.N. New insights for development of a safe and protective RSV vaccine. Hum. Vaccines 2010, 6, 482-492.

26. Delgado, M.F.; Coviello, S.; Monsalvo, A.C.; Melendi, G.A.; Hernandez, J.Z.; Batalle, J.P.; Diaz, L.; Trento, A.; Chang, H.Y.; Mitzner, W.; et al. Lack of antibody affinity maturation due to poor Toll-like receptor stimulation leads to enhanced respiratory syncytial virus disease. Nat. Med. 2009, 15, 34-41.

27. Shaw, C.A.; Otten, G.; Wack, A.; Palmer, G.A.; Mandl, C.W.; Mbow, M.L.; Valiante, N.; Dormitzer, P.R. Antibody affinity maturation and respiratory syncytial virus disease. Nat. Med. 2009, 15, 725-726.

28. Polack, F.P.; Teng, M.N.; Collins, P.L.; Prince, G.A.; Exner, M.; Regele, H.; Lirman, D.D.; Rabold, R.; Hoffman, S.J.; Karp, C.L.; et al. A role for immune complexes in enhanced respiratory syncytial virus disease. J. Exp. Med. 2002, 196, 859-865.

29. Polack, F.P. Atypical measles and enhanced respiratory syncytial virus disease (ERD) made simple. Pediatr. Res. 2007, 62, 111-115.

30. Kakuk, T.J.; Soike, K.; Brideau, R.J.; Zaya, R.M.; Cole, S.L.; Zhang, J.Y.; Roberts, E.D.; Wells, P.A.; Wathen, M.W. A human respiratory syncytial virus (RSV) primate model of enhanced pulmonary pathology induced with a formalin-inactivated RSV vaccine but not a recombinant FG subunit vaccine. J. Infect. Dis. 1993, 167, 553-561.

31. Walzl, G.; Matthews, S.; Kendall, S.; Gutierrez-Ramos, J.C.; Coyle, A.J.; Openshaw, P.J.; Hussell, T. Inhibition of T1/ST2 during respiratory syncytial virus infection prevents $\mathrm{T}$ helper cell type 2 (Th2)- but not Th1-driven immunopathology. J. Exp. Med. 2001, 193, 785-792.

32. Rosenberg, H.F.; Dyer, K.D.; Domachowske, J.B. Respiratory viruses and eosinophils: Exploring the connections. Antiviral Res. 2009, 83, 1-9.

33. Becker, Y. Respiratory syncytial virus (RSV) evades the human adaptive immune system by skewing the Th1/Th2 cytokine balance toward increased levels of Th2 cytokines and IgE, markers of allergy-A review. Virus Genes 2006, 33, 235-252.

34. Srikiatkhachorn, A.; Braciale, T.J. Virus-specific CD8+ T lymphocytes downregulate T helper cell type 2 cytokine secretion and pulmonary eosinophilia during experimental murine respiratory syncytial virus infection. J. Exp. Med. 1997, 186, 421-432.

35. Legg, J.P.; Hussain, I.R.; Warner, J.A.; Johnston, S.L.; Warner, J.O. Type 1 and type 2 cytokine imbalance in acute respiratory syncytial virus bronchiolitis. Am. J Respir. Crit. Care Med. 2003, 168, 633-639.

36. McLellan, J.S.; Correia, B.E.; Chen, M.; Yang, Y.; Graham, B.S.; Schief, W.R.; Kwong, P.D. Design and characterization of epitope-scaffold immunogens that present the motavizumab epitope from respiratory syncytial virus. J Mol. Biol. 2011, 409, 853-866. 
37. Prince, G.A.; Curtis, S.J.; Yim, K.C.; Porter, D.D. Vaccine-enhanced respiratory syncytial virus disease in cotton rats following immunization with Lot 100 or a newly prepared reference vaccine. J. Gen. Virol. 2001, 82, 2881-2888.

38. Chu, V.T.; Frohlich, A.; Steinhauser, G.; Scheel, T.; Roch, T.; Fillatreau, S.; Lee, J.J.; Lohning, M.; Berek, C. Eosinophils are required for the maintenance of plasma cells in the bone marrow. Nat. Immunol. 2011, 12, 151-159.

39. Olson, M.R.; Varga, S.M. CD8 T cells inhibit respiratory syncytial virus (RSV) vaccine-enhanced disease. J. Immunol. 2007, 179, 5415-5424.

40. Power, U.F.; Plotnicky, H.; Blaecke, A.; Nguyen, T.N. The immunogenicity, protective efficacy and safety of BBG2Na, a subunit respiratory syncytial virus (RSV) vaccine candidate, against RSV-B. Vaccine 2003, 22, 168-176.

41. Graham, B.S. Biological challenges and technological opportunities for respiratory syncytial virus vaccine development. Immunol. Rev. 2011, 239, 149-166.

42. McLellan, J.S. F Protein Structures and Their Implications for Humoral Immunity. Presented at the 8th Respiratory Syncytial Virus Symposium, Santa Fe, NM, 2012.

43. Fishaut, M.; Tubergen, D.; McIntosh, K. Cellular response to respiratory viruses with particular reference to children with disorders of cell-mediated immunity. J. Pediatr. 1980, 96, 179-186.

44. Hall, C.B.; Powell, K.R.; MacDonald, N.E.; Gala, C.L.; Menegus, M.E.; Suffin, S.C.; Cohen, H.J. Respiratory syncytial viral infection in children with compromised immune function. N. Engl. J Med. 1986, 315, 77-81.

45. Phipps, S.; Lam, C.E.; Mahalingam, S.; Newhouse, M.; Ramirez, R.; Rosenberg, H.F.; Foster, P.S.; Matthaei, K.I. Eosinophils contribute to innate antiviral immunity and promote clearance of respiratory syncytial virus. Blood 2007, 110, 1578-1586.

46. Welliver, R.C., Sr. The immune response to respiratory syncytial virus infection: Friend or foe? Clin. Rev. Allergy Immunol. 2008, 34, 163-173.

47. DeVincenzo, J.; Lambkin-Williams, R.; Wilkinson, T.; Cehelsky, J.; Nochur, S.; Walsh, E.; Meyers, R.; Gollob, J.; Vaishnaw, A. A randomized, double-blind, placebo-controlled study of an RNAi-based therapy directed against respiratory syncytial virus. Proc. Natl. Acad. Sci. USA 2010, 107, 8800-8805.

48. Rudraraju, R.; Surman, S.L.; Jones, B.G.; Sealy, R.; Woodland, D.L.; Hurwitz, J.L. Reduced frequencies and heightened CD103 expression among virus-induced CD8(+) $\mathrm{T}$ cells in the respiratory tract airways of vitamin A-deficient mice. Clin. Vaccine Immunol. 2012, 19, 757-765.

49. Surman, S.L.; Rudraraju, R.; Sealy, R.; Jones, B.; Hurwitz, J.L. Vitamin A deficiency disrupts vaccine-induced antibody-forming cells and the balance of $\operatorname{IgA} / \operatorname{IgG}$ isotypes in the upper and lower respiratory tract. Viral Immunol. 2012, 25, 341-344.

50. Falsey AR. RSV In Adults: What Do We Know? What Do We Need to Know? Where Are We Going? In Proceedings of the 7th International Respiratory Syncytial Virus Symposium, Rotterdam, The Netherlands, 2 December 2010. 
51. Graham, B.S. Future of RSV vaccine development. Presented at the 8th Respiratory Syncytial Virus Symposium, Santa Fe, NM, USA, 2012.

52. Moss, B. Smallpox vaccines: Targets of protective immunity. Immunol. Rev. 2011, 239, 8-26.

53. Murphy, K.; Travers, P.; Walport, M. Janeway's Immunobiology, 7th ed.; Garland Science: New York, NY, USA, 2008.

54. Peters, A.; Lee, Y.; Kuchroo, V.K. The many faces of Th17 cells. Curr. Opin. Immunol. 2011, 23, 702-706.

55. Kelso, A. Th1 and Th2 subsets: Paradigms lost? Immunol. Today 1995, 16, 374-379.

56. Mosmann, T.R.; Cherwinski, H.; Bond, M.W.; Giedlin, M.A.; Coffman, R.L. Two types of murine helper $\mathrm{T}$ cell clone. I. Definition according to profiles of lymphokine activities and secreted proteins. J. Immunol. 1986, 136, 2348-2357.

57. Crotty, S. Follicular helper CD4 T cells (TFH). Annu. Rev. Immunol. 2011, 29, 621-663.

58. Crotty, S.; Felgner, P.; Davies, H.; Glidewell, J.; Villarreal, L.; Ahmed, R. Cutting edge: Long-term B cell memory in humans after smallpox vaccination. J Immunol. 2003, 171, 4969-4973.

59. Lerch, R.A.; Anderson, K.; Amann, V.L.; Wertz, G.W. Nucleotide sequence analysis of the bovine respiratory syncytial virus fusion protein mRNA and expression from a recombinant vaccinia virus. Virology 1991, 181, 118-131.

60. Schmidt, A.C.; McAuliffe, J.M.; Murphy, B.R.; Collins, P.L. Recombinant bovine/human parainfluenza virus type 3 (B/HPIV3) expressing the respiratory syncytial virus (RSV) G and F proteins can be used to achieve simultaneous mucosal immunization against RSV and HPIV3. J. Virol. 2001, 75, 4594-4603.

61. Stevens, W.W.; Sun, J.; Castillo, J.P.; Braciale, T.J. Pulmonary eosinophilia is attenuated by early responding CD8(+) memory $\mathrm{T}$ cells in a murine model of RSV vaccine-enhanced disease. Viral Immunol. 2009, 22, 243-251.

62. Jones, B.; Zhan, X.; Mishin, V.; Slobod, K.S.; Surman, S.; Russell, C.J.; Portner, A.; Hurwitz, J.L. Human PIV-2 recombinant Sendai virus (rSeV) elicits durable immunity and combines with two additional rSeVs to protect against hPIV-1, hPIV-2, hPIV-3, and RSV. Vaccine 2009, 27, 1848-1857.

63. Zhan, X.; Slobod, K.S.; Krishnamurthy, S.; Luque, L.E.; Takimoto, T.; Jones, B.; Surman, S.; Russell, C.J.; Portner, A.; Hurwitz, J.L. Sendai virus recombinant vaccine expressing hPIV-3 HN or $\mathrm{F}$ elicits protective immunity and combines with a second recombinant to prevent hPIV-1, hPIV-3 and RSV infections. Vaccine 2008, 26, 3480-3488.

64. Crowe, J.E., Jr.; Randolph, V.; Murphy, B.R. The live attenuated subgroup B respiratory syncytial virus vaccine candidate RSV $2 \mathrm{~B} 33 \mathrm{~F}$ is attenuated and immunogenic in chimpanzees, but exhibits partial loss of the ts phenotype following replication in vivo. Virus Res. 1999, 59, $13-22$. 
65. De, W.L.; Power, U.F.; Yuksel, S.; Van, A.G.; Nguyen, T.N.; Niesters, H.G.; de Swart, R.L.; Osterhaus, A.D. Evaluation of BBG2Na in infant macaques: Specific immune responses after vaccination and RSV challenge. Vaccine 2004, 22, 915-922.

66. Jones, B.G.; Sealy, R.E.; Rudraraju, R.; Traina-Dorge, V.L.; Finneyfrock, B.; Cook, A.; Takimoto, T.; Portner, A.; Hurwitz, J.L. Sendai virus-based RSV vaccine protects African green monkeys from RSV infection. Vaccine 2012, 30, 959-968.

67. Karron, R.A.; Wright, P.F.; Crowe, J.E., Jr.; Clements-Mann, M.L.; Thompson, J.; Makhene, M.; Casey, R.; Murphy, B.R. Evaluation of two live, cold-passaged, temperature-sensitive respiratory syncytial virus vaccines in chimpanzees and in human adults, infants, and children. J. Infect. Dis. 1997, 176, 1428-1436.

68. Friedewald, W.T.; Forsyth, B.R.; Smith, C.B.; Gharpure, M.A.; Chanock, R.M. Low-temperature-grown RS virus in adult volunteers. JAMA 1968, 204, 690-694.

69. Schickli, J.H.; Kaur, J.; Tang, R.S. Nonclinical phenotypic and genotypic analyses of a Phase 1 pediatric respiratory syncytial virus vaccine candidate MEDI-559 (rA2cp248/404/1030DeltaSH) at permissive and non-permissive temperatures. Virus Res. 2012, 169, 38-47.

70. Wright, P.F.; Karron, R.A.; Belshe, R.B.; Thompson, J.; Crowe, J.E., Jr.; Boyce, T.G.; Halburnt, L.L.; Reed, G.W.; Whitehead, S.S.; Anderson, E.L.; et al. Evaluation of a live, cold-passaged, temperature-sensitive, respiratory syncytial virus vaccine candidate in infancy. J. Infect. Dis. 2000, 182, 1331-1342.

71. Firestone, C.Y.; Whitehead, S.S.; Collins, P.L.; Murphy, B.R.; Crowe, J.E., Jr. Nucleotide sequence analysis of the respiratory syncytial virus subgroup A cold-passaged (cp) temperature sensitive (ts) cpts-248/404 live attenuated virus vaccine candidate. Virology 1996, 225, 419-422.

72. Karron, R.A.; Wright, P.F.; Belshe, R.B.; Thumar, B.; Casey, R.; Newman, F.; Polack, F.P.; Randolph, V.B.; Deatly, A.; Hackell, J. Identification of a recombinant live attenuated respiratory syncytial virus vaccine candidate that is highly attenuated in infants. J. Infect. Dis. 2005, 191, 1093-1104.

73. Buchholz, U.J.; Luongo, C.; Winter, C.C.; Tang, R.S.; Karron, R.A.; Schickli, J.H.; Collins, P.L. Live-Attenuated RSV Vaccine Candidates for Clinical Studies: Improving Genetic Stability and Backup Plans. Presented at the 8th Respiratory Syncytial Virus Symposium, Santa Fe, NM, USA, 2012.

74. Luongo, C.; Winter, C.C.; Collins, P.L.; Buchholz, U.J. Respiratory syncytial virus modified by deletions of the NS2 gene and amino acid S1313 of the L polymerase protein is a temperature sensitive live-attenuated vaccine candidate that is phenotypically stable at physiological temperature. J. Virol. 2012, 87, 1985-96.

75. Nagai, Y.; Kato, A. Paramyxovirus reverse genetics is coming of age. Microbiol. Immunol. 1999, 43, 613-624.

76. Jin, H.; Zhou, H.; Cheng, X.; Tang, R.; Munoz, M.; Nguyen, N. Recombinant respiratory syncytial viruses with deletions in the NS1, NS2, SH, and M2-2 genes are attenuated in vitro and in vivo. Virology 2000, 273, 210-218. 
77. Cheng, X.; Zhou, H.; Tang, R.S.; Munoz, M.G.; Jin, H. Chimeric subgroup A respiratory syncytial virus with the glycoproteins substituted by those of subgroup B and RSV without the M2-2 gene are attenuated in African green monkeys. Virology 2001, 283, 59-68.

78. Schepens, B.; de Baets, S.; Sedyen, K.; Bogaert, P.; Gilbert, B.; Piedra, P.A.; Fiers, W.; Saelens, X. She's a novel target for RSV vaccination. Presented at the 8th Respiratory Syncytial Virus Symposium, Santa Fe, NM, USA, 2012.

79. Swanson, K.A.; Settembre, E.C.; Shaw, C.A.; Dey, A.K.; Rappuoli, R.; Mandl, C.W.; Dormitzer, P.R.; Carfi, A. Structural basis for immunization with postfusion respiratory syncytial virus fusion F glycoprotein (RSV F) to elicit high neutralizing antibody titers. Proc. Natl. Acad. Sci. USA 2011, 108, 9619-9624.

80. Zhang, W.; Choi, Y.; Haynes, L.M.; Harcourt, J.L.; Anderson, L.J.; Jones, L.P.; Tripp, R.A. Vaccination to induce antibodies blocking the CX3C-CX3CR1 interaction of respiratory syncytial virus $\mathrm{G}$ protein reduces pulmonary inflammation and virus replication in mice. $J$. Virol. 2010, 84, 1148-1157.

81. Sealy, R.; Chaka, W.; Surman, S.; Brown, S.A.; Cresswell, P.; Hurwitz, J.L. Target peptide sequence within infectious human immunodeficiency virus type 1 does not ensure envelope-specific T-helper cell reactivation: Influences of cysteine protease and gamma interferon-induced thiol reductase activities. Clin. Vaccine Immunol. 2008, 15, 713-719.

82. Colman, P.M.; Laver, W.G.; Varghese, J.N.; Baker, A.T.; Tulloch, P.A.; Air, G.M.; Webster, R.G. Three-dimensional structure of a complex of antibody with influenza virus neuraminidase. Nature 1987, 326, 358-363.

83. Arnold, P.Y.; La Gruta, N.L.; Miller, T.; Vignali, K.M.; Adams, P.S.; Woodland, D.L.; Vignali, D.A. The majority of immunogenic epitopes generate CD4+ T cells that are dependent on MHC class II-bound peptide-flanking residues. J. Immunol. 2002, 169, 739-749.

84. Moudgil, K.D.; Sercarz, E.E.; Grewal, I.S. Modulation of the immunogenicity of antigenic determinants by their flanking residues. Immunol. Today 1998, 19, 217-220.

85. Correia, B.E.; Bates, J.T.; Loomis, R.; Connell, M.J.; Baneyx, G.; Jardine, J.G.; Rupert, P.; Correnti, C.; Vittal, V.; Kalyuzhniy, O.; et al. A Computationally Designed Experimental RSV F Protein Epitope Vaccine that Induces Potent Neutralizing Antibodies in Nonhuman Primates. Presented at the 8th Respiratory Syncytial Virus Symposium, Santa Fe, NM, USA, 2012.

86. Makidon, P.E.; Bitko, V.; Simon, J.K.; Lukacs, N.W.; Hamouda, T.; O’Konek, J.J.; Fattom, A.; Baker, J.R., Jr. Immunogenicity and Efficacy of Line 19 RSV Vaccine in Cotton Rats. Presented at the 8th Respiratory Syncytial Virus Symposium, Santa Fe, NM, 2012.

87. Grasso, S.; Santi, L. Viral nanoparticles as macromolecular devices for new therapeutic and pharmaceutical approaches. Int. J. Physiol. Pathophysiol. Pharmacol. 2010, 2, 161-178.

88. Garg, R.; Brownlie, R.; Latimer, L.; Connor, W.; Simko, E.; Gerdts, V.; Babiuk, L.A.; Potter, A.; van Drunen Littel-van den Hurk, S. Intranasal immunization with the RSV fusion protein formulated with novel combination adjuvants induces protective immunity. Presented at the 8th Respiratory Syncytial Virus Symposium, Santa Fe, NM, USA, 2012. 
89. Garlapati, S.; Garg, R.; Brownlie, R.; Latimer, L.; Simko, E.; Hancock, R.E.; Babiuk, L.A.; Gerdts, V.; Potter, A.; van Drunen Littel-van den Hurk, S. Enhanced immune responses and protection by vaccination with respiratory syncytial virus fusion protein formulated with $\mathrm{CpG}$ oligodeoxynucleotide and innate defense regulator peptide in polyphosphazene microparticles. Vaccine 2012, 30, 5206-5214.

90. Glenn, G.M.; Smith, G.; Raghunandan, R.; Li, H.; Zhou, B.; Thomas, D.N.; Fries, L. Immunogenicity of an Sf9 insect cell-derived respiratory syncytial virus fusion protein nanoparticle vaccine: Insights into pathogenicity. Presented at the 8th Respiratory Syncytial Virus Symposium, Santa Fe, NM, USA, 2012.

91. Smith, G.; Raghunandan, R.; Wu, Y.; Liu, Y.; Massare, M.; Nathan, M.; Zhou, B.; Lu, H.; Boddapati, S.; Li, J.; Respiratory syncytial virus fusion glycoprotein expressed in insect cells form protein nanoparticles that induce protective immunity in cotton rats. PLoS One 2012, 7, e50852.

92. Simoes, E.A.; Groothuis, J.R.; Carbonell-Estrany, X.; Rieger, C.H.; Mitchell, I.; Fredrick, L.M.; Kimpen, J.L. Palivizumab prophylaxis, respiratory syncytial virus, and subsequent recurrent wheezing. J. Pediatr. 2007, 151, 34-42.

93. Kamphuis, T.; Meijerhof, T.; Stegmann, T.; Lederhofer, J.; Wilschut, J.; De, H.A. Immunogenicity and protective capacity of a virosomal respiratory syncytial virus vaccine adjuvanted with monophosphoryl lipid A in mice. PLoS One 2012, 7, e36812.

94. Bernstein, D.I.; Malkin, E.; Abughali, N.; Falloon, J.; Yi, T.; Dubovsky, F. Phase 1 study of the safety and immunogenicity of a live, attenuated respiratory syncytial virus and parainfluenza virus type 3 vaccine in seronegative children. Pediatr. Infect. Dis. J. 2012, 31, 109-114.

95. Tang, R.S.; Spaete, R.R.; Thompson, M.W.; MacPhail, M.; Guzzetta, J.M.; Ryan, P.C.; Reisinger, K.; Chandler, P.; Hilty, M.; Walker, R.E.; et al. Development of a PIV-vectored RSV vaccine: Preclinical evaluation of safety, toxicity, and enhanced disease and initial clinical testing in healthy adults. Vaccine 2008, 26, 6373-6382.

96. Tang, R.S.; MacPhail, M.; Schickli, J.H.; Kaur, J.; Robinson, C.L.; Lawlor, H.A.; Guzzetta, J.M.; Spaete, R.R.; Haller, A.A. Parainfluenza virus type 3 expressing the native or soluble fusion (F) Protein of Respiratory Syncytial Virus (RSV) confers protection from RSV infection in African green monkeys. J. Virol. 2004, 78, 11198-11207.

97. Tang, R.S.; Malkin, E.; Stillman, E.; Nelson, C.; Yang, C.-F.; Song, E.; Liang, B.; Shambaugh, C.; Zuo, F.; Liem, A.; et al. Implication of genetic changes observed in Phase 1 evaluation of MEDI534, a live attenuated chimeric bovine human parainfluenza type 3 vectored RSV vaccine. Presented at the 8th Respiratory Syncytial Virus Symposium, Santa Fe, NM, USA 2012.

98. Power, U.F.; Ryan, K.W.; Portner, A. Sequence characterization and expression of the matrix protein gene of human parainfluenza virus type 1. Virology 1992, 191, 947-952.

99. Lyn, D.; Gill, D.S.; Scroggs, R.A.; Portner, A. The nucleoproteins of human parainfluenza virus type 1 and Sendai virus share amino acid sequences and antigenic and structural determinants. J. Gen. Virol. 1991, 72, 983-987. 
100. Sangster, M.; Smith, F.S.; Coleclough, C.; Hurwitz, J.L. Human parainfluenza virus-type 1 immunization of infant mice protects from subsequent Sendai virus infection. Virology 1995, 212, 13-19.

101. Dave, V.P.; Allan, J.E.; Slobod, K.S.; Smith, S.F.; Ryan, K.; Powell, U.; Portner, A.; Hurwitz, J.L. Viral cross-reactivity and antigenic determinants recognized by human parainfluenza virus type 1-specific cytotoxic T-cells. Virology 1994, 199, 376-383.

102. Smith, F.S.; Portner, A.; Leggiadro, R.J.; Turner, E.V.; Hurwitz, J.L. Age-related development of human memory T-helper and B-cell responses toward parainfluenza virus type-1. Virology 1994, 205, 453-461.

103. Bousse, T.; Chambers, R.L.; Scroggs, R.A.; Portner, A.; Takimoto, T. Human parainfluenza virus type 1 but not Sendai virus replicates in human respiratory cells despite IFN treatment. Virus Res. 2006, 121, 23-32.

104. Zhan, X.; Hurwitz, J.L.; Krishnamurthy, S.; Takimoto, T.; Boyd, K.; Scroggs, R.A.; Surman, S.; Portner, A.; Slobod, K.S. Respiratory syncytial virus (RSV) fusion protein expressed by recombinant Sendai virus elicits B-cell and T-cell responses in cotton rats and confers protection against RSV subtypes A and B. Vaccine 2007, 25, 8782-8793.

105. Slobod, K.S.; Shenep, J.L.; Lujan-Zilbermann, J.; Allison, K.; Brown, B.; Scroggs, R.A.; Portner, A.; Coleclough, C.; Hurwitz, J.L. Safety and immunogenicity of intranasal murine parainfluenza virus type 1 (Sendai virus) in healthy human adults. Vaccine 2004, 22, 3182-3186.

106. Chen, M.; Hu, K.F.; Rozell, B.; Orvell, C.; Morein, B.; Liljestrom, P. Vaccination with recombinant alphavirus or immune-stimulating complex antigen against respiratory syncytial virus. J. Immunol. 2002, 169, 3208-3216.

107. Mok, H.; Lee, S.; Utley, T.J.; Shepherd, B.E.; Polosukhin, V.V.; Collier, M.L.; Davis, N.L.; Johnston, R.E.; Crowe, J.E., Jr. Venezuelan equine encephalitis virus replicon particles encoding respiratory syncytial virus surface glycoproteins induce protective mucosal responses in mice and cotton rats. J. Virol. 2007, 81, 13710-13722.

108. Elliott, M.B.; Chen, T.; Terio, N.B.; Chong, S.Y.; Abdullah, R.; Luckay, A.; Egan, M.A.; Boutilier, L.A.; Melville, K.; Lerch, R.A.; et al. Alphavirus replicon particles encoding the fusion or attachment glycoproteins of respiratory syncytial virus elicit protective immune responses in BALB/c mice and functional serum antibodies in rhesus macaques. Vaccine 2007, $25,7132-7144$.

109. Kohlmann, R.; Schwannecke, S.; Tippler, B.; Ternette, N.; Temchura, V.V.; Tenbusch, M.; Uberla, K.; Grunwald, T. Protective efficacy and immunogenicity of an adenoviral vector vaccine encoding the codon-optimized F protein of respiratory syncytial virus. J. Virol. 2009, 83, 12601-12610.

110. Johnson, T.R.; Rangel, D.; Liao, G.; Eastman, E.M.; Gall, J.G. Induction of Broadly Neutralizing Humoral Responses by Immunization with Respiratory Syncytial Virus F Expressing Adenovirus Vectors. Presented at the 8th Respiratory Syncytial Virus Symposium, Santa Fe, NM, USA, 2012. 
111. De Baets, S.; Schepens, B.; Sedeyn, K.; Schotsaert, M.; Bogaert, P.; Fiers, W.; Saelens, X. Recombinant influenza virus carrying an RSV CTL epitope protects mice against respiratory syncytial virus infection. Presented at the 8th Respiratory Syncytial Virus Symposium, Santa Fe, NM, USA, 2012.

112. Sawada, A.; Komase, K.; Nakayama, T. AIK-C measles vaccine expressing fusion protein of respiratory syncytial virus induces protective antibodies in cotton rats. Vaccine 2011, 29, 1481-1490.

113. McGinnes, L.W.; Gravel, K.A.; Finberg, R.W.; Kurt-Jones, E.A.; Massare, M.J.; Smith, G.; Schmidt, M.R.; Morrison, T.G. Assembly and immunological properties of Newcastle disease virus-like particles containing the respiratory syncytial virus $\mathrm{F}$ and $\mathrm{G}$ proteins. J. Virol. 2011, 85, 366-377.

114. Andersson, C.; Liljestrom, P.; Stahl, S.; Power, U.F. Protection against respiratory syncytial virus (RSV) elicited in mice by plasmid DNA immunisation encoding a secreted RSV G protein-derived antigen. FEMS Immunol. Med. Microbiol. 2000, 29, 247-253.

115. Hurwitz, J.L. Respiratory syncytial virus vaccine development. Expert. Rev. Vaccines 2011, 10, 1415-1433.

116. Lau, J.M.; Korban, S.S. Transgenic apple expressing an antigenic protein of the human respiratory syncytial virus. J. Plant Physiol. 2010, 167, 920-927.

117. Haijema, B.J.; Leenhouts, K.; Widjaja, I.; Rigter, A.; de Haan, X.; Rottier, P. Intranasal administration of bacterium-like particles carrying RSV F antigen (SynGEM) induces a safe and protective RSV-specific immune response. Presented at the 8th Respiratory Syncytial Virus Symposium, Santa Fe, NM, USA, 2012.

118. Taylor, G.; Thom, M.; Herbert, B.; Pierantoni, A.; Capone, S.; Nicosia, A.; Vitelli, A. Novel replication-defective chimpanzee adenovirus (ChAD) and modified vaccinia Ankara (MVA) vectors expressing human $(\mathrm{H}) \mathrm{RSV}$ antigens protect calves against BRSV infection. Presented at the 8th Respiratory Syncytial Virus Symposium, Santa Fe, NM, USA, 2012.

119. Grunwald, T.; Tenbusch, M.; Schulte, R.; Franz, M.; Hannaman, D.; Tippler, B.; de Swart, R.L.; Steinman, R.; Stahl-Hennig, C.; Uberla, K. Immunogenicity and efficacy of genetic RSV vaccines in rhesus monkeys. Presented at the 8th Respiratory Syncytial Virus Symposium, Santa Fe, NM, USA, 2012.

120. Shafique, M.; Wilschut, J.; De, H.A. Induction of mucosal and systemic immunity against respiratory syncytial virus by inactivated virus supplemented with TLR9 and NOD2 ligands. Vaccine 2012, 30, 597-606.

121. Stegmann, T.; Kamphuis, T.; Meijerhof, T.; Goud, E.; De, H.A.; Wilschut, J. Lipopeptide-adjuvanted respiratory syncytial virus virosomes: A safe and immunogenic non-replicating vaccine formulation. Vaccine 2010, 28, 5543-5550. 
122. Murawski, M.R.; McGinnes, L.W.; Finberg, R.W.; Kurt-Jones, E.A.; Massare, M.J.; Smith, G.; Heaton, P.M.; Fraire, A.E.; Morrison, T.G. Newcastle disease virus-like particles containing respiratory syncytial virus $\mathrm{G}$ protein induced protection in BALB/c mice, with no evidence of immunopathology. J. Virol. 2010, 84, 1110-1123.

123. Cautivo, K.M.; Bueno, S.M.; Cortes, C.M.; Wozniak, A.; Riedel, C.A.; Kalergis, A.M. Efficient lung recruitment of respiratory syncytial virus-specific Th1 cells induced by recombinant bacillus Calmette-Guerin promotes virus clearance and protects from infection. J. Immunol. 2010, 185, 7633-7645.

124. Xie, C.; He, J.S.; Zhang, M.; Xue, S.L.; Wu, Q.; Ding, X.D.; Song, W.; Yuan, Y.; Li, D.L.; Zheng, X.X.; et al. Oral respiratory syncytial virus (RSV) DNA vaccine expressing RSV F protein delivered by attenuated Salmonella typhimurium. Hum. Gene Ther. 2007, 18, 746-752.

125. Falcone, V.; Mihm, D.; Neumann-Haefelin, D.; Costa, C.; Nguyen, T.; Pozzi, G.; Ricci, S. Systemic and mucosal immunity to respiratory syncytial virus induced by recombinant Streptococcus gordonii surface-displaying a domain of viral glycoprotein G. FEMS Immunol. Med. Microbiol. 2006, 48, 116-122.

126. Schickli, J.H.; Dubovsky, F.; Tang, R.S. Challenges in developing a pediatric RSV vaccine. Hum. Vaccin. 2009, 5, 582-591. 
MDPI AG

Klybeckstrasse 64

4057 Basel, Switzerland

Tel. +41616837734

Fax +41613028918

http://www.mdpi.com/

Viruses Editorial Office

E-mail: viruses@mdpi.com http://www.mdpi.com/journal/viruses 


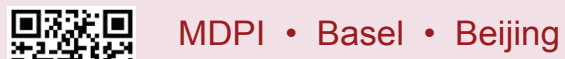
7 IS ISBN 978-3-03842-049-1 教 www.mdpi.com 